

TRABAJO FIN DE MÁSTER EN INGENIERÍA DE ESTRUCTURAS

UgV Universidad

\section{REACONDICIONAMIENTO SÍSMICO DE ESTRUCTURAS PORTICADAS DE HORMIGÓN ARMADO CON DISIPADORES DE ENERGÍA DE TIPO HISTERÉTICO}

Alumna

Carolina Cabrera González

Arquitecta e Ingeniera Técnica Industrial

Tutor

D. Amadeo Benavent Climent

Doctor Arquitecto, Catedrático del Departamento de Mecánica de Estructuras e Ingeniería Hidráulica de la Universidad de Granada

Granada - diciembre de 2011 


\section{AGRADECIMIENTOS}

No quisiera presentar esta memoria sin expresar mi sincero reconocimiento a todas aquellas personas que han contribuido con su respaldo, interés, apoyo y dedicación, a la realización de este trabajo de investigación.

Al Dr. D. Amadeo Benavent Climent, Catedrático del Departamento de Mecánica de Estructuras e Ingeniería Hidráulica de la Universidad de Granada. Aportando, sus consejos y su trabajo para la realización de este estudio de investigación.

Ha sido para mí un orgullo contar en este trabajo con uno de los mejores especialistas en esta disciplina.

He de agradecer la valiosa colaboración del Dr. Rafael Bravo Pareja, profesor del Departamento de Mecánica de Estructuras e Ingeniería Hidráulica de la Universidad de Granada, por su disposición desinteresada, su ayuda inestimable y apoyo. En el que siempre he encontrado un consejo valioso con la generosidad que le caracteriza.

A los profesores del Máster en Ingeniería de Estructuras a quienes agradezco los conocimientos adquiridos, el trato dispensado y su ayuda en la resolución de dudas.

A la Universidad de Granada, como institución, por hacer posible este trabajo.

A mis compañeros de este Máster y amigos por su apoyo y ánimo.

Pero este trabajo se debe, por encima de todo, a mi familia, y a ellos va dedicado, pues mis padres, José Antonio y Concha, que son mi modelo, por sus conocimientos, su apoyo incondicional y claridad de ideas han sabido siempre, sacar lo mejor de mí, mi hijo Manuel, mi motor y estímulo y del que estoy tan orgullosa y por último, por ser la persona más importante en mi vida, el que de verdad sabe cuánto trabajo y esfuerzo hay en estas páginas, esta Tesina está dedicada a Paco, mi marido. 



\section{ÍNDICE GENERAL}

Capítulo 1. Introducción. Motivación. Objetivos. Metodología. $\quad 1$

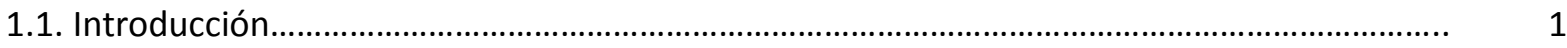

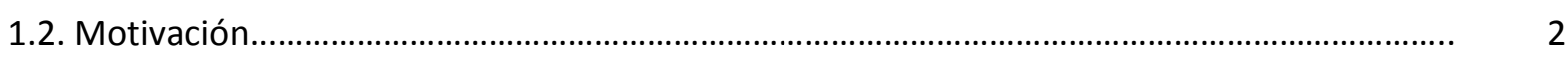

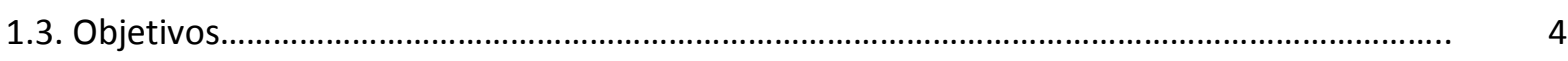

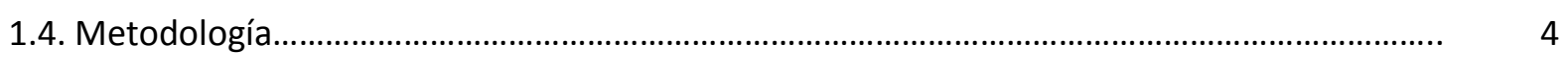

$\begin{array}{ll}\text { Capítulo 2. Estado del arte. } & 7\end{array}$

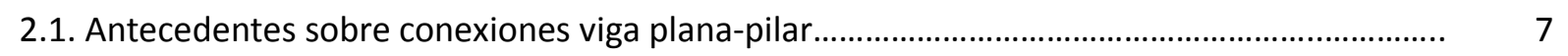

2.2. Proyecto sismorresistente basado en la teoría de la energía de Housner-Akiyama................ 8

2.3. Reacondicionamiento sísmico de estructuras con disipadores de energía.............................. 12

2.3.1. Definición de disipador de energía................................................................................ 12

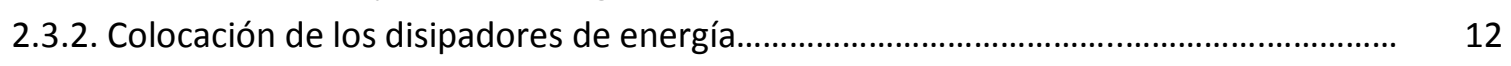

2.3.3. Utilidad de los disipadores de energía en edificios........................................................ 13

2.3.4. Cualidades exigibles a los disipadores de energía................................................................ 15

2.3.5. Tipos de elementos disipadores de energía.................................................................... 15

Capítulo 3. Determinación de prototipos de estructuras existentes de hormigón armado construidos en los años 70-90 en España. 17

3.1. Definición de los prototipos de pórticos con vigas planas......................................................... 17

3.2. Parámetros de proyecto y normativa empleada...................................................................... 21

Capítulo 4. Selección de acelerogramas. 23

4.0. Introducción ........................................................................................................................... 23

4.1. Aceleración sísmica de cálculo (NCSE-02)............................................................................. 23

4.1.1. Aceleración sísmica de cálculo para un edificio ubicado en Granada y suelo tipo I...... 24

4.1.2. Aceleración sísmica de cálculo para un edificio ubicado en Granada y suelo tipo II..... 25

4.1.3. Aceleración sísmica de cálculo para un edificio ubicado en Granada y suelo tipo III.... 25

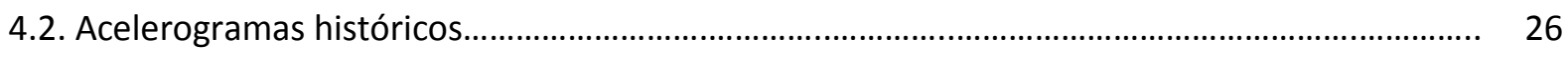

4.2.1. Características de los acelerogramas históricos inicialmente seleccionados.................. 27

4.2.2. Características espectrales de los acelerogramas escalados correspondientes a los periodos fundamentales de las estructuras analizadas...................................................... 28

4.2.3. Tabla resumen de los periodos fundamentales de vibración............................................. 29

4.3. Escalado, espectro elástico de respuesta y espectro de input de energía de los acelerogramas 30

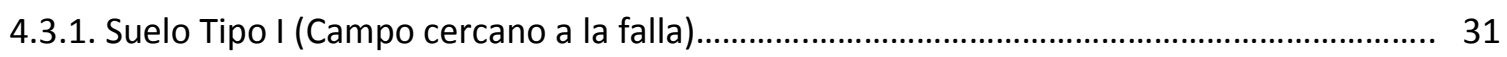

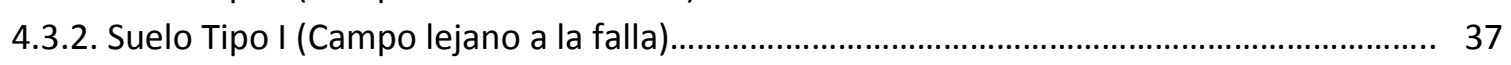

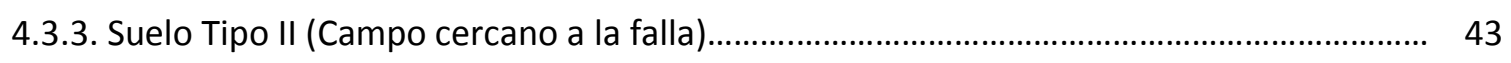

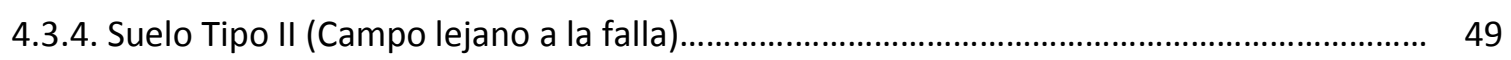

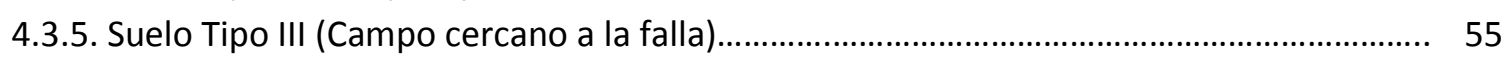

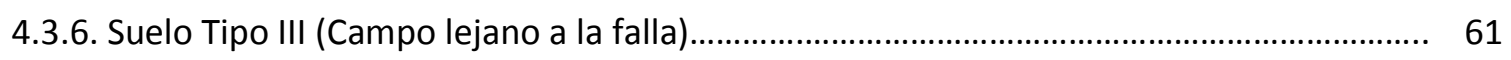




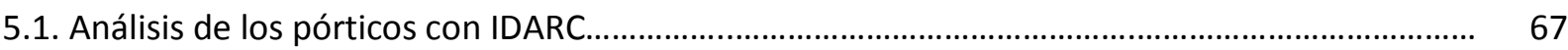

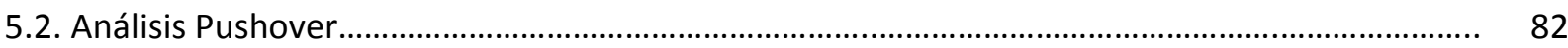

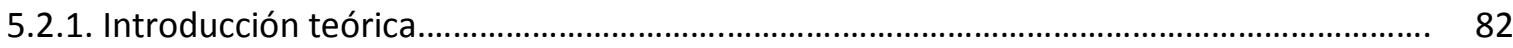

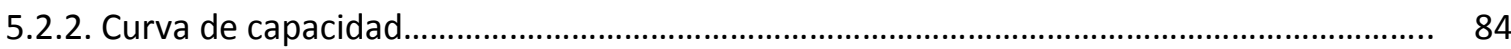

5.2.3. Relación entre fuerza cortante de la planta y desplazamiento entre forjados que delimitan dicha planta.................................................................................................... 86

5.2.4. Rigidez inicial y desplazamiento de fluencia de cada planta............................................ 92

5.2.4.1. Tabla resumen Rigidez inicia-Desplazamiento de fluencia para cada pórtico..... 105

5.2.5. Periodos de vibración obtenidos con el programa IDARC y con el programa LumpST..... 105

5.2.5.1. Periodos de vibración sin disipadores obtenidos con el programa IDARC.......... 106

5.2.5.2. Periodos de vibración sin disipadores obtenidos con el programa LumpST....... 106

5.2.5.3. Tabla resumen periodos de vibración sin disipadores de cada pórtico............... 112

5.2.5.4. Periodos de vibración con disipadores obtenidos con el programa LumpST...... 113

5.2.5.5. Tabla resumen frecuencia y periodos de vibración con disipadores de cada

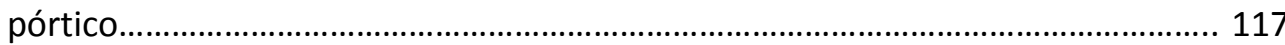

5.2.5.6. Tabla resumen de los periodos fundamentales de vibración................................ 118

5.3. Automatización del programa LumpST con Matlab..................................................................... 119

5.3.1. Secuencia de comandos en el problema directo para un pórtico de 3 plantas.................. 120

5.3.2. Secuencia de comandos en el problema inverso para un pórtico de 3 plantas.................. 122

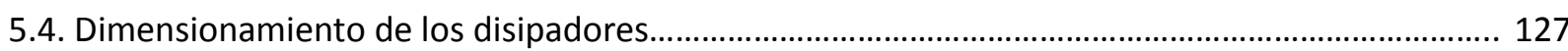

5.4.1. Criterio para establecer la rigidez lateral de los disipadores........................................... 127

5.4.2. Criterio para fijar la resistencia lateral de los disipadores................................................ 127

5.4.3. Obtención de los desplazamientos de fluencia de los disipadores y los desplazamientos máximos absolutos entre plantas.............................................................................. 128

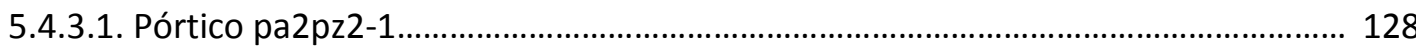

5.4.3.1.1. Suelo Tipo I (Campo lejano a la falla)................................................. 130

5.4.3.1.2. Suelo Tipo II (Campo cercano a la falla)............................................... 131

5.4.3.1.3. Suelo Tipo II (Campo lejano a la falla)................................................. 132

5.4.3.1.4. Suelo Tipo III (Campo cercano a la falla)............................................... 133

5.4.3.1.5. Suelo Tipo III (Campo lejano a la falla).................................................. 134

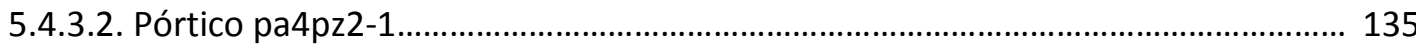

5.4.3.2.1. Suelo Tipo I (Campo lejano a la falla)...................................................... 137

5.4.3.2.2. Suelo Tipo II (Campo cercano a la falla)................................................. 138

5.4.3.2.3. Suelo Tipo II (Campo lejano a la falla).................................................. 139

5.4.3.2.4. Suelo Tipo III (Campo cercano a la falla)............................................... 140

5.4.3.2.5. Suelo Tipo III (Campo lejano a la falla)............................................... 140

5.4.3.3. Pórtico pa6pz2-1............................................................................................... 141

5.4.3.3.1. Suelo Tipo I (Campo lejano a la falla)...................................................... 144

5.4.3.3.2. Suelo Tipo II (Campo cercano a la falla)................................................. 145

5.4.3.3.3. Suelo Tipo III (Campo cercano a la falla).............................................. 145

5.4.3.3.4. Suelo Tipo III (Campo lejano a la falla)................................................ 146

5.5. Resistencia lateral y coeficientes de fuerza cortante.............................................................. 150

5.5.1. Resistencia lateral de los disipadores de una planta $i$ dada.............................................. 150

5.5.2. Coeficiente de fuerza cortante de fluencia de los disipadores de una planta $i$ dada ....... 150 
5.5.3. Resistencia lateral aportada por la parte flexible (pórtico) cuando los disipadores empiezan a plastificar

5.5.4. Coeficiente de fuerza cortante en la parte flexible (pórtico) cuando los disipadores empiezan a plastificar

5.5.5. Coeficiente de cortante total de la planta.

5.5.6. Obtención de los definidos en los apartados 5.5.1 a 5.5.5 para cada uno de los pórticos

5.5.6.1. Pórtico pa2pz2-1 151

5.5.6.1.1. Suelo Tipo I (Campo lejano a la falla). 152

5.5.6.1.2. Suelo Tipo II (Campo cercano a la falla).................................................. 158

5.5.6.1.3. Suelo Tipo II (Campo lejano a la falla)..................................................... 163

5.5.6.1.4. Suelo Tipo III (Campo cercano a la falla).................................................. 168

5.5.6.1.5. Suelo Tipo III (Campo lejano a la falla)................................................ 175

5.5.6.2. Pórtico pa4pz2-1................................................................................................. 180

5.5.6.2.1. Suelo Tipo I (Campo lejano a la falla)................................................. 180

5.5.6.2.2. Suelo Tipo II (Campo cercano a la falla)................................................ 187

5.5.6.2.3. Suelo Tipo II (Campo lejano a la falla)....................................................... 192

5.5.6.2.4. Suelo Tipo III (Campo cercano a la falla)................................................. 197

5.5.6.2.5. Suelo Tipo III (Campo lejano a la falla)..................................................... 203

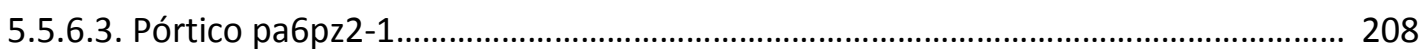

5.5.6.3.1. Suelo Tipo I (Campo lejano a la falla).................................................. 209

5.5.6.3.2. Suelo Tipo II (Campo cercano a la falla)............................................... 216

5.5.6.3.3. Suelo Tipo III (Campo cercano a la falla)................................................. 221

5.5.6.3.4. Suelo Tipo III (Campo lejano a la falla).................................................... 231

5.6. Comparación de las distribuciones de resistencia obtenidas con fórmulas propuestas en la literatura. 239

5.6.1. Distribución óptima del coeficiente de fuerza cortante de fluencia definida por Akiyama para una estructura de tres plantas $\alpha_{i} / \alpha_{1}$ 239

5.6.2. Distribución de resistencia entre plantas para el pórtico pa2pz2-1, suelo tipo I, campo lejano $\alpha_{i} / \alpha_{1}$

5.6.3. Distribución de resistencia entre plantas para el pórtico pa2pz2-1, suelo tipo II, campo cercano $\alpha_{i} / \alpha_{1}$ 241

5.6.4. Distribución de resistencia entre plantas para el pórtico pa2pz2-1, suelo tipo II, campo lejano $\alpha_{i} / \alpha_{1}$

5.6.5. Distribución de resistencia entre plantas para el pórtico pa2pz2-1, suelo tipo III, campo cercano $\alpha_{i} / \alpha_{1}$

5.6.6. Distribución de resistencia entre plantas para el pórtico pa2pz2-1, suelo tipo III, campo lejano $\alpha_{i} / \alpha_{1}$

5.6.7. Distribución de resistencia entre plantas para el pórtico pa4pz2-1, suelo tipo I, campo lejano $\alpha_{i} / \alpha_{1}$

5.6.8. Distribución de resistencia entre plantas para el pórtico pa4pz2-1, suelo tipo II, campo cercano $\alpha_{i} / \alpha_{1}$

5.6.9. Distribución de resistencia entre plantas para el pórtico pa4pz2-1, suelo tipo II, campo lejano $\alpha_{i} / \alpha_{1}$ 
5.6.10. Distribución de resistencia entre plantas para el pórtico pa4pz2-1, suelo tipo III, campo cercano $\alpha_{i} / \alpha_{1}$

5.6.11. Distribución de resistencia entre plantas para el pórtico pa4pz2-1, suelo tipo III, campo lejano $\alpha_{i} / \alpha_{1}$ 250

5.6.12. Distribución óptima del coeficiente de fuerza cortante de fluencia definida por Akiyama para una estructura de seis plantas $\alpha_{i} / \alpha_{1}$

5.6.13. Distribución de resistencia entre plantas para el pórtico pa6pz2-1, suelo tipo I, campo lejano $\alpha_{i} / \alpha_{1}$

5.6.14. Distribución de resistencia entre plantas para el pórtico pa6pz2-1, suelo tipo II, campo cercano $\alpha_{i} / \alpha_{1}$

5.6.15. Distribución de resistencia entre plantas para el pórtico pa6pz2-1, suelo tipo III, campo cercano $\alpha_{i} / \alpha_{1}$

5.6.16. Distribución de resistencia entre plantas para el pórtico pa6pz2-1, suelo tipo III, campo lejano $\alpha_{i} / \alpha_{1}$ .256

5.6.17. Valoración general. 257

Capítulo 6. Conclusiones

6.1. Resumen del trabajo realizado. .261

6.2. Conclusiones. 262

6.3. Futuras líneas de investigación. 


\section{ÍNDICE DE FIGURAS}

1.2.1. Detalle de conexiones viga plana-pilar....................................................................................... 2

1.2.2. Mecanismo de transmisión de momentos flectores de viga plana a pilar.................................... 3

2.2.1. Sistema real sometido a un movimiento en su base...................................................................... 8

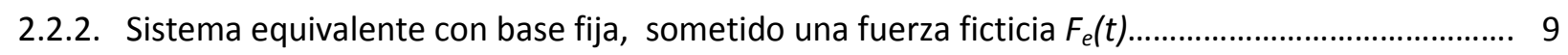

2.3.1. Colocación de disipadores de energía en estructuras porticadas................................................... 12

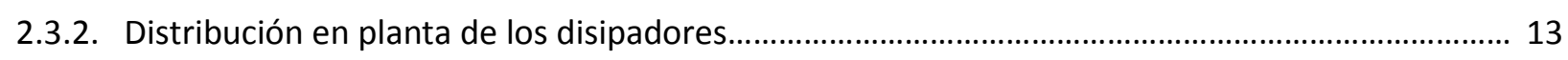

2.3.3. Ejemplos de organizaciones estructurales.................................................................................. 13

2.3.4. Espectros elásticos de respuesta. Comparación para edificios con periodos de vibración altos (flexibles)...

2.3.5. Espectros elásticos de respuesta. Comparación para edificios de periodos de vibración bajos

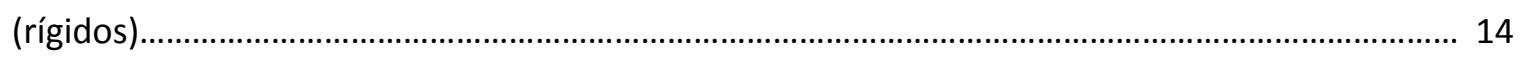

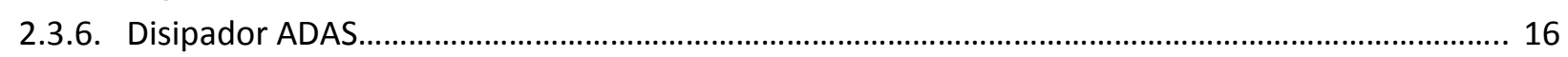

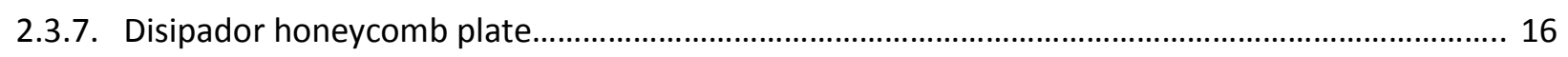

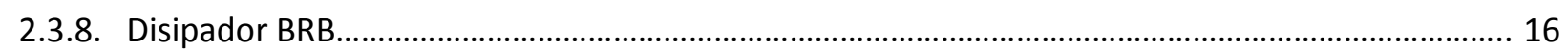

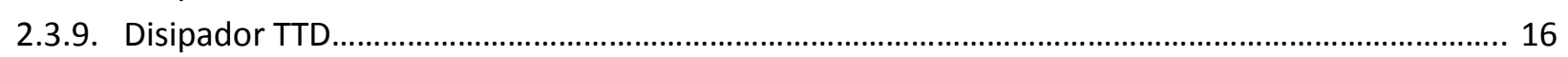

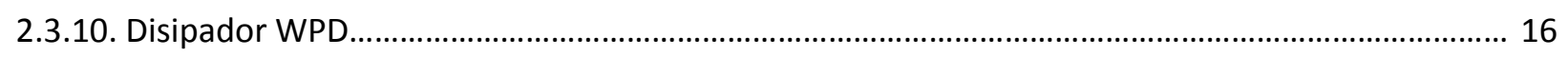

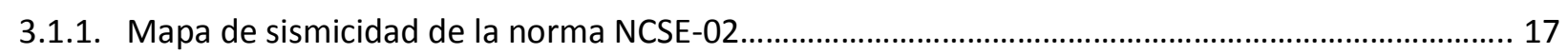

3.1.2. Edificio de 3 plantas, Zaidin, Plaza de las Palomas (Granada)....................................................... 18

3.1.3. Edificio de 6 plantas, Camino de Ronda (Granada)...................................................................... 18

3.1.4. Edificio de 6 plantas, Rivera del Violón (Granada) ........................................................................... 18

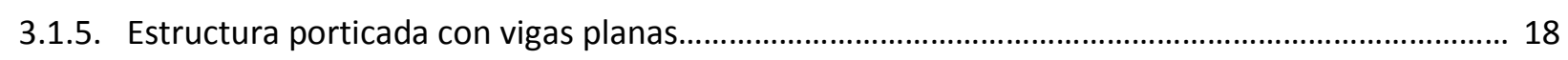

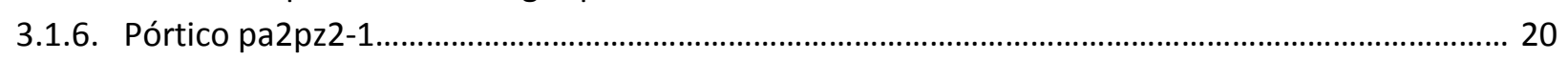

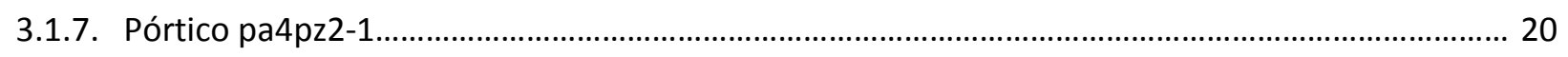

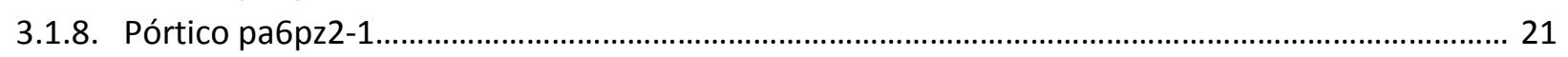

4.3.1. Acelerograma $\mathrm{N}^{\circ} 2$ (Ardal), suelo tipo I, campo cercano, sin escalar.............................................. 31

4.3.2. Acelerograma №2 (Ardal), suelo tipo I, campo cercano, escalado a $P G A=200 \mathrm{~cm} / \mathrm{s}^{2} \ldots \ldots \ldots \ldots \ldots \ldots . . . . . . .31$

4.3.3. Espectro elástico de respuesta del acelerograma №2 (Ardal) escalado a $P G A=200 \mathrm{~cm} / \mathrm{s}^{2}$.......... 32

4.3.4. Espectro de energía introducida del acelerograma №2 (Ardal) escalado a $P G A=200 \mathrm{~cm} / \mathrm{s}^{2} \ldots \ldots . .32$

4.3.5. Acelerograma $\mathrm{N}^{\circ} 6$ (Timfristos), suelo tipo I, campo cercano, sin escalar.................................... 33

4.3.6. Acelerograma $\mathrm{N}^{\circ} 6$ (Timfristos), suelo tipo I, campo cercano, escalado a $\mathrm{PGA}=200 \mathrm{~cm} / \mathrm{s}^{2} \ldots \ldots \ldots \ldots . . . . .33$

4.3.7. Espectro elástico de respuesta del acelerograma $N^{\circ} 6$ (Timfristos) escalado a $P G A=200 \mathrm{~cm} / \mathrm{s}^{2} \ldots .34$

4.3.8. Espectro de energía introducida del acelerograma $N^{\circ} 6$ (Timfristos) escalado a $P G A=200 \mathrm{~cm} / \mathrm{s}^{2} .34$

4.3.9. Acelerograma $\mathrm{N}^{0} 7$ (Timfristos), suelo tipo I, campo cercano, sin escalar..................................... 35

4.3.10. Acelerograma $\mathrm{N}^{0} 7$ (Timfristos), suelo tipo I, campo cercano, escalado a $\mathrm{PGA}=200 \mathrm{~cm} / \mathrm{s}^{2} \ldots \ldots \ldots \ldots . . . .35$

4.3.11. Espectro elástico de respuesta del acelerograma $N^{\circ} 7$ (Timfristos) escalado a $P G A=200 \mathrm{~cm} / \mathrm{s}^{2} \ldots . .36$

4.3.12. Espectro de energía introducida del acelerograma $N^{0} 7$ (Timfristos) escalado a $P G A=200 \mathrm{~cm} / \mathrm{s}^{2} . .36$

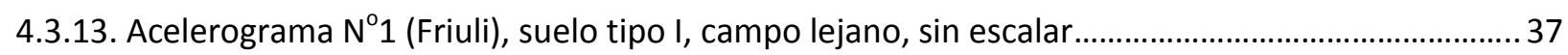

4.3.14. Acelerograma $N^{\circ} 1$ (Friuli), suelo tipo I, campo lejano, escalado a $P G A=200 \mathrm{~cm} / \mathrm{s}^{2} \ldots \ldots \ldots \ldots \ldots \ldots \ldots . . . . . . . . . . .37$

4.3.15. Espectro elástico de respuesta del acelerograma $N^{\circ} 1$ (Friuli) escalado a $P G A=200 \mathrm{~cm} / \mathrm{s}^{2} \ldots \ldots \ldots \ldots . . . .38$

4.3.16. Espectro de energía introducida del acelerograma $N^{\circ} 1$ (Friuli) escalado a $P G A=200 \mathrm{~cm} / \mathrm{s}^{2} \ldots \ldots \ldots . . .38$ 
4.3.17. Acelerograma $\mathrm{N}^{\circ} 4$ (Montenegro), suelo tipo I, campo lejano, sin escalar. .39

4.3.18. Acelerograma $\mathrm{N}^{\circ} 4$ (Montenegro), suelo tipo I, campo lejano, escalado a $\mathrm{PGA}=200 \mathrm{~cm} / \mathrm{s}^{2} \ldots \ldots \ldots . . . .39$

4.3.19. Espectro elástico de respuesta del acelerograma $N^{\circ} 4$ (Montenegro) escalado a $P G A=200 \mathrm{~cm} / \mathrm{s}^{2} 40$

4.3.20. Espectro de energía introducida del acelerograma $\mathrm{N}^{\circ} 4$ (Montenegro) escalado a $P G A=200 \mathrm{~cm} / \mathrm{s}^{2} \ldots . .40$

4.3.21. Acelerograma $\mathrm{N}^{\circ} 5$ (Campano Lucano), suelo tipo I, campo lejano, sin escalar............................. 41

4.3.22. Acelerograma $\mathrm{N}^{\circ} 5$ (Campano alucano), suelo tipo I, campo lejano, escalado a $P G A=200 \mathrm{~cm} / \mathrm{s}^{2} \ldots 41$

4.3.23. Espectro elástico de respuesta del acelerograma $N^{\circ} 5$ (Campano Lucano) escalado a $P G A=200 \mathrm{~cm} / \mathrm{s}^{2} 42$

4.3.24. Espectro de energía introducida del acelerograma $N^{\circ} 5$ (Campano Lucano) escalado a $P G A=200 \mathrm{~cm} / \mathrm{s}^{2} \ldots .42$

4.3.25. Acelerograma $\mathrm{N}^{0} 1$ (Friuli aftershock), suelo tipo II, campo cercano, sin escalar............................ 43

4.3.26. Acelerograma $N^{\circ} 1$ (Friuli aftershock), suelo tipo II, campo cercano, escalado a $P G A=230,5 \mathrm{~cm} / \mathrm{s}^{2} \ldots . . .43$

4.3.27. Espectro elástico de respuesta del acelerograma $N^{\circ} 1$ (Friuli aftershock) escalado a $P G A=230,5 \mathrm{~cm} / \mathrm{s}^{2} \ldots . . .44$

4.3.28. Espectro de energía introducida del acelerograma $N^{\circ} 1$ (Friuli aftershock) escalado a $P G A=230,5 \mathrm{~cm} / \mathrm{s}^{2} \ldots .44$

4.3.29. Acelerograma $\mathrm{N}^{\circ} 8$ (Friuli aftershock), suelo tipo II, campo cercano, sin escalar........................... 45

4.3.30. Acelerograma $\mathrm{N}^{\circ} 8$ (Friuli aftershock), suelo tipo II, campo cercano, escalado a $P G A=230,5 \mathrm{~cm} / \mathrm{s}^{2} \ldots . . .45$

4.3.31. Espectro elástico de respuesta del acelerograma $N^{\circ} 8$ (Friuli aftershock) escalado a $P G A=230,5 \mathrm{~cm} / \mathrm{s}^{2} \ldots . . .46$

4.3.32. Espectro de energía introducida del acelerograma $N^{\circ} 8$ (Friuli aftershock) escalado a $P G A=230,5 \mathrm{~cm} / \mathrm{s}^{2} \ldots .46$

4.3.33. Acelerograma $\mathrm{N}^{\circ} 14$ (Montenegro aftershock), suelo tipo II, campo cercano, sin escalar..............47

4.3.34. Acelerograma $\mathrm{N}^{0} 14$ (Montenegro aftershock), suelo tipo II, campo cercano, escalado a $\mathrm{PGA}=230,5 \mathrm{~cm} / \mathrm{s}^{2} \ldots \ldots . .47$

4.3.35. Espectro elástico de respuesta del acelerograma $N^{\circ} 14$ (Montenegro aftershock) escalado a $P G A=230,5 \mathrm{~cm} / \mathrm{s}^{2} .48$

4.3.36. Espectro de energía introducida del acelerograma $\mathrm{N}^{\circ} 14$ (Montenegro aftershock) escalado a $P G A=230,5 \mathrm{~cm} / \mathrm{s}^{2} \ldots \ldots .48$

4.3.37. Acelerograma $\mathrm{N}^{0} 2$ (Friuli aftershock), suelo tipo II, campo lejano, sin escalar.............................. 49

4.3.38. Acelerograma $\mathrm{N}^{\circ} 2$ (Friuli aftershock), suelo tipo II, campo lejano, escalado a $P G A=230,5 \mathrm{~cm} / \mathrm{s}^{2} \ldots \ldots \ldots .49$

4.3.39. Espectro elástico de respuesta del acelerograma $N^{\circ} 2$ (Friuli aftershock) escalado a $P G A=230,5 \mathrm{~cm} / \mathrm{s}^{2} \ldots . .50$

4.3.40. Espectro de energía introducida del acelerograma $N^{\circ} 2$ (Friuli aftershock) escalado a $P G A=230,5 \mathrm{~cm} / \mathrm{s}^{2} \ldots . .50$

4.3.41. Acelerograma $\mathrm{N}^{\circ} 4$ (Friuli aftershock), suelo tipo II, campo lejano, sin escalar............................... 51

4.3.42. Acelerograma $\mathrm{N}^{\circ} 4$ (Friuli aftershock), suelo tipo II, campo lejano, escalado a $\mathrm{PGA}=230,5 \mathrm{~cm} / \mathrm{s}^{2} \ldots \ldots \ldots . .51$

4.3.43. Espectro elástico de respuesta del acelerograma $N^{\circ} 4$ (Friuli aftershock) escalado a $P G A=230,5 \mathrm{~cm} / \mathrm{s}^{2} \ldots . .52$

4.3.44. Espectro de energía introducida del acelerograma $N^{\circ} 4$ (Friuli aftershock) escalado a $P G A=230,5 \mathrm{~cm} / \mathrm{s}^{2} \ldots .52$

4.3.45. Acelerograma $\mathrm{N}^{0} 12$ (Montenegro aftershock), suelo tipo II, campo lejano, sin escalar.................53

4.3.46. Acelerograma $\mathrm{N}^{\circ} 12$ (Montenegro aftershock), suelo tipo II, campo lejano, escalado a $P G A=230,5 \mathrm{~cm} / \mathrm{s}^{2} \ldots \ldots \ldots . . . .53$

4.3.47. Espectro elástico de respuesta del acelerograma $N^{\circ} 12$ (Montenegro aftershock) escalado a $P G A=230,5 \mathrm{~cm} / \mathrm{s}^{2} .54$

4.3.48. Espectro de energía introducida del acelerograma $N^{\circ} 12$ (Montenegro aftershock) escalado a $P G A=230,5 \mathrm{~cm} / \mathrm{s}^{2} \ldots \ldots . .54$

4.3.49. Acelerograma $\mathrm{N}^{\circ} 2$ (Friuli aftershock), suelo tipo III, campo cercano, sin escalar........................... 55

4.3.50. Acelerograma $\mathrm{N}^{\circ} 2$ (Friuli aftershock), suelo tipo III, campo cercano, escalado a $P G A=261,4 \mathrm{~cm} / \mathrm{s}^{2} \ldots . .55$

4.3.51. Espectro elástico de respuesta del acelerograma $N^{\circ} 2$ (Friuli aftershock) escalado a $P G A=261,4 \mathrm{~cm} / \mathrm{s}^{2} \ldots . . .56$

4.3.52. Espectro de energía introducida del acelerograma $N^{\circ} 2$ (Friuli aftershock) escalado a $P G A=261,4 \mathrm{~cm} / \mathrm{s}^{2} \ldots .56$

4.3.53. Acelerograma $\mathrm{N}^{\circ} 6$ (Kalamata aftershock), suelo tipo III, campo cercano, sin escalar.....................57

4.3.54. Acelerograma $N^{\circ} 6$ (Kalamata aftershock), suelo tipo III, campo cercano, escalado a $P G A=261,4 \mathrm{~cm} / \mathrm{s}^{2} \ldots . .57$

4.3.55. Espectro elástico de respuesta del acelerograma $N^{\circ} 6$ (Kalamata aftershock) escalado a $P G A=261,4 \mathrm{~cm} / \mathrm{s}^{2} \ldots \ldots . .58$

4.3.56. Espectro de energía introducida del acelerograma $N^{\circ} 6$ (Kalamata aftershock) escalado a $P G A=261,4 \mathrm{~cm} / \mathrm{s}^{2} \ldots . . .58$

4.3.57. Acelerograma $\mathrm{N}^{0} 12$ (Duzce 1), suelo tipo III, campo cercano, sin escalar...................................... 59

4.3.58. Acelerograma $\mathrm{N}^{\circ} 12$ (Duzce 1), suelo tipo III, campo cercano, escalado a $\mathrm{PGA}=261,4 \mathrm{~cm} / \mathrm{s}^{2} \ldots \ldots \ldots . .59$

4.3.59. Espectro elástico de respuesta del acelerograma $N^{\circ} 12$ (Duzce 1) escalado a $P G A=261,4 \mathrm{~cm} / \mathrm{s}^{2} \ldots 60$

4.3.60. Espectro de energía introducida del acelerograma $N^{\circ} 12$ (Duzce 1) escalado a $P G A=261,4 \mathrm{~cm} / \mathrm{s}^{2} 60$

4.3.61. Acelerograma $\mathrm{N}^{0} 3$ (Montenegro), suelo tipo III, campo lejano, sin escalar................................... 61

4.3.62. Acelerograma $N^{0} 3$ (Montenegro), suelo tipo III, campo lejano, escalado a $P G A=261,4 \mathrm{~cm} / \mathrm{s}^{2} \ldots \ldots .61$ 
4.3.63. Espectro elástico de respuesta del acelerograma $N^{\circ} 3$ (Montenegro) escalado a $P G A=261,4 \mathrm{~cm} / \mathrm{s}^{2} \ldots .62$

4.3.64. Espectro de energía introducida del acelerograma $N^{\circ} 3$ (Montenegro) escalado a $P G A=261,4 \mathrm{~cm} / \mathrm{s}^{2} . .62$

4.3.65. Acelerograma $\mathrm{N}^{\circ} 8$ (Sicilia-Orientale), suelo tipo III, campo lejano, sin escalar.............................. 63

4.3.66. Acelerograma $\mathrm{N}^{\circ} 8$ (Sicilia-Orientale), suelo tipo III, campo lejano, escalado a $P G A=261,4 \mathrm{~cm} / \mathrm{s}^{2} .63$

4.3.67. Espectro elástico de respuesta del acelerograma $N^{\circ} 8$ (Sicilia-Orientale) escalado a $P G A=261,4 \mathrm{~cm} / \mathrm{s}^{2} \ldots \ldots .64$

4.3.68. Espectro de energía introducida del acelerograma $N^{\circ} 8$ (Sicilia-Orientale) escalado a $P G A=261,4 \mathrm{~cm} / \mathrm{s}^{2} \ldots .64$

4.3.69. Acelerograma $\mathrm{N}^{\circ} 14$ (Ano Liosia), suelo tipo III, campo lejano, sin escalar..................................... 65

4.3.70. Acelerograma $N^{0} 14$ (Ano Liosia), suelo tipo III, campo lejano, escalado a PGA $=261,4 \mathrm{~cm} / \mathrm{s}^{2} \ldots \ldots \ldots .65$

4.3.71. Espectro elástico de respuesta del acelerograma $N^{\circ} 14$ (Ano Liosia) escalado a $P G A=261,4 \mathrm{~cm} / \mathrm{s}^{2} \ldots . . .66$

4.3.72. Espectro de energía introducida del acelerograma $N^{\circ} 14$ (Ano Liosia) escalado a $P G A=261,4 \mathrm{~cm} / \mathrm{s}^{2} \ldots .66$

5.1.1. Diagrama de interacción Axil - Momento................................................................................ 69

5.1.2. Notation for user Trilinear Envelopes..................................................................................... 70

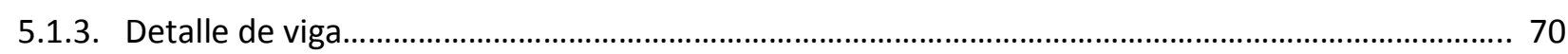

5.2.1. Desplazamiento de una estructura solicitada por fuerza horizontal.............................................. 82

5.2.2. Incremento de los daños con la intensidad................................................................................ 83

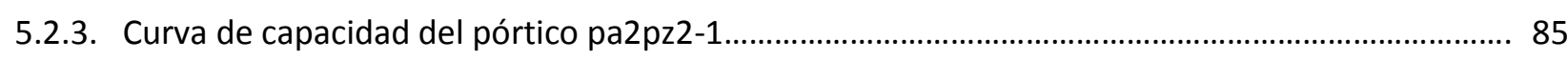

5.2.4. Curva de capacidad del pórtico pa4pz2-1................................................................................... 85

5.2.5. Curva de capacidad del pórtico pa6pz2-1.............................................................................. 86

5.2.6. Fuerza cortante-Desplazamiento entre forjados, pórtico pa2pz2-1, planta 1a.............................. 87

5.2.7. Fuerza cortante-Desplazamiento entre forjados, pórtico pa2pz2-1, planta 2a.............................. 87

5.2.8. Fuerza cortante-Desplazamiento entre forjados, pórtico pa2pz2-1, planta 3a.............................. 88

5.2.9. Fuerza cortante-Desplazamiento entre forjados, pórtico pa4pz2-1, planta 1a............................. 88

5.2.10. Fuerza cortante-Desplazamiento entre forjados, pórtico pa4pz2-1, planta 2a............................. 89

5.2.11. Fuerza cortante-Desplazamiento entre forjados, pórtico pa4pz2-1, planta 3a.............................. 89

5.2.12. Fuerza cortante-Desplazamiento entre forjados, pórtico pa6pz2-1, planta 1 1 a.............................. 90

5.2.13. Fuerza cortante-Desplazamiento entre forjados, pórtico pa6pz2-1, planta 2a............................. 90

5.2.14. Fuerza cortante-Desplazamiento entre forjados, pórtico pa6pz2-1, planta 3a.............................. 91

5.2.15. Fuerza cortante-Desplazamiento entre forjados, pórtico pa6pz2-1, planta 4a.............................. 91

5.2.16. Fuerza cortante-Desplazamiento entre forjados, pórtico pa6pz2-1, planta 5a.............................. 92

5.2.17. Fuerza cortante-Desplazamiento entre forjados, pórtico pa6pz2-1, planta 6a............................... 92

5.2.19. Rigidez-Desplazamiento, pórtico pa2pz2-1, planta 12................................................................ 93

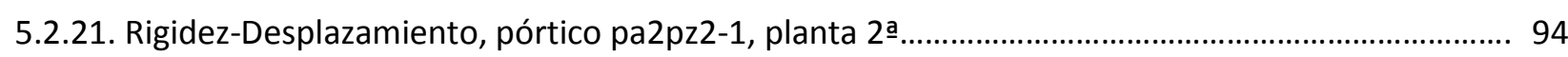

5.2.23. Rigidez-Desplazamiento, pórtico pa2pz2-1, planta 3a................................................................... 95

5.2.25. Rigidez-Desplazamiento, pórtico pa4pz2-1, planta 1'.................................................................. 96

5.2.27. Rigidez-Desplazamiento, pórtico pa4pz2-1, planta 2a................................................................. 97

5.2.29. Rigidez-Desplazamiento, pórtico pa4pz2-1, planta 3a................................................................ 98

5.2.31. Rigidez-Desplazamiento, pórtico pa6pz2-1, planta 1'_................................................................. 99

5.2.33. Rigidez-Desplazamiento, pórtico pa6pz2-1, planta 2 a................................................................ 100

5.2.35. Rigidez-Desplazamiento, pórtico pa6pz2-1, planta 3a.................................................................... 101

5.2.37. Rigidez-Desplazamiento, pórtico pa6pz2-1, planta 4a................................................................. 102

5.2.39. Rigidez-Desplazamiento, pórtico pa6pz2-1, planta 5a................................................................ 103

5.2.41. Rigidez-Desplazamiento, pórtico pa6pz2-1, planta 6a................................................................... 104

5.3.1. Esquema de funcionamiento del problema directo e inverso.................................................................. 119

5.3.2. Ejemplo de la convergencia de un método de búsqueda directa en la función Broyden.............. 120

5.4.1. Subsistemas estructurales de una estructura sismorresistente con disipadores de energía....... 127

5.4.2. Curva Desplazamiento de fluencia - Resistencia lateral del disipador.......................................... 128 
5.4.4. Desplazamientos máximos absolutos entre plantas, pórtico pa2pz2-1, suelo tipo I (muy duro). 131

5.4.5. Desplazamientos máximos absolutos entre plantas, pórtico pa2pz2-1, suelo tipo II (duro)........ 133

5.4.6. Desplazamientos máximos absolutos entre plantas, pórtico pa2pz2-1, suelo tipo III (medio).... 135

5.4.8. Desplazamientos máximos absolutos entre plantas, pórtico pa4pz2-1, suelo tipo I (muy duro). 138

5.4.9. Desplazamientos máximos absolutos entre plantas, pórtico pa4pz2-1, suelo tipo II (duro)........ 139

5.4.10. Desplazamientos máximos absolutos entre plantas, pórtico pa4pz2-1, suelo tipo III (medio).... 141

5.4.12. Desplazamientos máximos absolutos entre plantas, pórtico pa6pz2-1, suelo tipo I (muy duro). 144

5.4.13. Desplazamientos máximos absolutos entre plantas, pórtico pa6pz2-1, suelo tipo II (duro)....... 145

5.4.14. Desplazamientos máximos absolutos entre plantas, pórtico pa6pz2-1, suelo tipo III (medio).... 147

5.5.2. Idealización de un modelo de masas del pórtico pa2pz2-1......................................................... 151

5.5.3. Resistencia lateral de los disipadores de cada planta $i$, pórtico, pa2pz2-1, suelo tipo I, campo lejano. 156

5.5.4. Coeficiente de fuerza cortante de fluencia de los disipadores de cada planta $i$, pórtico pa2pz2-1, suelo tipo I, campo lejano

5.5.5. Resistencia lateral aportada por la parte flexible (pórtico) cuando los disipadores empiezan a plastificar, pórtico pa2pz2-1, suelo tipo I, campo lejano

5.5.6. Coeficiente cortante en la parte flexible (pórtico) cuando los disipadores empiezan a plastificar, pórtico pa2pz2-1, suelo tipo I, campo lejano

5.5.7. Coeficiente de fuerza cortante total de planta, pórtico pa2pz2-1, suelo tipo I, campo lejano..... 158

5.5.8. Resistencia lateral de los disipadores de cada planta $i$, pórtico, pa2pz2-1, suelo tipo II, campo cercano.

5.5.9. Coeficiente de fuerza cortante de fluencia de los disipadores de cada planta $i$, pórtico pa2pz2-1, suelo tipo II, campo cercano.

5.5.10. Resistencia lateral aportada por la parte flexible (pórtico) cuando los disipadores empiezan a plastificar, pórtico pa2pz2-1, suelo tipo II, campo cercano.

5.5.11. Coeficiente cortante en la parte flexible (pórtico) cuando los disipadores empiezan a plastificar, pórtico pa2pz2-1, suelo tipo II, campo cercano.

5.5.12. Coeficiente de fuerza cortante total de planta, pórtico pa2pz2-1, suelo tipo II, campo cercano. 163

5.5.13. Resistencia lateral de los disipadores de cada planta $i$, pórtico, pa2pz2-1, suelo tipo II, campo lejano

5.5.14. Coeficiente de fuerza cortante de fluencia de los disipadores de cada planta $i$, pórtico pa2pz2-1, suelo tipo II, campo lejano.

5.5.15. Resistencia lateral aportada por la parte flexible (pórtico) cuando los disipadores empiezan a plastificar, pórtico pa2pz2-1, suelo tipo II, campo lejano.

5.5.16. Coeficiente cortante en la parte flexible (pórtico) cuando los disipadores empiezan a plastificar, pórtico pa2pz2-1, suelo tipo II, campo lejano

5.5.17. Coeficiente de fuerza cortante total de planta, pórtico pa2pz2-1, suelo tipo II, campo lejano.... 168

5.5.18. Resistencia lateral de los disipadores de cada planta $i$, pórtico pa2pz2-1, suelo tipo III, campo cercano

5.5.19. Coeficiente de fuerza cortante de fluencia de los disipadores de cada planta $i$, pórtico pa2pz2-1, suelo tipo III, campo cercano

5.5.20. Resistencia lateral aportada por la parte flexible (pórtico) cuando los disipadores empiezan a plastificar, pórtico pa2pz2-1, suelo tipo III, campo cercano.

5.5.21. Coeficiente cortante en la parte flexible (pórtico) cuando los disipadores empiezan a plastificar, pórtico pa2pz2-1, suelo tipo III, campo cercano

5.5.22. Coeficiente de fuerza cortante total de planta, pórtico pa2pz2-1, suelo tipo III, campo cercano 175 
5.5.23. Resistencia lateral de los disipadores de cada planta $i$, pórtico pa2pz2-1, suelo tipo III, campo lejano.

5.5.24. Coeficiente de fuerza cortante de fluencia de los disipadores de cada planta $i$, pórtico pa2pz2-1, suelo tipo III, campo lejano.

5.5.25. Resistencia lateral aportada por la parte flexible (pórtico) cuando los disipadores empiezan a plastificar, pórtico pa2pz2-1, suelo tipo III, campo lejano

5.5.26. Coeficiente cortante en la parte flexible (pórtico) cuando los disipadores empiezan a plastificar, pórtico pa2pz2-1, suelo tipo III, campo lejano

5.5.27. Coeficiente de fuerza cortante total de planta, pórtico pa2pz2-1, suelo tipo III, campo lejano... 180

5.5.28. Idealización de un modelo de masas del pórtico pa4pz2-1 180

5.5.29. Resistencia lateral de los disipadores de cada planta $i$, pórtico pa4pz2-1, suelo tipo I, campo lejano

5.5.30. Coeficiente de fuerza cortante de fluencia de los disipadores de cada planta $i$, pórtico pa4pz2-1, suelo tipo I, campo lejano.

5.5.31. Resistencia lateral aportada por la parte flexible (pórtico) cuando los disipadores empiezan a plastificar, pórtico pa4pz2-1, suelo tipo I, campo lejano

5.5.32. Coeficiente cortante en la parte flexible (pórtico) cuando los disipadores empiezan a plastificar, pórtico pa4pz2-1, suelo tipo I, campo lejano

5.5.33. Coeficiente de fuerza cortante total de planta, pórtico pa4pz2-1, suelo tipo I, campo lejano..... 187

5.5.34. Resistencia lateral de los disipadores de cada planta $i$, pórtico pa4pz2-1, suelo tipo II, campo cercano.

5.5.35. Coeficiente de fuerza cortante de fluencia de los disipadores de cada planta $i$, pórtico pa4pz2-1, suelo tipo II, campo cercano

5.5.36. Resistencia lateral aportada por la parte flexible (pórtico) cuando los disipadores empiezan a plastificar, pórtico pa4pz2-1, suelo tipo II, campo cercano.

5.5.37. Coeficiente cortante en la parte flexible (pórtico) cuando los disipadores empiezan a plastificar, pórtico pa4pz2-1, suelo tipo II, campo cercano

5.5.38. Coeficiente de fuerza cortante total de planta, pórtico pa4pz2-1, suelo tipo II, campo cercano. 192

5.5.39. Resistencia lateral de los disipadores de cada planta $i$, pórtico pa4pz2-1, suelo tipo II, campo lejano.

5.5.40. Coeficiente de fuerza cortante de fluencia de los disipadores de cada planta $i$, pórtico pa4pz2-1, suelo tipo II, campo lejano

5.5.41. Resistencia lateral aportada por la parte flexible (pórtico) cuando los disipadores empiezan a plastificar, pórtico pa4pz2-1, suelo tipo II, campo lejano

5.5.42. Coeficiente cortante en la parte flexible (pórtico) cuando los disipadores empiezan a plastificar, pórtico pa4pz2-1, suelo tipo II, campo lejano

5.5.43. Coeficiente de fuerza cortante total de planta, pórtico pa4pz2-1, suelo tipo II, campo lejano.... 197

5.5.44. Resistencia lateral de los disipadores de cada planta $i$, pórtico pa4pz2-1, suelo tipo III, campo cercano.

5.5.45. Coeficiente de fuerza cortante de fluencia de los disipadores de cada planta $i$, pórtico pa4pz2-1, suelo tipo III, campo cercano.

5.5.46. Resistencia lateral aportada por la parte flexible (pórtico) cuando los disipadores empiezan a plastificar, pórtico pa4pz2-1, suelo tipo III, campo cercano.

5.5.47. Coeficiente cortante en la parte flexible (pórtico) cuando los disipadores empiezan a plastificar, pórtico pa4pz2-1, suelo tipo III, campo cercano.

5.5.48. Coeficiente de fuerza cortante total de planta, pórtico pa4pz2-1, suelo tipo III, campo cercano 203 
5.5.49. Resistencia lateral de los disipadores de cada planta i, pórtico pa4pz2-1, suelo tipo III, campo lejano.

5.5.50. Coeficiente de fuerza cortante de fluencia de los disipadores de cada planta $i$, pórtico pa4pz2-1, suelo tipo III, campo lejano....

5.5.51. Resistencia lateral aportada por la parte flexible (pórtico) cuando los disipadores empiezan a plastificar, pórtico pa4pz2-1, suelo tipo III, campo lejano

5.5.52. Coeficiente cortante en la parte flexible (pórtico) cuando los disipadores empiezan a plastificar, pórtico pa4pz2-1, suelo tipo III, campo lejano

5.5.53. Coeficiente de fuerza cortante total de planta, pórtico pa4pz2-1, suelo tipo III, campo lejano... 208

5.5.54. Idealización de un modelo de masas del pórtico pa6pz2-1 208

5.5.55. Resistencia lateral de los disipadores de cada planta $i$, pórtico pa6pz2-1, suelo tipo I, campo lejano....

5.5.56. Coeficiente de fuerza cortante de fluencia de los disipadores de cada planta $i$, pórtico pa6pz2-1, suelo tipo I, campo lejano....

5.5.57. Resistencia lateral aportada por la parte flexible (pórtico) cuando los disipadores empiezan a plastificar, pórtico pa6pz2-1, suelo tipo I, campo lejano

5.5.58. Coeficiente cortante en la parte flexible (pórtico) cuando los disipadores empiezan a plastificar, pórtico pa6pz2-1, suelo tipo I, campo lejano

5.5.59. Coeficiente de fuerza cortante total de planta, pórtico pa6pz2-1, suelo tipo I, campo lejano..... 216

5.5.60. Resistencia lateral de los disipadores de cada planta $i$, pórtico pa6pz2-1, suelo tipo II, campo cercano

5.5.61. Coeficiente de fuerza cortante de fluencia de los disipadores de cada planta $i$, pórtico pa6pz2-1, suelo tipo II, campo cercano

5.5.62. Resistencia lateral aportada por la parte flexible (pórtico) cuando los disipadores empiezan a plastificar, pórtico pa6pz2-1, suelo tipo II, campo cercano.

5.5.63. Coeficiente cortante en la parte flexible (pórtico) cuando los disipadores empiezan a plastificar, pórtico pa6pz2-1, suelo tipo II, campo cercano

5.5.64. Coeficiente de fuerza cortante total de planta, pórtico pa6pz2-1, suelo tipo II, campo cercano. 221

5.5.65. Resistencia lateral de los disipadores de cada planta $i$, pórtico pa6pz2-1, suelo tipo III, campo cercano

5.5.66. Coeficiente de fuerza cortante de fluencia de los disipadores de cada planta $i$, pórtico pa6pz2-1, suelo tipo III, campo cercano.

5.5.67. Resistencia lateral aportada por la parte flexible (pórtico) cuando los disipadores empiezan a plastificar, pórtico pa6pz2-1, suelo tipo III, campo cercano.

5.5.68. Coeficiente cortante en la parte flexible (pórtico) cuando los disipadores empiezan a plastificar, pórtico pa6pz2-1, suelo tipo III, campo cercano

5.5.69. Coeficiente de fuerza cortante total de planta, pórtico pa6pz2-1, suelo tipo III, campo cercano 231

5.5.70. Resistencia lateral de los disipadores de cada planta $i$, pórtico pa6pz2-1, suelo tipo III, campo lejano

5.5.71. Coeficiente de fuerza cortante de fluencia de los disipadores de cada planta $i$, pórtico pa6pz2-1, suelo tipo III, campo lejano.

5.5.72. Resistencia lateral aportada por la parte flexible (pórtico) cuando los disipadores empiezan a plastificar, pórtico pa6pz2-1, suelo tipo III, campo lejano

5.5.73. Coeficiente cortante en la parte flexible (pórtico) cuando los disipadores empiezan a plastificar, pórtico pa6pz2-1, suelo tipo III, campo lejano

5.5.74. Coeficiente de fuerza cortante total de planta, pórtico pa6pz2-1, suelo tipo III, campo lejano... 236 
5.6.1. Comparación de las distribuciones de resistencia, pórtico pa2pz2-1, suelo tipo I, campo lejano 241

5.6.2. Comparación de las distribuciones de resistencia, pórtico pa2pz2-1, suelo tipo II, campo cercano

5.6.3. Comparación de las distribuciones de resistencia, pórtico pa2pz2-1, suelo tipo II, campo lejano

5.6.4. Comparación de las distribuciones de resistencia, pórtico pa2pz2-1, suelo tipo III, campo cercano

5.6.5. Comparación de las distribuciones de resistencia, pórtico pa2pz2-1, suelo tipo III, campo lejano

5.6.6. Comparación de las distribuciones de resistencia, pórtico pa4pz2-1, suelo tipo I, campo lejano.

5.6.7. Comparación de las distribuciones de resistencia, pórtico pa4pz2-1, suelo tipo II, campo cercano

5.6.8. Comparación de las distribuciones de resistencia, pórtico pa4pz2-1, suelo tipo II, campo lejano.

5.6.9. Comparación de las distribuciones de resistencia, pórtico pa4pz2-1, suelo tipo III, campo cercano .250

5.6.10. Comparación de las distribuciones de resistencia, pórtico pa4pz2-1, suelo tipo III, campo lejano

5.6.11. Comparación de las distribuciones de resistencia, pórtico pa6pz2-1, suelo tipo I, campo lejano

5.6.12. Comparación de las distribuciones de resistencia, pórtico pa6pz2-1, suelo tipo II, campo cercano.

5.6.13. Comparación de las distribuciones de resistencia, pórtico pa6pz2-1, suelo tipo III, campo cercano.

5.6.14. Comparación de las distribuciones de resistencia, pórtico pa6pz2-1, suelo tipo III, campo lejano. 


\section{ÍNDICE DE TABLAS}

3.1.1. Características de las estructuras existentes con vigas planas construidas................................... 19

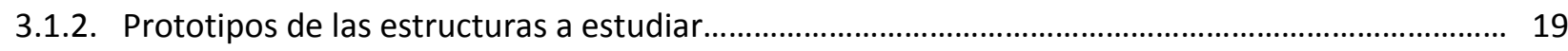

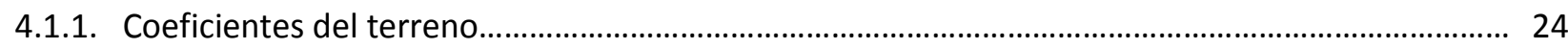

4.1.2. Aceleración sísmica de cálculo para un edificio ubicado en Granada y para cada tipo de suelo.. 26

4.2.1. Terremotos históricos registrados en suelo tipo I (muy duro)...................................................... 27

4.2.2. Terremotos históricos registrados en suelo tipo II (duro) ............................................................... 27

4.2.3. Terremotos históricos registrados en suelo tipo III (medio)............................................................ 28

4.2.4. Ordenadas espectrales de los terremotos históricos escalados registrados en suelo tipo I.......... 28

4.2.5. Ordenadas espectrales de los terremotos históricos escalados registrados en suelo tipo II.......... 29

4.2.6. Ordenadas espectrales de los terremotos históricos escalados registrados en suelo tipo III........ 29

4.2.7. Periodos fundamentales de vibración....................................................................................... 29

5.1.1. Archivo de entrada para el pórtico pa2pz2-1 de tres plantas (pa2pz2-1.DAT) IDARC.................... 72

5.1.2. Archivo de entrada para el pórtico pa4pz2-1 de tres plantas (pa4pz2-1.DAT) IDARC.................... 74

5.1.3. Archivo de entrada para el pórtico pa6pz2-1 de seis plantas (pa6pz2-1.DAT) IDARC.................... 77

5.2.1. Altura total y peso de cada uno de los pórticos.............................................................................. 84

5.2.5. Cortante basal y desplazamiento para los que se produce el colapso en cada uno de los pórticos..... 86

5.2.18. Rigidez inicial y desplazamiento de fluencia para cada planta de cada uno de los pórticos........... 105

5.2.19. Frecuencias y periodos de vibración sin disipadores obtenidos con IDARC para cada uno de los pórticos. 106

5.2.20. Frecuencias sin disipadores expresadas en (rad/s) obtenidas con LumpST para el pórtico pa2pz2-1. 107

5.2.21. Frecuencias y periodos de vibración sin disipadores obtenidos con LumpST para el pórtico pa2pz2-1...... 107

5.2.22. Frecuencias sin disipadores expresadas en (rad/s) obtenidas con LumpST para el pórtico pa4pz2-1. 109

5.2.23. Frecuencias y periodos de vibración sin disipadores obtenidos con LumpST para el pórtico pa4pz2-1...... 109

5.2.24. Frecuencias sin disipadores expresadas en ( $\mathrm{rad} / \mathrm{s}$ ) obtenidas con LumpST para el pórtico pa4pz2-1.111

5.2.25. Frecuencias y periodos de vibración sin disipadores obtenidos con LumpST para el pórtico pa6pz2-1...... 112

5.2.26. Comparación periodos de vibración sin disipadores, obtenidos con IDARC y LumpST, pórtico pa2pz2-1...113

5.2.27. Comparación periodos de vibración sin disipadores, obtenidos con IDARC y LumpST, pórtico pa4pz2-1...113

5.2.28. Comparación periodos de vibración sin disipadores, obtenidos con IDARC y LumpST, pórtico pa6pz2-1... 113

5.2.29. Frecuencias con disipadores expresadas en ( $\mathrm{rad} / \mathrm{s}$ ) obtenidas con LumpST para el pórtico pa2pz2-1114

5.2.30. Frecuencias y periodos de vibración con disipadores para el pórtico pa2pz2-1.............................. 115

5.2.31. Frecuencias con disipadores expresadas en (rad/s) obtenidas con LumpST para el pórtico pa4pz2-1115

5.2.32. Frecuencias y periodos de vibración con disipadores para el pórtico pa4pz2-1............................116

5.2.33. Frecuencias con disipadores expresadas en ( $\mathrm{rad} / \mathrm{s}$ ) obtenidas con LumpST para el pórtico pa4pz2-1117

5.2.34. Frecuencias y periodos de vibración con disipadores para el pórtico pa6pz2-1............................. 118

5.2.35. Frecuencias y periodos de vibración con disipadores para cada uno de los pórticos...................... 118

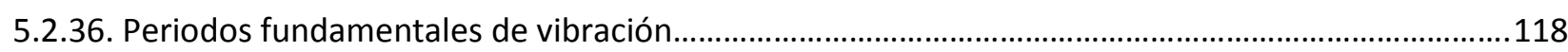

5.4.1. Desplazamientos de fluencia del disipador y desplazamientos máximos absolutos entre plantas, pórtico pa2pz2-1, acelerograma no1, suelo tipo I, campo lejano........................................130

5.4.2. Desplazamientos de fluencia del disipador y desplazamientos máximos absolutos entre plantas, pórtico pa2pz2-1, acelerograma nㄴ, suelo tipo I, campo lejano. .130 
5.4.3. Desplazamientos de fluencia del disipador y desplazamientos máximos absolutos entre plantas, pórtico pa2pz2-1, acelerograma no5, suelo tipo I, campo lejano 130

5.4.4. Desplazamientos de fluencia del disipador y desplazamientos máximos absolutos entre plantas, pórtico pa2pz2-1, acelerograma $\mathrm{n} \cong 1$, suelo tipo II, campo cercano.

5.4.5. Desplazamientos de fluencia del disipador y desplazamientos máximos absolutos entre plantas, pórtico pa2pz2-1, acelerograma nำ14, suelo tipo II, campo cercano.

5.4.6. Desplazamientos de fluencia del disipador y desplazamientos máximos absolutos entre plantas, pórtico pa2pz2-1, acelerograma n2, suelo tipo II, campo lejano.

5.4.7. Desplazamientos de fluencia del disipador y desplazamientos máximos absolutos entre plantas, pórtico pa2pz2-1, acelerograma no12, suelo tipo II, campo lejano.

5.4.8. Desplazamientos de fluencia del disipador y desplazamientos máximos absolutos entre plantas, pórtico pa2pz2-1, acelerograma $\mathrm{n}=2$, suelo tipo III, campo cercano

5.4.9. Desplazamientos de fluencia del disipador y desplazamientos máximos absolutos entre plantas, pórtico pa2pz2-1, acelerograma n6, suelo tipo III, campo cercano

5.4.10. Desplazamientos de fluencia del disipador y desplazamientos máximos absolutos entre plantas, pórtico pa2pz2-1, acelerograma no12, suelo tipo III, campo cercano

5.4.11. Desplazamientos de fluencia del disipador y desplazamientos máximos absolutos entre plantas, pórtico pa2pz2-1, acelerograma $\mathrm{n}$ 으, suelo tipo III, campo lejano

5.4.12. Desplazamientos de fluencia del disipador y desplazamientos máximos absolutos entre plantas, pórtico pa2pz2-1, acelerograma no14, suelo tipo III, campo lejano.

5.4.13. Desplazamientos de fluencia del disipador y desplazamientos máximos absolutos entre plantas, pórtico pa4pz2-1, acelerograma no1, suelo tipo I, campo lejano

5.4.14. Desplazamientos de fluencia del disipador y desplazamientos máximos absolutos entre plantas, pórtico pa4pz2-1, acelerograma no4, suelo tipo I, campo lejano

5.4.15. Desplazamientos de fluencia del disipador y desplazamientos máximos absolutos entre plantas, pórtico pa4pz2-1, acelerograma nํ5, suelo tipo I, campo lejano

5.4.16. Desplazamientos de fluencia del disipador y desplazamientos máximos absolutos entre plantas, pórtico pa4pz2-1, acelerograma nำ1, suelo tipo II, campo cercano

5.4.17. Desplazamientos de fluencia del disipador y desplazamientos máximos absolutos entre plantas, pórtico pa4pz2-1, acelerograma no14, suelo tipo II, campo cercano

5.4.18. Desplazamientos de fluencia del disipador y desplazamientos máximos absolutos entre plantas, pórtico pa4pz2-1, acelerograma n2, suelo tipo II, campo lejano.

5.4.19. Desplazamientos de fluencia del disipador y desplazamientos máximos absolutos entre plantas, pórtico pa4pz2-1, acelerograma nำ12, suelo tipo II, campo lejano.

5.4.20. Desplazamientos de fluencia del disipador y desplazamientos máximos absolutos entre plantas, pórtico pa4pz2-1, acelerograma n2, suelo tipo III, campo cercano

5.4.21. Desplazamientos de fluencia del disipador y desplazamientos máximos absolutos entre plantas, pórtico pa4pz2-1, acelerograma $\mathrm{n}=6$, suelo tipo III, campo cercano

5.4.22. Desplazamientos de fluencia del disipador y desplazamientos máximos absolutos entre plantas, pórtico pa4pz2-1, acelerograma nำ12, suelo tipo III, campo cercano

5.4.23. Desplazamientos de fluencia del disipador y desplazamientos máximos absolutos entre plantas, pórtico pa4pz2-1, acelerograma no3, suelo tipo III, campo lejano

5.4.24. Desplazamientos de fluencia del disipador y desplazamientos máximos absolutos entre plantas, pórtico pa4pz2-1, acelerograma n14, suelo tipo III, campo lejano

5.4.25. Desplazamientos de fluencia del disipador y desplazamientos máximos absolutos entre plantas, pórtico pa6pz2-1, acelerograma n누, suelo tipo I, campo lejano 
5.4.26. Desplazamientos de fluencia del disipador y desplazamientos máximos absolutos entre plantas, pórtico pa6pz2-1, acelerograma nำ5, suelo tipo I, campo lejano

5.4.27. Desplazamientos de fluencia del disipador y desplazamientos máximos absolutos entre plantas, pórtico pa6pz2-1, acelerograma nำ14, suelo tipo II, campo lejano.

5.4.28. Desplazamientos de fluencia del disipador y desplazamientos máximos absolutos entre plantas, pórtico pa6pz2-1, acelerograma $\mathrm{n}=2$, suelo tipo III, campo cercano

5.4.29. Desplazamientos de fluencia del disipador y desplazamientos máximos absolutos entre plantas, pórtico pa6pz2-1, acelerograma n6, suelo tipo III, campo cercano

5.4.30. Desplazamientos de fluencia del disipador y desplazamientos máximos absolutos entre plantas, pórtico pa6pz2-1, acelerograma nำ12, suelo tipo III, campo cercano

5.4.31. Desplazamientos de fluencia del disipador y desplazamientos máximos absolutos entre plantas, pórtico pa6pz2-1, acelerograma $\mathrm{n}$ 3, suelo tipo III, campo lejano

5.4.32. Desplazamientos de fluencia del disipador, desplazamiento máximo absoluto entre plantas, input de energía expresado en forma de pseudovelocidad equivalente para cada acelerograma

5.5.1. Resistencia lateral de los disipadores, coeficiente de fuerza cortante de fluencia de los disipadores, resistencia lateral aportada por la parte flexible (pórtico) cuando los disipadores empiezan a plastificar, coeficiente cortante en la parte flexible (pórtico) cuando los disipadores empiezan a plastificar, coeficiente de fuerza cortante total.

5.6.1. Distribución de resistencia entre plantas para cada pórtico y tipo de suelo y distribución óptima del coeficiente de fuerza cortante de fluencia propuesta por Akiyama 


\section{CAPÍTULO 1 \\ INTRODUCCIÓN - MOTIVACIÓN - OBJETIVOS - METODOLOGÍA}

\subsection{INTRODUCCIÓN}

Existen diferentes y diversas estrategias y metodologías de proyecto sismorresistente, que se han aplicado a lo largo de la historia para paliar los efectos del sismo que afectan a las estructuras de edificación. A continuación citamos algunas de ellas:

1a) Metodologías y estrategias basadas en proporcionar resistencia lateral a la estructura

Hasta principios de 1997 en que dejó de ser aplicable la primera norma sísmica PDS-74 [1], las estructuras de edificación construidas en España fueron proyectadas aplicando la filosofía de los primeros métodos de cálculo sísmico basados exclusivamente en la resistencia. Estos métodos trataban de conseguir seguridad estructural en caso de terremoto severo proporcionando únicamente resistencia lateral a la estructura, y no consideraba otro aspecto fundamental como es el de su capacidad de deformación plástica o ductilidad. Además, la resistencia lateral que exigía la norma PDS-74 era relativamente pequeña (y en la gran mayoría de los casos menor que la que exige la norma actual NCSE02) [2], lo que hacía que el dimensionado de las estructuras estuviese gobernado por las acciones gravitatorias.

2a) Metodologías y estrategias basadas en el control del desplazamiento lateral del edificio

En estas metodologías se exige que tanto el desplazamiento lateral entre plantas (es decir entre los dos forjados que delimitan la planta), como el desplazamiento lateral total del edificio (es decir respecto al suelo), esté limitado, entre otras, por las siguientes razones:

a) Para controlar los daños en elementos estructurales.

b) Para controlar los daños en elementos no estructurales (tabiquería, etc).

c) Para controlar el efecto P- $\Delta$.

d) Para evitar colisiones con edificios contiguos.

3a) Metodologías basadas en la resistencia y ductilidad (capacidad de disipación de energía)

Actualmente, la capacidad de disipación de la energía sísmica de la estructura esta comúnmente aceptado como un factor clave para conseguir seguridad estructural con un coste económico razonable. Las normativas actuales permiten que bajo el terremoto más severo esperable con una probabilidad razonable, las estructuras disipen energía mediante deformaciones plásticas. En las estructuras porticadas convencionales estas deformaciones plásticas se permiten en los extremos de las vigas y en los arranques de los pilares de planta baja, lo que tiene como principal inconveniente que la estructura principal del edificio puede quedar con daños importantes después del terremoto. En las últimas décadas se han desarrollado estrategias avanzadas de proyecto sismorresistente de estructuras [15] orientadas a incrementar la capacidad de disipación de energía de cada planta. Una manera de incrementar globalmente la capacidad límite última de disipación de energía de la estructura, consiste en, instalar en cada planta elementos disipadores de energía. 
Estos disipadores son elementos especiales capaces de disipar de forma estable cantidades muy elevadas de energía hasta la rotura. Existe una variedad muy amplia de disipadores de energía. Entre los más populares por su relativamente reducido coste económico son aquellos cuya fuente de disipación de energía se basa en hacer plastificar aceros, y a ellos de les denomina comúnmente disipadores de energía de tipo histerético.

La capacidad de disipación de energía de una estructura es una manera muy adecuada de caracterizar su sismorresistencia, y ello constituye uno de los fundamentos de los modernos métodos de proyecto sismorresistente basados en la teoría de Housne-Akiyama (Housner, 1956; Akiyama, 1980) [14].

En esta Tesina se aborda el uso de los disipadores de energía de tipo histerético como solución para aumentar la capacidad sismorresistente de estructuras existentes proyectadas en base a normativas antiguas. El estudio se centra concretamente en el reacondicionamiento sísmico mediante disipadores, de estructuras porticadas existentes de hormigón armado con vigas planas.

\subsection{MOTIVACIÓN}

La primera razón que justifica este estudio es la situación de elevada vulnerabilidad sísmica en la que se encuentran muchas estructuras porticadas con vigas planas proyectadas y construías en muchas regiones del sur de España, en base a normativas sísmicas obsoletas, como la PDS-74 [1].

En España el primer código sísmico nacional PDS-74 [1] no decía nada respecto al uso de vigas planas, pero la norma posterior de 1994, NCSE-94 [13] que la sustituyó, prohibió su uso en zonas como Granada, donde la aceleración máxima del suelo, $a_{c}$, era superior a $0.16 \mathrm{~g}$, siendo g la aceleración de la gravedad. Actualmente, la norma sísmica española vigente NCSE-02 [2] permite utilizar vigas planas en conexiones exteriores si el canto de la viga transversal $\left(h_{s}\right)$ es mayor que el canto de la viga plana $\left(h_{b}\right)$ y si $b_{b} \leq\left(b_{c}+h_{s}\right) ; y$ en conexiones interiores siempre y cuando $b_{b} \leq\left(b_{c}+h_{b}\right)$ [15].

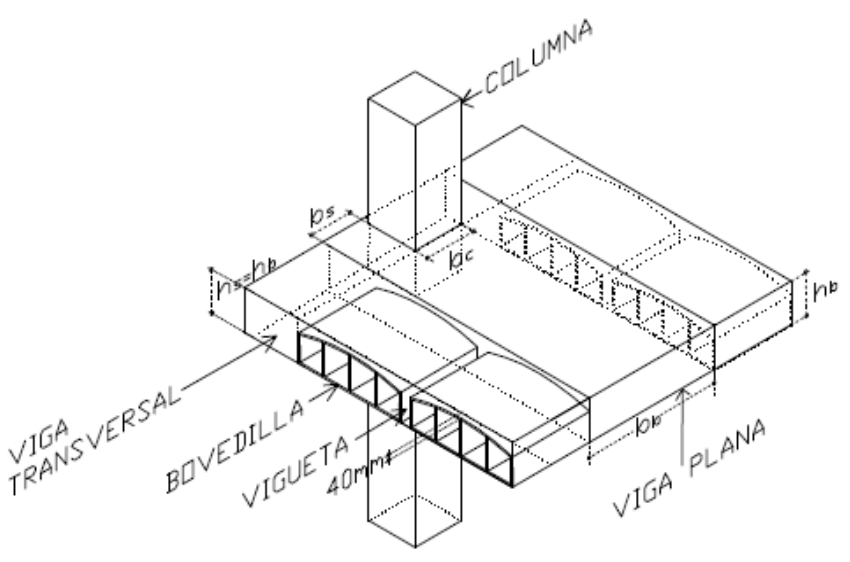

a)

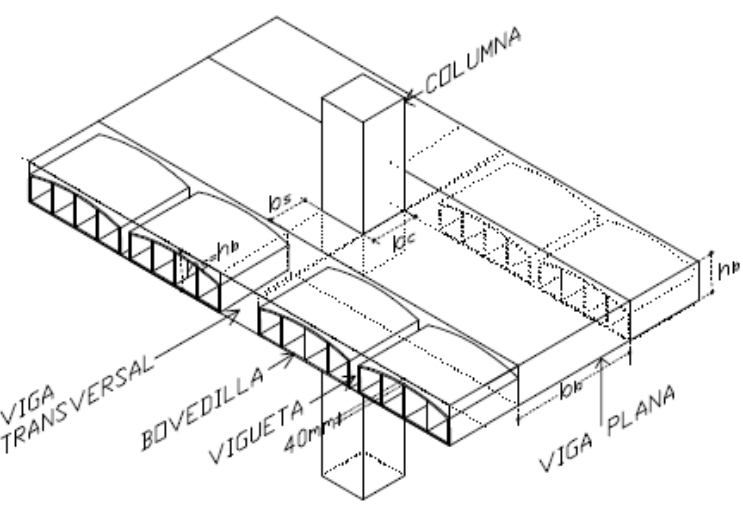

b)

Fig. 1.2.1: Detalle de conexiones viga plana-pilar.

a) Pilar exterior. b) Pilar interior

La característica principal de las denominadas "vigas planas" es que su anchura $\left(b_{b}\right)$ es mayor que la del pilar al que acomete $\left(b_{c}\right)$, de manera que parte de la armadura longitudinal está anclada o 
solapada fuera del nudo (Fig. 1.2.1). Este tipo de vigas se emplea (y se ha empleado en el pasado) en muy pocos países (España, Francia e Italia), incluso en zonas de cierta peligrosidad sísmica, a pesar de sus importantes inconvenientes desde el punto de vista sismorresistente, $y$ del insuficiente conocimiento que se tienen, hoy por hoy, sobre su comportamiento en caso de sismos intensos.

Por lo tanto, emplear pórticos con vigas planas como sistema sismorresistente es una solución en general desaconsejable por las siguientes razones:

- Reducida rigidez lateral, lo que hace que los pórticos sean muy deformables horizontalmente.

- Deficiente transmisión de los momentos flectores desde la viga a los pilares (ver Fig. 1.2.2).

- Reducida capacidad de disipación de energía.

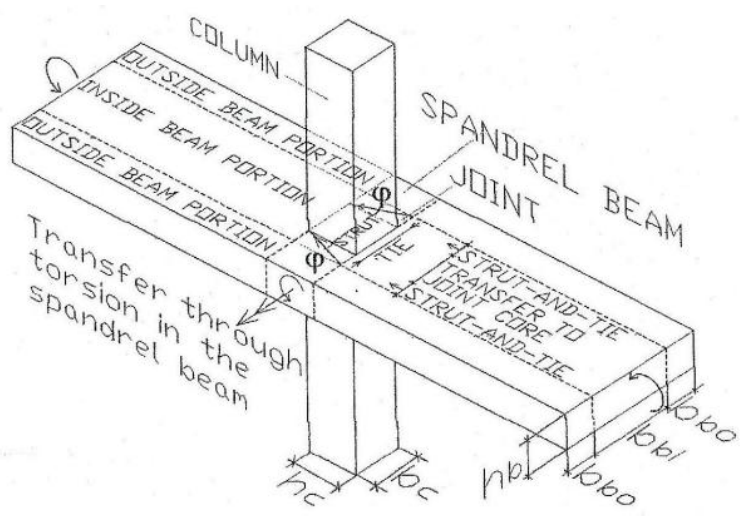

Fig. 1.2.2: Mecanismo de transmisión de momentos flectores de viga plana a pilar

La segunda razón que justifica este trabajo es la existencia de soluciones innovadoras consistentes en el uso de disipadores de energía de tipo histerético, que se han venido desarrollando y aplicando al reacondicionamiento sísmico de estructuras existentes en las últimas décadas. En Europa, esta aplicación de los disipadores se ha empezado a extender en los últimos 10 años, especialmente en Italia. Como hemos dicho anteriormente, existen varios tipos de disipadores de energía, algunos de ellos los citamos a continuación:

- Disipadores histeréticos:

Logran la disipación de energía, plastificando metales dúctiles (acero, plomo o cobre). Son robustos y baratos.

- Disipadores friccionales:

Tienen un comportamiento histéretico que se basa en la fricción seca entre dos metales. El principio básico de los disipadores friccionales consiste en utilizar la deformación relativa entre dos puntos de una estructura para disipar energía a través de fricción.

- Disipadores viscosos y viscoelásticos:

El principio básico de funcionamiento consiste en movilizar un elemento a través de un fluido viscoso. Esto genera fuerzas que se oponen al movimiento del elemento, de magnitud proporcional a la velocidad. Los fluidos viscosos (FV), tales como siliconas, aceites, etc. han sido utilizados con eficiencia en la generación de dispositivos disipadores de energía hace ya varias décadas en la industria militar y aeroespacial. 
- Con aleaciones con memoria de forma

Estos tipos de disipadores recuperan su forma a una determinada temperatura. Disipan energía no por plastificación sino por cambio de fase, por tanto no se fatigan. No es necesario cambiarlos nunca pero son caros.

- Amortiguador mecatrónico:

La energía mecánica se transforma en eléctrica. El factor de amortiguamiento se puede ajustar y tiene un rendimiento óptimo a bajas frecuencias y grandes amplitudes.

- Disipadores electro-inductivos:

La energía mecánica se transforma en eléctrica (calor), cuyo funcionamiento es similar a un amortiguador viscoso. No requieren mantenimiento y tienen un comportamiento pasivo o semiactivo.

En el presente trabajo, utilizaremos los disipadores de energía de tipo histeréticos instalados en forma de barras diagonales, por su bajo coste y sencillez de fabricación y de instalación, así como por no requerir mantenimiento. Este tipo de disipadores son aptos para emplearlos en estructuras de edificación antiguas puesto que no supone una gran inversión.

\subsection{OBJETIVOS}

En la línea de lo anteriormente expuesto, el objetivo general de este trabajo es investigar el uso de los disipadores de energía de tipo histerético para reacondionar sísmicamente estructuras porticadas existentes de hormigón armado con vigas planas. Concretamente, este trabajo se centra en determinar la resistencia mínima que deberían tener los disipadores de energía histeréticos colocados en cada una de las plantas de estructuras porticadas existentes con vigas planas, para que bajo el terremoto de proyecto sean capaces de limitar los desplazamientos máximos entre plantas a unos valores preestablecidos.

Como objetivo de desplazamiento máximo entre plantas, se fija un valor del $0,5 \%$ (en valor absoluto) de la altura entre plantas. Limitando el valor del desplazamiento máximo a este valor del $0.5 \%$ (con una tolerancia aproximada de $+/$ - el $10 \%$ ) se garantiza que el pórtico principal se va a mantener básicamente dentro del dominio elástico, y por lo tanto sin daños.

\subsection{METODOLOGÍA}

La metodología seguida para conseguir el objetivo anteriormente expuesto ha sido la siguiente:

1.- Se han definido varios prototipos de pórticos con vigas planas de estructuras existentes de hormigón armado, construidos en los años 70-90, empleados con mucha frecuencia en países del área mediterránea como España.

2.- Se ha elegido un conjunto de terremotos históricos de diferentes características y registrados en distintos tipos de suelo, y se han escalado con un determinado criterio para que representen la sismicidad de la región donde se han supuesto ubicadas las estructuras estudiadas. Uno de los problemas básicos en cualquier estudio de vulnerabilidad sísmica es la elección adecuada y en un número suficientemente elevado de terremotos. En esta investigación se seleccionó inicialmente un total de 18 terremotos históricos registrados en Europa en campo libre sobre distintos tipos de suelo, 
con el criterio de que cubriesen un amplio espectro de respuesta. Los acelerogramas se obtuvieron de una base de datos europea accesible gratuitamente a través de la red [3].

Los acelerogramas empleados deben escalarse en tiempo y en amplitud para que sean compatibles, respecto a la aceleración máxima del suelo (PGA) y a la forma de los espectros elásticos de respuesta, con los niveles de peligrosidad sísmica que prescribe la norma NCSE-02 [2].

3.- Los prototipos de pórticos con vigas planas seleccionados se modelizaron para poder ser analizados con el programa de análisis no lineal en dos dimensiones IDARC versión 7.0, que permite realizar tanto cálculos dinámicos directos como cálculos estáticos no lineales aplicando el método del empuje incremental (pushover analysis).

4.- Se realizaron cálculos estáticos no lineales aplicando el método del empuje incremental (pushover analysis) con control en fuerzas y empleando el primer modo de vibración. Estos cálculos permitieron obtener la curva de capacidad global de cada pórtico (relación entre cortante basal y desplazamiento de la última planta), y para cada planta de cada pórtico la relación entre fuerza cortante de la planta y el desplazamiento entre forjados que delimitan dicha planta.

De estas últimas curvas se obtuvo la rigidez inicial y el desplazamiento de fluencia de cada una de las plantas.

5.- A los modelos numéricos de los pórticos con vigas planas se le añadieron disipadores de energía de tipo histerético en cada planta. El criterio considerado para establecer la rigidez lateral de los disipadores de cada planta es que la rigidez lateral que aportan los disipadores instalados en una determinada planta sea cinco veces la rigidez del pórtico sin disipadores [4]. Este es un valor que, a la luz de investigaciones previas, se considera adecuado para garantizar que plastifique antes el disipador que el pórtico principal. El criterio para fijar la resistencia lateral de los disipadores es que el desplazamiento máximo entre plantas, sea igual al 0,5\% en valor absoluto de la altura entre plantas. Se adopta este valor, con una tolerancia del $\pm 10 \%$, para garantizar que el pórtico se mantenga básicamente dentro del dominio elástico. La determinación de la resistencia lateral de los disipadores se ha determinado a base de cálculos dinámicos directos iterativos, modificando la resistencia lateral de cada planta, o lo que es lo mismo, el desplazamiento de fluencia de los disipadores puesto que la rigidez lateral de los disipadores ha sido previamente fijada.

Debido al gran número de iteraciones que hay que realizar para obtener los desplazamientos de fluencia de los disipadores que garantizan que los desplazamientos laterales máximos de la estructura se mantienen en los límites fijados, se decidió automatizar el programa LumpST mediante la aplicación del módulo de optimización de Matlab, para agilizar los cálculos.

Obtenidos los desplazamientos de fluencia de los disipadores, se ha calculado la resistencia lateral de los disipadores de cada una de las plantas de los modelos estructurales estudiados y el coeficiente cortante de fluencia de los disipadores para cada uno de los pórticos. Se ha determinado también, la resistencia lateral aportada por el pórtico principal (sin disipadores) en cada planta, y se ha expresado en forma de coeficiente de fuerza cortante (normalizando por el peso del edificio por encima de la planta considerada). Finalmente se ha obtenido el coeficiente de fuerza cortante total como suma del coeficiente de fluencia cortante aportado por los disipadores de cada planta, y el coeficiente de fuerza cortante desarrollado por el pórtico principal, con el objetivo de comparalo con distribuciones de coeficiente de fuerza cortante óptimos propuestos en la literatura. 


\section{CAPÍTULO 2 \\ ESTADO DEL ARTE}

\subsection{ANTECEDENTES SOBRE CONEXIONES VIGA PLANA-PILAR}

A continuación, se resumen brevemente los principales estudios experimentales llevados a cabo en el pasado sobre conexiones exteriores viga plana-pilar y que han sido publicados en la literatura científica. Debido a que las vigas planas son poco empleadas fuera de España, Italia y parte de Francia, los estudios publicados sobre este tema son muy escasos.

Gentry y Wight [5] ensayaron en 1994 cuatro conexiones exteriores viga plana-pilar bajo cargas cíclicas de tipo estático. Estos autores encontraron que para que su comportamiento sea satisfactorio es fundamental controlar la demanda a torsión en las vigas transversales. Dicho de otra manera, la resistencia y rigidez torsional de las vigas transversales acaban gobernando el comportamiento global de la conexión.

Por su parte, LaFave and Wight [6] ensayaron en 1999, bajo cargas cíclicas estáticas, tres conexiones exteriores viga plana-pilar que cumplían los requisitos de la normativa $\mathrm{ACl}$ 318-95 [7]. Estos autores concluyeron que si dicha norma era satisfecha, las conexiones se comportaban adecuadamente bajo cargas cíclicas. En un estudio posterior (2001) [8], estos mismos autores compararon el comportamiento de las tres conexiones con una conexión convencional viga de canto-pilar y concluyeron que las diferencias de comportamiento no eran sustanciales.

Es muy importante decir, sin embargo, que en todos los estudios experimentales arriba citados, las vigas planas estaban provistas de vigas transversales de canto considerable $\left(h_{s} / h_{b}>>1\right)$ y fuertemente armadas a torsión. Esta circunstancia no se da en las conexiones exteriores viga plana-pilar construidas en el último tercio del siglo XX en el sur de España, en que las vigas transversales solían tener el mismo canto que la viga plana y no se armaban a torsión. Esta diferencia es determinante y hace que las conclusiones de los estudios antes referidos no sean extrapolables a España.

En una investigación más reciente (2007), Benavent-Climent [9] ensayó una conexión viga planapilar con vigas transversales del mismo canto que la viga plana y sin ninguna armadura a torsión. El espécimen se sometió a cargas dinámicas en una mesa sísmica del Laboratorio de Estructuras de EnelHydro-Ismes de Italia, hasta su colapso. Este autor encontró que la capacidad límite última de disipación de energía de estas vigas se situaba entre la mitad y quince veces inferior a la que puede alcanzar una conexión convencional con vigas de canto.

Finalmente hay que señalar, que en ninguna de las investigaciones anteriores se aplicaron cargas gravitatorias adicionales sobre la viga plana (más allá de su propio peso) antes de aplicar las cargas laterales. El nivel de carga gravitatoria sobre la viga, puede influir de forma importante en su capacidad de deformación lateral y conviene por lo tanto ser investigada. 


\subsection{PROYECTO SISMORRESISTENTE BASADO EN LA TEORÍA DE LA ENERGÍA DE HOUSNER-AKIYAMA}

La Fig. 2.2.1 muestra un sistema estructural de un grado de libertad sometido a un movimiento en su base. La ecuación de equilibrio dinámico de fuerzas que gobierna este sistema es la siguiente [15]:

$$
f_{I}(t)+f_{D}(t)+f_{S}(t)=0
$$

Siendo:

$f_{l}(t)$ : Fuerza ficticia denominada fuerza de inercia que resiste la aceleración y que viene dada por la expresión (2.2.2), donde $m$ es la masa y $\ddot{v}$ la aceleración absoluta del sistema. Adoptamos como sentido positivo de $f_{l}$ el que se corresponde con el sentido negativo de los desplazamientos.

$$
f_{I}(t)=m \ddot{v}
$$

$f_{D}(t)$ : Fuerza de amortiguamiento.

$f_{s}(t)$ : Fuerza restauradora del sistema, es decir, la fuerza que opone la estructura a ser deformada.

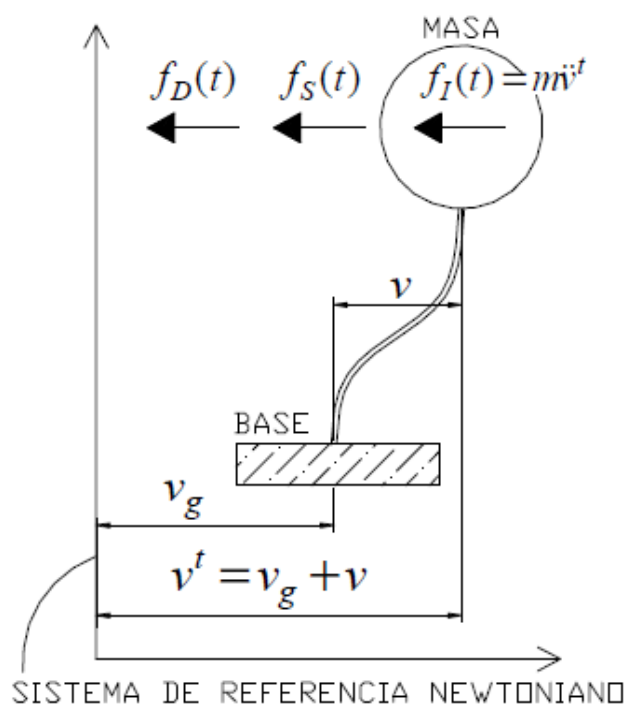

Fig. 2.2.1: Sistema real sometido a un movimiento en su base

Tal como veremos más adelante, a la hora de interpretar la ecuación del balance energético, resulta ventajoso transformar el sistema dinámico real representado en la Fig. 2.2.1 y gobernado por la ecuación (2.2.1), en un sistema dinámico ficticio equivalente que nos proporcione los mismos desplazamientos relativos $v(t)$. Esta transformación se apoya en el hecho de que $f_{D}$ y $f_{S}$ dependen de la velocidad y del desplazamiento relativos del punto másico (y no de los valores totales o absolutos), y que tal como se indica en la Fig. 2.2.1, el desplazamiento total absoluto, $v^{t}(t)$, es la suma del desplazamiento del suelo debido al terremoto, $v_{g}(t)$, y el desplazamiento relativo de la masa respecto al suelo, $v(t)$, con lo que:

$$
\ddot{v}=\ddot{v}_{g}+\ddot{v}
$$


Sustituyendo la ecuación (2.2.3) en (2.2.2), ésta a su vez en (2.2.1), y operando se llega a:

$$
\ddot{m} \ddot{v}+f_{D}(t)+f_{S}(t)=-m \ddot{v}_{g}
$$

La ecuación (2.2.4) proporciona obviamente los mismos desplazamientos relativos $v(t)$ que la ecuación (2.2.1), pero corresponde a un sistema dinámico equivalente al de la Fig. 2.2.1, con la base fija y el punto másico sometido a una fuerza externa ficticia $F_{e}(t)$ de valor $F_{e}(t)=-m \ddot{v_{g}}$, tal como se ilustra en la Fig. 2.2.2. En otras palabras, el desplazamiento relativo $v(t)$ del punto másico respecto a la base cuando ésta se ve sometida a un movimiento sísmico del suelo de aceleración $\ddot{v}_{g}$, es exactamente el mismo que experimentaría esa misma estructura si su base no se moviese y a cambio se viese sometida a una fuerza ficticia de valor $F_{e}(t)=-m \ddot{v}_{g}$, y que llamaremos fuerza sísmica.

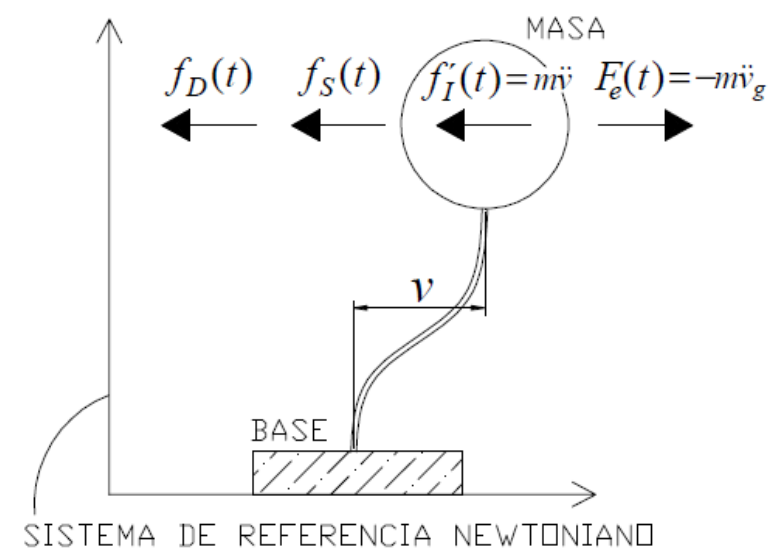

Fig. 2.2.2: Sistema equivalente con base fija, sometido una fuerza ficticia $F_{e}(t)$

Resolviendo la ecuación (2.2.4) se obtiene la magnitud más importante de la respuesta de la estructura que es el desplazamiento relativo $v(t)$. Conocido $v(t)$, la ecuación de equilibrio dinámico de fuerzas se puede transformar en una ecuación de balance de energía tal y como se expone a continuación.

Multiplicando los dos miembros de la ecuación (2.2.4) por un incremento diferencial de desplazamiento relativo $\mathrm{dv}$, o lo que lo mismo por $d v=\dot{v} d t$, se llega a:

$$
m \ddot{v} \dot{v} d t+f_{D}(t) \dot{v} d t+f_{S}(t) \dot{v} d t=-m \ddot{v} \dot{v} d t
$$

Si ahora integramos la ecuación (2.2.5) desde el instante $t=0$ en que comienza el movimiento del suelo, hasta el instante $t_{0}$ en que termina el terremoto, se obtiene:

$$
\underbrace{\int_{0}^{t_{0}} m \ddot{v} \dot{v} d t}_{W_{e k}}+\underbrace{\int_{W_{e s}}^{t_{0}} f_{D}(t) \dot{v} d t}_{W_{D}}+\underbrace{\int_{0}^{t_{0}} f_{S}(t) \dot{v} d t}_{W_{S}}=\underbrace{\int_{0}^{t_{0}}-m \ddot{v} \dot{v} d t}_{W_{E}}
$$


La primera integral del primer miembro de (2.2.6) se puede resolver directamente. Si además asumimos que en el instante $t=0$ la velocidad relativa de la estructura es nula, $\dot{v}(0)=0$, la solución de dicha integral conduce a la siguiente expresión que representa la energía cinética del sistema en el instante $t_{0}$, y que designaremos con $W_{e k}$.

$$
W_{e k}=\int_{0}^{t_{0}} m \ddot{v} \dot{v} d t=\frac{m \dot{v}^{2}\left(t_{0}\right)}{2}
$$

La segunda integral del primer miembro de (2.2.6) representa la energía consumida por la estructura mediante amortiguamiento y se designa por $W_{D}$, es decir:

$$
W_{D}=\int_{0}^{t_{0}} f_{D}(t) \dot{v} d t
$$

La tercera integral del primer miembro de (2.2.6) representa la energía de deformación total almacenada y/o disipada por la estructura hasta el instante $t_{0}$ que designaremos por $W_{S}$. Dicha $W_{S}$ es la suma de la energía almacenada por la estructura mediante deformaciones elásticas, $W_{e s}$, y la energía disipada por la misma mediante deformaciones plásticas, $W_{p}$, es decir:

$$
W_{S}=\int_{0}^{t_{0}} f_{S}(t) \dot{v} d t=W_{e s}+W_{p}
$$

El término $W_{p}$ representa de forma cuantitativa el daño estructural inducido en la estructura por el terremoto. La suma de $W_{e k}$ y $W_{e s}$ constituye lo que se denomina energía de vibración elástica del sistema que designaremos con $W_{e}$, es decir:

$$
W_{e}=W_{e k}+W_{e s}
$$

Por último, la integral del segundo miembro de (2.2.6) representa el trabajo total realizado por la fuerza sísmica $F_{e}(t)$ hasta el instante $t_{0}$ en que termina el terremoto, asociado al desplazamiento relativo $v(t)$ de la estructura. Dicho trabajo se define como el input de energía total introducida por el terremoto en la estructura, y emplearemos para designarlo la letra $E$, es decir:

$$
E=\int_{0}^{t_{0}}-m \ddot{\nu_{g}} \dot{v} d t
$$

Sustituyendo las ecuaciones (2.2.7), (2.2.8), (2.2.9) y (2.2.11) en (2.2.6) y teniendo en cuenta (2.2.10), se llega a la siguiente expresión, que se conoce como la ecuación del balance energético de la estructura en el instante $t_{0}$ en que termina el terremoto.

$$
W_{e}+W_{D}+W_{p}=E
$$

El primer miembro de la ecuación (2.2.12) se puede interpretar como la capacidad sismorresistente de la estructura y el segundo como el efecto de carga del terremoto, ambos expresados en términos de energía. La expresión (2.2.12) constituye la ecuación fundamental de la denominada metodología de proyecto sismorresistente basada en el balance energético de Housner-Akiyama. El criterio de proyecto fundamental en dicha metodología consiste en hacer que el primer miembro de la 
ecuación (2.2.12) sea mayor o igual que el segundo miembro. Ello se podrá lograr con relativa sencillez debido a que, la capacidad sismorresistente de la estructura y el efecto de carga de terremoto son dos aspectos que pueden definirse de forma básicamente independiente.

La condición que establecen estos métodos para que la estructura sobreviva al terremoto es que el primer miembro tendrá que ser mayor o igual que el segundo, es decir:

$$
W_{e}+W_{D}+W_{p} \geq E
$$

Para que la estructura sobreviva al sismo manteniéndose dentro del dominio elástico, la energía de vibración elástica $W_{e}$ que debe ser capaz de almacenar la estructura debe ser:

$$
W_{e} \geq\left(E-W_{D}\right)
$$

Esto se consigue dando resistencia (en sentido estricto) a la estructura.

Para que la estructura sobreviva al sismo permitiendo deformaciones plásticas en la misma, la energía de deformación plástica, $W_{p}$, que la estructura en su totalidad debe ser capaz de disipar hasta el instante en que se produce el colapso deberá ser:

$$
W_{p} \geq E-W_{D}-W_{e}
$$

Las ecuaciones anteriores junto con el control de los desplazamientos laterales de la estructura, forman un marco teórico en base al cual se pueden plantear distintas estrategias de proyecto sismorresistente (convencionales y avanzadas).

Los disipadores de energía aparecen pues como dispositivos capaces de mejorar las capacidades en el rango plástico de las construcciones, absorbiendo gran parte de esta energía $W_{p}$, o incluso toda, de forma que la estructura principal quedaría intacta tras un evento severo. 


\subsection{REACONDICIONAMIENTO SÍSMICO DE ESTRUCTURAS CON DISIPADORES DE ENERGÍA}

\subsubsection{Definición de disipador de energía}

Son elementos (pasivos) relativamente ajenos a la estructura (en el sentido en que no participan en la resistencia a cargas verticales y son reemplazables una vez dañados) que tienen como misión absorber energía al deformarse; para ello deben instalarse en lugares en que se produzcan deformaciones (apreciables) debidas a la acción que se desea controlar.

Los disipadores deben dimensionarse para que los daños se concentren en éstos antes que en la estructura, es decir, actúan como fusibles estructurales.

Se utilizan principalmente para reducir los daños generados en edificios por movimientos sísmicos pero también pueden ser útiles para amortiguar las oscilaciones debidas al viento.

Los disipadores responden a la filosofía del diseño por capacidad llevada a sus últimas consecuencias ya que el modo de colapso no solamente es dúctil sino que los daños se producen sólo en elementos ajenos a la estructura principal (y fácilmente reemplazables).

Los disipadores se pueden utilizar en los casos en que el aislamiento de base no resulta aplicable (viento, suelo blando y edificios altos y/o flexibles).

Los disipadores pueden ser más simples que los aisladores ya que no precisan soportar el peso del edificio; son más fáciles de instalar que éstos (especialmente en edificios existentes) lo que los hace particularmente convenientes para su uso masivo en países en desarrollo.

\subsubsection{Colocación de los disipadores de energía}

Los disipadores de energía, hay que colocarlos de tal forma que se produzcan desplazamientos relativos en todos los puntos. Siendo más sencillo situarlos en edificios de pórticos.

Si los disipadores se colocan como complemento de aisladores, deben estar preparados para soportar grandes desplazamientos; en caso contrario, las deformaciones a absorber son pequeñas.

Estos elementos se colocan en las estructuras porticadas como una barra estándar diagonal como se muestra en la Fig. 2.3.1 [19].
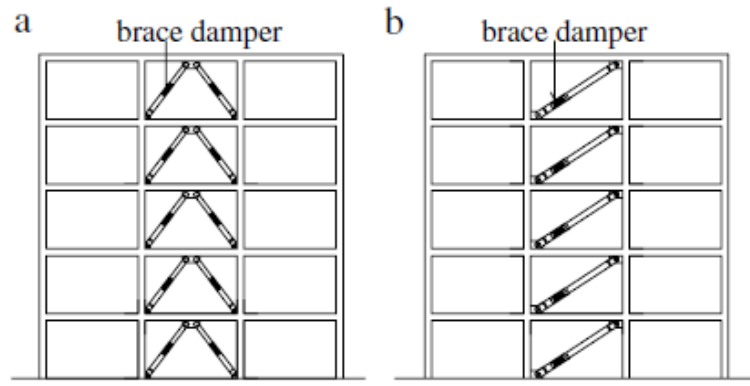

Fig. 2.3.1: Colocación de disipadores de energía en estructuras porticadas 
Distribución en planta:

Se trata de arriostramientos (aunque sean disipativos) y por ello deben satisfacer los requerimientos habituales de éstos:

- Debe haber al menos dos arriostramientos en cada dirección, situados simétricamente y lo más alejados que sea posible.

- Redundancia en cada dirección (para sismos fuertes).
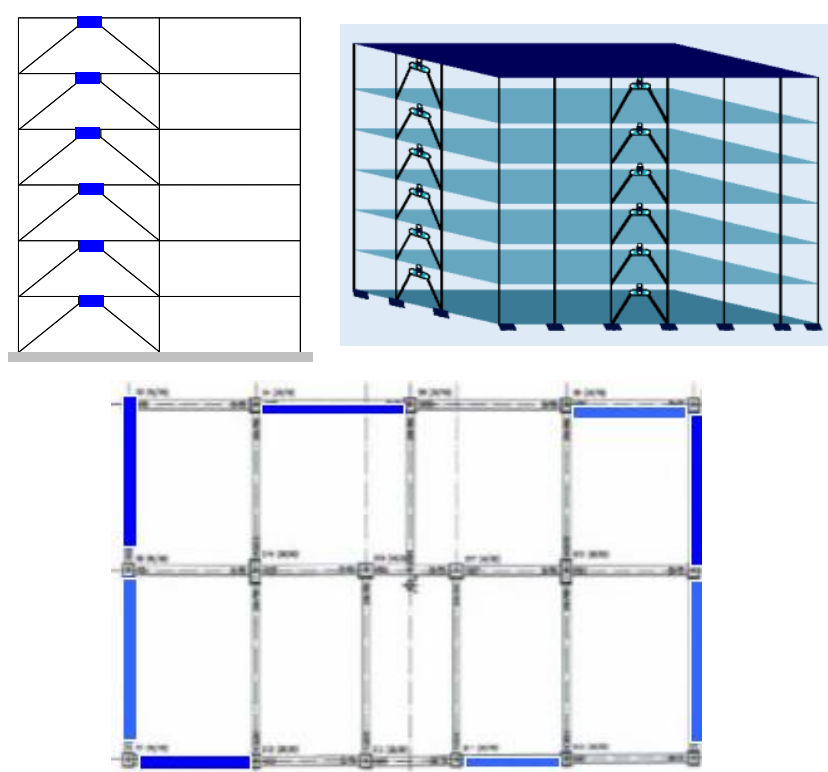

Fig. 2.3.2: Distribución en planta de los disipadores

\subsubsection{Utilidad de los disipadores de energía en edificios}

Los disipadores de energía se utilizan para dos finalidades: 1) para proteger a la estructura de los efectos del viento y 2) para protegerla del sismo. Éstos actúan como fusibles estructurales, es decir, se trata de lograr un mecanismo de colapso en el que, cuando la estructura se vea afectada por uno de estos efectos anteriormente citados, no produzcan daños importantes en los elementos estructurales siendo los disipadores los que se vean dañados.

Si comparamos los tres ejemplos de organizaciones estructurales, descritos en la Fig. 2.3.3, como son: pórtico desnudo o flexible; pórtico protegido con disipadores y pórtico arriostrado con uniones rígidas. Veremos en el espectro elástico de respuesta, Fig. 2.3.5 y 2.3.6, cuáles de ellos son más convenientes.
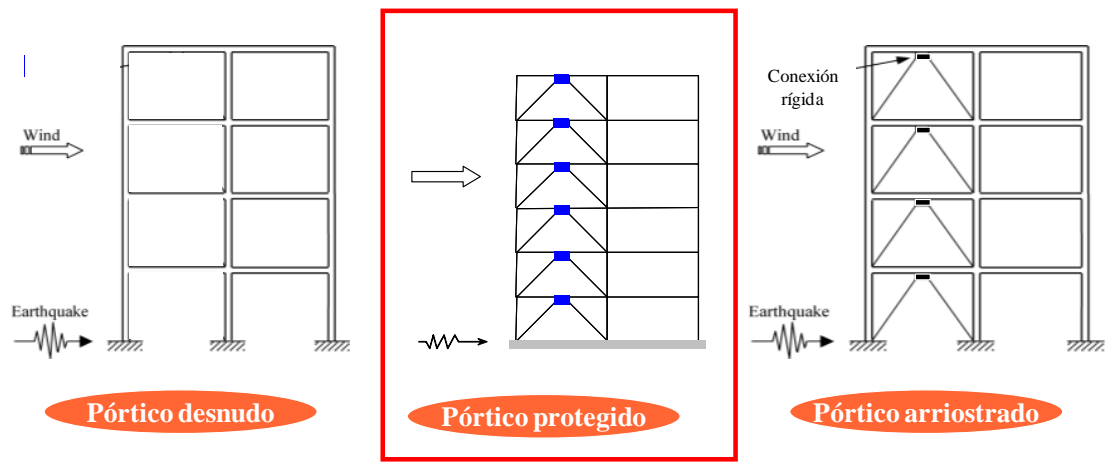

Fig. 2.3.3: Ejemplos de organizaciones estructurales 
- Para edificios con periodos de vibración altos, el pórtico arriostrado, se ve sometido a una acción sísmica mayor pero tiene más resistencia. En el pórtico protegido con disipadores, se ha incrementado un poco la rigidez, también se aprecia que no se ha producido un cambio importante en el periodo de vibración con respecto al pórtico desnudo, sin embargo el amortiguamiento ha aumentado.

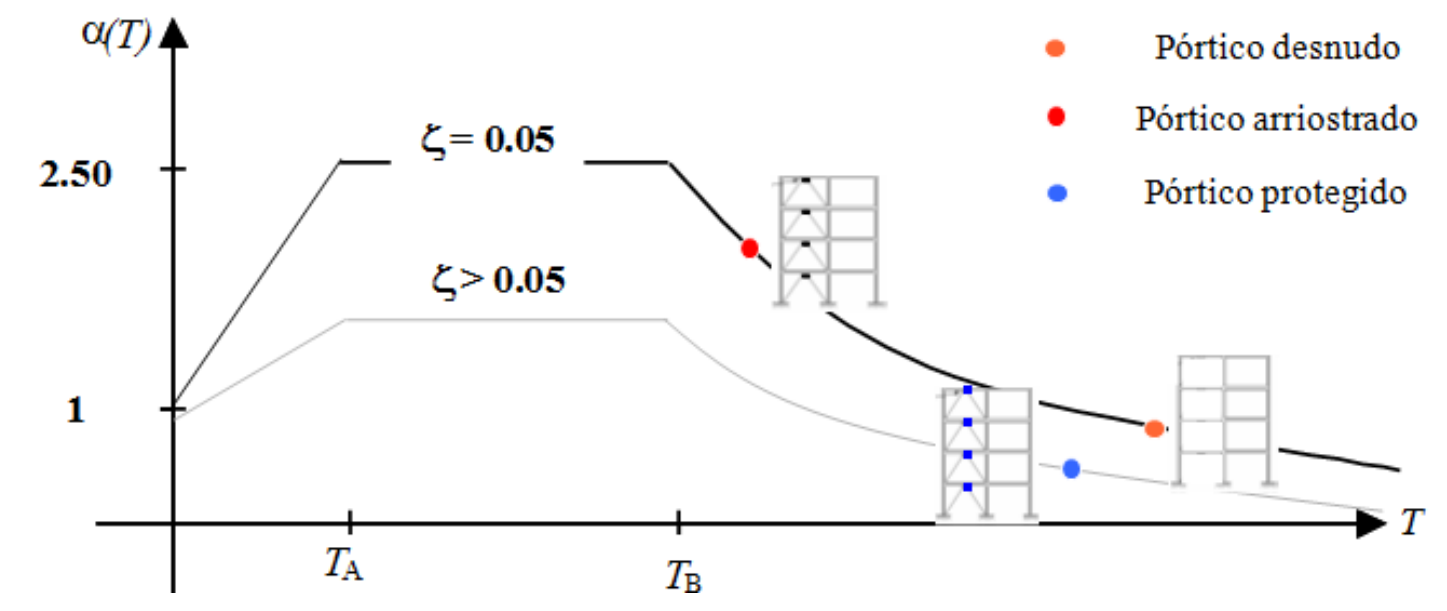

Fig. 2.3.4: Espectros elásticos de respuesta. Comparación para edificios con periodos de vibración altos (flexibles)

- Para edificios de periodos de vibración cortos, rigidizar es beneficioso si no aumenta la fuerza pero sí la resistencia. El pórtico desnudo es el que mayor fuerza tiene y la misma rigidez que el pórtico protegido con disipadores, siendo el pórtico arriostrado el que presenta una mayor rigidez.

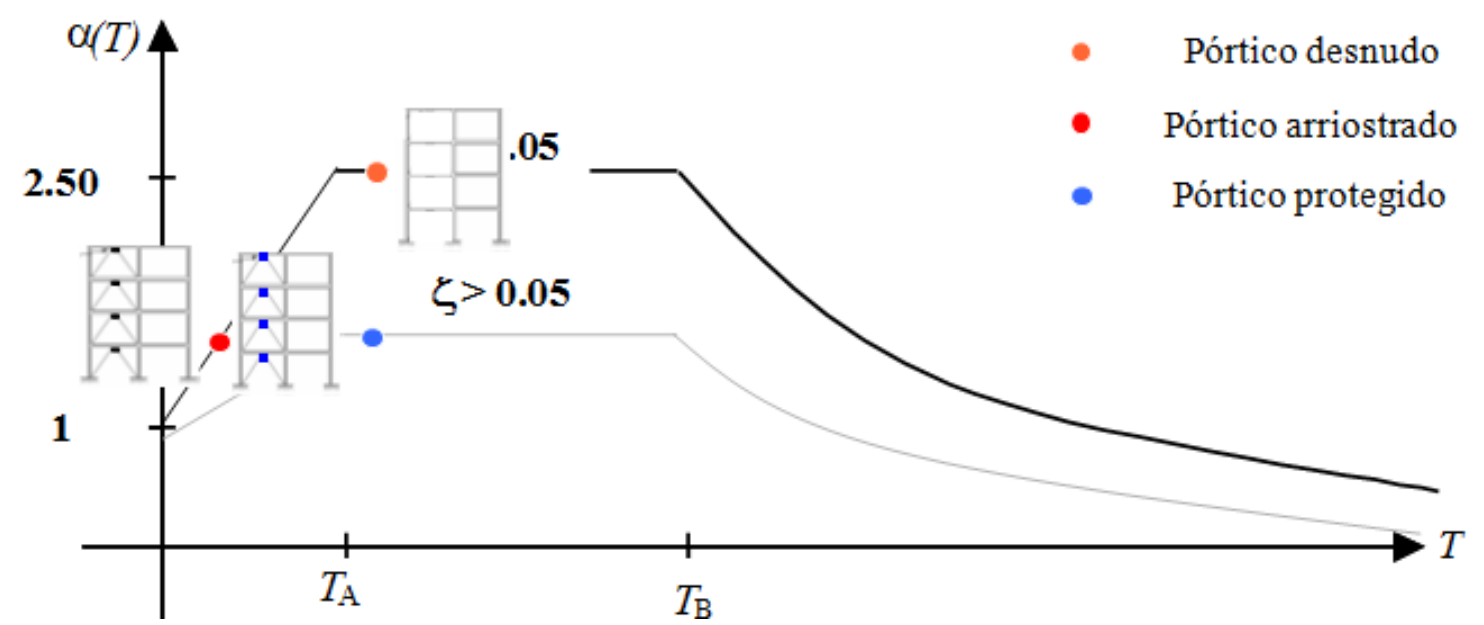

Fig. 2.3.5: Espectros elásticos de respuesta. Comparación para edificios de periodos de vibración cortos (rígidos) 


\subsubsection{Cualidades exigibles a los disipadores de energía}

Son diversas las cualidades exigibles a los disipadores de energía, algunas de ellas las citamos a continuación: a los disipadores de energía se les exige una alta capacidad de disipación de energía por ciclo y total; los ciclos de histéresis deben ser estables y estar bien caracterizados; se debe producir el inicio de la disipación de energía para pequeños desplazamientos de la estructura a proteger; también es importante la simplicidad, economía así como el bajo mantenimiento; la instalación deberá ser sencilla y con bajo impacto arquitectónico; otras cualidades exigibles a los disipadores son la robustez, durabilidad, fiabilidad y facilidad de sustitución en caso de daño.

\subsubsection{Tipos de elementos disipadores de energía}

Los disipadores de energía tienen una historia de unos 35 años y se empezaron a emplear en instalaciones de centrales nucleares. Su desarrollo y aplicación en la ingeniería sísmica de estructuras comenzó fundamentalmente en la última década del siglo pasado y su crecimiento está siendo exponencial. Los disipadores de energía forman parte de los denominados sistemas de control pasivo de estructuras. Estos sistemas disipan energía de distintas formas: 1) cambiando la microestructura de un metal y aumentando su temperatura "disipadores histeréticos"; 2) deformando materiales especiales con elevados niveles de amortiguamiento intrínseco o aumentando la temperatura de fluidos "disipadores viscosos, viscoelásticos o fluídos"; o 3) transfiriendo parte de la energía de vibración a una masa auxiliar (un bloque de acero, hormigón, o fluído) sintonizada con la estructura principal "amortiguador de masa".

Los disipadores de energía de tipo histerético suelen ser los más empleados en aplicaciones sísmicas por su bajo coste y sencillez, y su funcionamiento puede basarse en la 1) plastificación, 2) fricción, 3) extrusión, o 4) corte de metales. Los basados en la plastificación o fricción son especialmente populares. Dentro de los primeros existen múltiples variantes. Unos emplean chaspas de acero en forma de $\mathrm{X}$ o triangular; es el caso del conocido ADAS (added damping and stiffness) (Fig. 2.3.6). Otros, especialmente empleados en Japón, utilizan chapas perforadas de acero (honeycomb o slit plates) (Fig. 2.3.7). Además del acero, se han investigado también otros materiales como el plomo o aleaciones especiales con memoria de forma (shape-memory allows). Una tercera variante de disipador histerético cuyo uso ha crecido notablemente en los últimos años, son las barras de pandeo restringido BRB (buckling restrained brace) que se instalan como si fueran barras diagonales convencionales (Fig. 2.3.8). Consiste en un perfil de acero que se hace plastificar bajo deformaciones axiles de tracción/compresión, y para evitar su pandeo se embebe dentro de un tubo metálico relleno de hormigón. Las figuras 2.3.9 y 2.3.10 muestran otros dos nuevos disipadores desarrollados recientemente por Benavent en la Universidad de Granada, que se pueden instalar también en la estructura como barras diagonales convencionales. El primero, disipador TTD (tube in tuve damper) se basa en la plastificación de las paredes de tubos de acero, y el segundo WPD (web plastification damper) en la plastificación del alma de perfiles IPE o HBE. [15] 

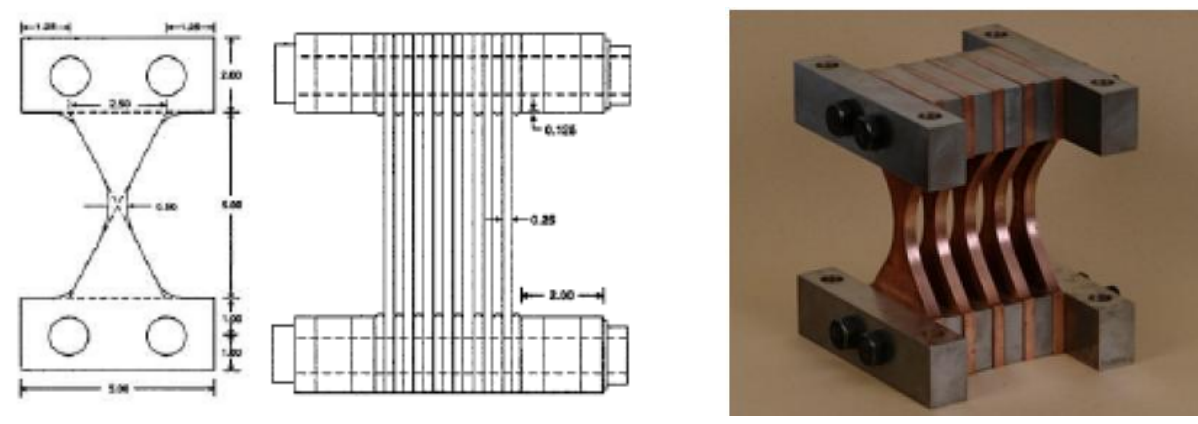

Fig. 2.3.6: Disipador ADAS
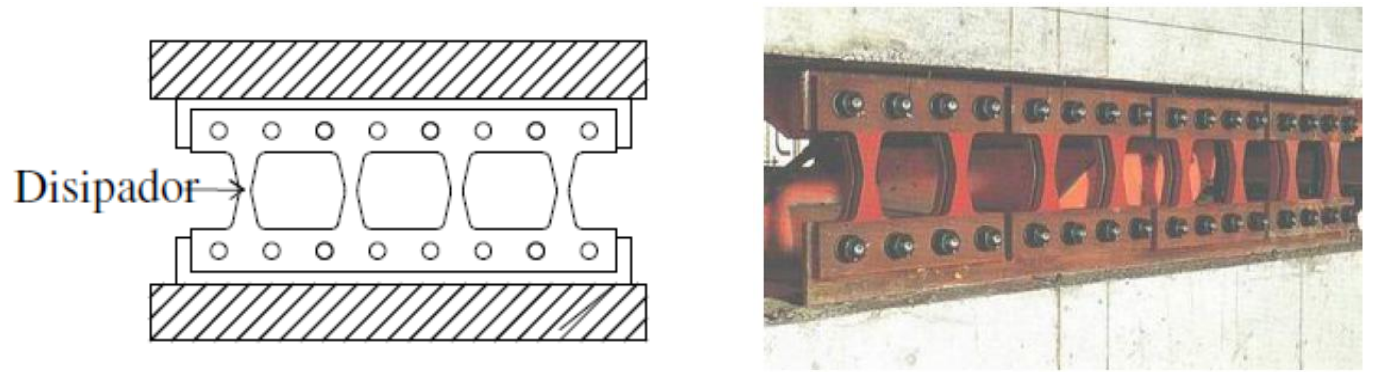

Fig. 2.3.7: Disipador honeycomb plate
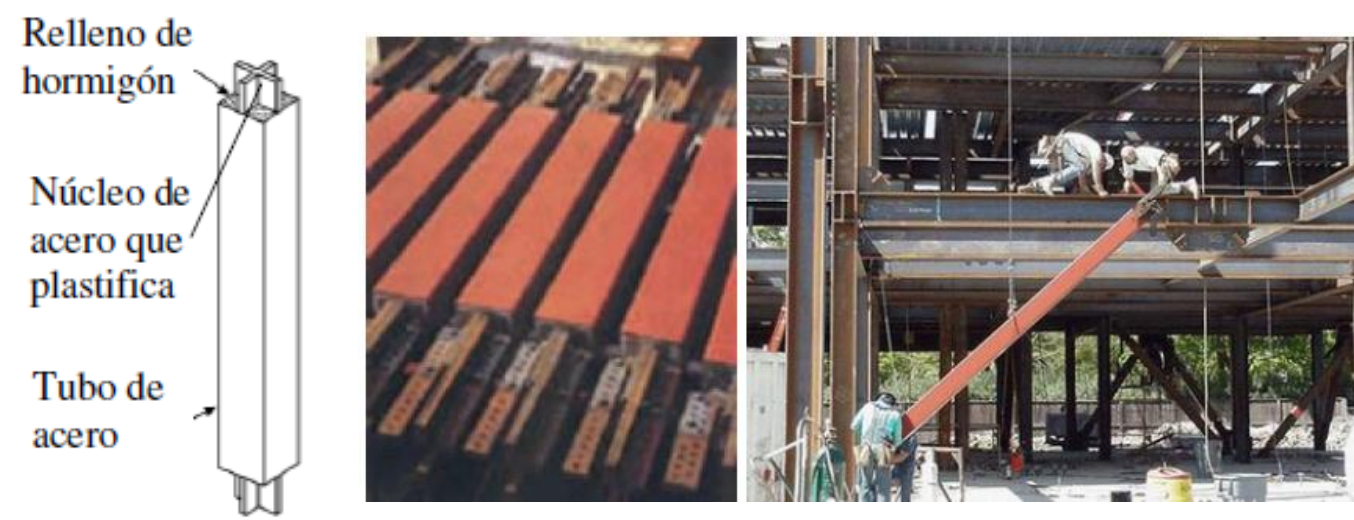

Fig. 2.3.8: Disipador BRB

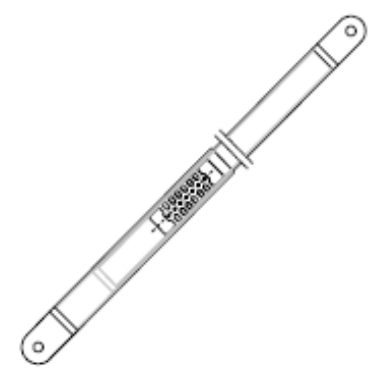

Fig. 2.3.9: Disipador TTD

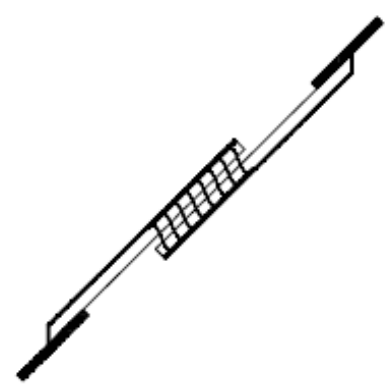

Fig. 2.3.10: Disipador WPD 


\section{CAPÍTULO 3 \\ DETERMINACIÓN DE PROTOTIPOS DE ESTRUCTURAS EXISTENTES DE HORMIGÓN ARMADO CONSTRUIDOS EN LOS AÑOS 70-90 EN ESPAÑA}

Los prototipos de estructuras porticadas con vigas planas a investigar se tomaron de un trabajo previo realizado por R. Zahran ("Predicción de la capacidad límite última de disipación de energía de estructuras porticadas con vigas planas existentes", Tesis Doctoral, Universidad de Granada, 2009). En este capítulo se describen sus características y criterios de proyecto.

\subsection{DEFINICIÓN DE LOS PROTOTIPOS DE PÓRTICOS CON VIGAS PLANAS}

A la hora de definir los prototipos de estructuras porticadas con vigas planas a estudiar, se ha elegido la zona de España de mayor sismicidad, tanto con la antigua norma PDS-74 [1] como con la actual norma NCSE-02 [2]. Se trata de la región de Granada clasificada por la PDS-74 como zona de intensidad sísmica "tercera", y a la que la NCSE-02 asigna una aceleración sísmica de cálculo que varía desde $0.16 \mathrm{~g}$ hasta $0.25 \mathrm{~g}$ (siendo g la aceleración de la gravedad), tal como se indica en la Fig. 3.1.1, tomada de esta norma.

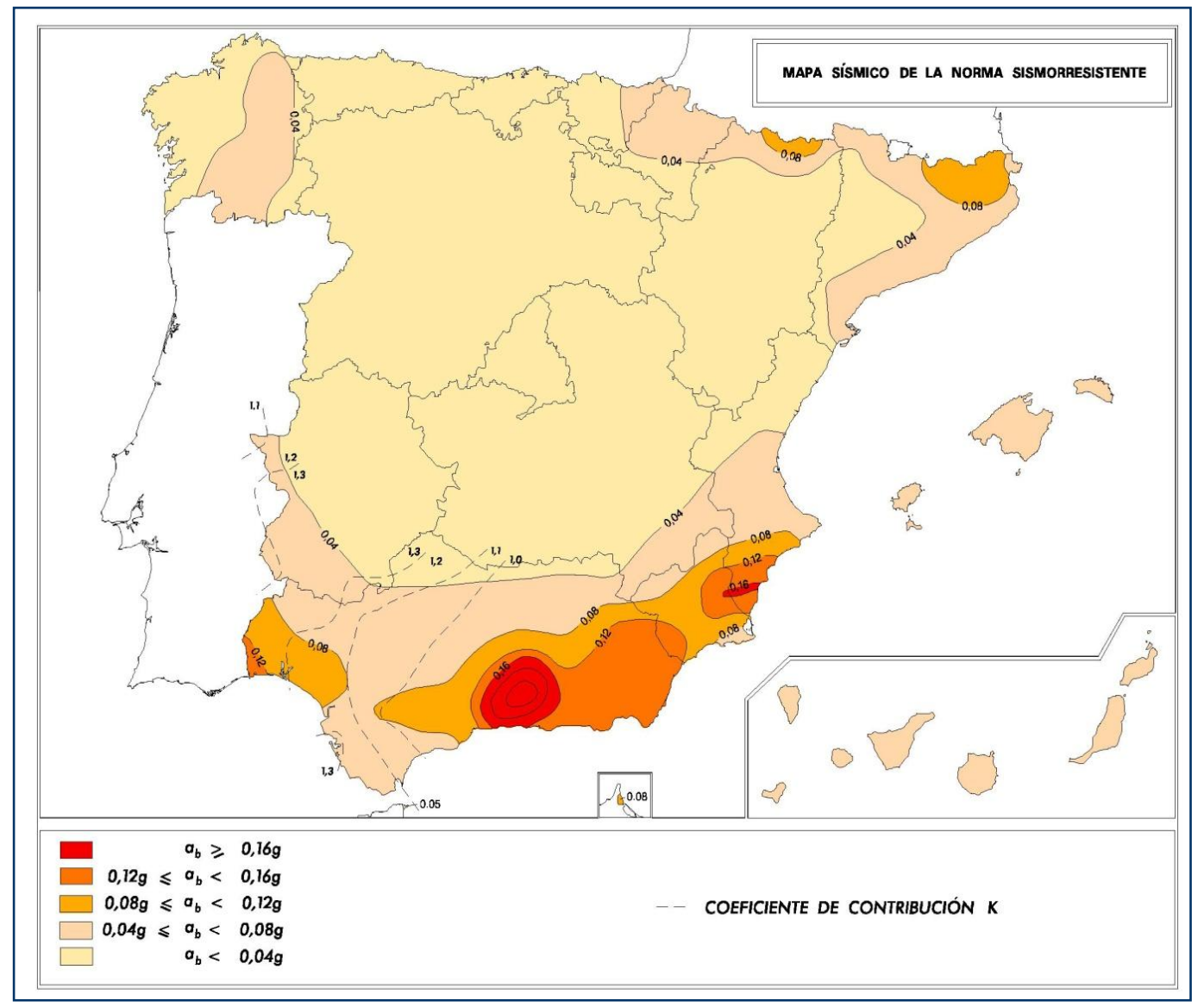

Fig. 3.1.1: Mapa de sismicidad de la norma NCSE-02 
Las características básicas de las estructuras de hormigón armado con vigas planas construidas entre 1974 y 1994 (luces, crujías, etc.) se han obtenido de consultas a arquitectos con amplia experiencia profesional en la región de Granada, de consultas a técnicos de entidades de control y mediante trabajo de campo. Las figuras 3.1.2, 3.1.3 y 3.1.4 muestran ejemplos de edificios resueltos con estructuras porticadas con vigas planas en la ciudad de Granada.

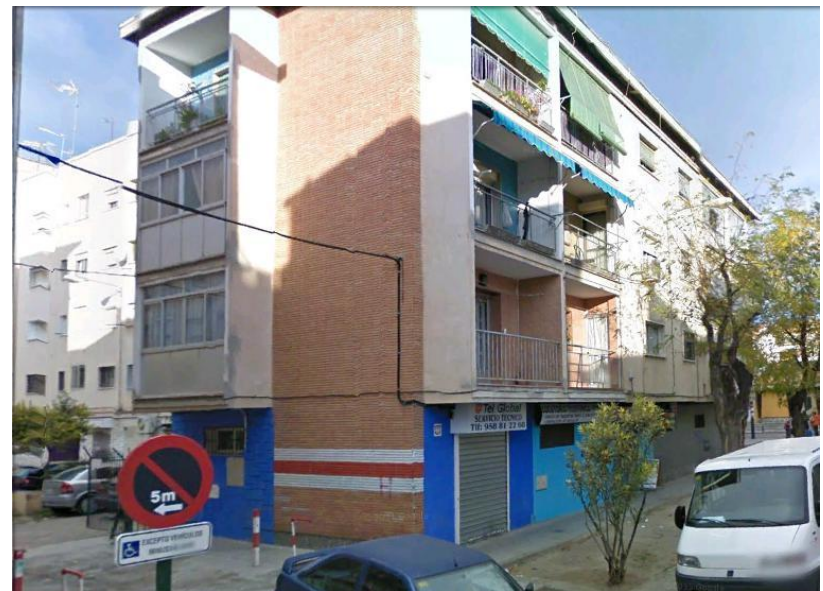

Fig. 3.1.2: Edificio de 3 plantas, Zaidin, Plaza de las Palomas (Granada)

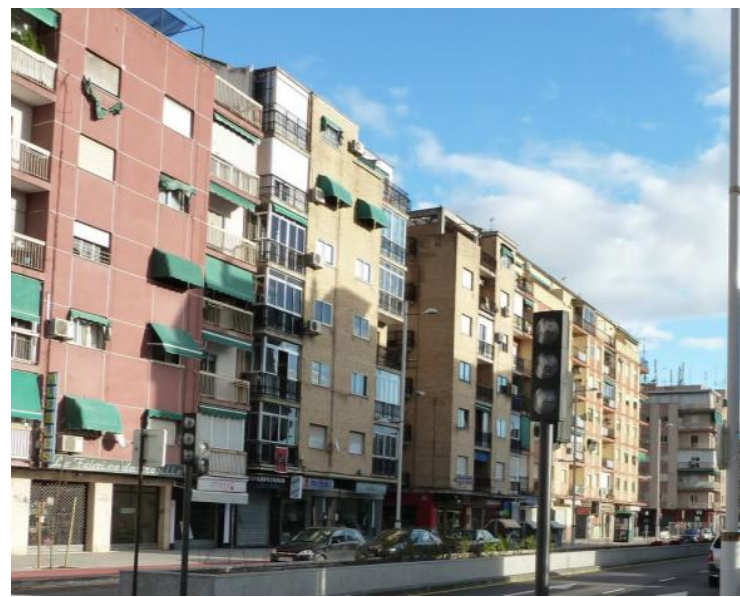

Fig. 3.1.3: Edificios de 6 plantas, Camino de Ronda (Granada)

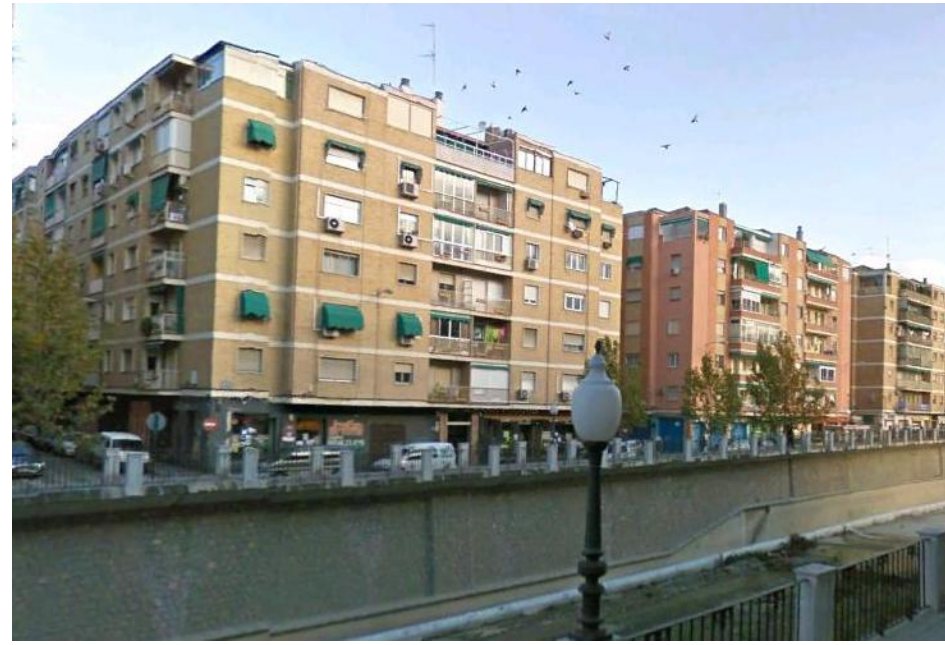

Fig. 3.1.4: Edificios de 6 plantas, Ribera del Violón (Granada)

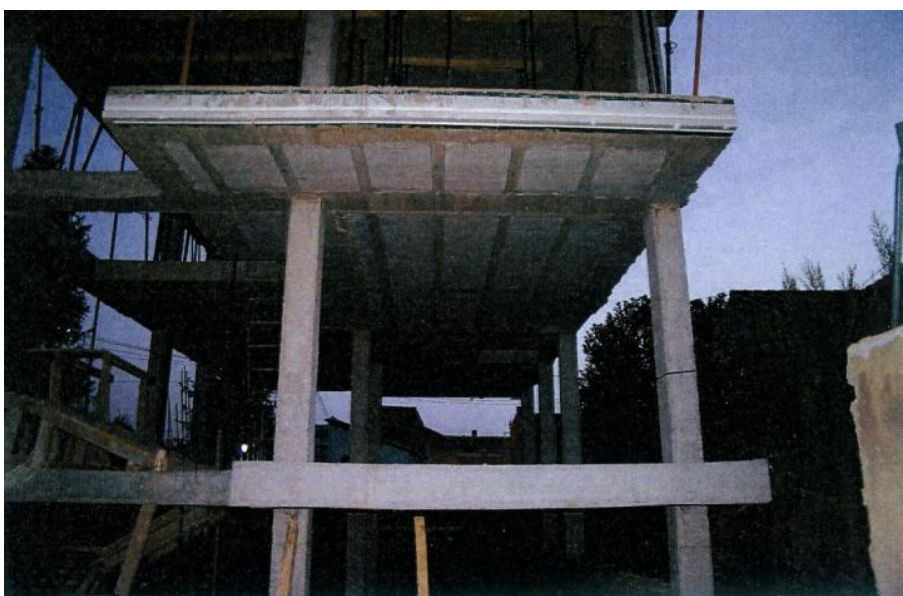

Fig. 3.1.5: Estructura porticada con vigas planas 
Estas características genéricas se resumen en la Tabla 3.1.1. A partir de esta información se definieron los tres prototipos de estructuras con vigas planas que se indican en la Tabla 3.1.2. Todos tiene 4 crujías y 4 vanos, y las plantas son doblemente simétricas. Los pórticos con vigas planas encargados de soportar las cargas gravitatorias de los forjados se disponen paralelamente en una dirección, en la cual se ha considerado aplicado el sismo. Debido a la simetría en planta de las estructuras, cada prototipo dio lugar a dos o tres pórticos distintos (en dimensiones y/o armado), unos exteriores que se han denominado pz1, y otros interiores que se han llamado pz2 o pz3. Los tres pórticos que se han definido, se han denominado: pa2pz2-1, pa4pz2-1 y pa6pz2-1. Su geometría se muestra en las figuras 3.1.6 a 3.1.8.

Tabla 3.1.1: Características de las estructuras existentes con vigas planas construidas

\begin{tabular}{|c|c|}
\hline Número de plantas & Oscilaba entre 1 y 6 plantas. \\
\hline Luces & Oscilaban entre los 5 y 6 metros. \\
\hline Canto del forjado & Oscilaba entre 27 y $30 \mathrm{~cm}$. \\
\hline $\begin{array}{l}\text { Número de vanos y } \\
\text { crujias }\end{array}$ & Variable, siendo frecuentes las 3 ó 4 crujías y vanos. \\
\hline Dimensiones de pilares & $\begin{array}{l}\text { Frecuentemente la sección transversal de los pilares se igualaba cada dos } \\
\text { o tres plantas consecutivas, siendo de } 30 \times 30 \mathrm{~cm} \text { en las dos últimas } \\
\text { plantas, y aumentando con incrementos de } 5010 \mathrm{~cm} \text { hacia las plantas } \\
\text { inferiores. }\end{array}$ \\
\hline Altura de plantas & $\begin{array}{l}\text { La altura de pilares (a ejes de vigas) estaba en torno a los } 3 \mathrm{~m} \text {, en todas } \\
\text { las plantas, excepto en la planta baja que oscilaba sobre los } 4.5 \mathrm{~m} \text {. }\end{array}$ \\
\hline Existencia de pantallas & Para edificios de hasta 6 plantas no se empleaban pantallas. \\
\hline $\begin{array}{l}\text { Propiedades mecánicas } \\
\text { de los materiales }\end{array}$ & $\begin{array}{l}\text { Resistencia característica del hormigón: } 175 \mathrm{kp} / \mathrm{cm}^{2} \\
\text { Resistencia característica del acero } 4100 \mathrm{kp} / \mathrm{cm}^{2} \text {. }\end{array}$ \\
\hline $\begin{array}{l}\text { Detalles constructivos y } \\
\text { organización de las } \\
\text { armaduras }\end{array}$ & $\begin{array}{l}\text { No se empleaban vigas transversales de canto en las conexiones } \\
\text { exteriores viga plana-pilar, sino simples zunchos de borde del mismo } \\
\text { canto de la viga, dimensionados para soportar únicamente las cargas } \\
\text { gravitatorias debidas al peso propio del zuncho y del cerramiento. } \\
\text { La armadura longitudinal, tanto inferior como superior, se colocaba } \\
\text { normalmente en una sola capa. Para vigas de ancho aproximado } 50 \mathrm{~cm} \text {, } \\
\text { era frecuente colocar como armadura de montaje } 4 \text { barras continuas en la } \\
\text { cara inferior y } 3 \text { en la cara superior, de diámetros de } 16 \mathrm{~mm} \text { o } 20 \mathrm{~mm} \text {. }\end{array}$ \\
\hline
\end{tabular}

Tabla 3.1.2: Prototipos de las estructuras a estudiar

\begin{tabular}{|c|c|c|c|c|c|}
\hline Prototipo & $\begin{array}{c}\text { No de plantas } \\
(N)\end{array}$ & $\begin{array}{c}\text { Luz de vigas } \\
L\end{array}$ & $\begin{array}{c}\text { Luz de crujía } \\
L^{\prime}\end{array}$ & $\begin{array}{c}\text { Canto de vigas } \\
(h)\end{array}$ & Pilares \\
\hline pa2 & 3 & 5 & 5 & 27 & Cuadrados \\
\hline pa4 & 3 & 5,50 & 5,50 & 30 & Cuadrados \\
\hline pa6 & 6 & 5 & 5 & 27 & Cuadrados \\
\hline
\end{tabular}




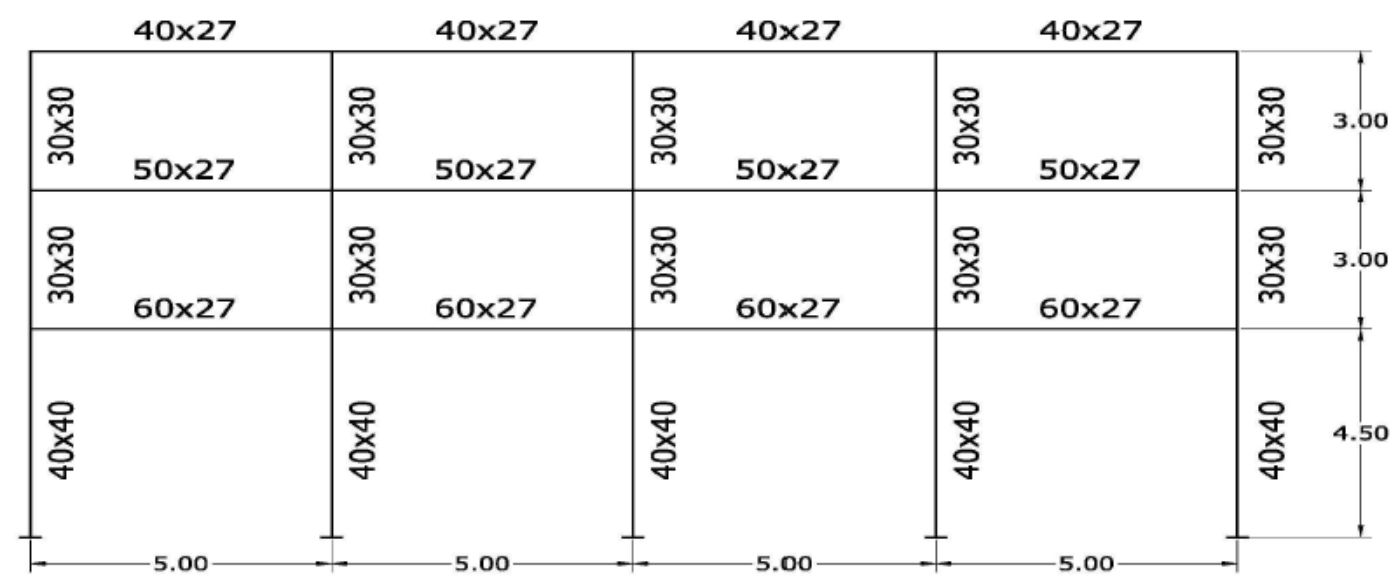

Fig. 3.1.6: Pórtico pa2pz2-1

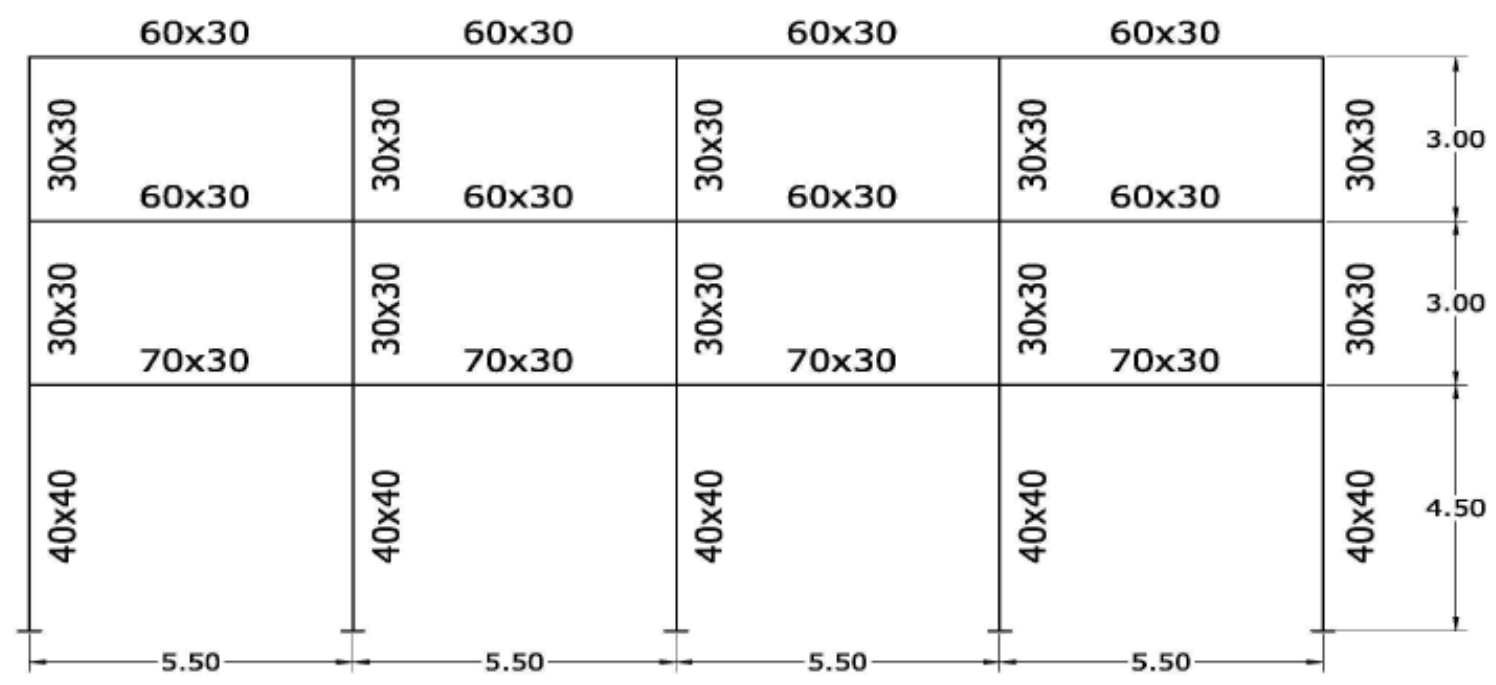

Fig. 3.1.7: Pórtico pa4pz2-1 


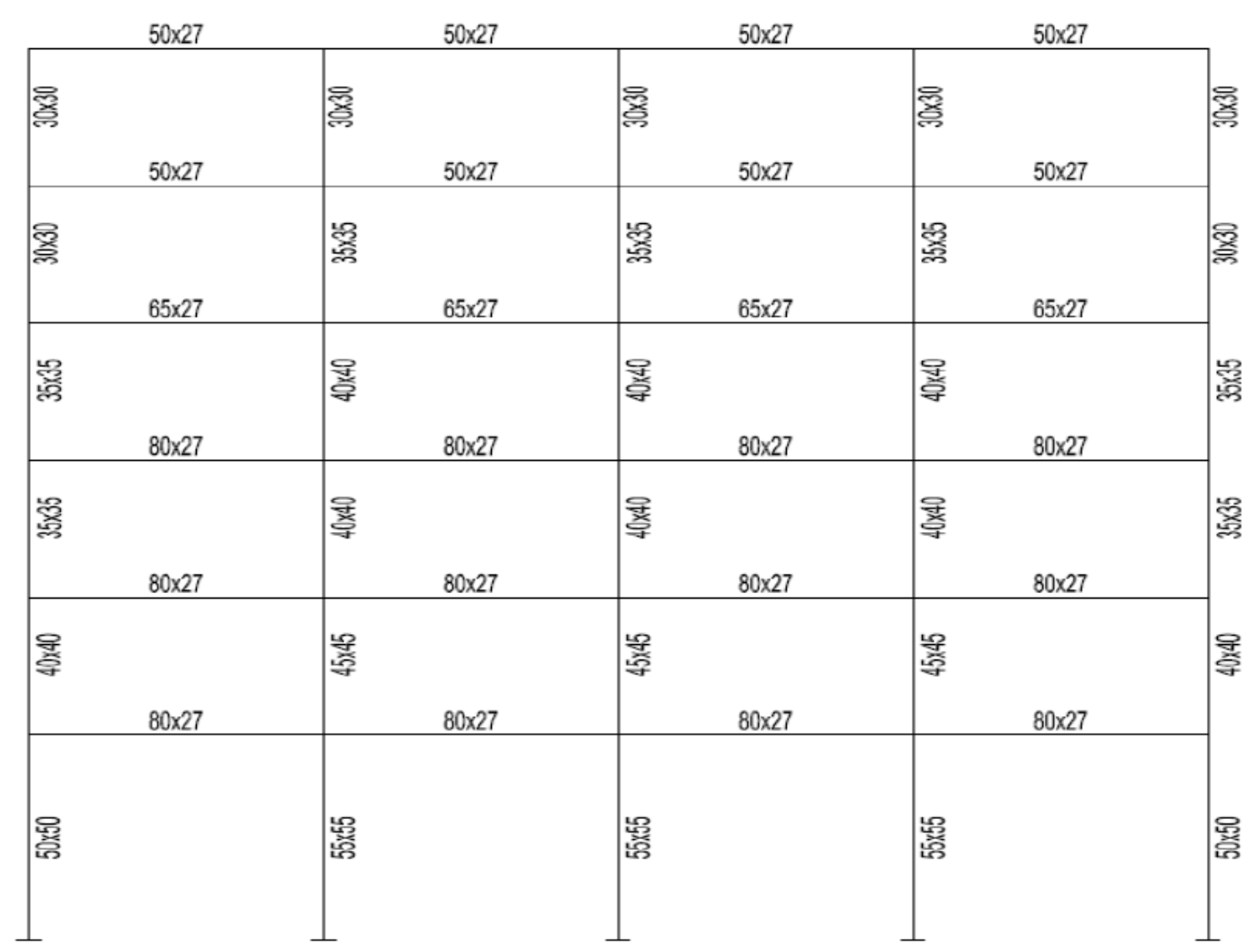

Fig. 3.1.8: Pórtico pa6pz2-1

\subsection{PARÁMETROS DE PROYECTO Y NORMATIVA EMPLEADA}

Las vigas y los pilares de los pórticos se dimensionaron y armaron de acuerdo con la Instrucción para el Proyecto y Ejecución de Obras de Hormigón Armado EH-91 [10]. La acción sísmica se caracterizó mediante un sistema de fuerzas horizontales que se calcularon con la norma PDS-74 [1], suponiendo las estructuras situadas en Granada, sobre un terreno de arcilla dura, y estando destinadas al uso de vivienda. Se supuso que los forjados eran indeformables en su plano. El valor de las cargas gravitatorias consideradas fue de $3.45 \mathrm{KN} / \mathrm{m}^{2}$ para el peso propio del forjado, $0.80 \mathrm{KN} / \mathrm{m}^{2}$ para las cargas debidas al solado y falsos techos, $1 \mathrm{KN} / \mathrm{m}^{2}$ para la carga de tabiquería y $2 \mathrm{KN} / \mathrm{m}^{2}$ como sobrecarga de uso. Para los materiales se adoptó una resistencia característica de $17.5 \mathrm{~N} / \mathrm{mm}^{2}$ para el hormigón, y de $400 \mathrm{~N} / \mathrm{mm}^{2}$ como límite elástico del acero para las armaduras. Los pórticos se dimensionaron con el programa informático CYPECAD versión 2008.1.c. 


\section{CAPÍTULO 4}

\section{SELECCIÓN DE ACELEROGRAMAS}

\subsection{INTRODUCCIÓN}

En este Capítulo se exponen las características de los acelerogramas empleados en los cálculos dinámicos, así como el criterio empleado para escalarlos.

Inicialmente se seleccionaron 18 acelerogramas, históricos europeos de la base de sismos europea [3].

Los acelerogramas se agruparon en tres tipos de suelo: tipo I que corresponde a suelo muy duro caracterizado por una velocidad de las ondas de corte superiores a $750 \mathrm{~m} / \mathrm{s}$; suelo tipo II que corresponde a suelo duro caracterizado por una velocidad de las ondas de corte entre 375 y $750 \mathrm{~m} / \mathrm{s}$; y suelo tipo III que corresponde a suelo medio caracterizado por una velocidad de las ondas de corte entre 175 y 375 m/s. Dentro de cada tipo de suelo los sismos se agruparon en dos clases: cercanos a falla y lejanos a falla, en función de que la distancia a la misma fuese menor o mayor de $10 \mathrm{~km}$, respectivamente.

Los terremotos se han escalado a la aceleración máxima del suelo (PGA) establecida por la norma sísmica española, (NCSE-02, capítulo II, 2.2) [2] para Granada, cuyo cálculo se detalla en el apartado 4.1.

En el apartado 4.2 se describen las características de los acelerogramas inicialmente seleccionados.

En el apartado 4.3 se muestran los acelerogramas originales, los acelerogramas escalados y sus correspondientes espectros elásticos de respuesta y espectros de energía introducida. Para cada uno de ellos se indica también en una Tabla las ordenadas espectrales de estos dos espectros correspondientes a los periodos de las estructuras analizadas $\left({ }_{f} T_{1(3 p)}=1.4 \mathrm{~s} ; \mathrm{T}_{1(3 \mathrm{p})}=0.5 \mathrm{~s} ; \mathrm{f}_{1(6 \mathrm{p})}=1.8 \mathrm{~s}\right.$ y $\left.\mathrm{T}_{1(6 \mathrm{p})}=0.7 \mathrm{~s}\right)$.

\subsection{ACELERACIÓN SÍSMICA DE CÁLCULO (NCSE - 02)}

Se expone a continuación la obtención de la aceleración de cálculo del suelo correspondiente a Granada, según la norma sísmica española NCSE-02, para tres tipos de suelo.

La aceleración sísmica de cálculo, $a_{c}$, se define como el producto:

Siendo:

$$
a_{c}=S \cdot \rho \cdot a_{b}
$$

$a_{b}$ : Aceleración sísmica básica definida en 2.1.

La lista del anejo 1 detalla por municipios los valores de la aceleración sísmica básica iguales o superiores a $0,04 \mathrm{~g}$, junto con los del coeficiente de contribución $\mathrm{K}$.

$\boldsymbol{\rho}$ : Coeficiente adimensional de riesgo, función de la probabilidad aceptable de que se exceda $a_{c}$ en el período de vida para el que se proyecta la construcción. Toma los siguientes valores:

- Construcciones de importancia normal $\rho=1,0$

- Construcciones de importancia especial $\rho=1,3$

$S$ : Coeficiente de amplificación del terreno. Toma el valor: 
- Para $\rho . a_{b} \leq 0,1 g$

$$
S=\frac{C}{1,25}
$$

- Para $0,1 g<\rho . a_{b}<0,4 g$

$$
S=\frac{C}{1,25}+3,33\left(\rho \frac{a_{b}}{g}-0,1\right)\left(1-\frac{C}{1,25}\right)
$$

- Para $0,4 g \leq \rho . a_{b}$

$$
S=0,1
$$

Siendo:

C: Coeficiente de terreno. Depende de las características geotécnicas del terreno de cimentación y se detalla en el apartado 2.4 .

En esta Norma NCSE-02, los terrenos se clasifican en los siguientes tipos:

- Terreno tipo I: Roca compacta, suelo cementado o granular muy denso. Velocidad de propagación de las ondas elásticas transversales o de cizalla, $v_{s}>750 \mathrm{~m} / \mathrm{s}$.

- Terreno tipo II: Roca muy fracturada, suelos granulares densos o cohesivos duros. Velocidad de propagación de las ondas elásticas transversales o de cizalla, $750 \mathrm{~m} / \mathrm{s} \geq v_{s}>400 \mathrm{~m} / \mathrm{s}$.

- Terreno tipo III: Suelo granular de compacidad media, o suelo cohesivo de consistencia firme. Velocidad de propagación de las ondas elásticas transversales o de cizalla, $400 \mathrm{~m} / \mathrm{s} \geq v_{s}>200 \mathrm{~m} / \mathrm{s}$.

- Terreno tipo IV: Suelo granular suelto, o suelo cohesivo blando. Velocidad de propagación de las ondas elásticas transversales o de cizalla, $v_{s} \leq 200 \mathrm{~m} / \mathrm{s}$.

Tabla 4.1.1: Coeficientes del terreno

\begin{tabular}{|c|c|}
\hline Tipo de terreno & Coeficiente C \\
\hline I & 1,0 \\
II & 1,3 \\
III & 1,6 \\
IV & 2,0 \\
\hline
\end{tabular}

4.1.1. Aceleración sísmica de cálculo para un edificio ubicado en Granada y suelo tipo I.

- Aceleración básica:

$$
a_{b} / g=0,23 \Rightarrow a_{b}=0,23 g
$$

- Coeficiente de contribución [Anejo 1, NCSE-02]:

$$
K=1,0
$$

- Coeficiente adimensional de riesgo:

$$
\rho=1,0 \rightarrow \text { Construcciones de importanci normal }
$$


- Coeficiente del terreno:

$$
C=1,0 \rightarrow \text { Para suelo tipo I }
$$

- Coeficiente de amplificación del terreno:

$$
\begin{aligned}
& \text { Como } \rho . a_{b}=1,0 \times 0,23 g=0,23 g \Rightarrow 0,1 g<\rho . a_{b}=0,23 g<0,4 g \\
& \qquad S=\frac{C}{1,25}+3,33\left(\rho \frac{a_{b}}{g}-0,1\right)\left(1-\frac{C}{1,25}\right)=\frac{1,0}{1,25}+3,33\left(1,0 \frac{0,23 g}{g}-0,1\right)\left(1-\frac{1,0}{1,25}\right)=0,886
\end{aligned}
$$

\section{- Aceleración sísmica de cálculo:}

$$
a_{c}=S \cdot \rho \cdot a_{b}=0,886 \times 1,0 \times 0,23 g=0,204 g \Rightarrow \underline{a_{c}=2,000 \mathrm{~m} / \mathrm{s}^{2}}
$$

4.1.2. Aceleración sísmica de cálculo para un edificio ubicado en Granada y suelo tipo II.

- Aceleración básica:

$$
a_{b} / g=0,23 \Rightarrow a_{b}=0,23 g
$$

- Coeficiente de contribución [Anejo 1, NCSE-02]:

$$
K=1,0
$$

- Coeficiente adimensional de riesgo:

$$
\rho=1,0 \rightarrow \text { Construcciones de importanci normal }
$$

- Coeficiente del terreno:

$$
C=1,3 \rightarrow \text { Para suelo tipo II }
$$

- Coeficiente de amplificación del terreno:

$$
\begin{aligned}
& \text { Como } 0,1 g<\rho . a_{b}=1,0 \times 0,23 g=0,23 g<0,4 g \\
& S=\frac{C}{1,25}+3,33\left(\rho \frac{a_{b}}{g}-0,1\right)\left(1-\frac{C}{1,25}\right)=\frac{1,3}{1,25}+3,33\left(1,0 \frac{0,23 g}{g}-0,1\right)\left(1-\frac{1,3}{1,25}\right)=1,02
\end{aligned}
$$

\section{- Aceleración sísmica de cálculo:}

$$
a_{c}=S . \rho \cdot a_{b}=1,02 \times 1,0 \times 0,23 g=0,235 g \Rightarrow \underline{a_{c}=2,305 \mathrm{~m} / \mathrm{s}^{2}}
$$

4.1.3. Aceleración sísmica de cálculo para un edificio ubicado en Granada y suelo tipo III.

- Aceleración básica:

$$
a_{b} / g=0,23 \Rightarrow a_{b}=0,23 g
$$

- Coeficiente de contribución [Anejo 1, NCSE-02]:

$$
K=1,0
$$

- Coeficiente adimensional de riesgo:

$$
\rho=1,0 \rightarrow \text { Construcciones de importanci normal }
$$


- Coeficiente del terreno:

$$
C=1,6 \rightarrow \text { Para suelo tipo III }
$$

- Coeficiente de amplificación del terreno:

$$
\begin{aligned}
& \text { Como } \rho . a_{b}=1,0 \times 0,23 g=0,23 g \Rightarrow 0,1 g<\rho . a_{b}=0,23 g<0,4 g \\
& \qquad S=\frac{C}{1,25}+3,33\left(\rho \frac{a_{b}}{g}-0,1\right)\left(1-\frac{C}{1,25}\right)=\frac{1,6}{1,25}+3,33\left(1,0 \frac{0,23 g}{g}-0,1\right)\left(1-\frac{1,6}{1,25}\right)=1,16
\end{aligned}
$$

\section{- Aceleración sísmica de cálculo:}

$$
a_{c}=S \cdot \rho \cdot a_{b}=1,16 \times 1,0 \times 0,23 g=0,266 g \Rightarrow \underline{a_{c}=2,614 m / s^{2}}
$$

Tabla 4.1.2 Aceleración sísmica de cálculo para un edificio ubicado en Granada y para cada tipo de suelo

\begin{tabular}{|l|c|}
\hline \multicolumn{1}{|c|}{ Tipo de suelo } & Aceleración sísmica de cálculo $\left(\mathrm{m} / \mathrm{s}^{2}\right)$ \\
\hline I (muy duro) & 2,000 \\
\hline II (duro) & 2,305 \\
\hline III (medio) & 2,614 \\
\hline
\end{tabular}

\subsection{ACELEROGRAMAS HISTÓRICOS}

La manera más directa de definir un terremoto histórico es mediante registros de la aceleración del suelo, comúnmente denominados acelerogramas históricos. Un acelerograma nos proporciona el valor de la aceleración del suelo a intervalos de tiempo determinados.

Los acelerogramas son registrados mediante un acelerógrafo y tiene tres componentes del movimiento del suelo: NS (norte-sur), EO (este-oeste) y UP (vertical).

Las características más importantes de los acelerogramas desde el punto de vista de su capacidad a la hora de excitar la estructura son:

a) La amplitud del acelerograma, que se puede caracterizar por el valor máximo de la aceleración ("peak ground acceleration" PGA).

b) El contenido de frecuencias, que se puede caracterizar contando el número de veces que se cruza el valor 0 en un segundo.

c) La duración, que se puede caracterizar por el tiempo transcurrido entre dos picos determinados del acelerograma.

Conocido el acelerograma, la respuesta de la estructura frente al mismo expresado en términos de desplazamientos, velocidades, aceleraciones, deformaciones, etc. se puede calcular con precisión mediante un cálculo dinámico directo.

Se resumen en las tablas siguientes los acelerogramas inicialmente seleccionados y sus principales características: Fecha y hora del registro; nombre de terremoto al que corresponde el registro; magnitud; estación donde se registró el acelerograma; tipo de suelo; distancia a la falla $\Delta$ en kilómetros; aceleración máxima horizontal del suelo PHA; aceleración máxima vertical del suelo PVA; aceleración máxima de respuesta $S_{a}$, correspondiente a los periodos fundamentales de vibración de las estructuras analizadas; y por último, velocidad equivalente correspondiente al input de energía que introducen los terremotos a la estructura, $V_{E}$, para los periodos fundamentales de vibración.

En otra tabla, se exponen los periodos fundamentales de las estructuras analizadas: ${ }_{f} T_{1}$, correspondiente al periodo del primer modo de la estructura sin disipadores, "periodo de la parte flexible" y $T_{1}$, periodo de la estructura completa con disipadores. 


\subsubsection{Características de los acelerogramas históricos inicialmente seleccionados.}

Tabla 4.2.1: Terremotos históricos registrados en suelo tipo I (muy duro)

\begin{tabular}{|c|c|c|c|c|c|c|c|c|c|}
\hline № & Fecha & $\begin{array}{c}\text { Tiempo } \\
\text { [UTC] }\end{array}$ & Nombre & Magnitud & Estación & $\begin{array}{c}\text { Geología } \\
\text { local }\end{array}$ & $\Delta[\mathrm{km}]$ & $\left|\begin{array}{c}\mathrm{PHA} \\
{\left[\mathrm{m} / \mathrm{s}^{2}\right]}\end{array}\right|$ & $\begin{array}{c}\text { PVA } \\
{\left[\mathrm{m} / \mathrm{s}^{2}\right]}\end{array}$ \\
\hline \multicolumn{10}{|c|}{ Cercanos a falla (near fault) $<10 \mathrm{Km}$} \\
\hline 2 & 06/04/1977 & $13: 36: 37$ & Ardal & $6 \mathrm{Mw}$ & Naghan 1 & rock & 5 & 8,907 & - \\
\hline 6 & $14 / 06 / 1986$ & 07:40:39 & Timfristos & $3,7 \mathrm{ML}$ & $\begin{array}{l}\text { Karpenisi- } \\
\text { Prefecture }\end{array}$ & rock & 9 & 3,019 & 2,292 \\
\hline 7 & & 09:49:18 & Timfristos & $3,3 \mathrm{ML}$ & $\begin{array}{l}\text { Karpenisi- } \\
\text { Prefecture }\end{array}$ & rock & 8 & 2,845 & 1,901 \\
\hline \multicolumn{10}{|c|}{ Lejanos a falla (far field) $>10 \mathrm{Km}$} \\
\hline 1 & 06/05/1976 & 20:00:13 & Friuli & $6,5 \mathrm{Mw}$ & $\begin{array}{l}\text { Tolmezzo-Diga } \\
\text { Ambiesta }\end{array}$ & rock & 27 & 3,499 & 2,623 \\
\hline 4 & $15 / 04 / 1979$ & 06:19:41 & Montenegro & 6,9Mw & \begin{tabular}{|l|} 
Hercegnovi \\
Novi-O.S.D. \\
Pavicic School
\end{tabular} & rock & 65 & 2,509 & 2,045 \\
\hline 5 & $23 / 11 / 1980$ & $18: 34: 52$ & $\begin{array}{c}\text { Campano } \\
\text { Lucano }\end{array}$ & $6,9 \mathrm{Mw}$ & Sturno & rock & 32 & 3,166 & 2,308 \\
\hline
\end{tabular}

Tabla 4.2.2: Terremotos históricos registrados en suelo tipo II (duro)

\begin{tabular}{|c|c|c|c|c|c|c|c|c|c|}
\hline No & Fecha & $\begin{array}{c}\text { Tiempo } \\
{[\text { UTC }]}\end{array}$ & Nombre & Magnitud & Estación & $\begin{array}{c}\text { Geología } \\
\text { local }\end{array}$ & $\Delta[\mathrm{km}]$ & $\begin{array}{c}\text { PHA } \\
{\left[\mathrm{m} / \mathrm{s}^{2}\right]}\end{array}$ & $\begin{array}{c}\text { PVA } \\
{\left[\mathrm{m} / \mathrm{s}^{2}\right]}\end{array}$ \\
\hline 1 & $11 / 05 / 76$ & $22: 44: 01$ & $\begin{array}{c}\text { Friuli } \\
\text { aftershock }\end{array}$ & $4,9 \mathrm{Mw}$ & $\begin{array}{l}\text { Forgaria- } \\
\text { Cornio }\end{array}$ & stiff soil & 3 & 3,001 & 1,404 \\
\hline 8 & $16 / 09 / 77$ & $23: 48: 08$ & $\begin{array}{c}\text { Friuli } \\
\text { aftershock }\end{array}$ & $5,4 \mathrm{Mw}$ & $\begin{array}{l}\text { Forgaria- } \\
\text { Cornio }\end{array}$ & stiff soil & 5 & 2,365 & 1,304 \\
\hline 14 & $24 / 05 / 79$ & $17: 23: 18$ & $\begin{array}{c}\text { Montenegro } \\
\text { aftershock }\end{array}$ & $6,2 \mathrm{Mw}$ & Budva-PTT & stiff soil & 8 & 2,624 & 1,652 \\
\hline 2 & $11 / 09 / 76$ & $16: 35: 03$ & $\begin{array}{c}\text { Friuli } \\
\text { aftershock }\end{array}$ & $5,5 \mathrm{Mw}$ & $\begin{array}{l}\text { Forgaria- } \\
\text { Cornio }\end{array}$ & stiff soil & 16 & 2,273 & 1,162 \\
\hline 4 & $15 / 09 / 76$ & $03: 15: 19$ & $\begin{array}{c}\text { Friuli } \\
\text { aftershock }\end{array}$ & $6 \mathrm{Mw}$ & $\begin{array}{l}\text { Forgaria- } \\
\text { Cornio }\end{array}$ & stiff soil & 17 & 2,586 & 0,965 \\
\hline 12 & $24 / 05 / 79$ & $17: 23: 18$ & $\begin{array}{l}\text { Montenegro } \\
\text { aftershock }\end{array}$ & $6,2 \mathrm{Mw}$ & $\begin{array}{l}\text { Bar-Skupstina } \\
\text { Opstine }\end{array}$ & stiff soil & 33 & 2,652 & 0,994 \\
\hline
\end{tabular}


Tabla 4.2.3: Terremotos históricos registrados en suelo tipo III (medio)

\begin{tabular}{|c|c|c|c|c|c|c|c|c|c|}
\hline № & Fecha & $\begin{array}{c}\text { Tiempo } \\
\text { [UTC] }\end{array}$ & Nombre & Magnitud & Estación & $\begin{array}{l}\text { Geología } \\
\text { local }\end{array}$ & $\Delta[\mathrm{km}]$ & $\begin{array}{c}\mathrm{PHA} \\
{\left[\mathrm{m} / \mathrm{s}^{2}\right]}\end{array}$ & $\begin{array}{c}\text { PVA } \\
{\left[\mathrm{m} / \mathrm{s}^{2}\right]}\end{array}$ \\
\hline \multicolumn{10}{|c|}{ Cercanos a falla (near fault) $<10 \mathrm{Km}$} \\
\hline 2 & $11 / 09 / 76$ & $16: 35: 03$ & $\begin{array}{c}\text { Friuli } \\
\text { aftershock }\end{array}$ & $5,5 \mathrm{Mw}$ & Buia & soft soil & 10 & 2,26 & 0,862 \\
\hline 6 & $15 / 09 / 86$ & $11: 41: 28$ & $\begin{array}{l}\text { Kalamata } \\
\text { aftershock }\end{array}$ & $4,9 \mathrm{Mw}$ & $\begin{array}{l}\text { Kalamata-OTE } \\
\text { Building }\end{array}$ & stiff soil & 3 & 2,355 & 1,017 \\
\hline 12 & 12/11/99 & $16: 57: 20$ & Duzce 1 & $7,2 \mathrm{Mw}$ & $\begin{array}{l}\text { Duzce- } \\
\text { Meteoroloji } \\
\text { Mudurlugu }\end{array}$ & soft soil & 8 & 5,036 & 3,382 \\
\hline \multicolumn{10}{|c|}{ Lejanos a falla (far field) $>10 \mathrm{Km}$} \\
\hline 3 & $15 / 04 / 79$ & 06:19:41 & Montenegro & $6,9 \mathrm{Mw}$ & $\begin{array}{l}\text { Ulcinj-Hotel } \\
\text { Olimpic }\end{array}$ & stiff soil & 24 & 2,88 & 4,49 \\
\hline 8 & $13 / 12 / 1990$ & $00: 24: 26$ & $\begin{array}{c}\text { Sicilia- } \\
\text { Orientale }\end{array}$ & $5,6 \mathrm{Mw}$ & Catania-Piana & soft soil & 24 & 2,483 & 0,682 \\
\hline 14 & 07/09/99 & $11: 56: 51$ & Ano Liosia & $6 \mathrm{Mw}$ & $\begin{array}{l}\text { Athens-Sepolia } \\
\text { (Garage) }\end{array}$ & stiff soil & 14 & 3,2 & 1,925 \\
\hline
\end{tabular}

\subsubsection{Características espectrales de los acelerogramas escalados correspondientes a los periodos} fundamentales de las estructuras analizadas $\left(_{f} T_{1(3 p)}=1.4 \mathrm{~s} ; T_{1(3 p)}=0.5 \mathrm{~s} ;{ }_{f} T_{1(6 p)}=1.8 \mathrm{~s}\right.$ y $\left.T_{1(6 p)}=0.7 \mathrm{~s}\right)$.

Tabla 4.2.4: Ordenadas espectrales de los terremotos históricos escalados registrados en suelo tipo I

\begin{tabular}{|c|c|c|c|c|c|c|c|c|c|c|c|}
\hline \multirow[b]{2}{*}{ No } & \multirow[b]{2}{*}{ Nombre } & \multirow[b]{2}{*}{$\begin{array}{c}\Delta \\
{[\mathrm{km}]}\end{array}$} & \multirow{2}{*}{$\begin{array}{l}\text { Factor } \\
\text { escala }\end{array}$} & \multicolumn{4}{|c|}{$\mathrm{S}_{\mathrm{a}}\left[\mathrm{cm} / \mathrm{s}^{2}\right]$} & \multicolumn{4}{|c|}{$V_{E}[\mathrm{~cm} / \mathrm{s}]$} \\
\hline & & & & $\begin{array}{c}{ }_{f} T_{1(3 p)} \\
1,4 s\end{array}$ & $\begin{array}{c}T_{1(3 p)} \\
0,5 s\end{array}$ & $\begin{array}{c}{ }_{f} T_{1(6 p)} \\
1,8 s\end{array}$ & $\begin{array}{c}T_{1(6 p)} \\
0,7 s \\
\end{array}$ & $\begin{array}{c}{ }_{f} T_{1(3 p)} \\
1,4 s\end{array}$ & $\begin{array}{c}T_{1(3 p)} \\
0,5 s \\
\end{array}$ & $\begin{array}{c}{ }_{f} T_{1(6 p)} \\
1,8 \mathrm{~s}\end{array}$ & $\begin{array}{c}T_{1(6 p)} \\
0,7 s \\
\end{array}$ \\
\hline \multicolumn{12}{|c|}{ Cercanos a falla (near fault) $<10 \mathrm{Km}$} \\
\hline 2 & Ardal & 5 & 0,2245 & 60 & 260 & 30 & 160 & 11 & 24 & 9 & 25 \\
\hline 6 & Timfristos & 9 & 0,6625 & $<10$ & 100 & $<10$ & 40 & 3,2 & 10,4 & 2,8 & 5,6 \\
\hline 7 & Timfristos & 8 & 0,7029 & $<10$ & 39 & $<10$ & 20 & 1,6 & 4 & 1,2 & 2,6 \\
\hline \multicolumn{12}{|c|}{ Lejanos a falla (far field) $>10 \mathrm{Km}$} \\
\hline 1 & Friuli & 27 & 0,5717 & 80 & 360 & 40 & 190 & 22 & 44 & 17 & 32 \\
\hline 4 & Montenegro & 65 & 0,9102 & 60 & 500 & 30 & 400 & 26 & 82 & 20 & 70 \\
\hline 5 & $\begin{array}{c}\text { Campano } \\
\text { Lucano }\end{array}$ & 32 & 0,9431 & 300 & 540 & 250 & 400 & 104 & 88 & 106 & 82 \\
\hline
\end{tabular}


Tabla 4.2.5: Ordenadas espectrales de los terremotos históricos escalados registrados en suelo tipo II

\begin{tabular}{|c|c|c|c|c|c|c|c|c|c|c|c|}
\hline \multirow[b]{2}{*}{ № } & \multirow[b]{2}{*}{ Nombre } & \multirow{2}{*}{$\begin{array}{c}\Delta \\
{[\mathrm{km}]}\end{array}$} & \multirow{2}{*}{$\begin{array}{l}\text { Factor } \\
\text { escala }\end{array}$} & \multicolumn{4}{|c|}{$\mathrm{S}_{\mathrm{a}}\left[\mathrm{cm} / \mathrm{s}^{2}\right]$} & \multicolumn{4}{|c|}{$\mathrm{V}_{\mathrm{E}}[\mathrm{cm} / \mathrm{s}]$} \\
\hline & & & & $\begin{array}{c}{ }_{f} T_{1(3 p)} \\
1,4 s\end{array}$ & $\begin{array}{l}T_{1(3 p)} \\
0,5 s\end{array}$ & $\begin{array}{c}{ }_{f} T_{1(6 p)} \\
1,8 \mathrm{~s}\end{array}$ & $\begin{array}{l}T_{1(6 p)} \\
0,7 s\end{array}$ & $\begin{array}{c}{ }_{f} T_{1(3 p)} \\
1,4 s\end{array}$ & $\begin{array}{l}T_{1(3 p)} \\
0,5 s\end{array}$ & $\begin{array}{c}f_{1(6 p)} \\
1,8 s\end{array}$ & $\begin{array}{l}T_{1(6 p)} \\
0,7 s \\
\end{array}$ \\
\hline \multicolumn{12}{|c|}{ Cercanos a falla (near fault) $<10 \mathrm{Km}$} \\
\hline 1 & $\begin{array}{c}\text { Friuli } \\
\text { aftershock }\end{array}$ & 3 & 1,2554 & 40 & 440 & 38 & 120 & 18 & 44 & 14 & 20 \\
\hline 8 & $\begin{array}{c}\text { Friuli } \\
\text { aftershock }\end{array}$ & 5 & 0,9745 & 30 & 260 & 20 & 140 & 12 & 34 & 10 & 20 \\
\hline 14 & $\begin{array}{c}\text { Montenegro } \\
\text { aftershock }\end{array}$ & 8 & 1,9662 & 360 & 720 & 240 & 920 & 105 & 130 & 75 & 155 \\
\hline \multicolumn{12}{|c|}{ Lejanos a falla (far field) $>10 \mathrm{Km}$} \\
\hline 2 & $\begin{array}{c}\text { Friuli } \\
\text { aftershock }\end{array}$ & 16 & 1,7929 & 50 & 500 & 20 & 300 & 20 & 56 & 12 & 38 \\
\hline 4 & $\begin{array}{c}\text { Friuli } \\
\text { aftershock }\end{array}$ & 17 & 0,8912 & 40 & 200 & 20 & 60 & 16 & 26 & 11 & 12 \\
\hline 12 & $\begin{array}{c}\text { Montenegro } \\
\text { aftershock }\end{array}$ & 33 & 1,1610 & 60 & 610 & 40 & 400 & 22 & 66 & 25 & 75 \\
\hline
\end{tabular}

Tabla 4.2.6: Ordenadas espectrales de los terremotos históricos escalados registrados en suelo tipo III

\begin{tabular}{|c|c|c|c|c|c|c|c|c|c|c|c|}
\hline \multirow[b]{2}{*}{ № } & \multirow[b]{2}{*}{ Nombre } & \multirow{2}{*}{$\begin{array}{c}\Delta \\
{[\mathrm{km}]}\end{array}$} & \multirow{2}{*}{$\begin{array}{l}\text { Factor } \\
\text { escala }\end{array}$} & \multicolumn{4}{|c|}{$\mathrm{S}_{\mathrm{a}}\left[\mathrm{cm} / \mathrm{s}^{2}\right]$} & \multicolumn{4}{|c|}{$\mathrm{V}_{\mathrm{E}}[\mathrm{cm} / \mathrm{s}]$} \\
\hline & & & & $\begin{array}{c}\mathrm{f}_{1(3 \mathrm{p})} \\
1,4 \mathrm{~s}\end{array}$ & $\begin{array}{l}T_{1(3 p)} \\
0,5 s\end{array}$ & $\begin{array}{c}\mathrm{f}_{1(6 \mathrm{p})} \\
1,8 \mathrm{~s}\end{array}$ & $\begin{array}{l}T_{1(6 p)} \\
0,7 s\end{array}$ & $\begin{array}{c}f_{1(3 p)} \\
1,4 s\end{array}$ & $\begin{array}{l}T_{1(3 p)} \\
0,5 s\end{array}$ & $\begin{array}{c}{ }_{f} T_{1(6 p)} \\
1,8 s\end{array}$ & $\begin{array}{l}T_{1(6 p)} \\
0,7 s \\
\end{array}$ \\
\hline \multicolumn{12}{|c|}{ Cercanos a falla (near fault) $<10 \mathrm{Km}$} \\
\hline 2 & $\begin{array}{c}\text { Friuli } \\
\text { aftershock }\end{array}$ & 10 & 1,1564 & 100 & 700 & 50 & 600 & 32 & 68 & 18 & 96 \\
\hline 6 & $\begin{array}{l}\text { Kalamata } \\
\text { aftershock }\end{array}$ & 3 & 1,1098 & 60 & 240 & 50 & 500 & 20 & 47 & 26 & 61 \\
\hline 12 & Duzce 1 & 8 & 0,7067 & 160 & 720 & 150 & 650 & 60 & 116 & 70 & 120 \\
\hline \multicolumn{12}{|c|}{ Lejanos a falla (far field) $>10 \mathrm{Km}$} \\
\hline 3 & Montenegro & 24 & 0,9077 & 470 & 500 & 220 & 335 & 60 & 116 & 70 & 120 \\
\hline 8 & $\begin{array}{c}\text { Sicilia- } \\
\text { Orientale }\end{array}$ & 24 & 1,0529 & 30 & 210 & 20 & 200 & 9 & 25 & 10 & 38 \\
\hline 14 & Ano Liosia & 14 & 0,8168 & 90 & 300 & 60 & 120 & 26 & 36 & 22 & 22 \\
\hline
\end{tabular}

\subsubsection{Tabla resumen de los periodos fundamentales de vibración.}

Cuyo cálculo se detalla en el apartado 5.2.5 de este capítulo.

Tabla 4.2.7: Periodos fundamentales de vibración

\begin{tabular}{c|c|c|c|}
\hline $\begin{array}{c}\text { Periodos } \\
\text { fundamentales }\end{array}$ & $\begin{array}{c}\text { Pórtico pa2pz2-1 } \\
\text { (3 plantas) }\end{array}$ & $\begin{array}{c}\text { Pórtico pa4pz2-1 } \\
\text { (3 plantas) }\end{array}$ & $\begin{array}{c}\text { Pórtico pa6pz2-1 } \\
\text { (6 plantas) }\end{array}$ \\
\hline Sin disipador ${ }_{\mathrm{f}} \mathrm{T}_{1}(\mathrm{~s})$ & $1,366 \approx \mathbf{1 , 4}$ & $1,391 \approx \mathbf{1 , 4}$ & $1,785 \approx \mathbf{1 , 8}$ \\
\hline Con disipador $\mathrm{T}_{1}(\mathrm{~s})$ & $0,558 \approx \mathbf{0 , 5}$ & $0,568 \approx \mathbf{0 , 5}$ & $0,740 \approx \mathbf{0 , 7}$ \\
\hline & & &
\end{tabular}




\subsection{ESCALADO, ESPECTRO ELÁSTICO DE RESPUESTA Y ESPECTRO DE INPUT DE ENERGÍA DE LOS ACELEROGRAMAS}

Los acelerogramas seleccionados se escalan a un determinado nivel (que será el que establezca la normativa sísmica de aplicación); el procedimiento más utilizado en la práctica es escalar la aceleración máxima.

Las aceleraciones máximas de cada uno de los acelerogramas históricos, PGA, se han escalado a las aceleraciones sísmicas de cálculo obtenidas en el apartado 4.1 de este capítulo, estas corresponden al tipo de suelo: $a_{c}=200 \mathrm{~cm} / \mathrm{s}^{2}$ suelo tipo I; $a_{c}=230,5 \mathrm{~cm} / \mathrm{s}^{2}$ suelo tipo II y $a_{c}=261,4 \mathrm{~cm} / \mathrm{s}^{2}$ suelo tipo III.

El espectro elástico de respuesta de aceleración absoluta $\mathrm{S}_{\mathrm{a}}-\mathrm{T}$, es un gráfico que no pasa nunca por el origen, ya que en el caso de una estructura infinitamente rígida (es decir con $T=0$ ) el espectro debe proporcionar la aceleración máxima que ha experimentado el suelo; otra característica de este espectro es que en el rango de periodos largos (es decir para valores de $T$ muy elevados) la aceleración absoluta tiende a decrecer a medida que aumenta $T$, en el caso límite de una estructura infinitamente flexible, no se transmitiría ningún movimiento a la masa y consecuentemente $S_{a}$ valdría cero.

Akiyama llegó a la siguiente conclusión, que es el fundamento de los métodos de proyecto sismorresistente basados en el balance energético: El input de energía total $E$ introducido por un terremoto determinado en un sistema, cantidad muy estable depende básicamente de la masa total del sistema y de su periodo de vibración $T$. Otros aspectos como la resistencia del sistema, el nivel de amortiguamiento, las características de la fuerza restauradora etc. apenas influyen en $E$ y a efectos de cálculo pueden despreciarse.

$E$ El input de energía total $E$ puede expresarse mediante una velocidad equivalente $V_{E}$ que se define como sigue [18]:

$$
V_{E}=\sqrt{\frac{2 E_{\text {input }}}{M_{\text {total }}}}
$$

Se denomina espectro de input de energía para un terremoto determinado y para un nivel de amortiguamiento dado, a la representación de $T$ y $V_{E}$ en unos ejes cartesianos. 


\subsubsection{Suelo Tipo I (Campo cercano a la falla)}

\section{- Acelerograma №2 (Ardal) $\Delta=5 \mathrm{Km}$}

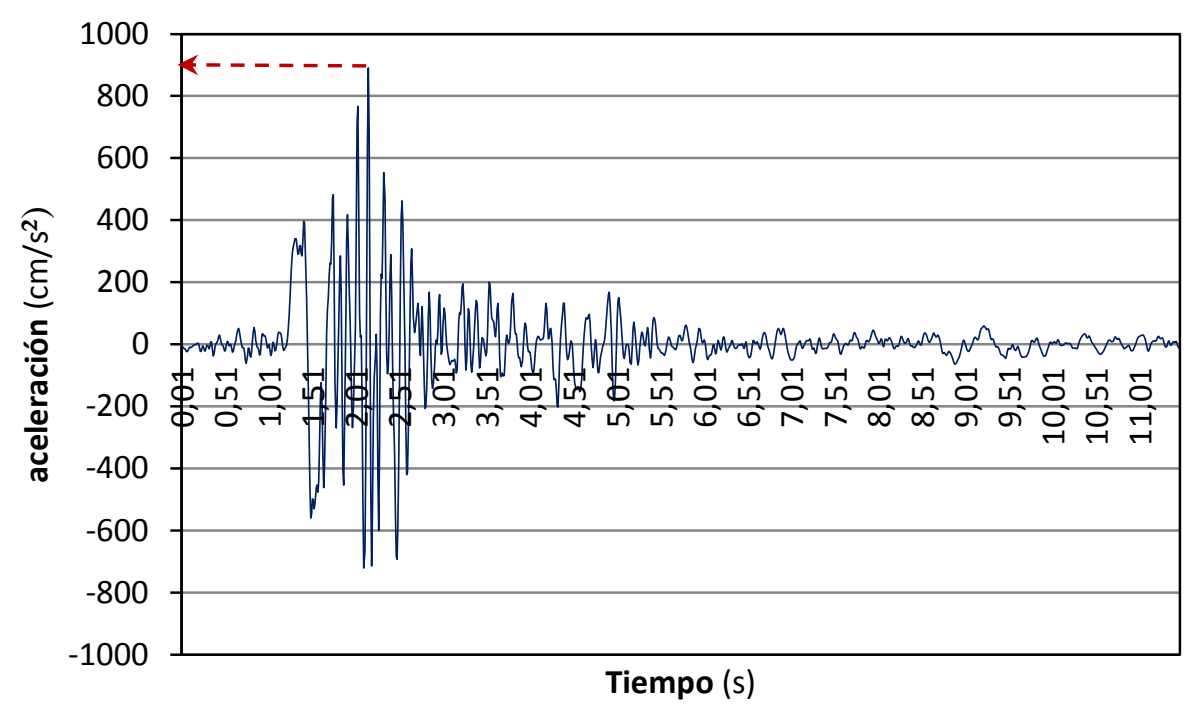

Fig. 4.3.1: Acelerograma $\mathrm{N}^{\circ} 2$ (Ardal), suelo tipo I, campo cercano, sin escalar

Aceleración sísmica de cálculo: $a_{c}=200 \mathrm{~cm} / \mathrm{s}^{2}$

Aceleración máxima: $P G A=890,74 \mathrm{~cm} / \mathrm{s}^{2}$

Factor de escala: $\frac{200 \mathrm{~cm} / \mathrm{s}^{2}}{890,74 \mathrm{~cm} / \mathrm{s}^{2}}=\underline{0,2245}$

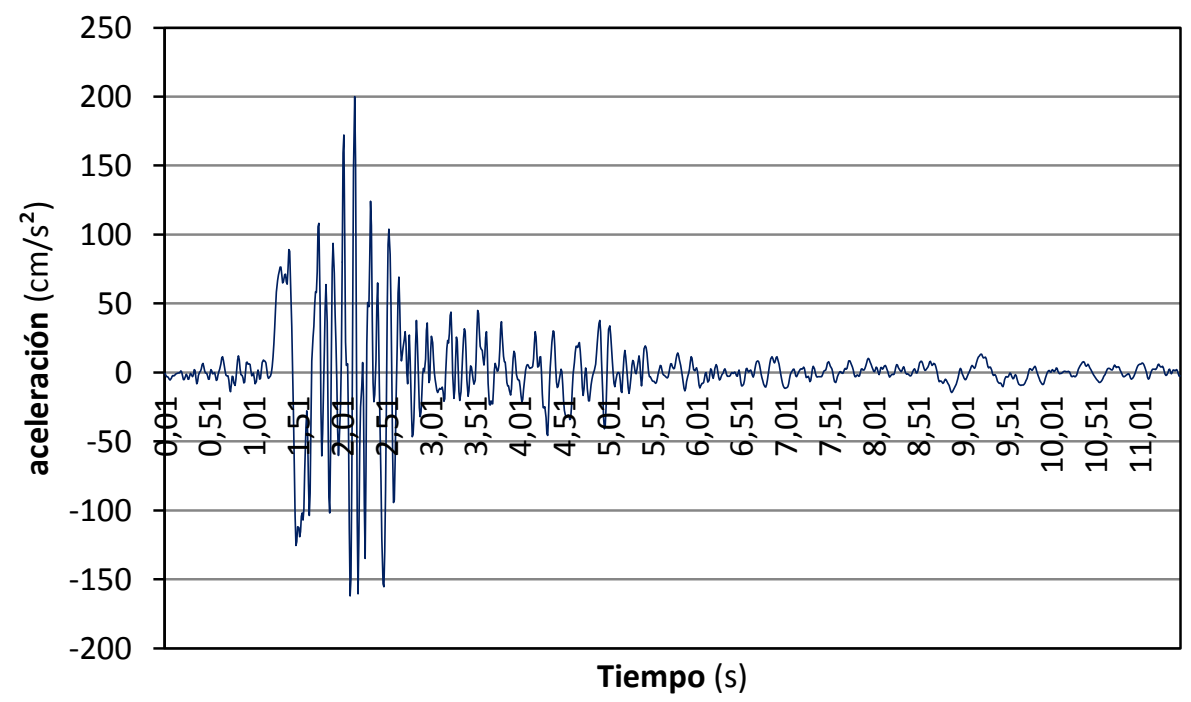

Fig. 4.3.2: Acelerograma №2 (Ardal), suelo tipo I, campo cercano, escalado a $P G A=200 \mathrm{~cm} / \mathrm{s}^{2}$ 


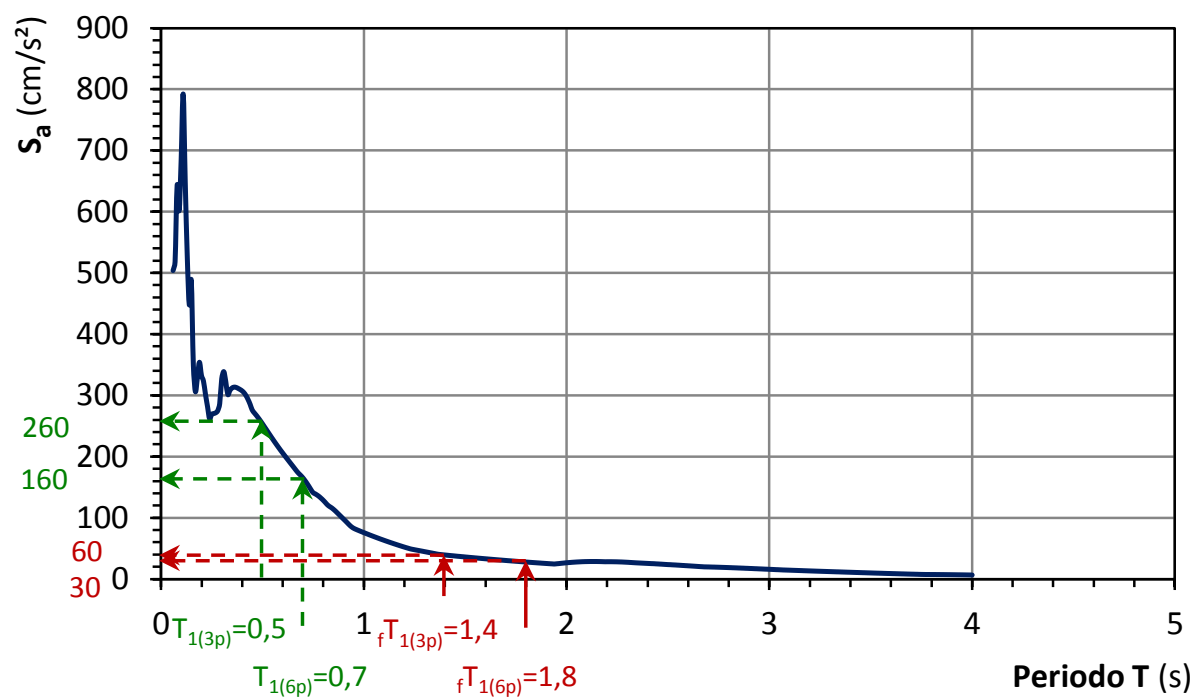

Fig. 4.3.3: Espectro elástico de respuesta del acelerograma №2 (Ardal) escalado a $P G A=200 \mathrm{~cm} / \mathrm{s}^{2}$

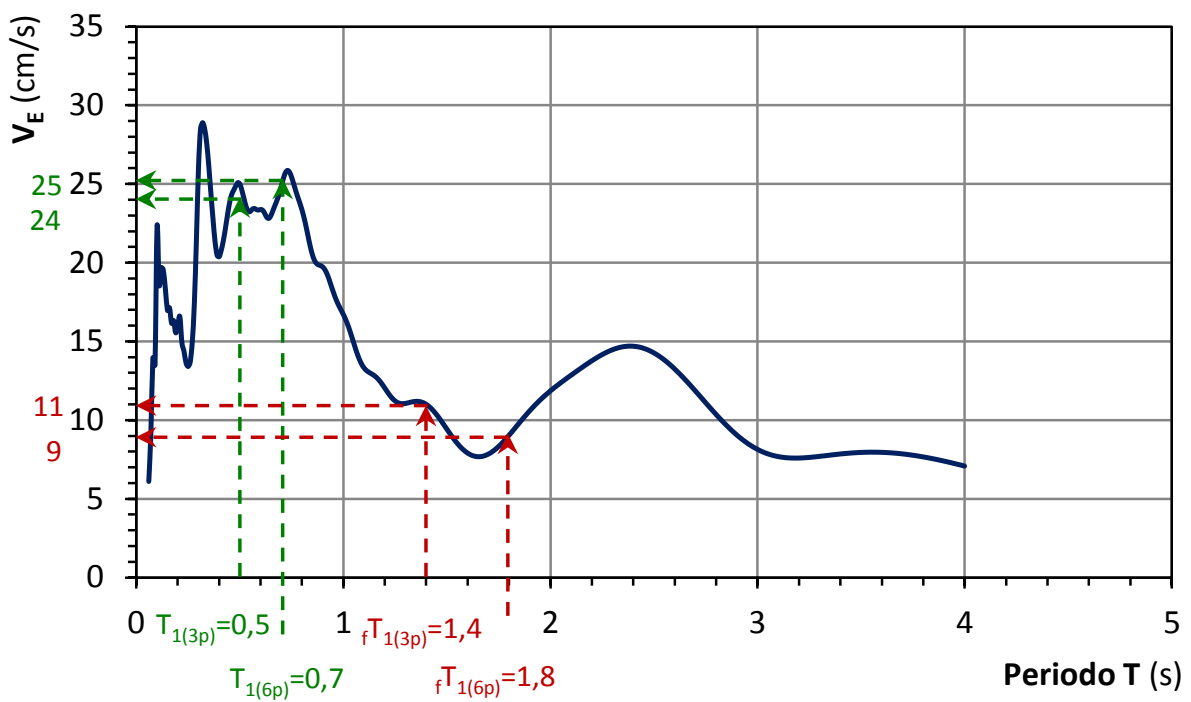

Fig. 4.3.4: Espectro de energía introducida del acelerograma №2 (Ardal) escalado a PGA $=200 \mathrm{~cm} / \mathrm{s}^{2}$

Comparando los valores de aceleración máxima de respuesta $S_{a}$ y de energía $V_{E}$, para los periodos de vibración anteriormente obtenidos.

Tabla 4.3.1: Ordenadas del espectro elástico de respuesta $S_{a}$ y del espectro de energía introducida, correspondientes a los periodos de las estructuras analizadas.

\begin{tabular}{l|c|c|c|c|} 
& $\mathrm{f}_{1(3 \mathrm{p})}=1,4 \mathrm{~s}$ & $\mathrm{~T}_{1(3 \mathrm{p})}=0,5 \mathrm{~s}$ & $\mathrm{f}_{1(6 \mathrm{p})}=1,8 \mathrm{~s}$ & $\mathrm{~T}_{1(6 \mathrm{p})}=0,7 \mathrm{~s}$ \\
\hline $\mathrm{S}_{\mathrm{a}}\left(\mathrm{cm} / \mathrm{s}^{2}\right)$ & 60 & 260 & 30 & 160 \\
\hline $\mathrm{V}_{\mathrm{E}}(\mathrm{cm} / \mathrm{s})$ & 11 & 24 & 9 & 25 \\
\hline & \multicolumn{2}{|c|}{} & \multicolumn{2}{|c}{}
\end{tabular}


- Acelerograma №6 (Timfristos) $\Delta=9 \mathrm{Km}$

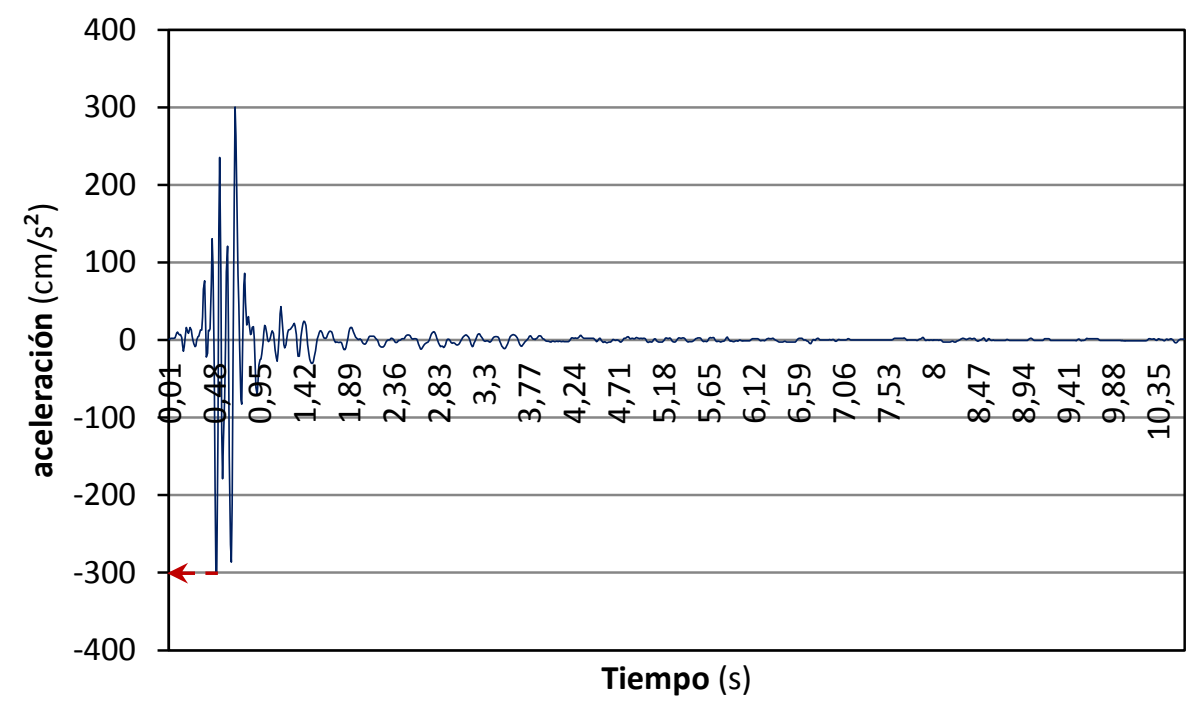

Fig. 4.3.5: Acelerograma $\mathrm{N}^{\circ} 6$ (Timfristos), suelo tipo I, campo cercano, sin escalar

Aceleración sísmica de cálculo: $a_{c}=200 \mathrm{~cm} / \mathrm{s}^{2}$

Aceleración máxima: $P G A=301,89 \mathrm{~cm} / \mathrm{s}^{2}$

Factor de escala: $\frac{200 \mathrm{~cm} / \mathrm{s}^{2}}{301,89 \mathrm{~cm} / \mathrm{s}^{2}}=\underline{0,6625}$

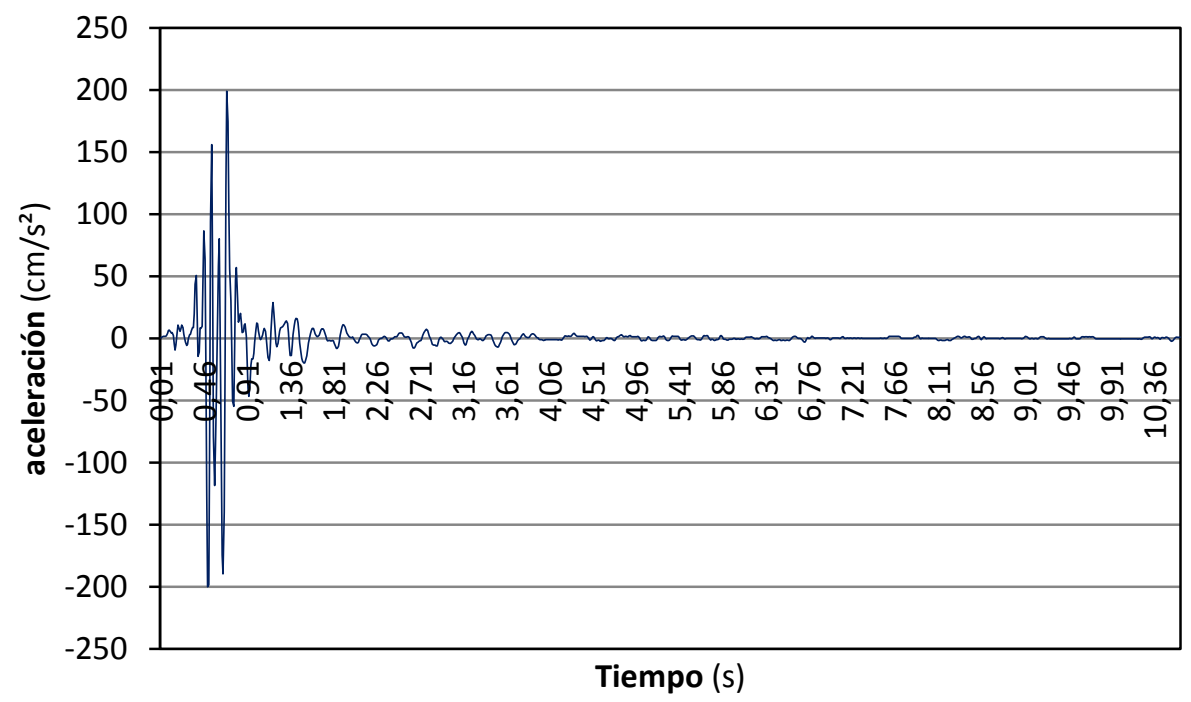

Fig. 4.3.6: Acelerograma $\mathrm{N}^{\circ} 6$ (Timfristos), suelo tipo I, campo cercano, escalado a PGA=200 $\mathrm{cm} / \mathrm{s}^{2}$ 


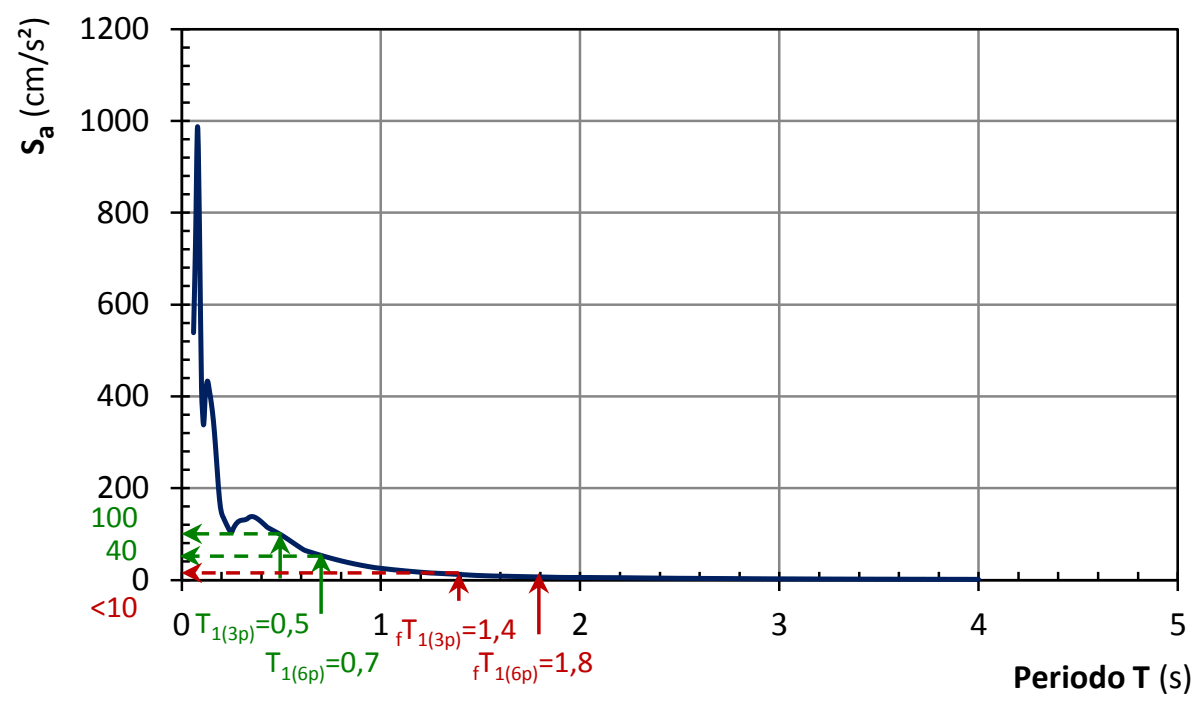

Fig. 4.3.7: Espectro elástico de respuesta del acelerograma $\mathrm{N}^{\circ} 6$ (Timfristos) escalado a $P G A=200 \mathrm{~cm} / \mathrm{s}^{2}$

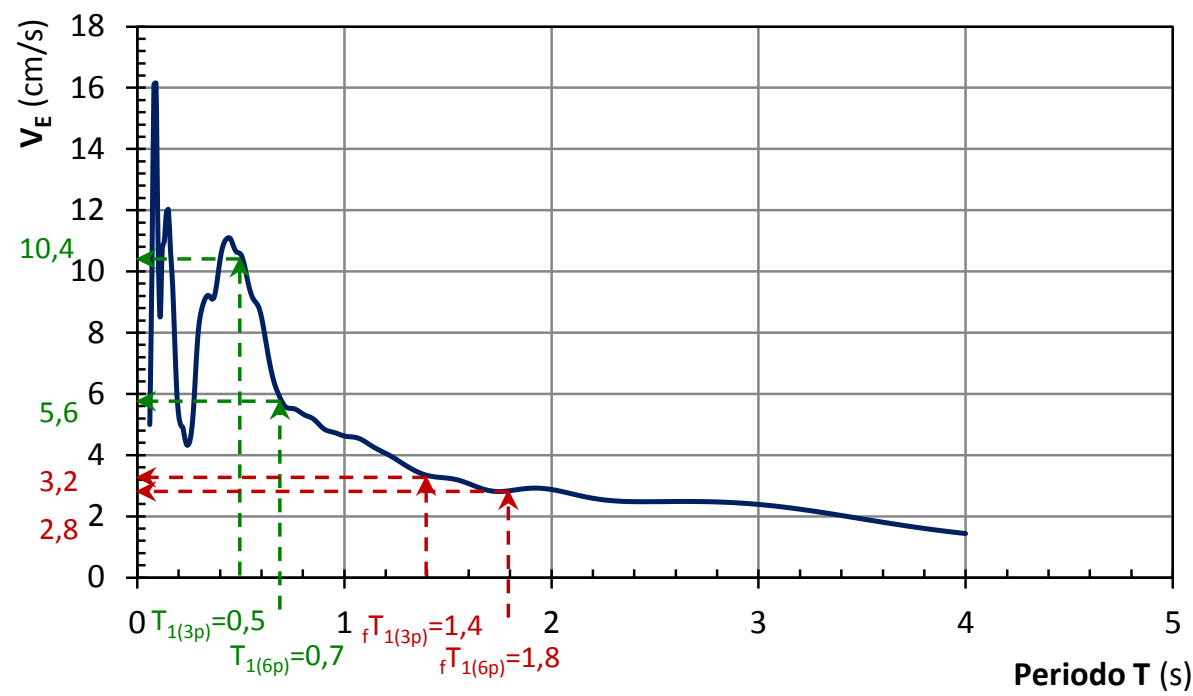

Fig. 4.3.8: Espectro de energía introducida del acelerograma $\mathrm{N}^{\circ} 6$ (Timfristos) escalado a $P G A=200 \mathrm{~cm} / \mathrm{s}^{2}$

Comparando los valores de aceleración máxima de respuesta $S_{a}$ y de energía $V_{E}$, para los periodos de vibración anteriormente obtenidos.

Tabla 4.3.2: Ordenadas del espectro elástico de respuesta $S_{a}$ y del espectro de energía introducida, correspondientes a los periodos de las estructuras analizadas.

\begin{tabular}{c|c|c|c|c|} 
& $\mathrm{f}_{1(3 \mathrm{p})}=1,4 \mathrm{~s}$ & $\mathrm{~T}_{1(3 \mathrm{p})}=0,5 \mathrm{~s}$ & $\mathrm{f}_{1(6 \mathrm{p})}=1,8 \mathrm{~s}$ & $\mathrm{~T}_{1(6 \mathrm{p})}=0,7 \mathrm{~s}$ \\
\hline $\mathrm{S}_{\mathrm{a}}\left(\mathrm{cm} / \mathrm{s}^{2}\right)$ & $<10$ & 100 & $<10$ & 40 \\
\hline $\mathrm{V}_{\mathrm{E}}(\mathrm{cm} / \mathrm{s})$ & 3,2 & 10,4 & 2,8 & 5,6 \\
\hline & \multicolumn{3}{|l}{} &
\end{tabular}


- Acelerograma №7 (Timfristos) $\Delta=8 \mathrm{Km}$

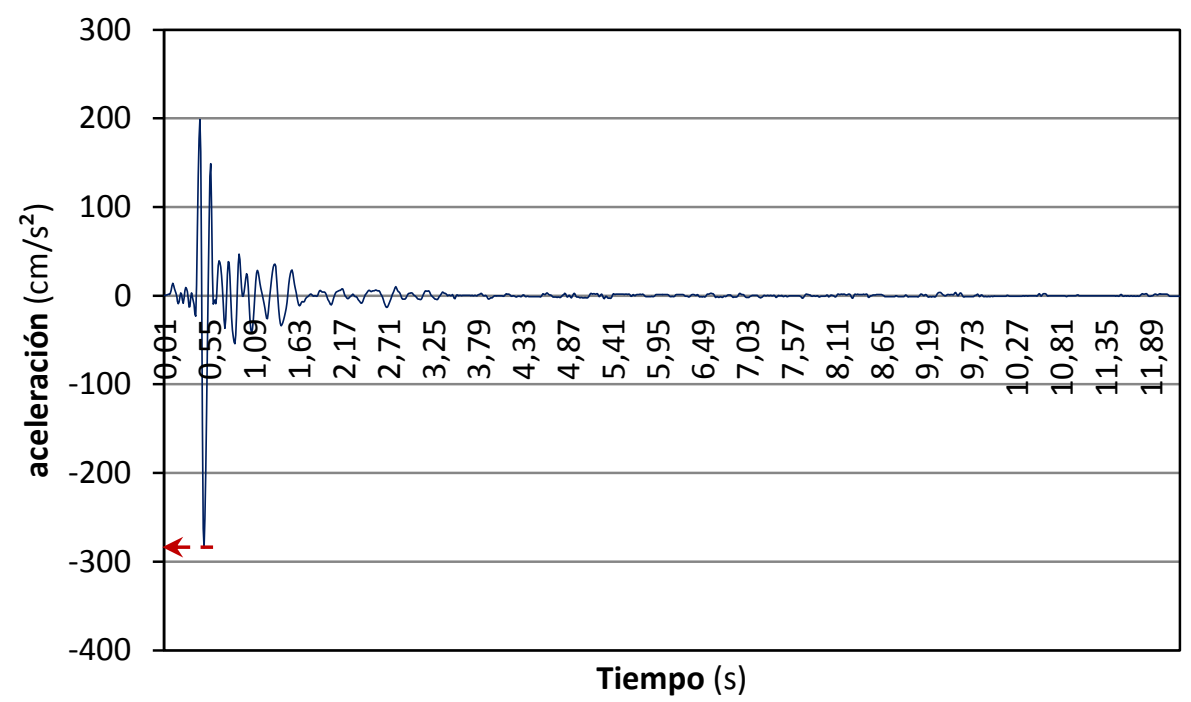

Fig. 4.3.9: Acelerograma $N^{\circ} 7$ (Timfristos), suelo tipo I, campo cercano, sin escalar

Aceleración sísmica de cálculo: $a_{c}=200 \mathrm{~cm} / \mathrm{s}^{2}$

Aceleración máxima: $P G A=284,55 \mathrm{~cm} / \mathrm{s}^{2}$

Factor de escala: $\frac{200 \mathrm{~cm} / \mathrm{s}^{2}}{284,55 \mathrm{~cm} / \mathrm{s}^{2}}=\underline{0,7029}$

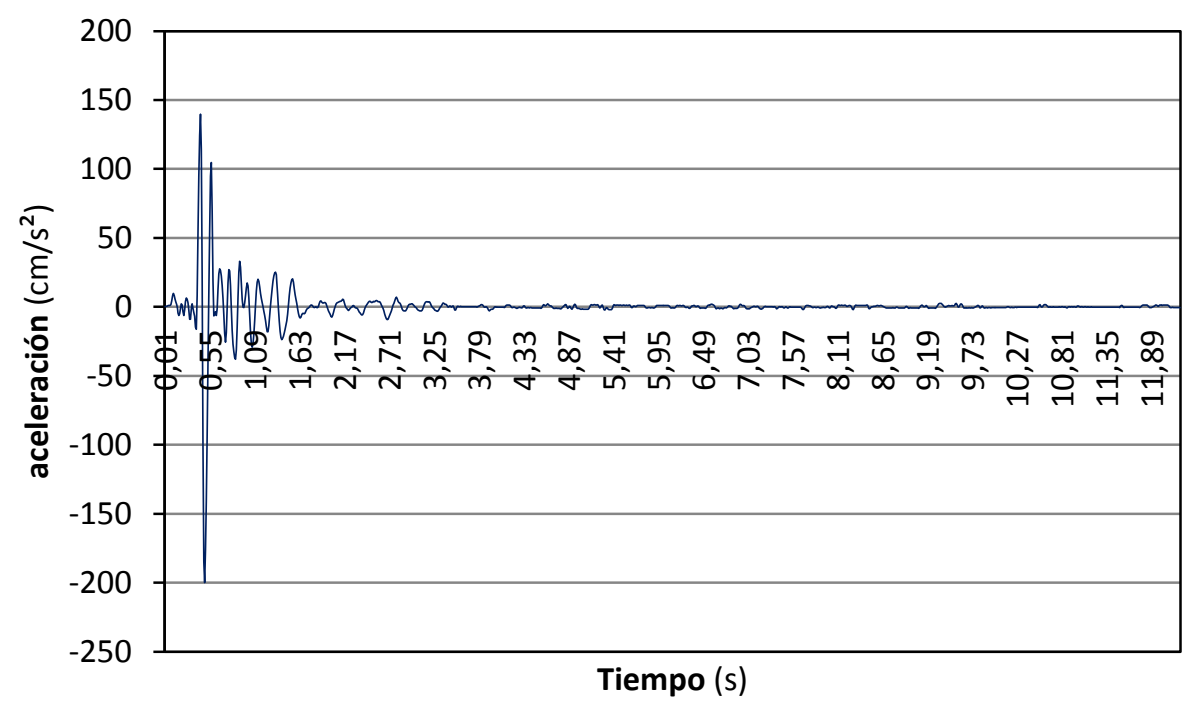

Fig. 4.3.10: Acelerograma $N^{\circ} 7$ (Timfristos), suelo tipo $I$, campo cercano, escalado a $P G A=200 \mathrm{~cm} / \mathrm{s}^{2}$ 


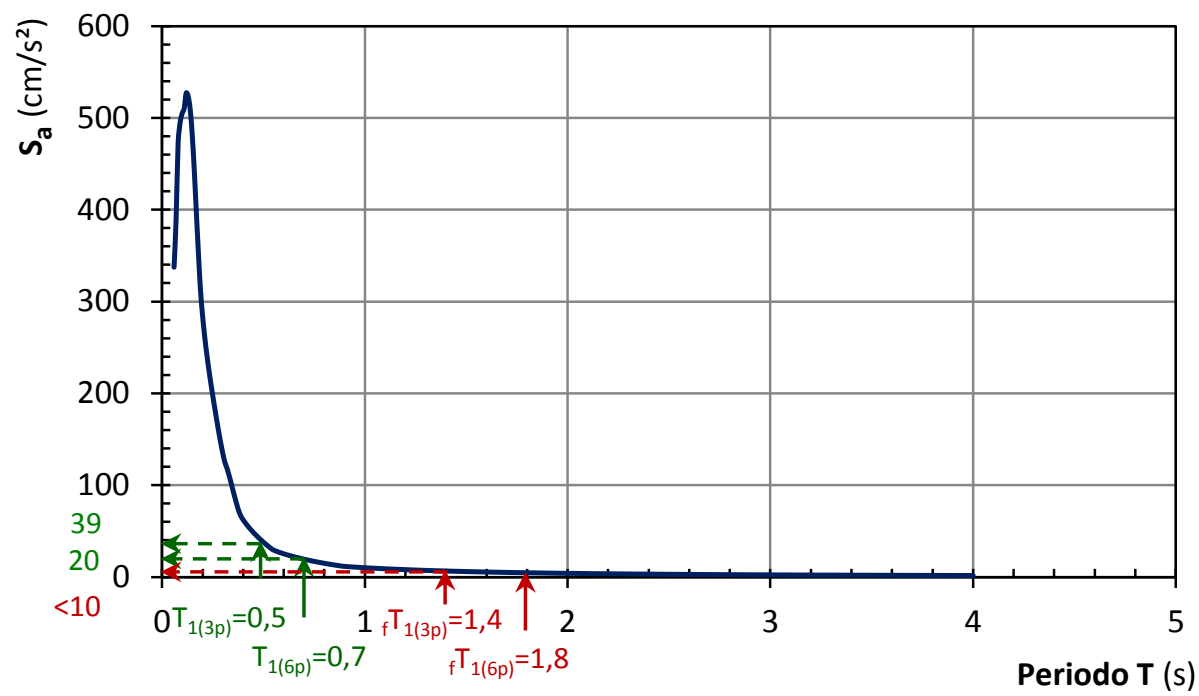

Fig. 4.3.11: Espectro elástico de respuesta del acelerograma $N^{\circ} 7$ (Timfristos) escalado a $P G A=200 \mathrm{~cm} / \mathrm{s}^{2}$

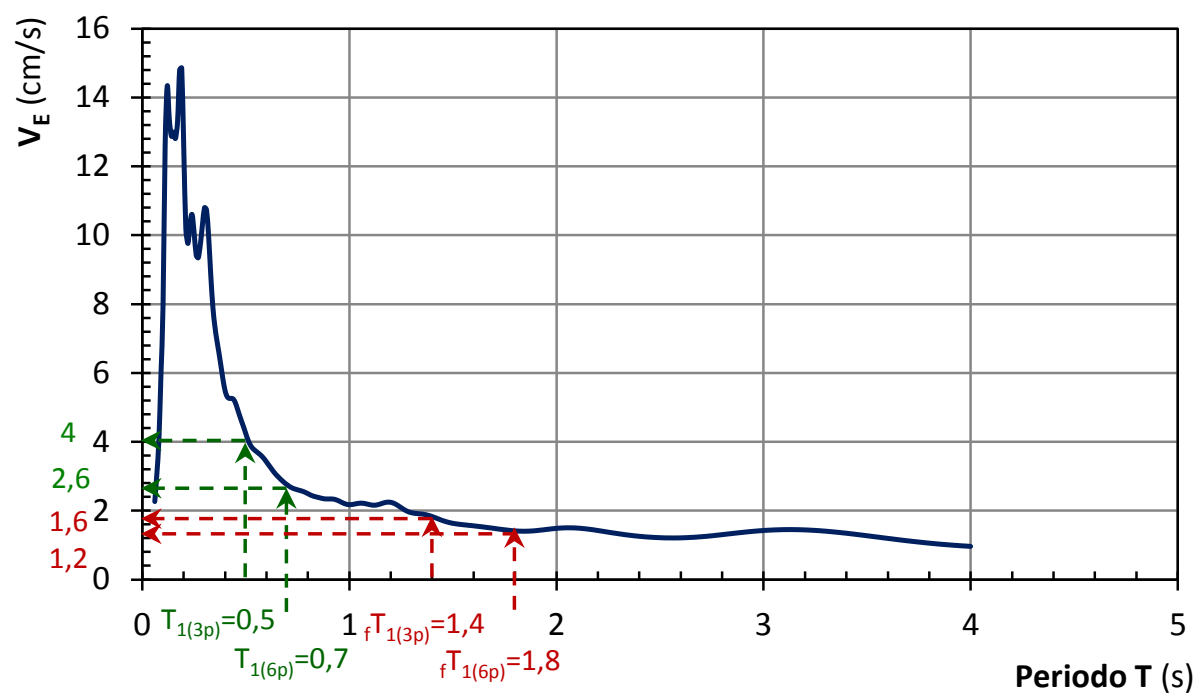

Fig. 4.3.12: Espectro de energía introducida del acelerograma $N^{\circ} 7$ (Timfristos) escalado a $P G A=200 \mathrm{~cm} / \mathrm{s}^{2}$

Comparando los valores de aceleración máxima de respuesta $S_{a}$ y de energía $V_{E}$, para los periodos de vibración anteriormente obtenidos.

Tabla 4.3.3: Ordenadas del espectro elástico de respuesta $S_{a}$ y del espectro de energía introducida, correspondientes a los periodos de las estructuras analizadas.

\begin{tabular}{c|c|c|c|c|} 
& $\mathrm{f}_{1(3 \mathrm{p})}=1,4 \mathrm{~s}$ & $\mathrm{~T}_{1(3 \mathrm{p})}=0,5 \mathrm{~s}$ & $\mathrm{f}_{1(6 \mathrm{p})}=1,8 \mathrm{~s}$ & $\mathrm{~T}_{1(6 \mathrm{p})}=0,7 \mathrm{~s}$ \\
\hline $\mathrm{S}_{\mathrm{a}}\left(\mathrm{cm} / \mathrm{s}^{2}\right)$ & $<10$ & 39 & $<10$ & 20 \\
\hline $\mathrm{V}_{\mathrm{E}}(\mathrm{cm} / \mathrm{s})$ & 1,6 & 4 & 1,2 & 2,6 \\
\hline & \multicolumn{2}{|l}{}
\end{tabular}




\subsubsection{Suelo Tipo I (Campo lejano a la falla)}

\section{- Acelerograma №1 (Friuli) $\Delta=27 \mathrm{Km}$}

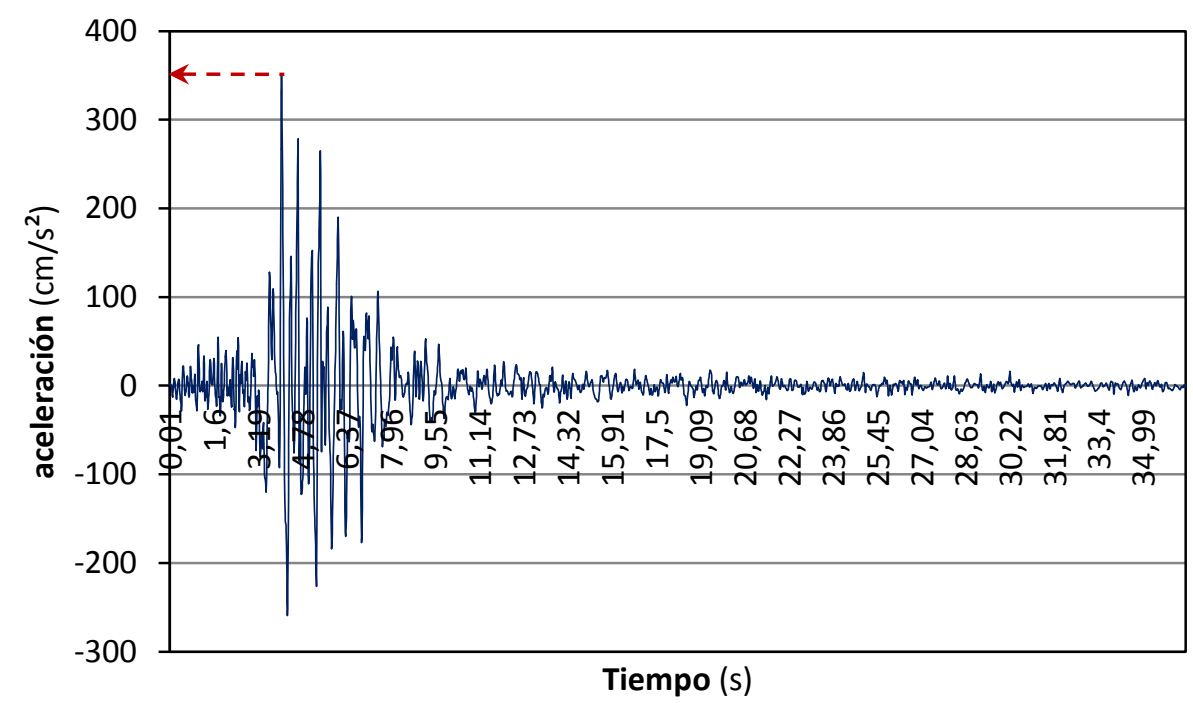

Fig. 4.3.13: Acelerograma $\mathrm{N}^{\circ} 1$ (Friuli), suelo tipo I, campo lejano, sin escalar

Aceleración sísmica de cálculo: $a_{c}=200 \mathrm{~cm} / \mathrm{s}^{2}$

Aceleración máxima: $P G A=349,85 \mathrm{~cm} / \mathrm{s}^{2}$

Factor de escala: $\frac{200 \mathrm{~cm} / \mathrm{s}^{2}}{349,85 \mathrm{~cm} / \mathrm{s}^{2}}=\underline{0,5717}$

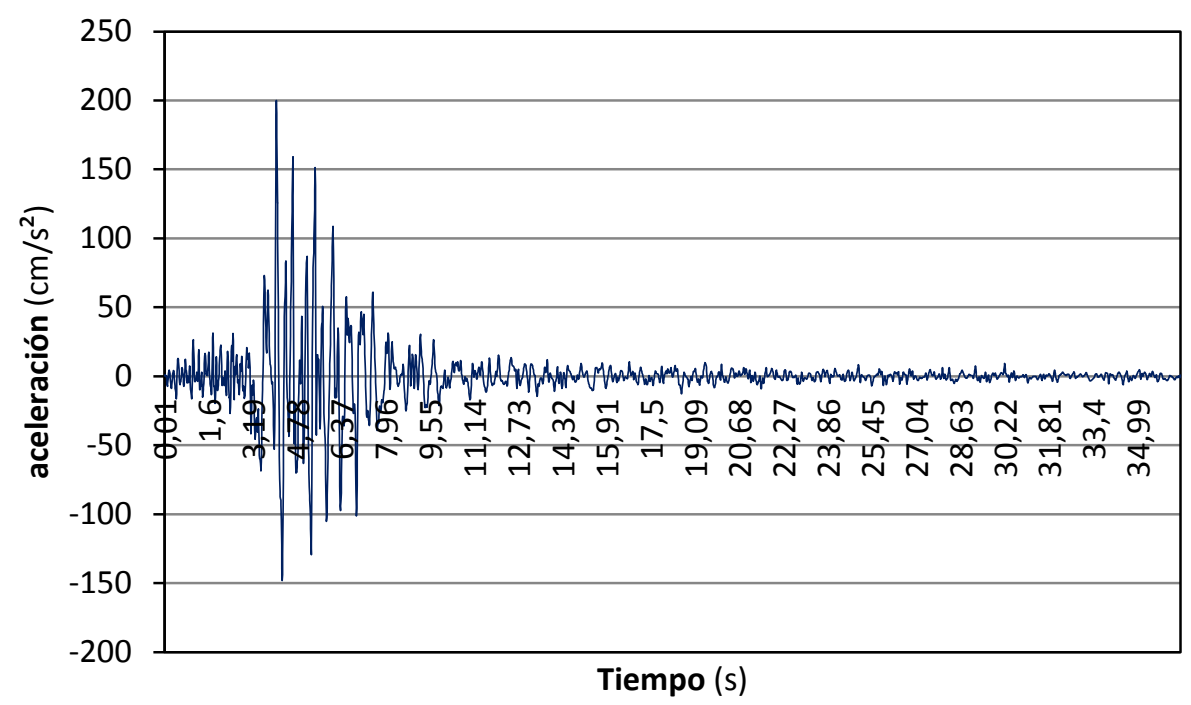

Fig. 4.3.14: Acelerograma $N^{\circ} 1$ (Friuli), suelo tipo I, campo lejano, escalado a $P G A=200 \mathrm{~cm} / \mathrm{s}^{2}$ 


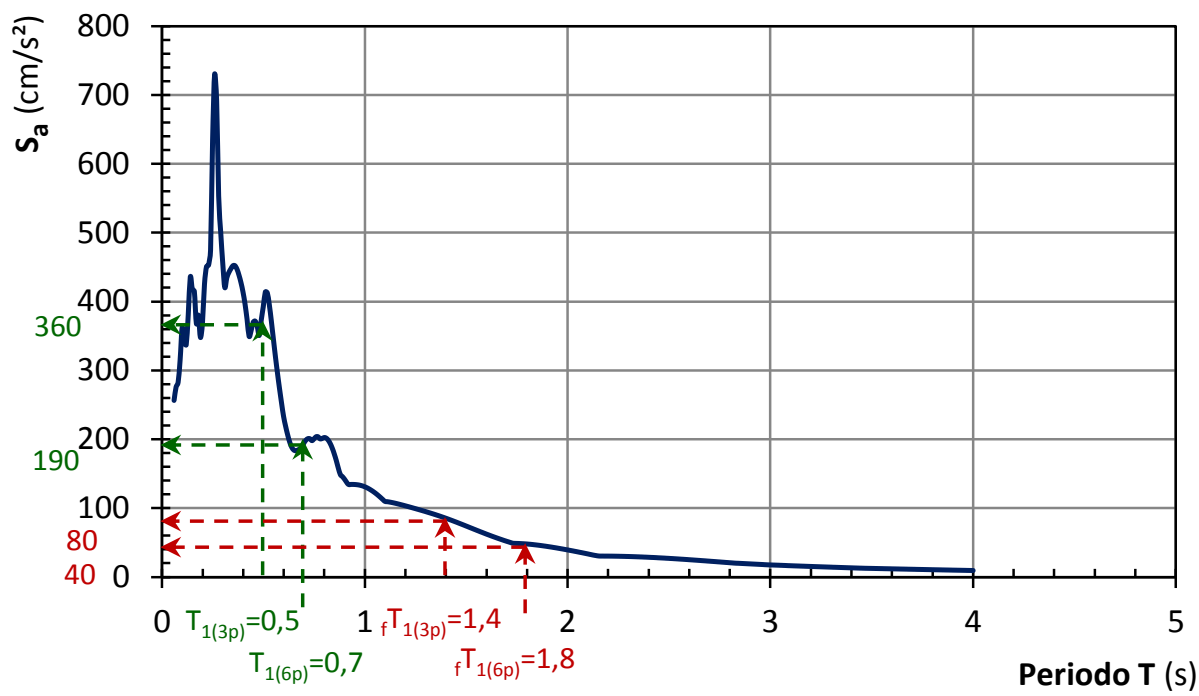

Fig. 4.3.15: Espectro elástico de respuesta del acelerograma $N^{\circ} 1$ (Friuli) escalado a $P G A=200 \mathrm{~cm} / \mathrm{s}^{2}$

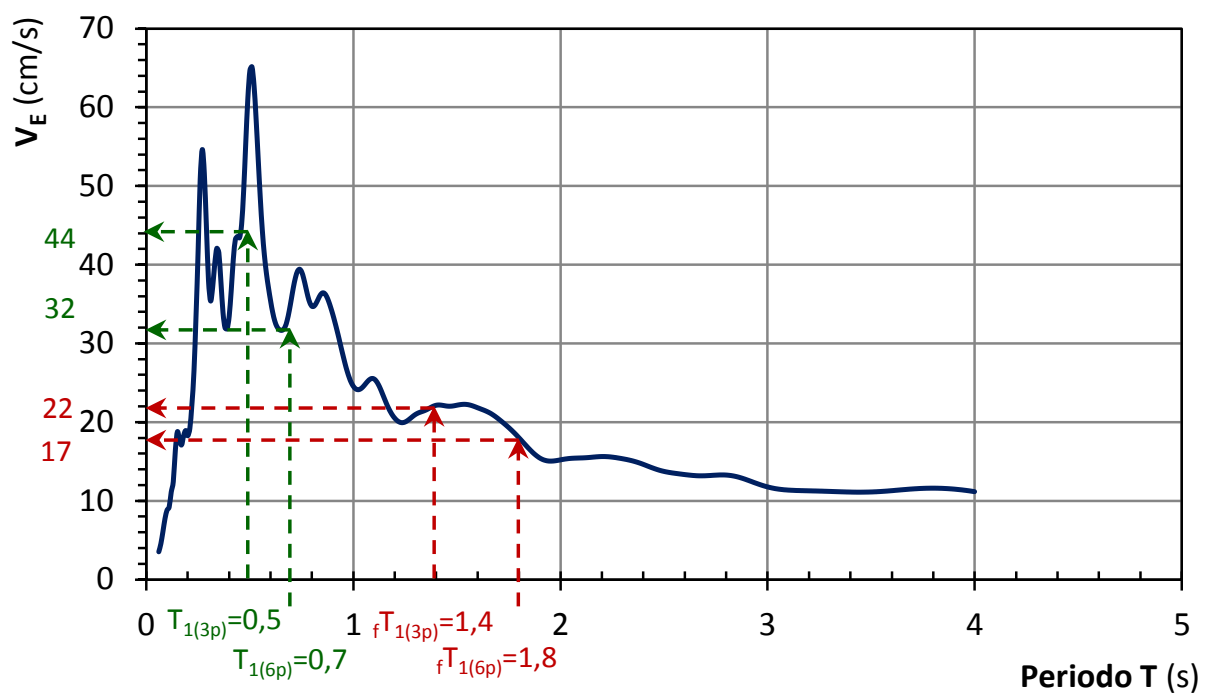

Fig. 4.3.16: Espectro de energía introducida del acelerograma $N^{\circ} 1$ (Friuli) escalado a $P G A=200 \mathrm{~cm} / \mathrm{s}^{2}$

Comparando los valores de aceleración máxima de respuesta $S_{a}$ y de energía $V_{E}$, para los periodos de vibración anteriormente obtenidos.

Tabla 4.3.4: Ordenadas del espectro elástico de respuesta $S_{a}$ y del espectro de energía introducida, correspondientes a los periodos de las estructuras analizadas.

\begin{tabular}{c|c|c|c|c|} 
& $\mathrm{f}_{1(3 \mathrm{p})}=1,4 \mathrm{~s}$ & $\mathrm{~T}_{1(3 \mathrm{p})}=0,5 \mathrm{~s}$ & $\mathrm{f}_{1(6 \mathrm{p})}=1,8 \mathrm{~s}$ & $\mathrm{~T}_{1(6 \mathrm{p})}=0,7 \mathrm{~s}$ \\
\hline $\mathrm{S}_{\mathrm{a}}\left(\mathrm{cm} / \mathrm{s}^{2}\right)$ & 80 & 360 & 40 & 190 \\
\hline $\mathrm{V}_{\mathrm{E}}(\mathrm{cm} / \mathrm{s})$ & 22 & 44 & 17 & 32 \\
\hline & \multicolumn{2}{|c|}{} & \multicolumn{2}{|c}{}
\end{tabular}




\section{- Acelerograma №4 (Montenegro) $\Delta=65 \mathrm{Km}$}

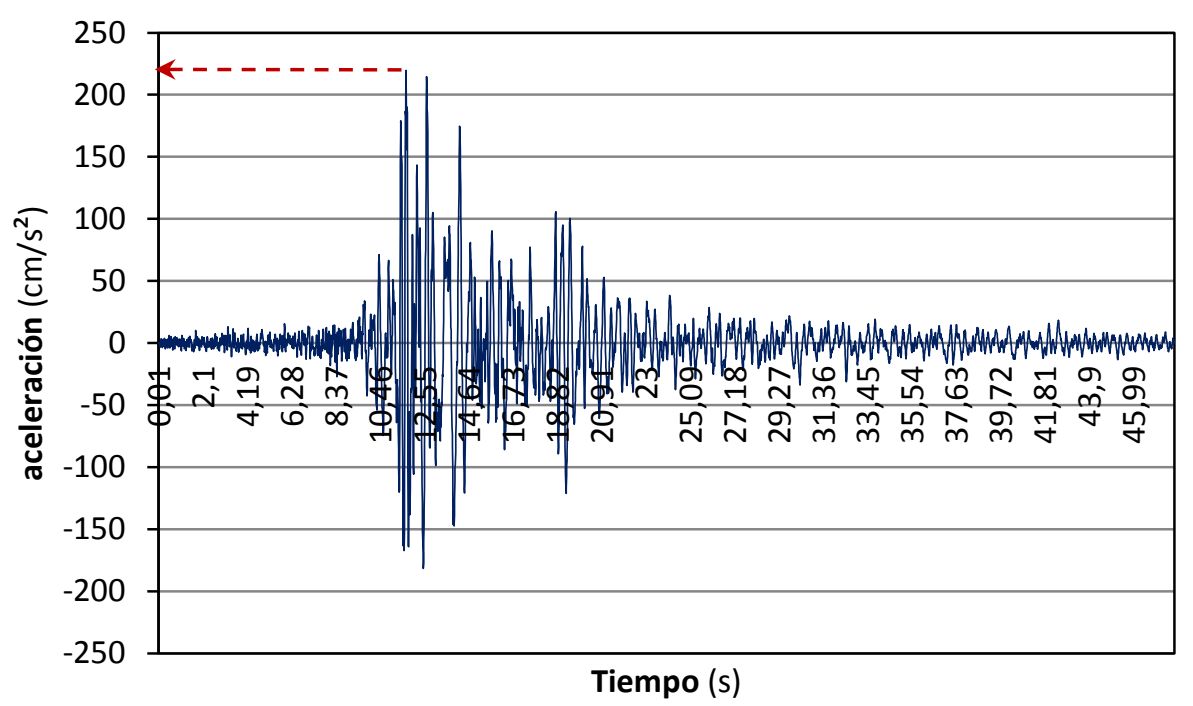

Fig. 4.3.17: Acelerograma $\mathrm{N}^{\circ} 4$ (Montenegro), suelo tipo I, campo lejano, sin escalar

Aceleración sísmica de cálculo: $a_{c}=200 \mathrm{~cm} / \mathrm{s}^{2}$

Aceleración máxima: $P G A=219,72 \mathrm{~cm} / \mathrm{s}^{2}$

Factor de escala: $\frac{200 \mathrm{~cm} / \mathrm{s}^{2}}{219,72 \mathrm{~cm} / \mathrm{s}^{2}}=\underline{0,9102}$

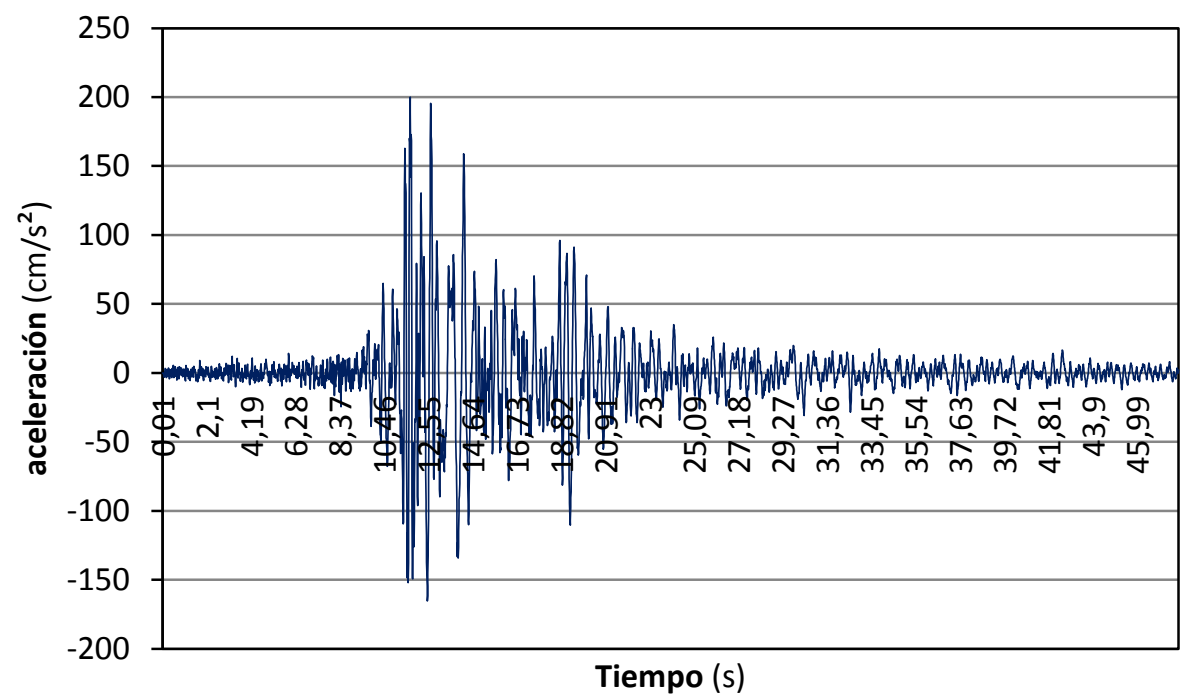

Fig. 4.3.18: Acelerograma $\mathrm{N}^{\circ} 4$ (Montenegro), suelo tipo I, campo lejano, escalado a $P G A=200 \mathrm{~cm} / \mathrm{s}^{2}$ 


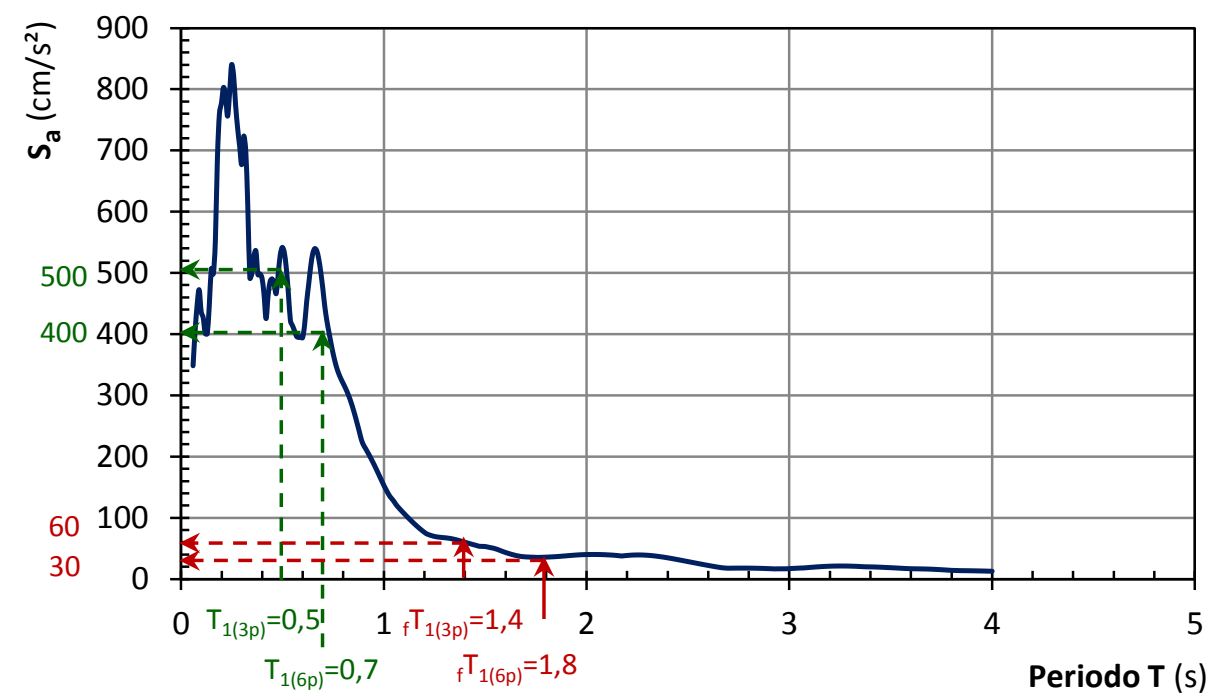

Fig. 4.3.19: Espectro elástico de respuesta del acelerograma $\mathrm{N}^{\circ} 4$ (Montenegro) escalado a $P G A=200 \mathrm{~cm} / \mathrm{s}^{2}$

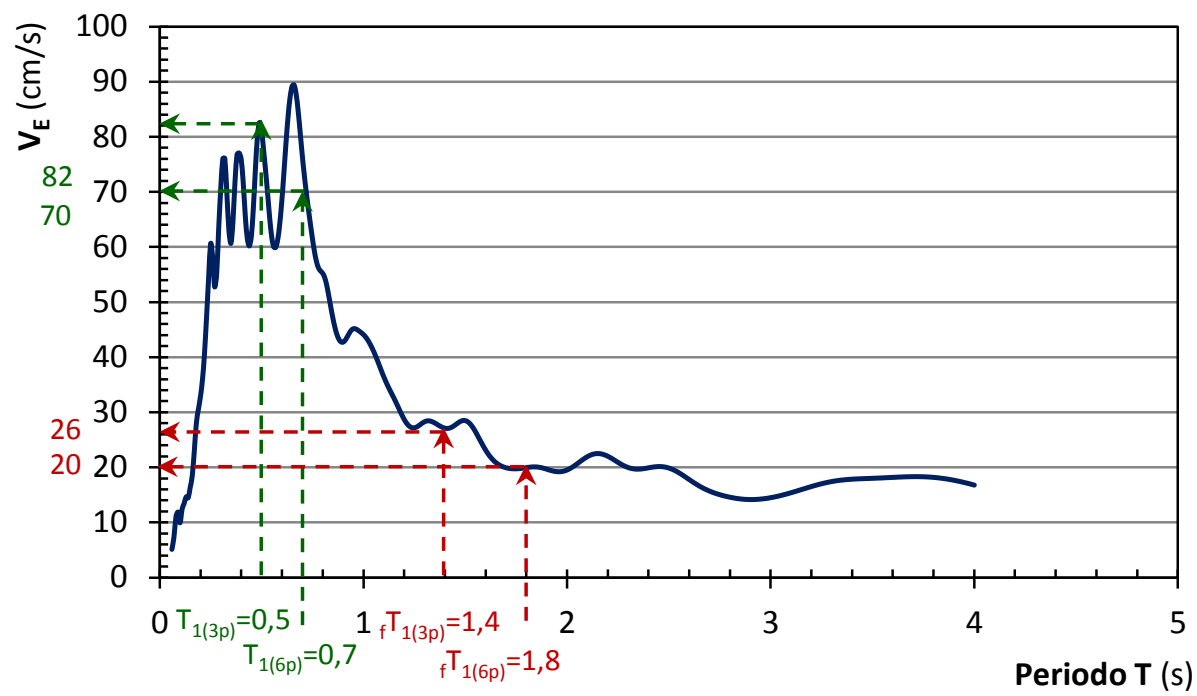

Fig. 4.3.20: Espectro de energía introducida del acelerograma $\mathrm{N}^{\circ} 4$ (Montenegro) escalado a $P G A=200 \mathrm{~cm} / \mathrm{s}^{2}$

Comparando los valores de aceleración máxima de respuesta $S_{a}$ y de energía $V_{E}$, para los periodos de vibración anteriormente obtenidos.

Tabla 4.3.5: Ordenadas del espectro elástico de respuesta $S_{\mathrm{a}}$ y del espectro de energía introducida, correspondientes a los periodos de las estructuras analizadas.

\begin{tabular}{l|c|c|c|c|} 
& $\mathrm{f}_{1(3 \mathrm{p})}=1,4 \mathrm{~s}$ & $\mathrm{~T}_{1(3 \mathrm{p})}=0,5 \mathrm{~s}$ & $\mathrm{f}_{1(6 \mathrm{p})}=1,8 \mathrm{~s}$ & $\mathrm{~T}_{1(6 \mathrm{p})}=0,7 \mathrm{~s}$ \\
\hline $\mathrm{S}_{\mathrm{a}}\left(\mathrm{cm} / \mathrm{s}^{2}\right)$ & 60 & 500 & 30 & 400 \\
\hline $\mathrm{V}_{\mathrm{E}}(\mathrm{cm} / \mathrm{s})$ & 26 & 82 & 20 & 70 \\
\hline & \multicolumn{3}{|l}{}
\end{tabular}


- Acelerograma №5 (Campano Lucano) $\Delta=32 \mathrm{Km}$

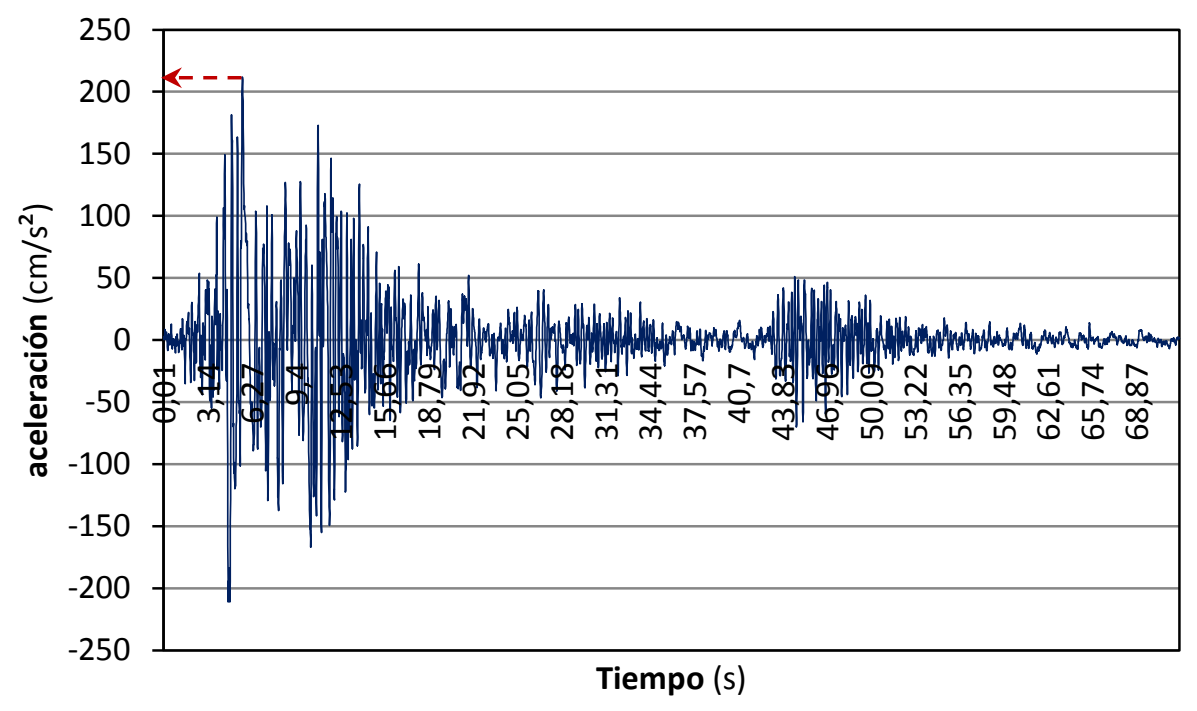

Fig. 4.3.21: Acelerograma $\mathrm{N}^{\circ} 5$ (Campano Lucano), suelo tipo I, campo lejano, sin escalar

Aceleración sísmica de cálculo: $a_{c}=200 \mathrm{~cm} / \mathrm{s}^{2}$

Aceleración máxima: $P G A=212,06 \mathrm{~cm} / \mathrm{s}^{2}$

Factor de escala: $\frac{200 \mathrm{~cm} / \mathrm{s}^{2}}{212,06 \mathrm{~cm} / \mathrm{s}^{2}}=\underline{0,9431}$

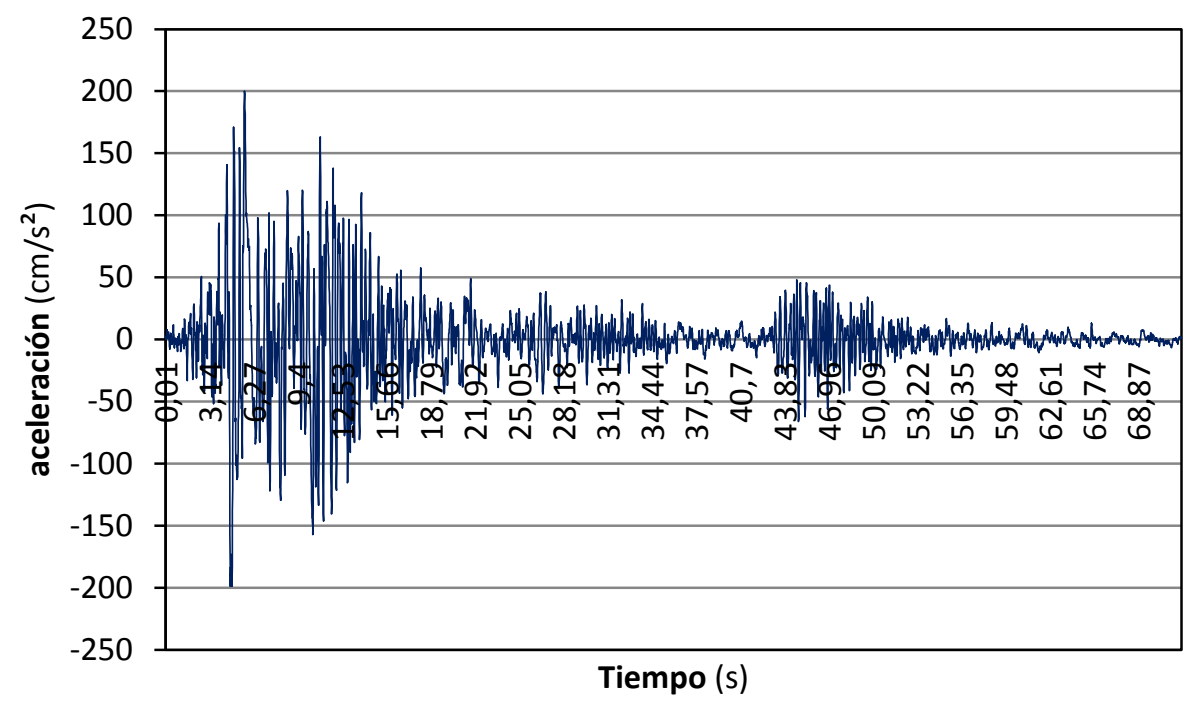

Fig. 4.3.22: Acelerograma $\mathrm{N}^{\circ} 5$ (Campano alucano), suelo tipo I, campo lejano, escalado a $P G A=200 \mathrm{~cm} / \mathrm{s}^{2}$ 


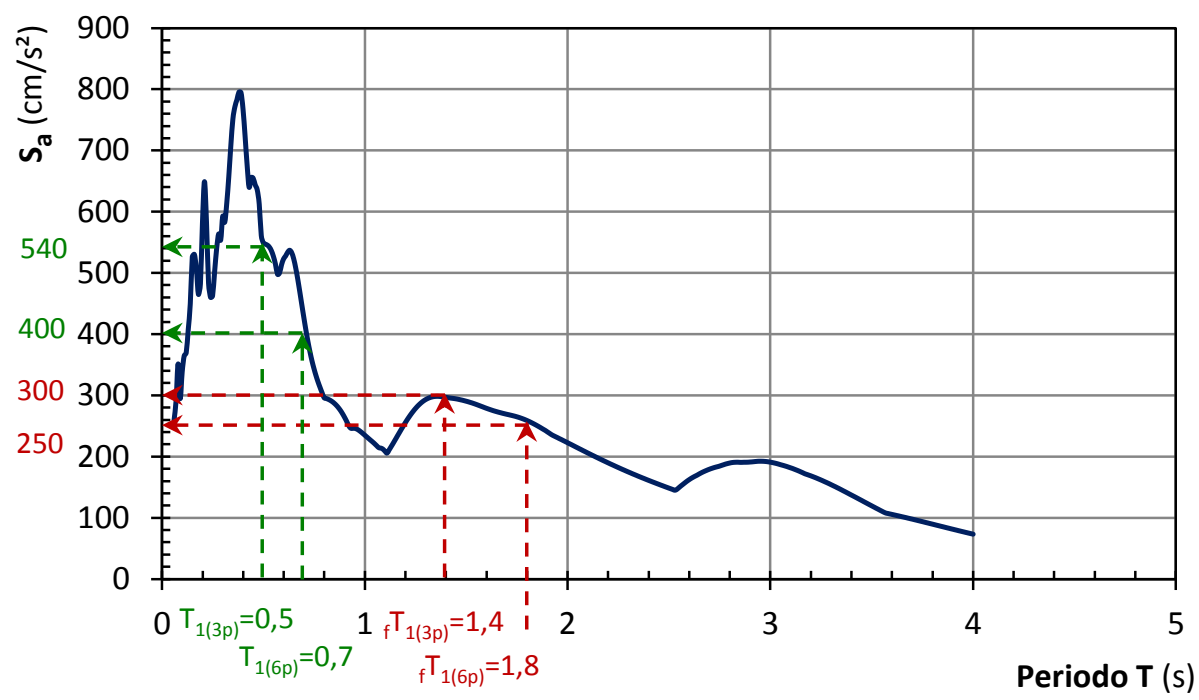

Fig. 4.3.23: Espectro elástico de respuesta del acelerograma $\mathrm{N}^{\circ} 5$ (Campano Lucano) escalado a $P G A=200 \mathrm{~cm} / \mathrm{s}^{2}$

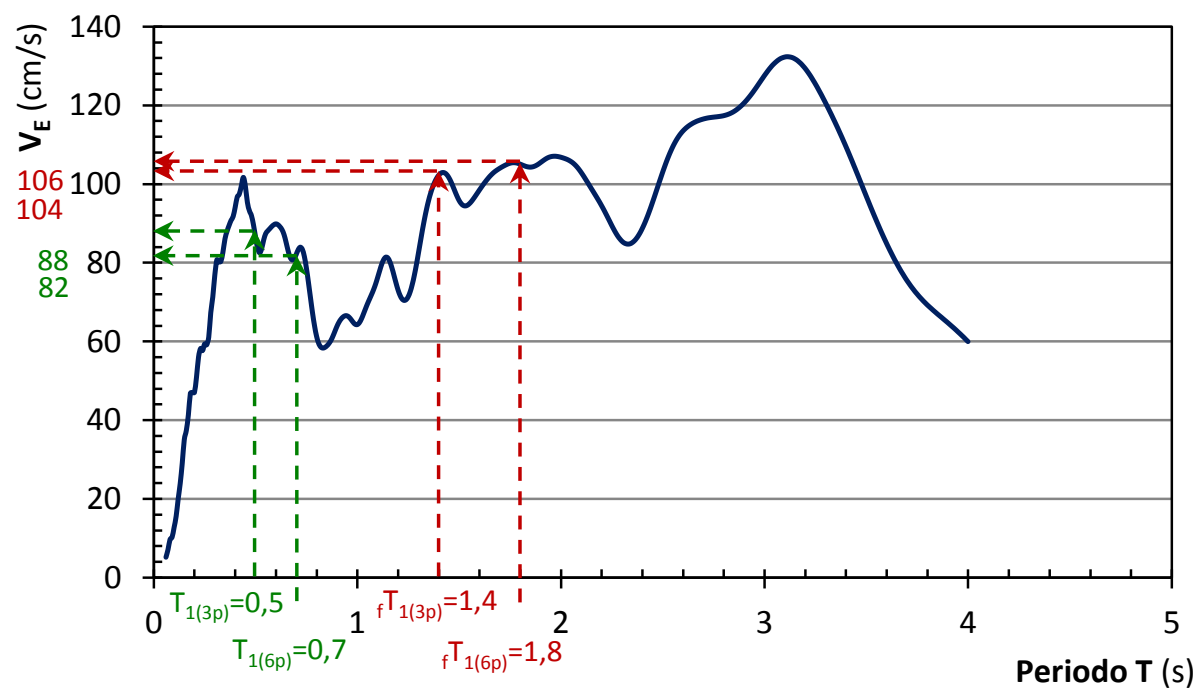

Fig. 4.3.24: Espectro de energía introducida del acelerograma $\mathrm{N}^{\circ} 5$ (Campano Lucano) escalado a $P G A=200 \mathrm{~cm} / \mathrm{s}^{2}$

Comparando los valores de aceleración máxima de respuesta $S_{a}$ y de energía $V_{E}$, para los periodos de vibración anteriormente obtenidos.

Tabla 4.3.6: Ordenadas del espectro elástico de respuesta $S_{a}$ y del espectro de energía introducida, correspondientes a los periodos de las estructuras analizadas.

\begin{tabular}{c|c|c|c|c|} 
& ${ }_{f} \mathrm{~T}_{1(3 \mathrm{p})}=1,4 \mathrm{~s}$ & $\mathrm{~T}_{1(3 \mathrm{p})}=0,5 \mathrm{~s}$ & $\mathrm{f}_{1(6 \mathrm{p})}=1,8 \mathrm{~s}$ & $\mathrm{~T}_{1(6 \mathrm{p})}=0,7 \mathrm{~s}$ \\
\hline $\mathrm{S}_{\mathrm{a}}\left(\mathrm{cm} / \mathrm{s}^{2}\right)$ & 300 & 540 & 250 & 400 \\
\hline $\mathrm{V}_{\mathrm{E}}(\mathrm{cm} / \mathrm{s})$ & 104 & 88 & 106 & 82 \\
\hline & \multicolumn{1}{|l}{}
\end{tabular}




\subsubsection{Suelo Tipo II (Campo cercano a la falla)}

\section{- Acelerograma №1 (Friuli aftershock) $\Delta=3 \mathrm{Km}$}

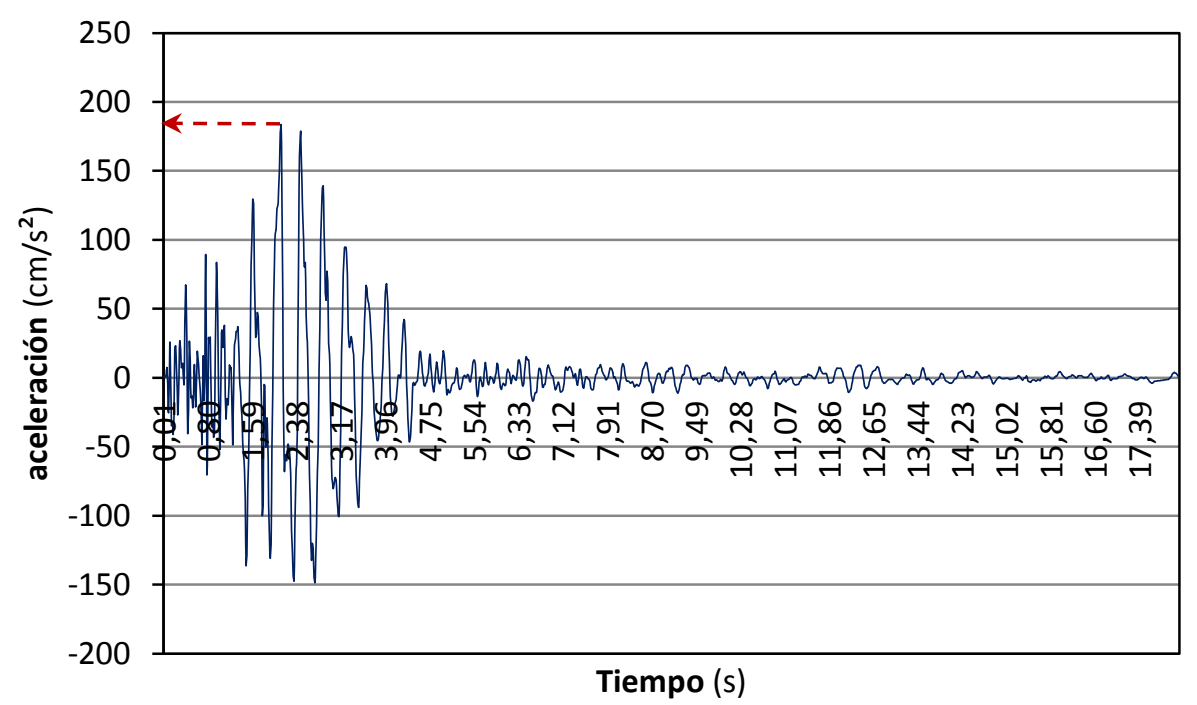

Fig. 4.3.25: Acelerograma $\mathrm{N}^{\circ} 1$ (Friuli aftershock), suelo tipo II, campo cercano, sin escalar

Aceleración sísmica de cálculo: $a_{c}=230,5 \mathrm{~cm} / \mathrm{s}^{2}$

Aceleración máxima: $P G A=183,61 \mathrm{~cm} / \mathrm{s}^{2}$

Factor de escala: $\frac{230,5 \mathrm{~cm} / \mathrm{s}^{2}}{183,61 \mathrm{~cm} / \mathrm{s}^{2}}=\underline{1,2554}$

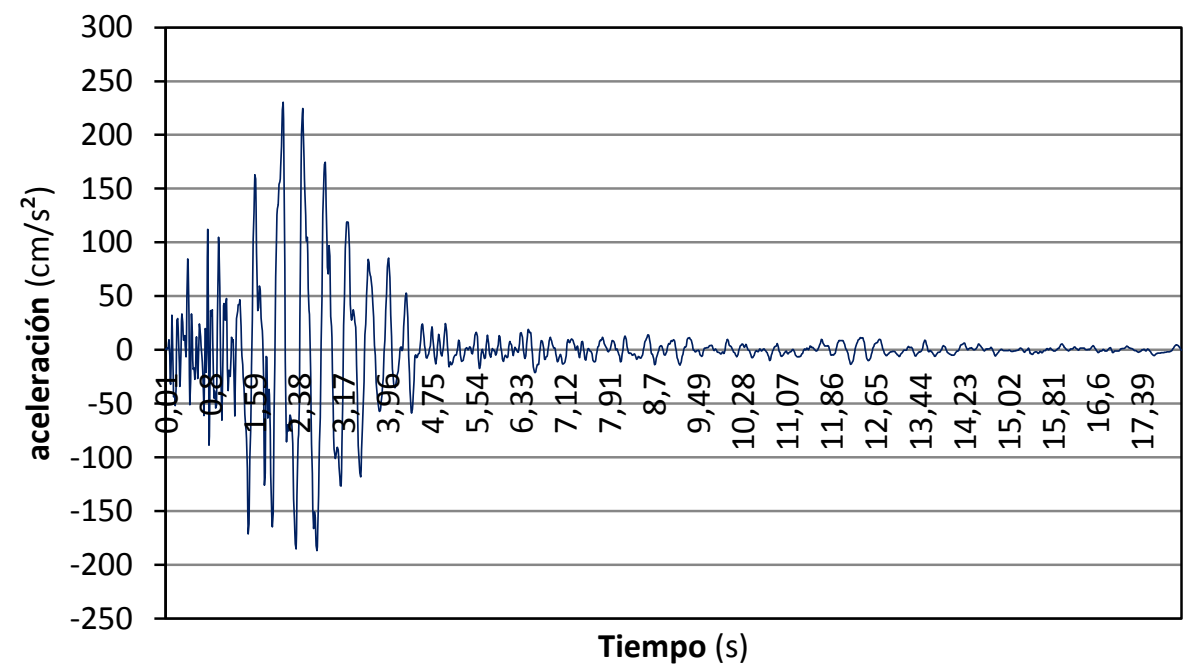

Fig. 4.3.26: Acelerograma $\mathrm{N}^{\circ} 1$ (Friuli aftershock), suelo tipo II, campo cercano, escalado a $P G A=230,5 \mathrm{~cm} / \mathrm{s}^{2}$ 


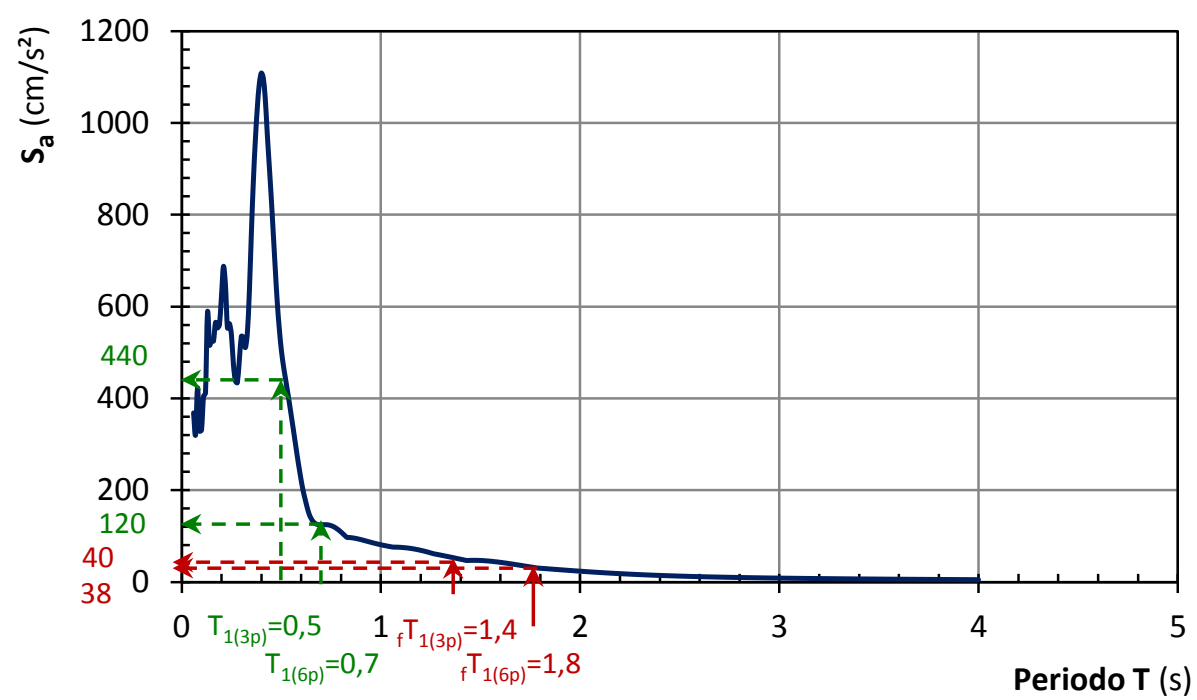

Fig. 4.3.27: Espectro elástico de respuesta del acelerograma $\mathrm{N}^{\circ} 1$ (Friuli aftershock) escalado a $P G A=230,5 \mathrm{~cm} / \mathrm{s}^{2}$

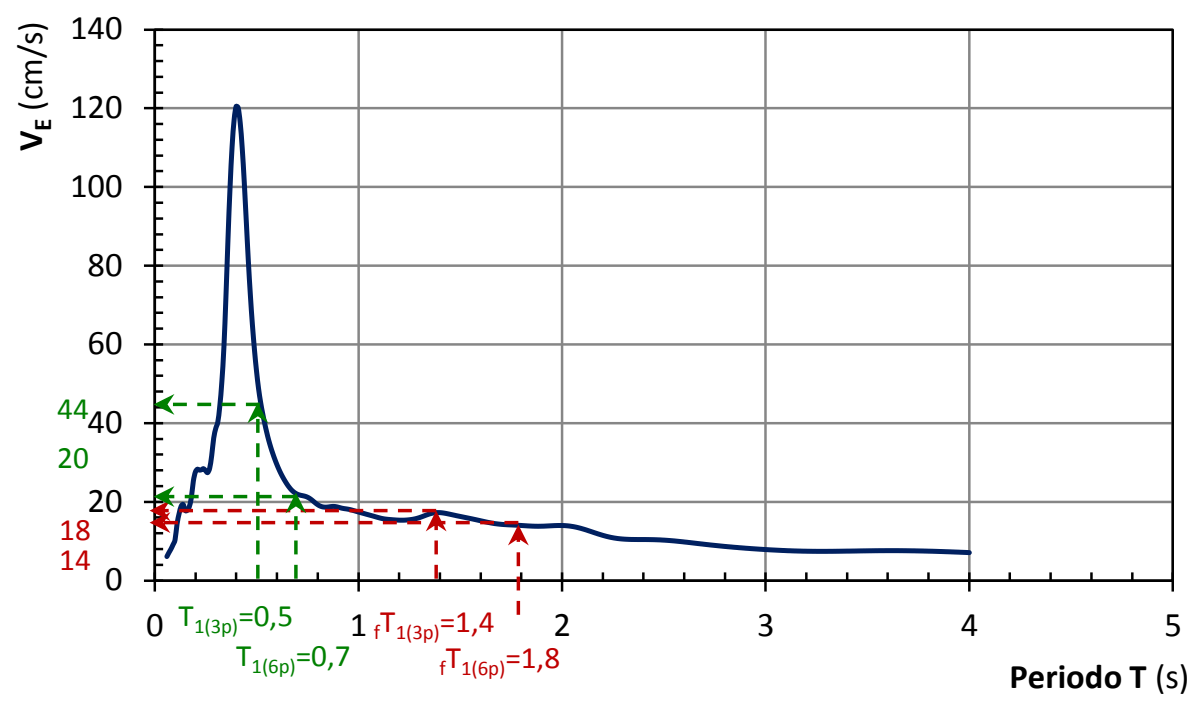

Fig. 4.3.28: Espectro de energía introducida del acelerograma $N^{\circ} 1$ (Friuli aftershock) escalado a $P G A=230,5 \mathrm{~cm} / \mathrm{s}^{2}$

Comparando los valores de aceleración máxima de respuesta $S_{a}$ y de energía $V_{E}$, para los periodos de vibración anteriormente obtenidos.

Tabla 4.3.7: Ordenadas del espectro elástico de respuesta $S_{a}$ y del espectro de energía introducida, correspondientes a los periodos de las estructuras analizadas.

\begin{tabular}{c|c|c|c|c|} 
& ${ }_{\mathrm{f}} \mathrm{T}_{1(3 \mathrm{p})}=1,4 \mathrm{~s}$ & $\mathrm{~T}_{1(3 \mathrm{p})}=0,5 \mathrm{~s}$ & $\mathrm{f}_{1(6 \mathrm{p})}=1,8 \mathrm{~s}$ & $\mathrm{~T}_{1(6 \mathrm{p})}=0,7 \mathrm{~s}$ \\
\hline $\mathrm{S}_{\mathrm{a}}\left(\mathrm{cm} / \mathrm{s}^{2}\right)$ & 40 & 440 & 38 & 120 \\
\hline $\mathrm{V}_{\mathrm{E}}(\mathrm{cm} / \mathrm{s})$ & 18 & 44 & 14 & 20 \\
\hline & \multicolumn{3}{|l}{}
\end{tabular}




\section{- Acelerograma №8 (Friuli aftershock) $\Delta=5 \mathrm{Km}$}

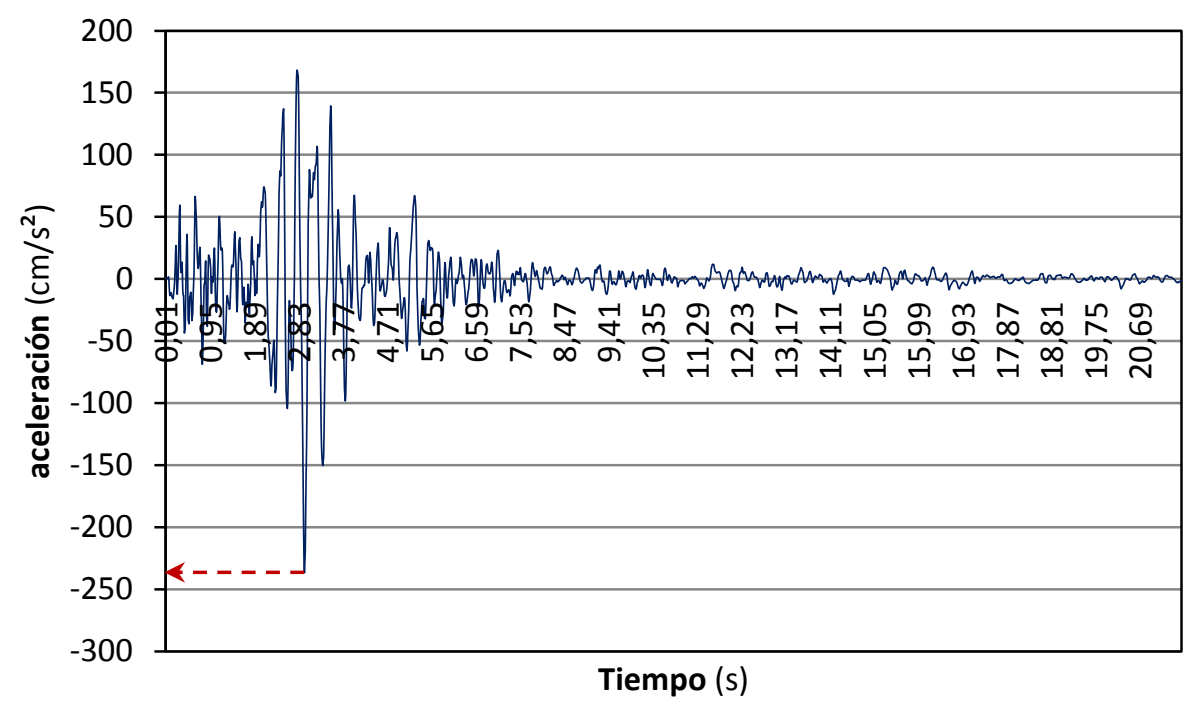

Fig. 4.3.29: Acelerograma $\mathrm{N}^{\circ} 8$ (Friuli aftershock), suelo tipo II, campo cercano, sin escalar

Aceleración sísmica de cálculo: $a_{c}=230,5 \mathrm{~cm} / \mathrm{s}^{2}$

Aceleración máxima: $P G A=236,53 \mathrm{~cm} / \mathrm{s}^{2}$

Factor de escala: $\frac{230,5 \mathrm{~cm} / \mathrm{s}^{2}}{236,53 \mathrm{~cm} / \mathrm{s}^{2}}=\underline{0,9745}$

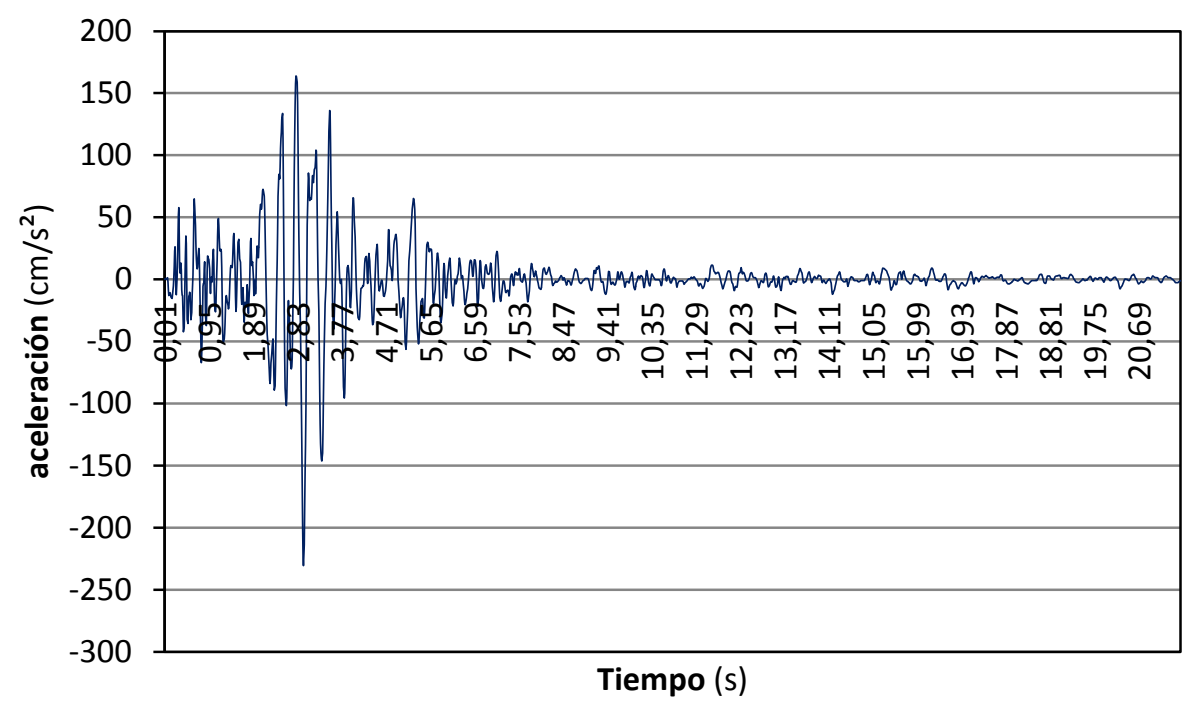

Fig. 4.3.30: Acelerograma $\mathrm{N}^{\circ} 8$ (Friuli aftershock), suelo tipo II, campo cercano, escalado a $P G A=230,5 \mathrm{~cm} / \mathrm{s}^{2}$ 


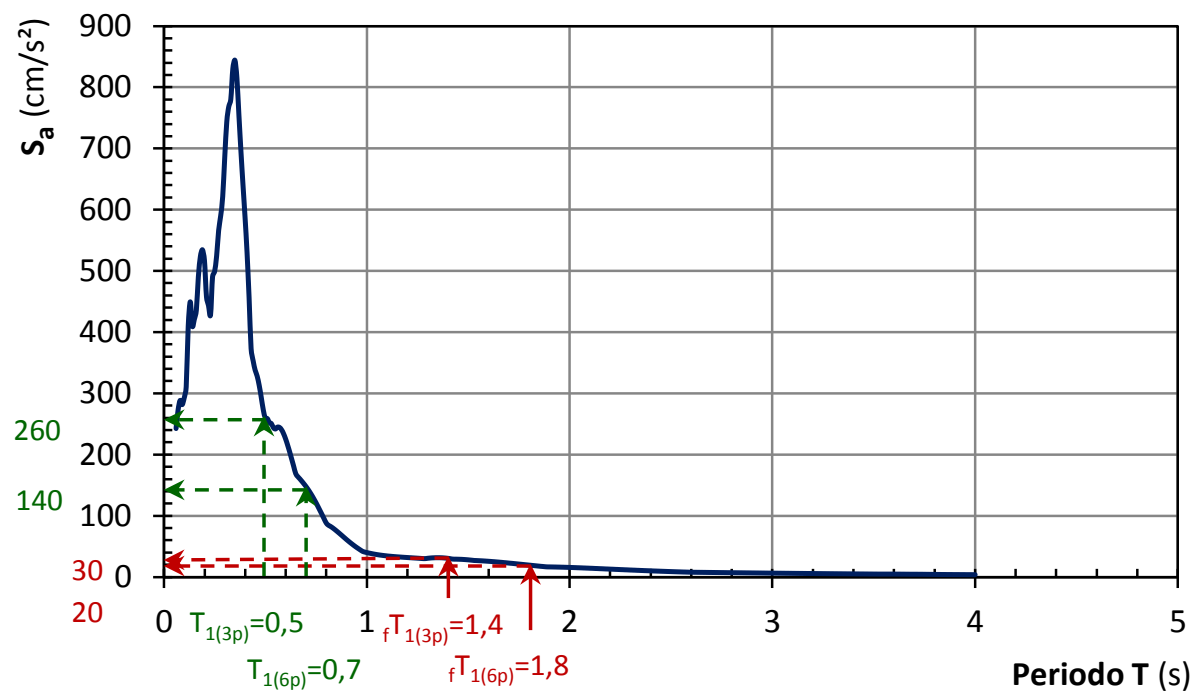

Fig. 4.3.31: Espectro elástico de respuesta del acelerograma $N^{\circ} 8$ (Friuli aftershock) escalado a $P G A=230,5 \mathrm{~cm} / \mathrm{s}^{2}$

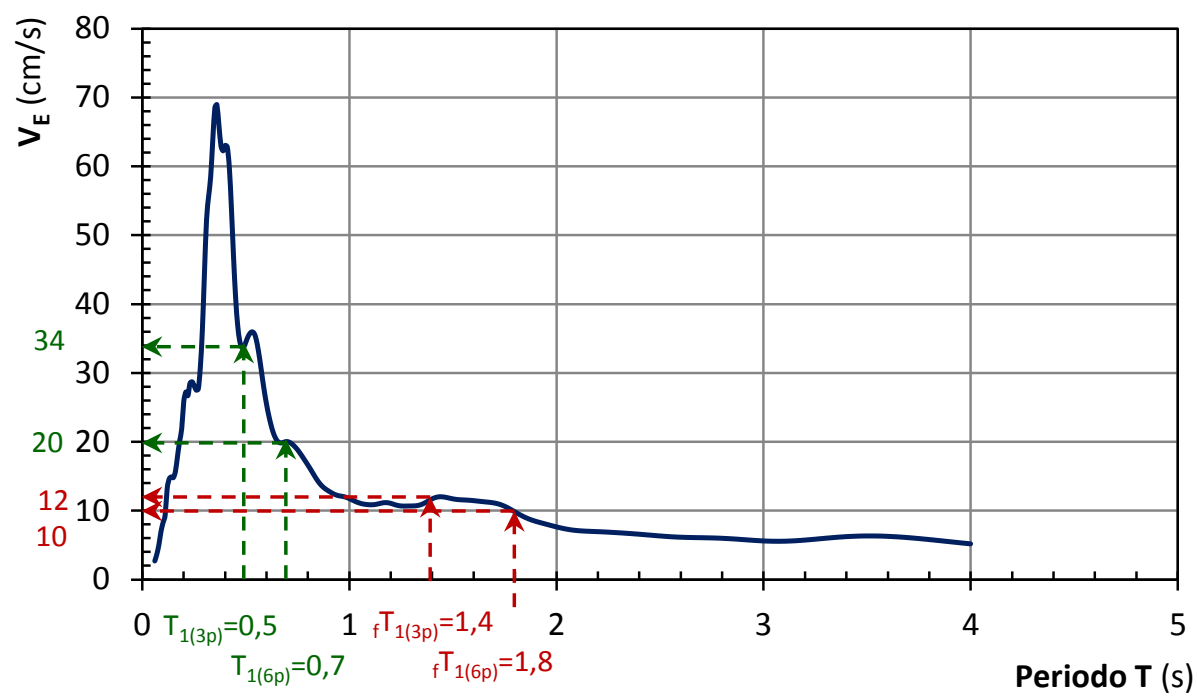

Fig. 4.3.32: Espectro de energía introducida del acelerograma $N^{\circ} 8$ (Friuli aftershock) escalado a $P G A=230,5 \mathrm{~cm} / \mathrm{s}^{2}$

Comparando los valores de aceleración máxima de respuesta $S_{a}$ y de energía $V_{E}$, para los periodos de vibración anteriormente obtenidos.

Tabla 4.3.8: Ordenadas del espectro elástico de respuesta $S_{a}$ y del espectro de energía introducida, correspondientes a los periodos de las estructuras analizadas.

\begin{tabular}{l|c|c|c|c|} 
& ${ }_{\mathrm{T}} \mathrm{T}_{1(3 \mathrm{p})}=1,4 \mathrm{~s}$ & $\mathrm{~T}_{1(3 \mathrm{p})}=0,5 \mathrm{~s}$ & ${ }_{\mathrm{f}} \mathrm{T}_{1(6 \mathrm{p})}=1,8 \mathrm{~s}$ & $\mathrm{~T}_{1(6 \mathrm{p})}=0,7 \mathrm{~s}$ \\
\hline $\mathrm{S}_{\mathrm{a}}\left(\mathrm{cm} / \mathrm{s}^{2}\right)$ & 30 & 260 & 20 & 140 \\
\hline $\mathrm{V}_{\mathrm{E}}(\mathrm{cm} / \mathrm{s})$ & 12 & 34 & 10 & 20 \\
\hline & \multicolumn{3}{|l}{}
\end{tabular}




\section{- Acelerograma №14 (Montenegro aftershock) $\Delta=8 \mathrm{Km}$}

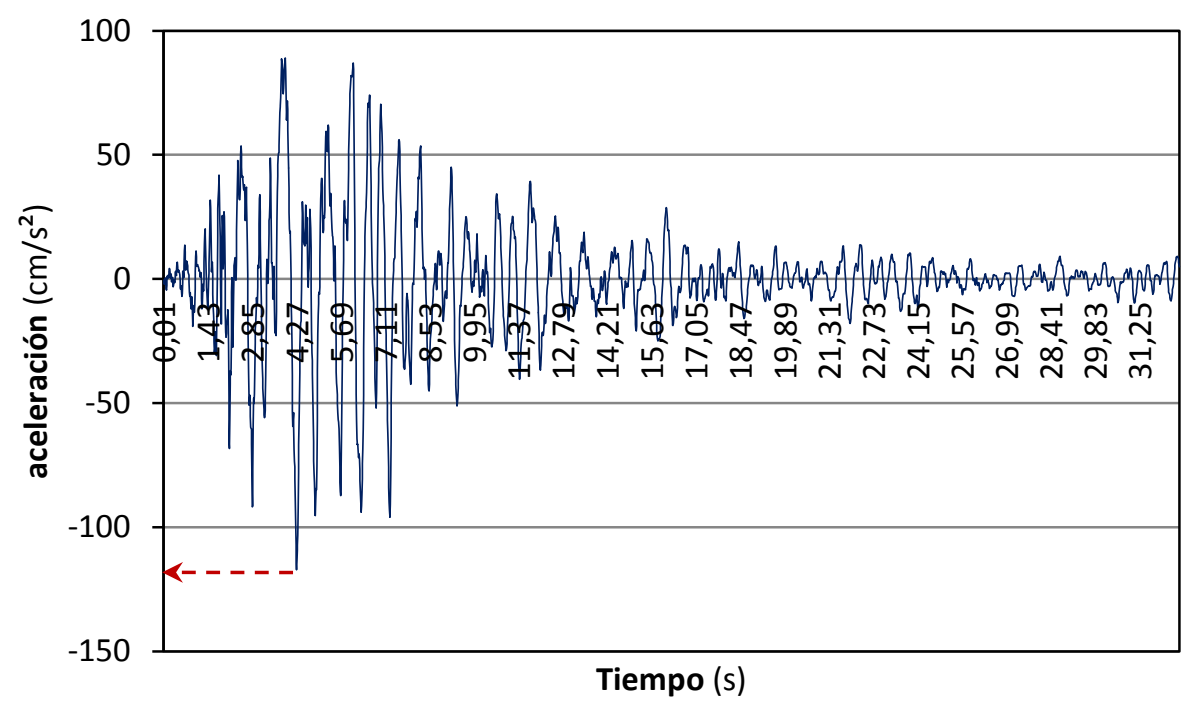

Fig. 4.3.33 Acelerograma $\mathrm{N}^{\circ} 14$ (Montenegro aftershock), suelo tipo II, campo cercano, sin escalar

Aceleración sísmica de cálculo: $a_{c}=230,5 \mathrm{~cm} / \mathrm{s}^{2}$

Aceleración máxima: $P G A=117,23 \mathrm{~cm} / \mathrm{s}^{2}$

Factor de escala: $\frac{230,5 \mathrm{~cm} / \mathrm{s}^{2}}{117,23 \mathrm{~cm} / \mathrm{s}^{2}}=\underline{1,9662}$

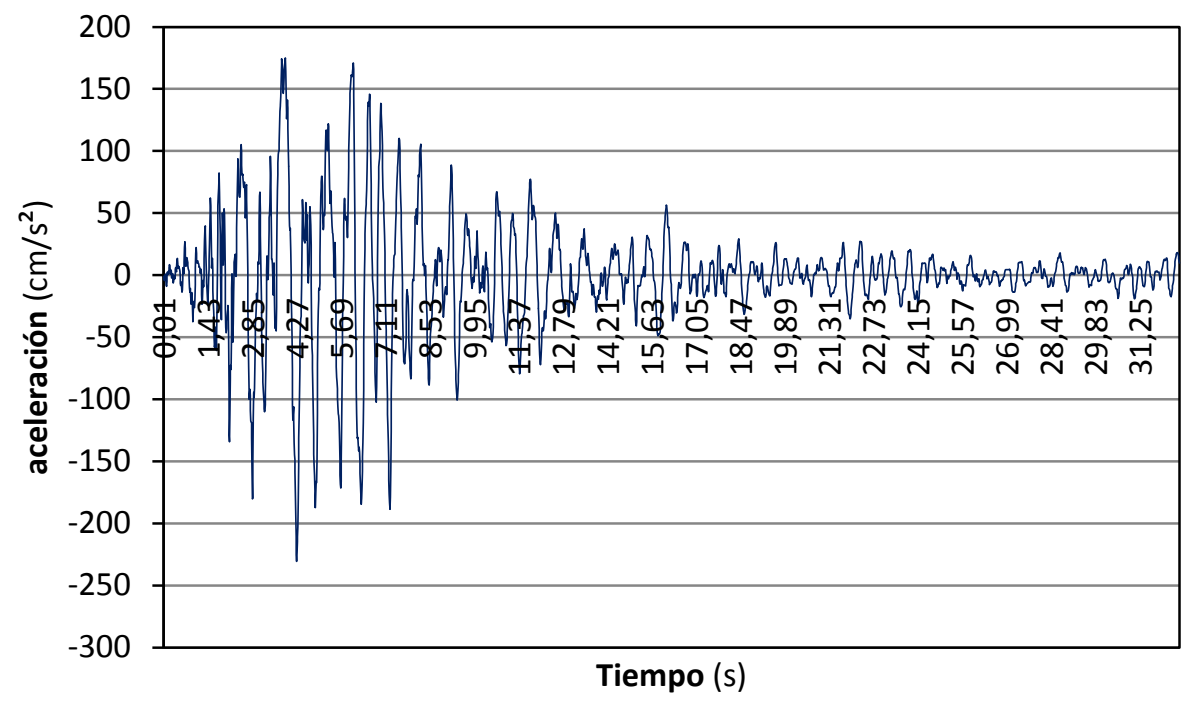

Fig. 4.3.34: Acelerograma $\mathrm{N}^{\circ} 14$ (Montenegro aftershock), suelo tipo II, campo cercano, escalado a $P G A=230,5 \mathrm{~cm} / \mathrm{s}^{2}$ 


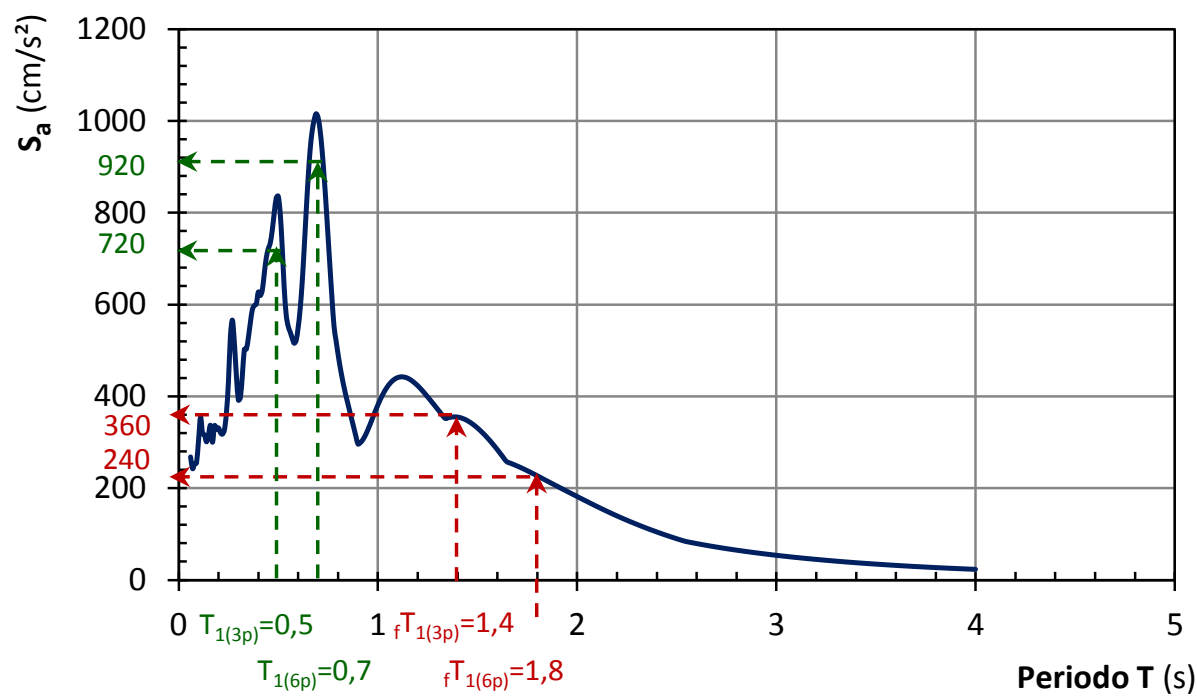

Fig. 4.3.35: Espectro elástico de respuesta del acelerograma $\mathrm{N}^{\circ} 14$ (Montenegro aftershock) escalado a $P G A=230,5 \mathrm{~cm} / \mathrm{s}^{2}$

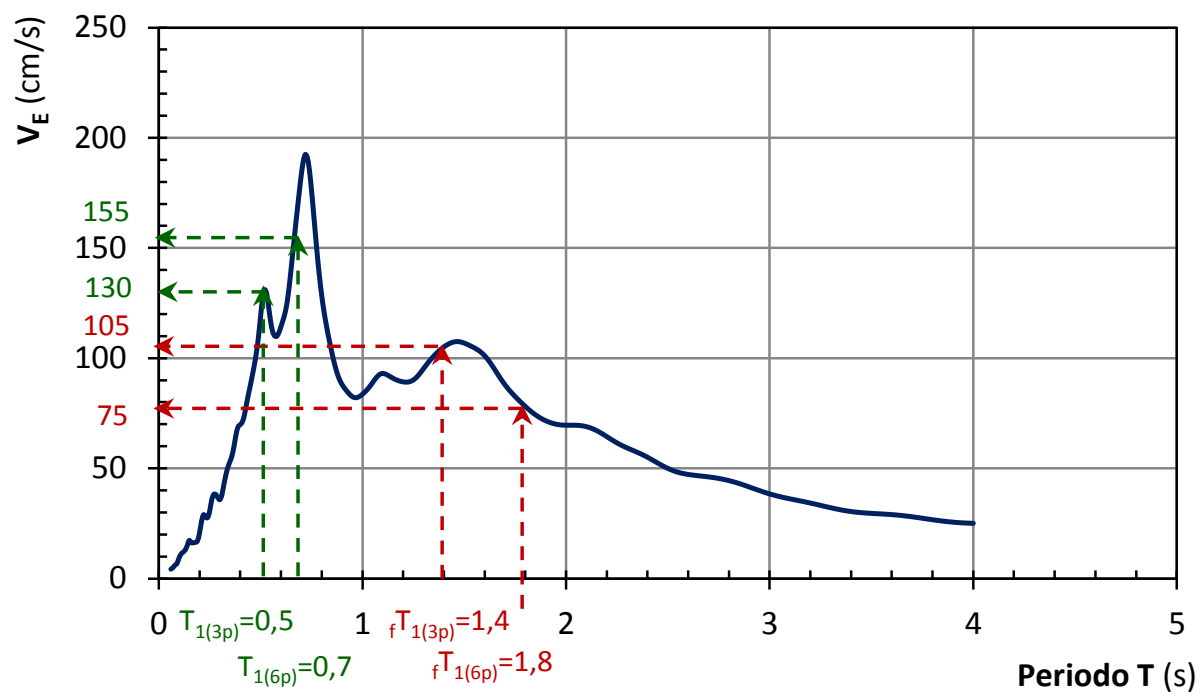

Fig. 4.3.36: Espectro de energía introducida del acelerograma $\mathrm{N}^{\circ} 14$ (Montenegro aftershock) escalado a $P G A=230,5 \mathrm{~cm} / \mathrm{s}^{2}$

Comparando los valores de aceleración máxima de respuesta $S_{a}$ y de energía $V_{E}$, para los periodos de vibración anteriormente obtenidos.

Tabla 4.3.9: Ordenadas del espectro elástico de respuesta $S_{a}$ y del espectro de energía introducida, correspondientes a los periodos de las estructuras analizadas.

\begin{tabular}{c|c|c|c|c|} 
& $\mathrm{f}_{1(3 \mathrm{p})}=1,4 \mathrm{~s}$ & $\mathrm{~T}_{1(3 \mathrm{p})}=0,5 \mathrm{~s}$ & $\mathrm{f}_{1(6 \mathrm{p})}=1,8 \mathrm{~s}$ & $\mathrm{~T}_{1(6 \mathrm{p})}=0,7 \mathrm{~s}$ \\
\hline $\mathrm{S}_{\mathrm{a}}\left(\mathrm{cm} / \mathrm{s}^{2}\right)$ & 360 & 720 & 240 & 920 \\
\hline $\mathrm{V}_{\mathrm{E}}(\mathrm{cm} / \mathrm{s})$ & 105 & 130 & 75 & 155 \\
\hline & \multicolumn{3}{|l}{} & \multicolumn{2}{|c}{}
\end{tabular}




\subsubsection{Suelo Tipo II (Campo lejano a la falla)}

\section{- Acelerograma №2 (Friuli aftershock) $\Delta=16 \mathrm{Km}$}

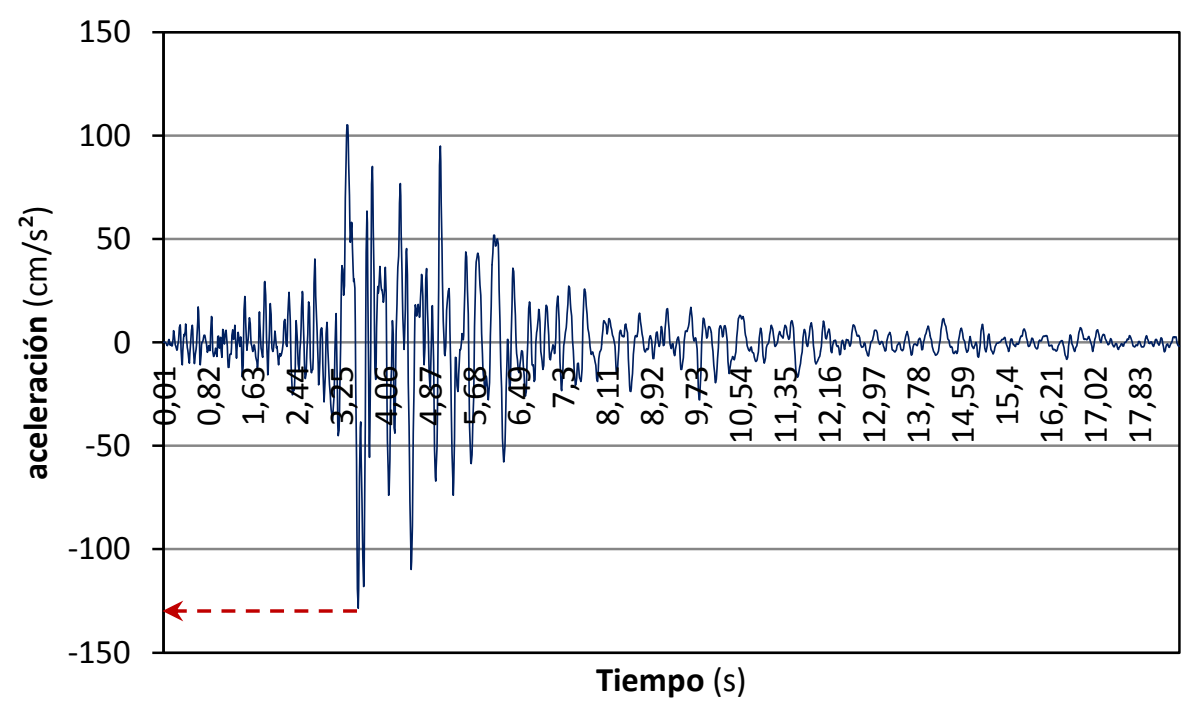

Fig. 4.3.37: Acelerograma $\mathrm{N}^{\circ} 2$ (Friuli aftershock), suelo tipo II, campo lejano, sin escalar

Aceleración sísmica de cálculo: $a_{c}=230,5 \mathrm{~cm} / \mathrm{s}^{2}$

Aceleración máxima: $P G A=128,56 \mathrm{~cm} / \mathrm{s}^{2}$

Factor de escala: $\frac{230,5 \mathrm{~cm} / \mathrm{s}^{2}}{128,56 \mathrm{~cm} / \mathrm{s}^{2}}=\underline{1,7929}$

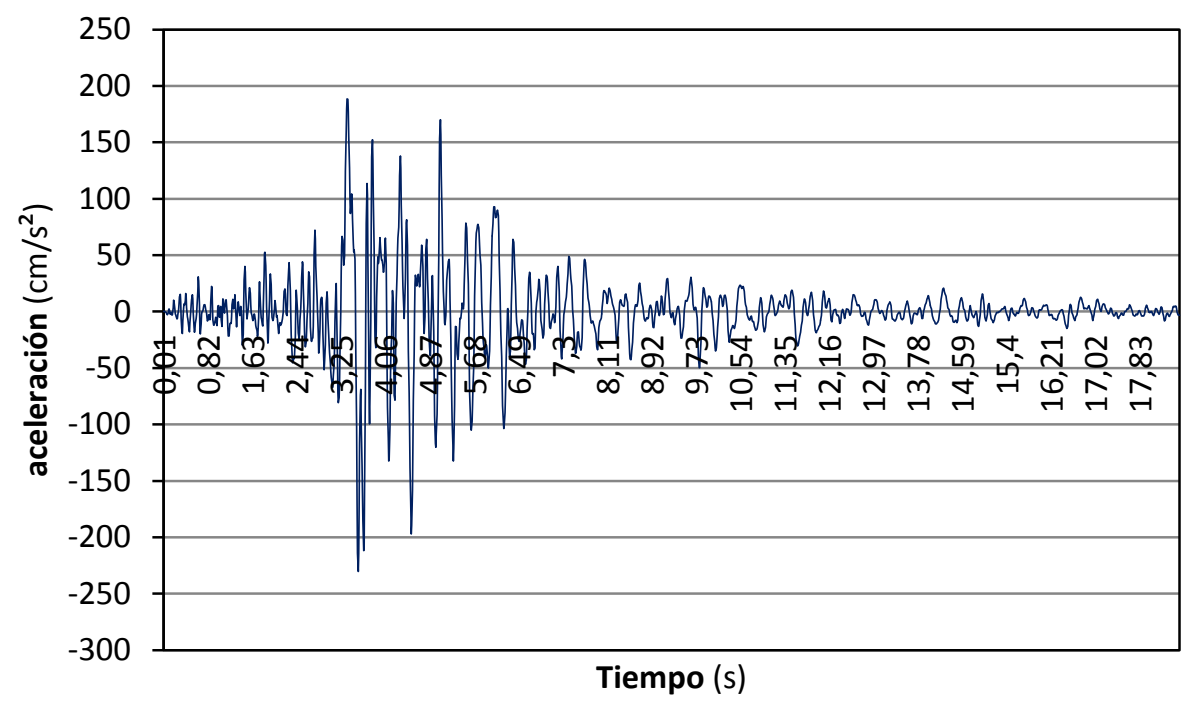

Fig. 4.3.38: Acelerograma $\mathrm{N}^{\circ} 2$ (Friuli aftershock), suelo tipo II, campo lejano, escalado a $P G A=230,5 \mathrm{~cm} / \mathrm{s}^{2}$ 


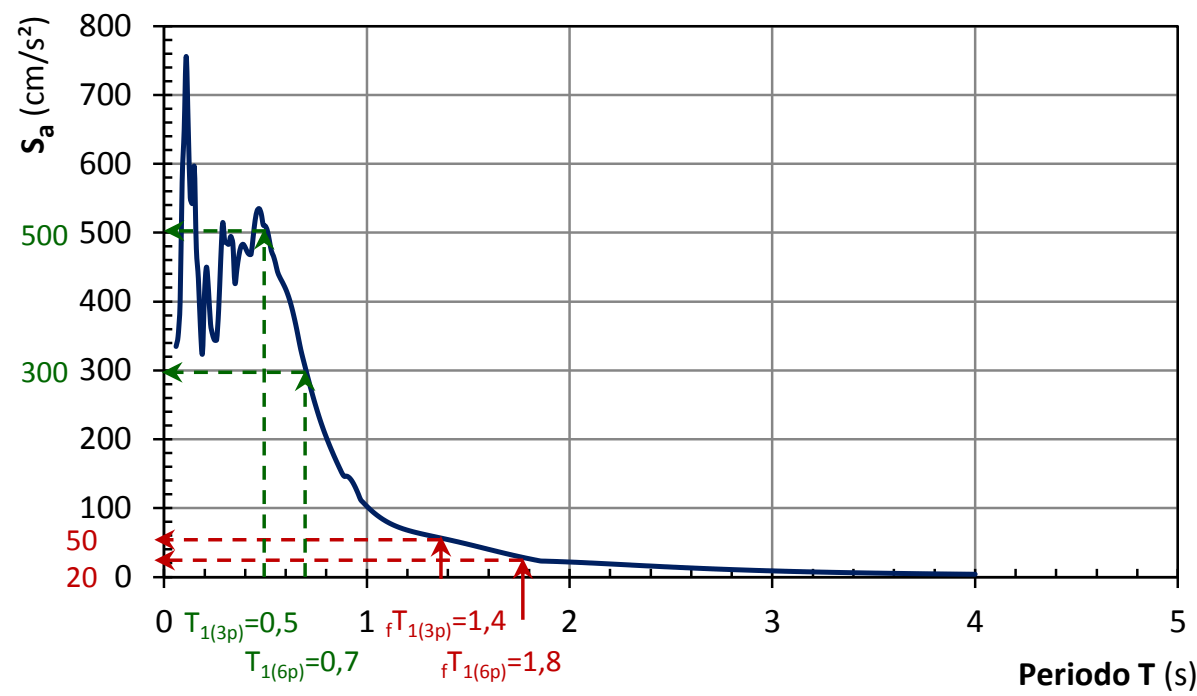

Fig. 4.3.39: Espectro elástico de respuesta del acelerograma $N^{\circ} 2$ (Friuli aftershock) escalado a $P G A=230,5 \mathrm{~cm} / \mathrm{s}^{2}$

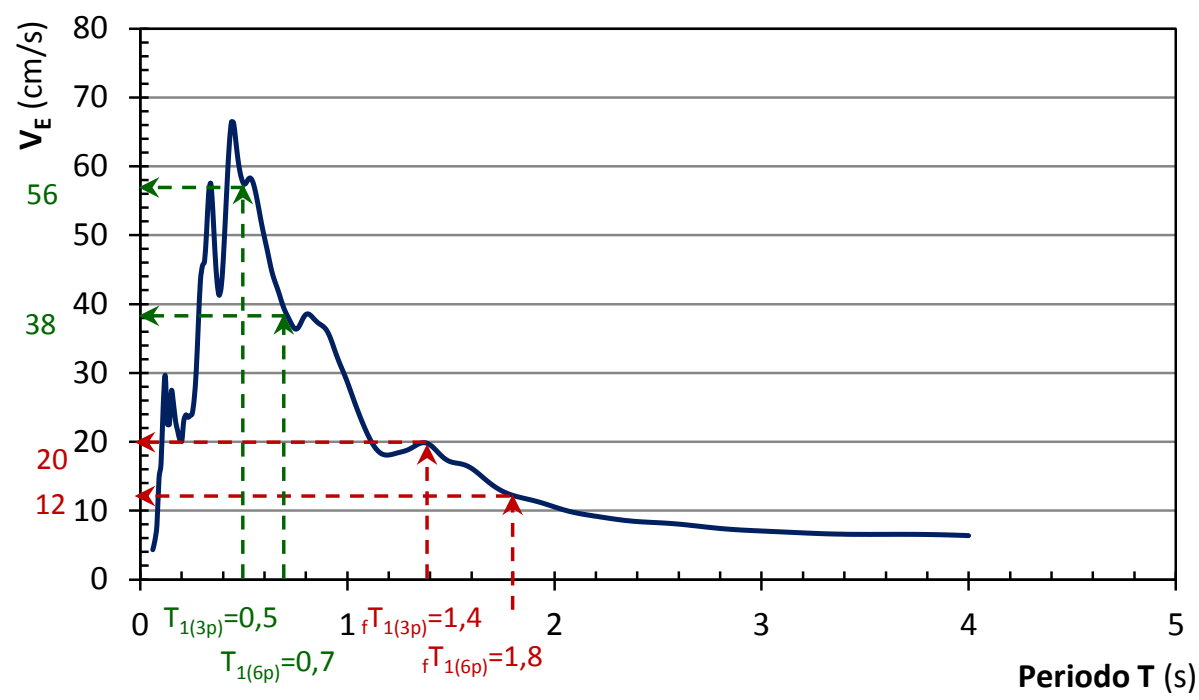

Fig. 4.3.40: Espectro de energía introducida del acelerograma $N^{\circ} 2$ (Friuli aftershock) escalado a $P G A=230,5 \mathrm{~cm} / \mathrm{s}^{2}$

Comparando los valores de aceleración máxima de respuesta $S_{a}$ y de energía $V_{E}$, para los periodos de vibración anteriormente obtenidos.

Tabla 4.3.10: Ordenadas del espectro elástico de respuesta $S_{a}$ y del espectro de energía introducida, correspondientes a los periodos de las estructuras analizadas.

\begin{tabular}{|c|c|c|c|c|}
\hline & ${ }_{f} T_{1(3 p)}=1,4 s$ & $T_{1(3 p)}=0,5 s$ & ${ }_{f} T_{1(6 p)}=1,8 s$ & $T_{1(6 \mathrm{p})}=0,7 \mathrm{~s}$ \\
\hline $\mathrm{S}_{\mathrm{a}}\left(\mathrm{cm} / \mathrm{s}^{2}\right)$ & 50 & 500 & 20 & 300 \\
\hline $\mathrm{V}_{\mathrm{E}}(\mathrm{cm} / \mathrm{s})$ & 20 & 56 & 12 & 38 \\
\hline
\end{tabular}


- Acelerograma №4 (Friuli aftershock) $\Delta=17 \mathrm{Km}$

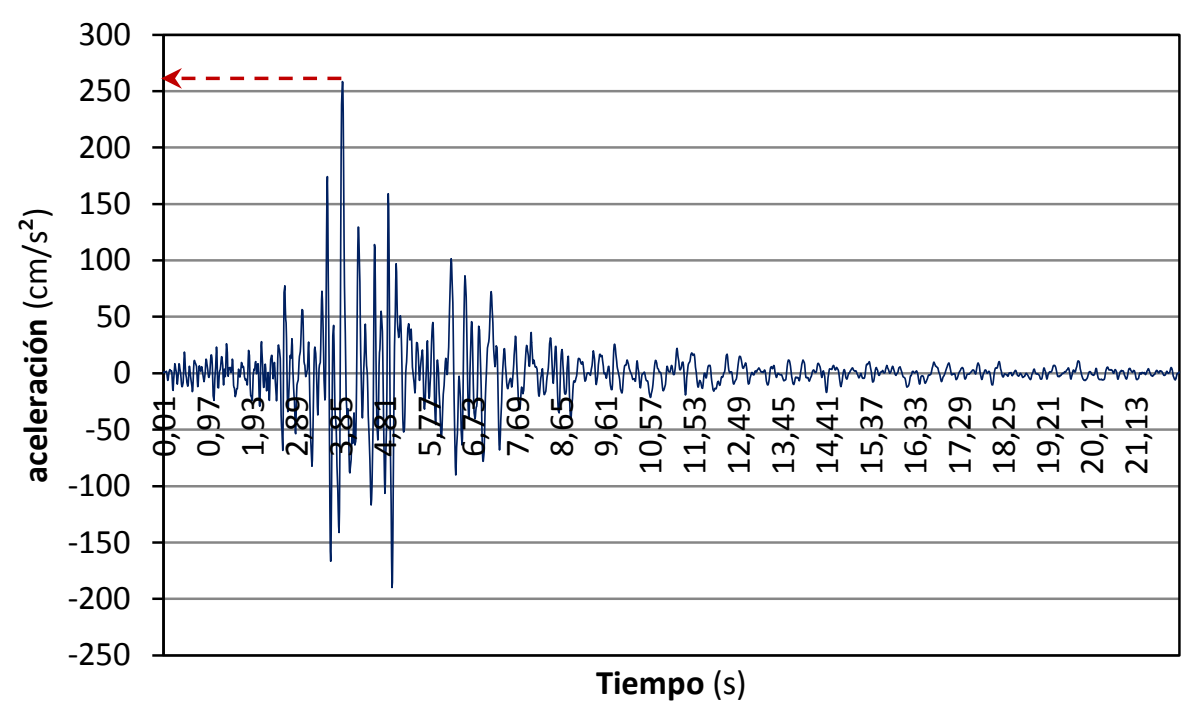

Fig. 4.3.41: Acelerograma $\mathrm{N}^{\circ} 4$ (Friuli aftershock), suelo tipo II, campo lejano, sin escalar

Aceleración sísmica de cálculo: $a_{c}=230,5 \mathrm{~cm} / \mathrm{s}^{2}$

Aceleración máxima: $P G A=258,64 \mathrm{~cm} / \mathrm{s}^{2}$

Factor de escala: $\frac{230,5 \mathrm{~cm} / \mathrm{s}^{2}}{258,64 \mathrm{~cm} / \mathrm{s}^{2}}=0,8912$

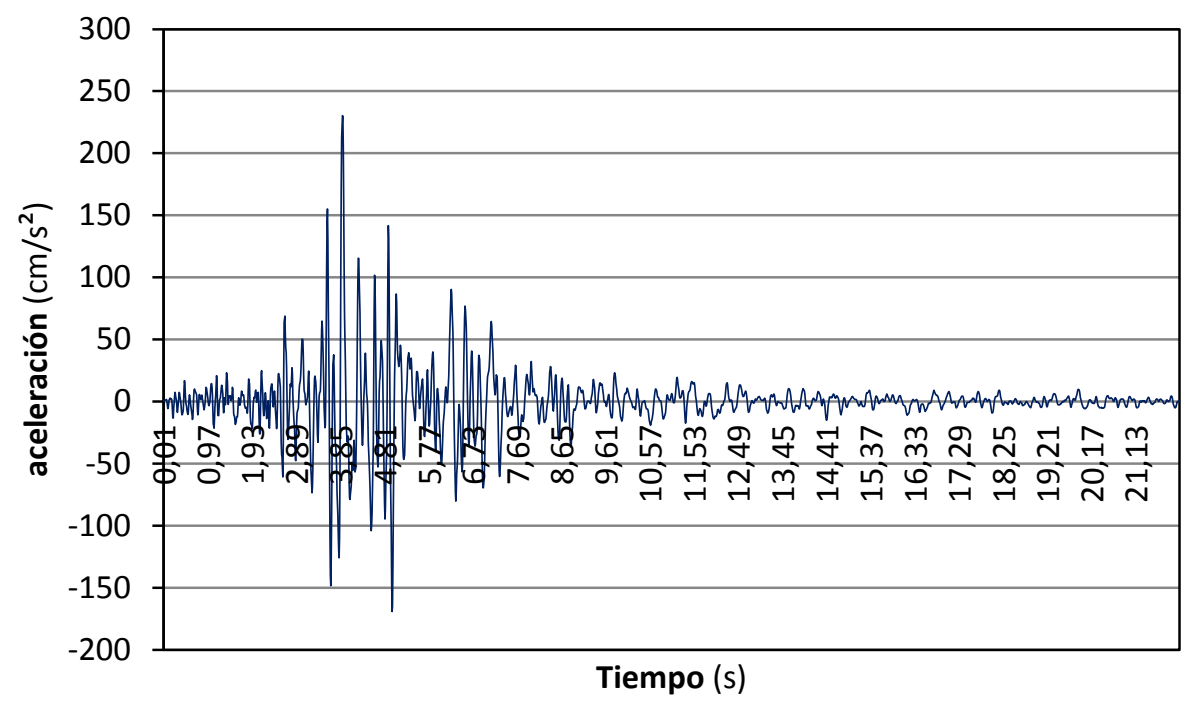

Fig. 4.3.42: Acelerograma $\mathrm{N}^{\circ} 4$ (Friuli aftershock), suelo tipo II, campo lejano, escalado a $P G A=230,5 \mathrm{~cm} / \mathrm{s}^{2}$ 


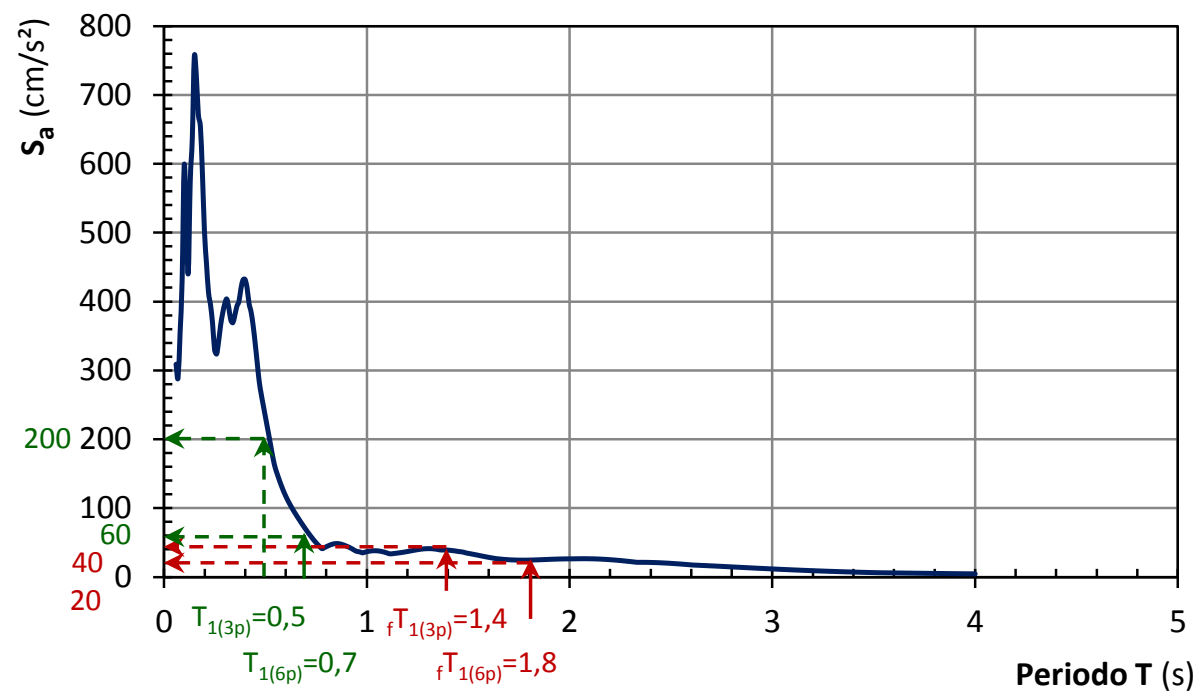

Fig. 4.3.43: Espectro elástico de respuesta del acelerograma $\mathrm{N}^{\circ} 4$ (Friuli aftershock) escalado a $P G A=230,5 \mathrm{~cm} / \mathrm{s}^{2}$

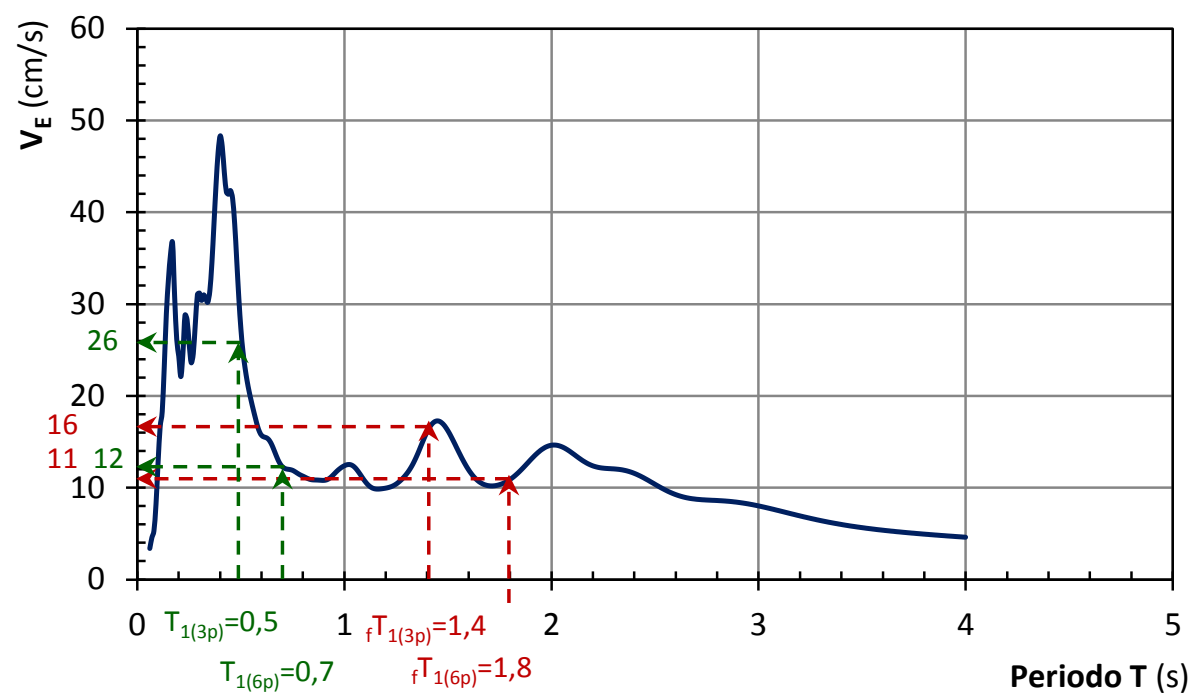

Fig. 4.3.44: Espectro de energía introducida del acelerograma $\mathrm{N}^{\circ} 4$ (Friuli aftershock) escalado a $P G A=230,5 \mathrm{~cm} / \mathrm{s}^{2}$

Comparando los valores de aceleración máxima de respuesta $S_{a}$ y de energía $V_{E}$, para los periodos de vibración anteriormente obtenidos.

Tabla 4.3.11: Ordenadas del espectro elástico de respuesta $S_{a}$ y del espectro de energía introducida, correspondientes a los periodos de las estructuras analizadas.

\begin{tabular}{l|c|c|c|c|} 
& $\mathrm{f}_{1(3 \mathrm{p})}=1,4 \mathrm{~s}$ & $\mathrm{~T}_{1(3 \mathrm{p})}=0,5 \mathrm{~s}$ & $\mathrm{f}_{1(6 \mathrm{p})}=1,8 \mathrm{~s}$ & $\mathrm{~T}_{1(6 \mathrm{p})}=0,7 \mathrm{~s}$ \\
\hline $\mathrm{S}_{\mathrm{a}}\left(\mathrm{cm} / \mathrm{s}^{2}\right)$ & 40 & 200 & 20 & 60 \\
\hline $\mathrm{V}_{\mathrm{E}}(\mathrm{cm} / \mathrm{s})$ & 16 & 26 & 11 & 12 \\
\hline & \multicolumn{2}{|c|}{} & &
\end{tabular}




\section{- Acelerograma №12 (Montenegro aftershock) $\Delta=33 \mathrm{Km}$}

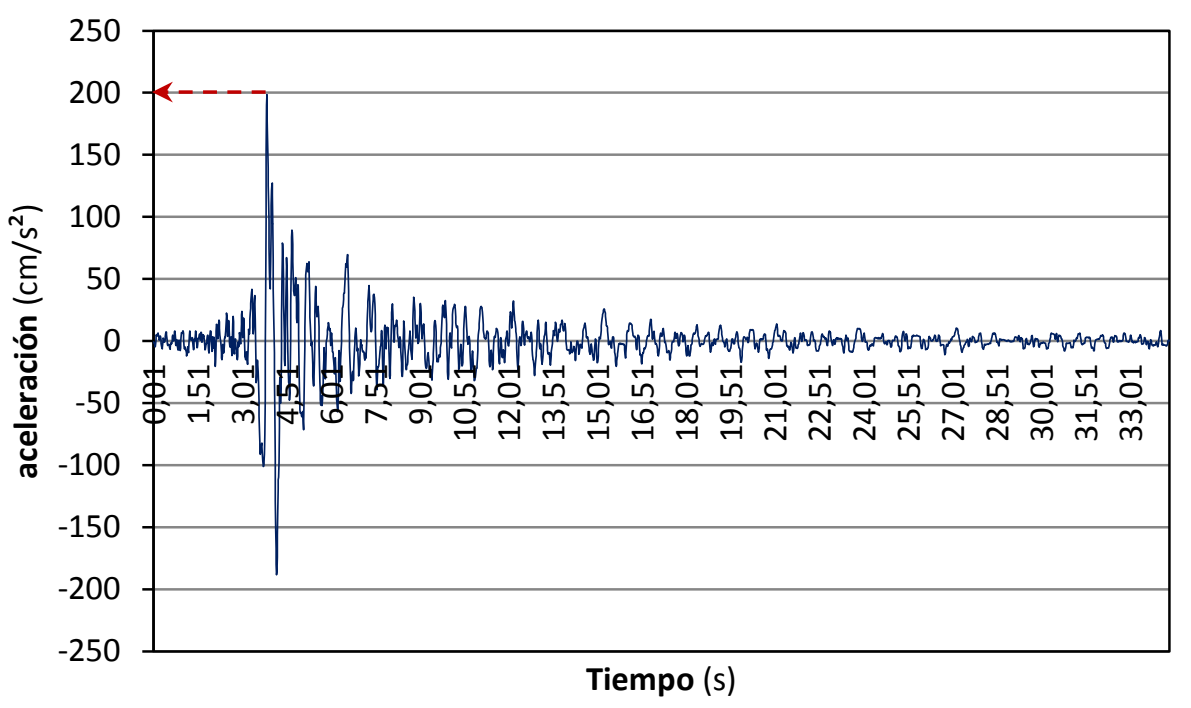

Fig. 4.3.45: Acelerograma $\mathrm{N}^{\circ} 12$ (Montenegro aftershock), suelo tipo II, campo lejano, sin escalar

Aceleración sísmica de cálculo: $a_{c}=230,5 \mathrm{~cm} / \mathrm{s}^{2}$

Aceleración máxima: $P G A=198,54 \mathrm{~cm} / \mathrm{s}^{2}$

Factor de escala: $\frac{230,5 \mathrm{~cm} / \mathrm{s}^{2}}{198,54 \mathrm{~cm} / \mathrm{s}^{2}}=\underline{1,1610}$

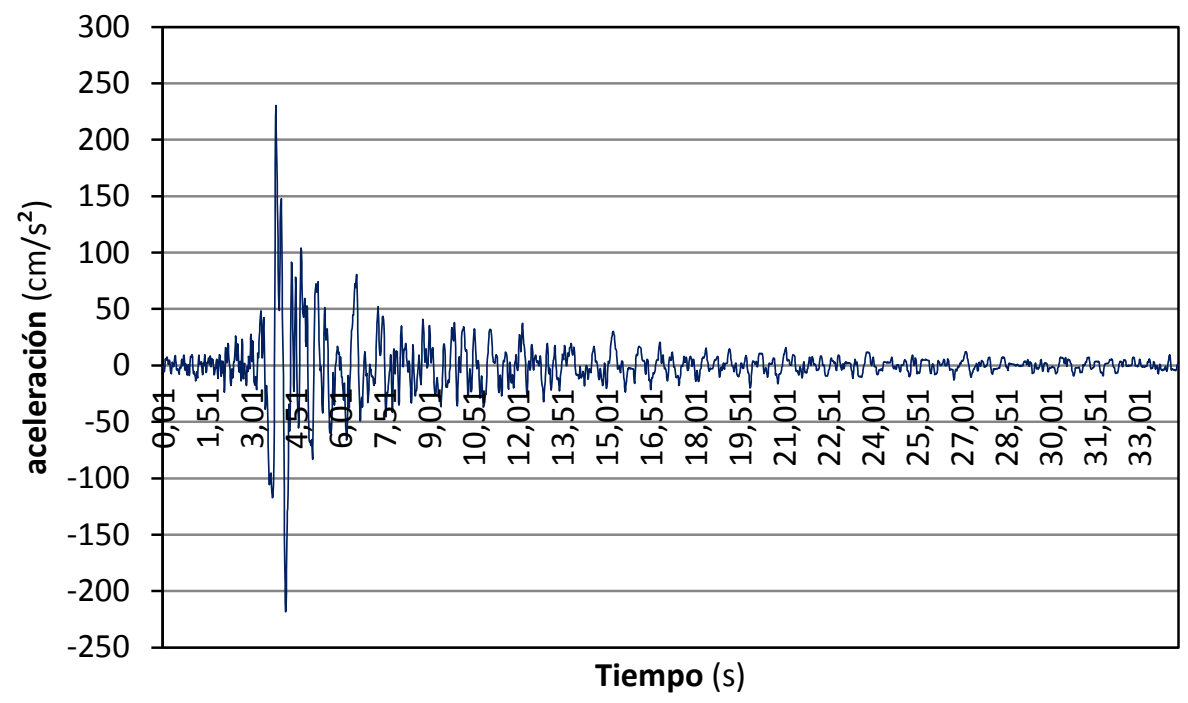

Fig. 4.3.46: Acelerograma $\mathrm{N}^{\circ} 12$ (Montenegro aftershock), suelo tipo II, campo lejano, escalado a $P G A=230,5 \mathrm{~cm} / \mathrm{s}^{2}$ 


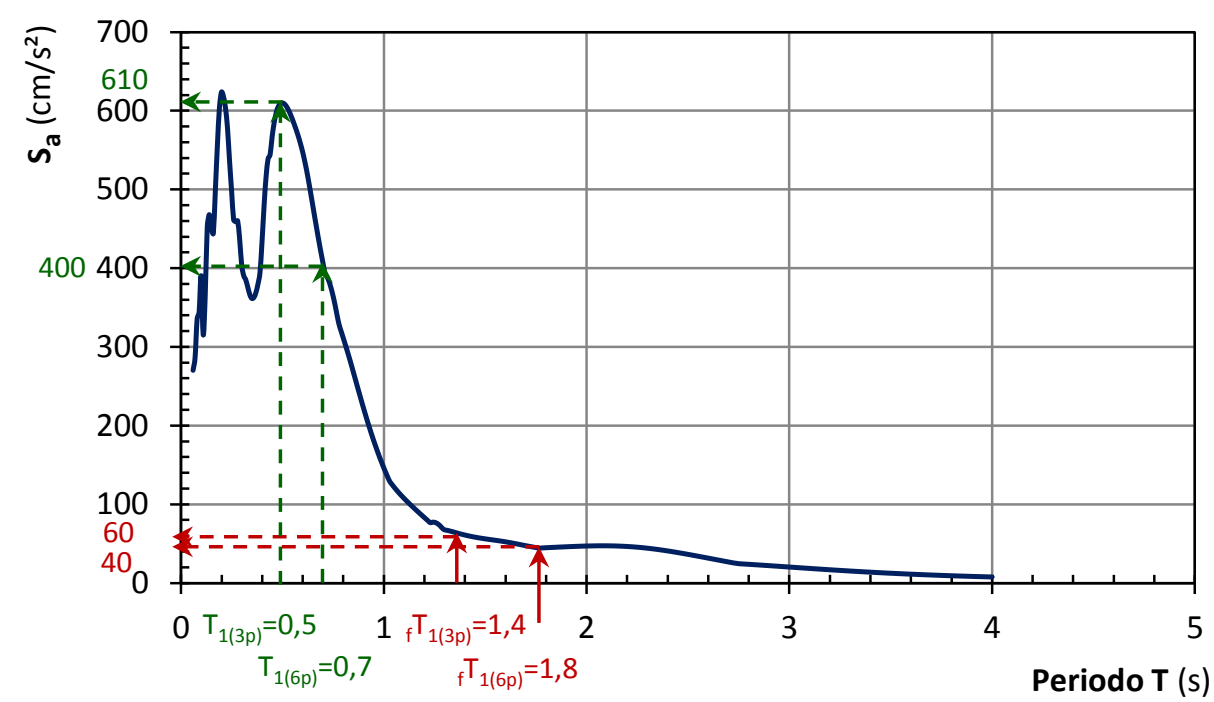

Fig. 4.3.47: Espectro elástico de respuesta del acelerograma $\mathrm{N}^{\circ} 12$ (Montenegro aftershock) escalado a $P G A=230,5 \mathrm{~cm} / \mathrm{s}^{2}$

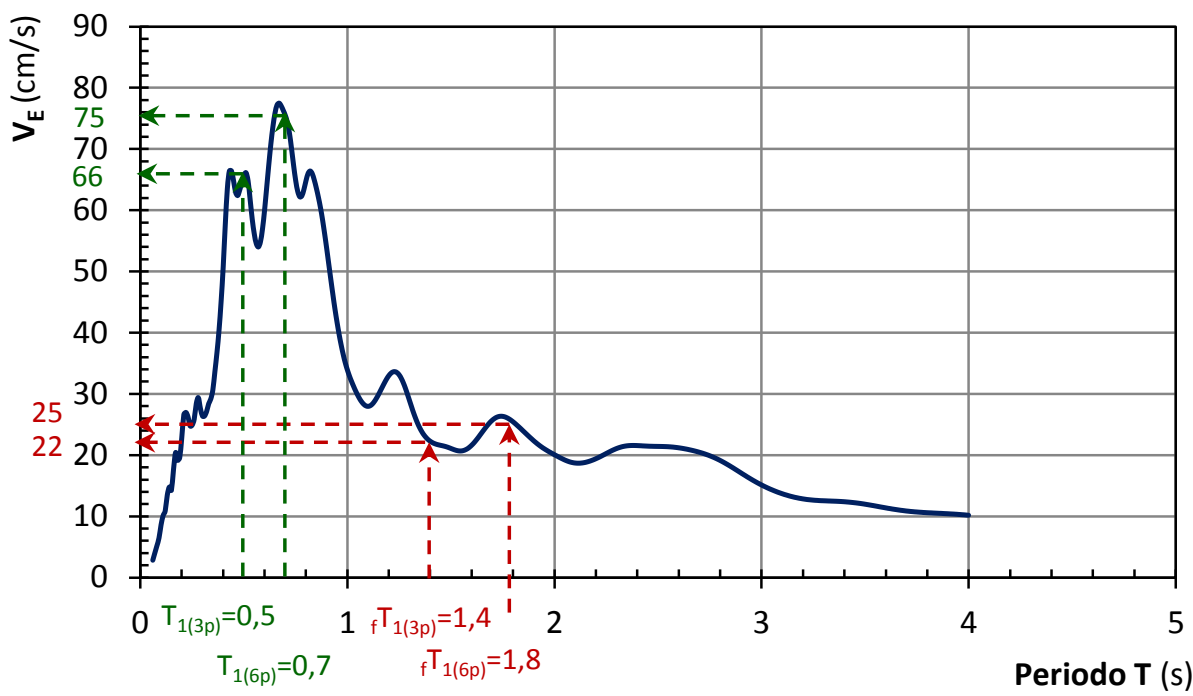

Fig. 4.3.48: Espectro de energía introducida del acelerograma $\mathrm{N}^{\circ} 12$ (Montenegro aftershock) escalado a $P G A=230,5 \mathrm{~cm} / \mathrm{s}^{2}$

Comparando los valores de aceleración máxima de respuesta $S_{a}$ y de energía $V_{E}$, para los periodos de vibración anteriormente obtenidos.

Tabla 4.3.12: Ordenadas del espectro elástico de respuesta $S_{a}$ y del espectro de energía introducida, correspondientes a los periodos de las estructuras analizadas.

\begin{tabular}{c|c|c|c|c|} 
& $\mathrm{fT}_{1(3 \mathrm{p})}=1,4 \mathrm{~s}$ & $\mathrm{~T}_{1(3 \mathrm{p})}=0,5 \mathrm{~s}$ & $\mathrm{f}_{1(6 \mathrm{p})}=1,8 \mathrm{~s}$ & $\mathrm{~T}_{1(6 \mathrm{p})}=0,7 \mathrm{~s}$ \\
\hline $\mathrm{S}_{\mathrm{a}}\left(\mathrm{cm} / \mathrm{s}^{2}\right)$ & 60 & 610 & 40 & 400 \\
\hline $\mathrm{V}_{\mathrm{E}}(\mathrm{cm} / \mathrm{s})$ & 22 & 66 & 25 & 75 \\
\hline & \multicolumn{3}{|c}{} &
\end{tabular}




\subsubsection{Suelo Tipo III (Campo cercano a la falla)}

\section{- Acelerograma №2 (Friuli aftershock) $\Delta=10 \mathrm{Km}$}

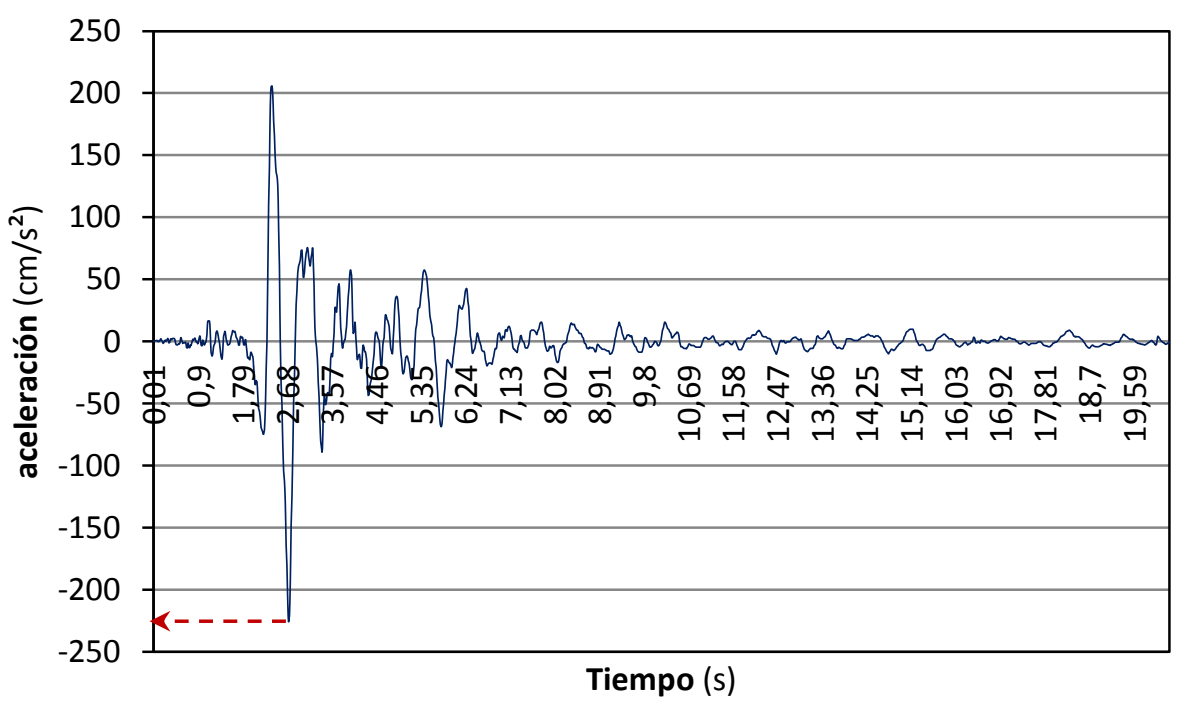

Fig. 4.3.49: Acelerograma $\mathrm{N}^{\circ} 2$ (Friuli aftershock), suelo tipo III, campo cercano, sin escalar

Aceleración sísmica de cálculo: $a_{c}=261,4 \mathrm{~cm} / \mathrm{s}^{2}$

Aceleración máxima: $P G A=226,05 \mathrm{~cm} / \mathrm{s}^{2}$

Factor de escala: $\frac{261,4 \mathrm{~cm} / \mathrm{s}^{2}}{226,05 \mathrm{~cm} / \mathrm{s}^{2}}=\underline{1,1564}$

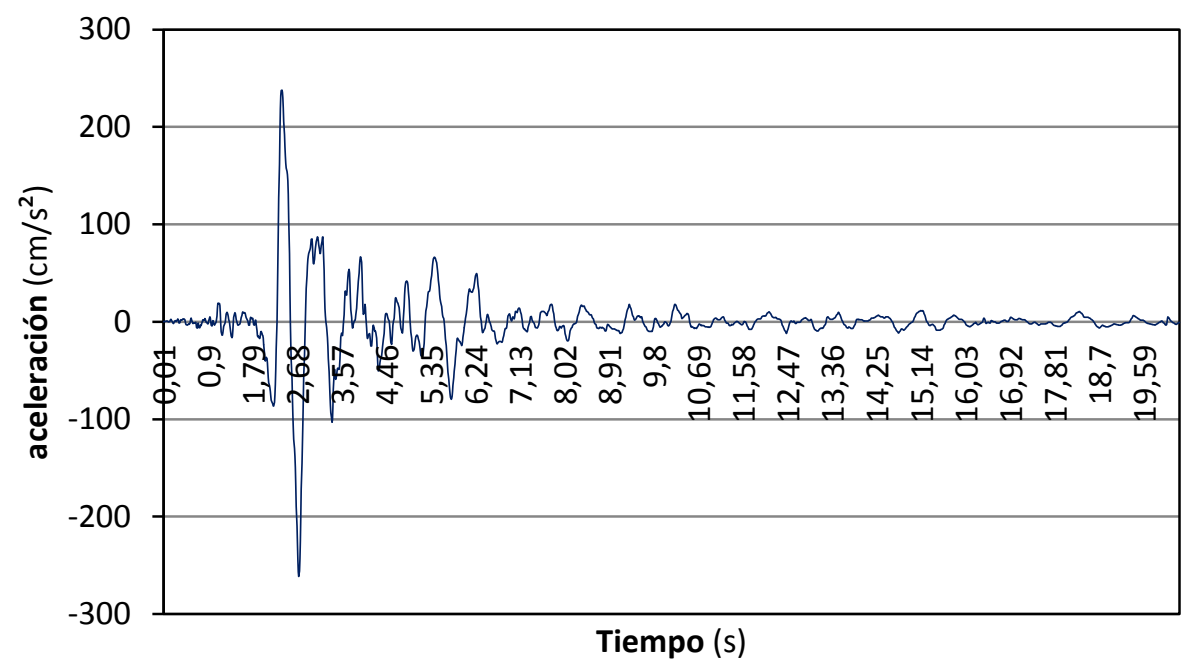

Fig. 4.3.50: Acelerograma $\mathrm{N}^{\circ} 2$ (Friuli aftershock), suelo tipo III, campo cercano, escalado a $P G A=261,4 \mathrm{~cm} / \mathrm{s}^{2}$ 


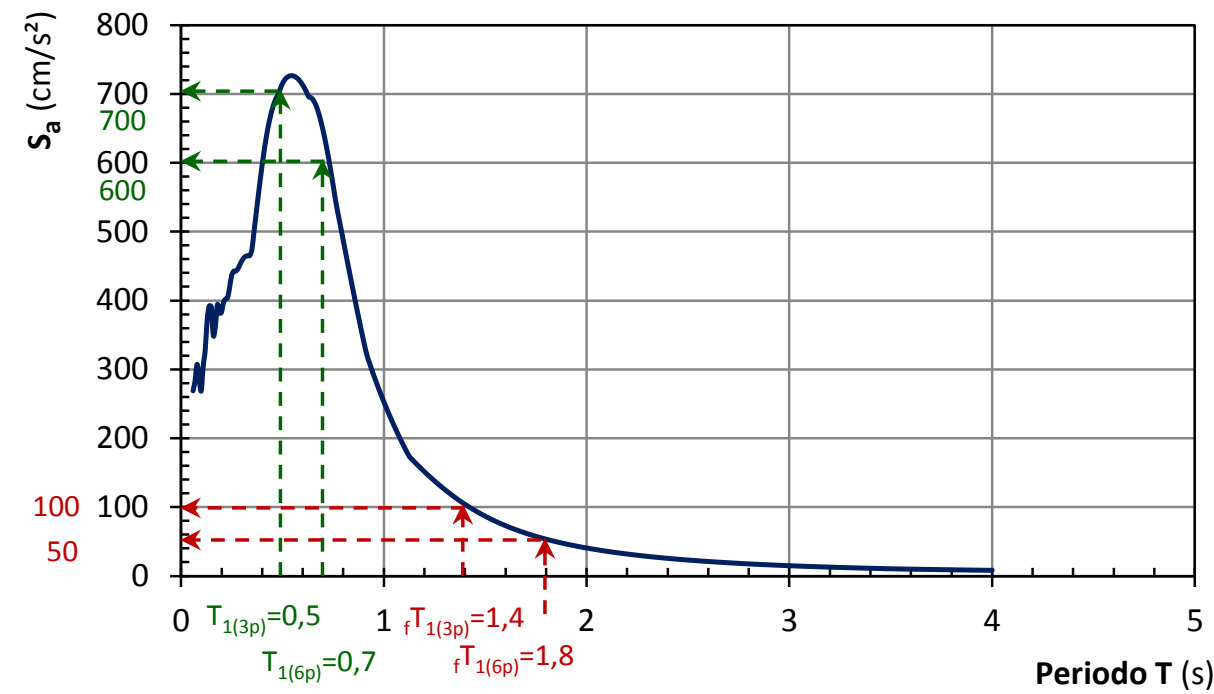

Fig. 4.3.51: Espectro elástico de respuesta del acelerograma $N^{\circ} 2$ (Friuli aftershock) escalado a PGA $=261,4 \mathrm{~cm} / \mathrm{s}^{2}$

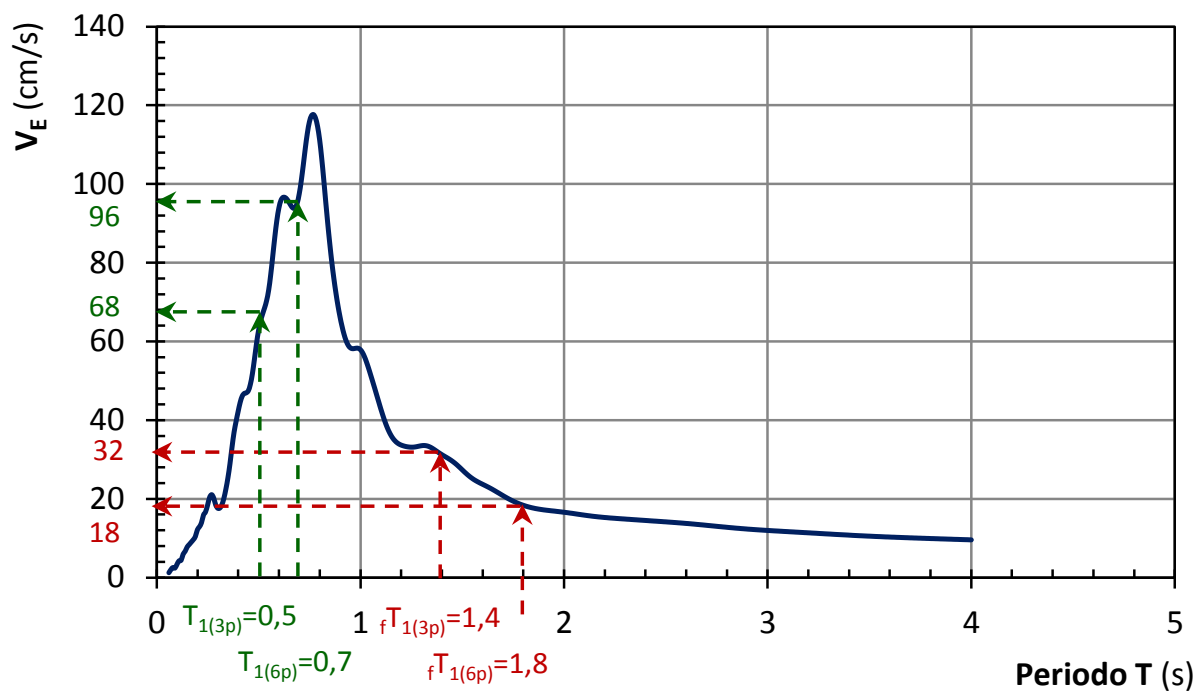

Fig. 4.3.52: Espectro de energía introducida del acelerograma $\mathrm{N}^{\circ} 2$ (Friuli aftershock) escalado a $P G A=261,4 \mathrm{~cm} / \mathrm{s}^{2}$

Comparando los valores de aceleración máxima de respuesta $S_{a}$ y de energía $V_{E}$, para los periodos de vibración anteriormente obtenidos.

Tabla 4.3.13: Ordenadas del espectro elástico de respuesta $S_{a}$ y del espectro de energía introducida, correspondientes a los periodos de las estructuras analizadas.

\begin{tabular}{l|c|c|c|c|} 
& ${ }_{f} T_{1(3 p)}=1,4 s$ & $T_{1(3 p)}=0,5 s$ & ${ }_{f} T_{1(6 p)}=1,8 s$ & $T_{1(6 p)}=0,7 s$ \\
\hline $\mathrm{S}_{\mathrm{a}}\left(\mathrm{cm} / \mathrm{s}^{2}\right)$ & 100 & 700 & 50 & 600 \\
\hline $\mathrm{V}_{\mathrm{E}}(\mathrm{cm} / \mathrm{s})$ & 32 & 68 & 18 & 96 \\
\hline & \multicolumn{4}{|c|}{}
\end{tabular}


- Acelerograma №6 (Kalamata aftershock) $\Delta=3 \mathrm{Km}$

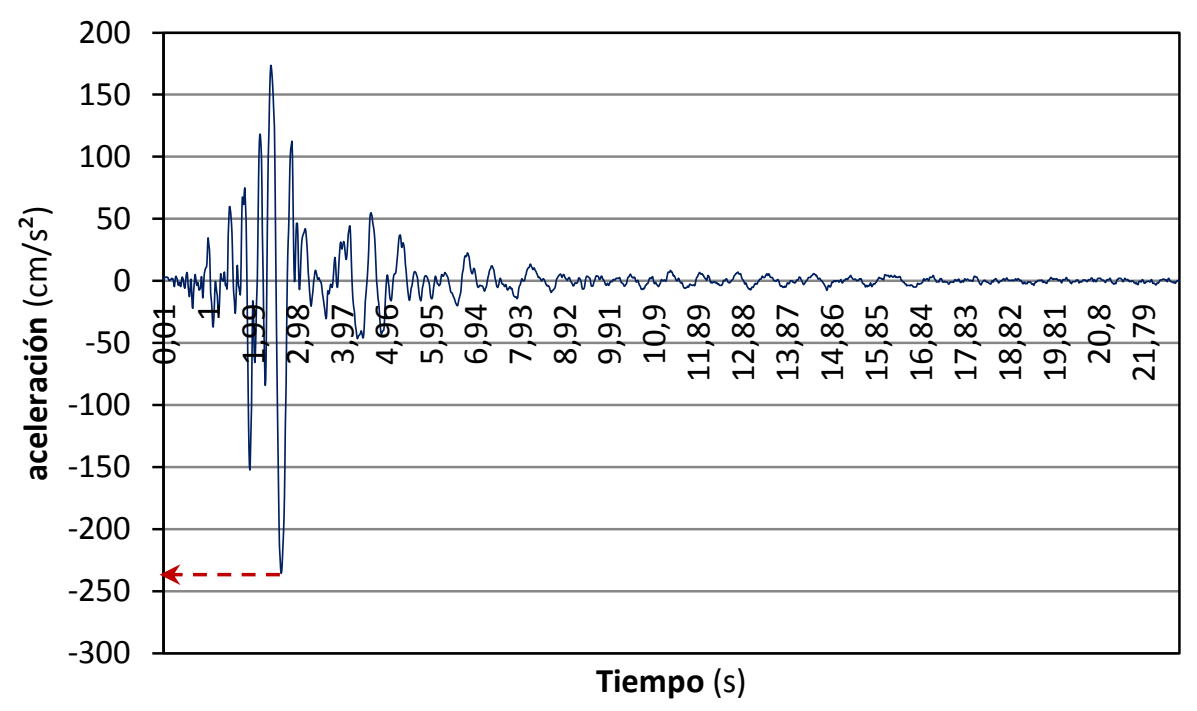

Fig. 4.3.53: Acelerograma $\mathrm{N}^{\circ} 6$ (Kalamata aftershock), suelo tipo III, campo cercano, sin escalar

Aceleración sísmica de cálculo: $a_{c}=261,4 \mathrm{~cm} / \mathrm{s}^{2}$

Aceleración máxima: $P G A=235,53 \mathrm{~cm} / \mathrm{s}^{2}$

Factor de escala: $\frac{261,4 \mathrm{~cm} / \mathrm{s}^{2}}{235,53 \mathrm{~cm} / \mathrm{s}^{2}}=\underline{1,1098}$

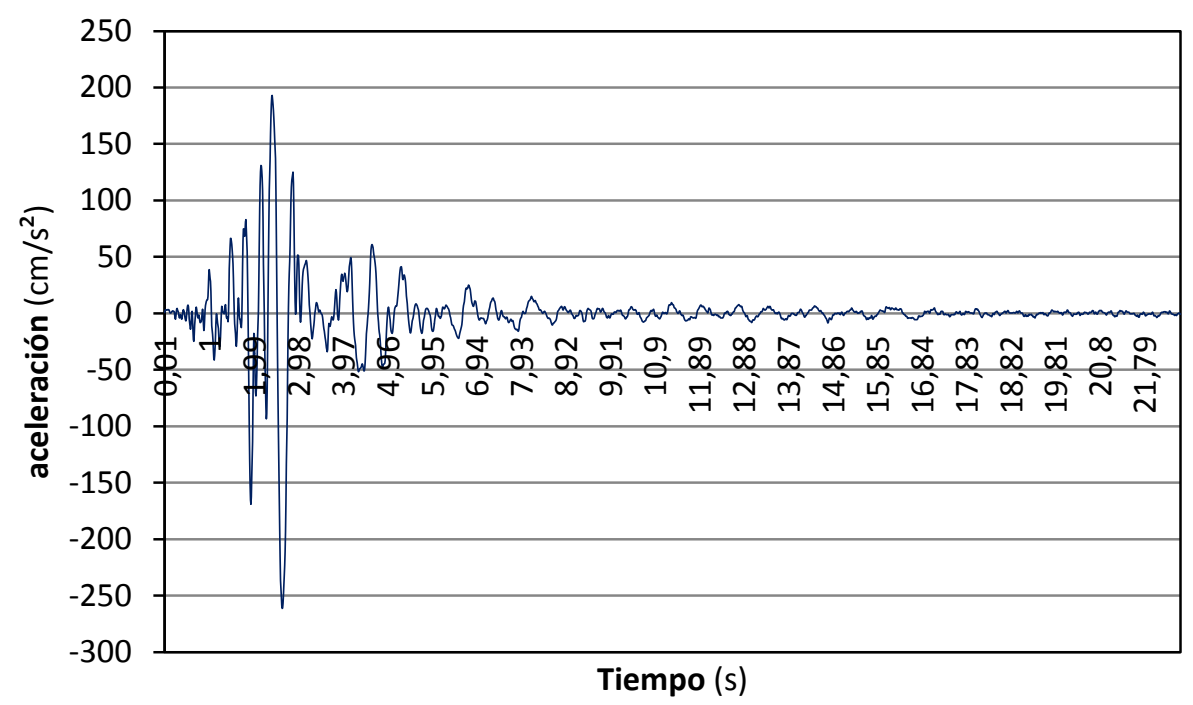

Fig. 4.3.54: Acelerograma $\mathrm{N}^{\circ} 6$ (Kalamata aftershock), suelo tipo III, campo cercano, escalado a $P G A=261,4 \mathrm{~cm} / \mathrm{s}^{2}$ 


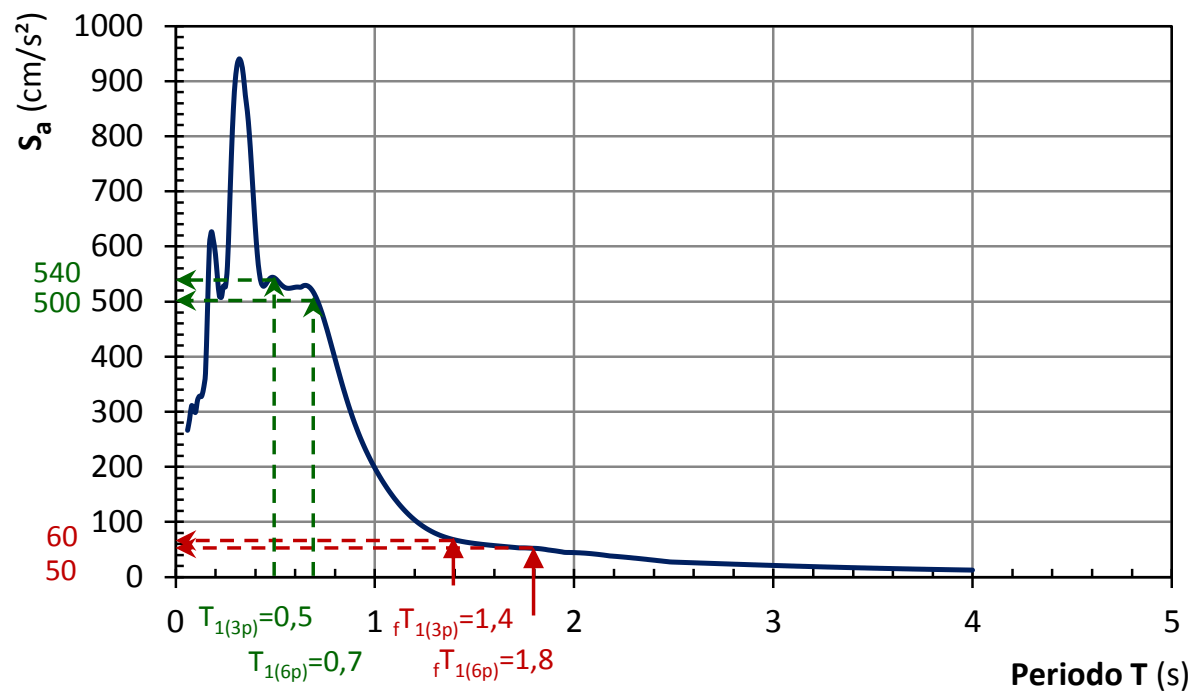

Fig. 4.3.55: Espectro elástico de respuesta del acelerograma $N^{\circ} 6$ (Kalamata aftershock) escalado a $P G A=261,4 \mathrm{~cm} / \mathrm{s}^{2}$

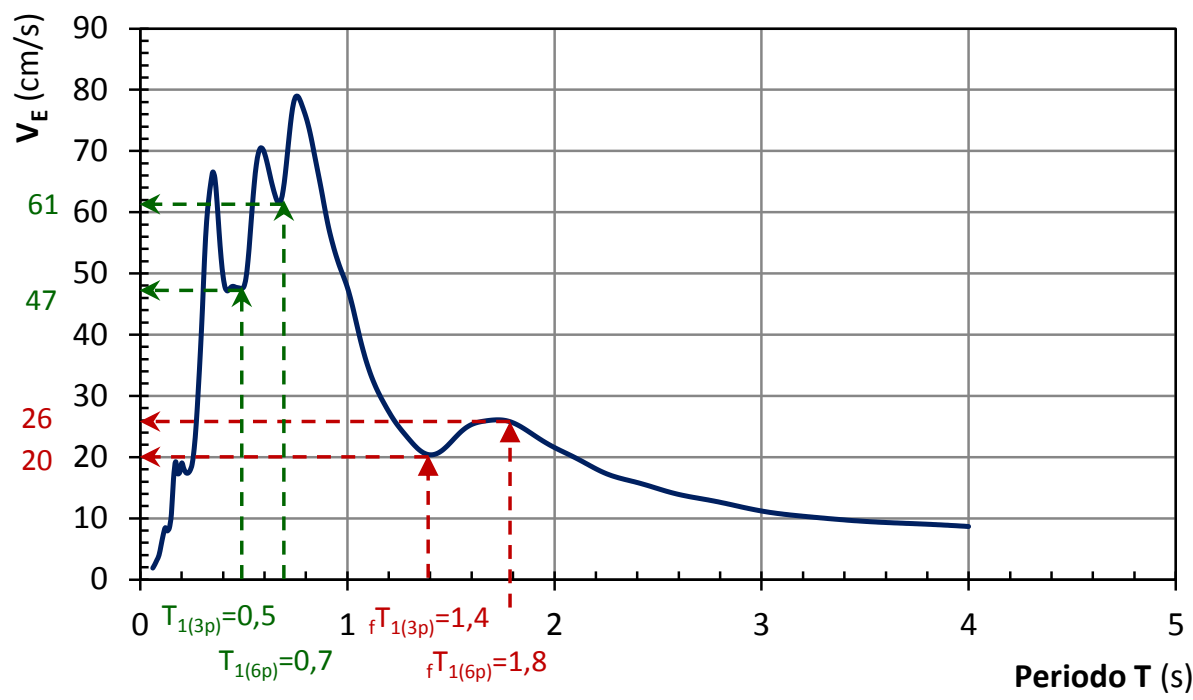

Fig. 4.3.56: Espectro de energía introducida del acelerograma $N^{\circ} 6$ (Kalamata aftershock) escalado a $P G A=261,4 \mathrm{~cm} / \mathrm{s}^{2}$

Comparando los valores de aceleración máxima de respuesta $S_{a}$ y de energía $V_{E}$, para los periodos de vibración anteriormente obtenidos.

Tabla 4.3.14: Ordenadas del espectro elástico de respuesta $S_{a}$ y del espectro de energía introducida, correspondientes a los periodos de las estructuras analizadas.

\begin{tabular}{c|c|c|c|c|} 
& ${ }_{\mathrm{f}} \mathrm{T}_{1(3 \mathrm{p})}=1,4 \mathrm{~s}$ & $\mathrm{~T}_{1(3 \mathrm{p})}=0,5 \mathrm{~s}$ & $\mathrm{f}_{1(6 \mathrm{p})}=1,8 \mathrm{~s}$ & $\mathrm{~T}_{1(6 \mathrm{p})}=0,7 \mathrm{~s}$ \\
\hline $\mathrm{S}_{\mathrm{a}}\left(\mathrm{cm} / \mathrm{s}^{2}\right)$ & 60 & 540 & 50 & 500 \\
\hline $\mathrm{V}_{\mathrm{E}}(\mathrm{cm} / \mathrm{s})$ & 20 & 47 & 26 & 61 \\
\hline & \multicolumn{3}{|c}{}
\end{tabular}




\section{- Acelerograma №12 (Duzce 1) $\Delta=8 \mathrm{Km}$}

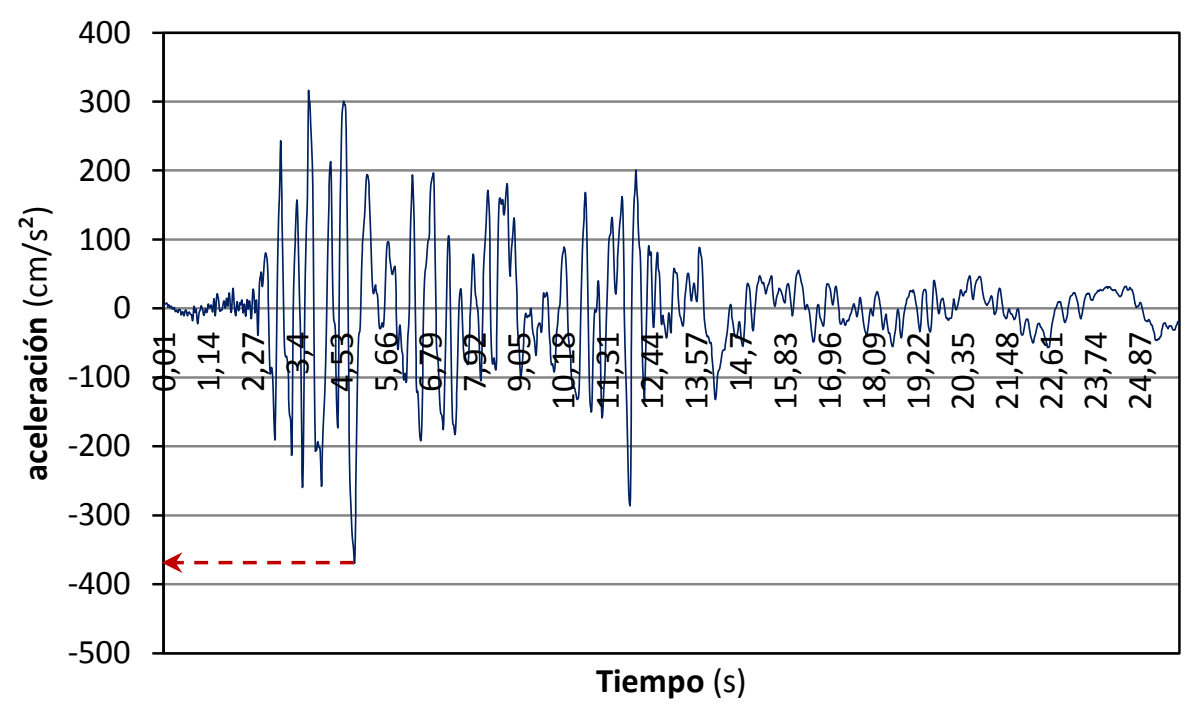

Fig. 4.3.57: Acelerograma $\mathrm{N}^{\circ} 12$ (Duzce 1), suelo tipo III, campo cercano, sin escalar

Aceleración sísmica de cálculo: $a_{c}=261,4 \mathrm{~cm} / \mathrm{s}^{2}$

Aceleración máxima: $P G A=369,88 \mathrm{~cm} / \mathrm{s}^{2}$

Factor de escala: $\frac{261,4 \mathrm{~cm} / \mathrm{s}^{2}}{369,88 \mathrm{~cm} / \mathrm{s}^{2}}=0,7067$

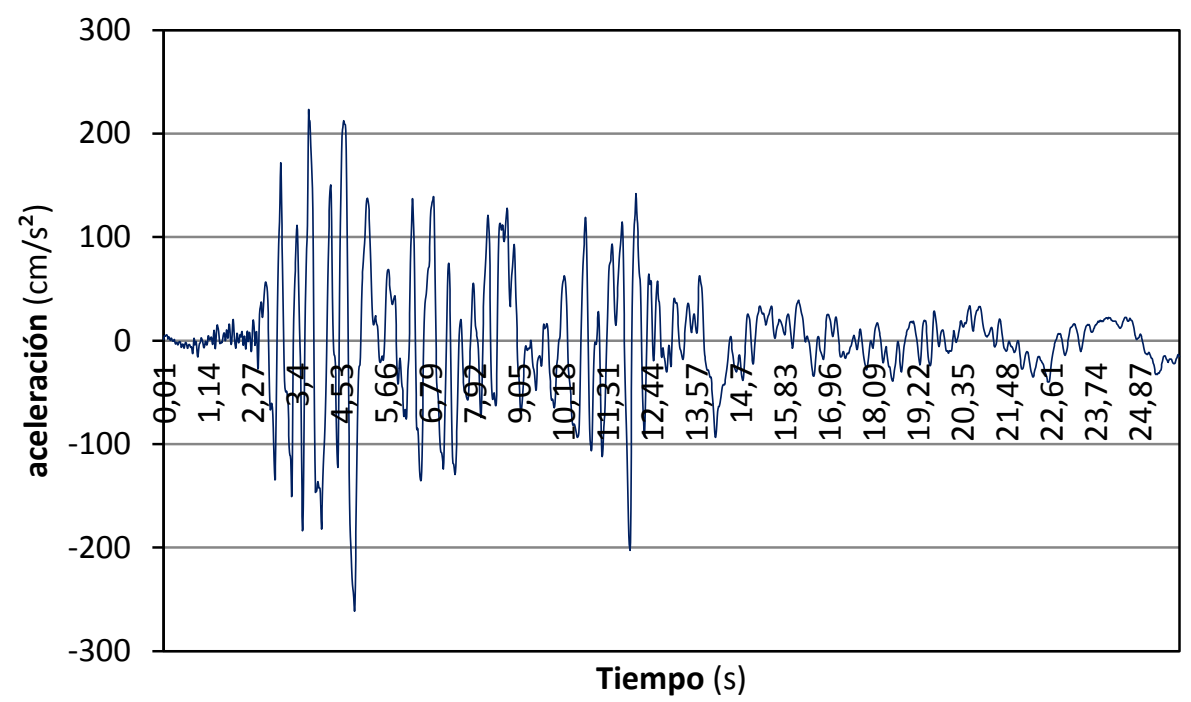

Fig. 4.3.58: Acelerograma $\mathrm{N}^{\circ} 12$ (Duzce 1), suelo tipo III, campo cercano, escalado a $P G A=261,4 \mathrm{~cm} / \mathrm{s}^{2}$ 


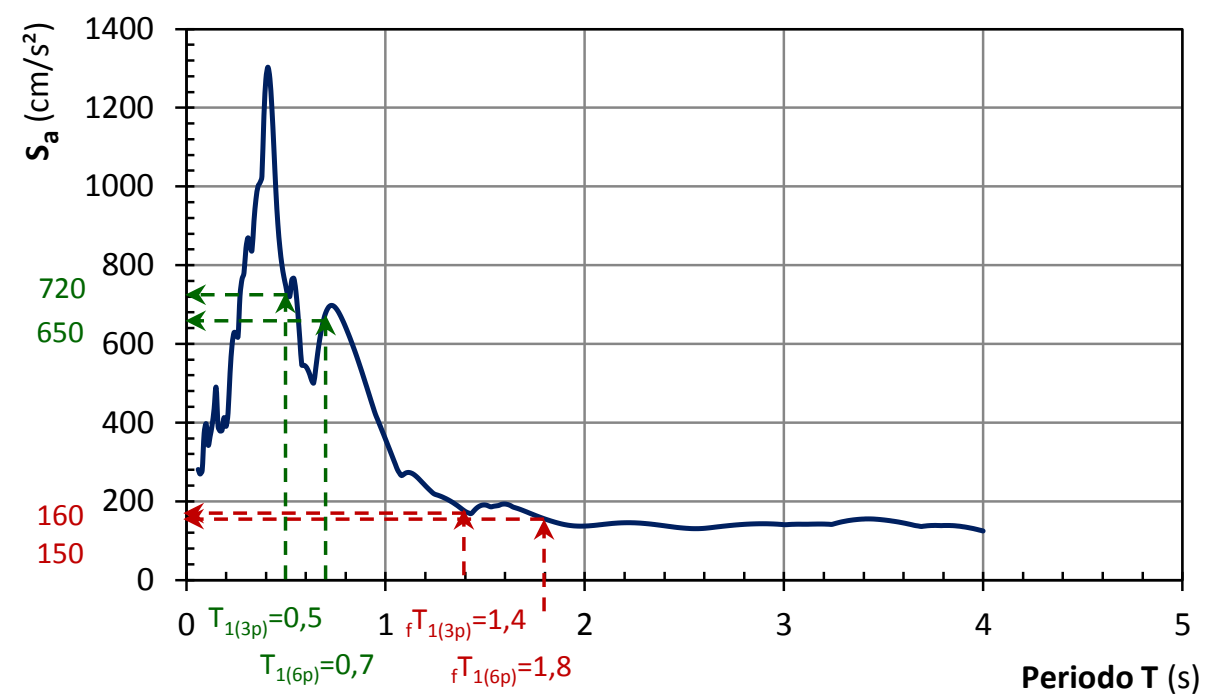

Fig. 4.3.59: Espectro elástico de respuesta del acelerograma $N^{\circ} 12$ (Duzce 1) escalado a $P G A=261,4 \mathrm{~cm} / \mathrm{s}^{2}$

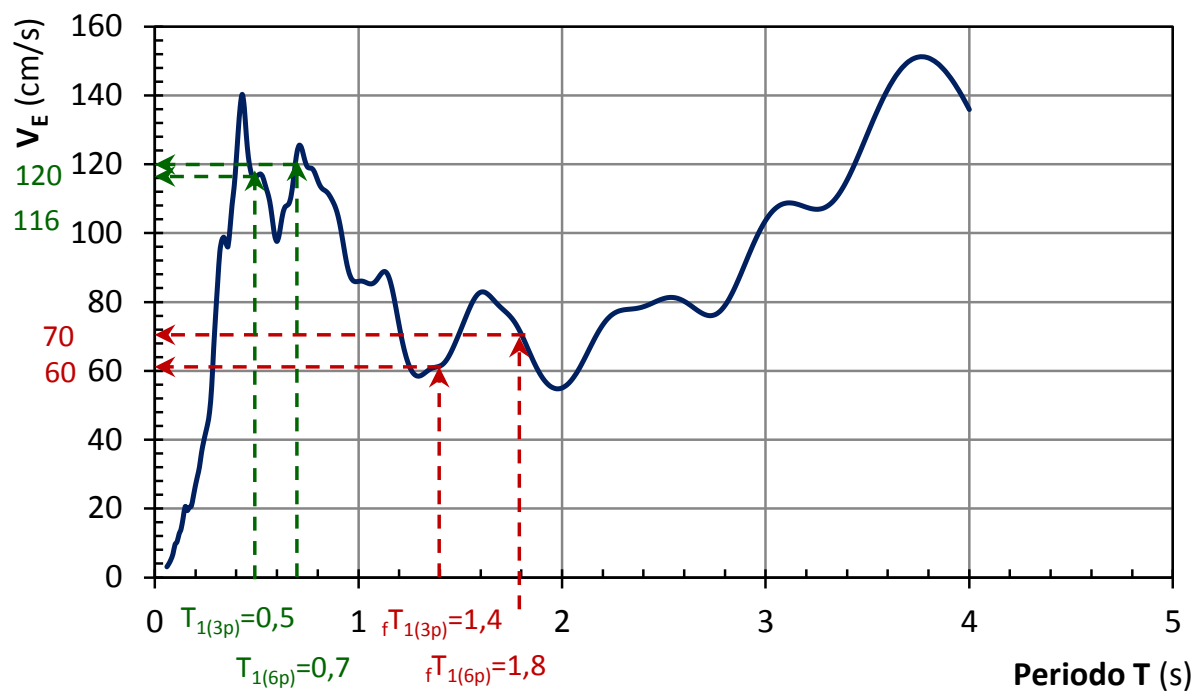

Fig. 4.3.60: Espectro de energía introducida del acelerograma $N^{\circ} 12$ (Duzce 1) escalado a $\mathrm{PGA}=261,4 \mathrm{~cm} / \mathrm{s}^{2}$

Comparando los valores de aceleración máxima de respuesta $S_{a}$ y de energía $V_{E}$, para los periodos de vibración anteriormente obtenidos.

Tabla 4.3.15: Ordenadas del espectro elástico de respuesta $S_{a}$ y del espectro de energía introducida, correspondientes a los periodos de las estructuras analizadas.

\begin{tabular}{c|c|c|c|c|} 
& ${ }_{\mathrm{f}} \mathrm{T}_{1(3 \mathrm{p})}=1,4 \mathrm{~s}$ & $\mathrm{~T}_{1(3 \mathrm{p})}=0,5 \mathrm{~s}$ & $\mathrm{f}_{1(6 \mathrm{p})}=1,8 \mathrm{~s}$ & $\mathrm{~T}_{1(6 \mathrm{p})}=0,7 \mathrm{~s}$ \\
\hline $\mathrm{S}_{\mathrm{a}}\left(\mathrm{cm} / \mathrm{s}^{2}\right)$ & 160 & 720 & 150 & 650 \\
\hline $\mathrm{V}_{\mathrm{E}}(\mathrm{cm} / \mathrm{s})$ & 60 & 116 & 70 & 120 \\
\hline & \multicolumn{1}{|c|}{} & & \multicolumn{2}{|c}{}
\end{tabular}




\subsubsection{Suelo Tipo III (Campo lejano a la falla)}

\section{- Acelerograma №3 (Montenegro) $\Delta=24 \mathrm{Km}$}

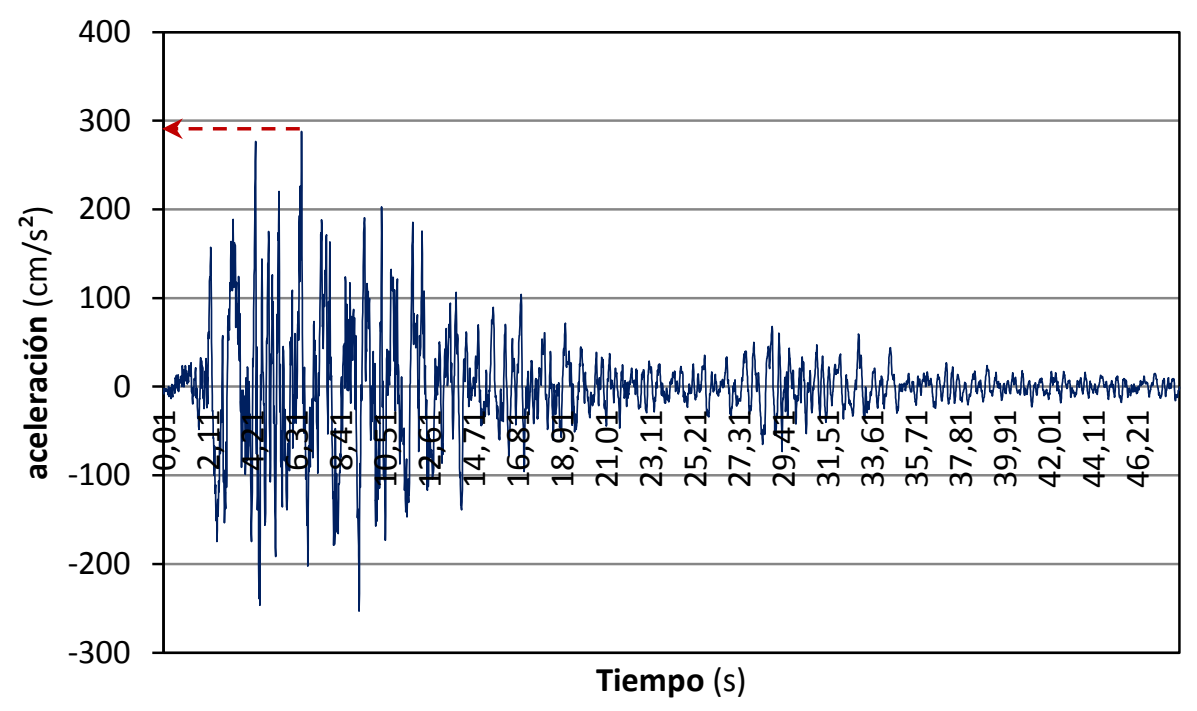

Fig. 4.3.61: Acelerograma $\mathrm{N}^{\circ} 3$ (Montenegro), suelo tipo III, campo lejano, sin escalar

Aceleración sísmica de cálculo: $a_{c}=261,4 \mathrm{~cm} / \mathrm{s}^{2}$

Aceleración máxima: $P G A=287,97 \mathrm{~cm} / \mathrm{s}^{2}$

Factor de escala: $\frac{261,4 \mathrm{~cm} / \mathrm{s}^{2}}{287,97 \mathrm{~cm} / \mathrm{s}^{2}}=\underline{0,9077}$

\section{Escalado acelerograma:}

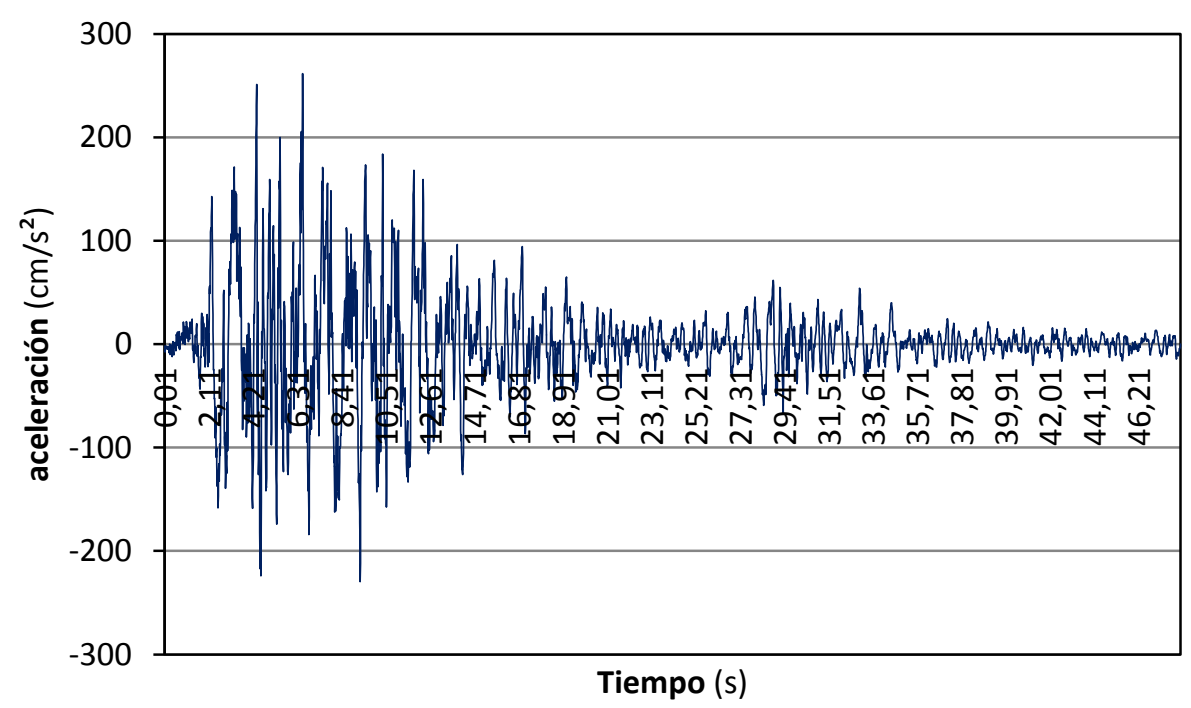

Fig. 4.3.62: Acelerograma $\mathrm{N}^{\circ} 3$ (Montenegro), suelo tipo III, campo lejano, escalado a $P G A=261,4 \mathrm{~cm} / \mathrm{s}^{2}$ 


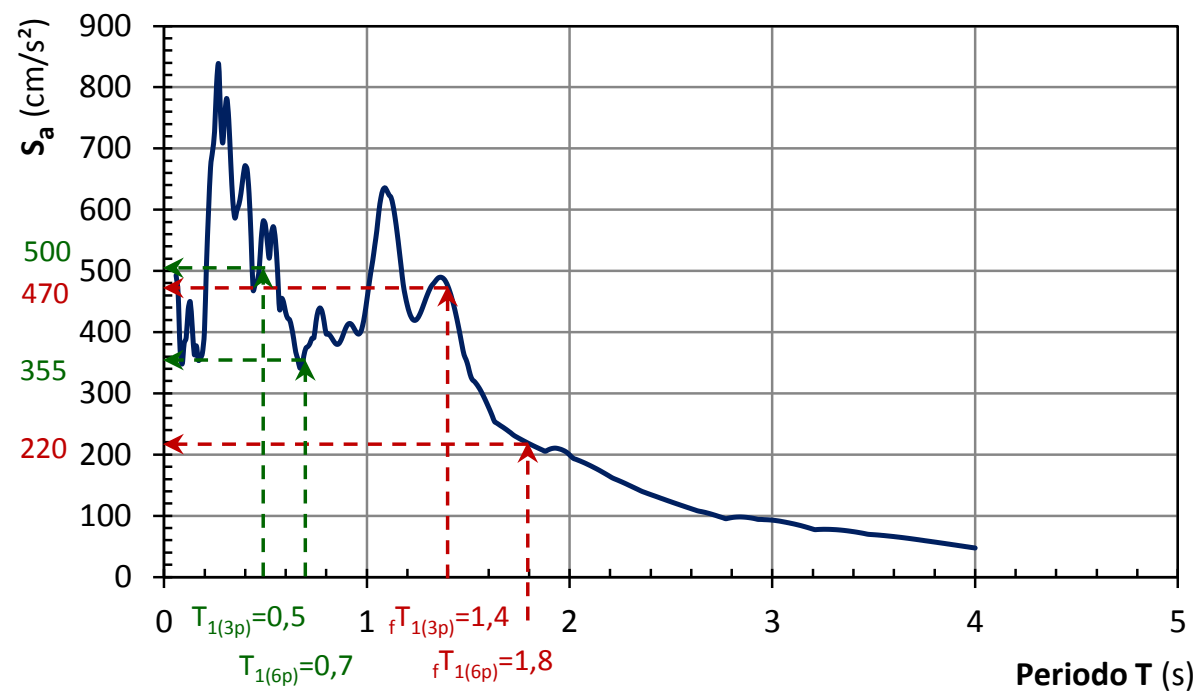

Fig. 4.3.63: Espectro elástico de respuesta del acelerograma $\mathrm{N}^{\circ} 3$ (Montenegro) escalado a $P G A=261,4 \mathrm{~cm} / \mathrm{s}^{2}$

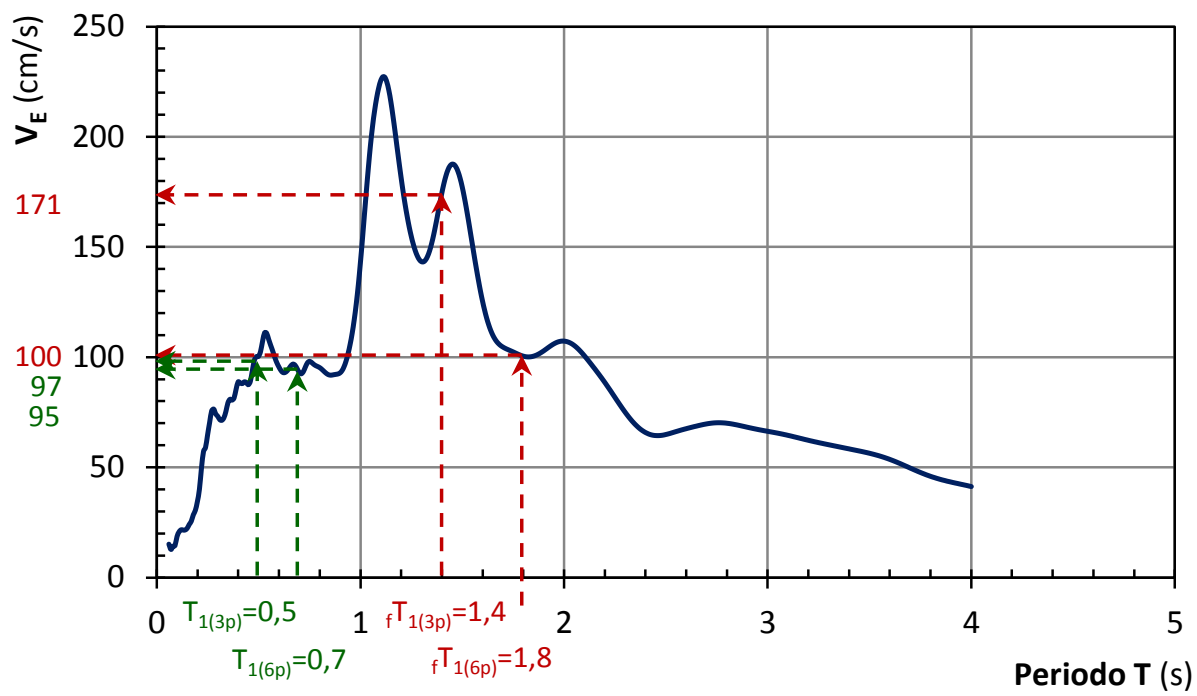

Fig. 4.3.64: Espectro de energía introducida del acelerograma $\mathrm{N}^{\circ} 3$ (Montenegro) escalado a $P G A=261,4 \mathrm{~cm} / \mathrm{s}^{2}$

Comparando los valores de aceleración máxima de respuesta $S_{a}$ y de energía $V_{E}$, para los periodos de vibración anteriormente obtenidos.

Tabla 4.3.16: Ordenadas del espectro elástico de respuesta $S_{a}$ y del espectro de energía introducida, correspondientes a los periodos de las estructuras analizadas.

\begin{tabular}{c|c|c|c|c|} 
& $\mathrm{f}_{1(3 \mathrm{p})}=1,4 \mathrm{~s}$ & $\mathrm{~T}_{1(3 \mathrm{p})}=0,5 \mathrm{~s}$ & $\mathrm{f}_{1(6 \mathrm{p})}=1,8 \mathrm{~s}$ & $\mathrm{~T}_{1(6 \mathrm{p})}=0,7 \mathrm{~s}$ \\
\hline $\mathrm{S}_{\mathrm{a}}\left(\mathrm{cm} / \mathrm{s}^{2}\right)$ & 470 & 500 & 220 & 335 \\
\hline $\mathrm{V}_{\mathrm{E}}(\mathrm{cm} / \mathrm{s})$ & 60 & 116 & 70 & 120 \\
\hline & \multicolumn{3}{|l}{}
\end{tabular}


- Acelerograma №8 (Sicilia-Orientale) $\Delta=24 \mathrm{Km}$

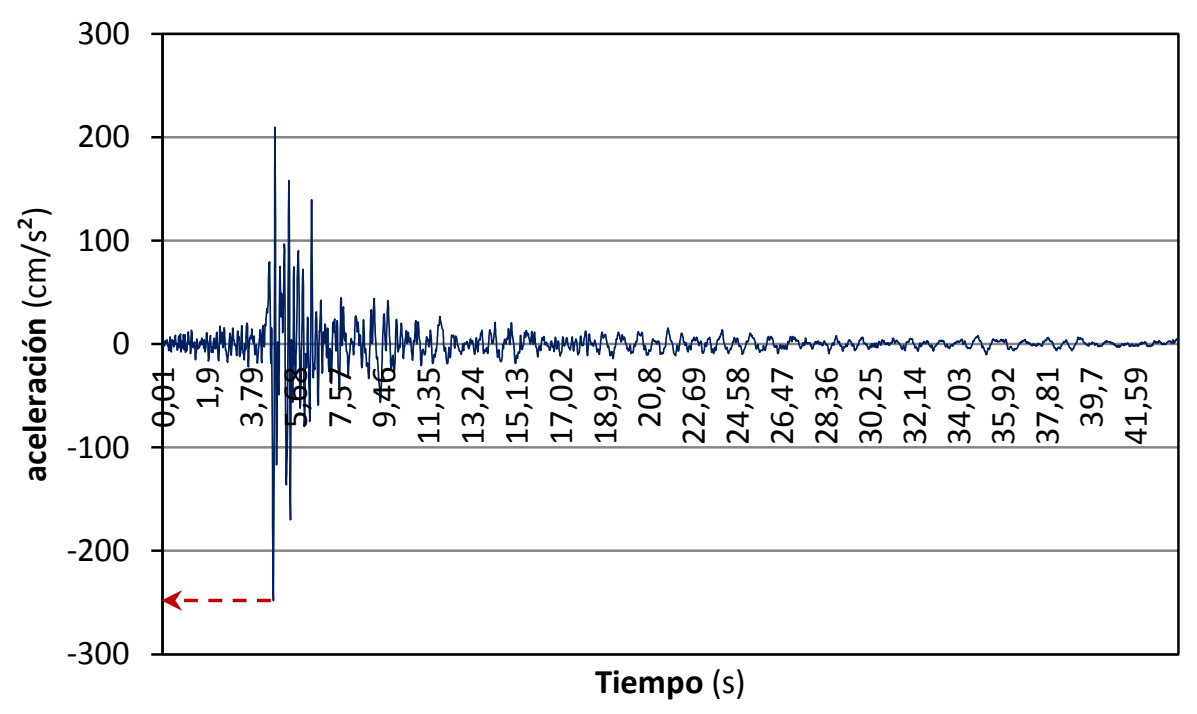

Fig. 4.3.65: Acelerograma $\mathrm{N}^{\circ} 8$ (Sicilia-Orientale), suelo tipo III, campo lejano, sin escalar

Aceleración sísmica de cálculo: $a_{c}=261,4 \mathrm{~cm} / \mathrm{s}^{2}$

Aceleración máxima: $P G A=248,27 \mathrm{~cm} / \mathrm{s}^{2}$

Factor de escala: $\frac{261,4 \mathrm{~cm} / \mathrm{s}^{2}}{248,27 \mathrm{~cm} / \mathrm{s}^{2}}=\underline{1,0529}$

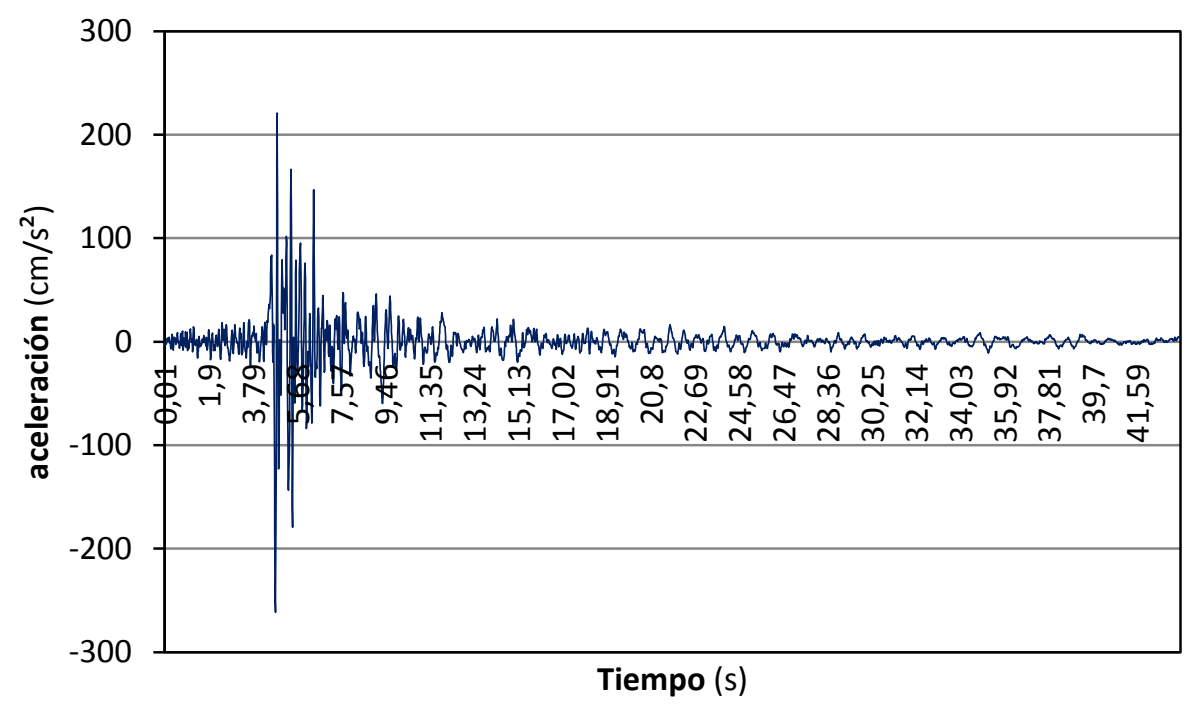

Fig. 4.3.66: Acelerograma $\mathrm{N}^{\circ} 8$ (Sicilia-Orientale), suelo tipo III, campo lejano, escalado a $P G A=261,4 \mathrm{~cm} / \mathrm{s}^{2}$ 


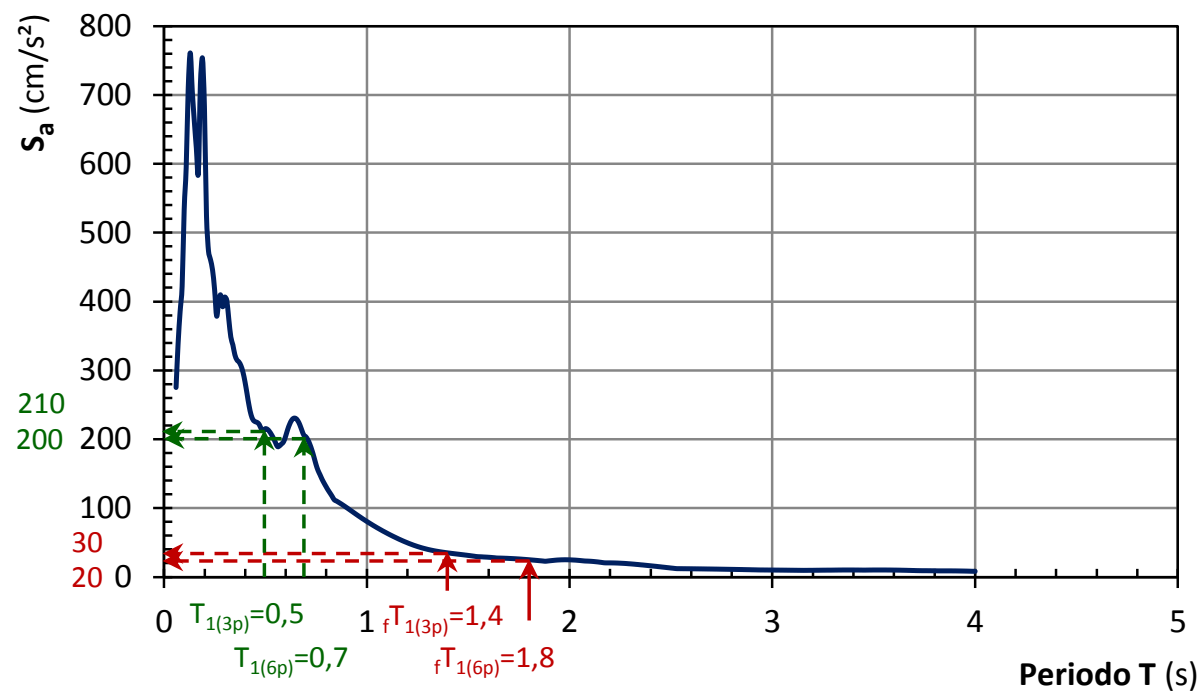

Fig. 4.3.67: Espectro elástico de respuesta del acelerograma $N^{\circ} 8$ (Sicilia-Orientale) escalado a $P G A=261,4 \mathrm{~cm} / \mathrm{s}^{2}$

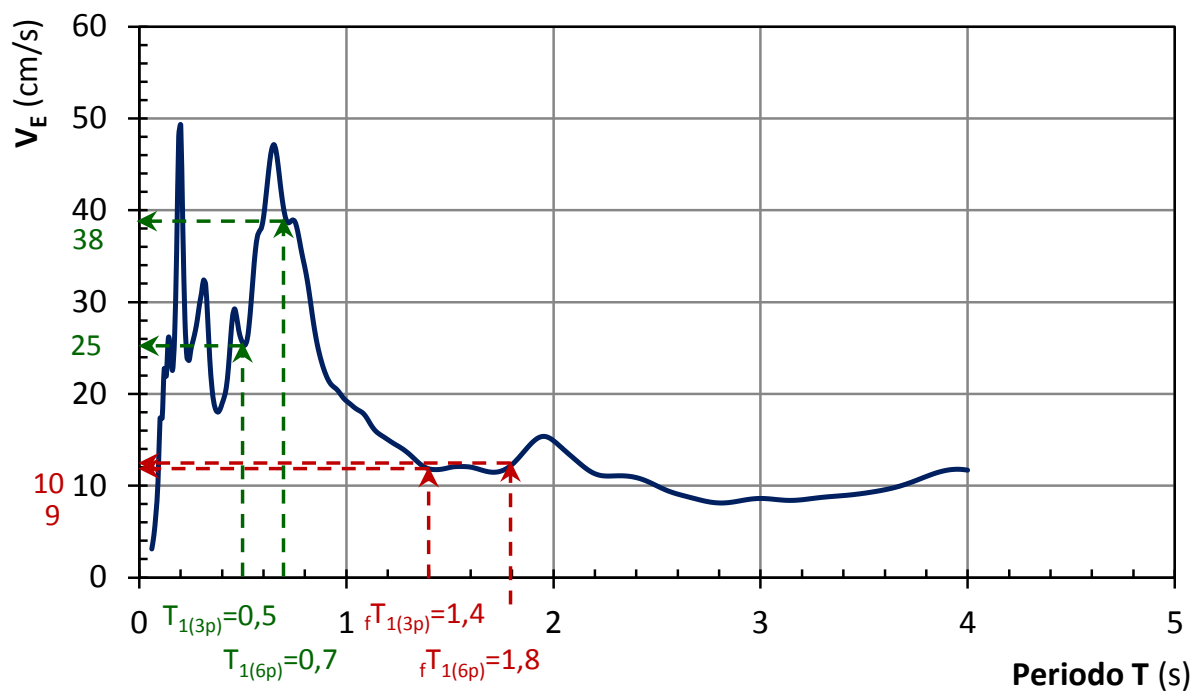

Fig. 4.3.68: Espectro de energía introducida del acelerograma $\mathrm{N}^{\circ} 8$ (Sicilia-Orientale) escalado a $P G A=261,4 \mathrm{~cm} / \mathrm{s}^{2}$

Comparando los valores de aceleración máxima de respuesta $S_{a}$ y de energía $V_{E}$, para los periodos de vibración anteriormente obtenidos.

Tabla 4.3.17: Ordenadas del espectro elástico de respuesta $S_{a}$ y del espectro de energía introducida, correspondientes a los periodos de las estructuras analizadas.

\begin{tabular}{c|c|c|c|c|} 
& $\mathrm{f}_{1(3 \mathrm{p})}=1,4 \mathrm{~s}$ & $\mathrm{~T}_{1(3 \mathrm{p})}=0,5 \mathrm{~s}$ & $\mathrm{f}_{1(6 \mathrm{p})}=1,8 \mathrm{~s}$ & $\mathrm{~T}_{1(6 \mathrm{p})}=0,7 \mathrm{~s}$ \\
\hline $\mathrm{S}_{\mathrm{a}}\left(\mathrm{cm} / \mathrm{s}^{2}\right)$ & 30 & 210 & 20 & 200 \\
\hline $\mathrm{V}_{\mathrm{E}}(\mathrm{cm} / \mathrm{s})$ & 9 & 25 & 10 & 38 \\
\hline & \multicolumn{3}{|l}{}
\end{tabular}


- Acelerograma №14 (Ano Liosia) $\Delta=14 \mathrm{Km}$

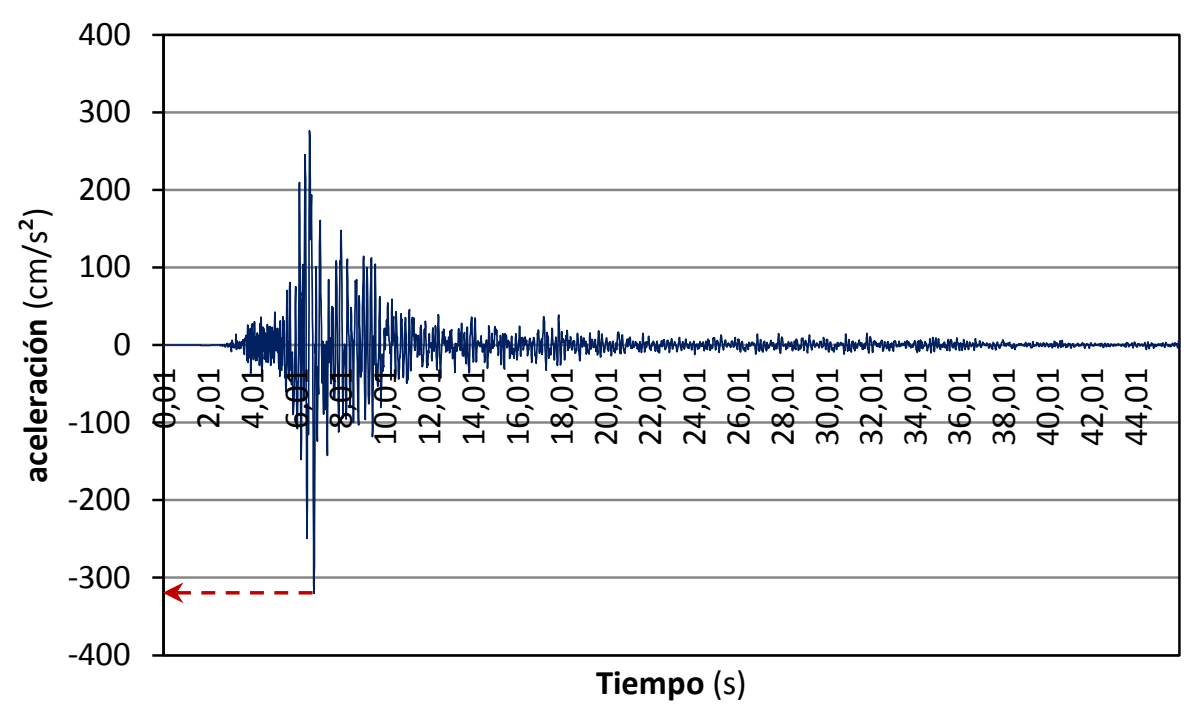

Fig. 4.3.69: Acelerograma $\mathrm{N}^{\circ} 14$ (Ano Liosia), suelo tipo III, campo lejano, sin escalar

Aceleración sísmica de cálculo: $a_{c}=261,4 \mathrm{~cm} / \mathrm{s}^{2}$

Aceleración máxima: $P G A=320,01 \mathrm{~cm} / \mathrm{s}^{2}$

Factor de escala: $\frac{261,4 \mathrm{~cm} / \mathrm{s}^{2}}{320,01 \mathrm{~cm} / \mathrm{s}^{2}}=0,8168$

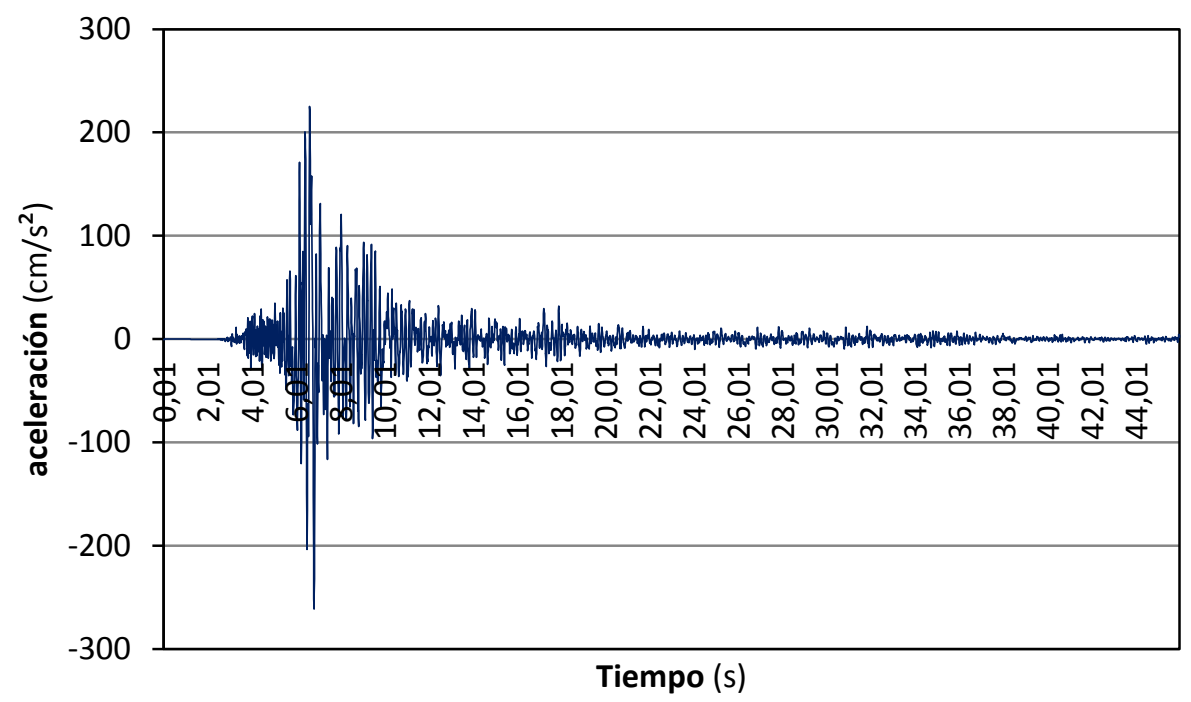

Fig. 4.3.70: Acelerograma $\mathrm{N}^{\circ} 14$ (Ano Liosia), suelo tipo III, campo lejano, escalado a $\mathrm{PGA}=261,4 \mathrm{~cm} / \mathrm{s}^{2}$ 


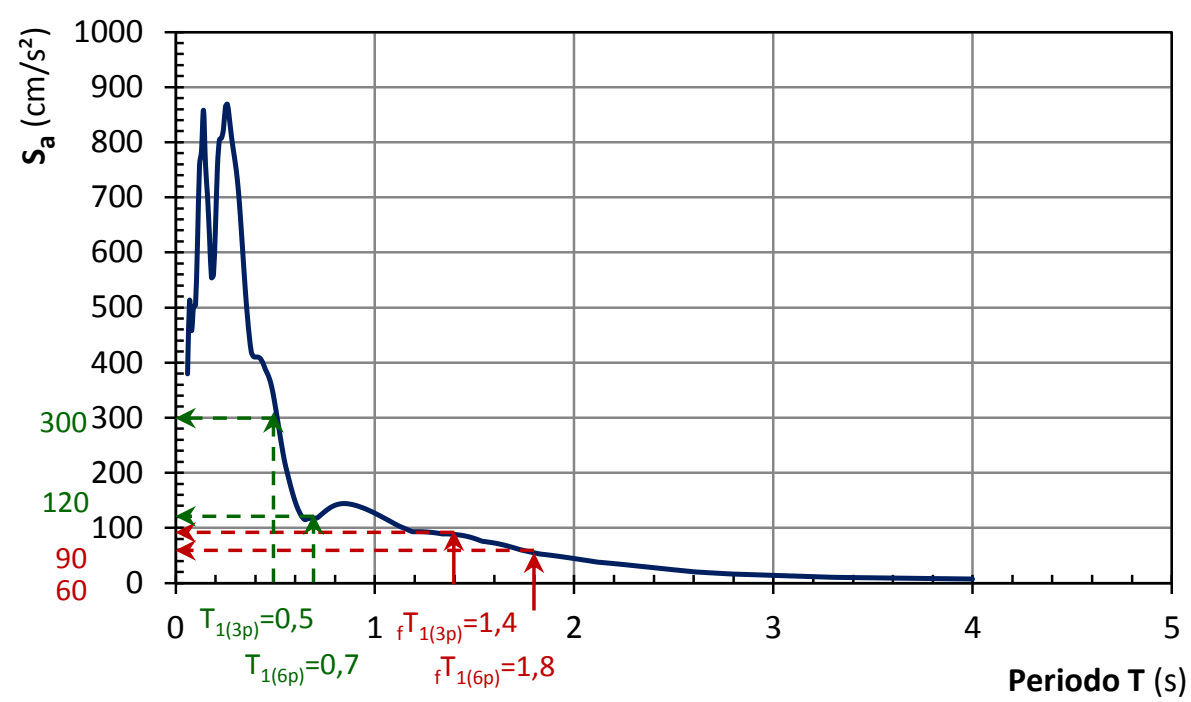

Fig. 4.3.71: Espectro elástico de respuesta del acelerograma $\mathrm{N}^{\circ} 14$ (Ano Liosia) escalado a $P G A=261,4 \mathrm{~cm} / \mathrm{s}^{2}$

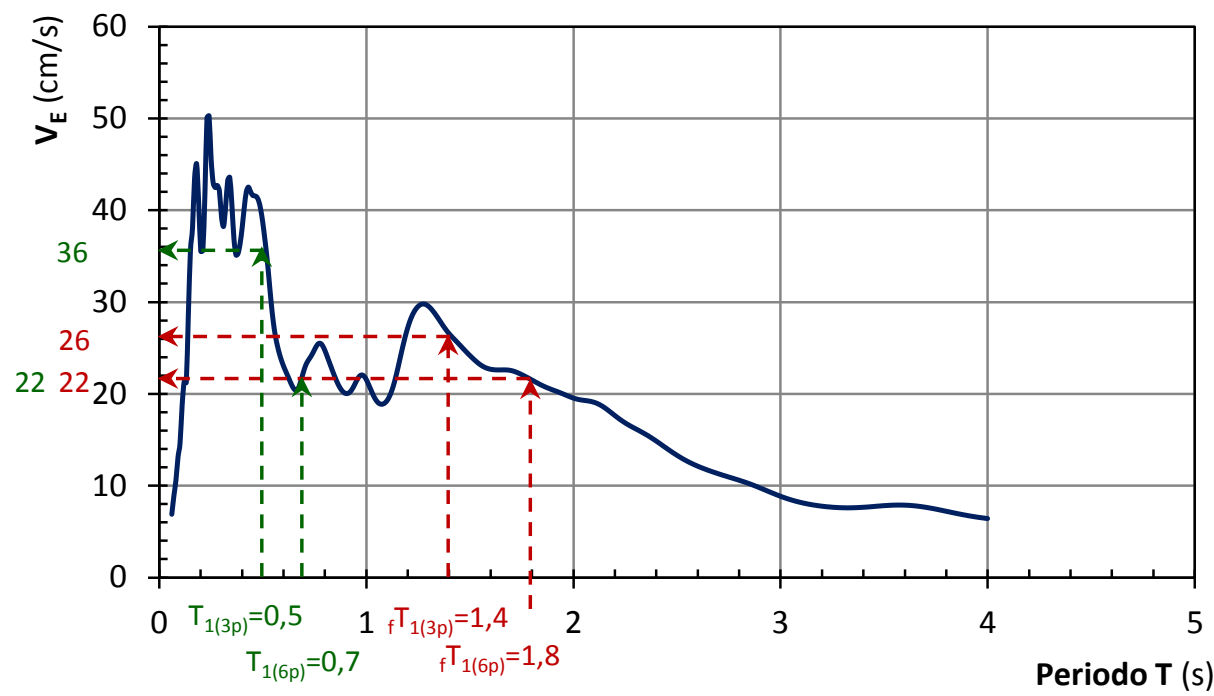

Fig. 4.3.72: Espectro de energía introducida del acelerograma $\mathrm{N}^{\circ} 14$ (Ano Liosia) escalado a $P G A=261,4 \mathrm{~cm} / \mathrm{s}^{2}$

Comparando los valores de aceleración máxima de respuesta $S_{a}$ y de energía $V_{E}$, para los periodos de vibración anteriormente obtenidos.

Tabla 4.3.18: Ordenadas del espectro elástico de respuesta $S_{a}$ y del espectro de energía introducida, correspondientes a los periodos de las estructuras analizadas.

\begin{tabular}{l|c|c|c|c|} 
& $\mathrm{f}_{1(3 \mathrm{p})}=1,4 \mathrm{~s}$ & $\mathrm{~T}_{1(3 \mathrm{p})}=0,5 \mathrm{~s}$ & $\mathrm{f}_{1(6 \mathrm{p})}=1,8 \mathrm{~s}$ & $\mathrm{~T}_{1(6 \mathrm{p})}=0,7 \mathrm{~s}$ \\
\hline $\mathrm{S}_{\mathrm{a}}\left(\mathrm{cm} / \mathrm{s}^{2}\right)$ & 90 & 300 & 60 & 120 \\
\hline $\mathrm{V}_{\mathrm{E}}(\mathrm{cm} / \mathrm{s})$ & 26 & 36 & 22 & 22 \\
\hline & & & \multicolumn{2}{|c}{}
\end{tabular}




\section{CAPÍTULO 5 \\ DETERMINACIÓN DE LA RESISTENCIA LATERAL REQUERIDA A LOS DISIPADORES}

\subsection{INTRODUCCIÓN}

En este capítulo se describen los cálculos dinámicos directos llevados a cabo para este trabajo, con el fin de averiguar el comportamiento sísmico de los pórticos construidos con vigas planas. Los cálculos se han realizado con modelos numéricos de barras en dos dimensiones, que son representativas de las estructuras construidas en España durante los años 70, 80, 90 del siglo XX, siguiendo las prácticas constructivas de ese momento y aplicando en su mayoría la antigua norma sísmica PDS-74 [1].

Para ello se han descrito los prototipos de estructura seleccionadas en el Capítulo 3 y su modelización para ser tratados con el programa IDARC en el apartado 5.1 de este capítulo. En el Capítulo 4, se han seleccionado un conjunto de terremotos históricos representativos de la sismicidad de la zona mediterránea y en este capítulo, se llevan a cabo un gran número de cálculos dinámicos directos, en los que se analizan y sintetizan los resultados de dichos cálculos desde diferentes puntos de vista (energía total disipada y desplazamiento máximo entre planta, obtenidos en el apartado 5.4; resistencia lateral requerida a los disipadores, definida en el apartado 5.5; así como la automatización del programa LumpST con Matlab especificada en el apartado 5.2).

\subsection{ANÁLISIS DE LOS PÓRTICOS CON IDARC}

El programa IDARC, desarrollado en la Universidad de Buffalo, Universidad Estatal de Nueva York, se introdujo por primera vez en 1987 como un programa de análisis de dos dimensiones para estudiar la respuesta no lineal de edificios de hormigón armado de varios niveles, con el propósito de analizar los daños del terremoto en edificios. Desde entonces, numerosas mejoras se han añadido, incluyendo la capacidad de analizar una amplia variedad de estructuras, materiales estructurales, y recientemente, dispositivos estructurales de amortiguación.

Se dimensionaron tres prototipos y se modelizaron para poder ser analizados con el programa de análisis no lineal en dos dimensiones IDARC versión 7.0. Éste incluye elementos lineales macroscópicos, tipo barra, que se pueden emplear para modelizar vigas y pilares. Estos modelos permiten reproducir numéricamente deformaciones no lineales de flexión y deformaciones elásticas por cortante y axil. Para implementar el comportamiento inelástico en los modelos macroscópicos, el programa incorpora un modelo de flexibilidad distribuida, que extiende y mejora los modelos de plasticidad concentrada que se emplean normalmente para elementos metálicos.

Este programa permite tanto cálculos estáticos como dinámicos en régimen no lineal. Una parte de los cálculos realizados con IDARC para esta Tesina son cálculos estáticos no lineales empleando el método del empuje incremental (análisis pushover) con control en fuerzas y empleando el primer modo de vibración. Este tipo de análisis se ha empleado para obtener: 
1) La curva de capacidad de cada pórtico (relación entre cortante basal y desplazamiento de la última planta.

2) La relación entre fuerza cortante de la planta y el desplazamiento entre forjados que delimitan dicha planta, para cada planta de cada pórtico.

El formato de entrada del programa está compuesto por una serie de secciones (SET), en las que se introduce la información requerida para el análisis. [16]

En primer lugar, se detalla la información general (SET A) de nueve parámetros: NSO; NFR; NCON; NSTL; NMSR; NPDEL; INFLEX; INFLEXDIST; IPC, en estos parámetros se detallan el número de plantas; número de pórticos distintos; tipos de hormigón; tipos de acero de refuerzo; tipos de tabiquería; efecto P- $\Delta$ (0 para ignorar este efecto y 1 para incluirlo); consideración de rótulas; rigidez de la viga (1 si es constante); sistema operativo (0 si el sistema operativo es Unix y 1 si se trata de DOS/WINDOWS).

El programa IDARC permite incorporar en los cálculos los efectos P- $\Delta$. Estos efectos son debidos a los momentos de vuelco adicionales que generan las cargas gravitatorias debido a los desplazamientos relativos de los forjados de cada planta. La forma más usual de tener en cuenta estos efectos consiste en evaluar las cargas axiles en los elementos verticales de la estructura y calcular una matriz de rigidez geométrica que se suma a la matriz de rigidez de las barras de la estructura. En el programa IDARC los efectos P- $\Delta$ se representan mediante un sistema de fuerzas laterales ficticias equivalentes que, igualan en magnitud a los momentos de vuelco que producen las cargas verticales gravitatorias al actuar excéntricamente debido, al desplazamiento relativo de los forjados (Wilson y Habibullah, 1987). Los efectos $\mathrm{P}-\Delta$ se han tenido en cuenta en los cálculos realizados para este trabajo.

Esta sección SET A, se compone de otras subsecciones, que se definen a continuación:

(SET A1) En esta sección se detallan los tipos de elementos, de diez parámetros: MCOL; MBEM; MWAL; MEDG; MTRN; MSPR; MBRV; MBRF; MBRH; MIW, en los que concretizamos el número de tipos de pilares; tipos de vigas; tipos de pantallas; tipos de pilares de borde; tipos de vigas de canto; tipos de muelles de torsión; tipos de amortiguadores; tipos de disipadores de fricción; tipos de disipadores histeréticos y tipos de tabiquería.

(SET A2) En este definimos el número de elementos, de nueve parámetros: NCOL; NBEM; NWAL; NEDG; NTRN; NSPR; NMR; NBR; NIW, como son el número de pilares; vigas; muros a cortante; pilares de borde; vigas transversales; muelles de torsión; rótulas; disipadores y tabiquería.

(SET A3) En este, se define el sistema de unidades, compuesto por un solo parámetro: IU (1, para unidades anglo sajonas y 2 para $m m, K N$ ).

(SET A4) Aquí se especifica la altura de la planta "i", desde la base a partir de la primera planta: HIGT(i).

(SET A5) En este se describen el número de pórticos iguales: NDUP(i)

(SET A6) En este set se introduce el número de líneas de pilares, de cada pórtico "i": NVLN(i).

(SET A7) Finalmente, se especifican los pesos nodales, estos se componen de tres parámetros: LEVEL; IFR(J); WVT(K), en los que se detallan el número de planta; el número de pórtico; y el peso nodal, respectivamente. 
A continuación, se especifican las propiedades de los materiales (SET B) que está compuesto por un parámetro: IUSER, en el que se consideran dos opciones, 0 para que el programa genere el diagrama momento-curvatura y 1 para que lo genere el usuario. Si se elige esta última opción, como es el caso, no son necesarios los SET B1, B2 y B3, e iríamos, para seguir el flujo de cálculo al apartado (SET C) directamente.

En esta sección (SET C), estudiaremos la respuesta histerética de las secciones de las barras que se controla a partir de la definición del esqueleto de la curva momento-curvatura. En primer lugar se define el número de modelos de disipadores NHYS que en el caso que nos ocupa es 3 , y seguidamente se especifican cada uno de los siete parámetros: IR; 1; HC; HBD; HBE; HS; IBILINEAR, correspondientes a cada uno de los modelos, IR; 1 para un modelo histerético multilineal; los cuatro parámetros siguientes $\operatorname{HC}(\alpha), \operatorname{HBD}\left(\beta_{1}\right), \operatorname{HBE}\left(\beta_{2}\right), \mathrm{HS}(\gamma)$, gobiernan los fenómenos de degradación de rigidez, efecto de pinzamiento de las curvas y degradación de resistencia; y por último el parámetro IBILINEAL en el que existen varias opciones, de la cuales se considera el valor 0 , es decir para un modelo trilineal.

A continuación, la propiedades de los pilares se definen en la sección (SET D), compuesta por un parámetro IUCOL, con dos opciones: 0 si se especifica las dimensiones y armado (número de redondos, etc.) y 1 en la que se especifica el diagrama momento-curvatura del pilar. Si se especifica esta última opción, como es el caso, se iría directamente al apartado (SET D3).

En esta sección (SET D3), se especifica la forma del pilar, mediante el parámetro ICTYPE, considerando el valor 1 , para el caso de pilares rectangulares.

Si ICTYPE=1, el flujo de cálculo nos dirige a la sección SET D3(a), que se compone de una primera línea de siete parámetros: KC; AN; ANY; ANB; AMLC; RAMC1; RAMC2, siendo KC el número de tipo de pilar, es decir, existirá una entrada por cada tipo de pilar, que en nuestro caso son 7 tipos, para los pórticos de tres plantas, pa2pz2-1 y pa4pz2-1, y 12 para el pórtico de 6 plantas pz6pz2-1. Los parámetros AN, ANY y ANB correspondientes a las fuerzas axiles, se pueden observar en la Fig. 5.1.1; y por último los parámetros RAMC1 y RAMC2, correspondientes a los brazos mecánicos de excentricidad del axil, para la sección inferior y superior respectivamente.

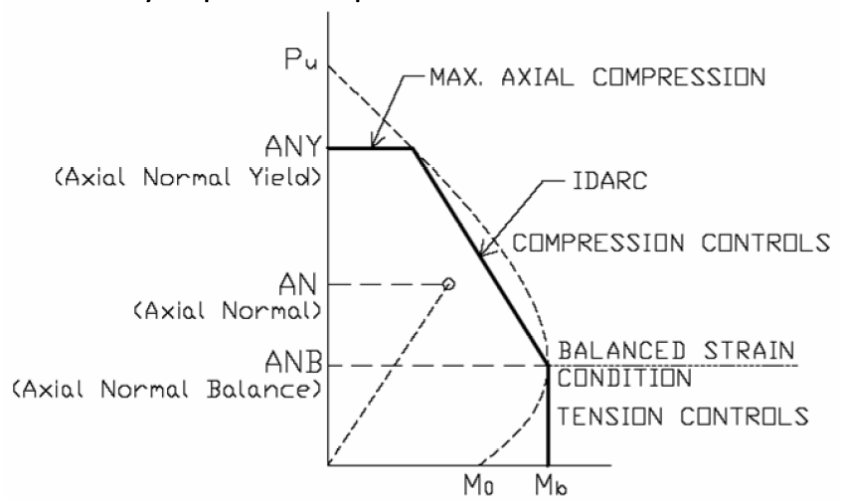

Fig. 5.1.1: Diagrama de interacción Axil - Momento

La segunda y tercera línea, corresponden a la sección inferior (bottom) y la cuarta y quinta línea a la sección superior (top). La segunda línea consta de ocho parámetros: KHYSC; El; EA; PCP; PYP; UYP; UUP; EI3P, siendo KHYSC la ley histerética, el resto de parámetros se representan en la Fig. 5.2. Los cinco parámetros de la tercera línea: PCN; PYN; UYN; UUN; EI3N, también están representados en la Fig. 5.1.2. 


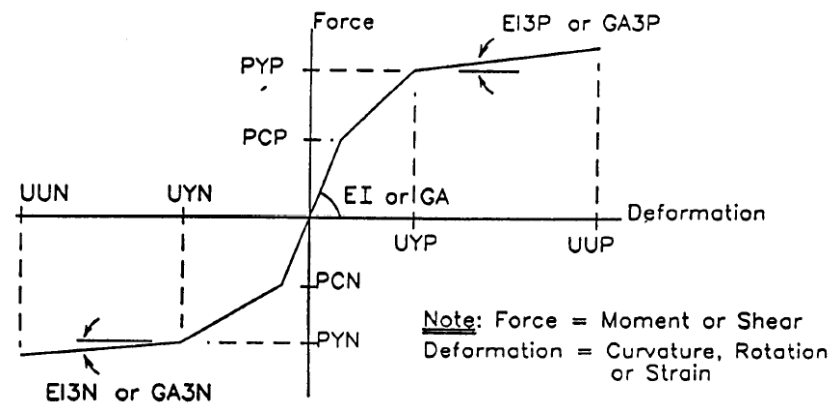

Fig. 5.1.2: Notation for user Trilinear Envelopes

Un valor de entrada de KHYSC con signo negativo para la sección inferior, implica valores simétricos en la sección superior.

A continuación, la propiedades de las vigas se definen en la sección (SET E), compuesta por un parámetro IUBEM, que tiene dos opciones: 0 si se especifica las dimensiones y armado (número de redondos, etc.) y 1 si especificamos el diagrama momento-curvatura de la viga. Si elegimos esta última opción, como es el caso, se iría directamente al apartado (SET E2).

En esta sección (SET E2), se especifica la forma de la viga, mediante el parámetro IBTYPE, considerando el valor 1 , para el caso de vigas rectangulares.

Si IBTYPE=1, según el flujo de cálculo, nos dirigimos a la sección SET E2(a), que se compone de una primera línea de cuatro parámetros: KB; $A M L B$; RAMB1 y RAMB2, siendo KB el número de tipo de viga es decir, existirá una entrada por cada tipo de viga, que en nuestro caso tendremos 9, para los pórticos de tres plantas, pa2pz2-1 y pa4pz2-1, y 18 para el pórtico de 6 plantas pz6pz2-1. AMLB, es la longitud de la viga; este, junto con los parámetros RAMB1, RAMB2 se especifican en la figura 5.1.3.

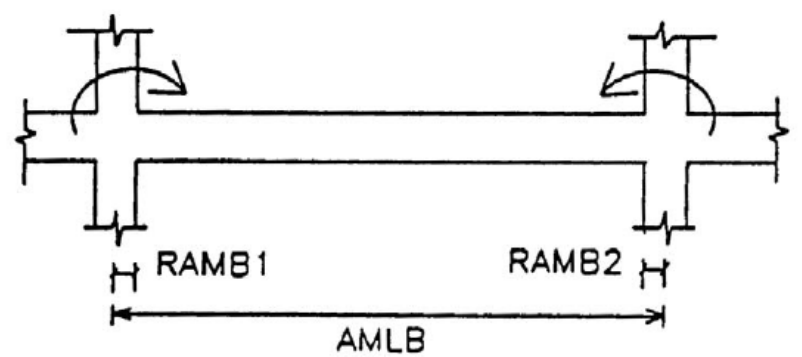

Fig. 5.1.3: Detalle de viga

La segunda y tercera línea, corresponden a la sección inferior (bottom) y la cuarta y quinta a la sección superior (top). La segunda y cuarta líneas tienen siete parámetros que son: KHYSB; EI; PCP; PYP; UYP; UUP; EI3P, siendo KHYSB la ley histerética y las líneas tercera y quinta, se componen de los cinco parámetros siguientes: PCN; PYN; UYN; UUN; EI3N.

De igual forma que en el pilar, un valor de entrada de KHYSB con signo negativo para la sección inferior, implica valores simétricos en la sección superior.

A continuación nos dirigimos a la sección (SET L1), en la que se concretiza la conexión de los pilares, cada línea está compuesta por seis parámetros: M; ITC; IC; JC; LBC; LTC, que corresponden al 
número de pilar; número de tipo de pilar; número de pórtico; línea de pilares a la que pertenece; nivel inferior desde el que arranca el pilar y por último el nivel superior hasta el que llega dicho pilar.

Igualmente en la sección (SET L2) se define la conexión de las vigas, en la que cada línea está compuesta por seis parámetros: $\mathrm{M}$; ITB; LB; IB; JLB; JRB, que corresponden al número de viga; número de tipo de viga; número de planta a la que pertenece; número de pórtico; número de línea de pilares del lado izquierdo a la que pertenece y número de línea de pilares del lado derecho a la que pertenece.

Por último, en la sección (SET M), definimos la opción de análisis que queremos realizar mediante el parámetro IOPT. Se considerará la opción IOPT=2 para efectuar un Monotonic "pushover" analysis including static loads.

Esta sección SET M, se compone de otras subsecciones, que se definen a continuación:

(SET M1) Aquí se detallan las cargas estáticas, que se componen de cuatro parámetros: NLU; NL; NLM; NLC, en los que definimos la carga distribuida en las vigas; la carga en los nudos; los momentos nodales y las cargas verticales concentradas. Se considerará el valor 0 para todos ellos.

(SET M2) Define, mediante el parámetro JOPT =1 la opción de análisis pushover que se considera, que es la de control de fuerza.

Elegida esta opción, JOPT=1, nos dirigimos a la sección (M2.1), que se compone de dos líneas, la primera línea se define mediante el parámetro ITYP $=1$, la opción de carga a considerar es lineal (triángulo invertido). En la segunda, se define el criterio de parada del programa, mediante tres parámetros: PMAX; MSTEPS; DRFLIM, es decir, el coeficiente de cortante basal; el número de pasos a considerar para llegar a PMAX; y el límite superior para el desplazamiento de la última planta (porcentaje de la altura del edificio).

Una vez introducidos todos estos datos de entrada, en la sección (SET N), controlamos el formato del output.

(SET N1.1), mediante el parámetro NPRNT, definimos el número adicional de "snapshots (instantáneas)" de la respuesta estructural durante el pushover.

(SET N1.3), compuesta por cinco parámetros: ICDPRNT(1); ICDPRNT(2); ICDPRNT(3); ICDPRNT(4); ICDPRNT(5), con estos podemos controlar la impresión de desplazamientos, tensión, estado de colapso, índices de deño, etc.

Mediante la sección (SET N2), controlamos el archivo de salida con cuatro parámetros. En una primera línea, compuesta por los tres primeros parámetros: NSOUT, es el número de historias de salida; DTOUT, tiempo de salida/intervalo de step; ISO(i), lista de números de historia de salida.

Con el cuarto parámetro FNAMES(i), damos nombre a los ficheros de salida que se generan, uno en cada línea.

Y finalmente en la sección (SET N3), definimos los elementos histeréticos de salida mediante seis parámetros: KCOUT; KBOUT; KWOUT; KSOUT; KBROUT; KIWOUT. 
Tabla 5.1.1: Archivo de entrada para el pórtico pa2pz2-1 de tres plantas (pa2pz2-1.DAT) IDARC

\begin{tabular}{|c|c|c|}
\hline SET A & $\begin{array}{l}\text { USER_TEXT } \\
\text { NSO,NFR,NCON,NSTL,NMSR,NPDEL,IFLEX,IFLEXDIST,IPC }\end{array}$ & $\begin{array}{l}\text { CONTROL DATA } \\
3,1,1,1,0,1,1,1,1\end{array}$ \\
\hline SET A1 & $\begin{array}{l}\text { USER_TEXT } \\
\text { MCOL,MBEM,MWAL,MEDG,MTRN,MSPR,MBRV,MBRF,MBRH,MIW }\end{array}$ & $\begin{array}{l}\text { ELEMENT TYPES } \\
7,9,0,0,0,0,0,0,0,0\end{array}$ \\
\hline SET A2 & $\begin{array}{l}\text { USER_TEXT } \\
\text { NCOL,NBEM,NWAL,NEDG,NTRN,NSPR,NMR,NBR,NIW }\end{array}$ & $\begin{array}{l}\text { ELEMENT DATA } \\
15,12,0,0,0,0,0,0,0\end{array}$ \\
\hline SET A3 & $\begin{array}{l}\text { USER_TEXT } \\
\text { IU }\end{array}$ & $\begin{array}{l}\text { UNITS SYSTEM KN-mm } \\
2\end{array}$ \\
\hline SET A4 & $\begin{array}{l}\text { USER_TEXT } \\
\text { HIGT(i) }\end{array}$ & $\begin{array}{l}\text { FLOOR ELEVATIONS } \\
4500.0,7500.0,10500.0\end{array}$ \\
\hline SET A5 & $\begin{array}{l}\text { USER_TEXT } \\
\text { NDUP(i) }\end{array}$ & $\begin{array}{l}\text { DESCRIPTION OF IDENTICAL FRAMES } \\
1\end{array}$ \\
\hline SET A6 & $\begin{array}{l}\text { USER_TEXT } \\
\text { NVLN(i) }\end{array}$ & $\begin{array}{l}\text { PLAN CONFIGURATION: NO OF COLUMN LINES } \\
5\end{array}$ \\
\hline SET A7 & $\begin{array}{l}\text { USER_TEXT } \\
\text { LEVEL,IFR(J),WVT(K) }\end{array}$ & $\begin{array}{l}\text { NODAL WEIGHTS } \\
1,1,118.75,162.5,162.5,162.5,118.75 \\
2,1,118.75,162.5,162.5,162.5,118.75 \\
3,1,118.75,162.5,162.5,162.5,118.75\end{array}$ \\
\hline SET B & $\begin{array}{l}\text { USER_TEXT } \\
\text { IUSER }\end{array}$ & $\begin{array}{l}\text { CODE FOR SPECIFICATION OF USER PROPERTIES } \\
1\end{array}$ \\
\hline SET C & $\begin{array}{l}\text { USER_TEXT } \\
\text { NHYS } \\
\text { IR,1,HC,HBD,HBE,HS,IBILINEAR }\end{array}$ & $\begin{array}{l}\text { HYSTERETIC MODELING RULES } \\
3 \\
1,1,3.1,0.0,0.4,0.20,0 \\
2,1,3.1,0.0,0.4,0.20,0 \\
3,1,1.8,0.0,0.6,0.20,0\end{array}$ \\
\hline SET D & $\begin{array}{l}\text { USER_TEXT } \\
\text { IUCOL }\end{array}$ & $\begin{array}{l}\text { MOMENT CURVATURE ENVELOPE GENERATION } \\
1\end{array}$ \\
\hline SET D3 & $\begin{array}{l}\text { USER_TEXT } \\
\text { ICTYPE }\end{array}$ & $\begin{array}{l}\text { COLUMN DIMENSIONS } \\
1\end{array}$ \\
\hline SET D3(a) & $\begin{array}{l}\text { KC,AN,ANY,ANB,AMLC,RAMC1,RAMC2 } \\
\text { KHYSC,EI,EA,PCP,PYP,UYP,UUP,EI3P } \\
\text { PCN,PYN,UYN,UUN,EI3N }\end{array}$ & 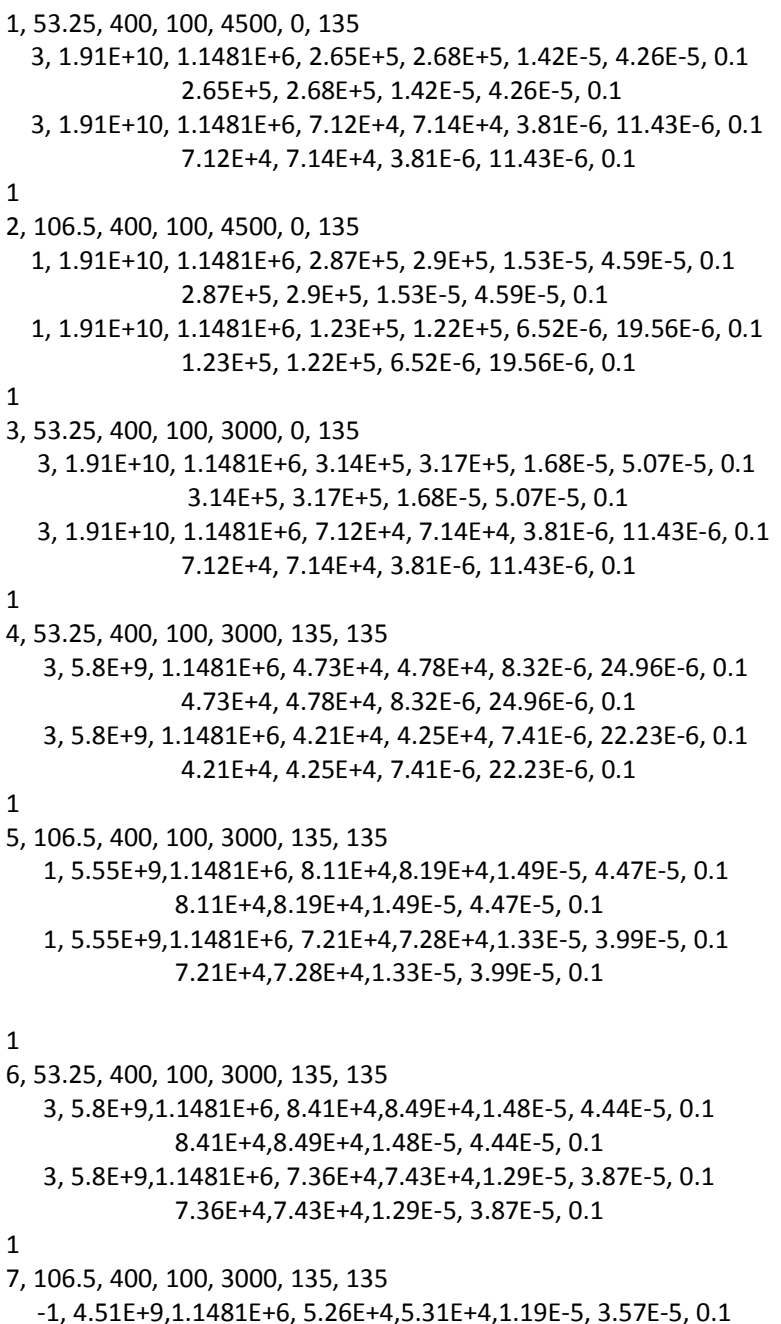 \\
\hline
\end{tabular}




\begin{tabular}{|c|c|c|}
\hline & & $5.26 \mathrm{E}+4,5.31 \mathrm{E}+4,1.19 \mathrm{E}-5,3.57 \mathrm{E}-5,0.1$ \\
\hline SETE & $\begin{array}{l}\text { USER_TEXT } \\
\text { IUBEM }\end{array}$ & $\begin{array}{l}\text { BEAM MOMENT CURVATURE ENVELOPE GENERATION } \\
1\end{array}$ \\
\hline SET E2 & $\begin{array}{l}\text { USER_TEXT } \\
\text { IBTYPE }\end{array}$ & $\begin{array}{l}\text { BEAM DIMENSIONS } \\
1\end{array}$ \\
\hline SET E2(a) & $\begin{array}{l}\text { KB,AMLB,RAMB1,RAMB2 } \\
\text { KHYSB,EI,PCP,PYP,UYP,UUP,EI3P } \\
\text { PCN,PYN,UYN,UUNEI3N }\end{array}$ & 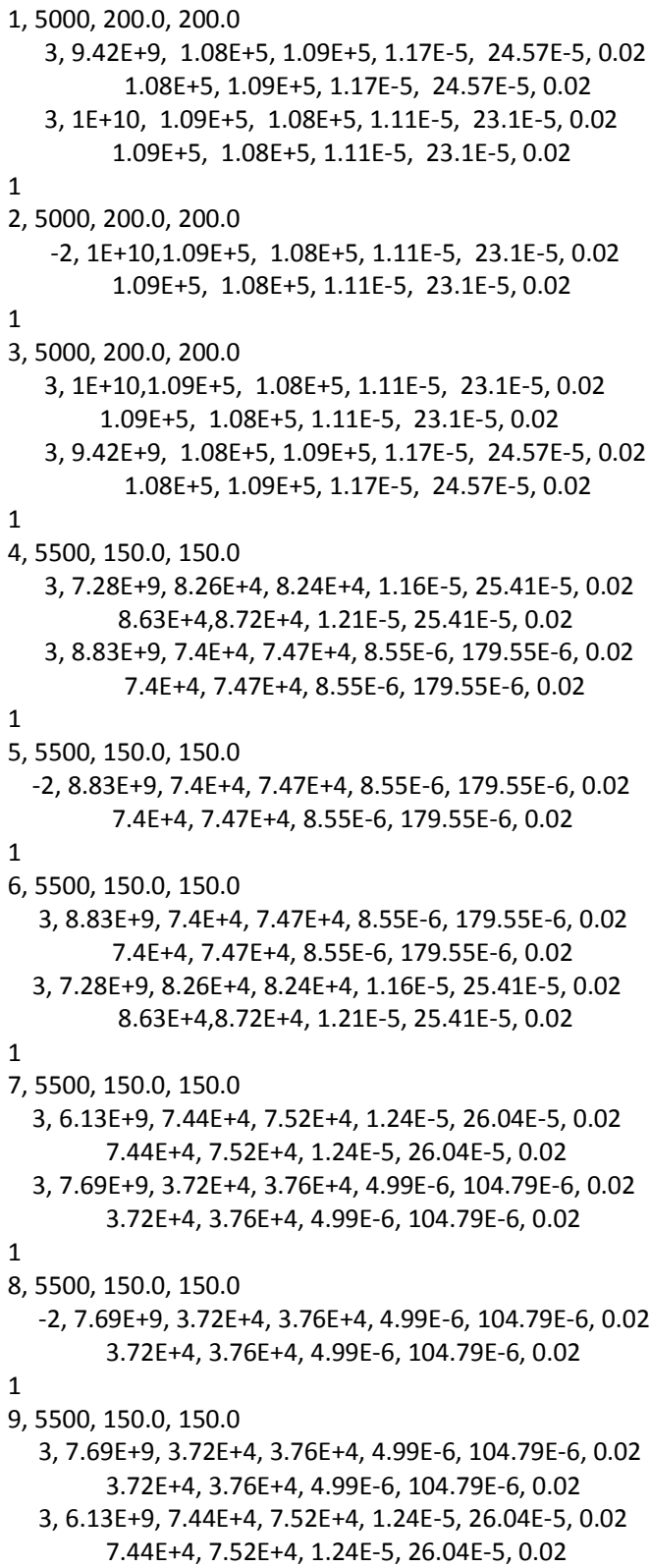 \\
\hline SET L1 & $\begin{array}{l}\text { USER_TEXT } \\
\text { M,ITC,IC,JC,LBC,LTC }\end{array}$ & $\begin{array}{c}\text { COLUMN CONNECTIVTY } \\
1,1,1,1,0,1 \\
2,2,1,2,0,1 \\
3,2,1,3,0,1 \\
4,2,1,4,0,1 \\
5,3,1,5,0,1 \\
6,4,1,1,1,2 \\
7,5,1,2,1,2 \\
8,5,1,3,1,2 \\
9,5,1,4,1,2 \\
10,4,1,5,1,2 \\
11,6,1,1,2,3 \\
12,7,1,2,2,3 \\
13,7,1,3,2,3 \\
14,7,1,4,2,3 \\
15,6,1,5,2,3 \\
\end{array}$ \\
\hline SET L2 & USER_TEXT & BEAM CONNECTIVITY \\
\hline
\end{tabular}




\begin{tabular}{|l|l|l|}
\hline & M,ITB,LB,IB,ILB,JRB & $1,1,1,1,1,2$ \\
& & $2,2,1,1,2,3$ \\
& & $3,2,1,1,4$ \\
& & $4,3,1,4,5$ \\
& & $5,4,2,1,2$ \\
& & $6,5,2,1,2,3$ \\
& & $7,5,2,1,3$ \\
& & $8,6,2,1,4,5$ \\
& & $9,7,3,1,1,2$ \\
& & $10,8,3,1,2,3$ \\
\hline SET M & USER_TEXT & $11,8,3,1,3,4$ \\
& IOPT & $12,9,3,1,4,5$ \\
\hline SET M1 & USER_TEXT & ANALYSIS OPTIONS \\
& NLU,NU,NLM,NLC & 2 \\
\hline SET M2 & USER_TEXT & LONG TERM \\
& JOPT & $0,0,0,0$ \\
\hline SET M2.1 & USER_TEXT & Monotonic Pushover Analysis \\
& ITYP & 1 \\
\hline SET N1.1 & PMAX,MSTEPS,DRFLIM & FORCE CONTROLLED ANALYSIS \\
& NPRNT & 1 \\
\hline SET N1.3 & ICDPRNT(1), ICDPRNT(2), ICDPRNT(3), ICDPRNT(4), ICDPRNT(5) & $0,0,0,0,0$ \\
\hline SET N2 & USER_TEXT & OUTPUT CONTROL \\
& NSOUT,DTOUT,ISO(i) & $3,1,1,2,3$ \\
& FNAMES(i) & story1 \\
& & story2 \\
& & story3 \\
\hline SET N3 & USER_TEXT & ELEMENT HYSTERESIS OUTPUT \\
& KCOUT,KBOUT,KWOUT,KSOUT,KBROUT,KIWOUT & $0,0,0,0,0,0$ \\
\hline
\end{tabular}

Tabla 5.1.2: Archivo de entrada para el pórtico pa4pz2-1 de tres plantas (pa4pz2-1.DAT) IDARC

\begin{tabular}{|c|c|c|}
\hline SET A & $\begin{array}{l}\text { USER_TEXT } \\
\text { NSO,NFR,NCON,NSTL,NMSR,NPDEL,IFLEX,IFLEXDIST,IPC }\end{array}$ & $\begin{array}{l}\text { CONTROL DATA } \\
3,1,1,1,0,1,1,1,1\end{array}$ \\
\hline SET A1 & $\begin{array}{l}\text { USER_TEXT } \\
\text { MCOL,MBEM,MWAL,MEDG,MTRN,MSPR,MBRV,MBRF,MBRH,MIW }\end{array}$ & $\begin{array}{l}\text { ELEMENT TYPES } \\
7,14,0,0,0,0,0,0,0,0\end{array}$ \\
\hline SET A2 & $\begin{array}{l}\text { USER_TEXT } \\
\text { NCOL,NBEM,NWAL,NEDG,NTRN,NSPR,NMR,NBR,NIW }\end{array}$ & $\begin{array}{l}\text { ELEMENT DATA } \\
15,24,0,0,0,0,0,0,0\end{array}$ \\
\hline SET A3 & $\begin{array}{l}\text { USER_TEXT } \\
\text { IU }\end{array}$ & $\begin{array}{l}\text { UNITS SYSTEM KN - mm } \\
2\end{array}$ \\
\hline SET A4 & $\begin{array}{l}\text { USER_TEXT } \\
\text { HIGT(i) }\end{array}$ & $\begin{array}{l}\text { FLOOR ELEVATIONS } \\
4500.0,7500.0,10500.0\end{array}$ \\
\hline SET A5 & $\begin{array}{l}\text { USER_TEXT } \\
\text { NDUP(i) }\end{array}$ & $\begin{array}{l}\text { DESCRIPTION OF IDENTICAL FRAMES } \\
1\end{array}$ \\
\hline SET A6 & $\begin{array}{l}\text { USER_TEXT } \\
\text { NVLN(i) }\end{array}$ & $\begin{array}{l}\text { PLAN CONFIGURATION: NO OF COLUMN LINES } \\
5\end{array}$ \\
\hline SET A7 & $\begin{array}{l}\text { USER_TEXT } \\
\text { LEVEL,IFR(J),WVT(K) }\end{array}$ & $\begin{array}{l}\text { NODAL WEIGHTS } \\
1,1,139.56,196.625,196.625,196.625,139.56 \\
2,1,139.56,196.625,196.625,196.625,139.56 \\
3,1,139.56,196.625,196.625,196.625,139.56\end{array}$ \\
\hline SET B & $\begin{array}{l}\text { USER_TEXT } \\
\text { IUSER }\end{array}$ & $\begin{array}{l}\text { CODE FOR SPECIFICATION OF USER PROPERTIES } \\
1\end{array}$ \\
\hline SET C & $\begin{array}{l}\text { USER_TEXT } \\
\text { NHYS } \\
\text { IR,1,HC,HBD,HBE,HS,IBILINEAR }\end{array}$ & $\begin{array}{l}\text { HYSTERETIC MODELING RULES } \\
3 \\
1,1,3.1,0.0,0.4,0.20,0 \\
2,1,3.1,0.0,0.4,0.20,0 \\
3,1,1.8,0.0,0.6,0.20,0\end{array}$ \\
\hline SET D & $\begin{array}{l}\text { USER_TEXT } \\
\text { IUCOL }\end{array}$ & $\begin{array}{l}\text { MOMENT CURVATURE ENVELOPE GENERATION } \\
1\end{array}$ \\
\hline SET D3 & $\begin{array}{l}\text { USER_TEXT } \\
\text { ICTYPE }\end{array}$ & $\begin{array}{l}\text { COLUMN DIMENSIONS } \\
1\end{array}$ \\
\hline SET D3(a) & $\begin{array}{l}\text { KC,AN,ANY,ANB,AMLC,RAMC1,RAMC2 } \\
\text { KHYSC,EI,EA,PCP,PYP,UYP,UUP,EI3P } \\
\text { PCN,PYN,UYN,UUN,EI3N }\end{array}$ & $\begin{array}{r}1,64.44,400,100,4500,0,150 \\
3,2.24 \mathrm{E}+10,1.1481 \mathrm{E}+6,3.64 \mathrm{E}+5,3.68 \mathrm{E}+5,1.66 \mathrm{E}-5,4.98 \mathrm{E}-5,0.1 \\
3.64 \mathrm{E}+5,3.68 \mathrm{E}+5,1.66 \mathrm{E}-5,4.98 \mathrm{E}-5,0.1 \\
3,2.24 \mathrm{E}+10,1.1481 \mathrm{E}+6,8.6 \mathrm{E}+4,8.69 \mathrm{E}+4,3.92 \mathrm{E}-6,11.76 \mathrm{E}-6,0.1 \\
8.6 \mathrm{E}+4,8.69 \mathrm{E}+4,3.92 \mathrm{E}-6,11.76 \mathrm{E}-6,0.1 \\
1\end{array}$ \\
\hline
\end{tabular}




\begin{tabular}{|c|c|c|}
\hline & & 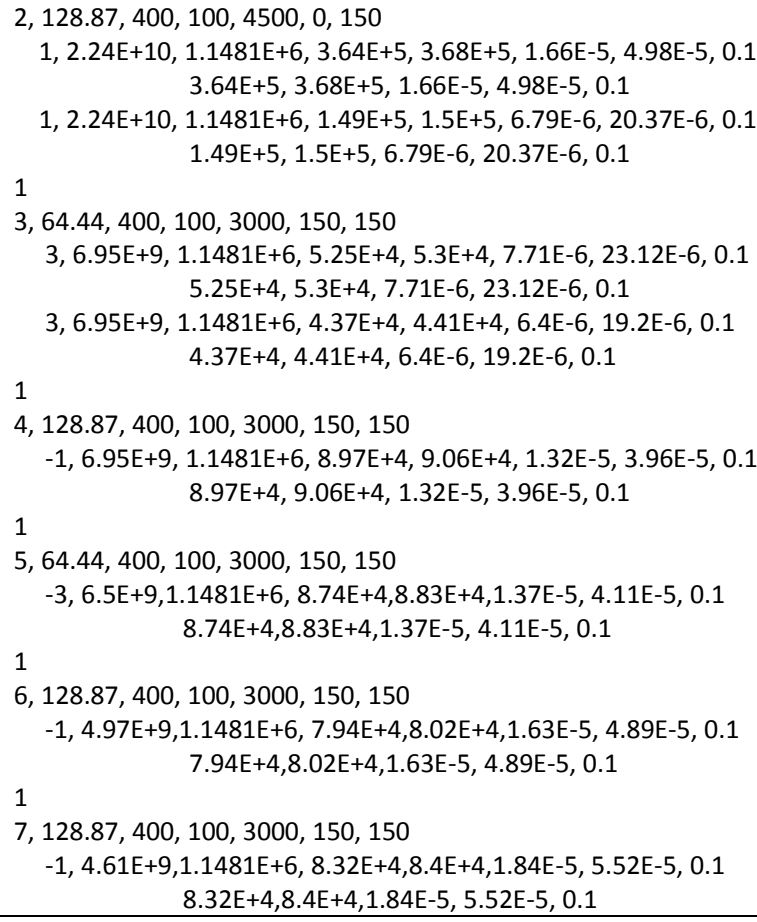 \\
\hline SETE & $\begin{array}{l}\text { USER_TEXT } \\
\text { IUBEM }\end{array}$ & $\begin{array}{l}\text { BEAM MOMENT CURVATURE ENVELOPE GENERATION } \\
1\end{array}$ \\
\hline SET E2 & $\begin{array}{l}\text { USER_TEXT } \\
\text { IBTYPE }\end{array}$ & $\begin{array}{l}\text { BEAM DIMENSIONS } \\
1\end{array}$ \\
\hline SET E2(a) & $\begin{array}{l}\text { KB,AMLB,RAMB1,RAMB2 } \\
\text { KHYSB,EI,PCP,PYP,UYP,UUP,EI3P } \\
\text { PCN,PYN,UYN,UUNEI3N }\end{array}$ & 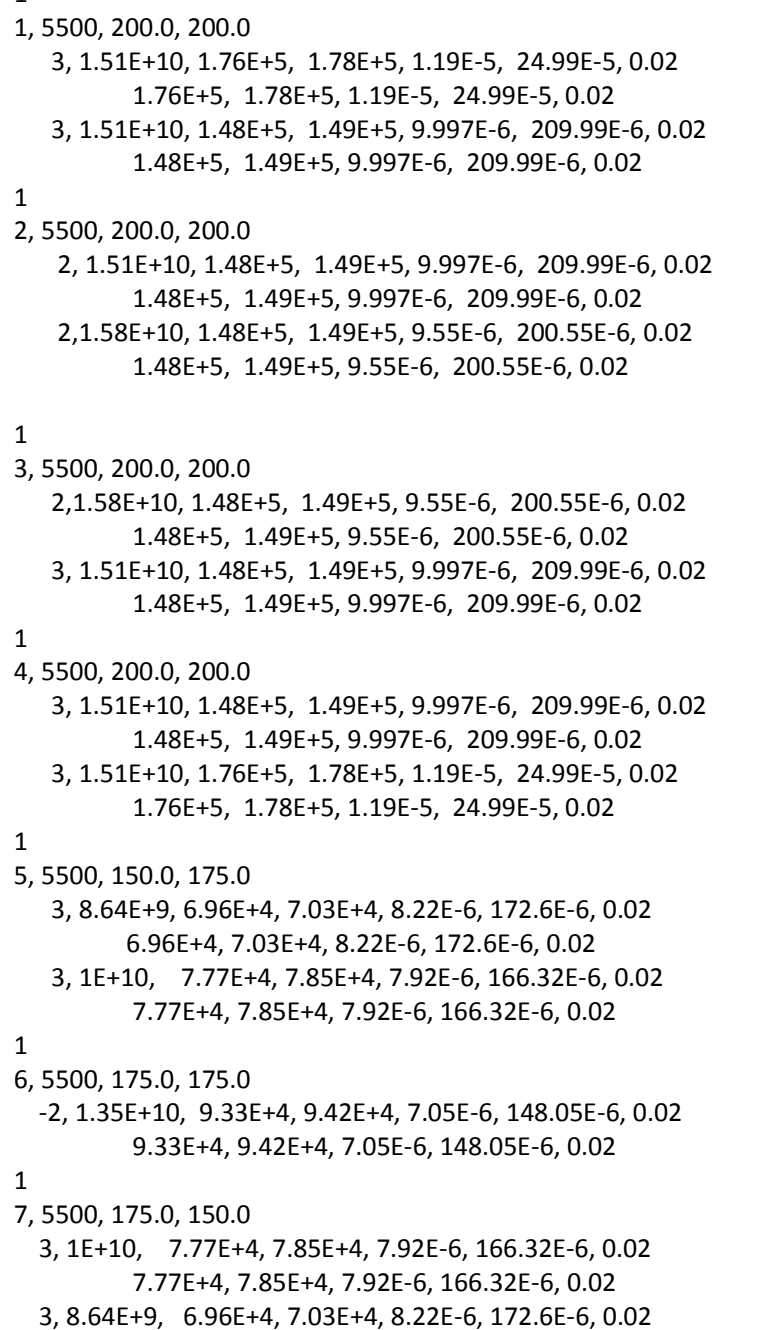 \\
\hline
\end{tabular}




\begin{tabular}{|c|c|c|}
\hline & & 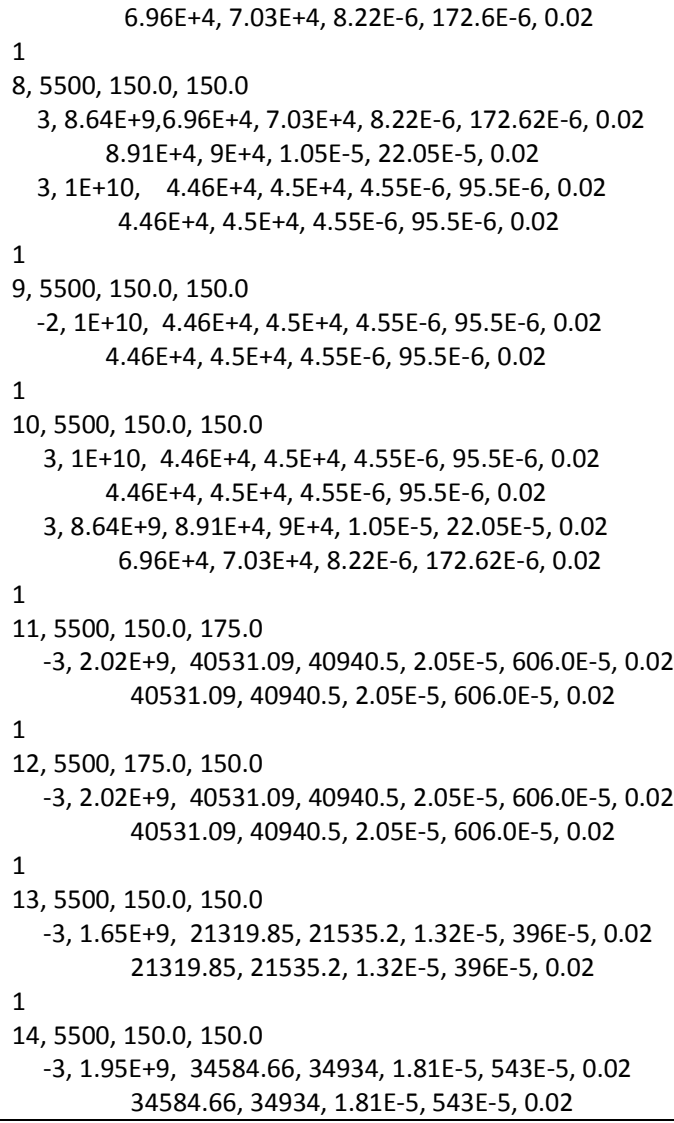 \\
\hline SET L1 & $\begin{array}{l}\text { USER_TEXT } \\
\text { M,ITC,IC,JC,LBC,LTC }\end{array}$ & $\begin{array}{l}\text { COLUMN CONNECTIVTY } \\
1,1,1,1,0,1 \\
2,2,1,2,0,1 \\
3,2,1,3,0,1 \\
4,2,1,4,0,1 \\
5,1,1,5,0,1 \\
6,3,1,1,1,2 \\
7,4,1,2,1,2 \\
8,4,1,3,1,2 \\
9,4,1,4,1,2 \\
10,3,1,5,1,2 \\
11,5,1,1,2,3 \\
12,6,1,2,2,3 \\
13,7,1,3,2,3 \\
14,6,1,4,2,3 \\
15,5,1,5,2,3 \\
\end{array}$ \\
\hline SET L2 & $\begin{array}{l}\text { USER_TEXT } \\
\text { M,ITB,LB,IB,JLB,JRB }\end{array}$ & $\begin{array}{c}\text { BEAM CONNECTIVITY } \\
1,1,1,1,1,2 \\
2,2,1,1,2,3 \\
3,3,1,1,3,4 \\
4,4,1,1,4,5 \\
5,5,2,1,1,2 \\
6,6,2,1,2,3 \\
7,6,2,1,3,4 \\
8,7,2,1,4,5 \\
9,8,3,1,1,2 \\
10,9,3,1,2,3 \\
11,9,3,1,3,4 \\
12,10,3,1,4,5 \\
13,11,2,1,1,2 \\
14,11,2,1,1,2 \\
15,12,2,1,4,5 \\
16,12,2,1,4,5 \\
17,13,3,1,1,2 \\
18,13,3,1,1,2 \\
19,14,3,1,2,3 \\
20,14,3,1,2,3 \\
\end{array}$ \\
\hline
\end{tabular}




\begin{tabular}{|l|l|l|}
\hline \multirow{2}{*}{} & & $21,14,3,1,3,4$ \\
& & $22,14,3,1,3,4$ \\
& & $23,13,3,1,4,5$ \\
& $24,13,3,1,4,5$ \\
\hline SET M & USER_TEXT & ANALYSIS OPTIONS \\
& IOPT & 2 \\
\hline SET M1 & USER_TEXT & LONG TERM \\
& NLU,NU,NLM,NLC & $0,0,0,0$ \\
\hline SET M2 & USER_TEXT & Monotonic Pushover Analysis \\
& JOPT & 1 \\
\hline SET M2.1 & USER_TEXT & FORCE CONTROLLED ANALYSIS \\
& ITYP & 1 \\
& PMAX,MSTEPS,DRFLIM & $0.5,60000,5$ \\
\hline SET N1.1 & USER_TEXT & Snapshot Control Data \\
& NPRNT & 0 \\
\hline SET N1.3 & ICDPRNT(1),ICDPRNT(2), ICDPRNT(3), ICDPRNT(4), ICDPRNT(5) & $0,0,0,0,0$ \\
\hline SET N2 & USER_TEXT & OUTPUT CONTROL \\
& NSOUT,DTOUT,ISO(i) & $3,1,1,2,3$ \\
& FNAMES(i) & story1 \\
& & story2 \\
& & story3 \\
\hline SET N3 & USER_TEXT & ELEMENT HYSTERESIS OUTPUT \\
& KCOUT,KBOUT,KWOUT,KSOUT,KBROUT,KIWOUT & $0,0,0,0,0,0$ \\
\hline
\end{tabular}

Tabla 5.1.3: Archivo de entrada para el pórtico pa6pz2-1 de seis plantas (pa6pz2-1.DAT) IDARC

\begin{tabular}{|c|c|c|}
\hline SET A & $\begin{array}{l}\text { USER_TEXT } \\
\text { NSO,NFR,NCON,NSTL,NMSR,NPDEL,IFLEX,IFLEXDIST,IPC }\end{array}$ & $\begin{array}{l}\text { CONTROL DATA } \\
6,1,1,1,0,1,1,1,1\end{array}$ \\
\hline SET A1 & $\begin{array}{l}\text { USER_TEXT } \\
\text { MCOL,MBEM,MWAL,MEDG,MTRN,MSPR,MBRV,MBRF,MBRH,MIW }\end{array}$ & $\begin{array}{l}\text { ELEMENT TYPES } \\
7,14,0,0,0,0,0,0,0,0\end{array}$ \\
\hline SET A2 & $\begin{array}{l}\text { USER_TEXT } \\
\text { NCOL,NBEM,NWAL,NEDG,NTRN,NSPR,NMR,NBR,NIW }\end{array}$ & $\begin{array}{l}\text { ELEMENT DATA } \\
12,26,0,0,0,0,0,0,0,0\end{array}$ \\
\hline SET A3 & $\begin{array}{l}\text { USER_TEXT } \\
\text { IU }\end{array}$ & $\begin{array}{l}\text { UNITS SYSTEM KN - mm } \\
2\end{array}$ \\
\hline SET A4 & $\begin{array}{l}\text { USER_TEXT } \\
\text { HIGT(i) }\end{array}$ & $\begin{array}{l}\text { FLOOR ELEVATIONS } \\
4500.0,7500.0,10500.0,13500.0,16500.0,19500.0\end{array}$ \\
\hline SET A5 & $\begin{array}{l}\text { USER_TEXT } \\
\text { NDUP(i) }\end{array}$ & $\begin{array}{l}\text { DESCRIPTION OF IDENTICAL FRAMES } \\
1\end{array}$ \\
\hline SET A6 & $\begin{array}{l}\text { USER_TEXT } \\
\text { NVLN(i) }\end{array}$ & $\begin{array}{l}\text { PLAN CONFIGURATION: NO OF COLUMN LINES } \\
5\end{array}$ \\
\hline SET A7 & $\begin{array}{l}\text { USER_TEXT } \\
\text { LEVEL,IFR(J),WVT(K) }\end{array}$ & $\begin{array}{l}\text { NODAL WEIGHTS } \\
1,1,118.75,162.5,162.5,162.5,118.75 \\
2,1,118.75,162.5,162.5,162.5,118.75 \\
3,1,118.75,162.5,162.5,162.5,118.75 \\
4,1,118.75,162.5,162.5,162.5,118.75 \\
5,1,118.75,162.5,162.5,162.5,118.75 \\
6,1,118.75,162.5,162.5,162.5,118.75\end{array}$ \\
\hline SET B & $\begin{array}{l}\text { USER_TEXT } \\
\text { IUSER }\end{array}$ & $\begin{array}{l}\text { CODE FOR SPECIFICATION OF USER PROPERTIES } \\
1\end{array}$ \\
\hline SET C & $\begin{array}{l}\text { USER_TEXT } \\
\text { NHYS } \\
\text { IR,1,HC,HBD,HBE,HS,IBILINEAR }\end{array}$ & $\begin{array}{l}\text { HYSTERETIC MODELING RULES } \\
3 \\
1,1,3.1,0.0,0.4,0.20,0 \\
2,1,3.1,0.0,0.4,0.20,0 \\
3,1,1.8,0.0,0.6,0.20,0\end{array}$ \\
\hline SET D & $\begin{array}{l}\text { USER_TEXT } \\
\text { IUCOL }\end{array}$ & $\begin{array}{l}\text { MOMENT CURVATURE ENVELOPE GENERATION } \\
1\end{array}$ \\
\hline SET D3 & $\begin{array}{l}\text { USER_TEXT } \\
\text { ICTYPE }\end{array}$ & \multirow[b]{2}{*}{ 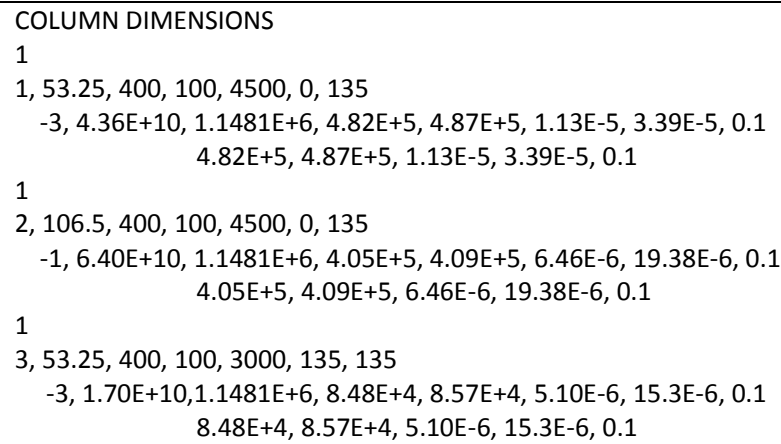 } \\
\hline SET D3(a) & $\begin{array}{l}\text { KC,AN,ANY,ANB,AMLC,RAMC1,RAMC2 } \\
\text { KHYSC,EI,EA,PCP,PYP,UYP,UUP,EI3P } \\
\text { PCN,PYN,UYN,UUN,EI3N }\end{array}$ & \\
\hline
\end{tabular}




\begin{tabular}{|c|c|c|}
\hline & & 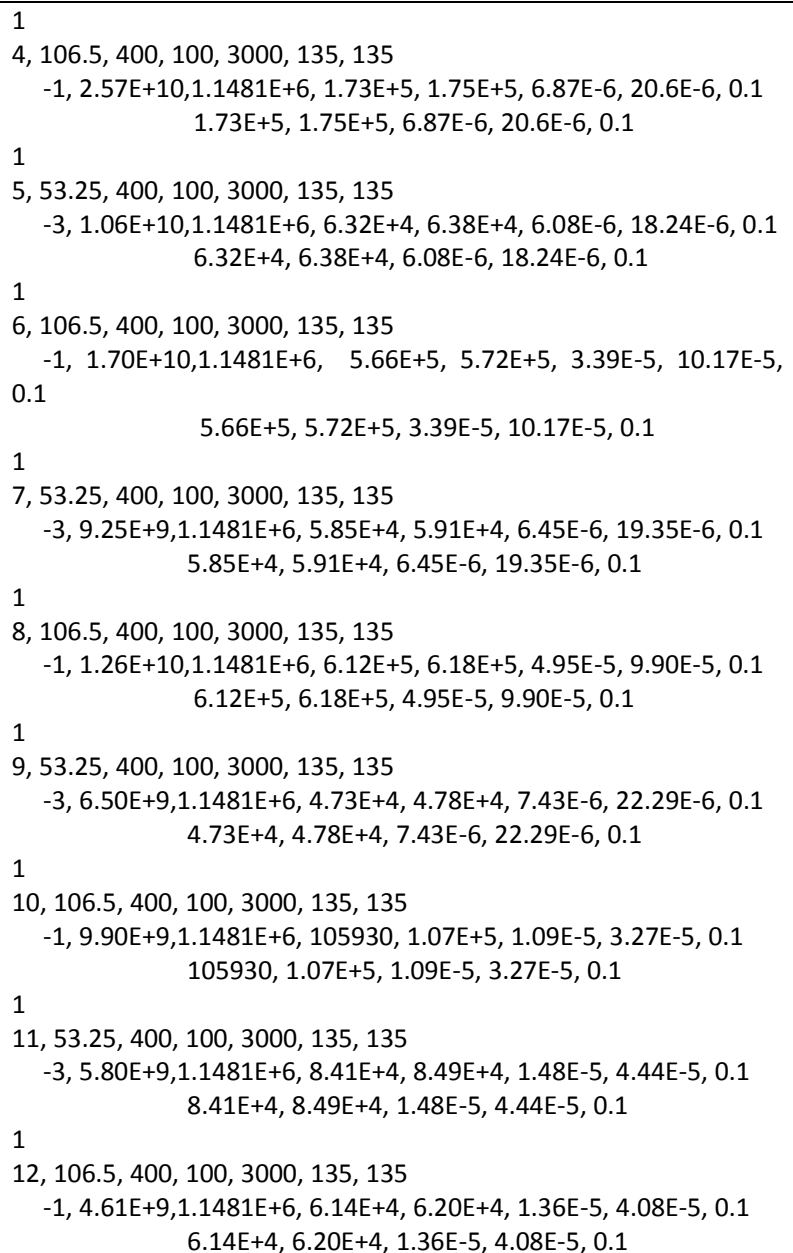 \\
\hline SET E & $\begin{array}{l}\text { USER_TEXT } \\
\text { IUBEM }\end{array}$ & $\begin{array}{l}\text { BEAM MOMENT CURVATURE ENVELOPE GENERATION } \\
1\end{array}$ \\
\hline SET E2 & $\begin{array}{l}\text { USER_TEXT } \\
\text { IBTYPE }\end{array}$ & $\begin{array}{l}\text { BEAM DIMENSIONS } \\
1\end{array}$ \\
\hline SET E2(a) & $\begin{array}{l}\text { KB,AMLB,RAMB1,RAMB2 } \\
\text { KHYSB,EI,PCP,PYP,UYP,UUP,EI3P } \\
\text { PCN,PYN,UYN,UUNEI3N }\end{array}$ & 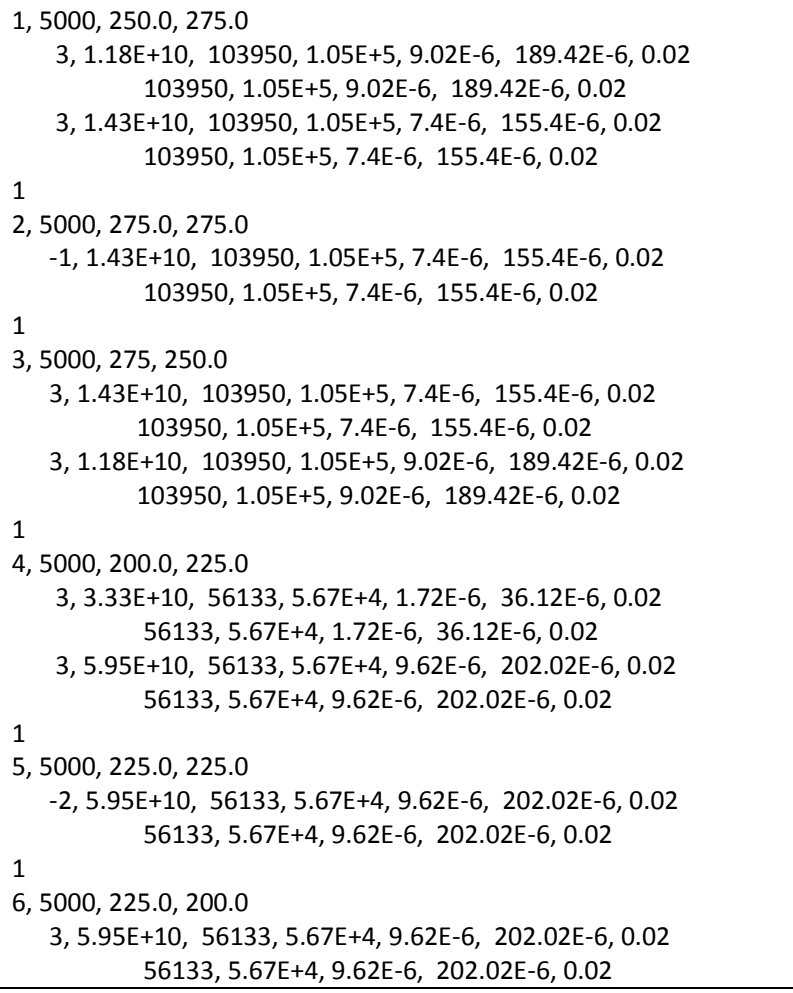 \\
\hline
\end{tabular}




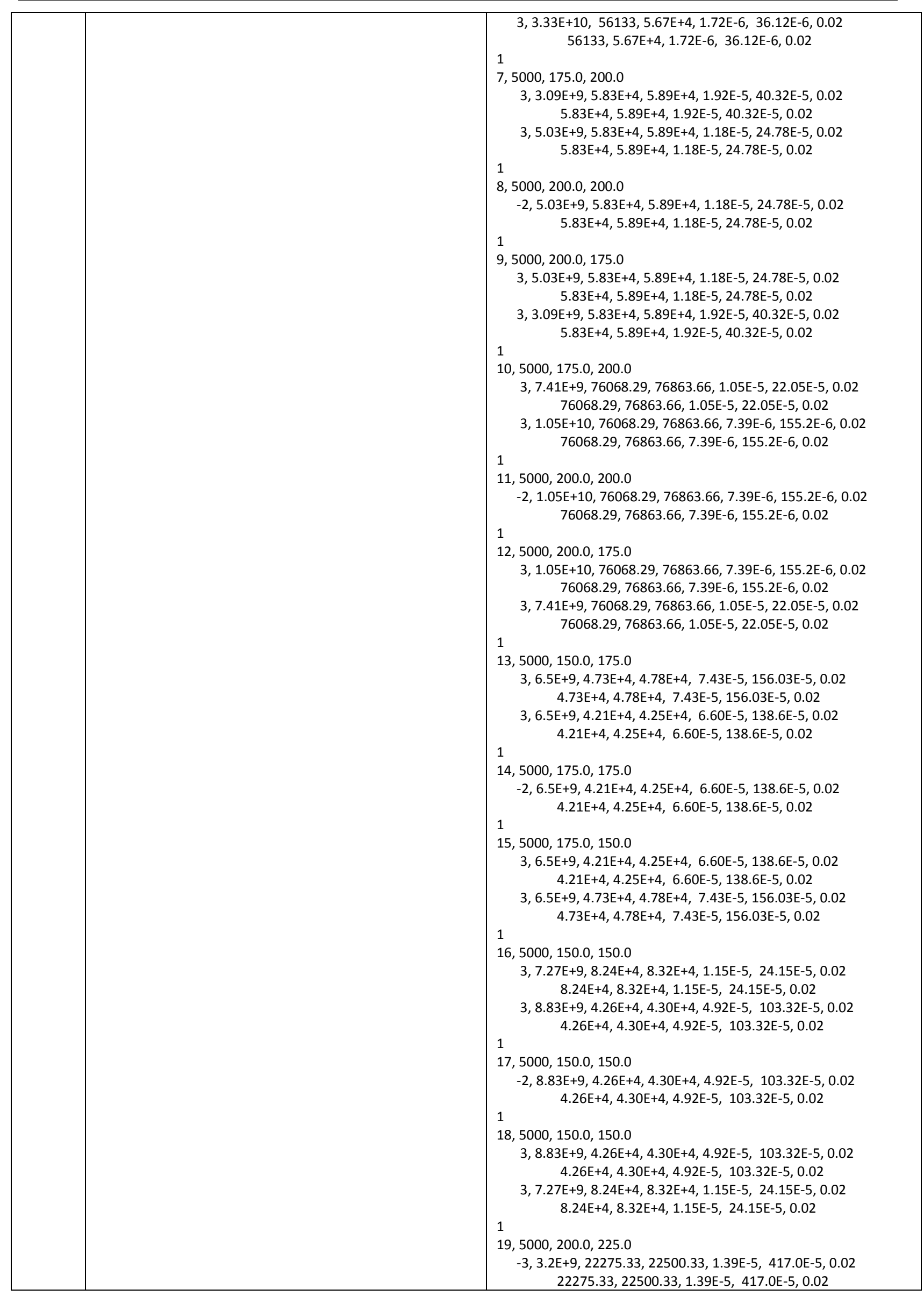




\begin{tabular}{|c|c|c|}
\hline & & 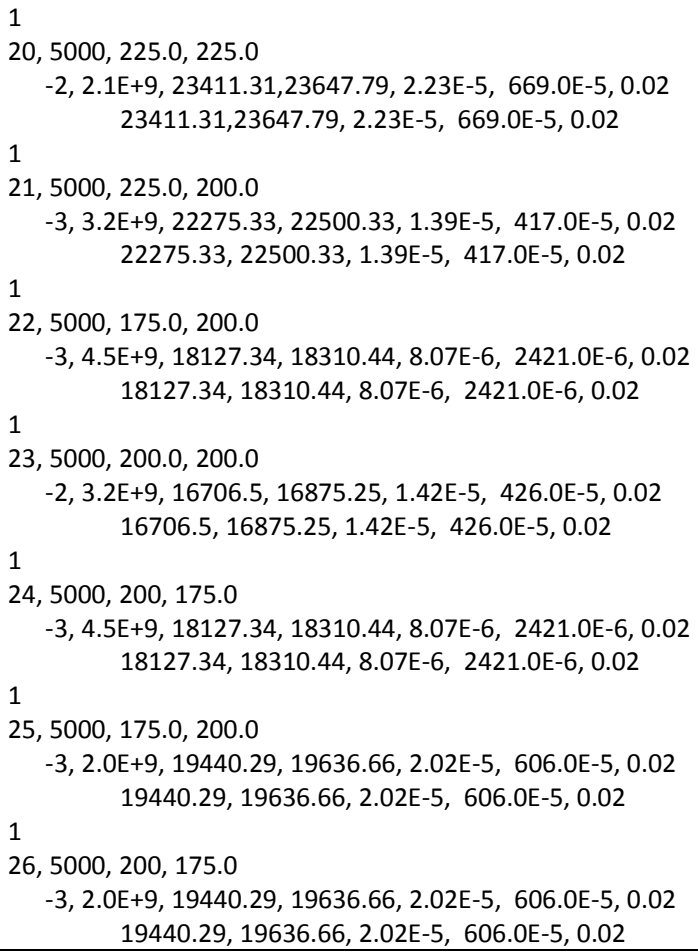 \\
\hline SET L1 & $\begin{array}{l}\text { USER_TEXT } \\
\text { M,ITC,IC,JC,LBC,LTC }\end{array}$ & $\begin{array}{l}\text { COLUMN CONNECTIVTY } \\
1,1,1,1,0,1 \\
2,2,1,2,0,1 \\
3,2,1,3,0,1 \\
4,2,1,4,0,1 \\
5,1,1,5,0,1 \\
6,3,1,1,1,2 \\
7,4,1,2,1,2 \\
8,4,1,3,1,2 \\
9,4,1,4,1,2 \\
10,3,1,5,1,2 \\
11,5,1,1,2,3 \\
12,6,1,2,2,3 \\
13,6,1,3,2,3 \\
14,6,1,4,2,3 \\
15,5,1,5,2,3 \\
16,7,1,1,3,4 \\
17,8,1,2,3,4 \\
18,8,1,3,3,4 \\
19,8,1,4,3,4 \\
20,7,1,5,3,4 \\
21,9,1,1,4,5 \\
22,10,1,2,4,5 \\
23,10,1,3,4,5 \\
24,10,1,4,4,5 \\
25,9,1,5,4,5 \\
26,11,1,1,5,6 \\
27,12,1,2,5,6 \\
28,12,1,3,5,6 \\
29,12,1,4,5,6 \\
30,11,1,5,5,6\end{array}$ \\
\hline SET L2 & $\begin{array}{l}\text { USER_TEXT } \\
\text { M,ITB,LB,IB,JLB,JRB }\end{array}$ & $\begin{array}{c}\text { BEAM CONNECTIVITY } \\
1,1,1,1,1,2 \\
2,2,1,1,2,3 \\
3,2,1,1,3,4 \\
4,3,1,1,4,5 \\
5,4,2,1,1,2 \\
6,5,2,1,2,3 \\
7,5,2,1,3,4 \\
8,6,2,1,4,5 \\
9,7,3,1,1,2 \\
10,8,3,1,2,3 \\
\end{array}$ \\
\hline
\end{tabular}




\begin{tabular}{|c|c|c|}
\hline & & $\begin{array}{l}11,8,3,1,3,4 \\
12,9,3,1,4,5 \\
13,10,4,1,1,2 \\
14,11,4,1,2,3 \\
15,11,4,1,3,4 \\
16,12,4,1,4,5 \\
17,13,5,1,1,2 \\
18,14,5,1,2,3 \\
19,14,5,1,3,4 \\
20,15,5,1,4,5 \\
21,16,6,1,1,2 \\
22,17,6,1,2,3 \\
23,17,6,1,3,4 \\
24,18,6,1,4,5 \\
25,19,2,1,1,2 \\
26,19,2,1,1,2 \\
27,20,2,1,2,3 \\
28,20,2,1,2,3 \\
29,20,2,1,3,4 \\
30,20,2,1,3,4 \\
31,21,2,1,4,5 \\
32,21,2,1,4,5 \\
33,22,3,1,1,2 \\
34,22,3,1,1,2 \\
35,23,3,1,2,3 \\
36,23,3,1,2,3 \\
37,23,3,1,3,4 \\
38,23,3,1,3,4 \\
39,24,3,1,4,5 \\
40,24,3,1,4,5 \\
41,25,4,1,1,2 \\
42,25,4,1,1,2 \\
43,26,4,1,4,5 \\
44,26,4,1,4,5 \\
\end{array}$ \\
\hline SET M & $\begin{array}{l}\text { USER_TEXT } \\
\text { IOPT }\end{array}$ & $\begin{array}{l}\text { ANALYSIS OPTIONS } \\
2\end{array}$ \\
\hline SET M1 & $\begin{array}{l}\text { USER_TEXT } \\
\text { NLU,NU,NLM,NLC }\end{array}$ & $\begin{array}{l}\text { LONG TERM } \\
0,0,0,0\end{array}$ \\
\hline SET M2 & $\begin{array}{l}\text { USER_TEXT } \\
\text { JOPT }\end{array}$ & $\begin{array}{l}\text { Monotonic Pushover Analysis } \\
1\end{array}$ \\
\hline SET M2.1 & $\begin{array}{l}\text { USER_TEXT } \\
\text { ITYP } \\
\text { PMAX,MSTEPS,DRFLIM }\end{array}$ & $\begin{array}{l}\text { FORCE CONTROLLED ANALYSIS } \\
1 \\
0.5,60000,5\end{array}$ \\
\hline SET N1.1 & $\begin{array}{l}\text { USER_TEXT } \\
\text { NPRNT }\end{array}$ & $\begin{array}{l}\text { Snapshot Control Data } \\
0\end{array}$ \\
\hline SET N1.3 & ICDPRNT(1), ICDPRNT(2), ICDPRNT(3), ICDPRNT(4), ICDPRNT(5) & $0,0,0,0,0$ \\
\hline SET N2 & $\begin{array}{l}\text { USER_TEXT } \\
\text { NSOUT,DTOUT,ISO(i) } \\
\text { FNAMES(i) }\end{array}$ & $\begin{array}{l}\text { OUTPUT CONTROL } \\
6,1,1,2,3,4,5,6 \\
\text { story1 } \\
\text { story2 } \\
\text { story3 } \\
\text { story4 } \\
\text { story5 } \\
\text { story6 } \\
\end{array}$ \\
\hline SET N3 & $\begin{array}{l}\text { USER_TEXT } \\
\text { KCOUT,KBOUT,KWOUT,KSOUT,KBROUT,KIWOUT }\end{array}$ & $\begin{array}{l}\text { ELEMENT HYSTERESIS OUTPUT } \\
0,0,0,0,0,0\end{array}$ \\
\hline
\end{tabular}




\subsection{ANÁLISIS PUSHOVER}

\subsubsection{Introducción teórica}

El análisis comúnmente llamado "pushover" en la literatura anglosajona consiste en efectuar un análisis estático no lineal en el que se aplica una distribución de cargas (fuerzas o desplazamientos impuestos) de forma monótonamente creciente e incremental. Es un tipo de análisis que intenta cuantificar los daños producidos en la estructura por determinados fenómenos (como pueden ser los sismos) al mismo tiempo que identifica dónde se producirán los mecanismos de fallo a medida que transcurre el fenómeno en estudio.

Es un método incremental porque al ser no lineal, el método numérico utilizado, que podría ser el de Newmark o aceleración lineal, calcula los efectos a partir de un incremento de los anteriores.

Al final se comprueba la formación de articulaciones o rótulas y el lugar donde ocurren.

En la Fig. 5.2.1 se representan los desplazamientos de la última planta de un edificio en función del cortante en la base: la línea OA muestra el comportamiento elástico de la estructura, en la que los desplazamientos se suponen proporcionales a la magnitud de la carga horizontal aplicada. El punto $D$ representa la aparición de la primera articulación plástica. A partir de este punto, el comportamiento deja de ser lineal, y en la medida en que se presentan más articulaciones, este comportamiento tiende a ser el que muestra la curva ODB, que suele simplificarse con la curva idealizada OEC.

A
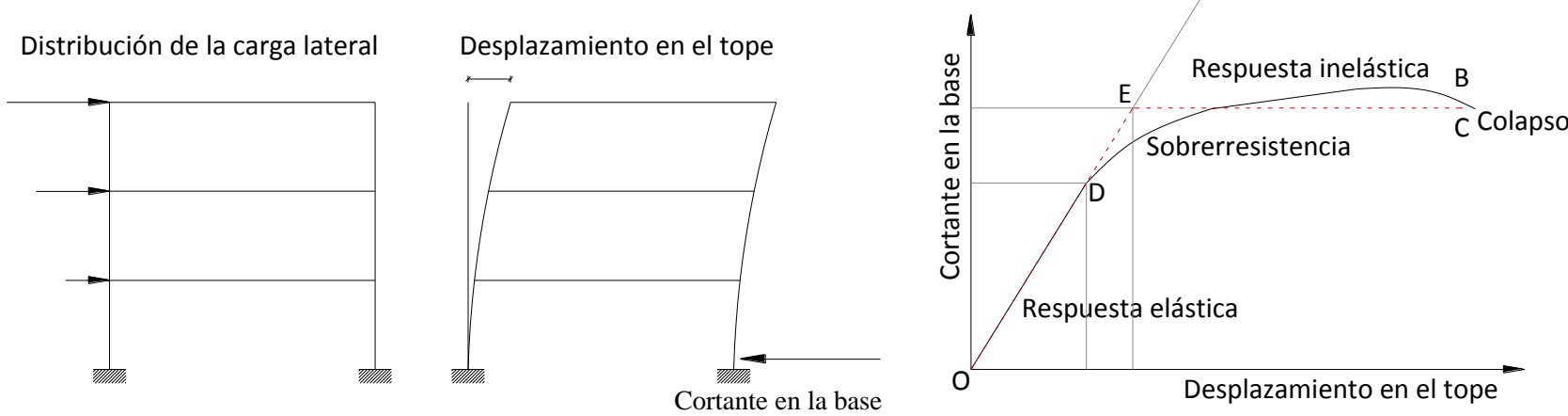

Fig. 5.2.1: Desplazamiento de una estructura solicitada por fuerza horizontal

En la Fig. 5.2.2 [12] se muestra el incremento de los daños con la intensidad. El Grado 1 corresponde a daños de despreciables a ligeros (ningún daño estructural, daños no estructurales ligeros). El Grado 2, daños moderados (daños estructurales ligeros, daños no estructurales moderados). El Grado 3, daños de importantes a graves (daños estructurales moderados, daños no estructurales graves). El Grado 4, daños muy graves (daños estructurales graves, daños no estructurales muy graves). Y el Grado 5, destrucción (daños estructurales muy graves). 


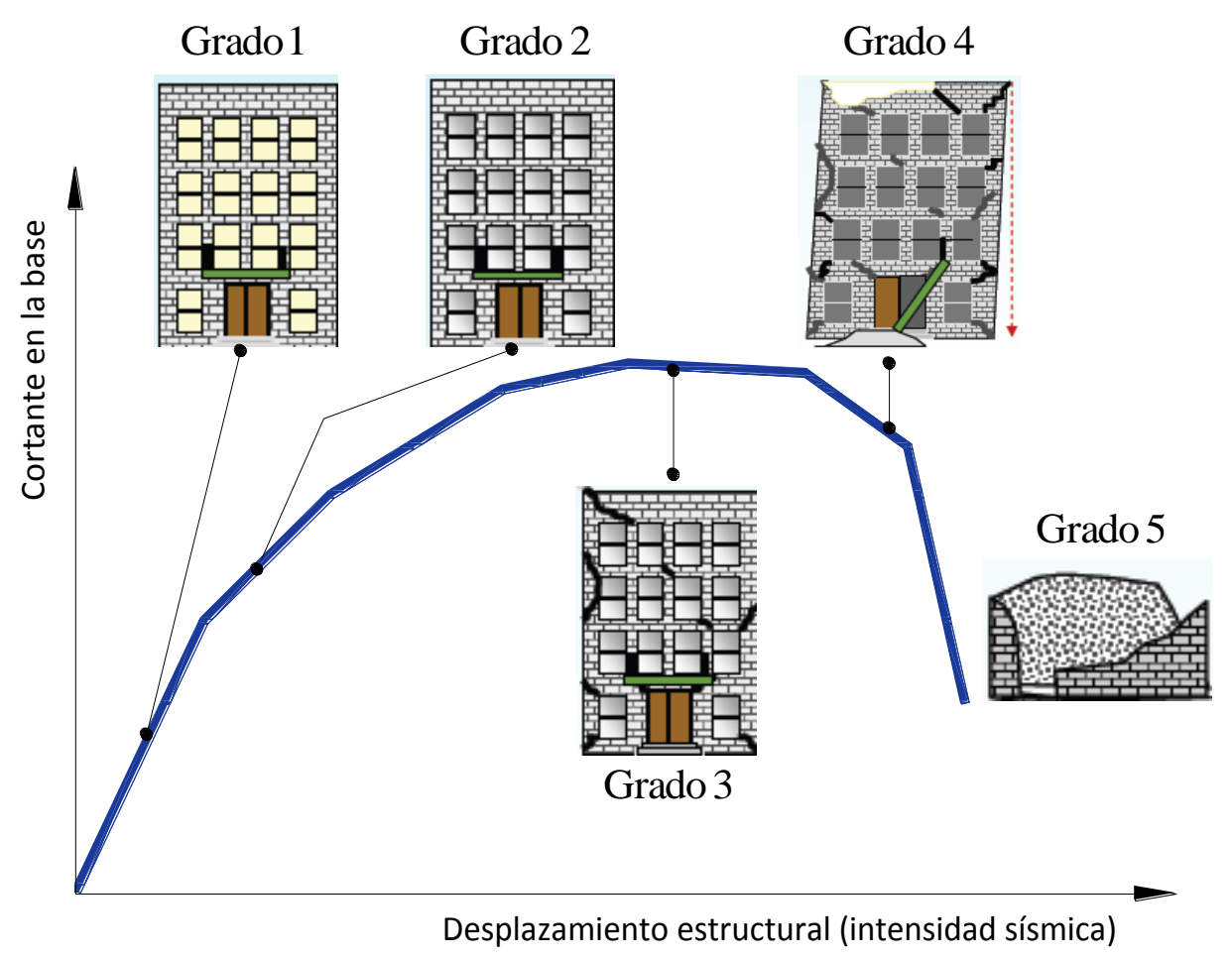

Grado 1

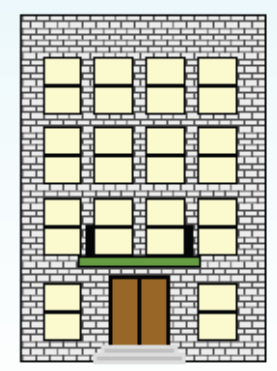

Funcional
Grado 2

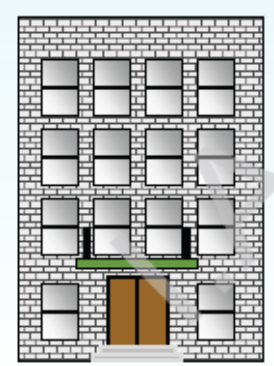

Ocupación

inmediata
Grado 3

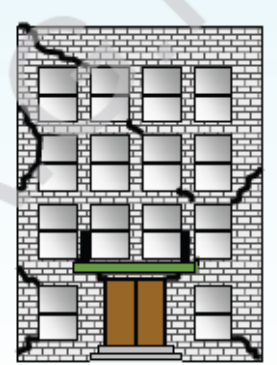

Sin peligro

para la vida
Grado 4

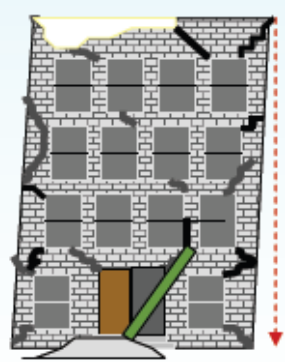

Prevención

colapso

Grado 5

Fig. 5.2.2: Incremento de los daños con la intensidad

Todos los pórticos definidos y modelizados en los apartados anteriores se sometieron a este tipo de análisis que permite determinar características globales de la respuesta de los pórticos en términos de fuerza y deformación lateral. En este apartado se ha obtenido la curva de capacidad de cada pórtico, la relación entre fuerza cortante de la planta y el desplazamiento entre forjados que delimitan dicha planta, para cada planta de cada pórtico y la rigidez inicial así como el desplazamiento de fluencia de cada planta. 


\subsubsection{Curva de capacidad}

Se obtienen a continuación las curvas de capacidad, es decir, la relación entre el cortante basal y el desplazamiento de la última planta para cada uno de pórticos que estamos analizando.

Mediante el programa IDARC se obtienen los siguientes parámetros: deformación promedio (overvall deformation) en \% y coeficiente cortante basal (base shear coefficient) adimensional. Para poder obtener las curvas de capacidad, expresadas en las Fig. 5.2.3, 5.2.4 y 5.2.5, como la relación entre el cortante basal en $\mathrm{KN}$ y el desplazamiento en $\mathrm{mm}$, consideraremos la altura total de cada uno de los pórticos así como su peso respectivamente. Estos valores se expresan en la tabla 5.2.1.

Tabla 5.2.1: Altura total y peso de cada uno

\begin{tabular}{c|c|c}
\multicolumn{3}{c}{ de los pórticos } \\
\hline Pórtico & $\begin{array}{c}\text { Altura } \\
(\mathrm{mm})\end{array}$ & $\begin{array}{c}\text { Peso } \\
(\mathrm{KN})\end{array}$ \\
\hline pa2pz2-1 & 10500 & 2175.000 \\
\hline pa4pz2-1 & 10500 & 2606.985 \\
\hline pa6pz2-1 & 19500 & 4350.000 \\
\hline
\end{tabular}

En esta curva vemos el comportamiento de la estructura. En primer lugar el comportamiento es elástico lineal, seguidamente empiezan a aparecer rótulas, hasta que se convierte en una curva no lineal. Cuando la estructura plastifica, necesita menos cortante lo que implica que se produzca un mayor desplazamiento. Se obtendrá el punto donde se produce el colapso de la estructura, a partir del cual la estructura no resiste un incremento de la fuerza. 
- Pórtico pa2pz2-1

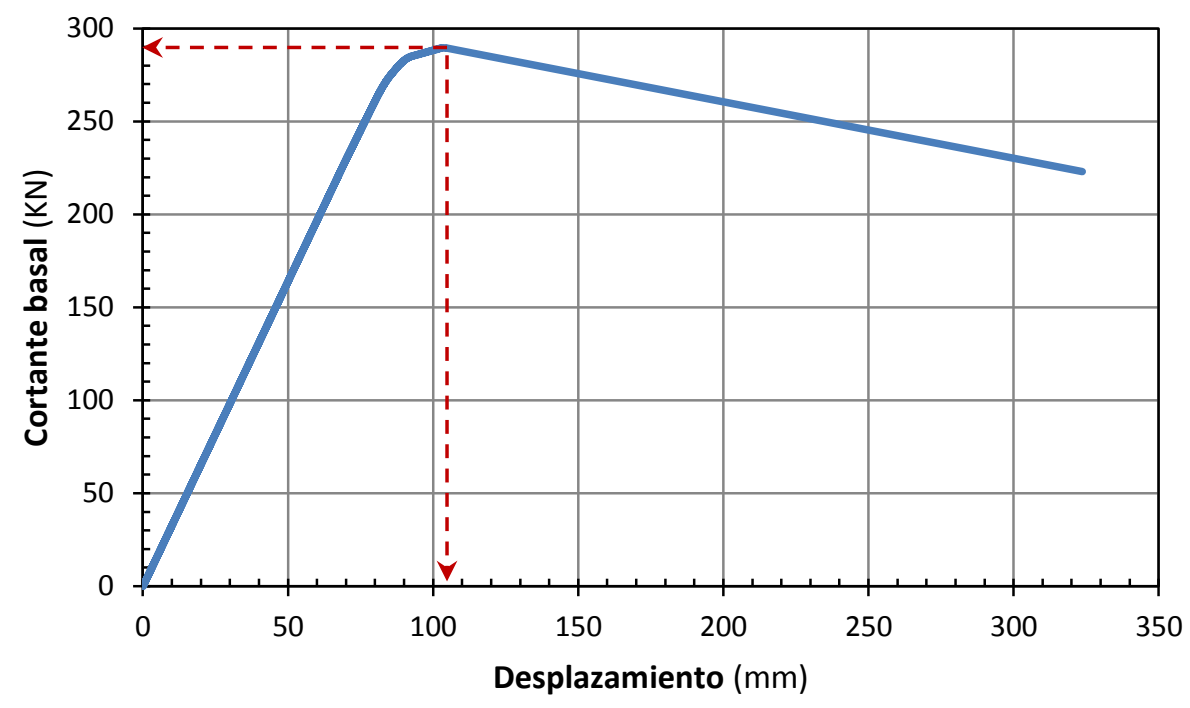

Fig. 5.2.3: Curva de capacidad del pórtico pa2pz2-1

Tabla 5.2.2: Cortante basal y desplazamiento para los que se produce el colapso en el pórtico pa2pz2-1

\begin{tabular}{l|c}
\hline Cortante basal $(\mathrm{KN})$ & 28 \\
\hline Desplazamiento $(\mathrm{mm})$ & 103 \\
\hline
\end{tabular}

- Pórtico pa4pz2-1

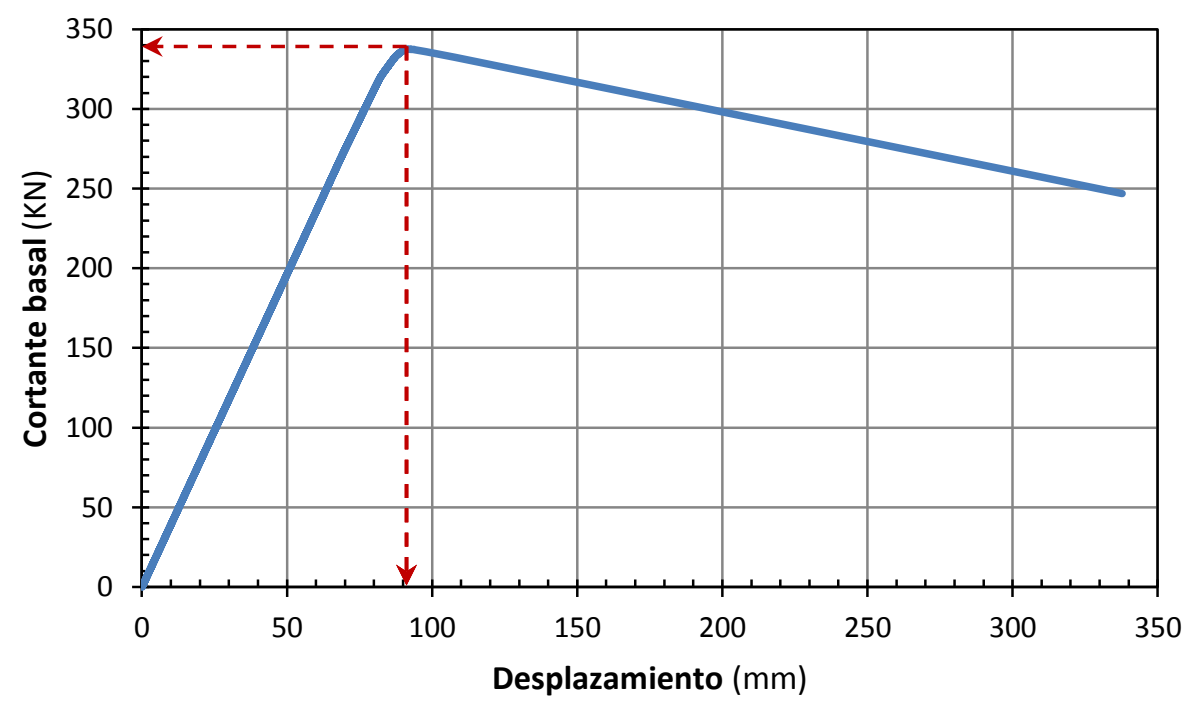

Fig. 5.2.4: Curva de capacidad del pórtico pa4pz2-1

Tabla 5.2.3: Cortante basal y desplazamiento para los que se produce el colapso en el pórtico pa4pz2-1

\begin{tabular}{l|c}
\hline Cortante basal $(\mathrm{KN})$ & 91 \\
\hline Desplazamiento $(\mathrm{mm})$ & 338 \\
\hline
\end{tabular}


- Pórtico pa6pz2-1

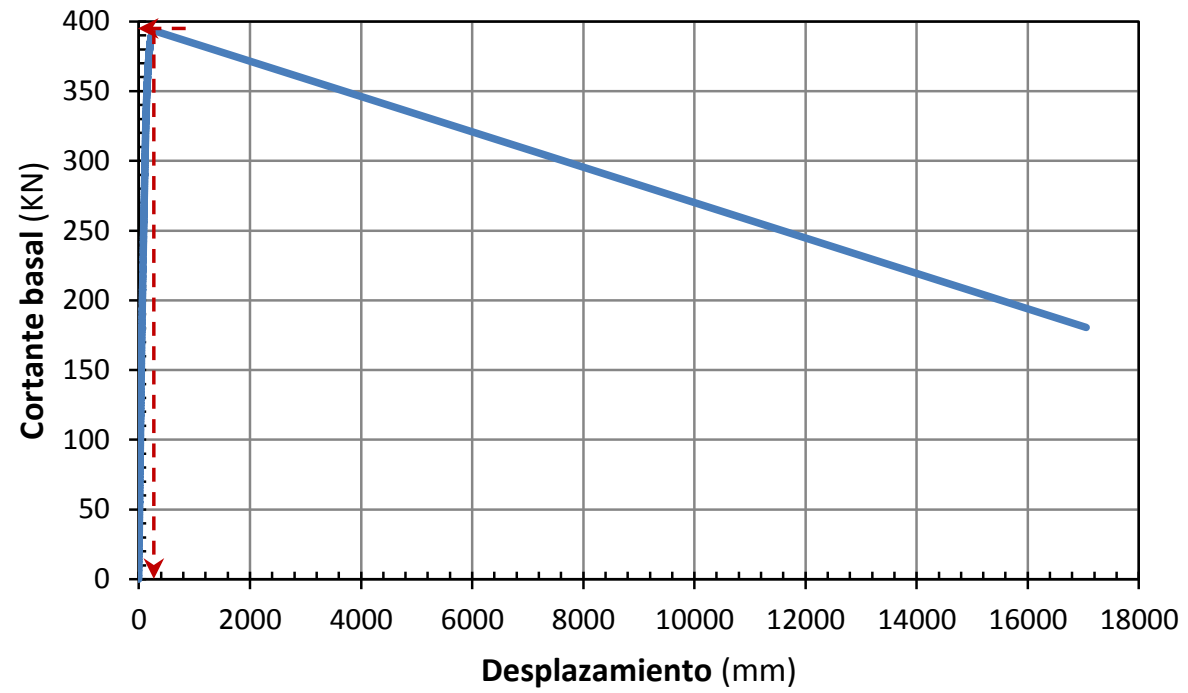

Fig. 5.2.5: Curva de capacidad del pórtico pa6pz2-1

Tabla 5.2.4: Cortante basal y desplazamiento para los que se produce el colapso en el pórtico pa6pz2-1

\begin{tabular}{l|l}
\hline Cortante basal (KN) & 392 \\
\hline Desplazamiento $(\mathrm{mm})$ & 347 \\
\hline
\end{tabular}

Tabla 5.2.5: Cortante basal y desplazamiento para los que se produce el colapso en cada uno de los pórticos

\begin{tabular}{c|c|c|c} 
Pórtico & pa2pz2-1 & pa4pz2-1 & pa6pz2-1 \\
\hline $\begin{array}{c}\text { Cortante basal } \\
(\mathrm{KN})\end{array}$ & 289 & 338 & 392 \\
\hline $\begin{array}{c}\text { Desplazamiento } \\
(\mathrm{mm})\end{array}$ & 103 & 91 & 347 \\
\hline & & &
\end{tabular}

\subsubsection{Relación entre fuerza cortante de la planta y desplazamiento entre forjados que delimitan dicha planta}

El programa IDARC, genera los ficheros de salida para cada una de las plantas de cada pórtico, en los que se encuentran los parámetros de Desplazamiento en mm (story drift) y Cortante en KN (story shear) que representaremos en las figuras 5.2.6 a 5.2.17 siguientes: 
- Pórtico pa2pz2-1

- Planta 1모 (story1)

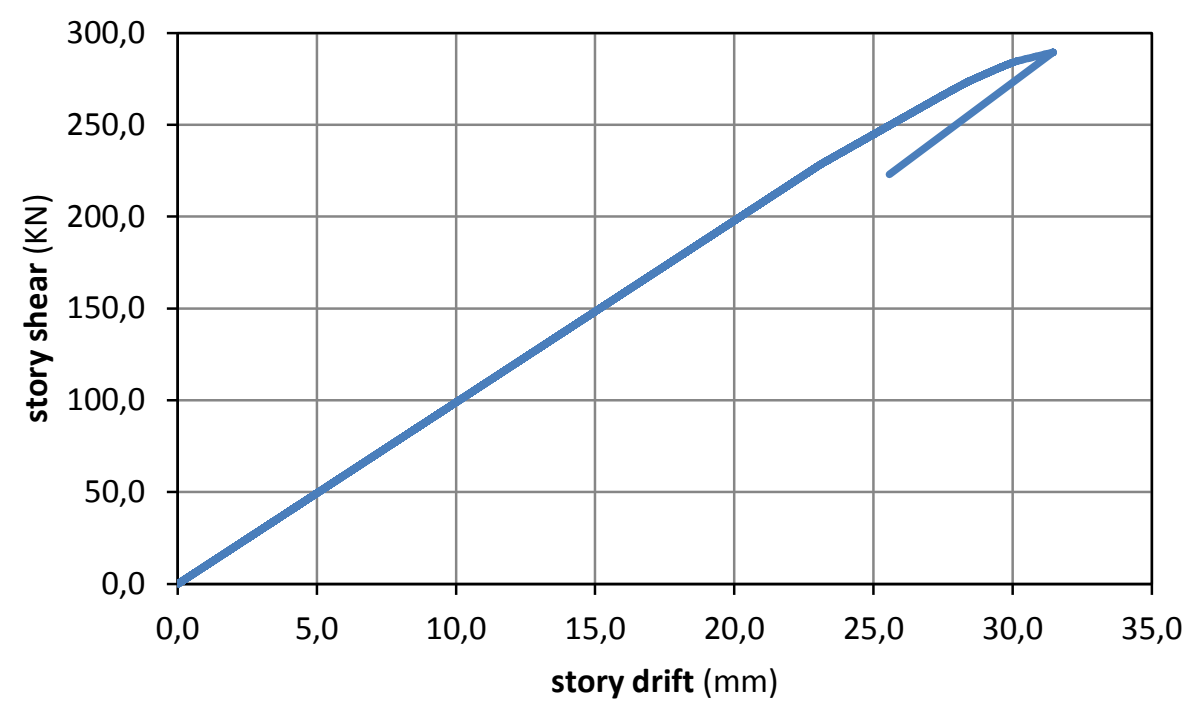

Fig. 5.2.6: Fuerza cortante-Desplazamiento entre forjados, pórtico pa2pz2-1, planta 1a

- Planta 2모 (story2)

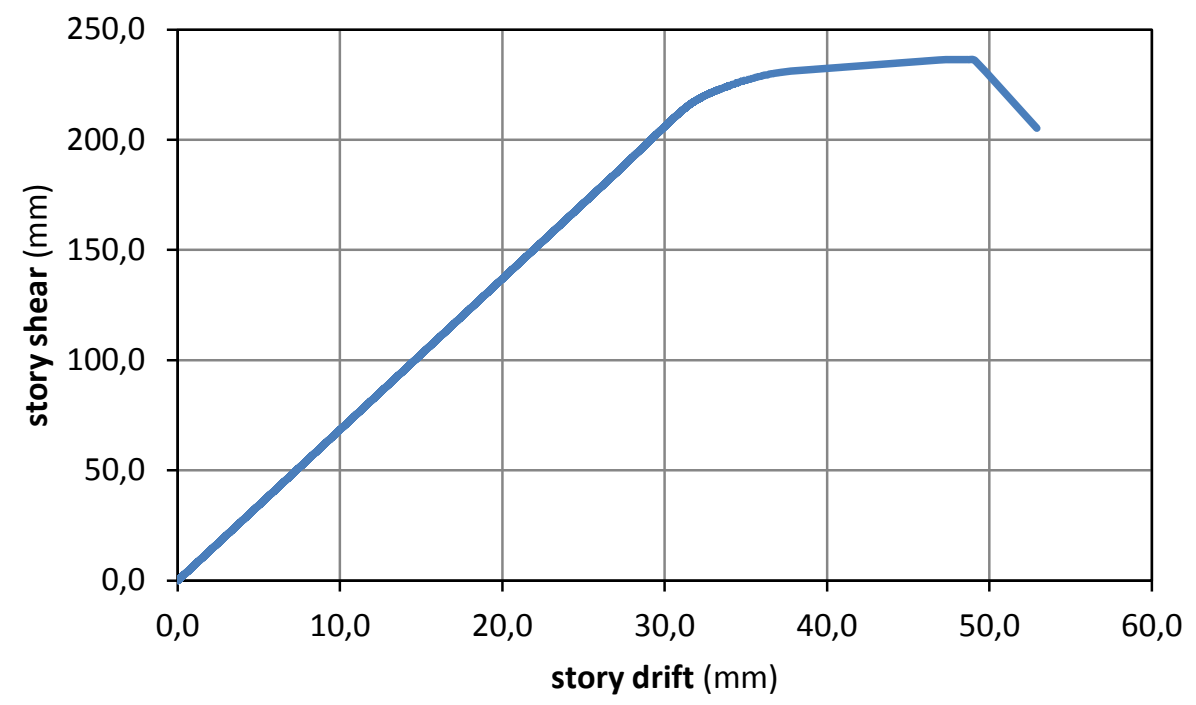

Fig. 5.2.7: Fuerza cortante-Desplazamiento entre forjados, pórtico pa2pz2-1, planta 2a 
- Planta 3모 (story3)

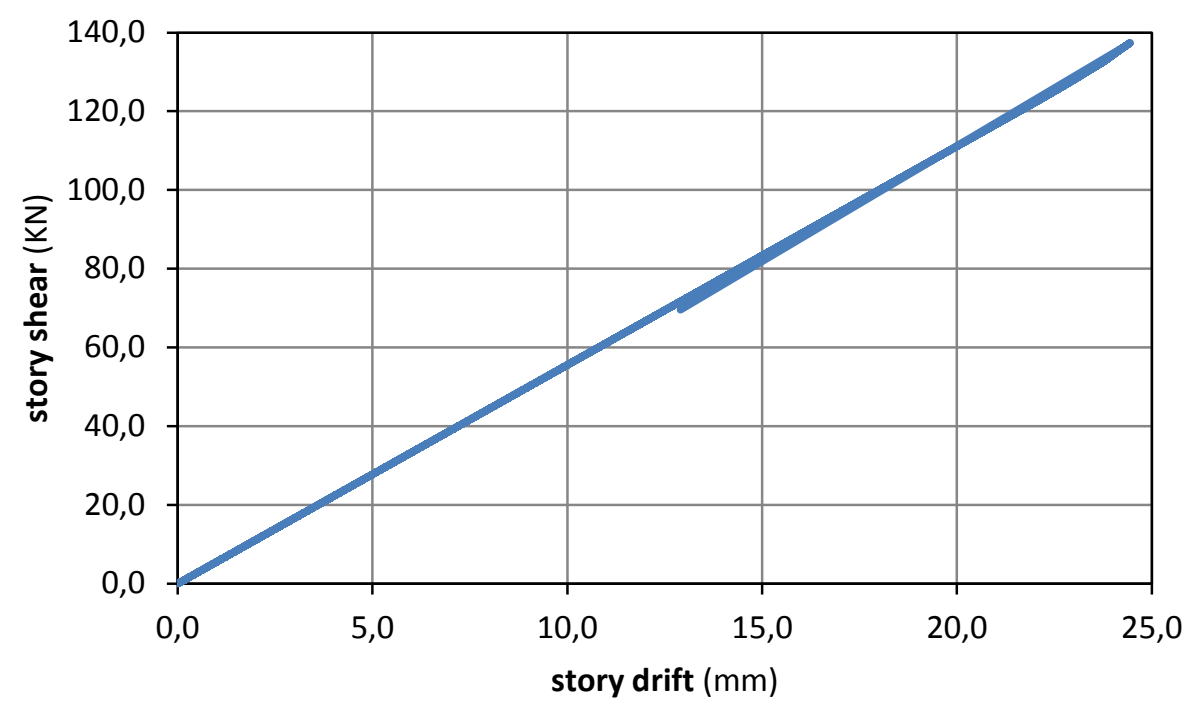

Fig. 5.2.8: Fuerza cortante-Desplazamiento entre forjados, pórtico pa2pz2-1, planta 3a

- Pórtico pa4pz2-1

- Planta 1모 (story1)

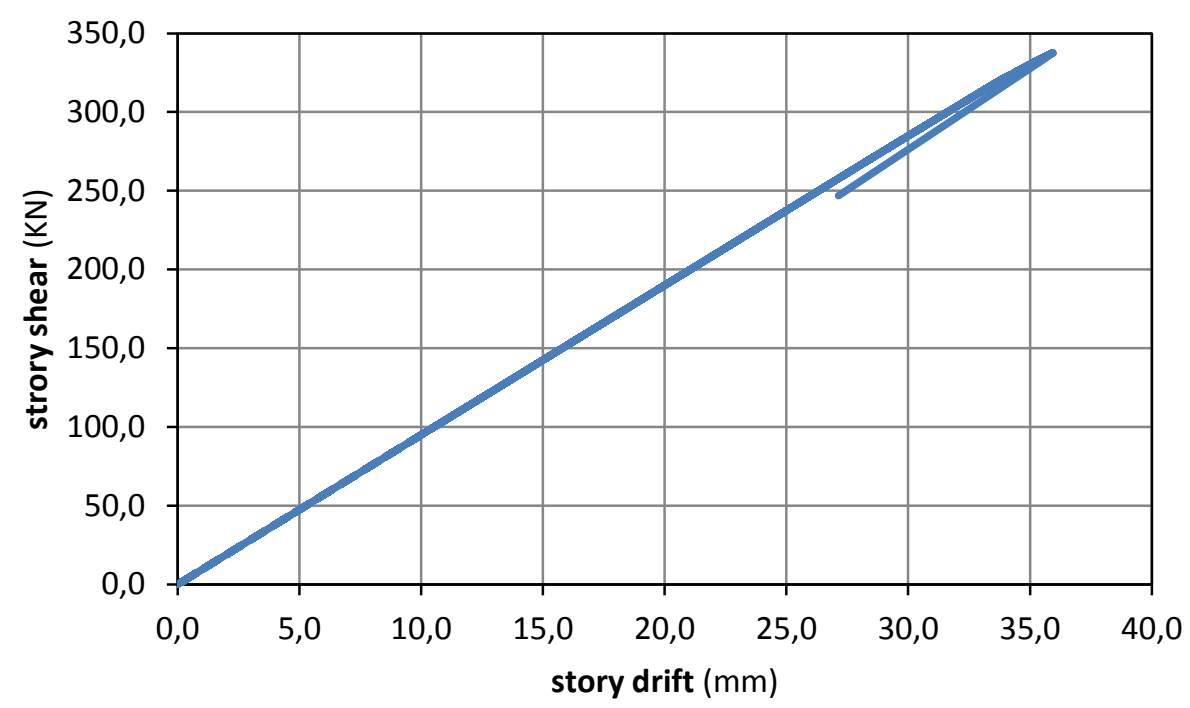

Fig. 5.2.9: Fuerza cortante-Desplazamiento entre forjados, pórtico pa4pz2-1, planta 1ạ 
- Planta 2 $\stackrel{a}{(\text { story2) }}$

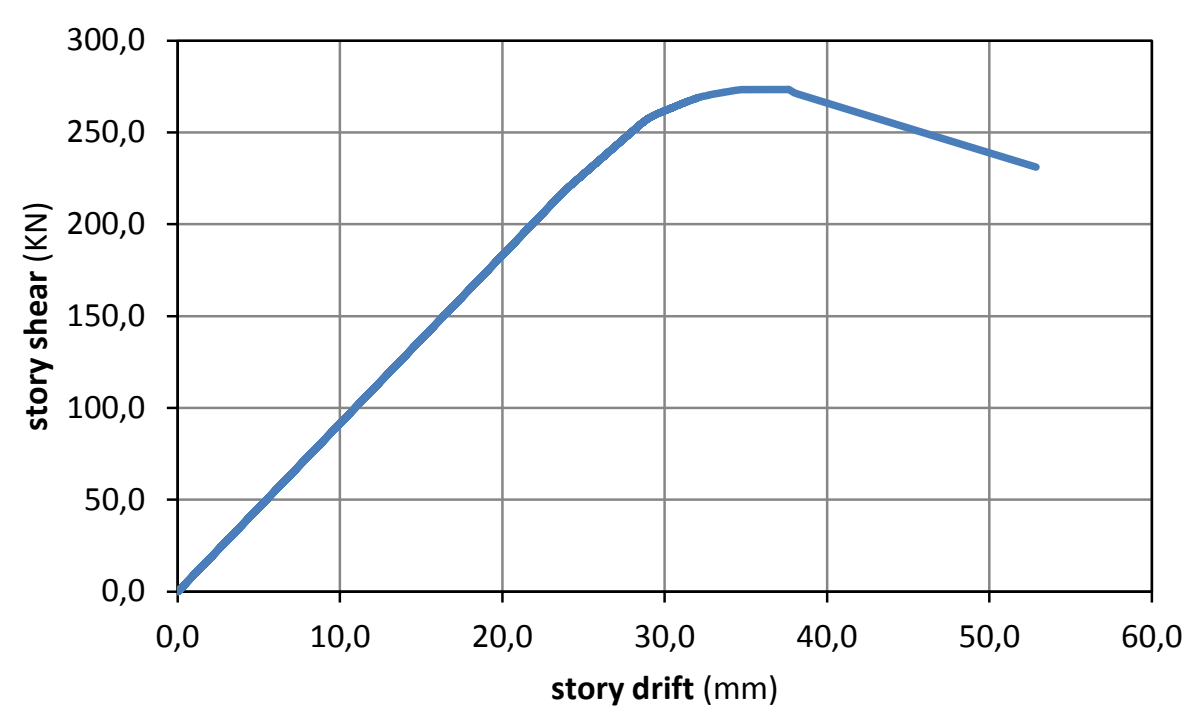

Fig. 5.2.10: Fuerza cortante-Desplazamiento entre forjados, pórtico pa4pz2-1, planta $2^{a}$

- Planta 3모 (story3)

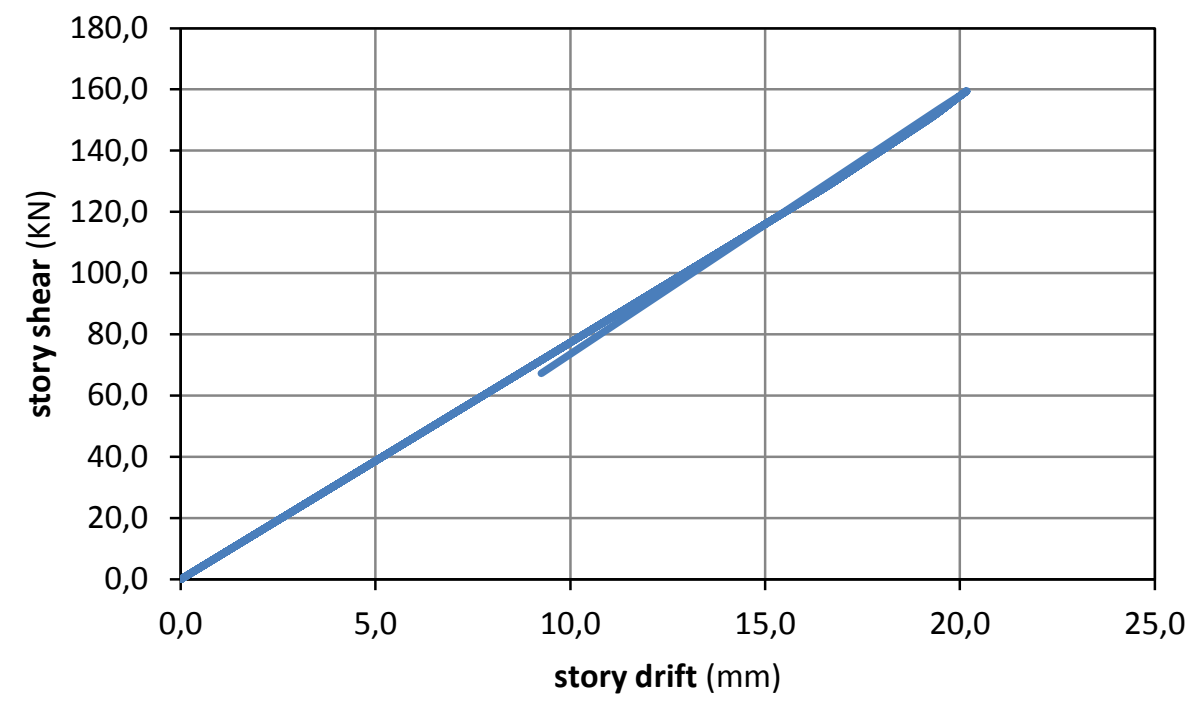

Fig. 5.2.11: Fuerza cortante-Desplazamiento entre forjados, pórtico pa4pz2-1, planta 3ạ 
- Pórtico pa6pz2-1

- Planta 1모 (story1)

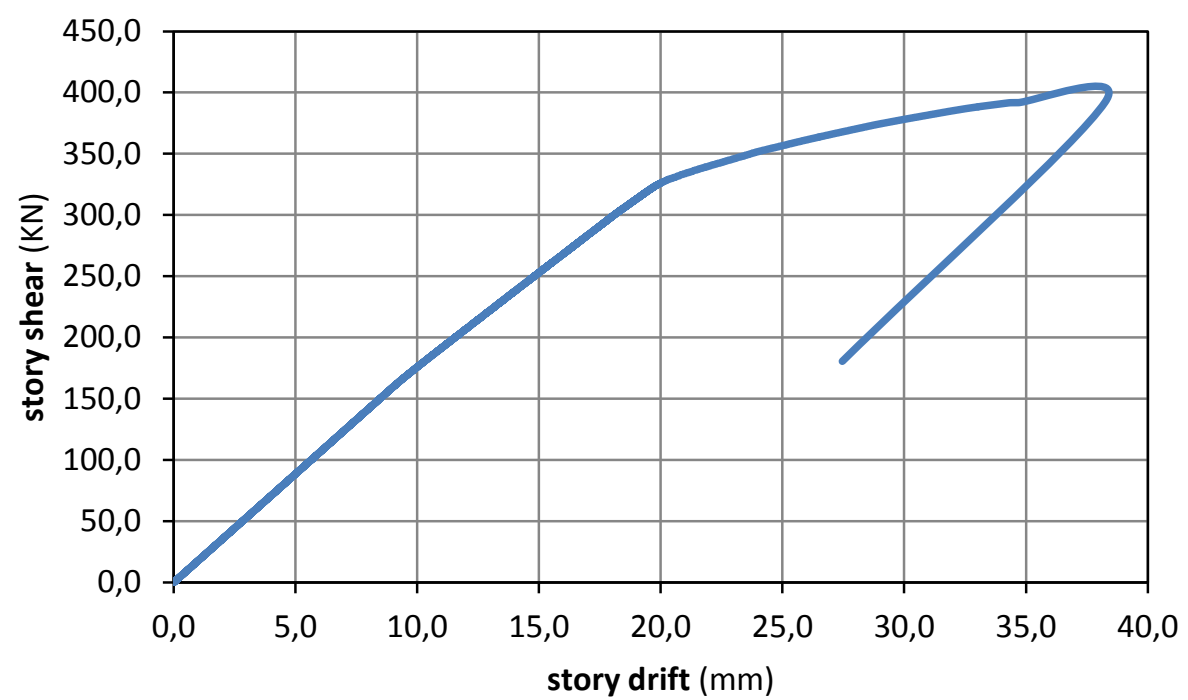

Fig. 5.2.12: Fuerza cortante-Desplazamiento entre forjados, pórtico pa6pz2-1, planta 1a

- Planta 2모 (story2)

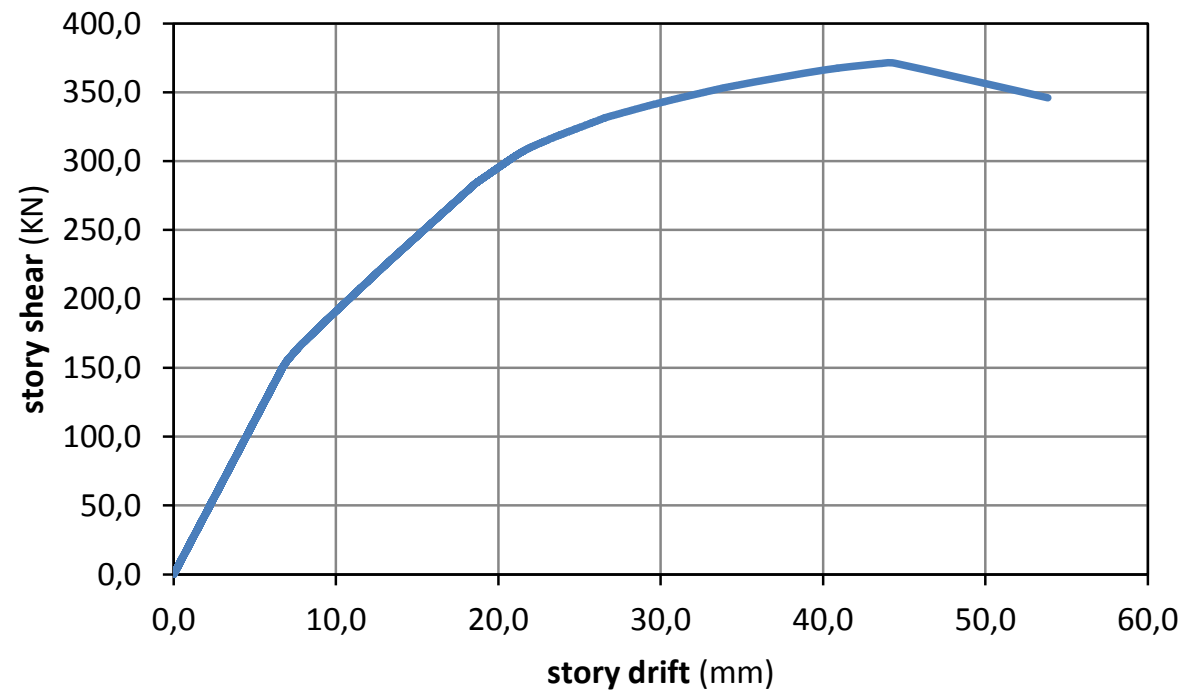

Fig. 5.2.13: Fuerza cortante-Desplazamiento entre forjados, pórtico pa6pz2-1, planta 2a 
- Planta 3모 (story3)

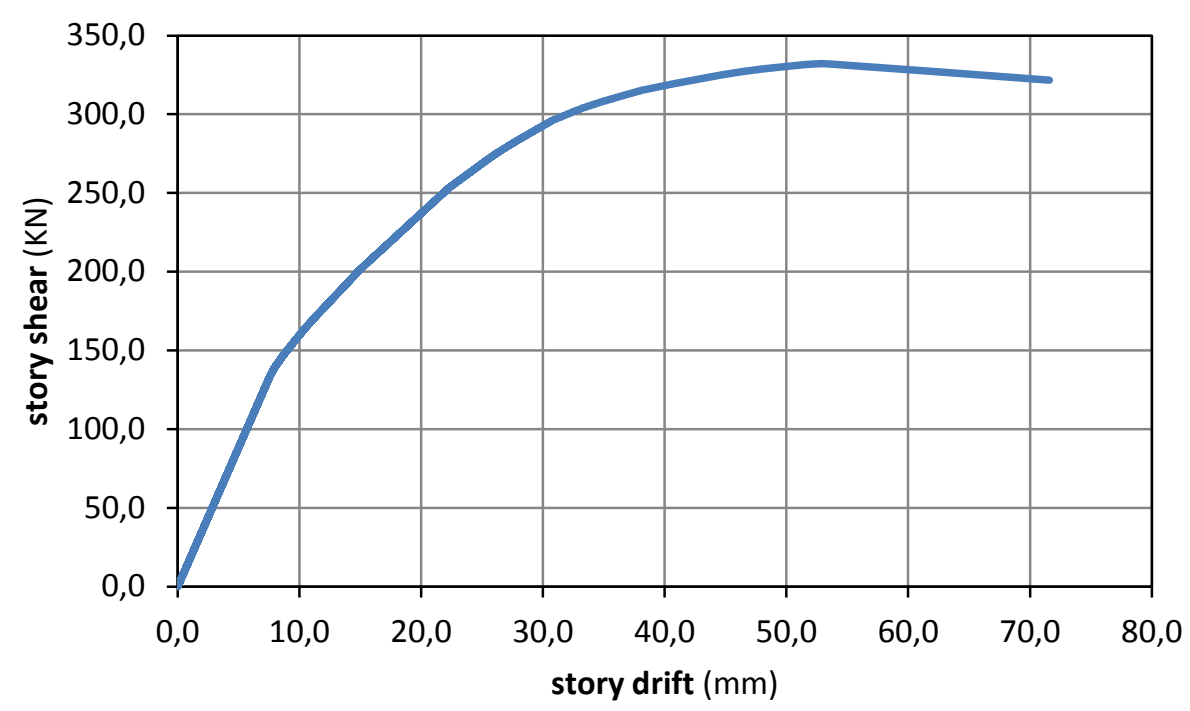

Fig. 5.2.14: Fuerza cortante-Desplazamiento entre forjados, pórtico pa6pz2-1, planta 3a

- Planta 4ㅁa (story4)

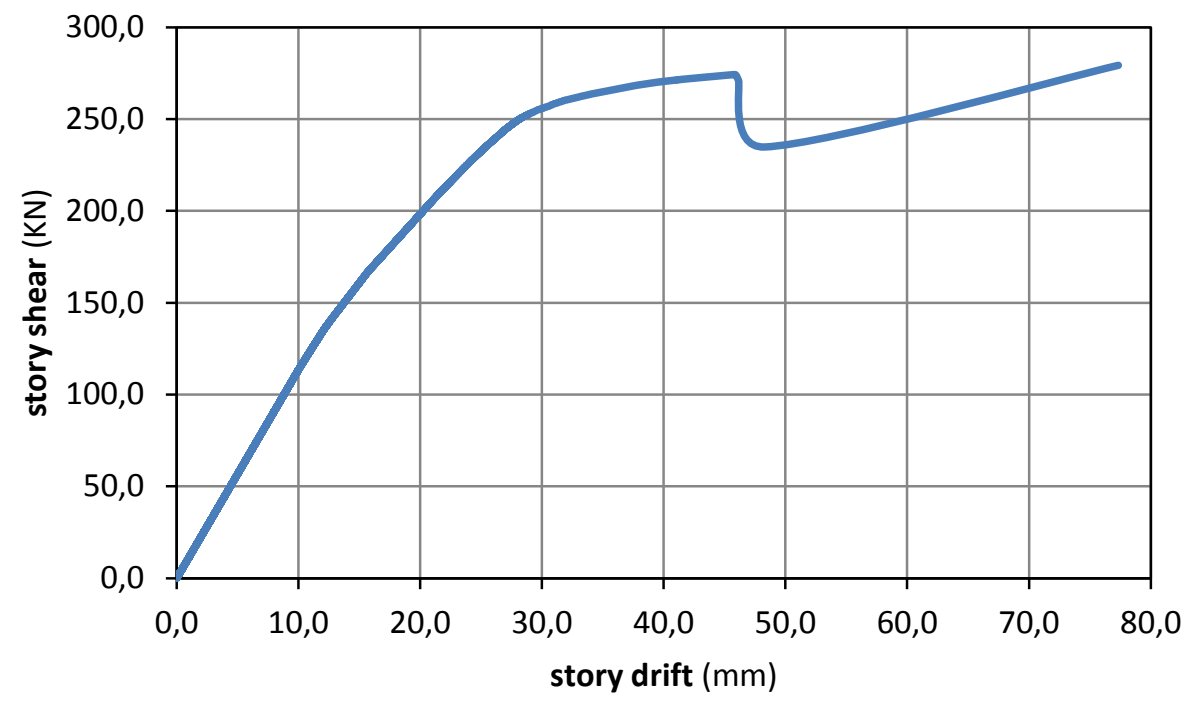

Fig. 5.2.15: Fuerza cortante-Desplazamiento entre forjados, pórtico pa6pz2-1, planta 4a 
- Planta 5ㅁ (story5)

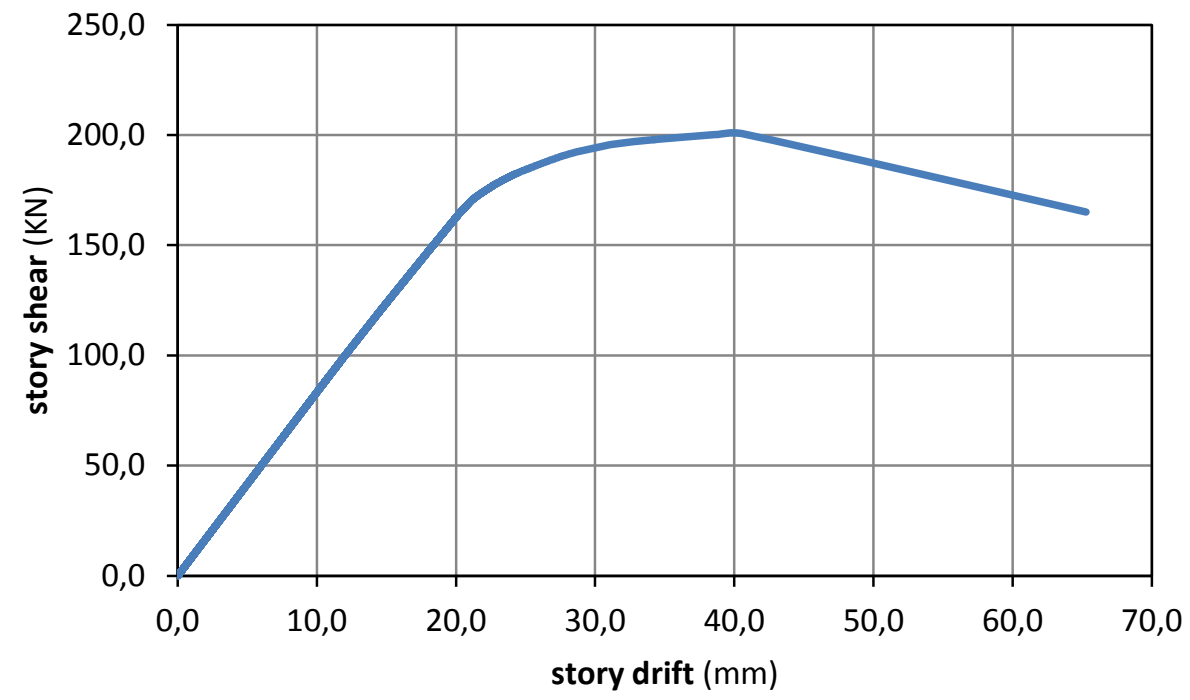

Fig. 5.2.16: Fuerza cortante-Desplazamiento entre forjados, pórtico pa6pz2-1, planta 5a

- Planta 6ㅁ (story6)

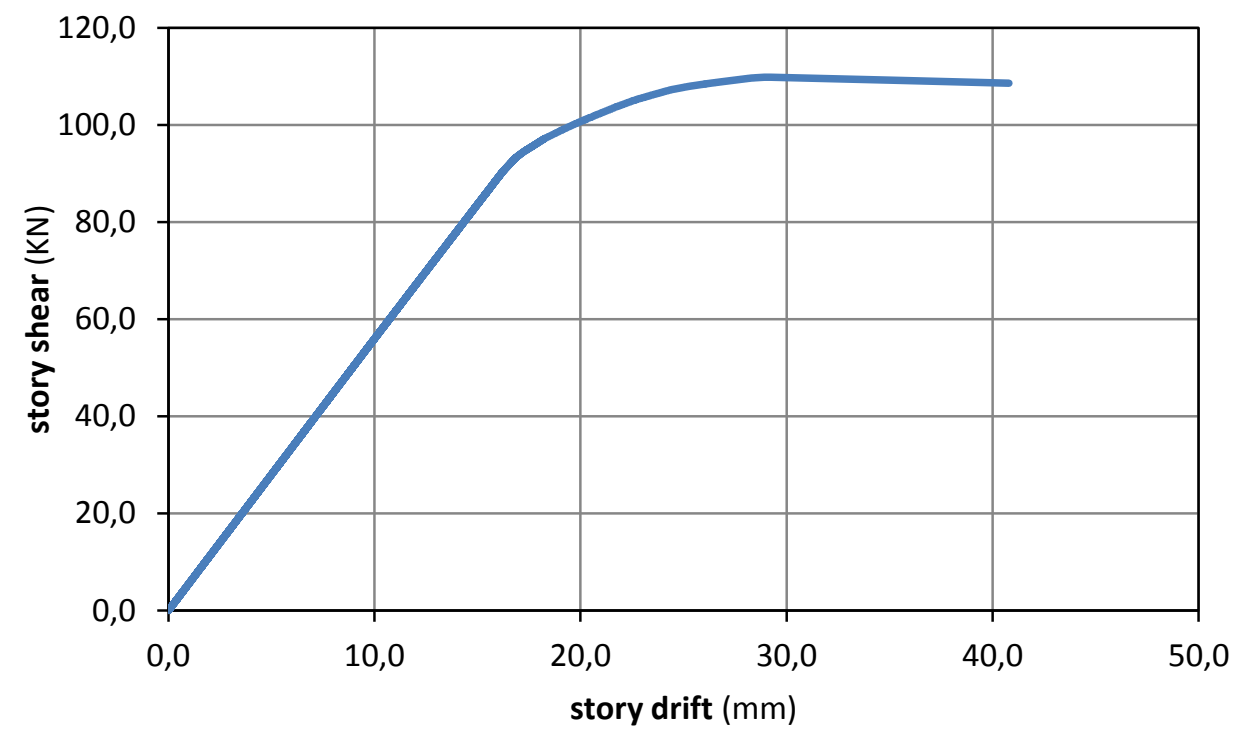

Fig. 5.2.17: Fuerza cortante-Desplazamiento entre forjados, pórtico pa6pz2-1, planta 6a

\subsubsection{Rigidez inicial y desplazamiento de fluencia de cada planta}

La rigidez inicial, es la tangente a la curva. Para obtener la rigidez inicial y el desplazamiento de fluencia de cada planta, partimos de los valores obtenidos en el apartado 5.2.3 de este capítulo, relación entre fuerza cortante de la planta y desplazamiento entre forjados que delimitan dicha planta, puesto que el desplazamiento de fluencia se produce cuando hay un cambio de rigidez.

En un principio, la rigidez permanece constante, hasta que al llegar a un determinado desplazamiento conforme la estructura va plastificando ésta disminuye. Sin embargo en las figuras 5.2.23 y 5.3.29 se observa un aumento de la rigidez debido a un endurecimiento por plastificación. 
- Pórtico pa2pz2-1

- Planta 1a (story1)

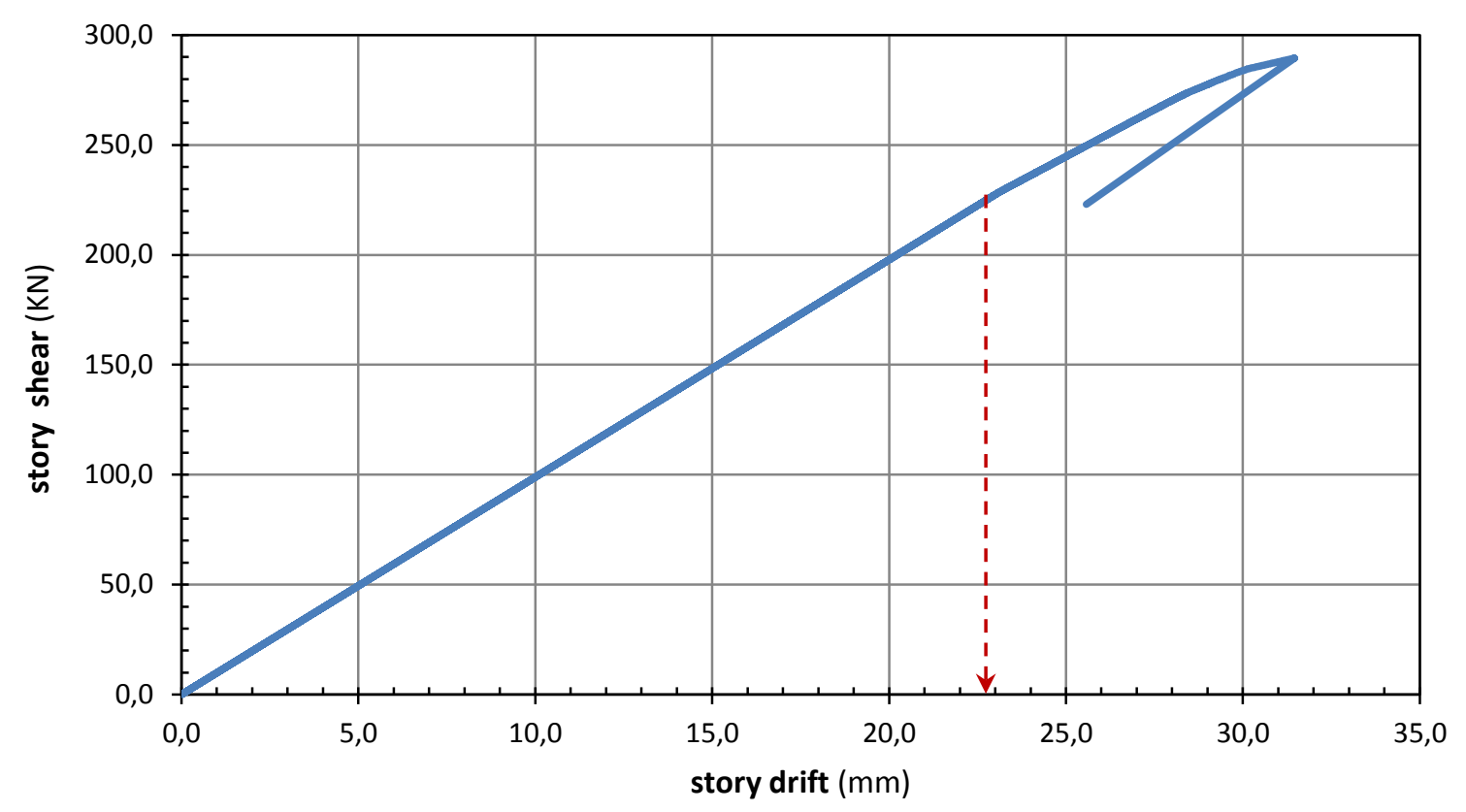

Fig. 5.2.18: Fuerza cortante-Desplazamiento entre forjados, pórtico pa2pz2-1, planta 1ạ

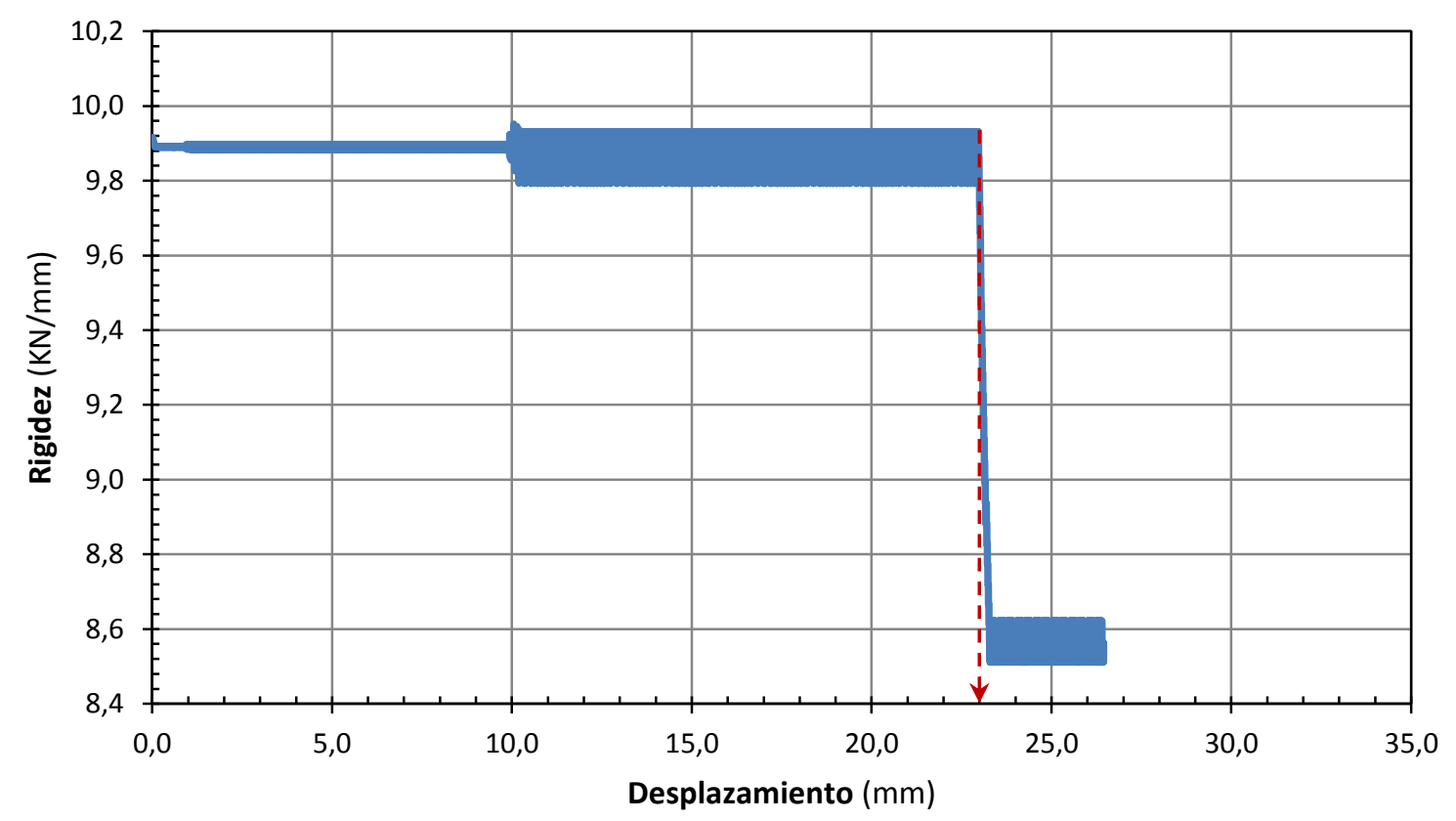

Fig. 5.2.19: Rigidez-Desplazamiento, pórtico pa2pz2-1, planta 1ạ

Tabla 5.2.6: Rigidez inicial y desplazamiento de fluencia, pórtico pa2pz2-1, planta 1a

\begin{tabular}{l|c}
\hline Rigidez inicial (KN/mm) & 9,9 \\
\hline Desplazamiento de fluencia $(\mathrm{mm})$ & 23 \\
\hline
\end{tabular}


- Planta 2모 (story2)

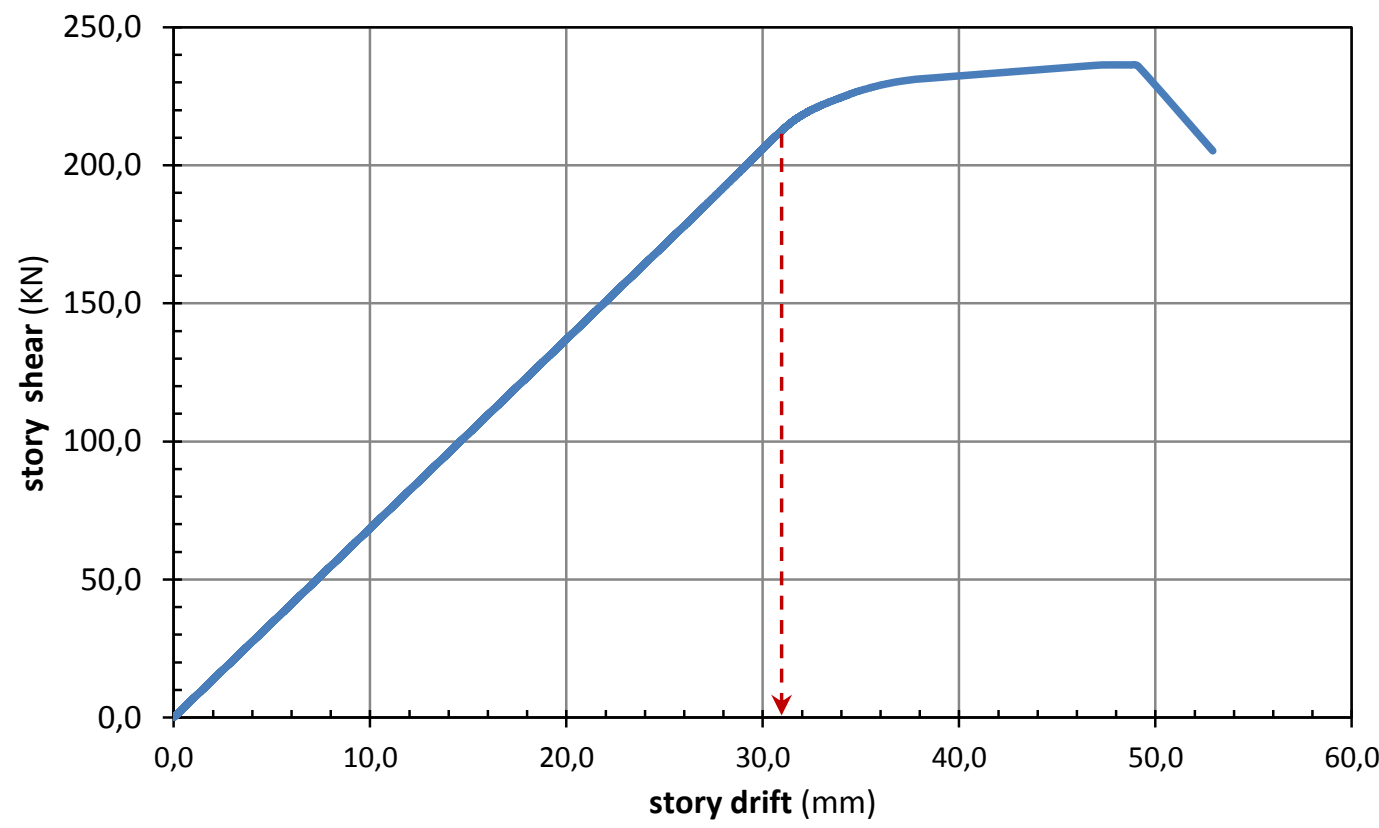

Fig. 5.2.20: Fuerza cortante-Desplazamiento entre forjados, pórtico pa2pz2-1, planta 2a

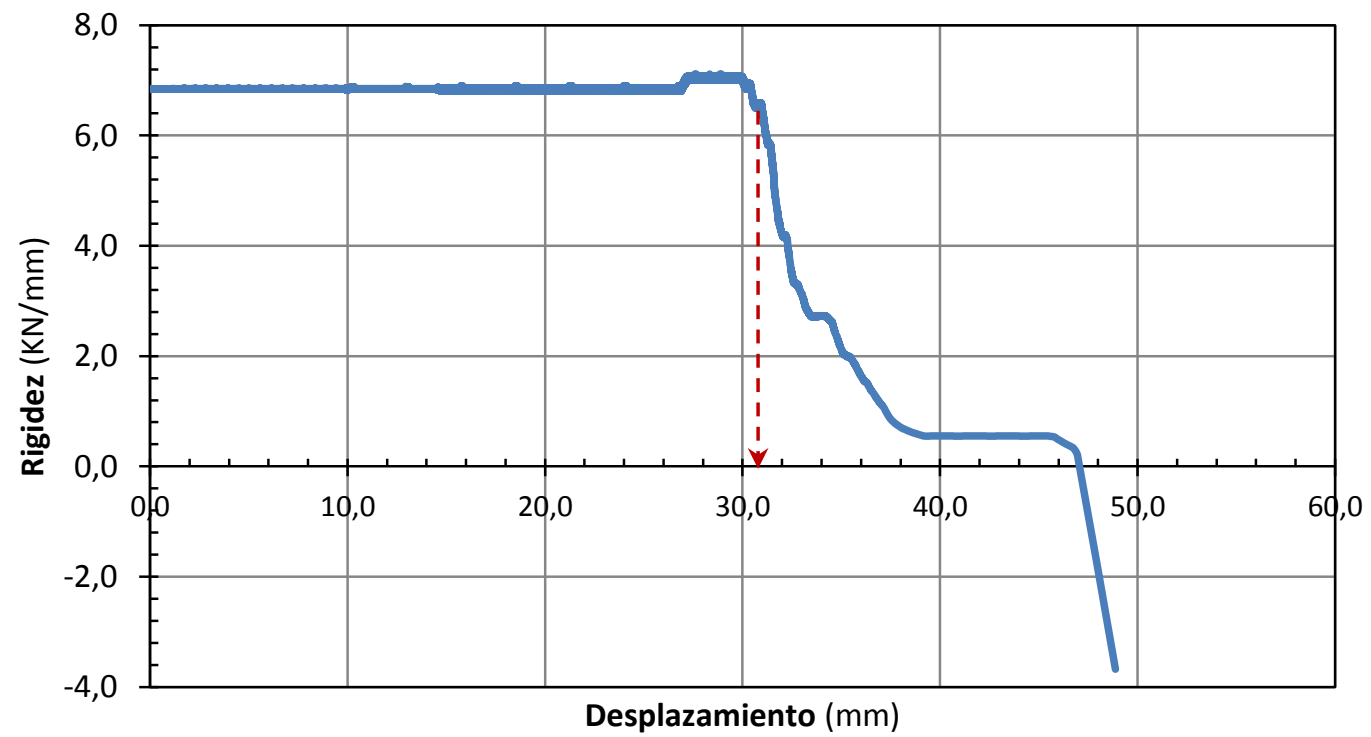

Fig. 5.2.21: Rigidez-Desplazamiento, pórtico pa2pz2-1, planta 2a

Tabla 5.2.7: Rigidez inicial y desplazamiento de fluencia, pórtico pa2pz2-1, planta 2ạ

\begin{tabular}{l|c}
\hline Rigidez inicial (KN/mm) & 6,8 \\
\hline Desplazamiento de fluencia $(\mathrm{mm})$ & 32 \\
\hline
\end{tabular}


- Planta 3모 (story3)

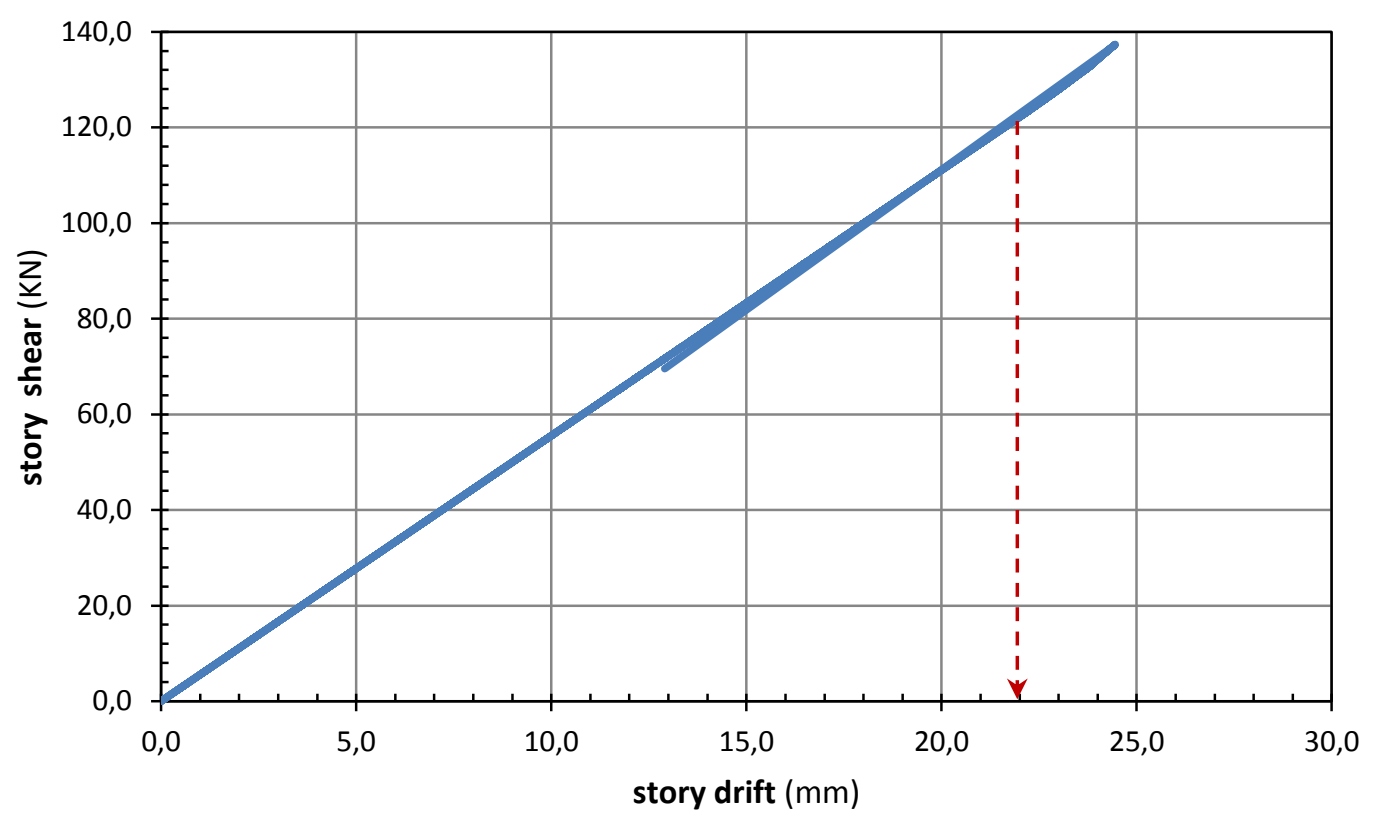

Fig. 5.2.22: Fuerza cortante-Desplazamiento entre forjados, pórtico pa2pz2-1, planta 3a

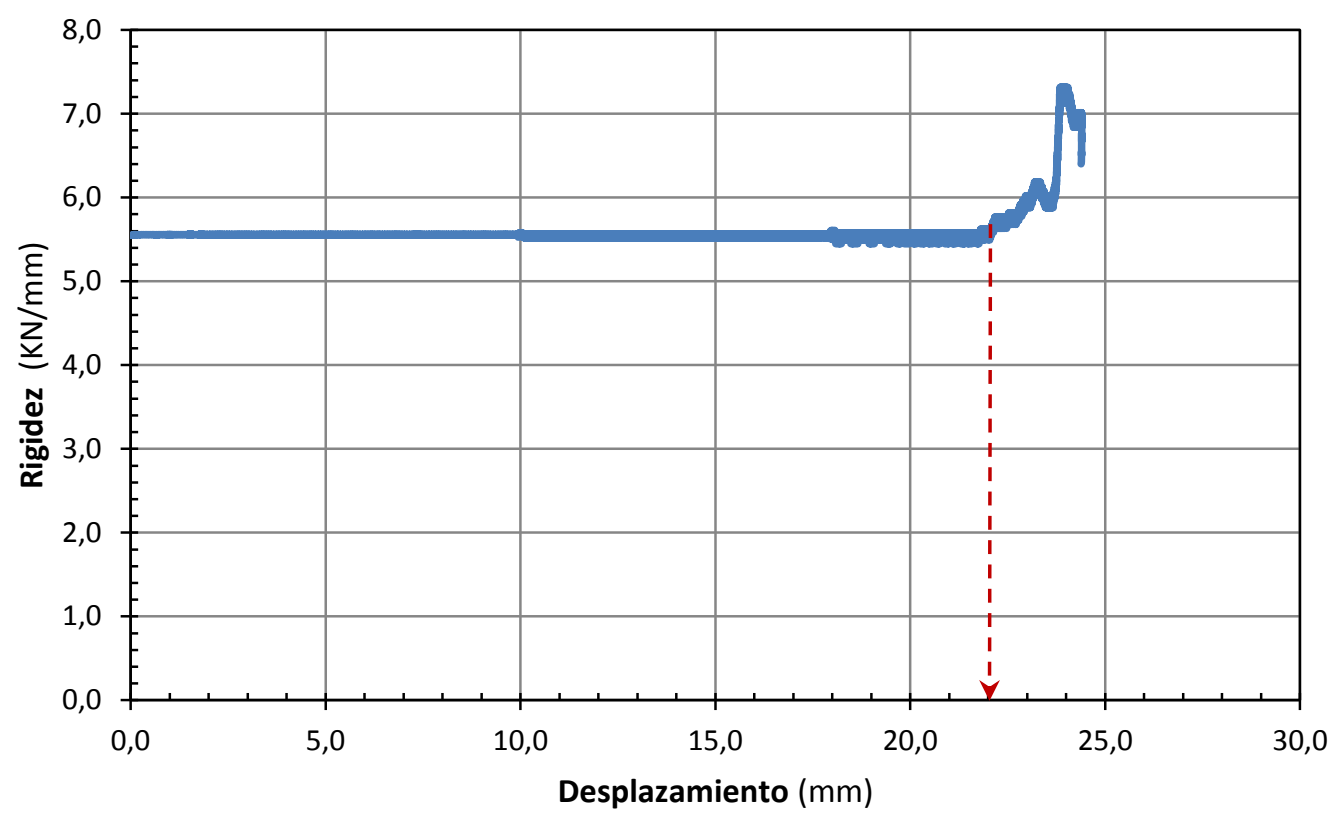

Fig. 5.2.23: Rigidez-Desplazamiento, pórtico pa2pz2-1, planta 3ạ

Tabla 5.2.8: Rigidez inicial y desplazamiento de fluencia, pórtico pa2pz2-1, planta 3a

\begin{tabular}{l|c}
\hline Rigidez inicial (KN/mm) & 5,5 \\
\hline Desplazamiento de fluencia $(\mathrm{mm})$ & 22 \\
\hline
\end{tabular}


- Pórtico pa4pz2-1

- Planta 1모 (story1)

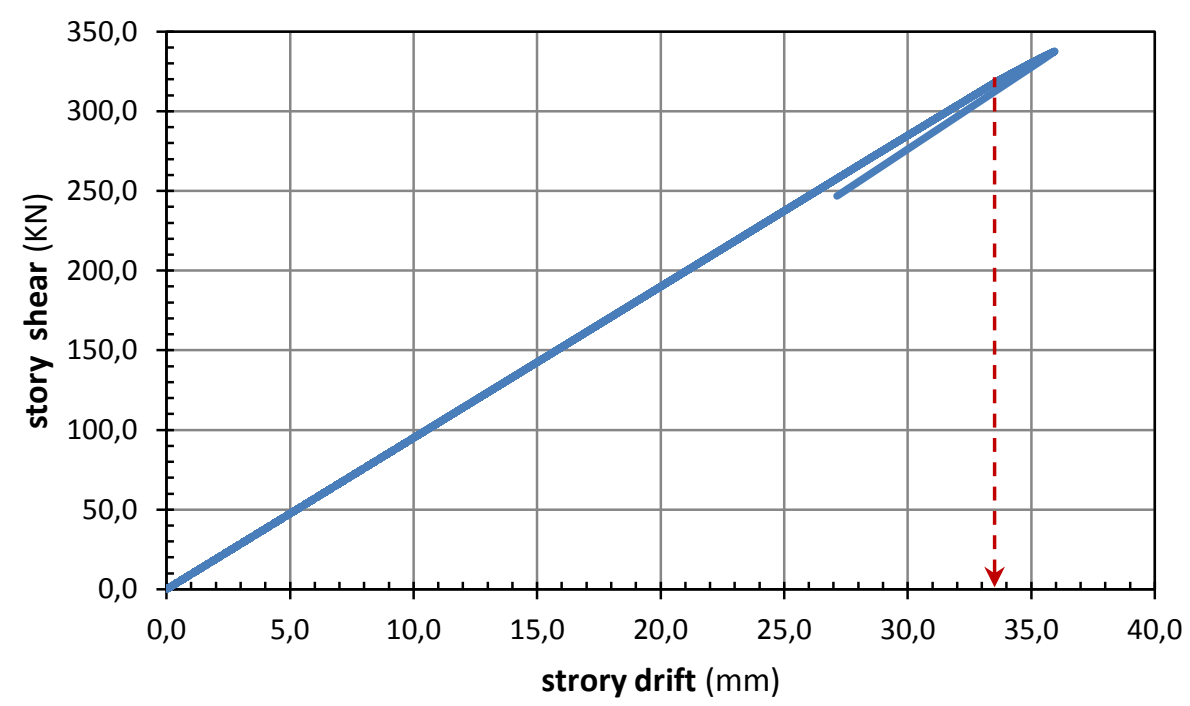

Fig. 5.2.24: Fuerza cortante-Desplazamiento entre forjados, pórtico pa4pz2-1, planta 1a

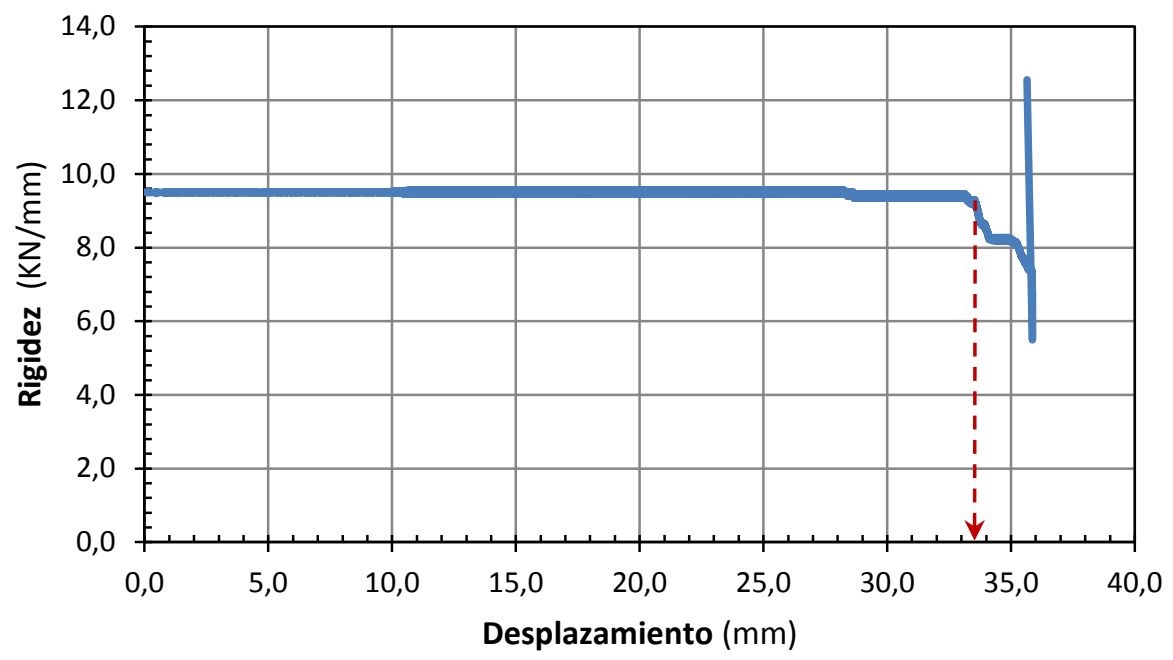

Fig. 5.2.25: Rigidez-Desplazamiento, pórtico pa4pz2-1, planta 1a

Tabla 5.2.9: Rigidez inicial y desplazamiento de fluencia, pórtico pa4pz2-1, planta 1ạ

\begin{tabular}{l|c}
\hline Rigidez inicial (KN/mm) & 9,5 \\
\hline Desplazamiento de fluencia $(\mathrm{mm})$ & 33 \\
\hline
\end{tabular}


- Planta 2모 (story2)

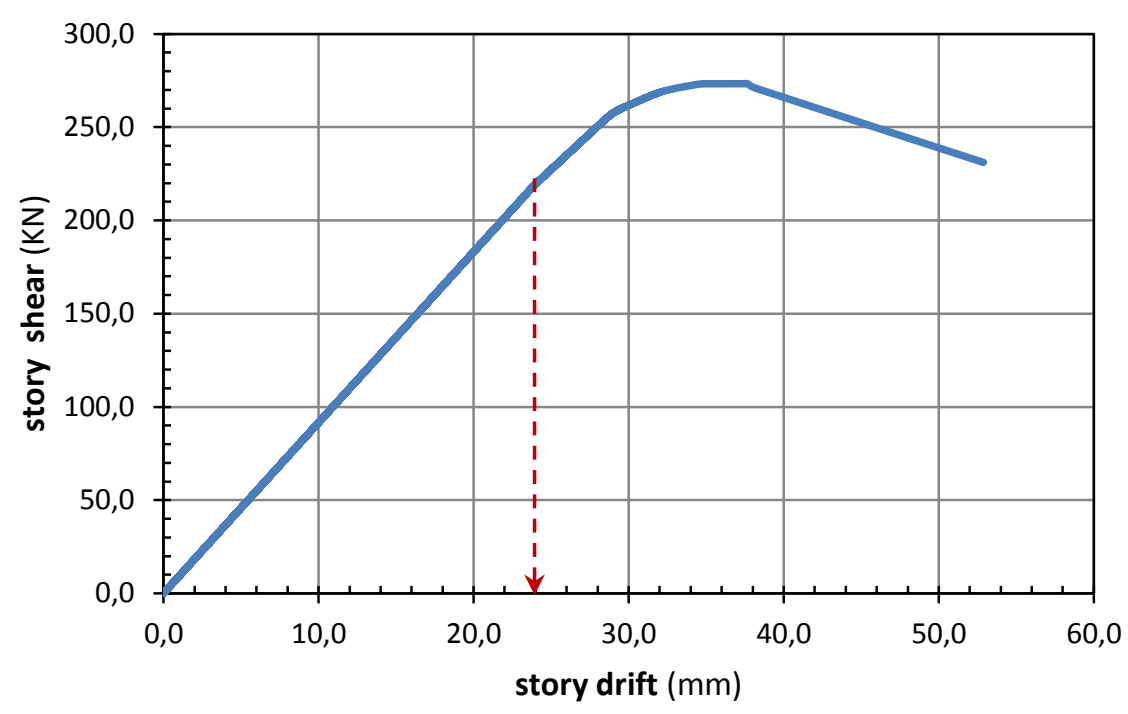

Fig. 5.2.26: Fuerza cortante-Desplazamiento entre forjados, pórtico pa4pz2-1, planta 2a

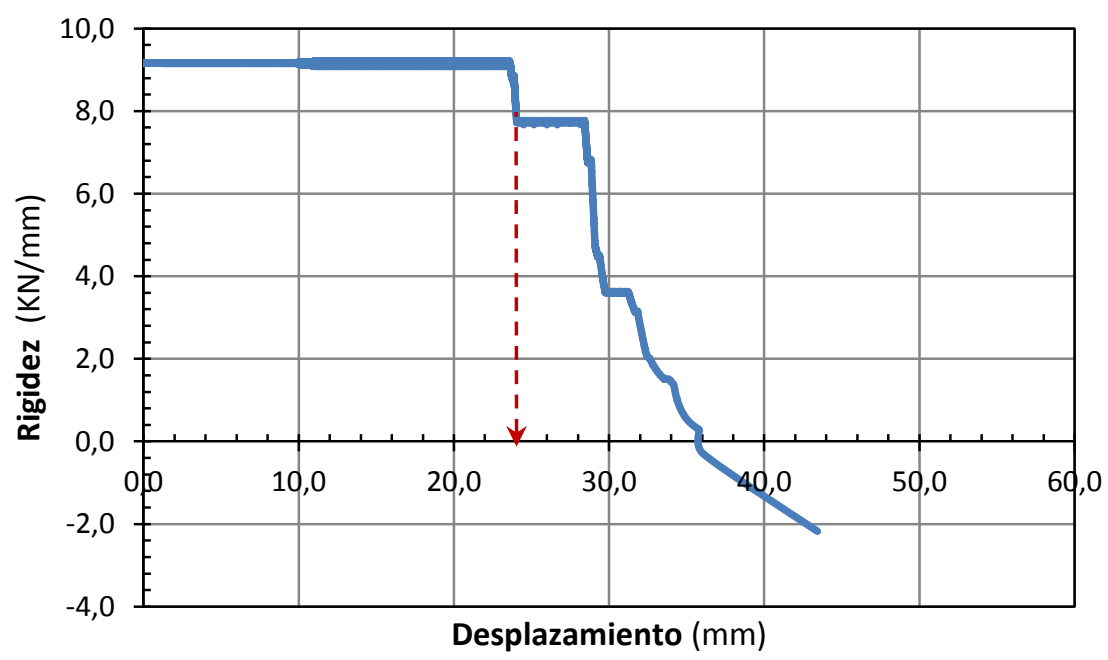

Fig. 5.2.27: Rigidez-Desplazamiento, pórtico pa4pz2-1, planta 2a

Tabla 5.2.10: Rigidez inicial y desplazamiento de fluencia, pórtico pa4pz2-1, planta 2a

\begin{tabular}{l|c}
\hline Rigidez inicial (KN/mm) & 9,1 \\
\hline Desplazamiento de fluencia $(\mathrm{mm})$ & 24 \\
\hline
\end{tabular}


- Planta 3모 (story3)

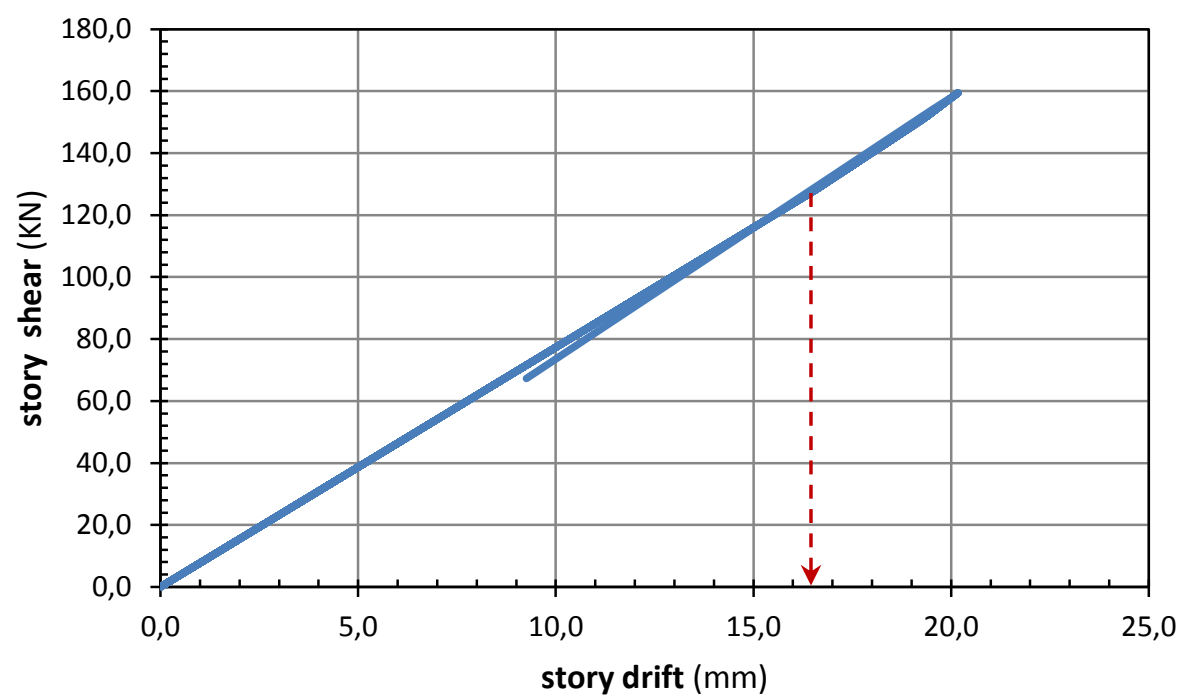

Fig. 5.2.28: Fuerza cortante-Desplazamiento entre forjados, pórtico pa4pz2-1, planta 3a

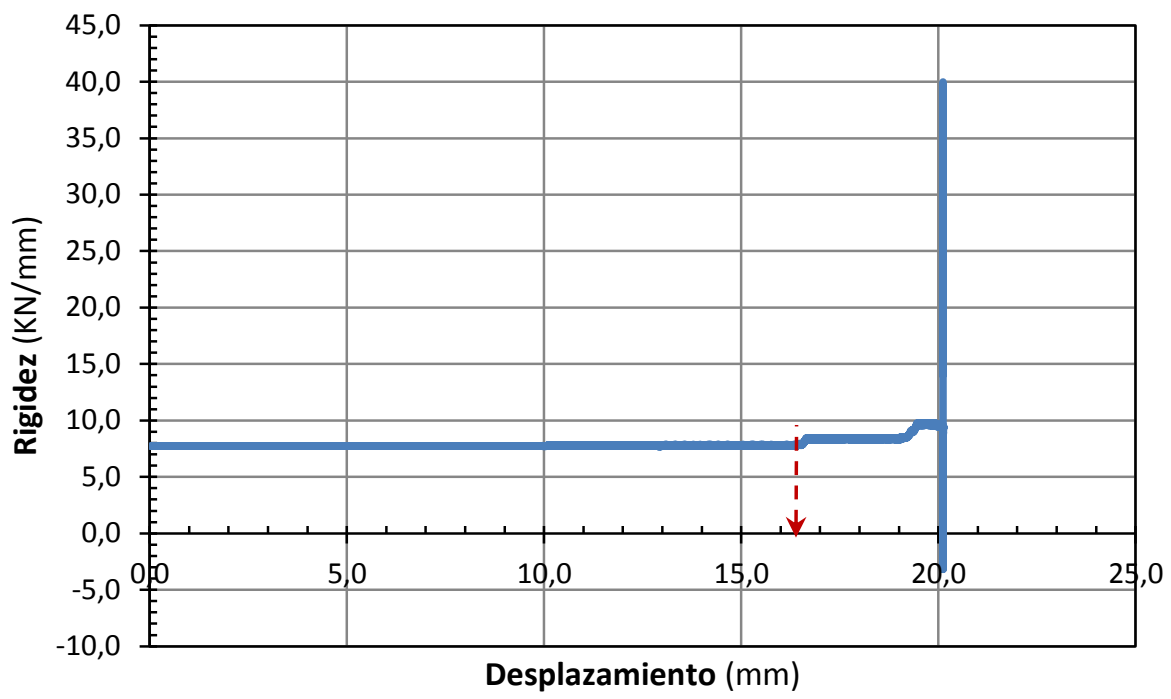

Fig. 5.2.29: Rigidez-Desplazamiento, pórtico pa4pz2-1, planta 3a

Tabla 5.2.11: Rigidez inicial y desplazamiento de fluencia, pórtico pa4pz2-1, planta 3a

\begin{tabular}{l|c}
\hline Rigidez inicial (KN/mm) & 7,7 \\
\hline Desplazamiento de fluencia $(\mathrm{mm})$ & 16,5 \\
\hline
\end{tabular}


- Pórtico pa6pz2-1

- Planta 1모 (story1)

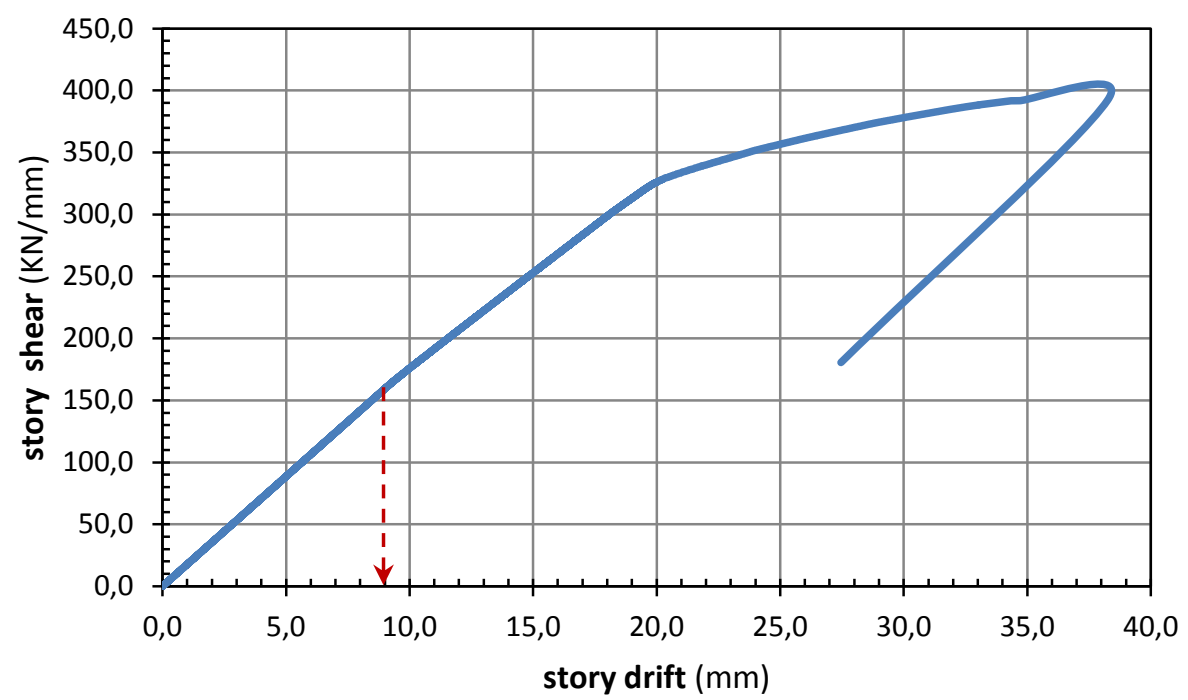

Fig. 5.2.30: Fuerza cortante-Desplazamiento entre forjados, pórtico pa6pz2-1, planta 1a

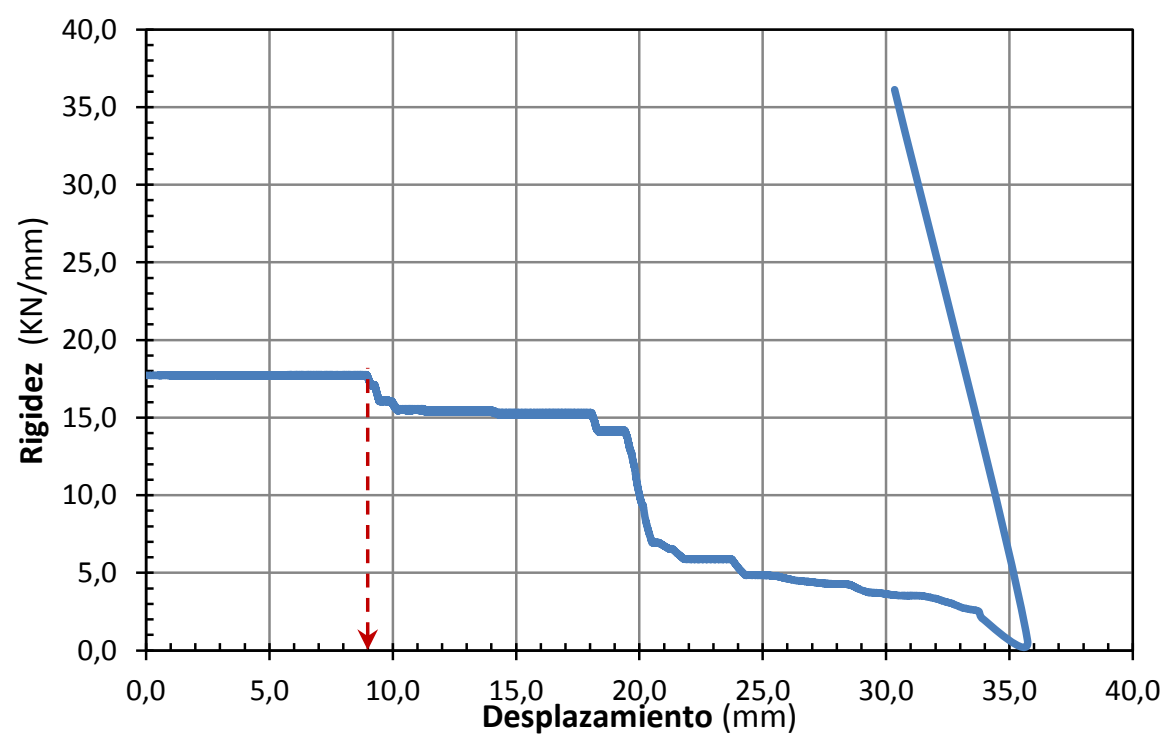

Fig. 5.2.31: Rigidez-Desplazamiento, pórtico pa6pz2-1, planta 1ạ

Tabla 5.2.12: Rigidez inicial y desplazamiento de fluencia, pórtico pa6pz2-1, planta 1a

\begin{tabular}{l|c}
\hline Rigidez inicial (KN/mm) & 17,5 \\
\hline Desplazamiento de fluencia $(\mathrm{mm})$ & 9 \\
\hline
\end{tabular}


- Planta $2^{a}$ (story2)

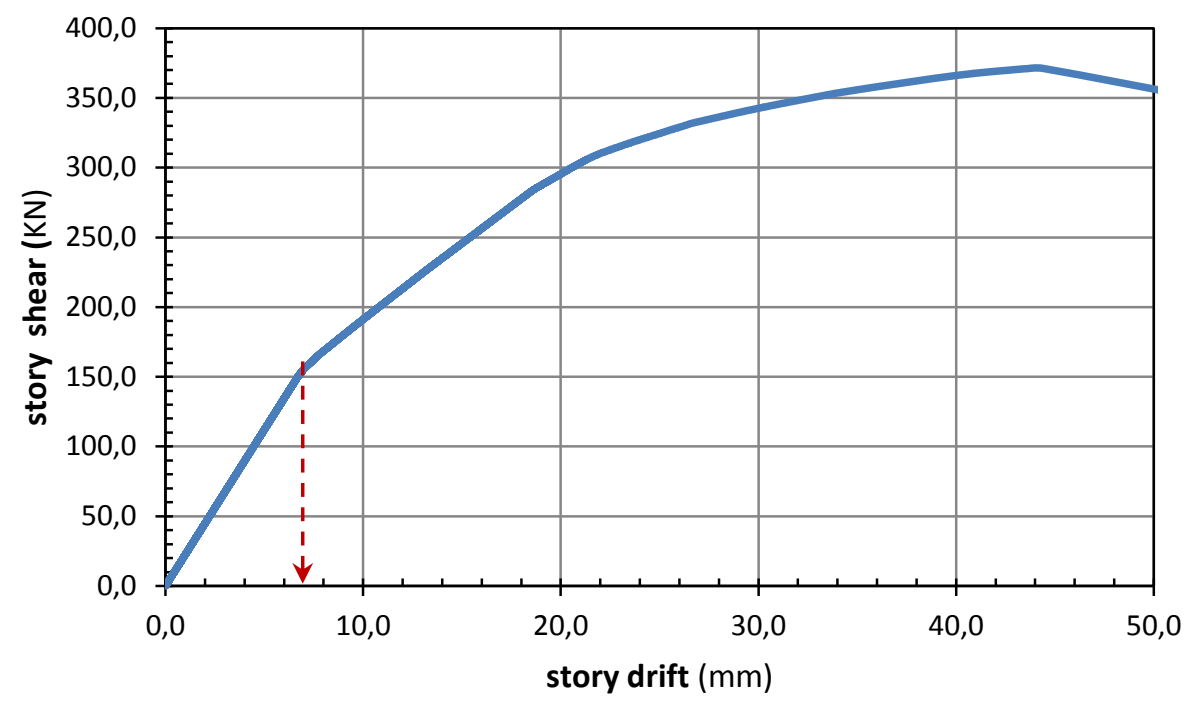

Fig. 5.2.32: Fuerza cortante-Desplazamiento entre forjados, pórtico pa6pz2-1, planta 2a

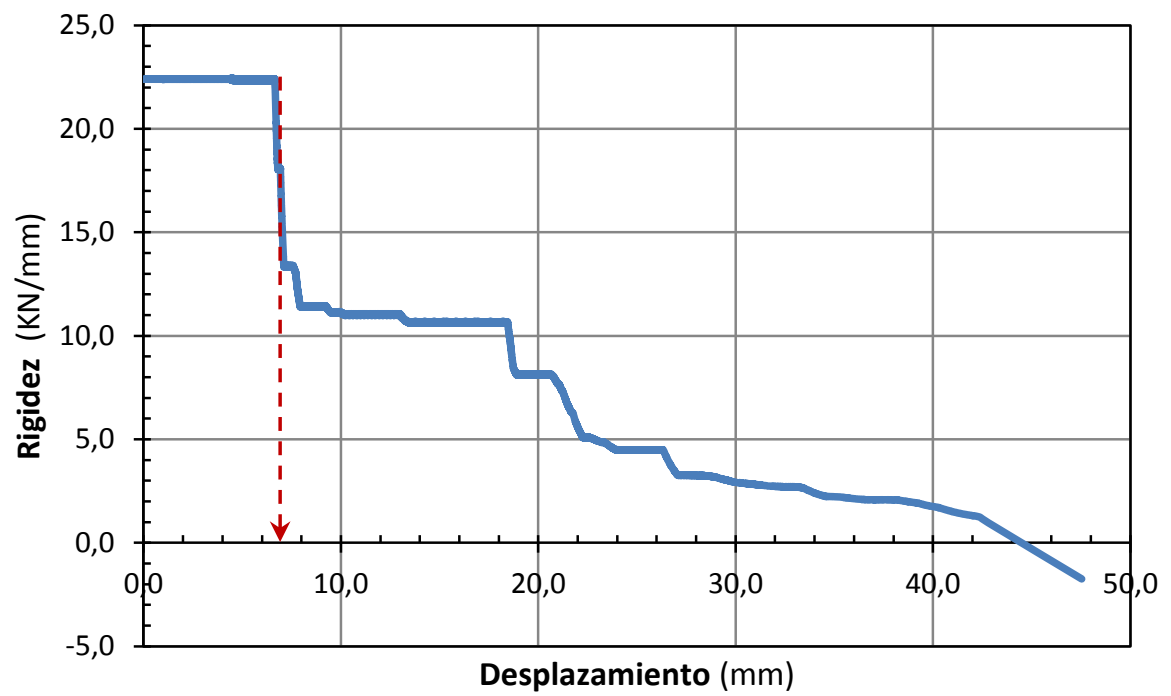

Fig. 5.2.33: Rigidez-Desplazamiento, pórtico pa6pz2-1, planta 2a

Tabla 5.2.13: Rigidez inicial y desplazamiento de fluencia, pórtico pa6pz2-1, planta 2 a

\begin{tabular}{l|c}
\hline Rigidez inicial (KN/mm) & 22,4 \\
\hline Desplazamiento de fluencia $(\mathrm{mm})$ & 7 \\
\hline
\end{tabular}


- Planta 3모 (story3)

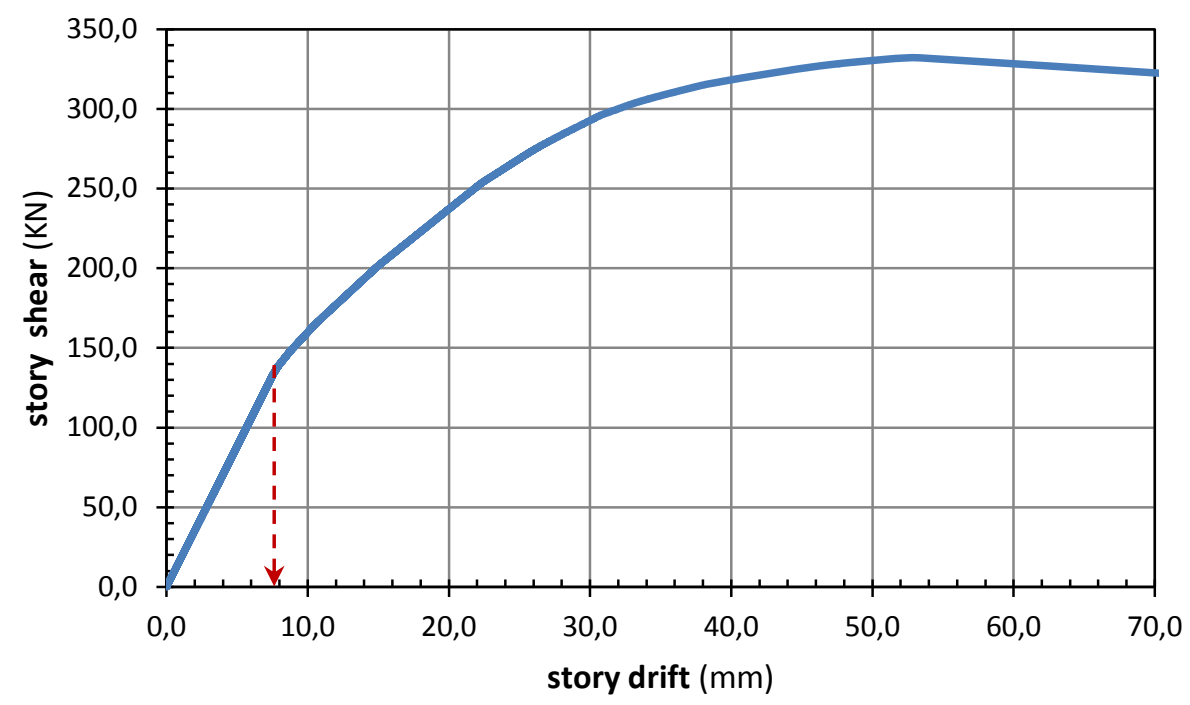

Fig. 5.2.34: Fuerza cortante-Desplazamiento entre forjados, pórtico pa6pz2-1, planta 3a

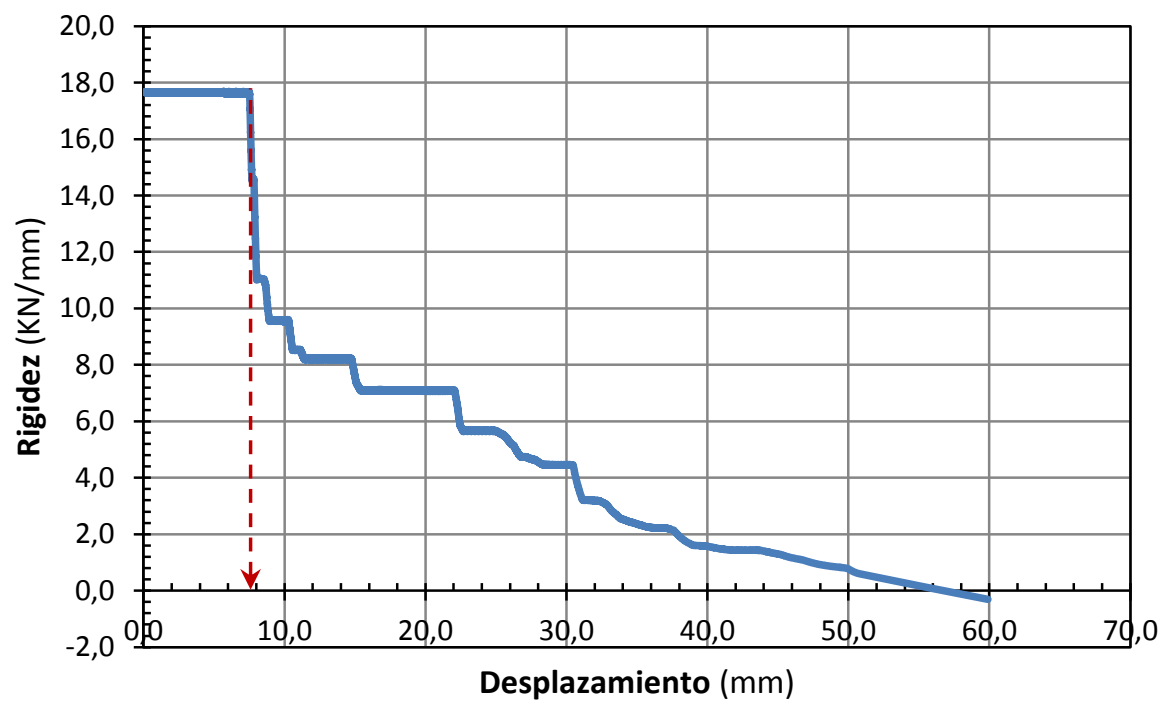

Fig. 5.2.35: Rigidez-Desplazamiento, pórtico pa6pz2-1, planta 3a

Tabla 5.2.14: Rigidez inicial y desplazamiento de fluencia, pórtico pa6pz2-1, planta 3a

\begin{tabular}{l|c}
\hline Rigidez inicial (KN/mm) & 17,7 \\
\hline Desplazamiento de fluencia $(\mathrm{mm})$ & 8 \\
\hline
\end{tabular}


- Planta $4 \underline{a}$ (story4)

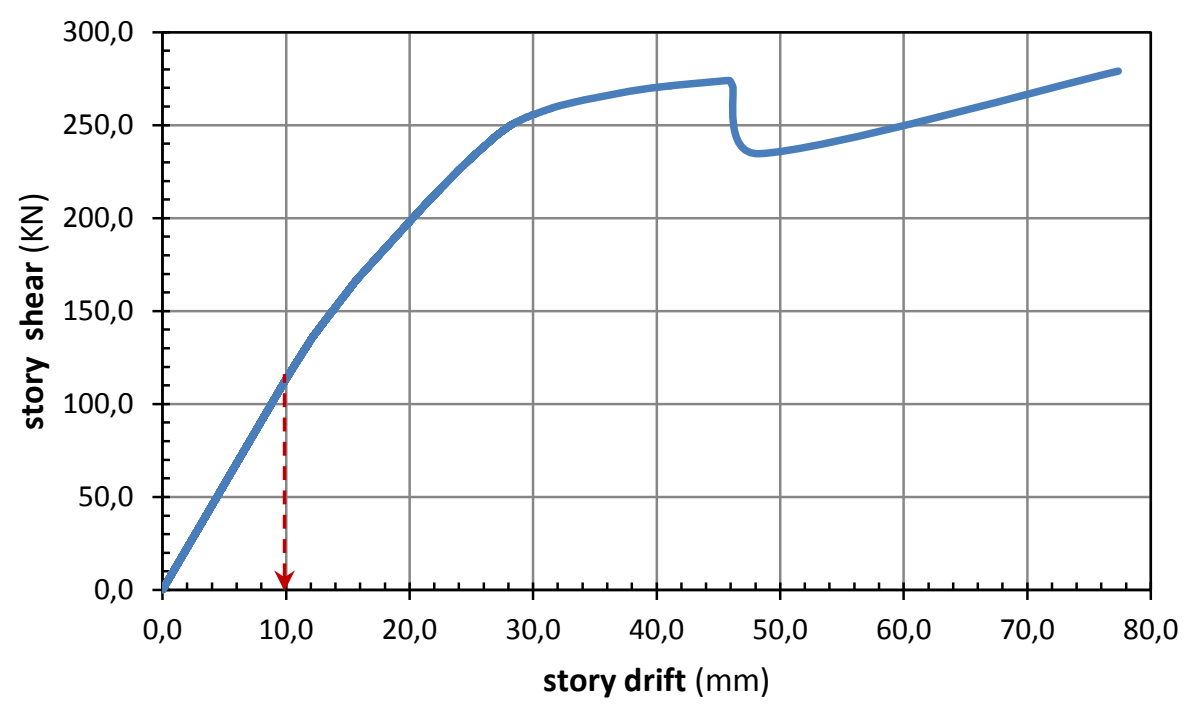

Fig. 5.2.36: Fuerza cortante-Desplazamiento entre forjados, pórtico pa6pz2-1, planta 4a

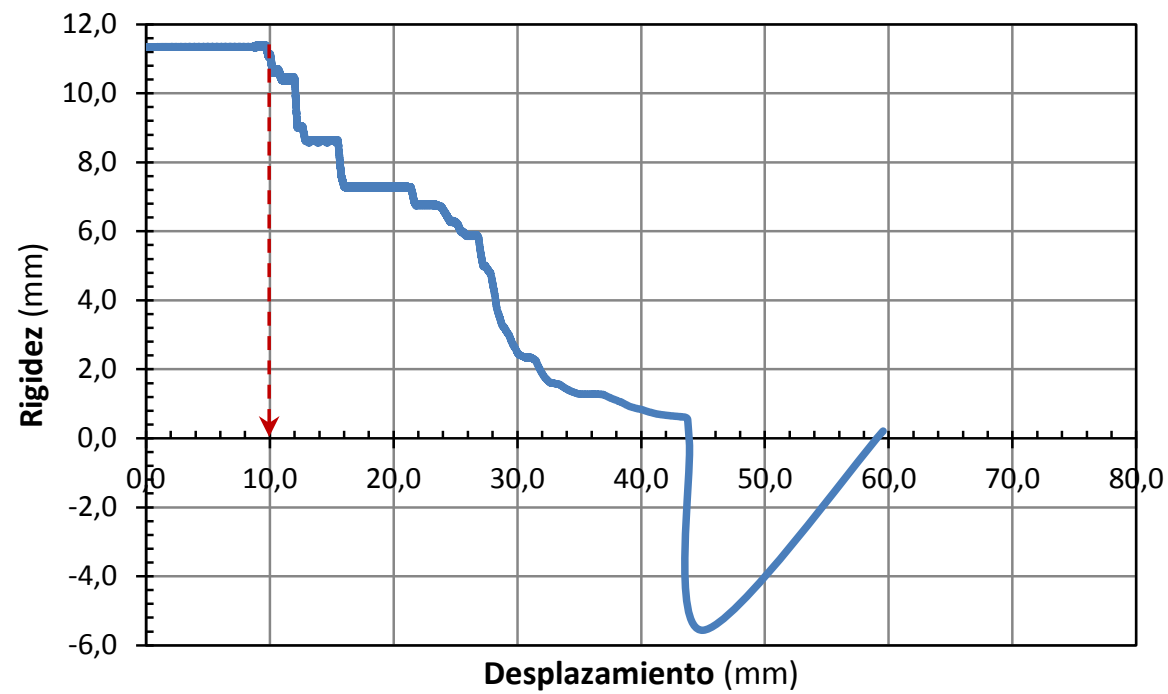

Fig. 5.2.37: Rigidez-Desplazamiento, pórtico pa6pz2-1, planta 4ạ

Tabla 5.2.15: Rigidez inicial y desplazamiento de fluencia, pórtico pa6pz2-1, planta 4a

\begin{tabular}{l|c}
\hline Rigidez inicial (KN/mm) & 11,3 \\
\hline Desplazamiento de fluencia $(\mathrm{mm})$ & 10 \\
\hline
\end{tabular}


- Planta 5a (story5)

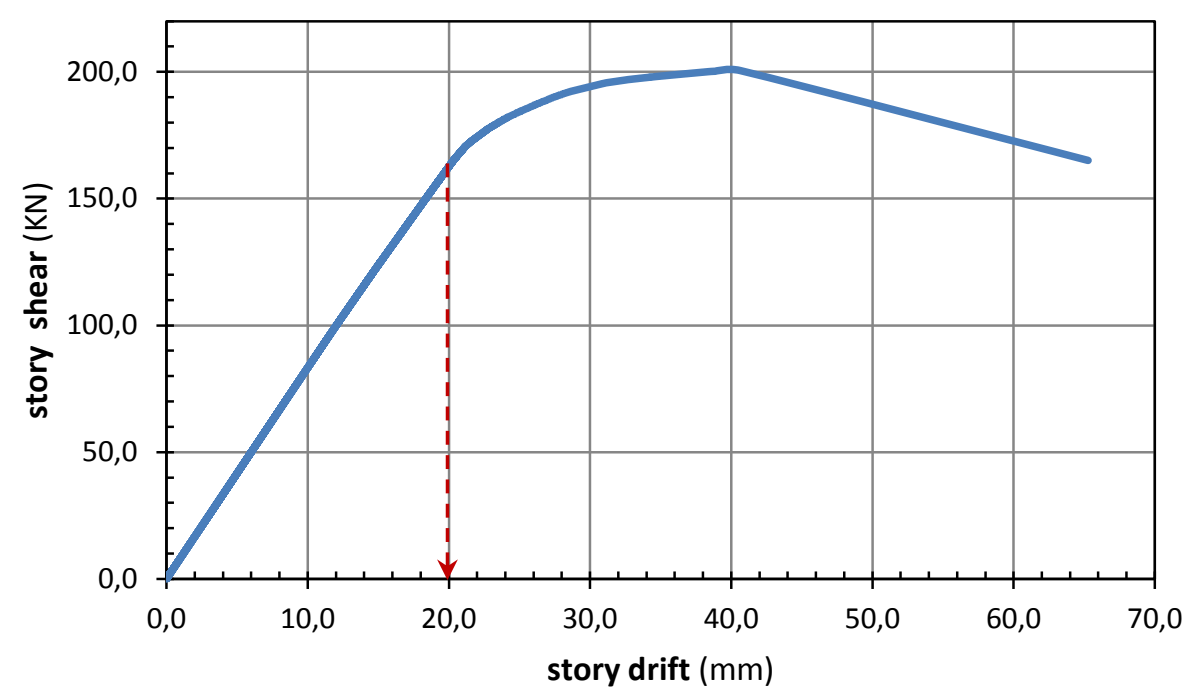

Fig. 5.2.38: Fuerza cortante-Desplazamiento entre forjados, pórtico pa6pz2-1, planta 5a

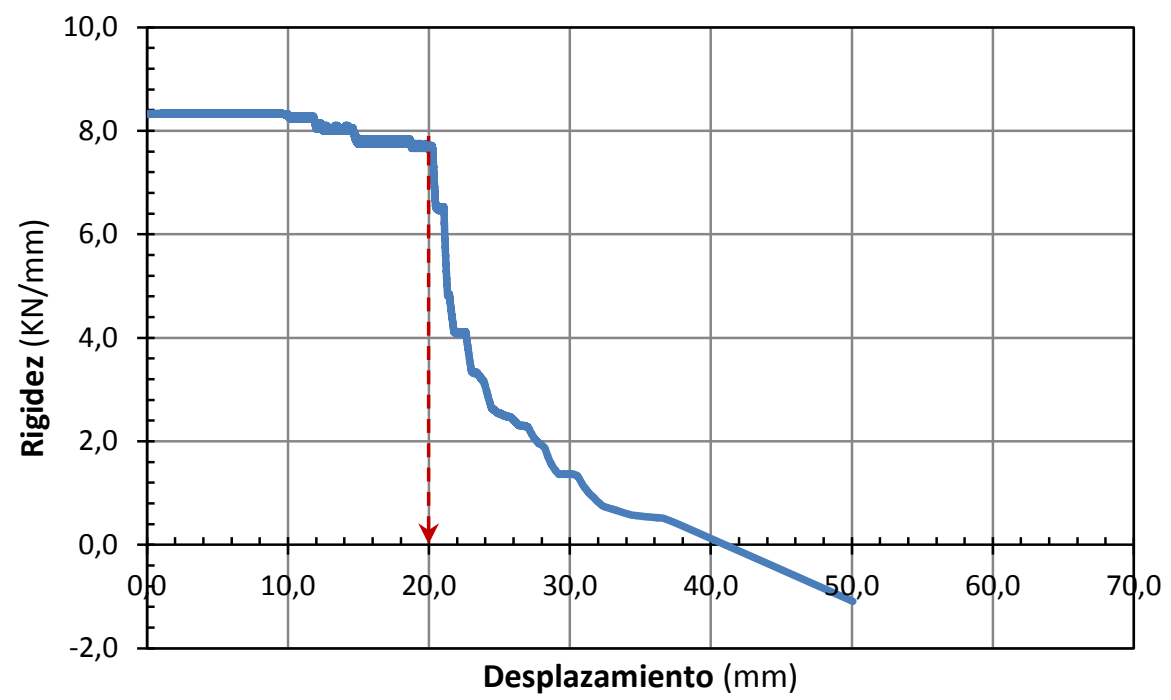

Fig. 5.2.39: Rigidez-Desplazamiento, pórtico pa6pz2-1, planta 5a

Tabla 5.2.16: Rigidez inicial y desplazamiento de fluencia, pórtico pa6pz2-1, planta 5a

\begin{tabular}{l|c}
\hline Rigidez inicial (KN/mm) & 8,3 \\
\hline Desplazamiento de fluencia $(\mathrm{mm})$ & 20 \\
\hline
\end{tabular}


- Planta 6 $\underline{a}$ (story6)

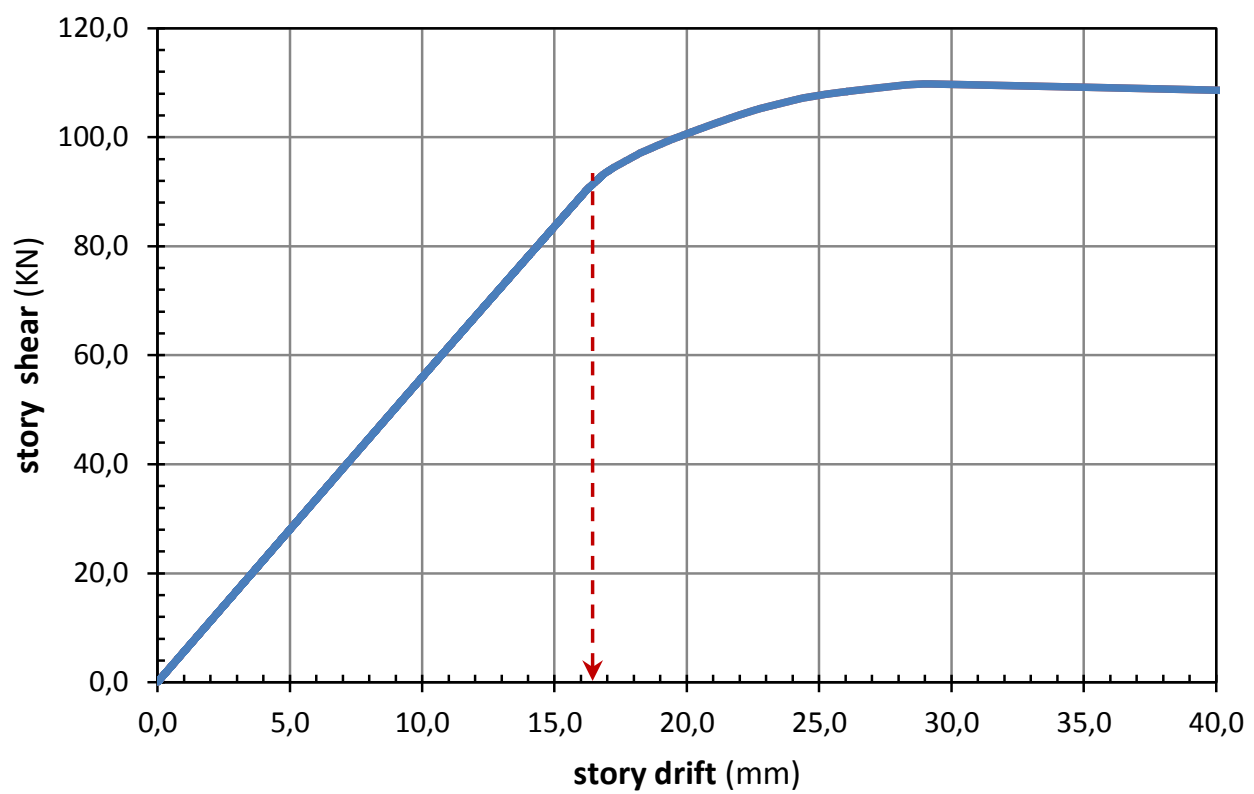

Fig. 5.2.40: Fuerza cortante-Desplazamiento entre forjados, pórtico pa6pz2-1, planta 6a

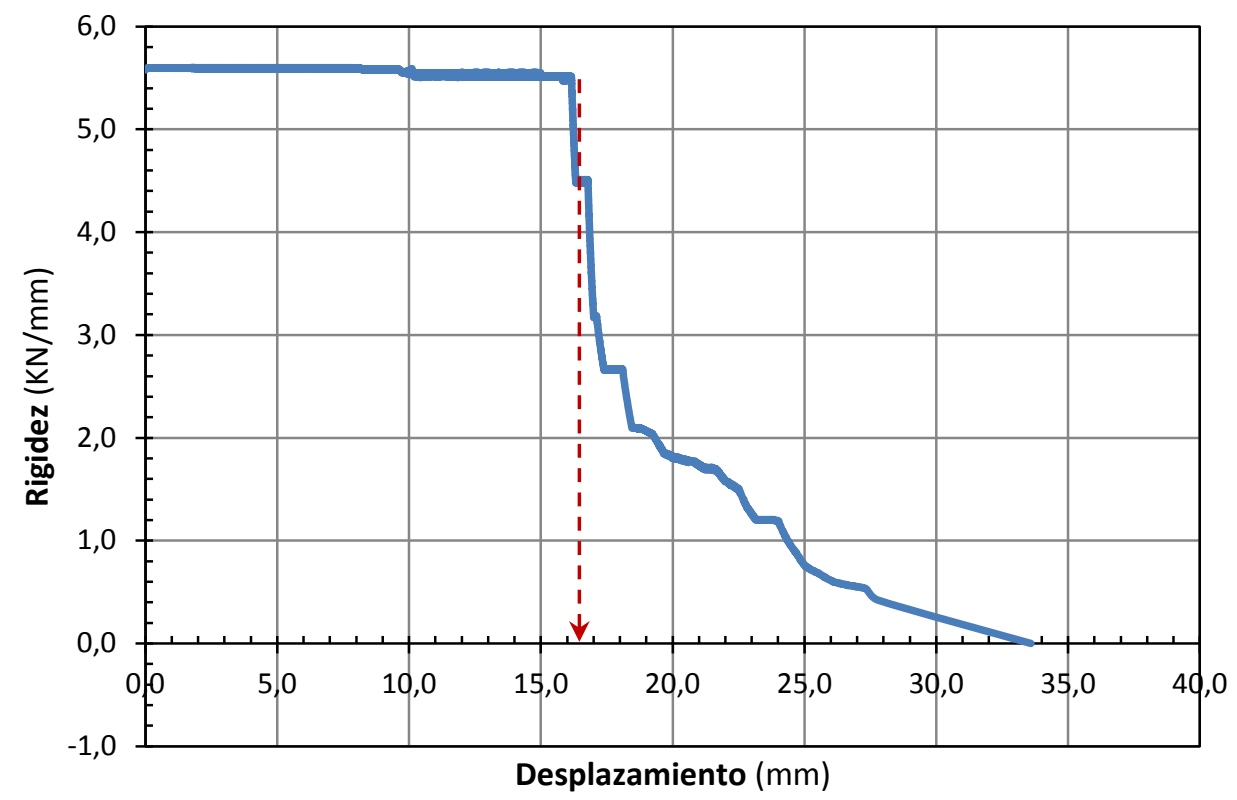

Fig. 5.2.41: Rigidez-Desplazamiento, pórtico pa6pz2-1, planta 6a

Tabla 5.2.17: Rigidez inicial y desplazamiento de fluencia, pórtico pa6pz2-1, planta 6 a

\begin{tabular}{l|c}
\hline Rigidez inicial (KN/mm) & 5,6 \\
\hline Desplazamiento de fluencia $(\mathrm{mm})$ & 16 \\
\hline
\end{tabular}




\subsubsection{Tabla resumen Rigidez inicial-Desplazamiento de fluencia para cada uno de los pórticos}

Tabla 5.2.18: Rigidez inicial y desplazamiento de fluencia para cada planta de cada uno de los pórticos

\begin{tabular}{c|c|c|c|c|c|c} 
& \multicolumn{2}{|c|}{ Pórtico pa2pz2-1 } & \multicolumn{2}{c|}{ Pórtico pa4pz2-1 } & \multicolumn{2}{c}{ Pórtico pa6pz2-1 } \\
\cline { 2 - 7 } & $\begin{array}{c}\text { Rigidez inicial } \\
(\mathrm{KN} / \mathrm{mm})\end{array}$ & $\begin{array}{c}\text { Desplaz. fluencia } \\
(\mathrm{mm})\end{array}$ & $\begin{array}{c}\text { Rigidez inicial } \\
(\mathrm{KN} / \mathrm{mm})\end{array}$ & $\begin{array}{c}\text { Desplaz. fluencia } \\
(\mathrm{mm})\end{array}$ & $\begin{array}{c}\text { Rigidez inicial } \\
(\mathrm{KN} / \mathrm{mm})\end{array}$ & $\begin{array}{c}\text { Desplaz. fluencia } \\
(\mathrm{mm})\end{array}$ \\
\hline Planta 1- & 9,9 & 23 & 9,5 & 33 & 17,7 & 9 \\
\hline Planta 2- & 6,8 & 32 & 9,1 & 24 & 22,4 & 7 \\
\hline Planta 3a & 5,5 & 22 & 7,7 & 16,5 & 17,7 & 8 \\
\hline Planta 4- & --- & --- & --- & --- & 11,3 & 10 \\
\hline Planta 5a & --- & --- & --- & --- & 8,3 & 20 \\
\hline Planta 6a & --- & --- & --- & -- & & 16 \\
\hline & & & & & &
\end{tabular}

\subsubsection{Periodos de vibración obtenidos con el programa IDARC y con el programa LumpST}

La estimación del periodo natural de vibración de una estructura de hormigón armado, es un procedimiento esencial en el diseño sísmico. A partir de esta característica puede obtenerse una buena apreciación de las demandas globales sobre una estructura sometida a una determinada acción sísmica.

Esta propiedad depende de la masa y la rigidez de la estructura, también influyen muchos otros factores, tales como la regularidad de la estructura, el número de plantas y huecos, las dimensiones de las secciones, el nivel de carga axial, las cuantías de refuerzo y del nivel de agrietamiento del hormigón.

El período es el tiempo en que tarda un objeto en cumplir un ciclo cuando vibra, es una característica única del objeto y no se altera a menos que sea forzado a cambiarlo.

La respuesta sísmica de un sistema depende de su periodo de vibración, lo que indica que la respuesta máxima de una estructura ante un temblor varíe principalmente por el periodo de vibración.

Para cambiar el período de vibración se debe variar la masa o la rigidez del edificio. En general, un proyectista tiene poca libertad para modificar la masa del edificio sin embargo sí se puede actuar sobre la rigidez lateral, principalmente dependiendo del sistema estructural que se elija, el cual puede ser relativamente flexible, cuando es a base de pórticos o muy rígido cuando tiene muros estructurales.

Por otra parte, los periodos de vibración de un edificio aumentan con el número de plantas, por lo que se acostumbra a numerarlos en orden decreciente; así el primer período $T_{1}$ (llamado periodo fundamental) tiene el mayor valor y el último, $T_{n}$, el menor.

La relación entre el periodo fundamental del edificio $\left(T_{E}\right)$ y el periodo dominante del suelo $\left(T_{S}\right)$ influye en la respuesta de una estructura real. Si se someten varios sistemas con diferentes periodos a un movimiento del terreno, cada uno responderá de manera diferente; la amplitud de su respuesta depende esencialmente de la relación entre el periodo de la estructura y el periodo dominante del movimiento del suelo $\left(T_{E} / T_{S}\right)$. La resonancia ocurre cuando esta relación esta cerca de la unidad, ya que la amplitud de la respuesta es mayor. Por ello, es conveniente evitar esta situación en los edificios, alejando el valor $T_{E}$ del $T_{S}$, ya que de ser así, estarían sujetos en cada sismo fuerzas grandes. Es recomendable procurar que la relación esté fuera del rango indicado en la Ecuación 5.2.1

$$
0,7 \leq \frac{T_{E}}{T_{S}} \leq 1,2
$$

Por lo general cuando el movimiento del terreno es lento, con periodos dominantes largos, son las estructuras altas y flexibles donde se amplifican las vibraciones y generan aceleraciones más elevadas y por ende fuerzas sísmicas mayores. Por el contrario, movimiento de periodo corto afectan más a las estructuras bajas y rígidas. 


\subsubsection{Periodos de vibración sin disipadores obtenidos con el programa IDARC}

En la Tabla 5.2.19 se exponen las frecuencias y periodos de vibración obtenidos del análisis modal para cada uno de los modos de vibración.

Tabla 5.2.19: Frecuencias y periodos de vibración sin disipadores obtenidos con IDARC para cada uno de los

\begin{tabular}{|c|c|c|c|c|c|c|}
\hline \multirow[b]{2}{*}{ Modo } & \multicolumn{2}{|c|}{ Pórtico pa2pz2-1 } & \multicolumn{2}{|c|}{ Pórtico pa4pz2-1 } & \multicolumn{2}{|c|}{ Pórtico pa6pz2-1 } \\
\hline & $\begin{array}{c}\text { Frecuencia } \\
(\mathrm{Hz})\end{array}$ & $\begin{array}{c}\text { Periodo } \\
\text { (s) }\end{array}$ & $\begin{array}{c}\text { Frecuencia } \\
(\mathrm{Hz})\end{array}$ & $\begin{array}{c}\text { Periodo } \\
\text { (s) }\end{array}$ & $\begin{array}{c}\text { Frecuencia } \\
(\mathrm{Hz})\end{array}$ & $\begin{array}{l}\text { Periodo } \\
\text { (s) }\end{array}$ \\
\hline 1 & 0.73348 & 1.36336 & 0.71982 & 1.38923 & 0.55290 & 1.80865 \\
\hline 2 & 2.23037 & 0.44836 & 2.19904 & 0.45474 & 1.40418 & 0.71216 \\
\hline 3 & 4.01774 & 0.24890 & 3.97820 & 0.25137 & 2.45124 & 0.40796 \\
\hline 4 & --- & --- & --- & --- & 3.88800 & 0.25720 \\
\hline 5 & --- & --- & $\begin{array}{ll}-- \\
\end{array}$ & --- & 5.52625 & 0.18095 \\
\hline 6 & --- & $-\cdots$ & --- & --- & 7.48868 & 0.13353 \\
\hline $\begin{array}{l}\text { Periodo fund. } \\
{ }_{f} T_{1}(s)\end{array}$ & \multicolumn{2}{|c|}{1.36336} & \multicolumn{2}{|c|}{1.38923} & \multicolumn{2}{|c|}{1.80865} \\
\hline
\end{tabular}

5.2.5.2. Periodos de vibración sin disipadores obtenidos con el programa LumpST

- Pórtico pa2pz2-1

Peso de una planta: $P=[118,75+(162,5 \times 3)+118,75] K N=725 K N$

Masa de una planta: $m=\frac{725000 \mathrm{~N}}{981 \mathrm{~cm} / \mathrm{s}^{2}}=739,04 \mathrm{Ns}^{2} / \mathrm{cm}$

Rigidez de cada una de las plantas:

$$
\begin{aligned}
& \text { Planta 3a: }{ }_{f} k_{3}=5,5 \mathrm{KN} / \mathrm{mm}=55000 \mathrm{~N} / \mathrm{cm} \\
& \text { Planta 2a: }{ }_{f} k_{2}=6,8 \mathrm{KN} / \mathrm{mm}=68000 \mathrm{~N} / \mathrm{cm} \\
& \text { Planta 1: }{ }_{f} k_{1}=9,9 \mathrm{KN} / \mathrm{mm}=99000 \mathrm{~N} / \mathrm{cm}
\end{aligned}
$$

Fichero model.dat programa LumpST:

$1,1,4,739.04,0,99000,1000 ., 0.01$

$2,1,4, \quad 0 ., \quad 0, \quad 0.1, \quad 0.1, \quad 0.01$

$3,2,1,739.04,0,68000,1000 ., 0.01$

$4,2,1, \quad 0 ., \quad 0, \quad 0.1, \quad 0.1, \quad 0.01$

$5,3,2,739.04,0,55000,1000 ., 0.01$

$6,3,2, \quad 0 ., \quad 0, \quad 0.1, \quad 0.1, \quad 0.01$

Las frecuencias para cada modo de vibración expresadas en (rad/s) para el pórtico pa2pz2-1 son: 
Tabla 5.2.20: Frecuencias sin disipadores expresadas en (rad/s) obtenidas con LumpST para el pórtico pa2pz2-1

\begin{tabular}{c|c} 
Modo & Frcuencia $(\mathrm{rad} / \mathrm{s})$ \\
\hline 1 & $.4599 \mathrm{D}+01$ \\
\hline 2 & $.1198 \mathrm{D}+02$ \\
\hline 3 & $.1738 \mathrm{D}+02$ \\
\hline
\end{tabular}

Obtenidas las frecuencias, podemos calcular los periodos de vibración para cada uno de los modos, mediante la siguiente ecuación:

$$
{ }_{f} f_{i}=\frac{{ }_{f} w_{i}}{2 \pi} \Rightarrow{ }_{f} T_{i}=\frac{1}{{ }_{f} f_{i}}
$$

Siendo:

${ }_{f} f_{i}$ : Frecuencia sin disipador para un determinado modo de vibración expresada en $(\mathrm{Hz})$

${ }_{f} W_{i}:$ Frecuencia sin disipador para un determinado modo de vibración expresada en ( $\mathrm{rad} / \mathrm{s}$ )

${ }_{f} T_{i}$ : Periodo de vibración sin disipador para un determinado modo de vibración expresado en (s)

- Modo 1:

$$
\begin{aligned}
& { }_{f} w_{1}=4,599 \mathrm{rad} / \mathrm{s} \\
& { }_{f} f_{1}=\frac{{ }_{f} w_{1}}{2 \pi}=\frac{4,599}{2 \pi}=0,73195 \mathrm{~Hz} \Rightarrow{ }_{f} T_{1}=\frac{1}{{ }_{f} f_{1}}=\frac{1}{0,73195} \Rightarrow{ }_{f} T_{1}=1,36621 \mathrm{~s}
\end{aligned}
$$

- Modo 2:

$$
\begin{aligned}
& { }_{f} w_{2}=11,98 \mathrm{rad} / \mathrm{s} \\
& { }_{f} f_{2}=\frac{{ }_{f} w_{2}}{2 \pi}=\frac{11,98}{2 \pi}=1,90668 \mathrm{~Hz} \Rightarrow{ }_{f} T_{2}=\frac{1}{{ }_{f} f_{2}}=\frac{1}{1,90668} \Rightarrow{ }_{f} T_{2}=0,52447 \mathrm{~s}
\end{aligned}
$$

- Modo 3:

$$
\begin{aligned}
& { }_{f} w_{3}=17,38 \mathrm{rad} / \mathrm{s} \\
& { }_{f} f_{3}=\frac{{ }_{f} w_{3}}{2 \pi}=\frac{17,38}{2 \pi}=2,76611 \mathrm{~Hz} \Rightarrow{ }_{f} T_{3}=\frac{1}{{ }_{f} f_{3}}=\frac{1}{2,76611} \Rightarrow{ }_{f} T_{3}=0,36152 \mathrm{~s}
\end{aligned}
$$

Tabla 5.2.21: Frecuencias y periodos de vibración sin disipadores obtenidos con LumpST para el pórtico pa2pz2-1

\begin{tabular}{c|c|c} 
& \multicolumn{2}{|c}{ Pórtico pa2pz2-1 } \\
\hline Modo & $\begin{array}{c}\text { Frecuencia } \\
(\mathrm{Hz})\end{array}$ & $\begin{array}{c}\text { Periodo } \\
(\mathrm{s})\end{array}$ \\
\hline 1 & 0.73185 & 1.36621 \\
\hline 2 & 1.90668 & 0.52447 \\
\hline 3 & 2.76610 & 0.36152 \\
\hline \multicolumn{3}{|c}{1.36621} \\
\hline $\begin{array}{c}\text { Periodo fund. } \\
\mathrm{fT}_{1}(\mathrm{~s})\end{array}$ & \multicolumn{2}{|c}{} \\
\hline
\end{tabular}


El error cometido para los periodos de vibración obtenidos con los programas IDARC y LumpST es el siguiente:

- $\quad$ Periodo Modo 1:

$$
\frac{(1,36621-1,36336)}{1,36336} \times 100=0,21 \%
$$

- Periodo Modo 2:

$$
\frac{(0,52447-0,44836)}{0,44836} \times 100=16,97 \%
$$

- Periodo Modo 3:

$$
\frac{(0,36152-0,24890)}{0,24890} \times 100=45,25 \%
$$

Vemos que el error cometido para los periodos fundamentales ${ }_{f} T_{1}$, correspondientes al modo 1 de vibración, obtenidos con los programas IDARC y LumpST es pequeño.

\section{- Pórtico pa4pz2-1}

Peso de una planta: $P=[139,56+(196,625 \times 3)+139,56] K N=868,995 K N$

Masa de una planta: $m=\frac{868995 \mathrm{~N}}{981 \mathrm{~cm} / \mathrm{s}^{2}}=885,82 \mathrm{Ns} / \mathrm{cm}$

Rigidez de cada una de las plantas:

$$
\begin{aligned}
& \text { Planta 3a: }{ }_{f} k_{3}=7,7 \mathrm{KN} / \mathrm{mm}=77000 \mathrm{~N} / \mathrm{cm} \\
& \text { Planta 2a: }{ }_{f} k_{2}=9,1 \mathrm{KN} / \mathrm{mm}=91000 \mathrm{~N} / \mathrm{cm} \\
& \text { Planta 1ㅁ: }{ }_{f} k_{1}=9,5 \mathrm{KN} / \mathrm{mm}=95000 \mathrm{~N} / \mathrm{cm}
\end{aligned}
$$

Fichero model.dat programa LumpST:

$1,1,4,885.82,0,95000,1000 ., 0.01$

$2,1,4, \quad 0 ., \quad 0, \quad 0.1, \quad 0.1, \quad 0.01$

$3,2,1,885.82,0,91000,1000 ., 0.01$

$4,2,1, \quad 0 ., \quad 0, \quad 0.1, \quad 0.1, \quad 0.01$

$5,3,2,885.82,0,77000,1000 ., 0.01$

$6,3,2, \quad 0 ., \quad 0, \quad 0.1, \quad 0.1, \quad 0.01$

Las frecuencias para cada modo de vibración expresadas en (rad/s) para el pórtico pa6pz2-1 son: 
Tabla 5.2.22: Frecuencias sin disipadores expresadas en (rad/s) obtenidas con LumpST para el pórtico pa4pz2-1

\begin{tabular}{c|c} 
Modo & Frcuencia (rad/s) \\
\hline 1 & $.4517 \mathrm{D}+01$ \\
\hline 2 & $.1213 \mathrm{D}+02$ \\
\hline 3 & $.1786 \mathrm{D}+02$ \\
\hline
\end{tabular}

De igual forma, obtenidas las frecuencias, podemos calcular los periodos de vibración para cada uno de los modos, mediante la ecuación (5.2.2):

- Modo 1:

$$
\begin{aligned}
& { }_{f} w_{1}=4,517 \mathrm{rad} / \mathrm{s} \\
& { }_{f} f_{1}=\frac{{ }^{w_{1}}}{2 \pi}=\frac{4,517}{2 \pi}=0,71890 \mathrm{~Hz} \Rightarrow{ }_{f} T_{1}=\frac{1}{{ }_{f} f_{1}}=\frac{1}{0,71890} \Rightarrow{ }_{f} T_{1}=1,39101 \mathrm{~s}
\end{aligned}
$$

- Modo 2:

$$
\begin{aligned}
& { }_{f} w_{2}=12,13 \mathrm{rad} / \mathrm{s} \\
& { }_{f} f_{2}=\frac{{ }_{s} w_{2}}{2 \pi}=\frac{12,13}{2 \pi}=1,93055 \mathrm{~Hz} \Rightarrow{ }_{f} T_{2}=\frac{1}{{ }_{f} f_{2}}=\frac{1}{1,93055} \Rightarrow{ }_{f} T_{2}=0,51799 \mathrm{~s}
\end{aligned}
$$

- Modo 3:

$$
\begin{aligned}
& { }_{f} w_{3}=17,86 \mathrm{rad} / \mathrm{s} \\
& { }_{f} f_{3}=\frac{{ }_{f} w_{3}}{2 \pi}=\frac{17,86}{2 \pi}=2,84251 \mathrm{~Hz} \Rightarrow{ }_{f} T_{3}=\frac{1}{{ }_{f} f_{3}}=\frac{1}{2,84251} \Rightarrow{ }_{f} T_{3}=0,35180 \mathrm{~s}
\end{aligned}
$$

Tabla 5.2.23: Frecuencias y periodos de vibración sin disipadores obtenidos con LumpST para el pórtico pa4pz2-1

\begin{tabular}{c|c|c} 
& \multicolumn{2}{|c}{ Pórtico pa4pz2-1 } \\
\hline Modo & $\begin{array}{c}\text { Frecuencia } \\
(\mathrm{Hz})\end{array}$ & $\begin{array}{c}\text { Periodo } \\
(\mathrm{s})\end{array}$ \\
\hline 1 & 0.71982 & 1.38923 \\
\hline 2 & 2.19904 & 0.45474 \\
\hline 3 & 3.97820 & 0.25137 \\
\hline $\begin{array}{c}\text { Periodo fund. } \\
\mathrm{f}_{1}(\mathrm{~s})\end{array}$ & \multicolumn{2}{|c}{1.38923} \\
\hline
\end{tabular}

El error cometido para los periodos de vibración obtenidos con los programas IDARC y LumpST es el siguiente:

- $\quad$ Periodo Modo 1:

$$
\frac{(1,39101-1,38923)}{1,38923} \times 100=0,13 \%
$$


- $\quad$ Periodo Modo 2:

$$
\frac{(0,51799-0,45474)}{0,45474} \times 100=13,91 \%
$$

- Periodo Modo 3:

$$
\frac{(0,35180-0,25137)}{0,25137} \times 100=39,95 \%
$$

También en este caso el error cometido para los periodos fundamentales ${ }_{f} T_{1}$, correspondientes al modo 1 de vibración, obtenidos con los programas IDARC y LumpST es pequeño.

- Pórtico pa6pz2-1

Peso de una planta: $P=[118,75+(162,5 \times 3)+118,75] K N=725 K N$

Masa de una planta: $m=\frac{725000 \mathrm{~N}}{981 \mathrm{~cm} / \mathrm{s}^{2}}=739,04 \mathrm{Ns}^{2} / \mathrm{cm}$

Rigidez de cada una de las plantas:

$$
\begin{aligned}
& \text { Planta 6a: }{ }_{f} k_{6}=5,6 \mathrm{KN} / \mathrm{mm}=56000 \mathrm{~N} / \mathrm{cm} \\
& \text { Planta 5ㄹ: }{ }_{f} k_{5}=8,3 \mathrm{KN} / \mathrm{mm}=83000 \mathrm{~N} / \mathrm{cm} \\
& \text { Planta 4ㄹ: }{ }_{f} k_{4}=11,3 \mathrm{KN} / \mathrm{mm}=113000 \mathrm{~N} / \mathrm{cm} \\
& \text { Planta 3a: }{ }_{f} k_{3}=17,7 \mathrm{KN} / \mathrm{mm}=177000 \mathrm{~N} / \mathrm{cm} \\
& \text { Planta 2a: }{ }_{f} k_{2}=22,4 \mathrm{KN} / \mathrm{mm}=224000 \mathrm{~N} / \mathrm{cm} \\
& \text { Planta 1ㅁ: }{ }_{f} k_{1}=17,5 \mathrm{KN} / \mathrm{mm}=175000 \mathrm{~N} / \mathrm{cm}
\end{aligned}
$$

Fichero model.dat programa LumpST:

$1,1,7,739.04,0,175000,1000 ., 0.01$

$2,1,7, \quad 0 ., \quad 0, \quad 0.1, \quad 0.1, \quad 0.01$

$3,2,1,739.04,0,224000,1000 ., 0.01$

$4,2,1, \quad 0 ., \quad 0, \quad 0.1, \quad 0.1, \quad 0.01$

$5,3,2,739.04,0,177000,1000 ., 0.01$

$6,3,2, \quad 0 ., \quad 0, \quad 0.1, \quad 0.1, \quad 0.01$

$7,4,3,739.04,0,133000,1000 ., 0.01$

$8,4,3, \quad 0 ., \quad 0, \quad 0.1, \quad 0.1, \quad 0.01$

$9,5,4,739.04,0,83000,1000 ., 0.01$

$10,5,4, \quad 0 ., \quad 0, \quad 0.1, \quad 0.1, \quad 0.01$

$11,6,5,739.04,0,56000,1000 ., 0.01$

$\begin{array}{llllll}12,6,5, & 0 ., \quad 0, & 0.1, & 0.1, & 0.01\end{array}$

Las frecuencias para cada modo de vibración expresadas en (rad/s) para el pórtico pa6pz2-1 son: 
Tabla 5.2.24: Frecuencias sin disipadores expresadas en (rad/s) obtenidas con LumpST para el pórtico pa6pz2-1

\begin{tabular}{c|c} 
Modo & Frcuencia $(\mathrm{rad} / \mathrm{s})$ \\
\hline 1 & $.3520 \mathrm{D}+01$ \\
\hline 2 & $.8445 \mathrm{D}+01$ \\
\hline 3 & $.1350 \mathrm{D}+02$ \\
\hline 4 & $.1794 \mathrm{D}+02$ \\
\hline 5 & $.2354 \mathrm{D}+02$ \\
\hline 6 & $.3027 \mathrm{D}+02$ \\
\hline
\end{tabular}

Calculamos ahora los periodos de vibración para cada uno de los modos, mediante la ecuación (5.2.2):

- Modo 1:

$$
\begin{aligned}
& { }_{f} w_{1}=3,520 \mathrm{rad} / \mathrm{s} \\
& { }_{f} f_{1}=\frac{{ }_{f} w_{1}}{2 \pi}=\frac{3,520}{2 \pi}=0,56022 \mathrm{~Hz} \Rightarrow{ }_{f} T_{1}=\frac{1}{{ }_{f} f_{1}}=\frac{1}{0,56022} \Rightarrow{ }_{f} T_{1}=1,78499 \mathrm{~s}
\end{aligned}
$$

- Modo 2:

$$
\begin{aligned}
& { }_{f} w_{2}=8,445 \mathrm{rad} / \mathrm{s} \\
& { }_{f} f_{2}=\frac{{ }_{f} w_{2}}{2 \pi}=\frac{8,455}{2 \pi}=1,34565 \mathrm{~Hz} \Rightarrow{ }_{f} T_{2}=\frac{1}{{ }_{f} f_{2}}=\frac{1}{1,34565} \Rightarrow{ }_{f} T_{2}=0,74313 \mathrm{~s}
\end{aligned}
$$

- Modo 3:

$$
\begin{aligned}
& { }_{f} w_{3}=13,50 \mathrm{rad} / \mathrm{s} \\
& { }_{f} f_{3}=\frac{{ }_{f} w_{3}}{2 \pi}=\frac{13,50}{2 \pi}=2,14859 \mathrm{~Hz} \Rightarrow{ }_{f} T_{3}=\frac{1}{{ }_{f} f_{3}}=\frac{1}{2,14859} \Rightarrow{ }_{f} T_{3}=0,46542 \mathrm{~s}
\end{aligned}
$$

- Modo 4:

$$
\begin{aligned}
& { }_{f} w_{4}=17,94 \mathrm{rad} / \mathrm{s} \\
& { }_{f} f_{4}=\frac{{ }_{f} w_{4}}{2 \pi}=\frac{17,94}{2 \pi}=2,85524 \mathrm{~Hz} \Rightarrow{ }_{f} T_{4}=\frac{1}{{ }_{f} f_{4}}=\frac{1}{2,85524} \Rightarrow{ }_{f} T_{4}=0,35023 \mathrm{~s}
\end{aligned}
$$

- Modo 5:

$$
\begin{aligned}
& { }_{f} w_{5}=23,54 \mathrm{rad} / \mathrm{s} \\
& { }_{f} f_{5}=\frac{{ }_{f} w_{5}}{2 \pi}=\frac{23,54}{2 \pi}=3,74651 \mathrm{~Hz} \Rightarrow{ }_{f} T_{5}=\frac{1}{{ }_{f} f_{5}}=\frac{1}{3,74651} \Rightarrow{ }_{f} T_{5}=0,26691 \mathrm{~s}
\end{aligned}
$$

- Modo 6:

$$
\begin{aligned}
& { }_{f} w_{6}=30,27 \mathrm{rad} / \mathrm{s} \\
& { }_{f} f_{6}=\frac{{ }_{f} w_{6}}{2 \pi}=\frac{30,27}{2 \pi}=4,81762 \mathrm{~Hz} \Rightarrow{ }_{f} T_{6}=\frac{1}{{ }_{f} f_{6}}=\frac{1}{4,81762} \Rightarrow{ }_{f} T_{6}=0,20757 \mathrm{~s}
\end{aligned}
$$


Tabla 5.2.25: Frecuencias y periodos de vibración sin disipadores obtenidos con LumpST para el pórtico pa6pz2-1

\begin{tabular}{c|c|c} 
& \multicolumn{2}{|c}{ Pórtico pa4pz2-1 } \\
\hline Modo & $\begin{array}{c}\text { Frecuencia } \\
(\mathrm{Hz})\end{array}$ & $\begin{array}{c}\text { Periodo } \\
(\mathrm{s})\end{array}$ \\
\hline 1 & 0.55290 & 1.80865 \\
\hline 2 & 1.40418 & 0.71216 \\
\hline 3 & 2.45124 & 0.40796 \\
\hline 4 & 3.88800 & 0.25720 \\
\hline 5 & 5.52625 & 0.18095 \\
\hline 6 & 7.48868 & 0.13353 \\
\hline $\begin{array}{c}\mid c \\
\text { Periodo fund. } \\
\mathrm{f}_{1} \text { (s) }\end{array}$
\end{tabular}

El error cometido para los periodos de vibración obtenidos con los programas IDARC y LumpST es el siguiente:

- Periodo Modo 1:

$$
\frac{(1,80865-1,78499)}{1,78499} \times 100=1,32 \%
$$

- Periodo Modo 2:

$$
\frac{(0,74313-0,71216)}{0,71216} \times 100=4,35 \%
$$

- Periodo Modo 3:

$$
\frac{(0,46542-0,40796)}{0,40796} \times 100=14,08 \%
$$

- Periodo Modo 4:

$$
\frac{(0,35023-0,25720)}{0,25720} \times 100=36,17 \%
$$

- Periodo Modo 5:

$$
\frac{(0,26691-0,18095)}{0,18095} \times 100=47,50 \%
$$

- Periodo Modo 6:

$$
\frac{(0,20757-0,13353)}{0,13353} \times 100=55,45 \%
$$


De igual forma el error cometido para los periodos fundamentales ${ }_{f} T_{1}$, correspondientes al modo 1 de vibración, obtenidos con los programas IDARC y LumpST es pequeño.

\subsubsection{Tabla resumen periodos de vibración sin disipadores para cada uno de los pórticos}

Tabla 5.2.26: Comparación periodos de vibración sin disipadores, obtenidos con IDARC y LumpST, pórtico pa2pz2-1

\begin{tabular}{|c|c|c|c|c|c|c|}
\hline & \multicolumn{6}{|c|}{ pórtico pa2pz2-1 } \\
\hline & IDARC & Lump & IDARC & Lump & IDARC & Lump \\
\hline \multirow{2}{*}{$\begin{array}{c}\text { Periodo } \\
\text { (s) }\end{array}$} & \multicolumn{2}{|c|}{$\mathrm{T}_{1}$} & \multicolumn{2}{|c|}{$T_{2}$} & \multicolumn{2}{|c|}{$\mathrm{T}_{3}$} \\
\hline & 1,36336 & 1,36621 & 0,44836 & 0,52447 & 0,24890 & 0,36152 \\
\hline $\begin{array}{c}\text { Error } \\
(\%)\end{array}$ & \multicolumn{2}{|c|}{0,21} & \multicolumn{2}{|c|}{16,97} & \multicolumn{2}{|c|}{45,25} \\
\hline
\end{tabular}

Tabla 5.2.27: Comparación periodos de vibración sin disipadores, obtenidos con IDARC y LumpST, pórtico pa4pz2-1

\begin{tabular}{|c|c|c|c|c|c|c|}
\hline & \multicolumn{6}{|c|}{ pórtico pa4pz2-1 } \\
\hline & IDARC & Lump & IDARC & Lump & IDARC & Lump \\
\hline \multirow{2}{*}{$\begin{array}{l}\text { Periodo } \\
\text { (s) }\end{array}$} & \multicolumn{2}{|c|}{$\mathrm{T}_{1}$} & \multicolumn{2}{|c|}{$\mathrm{T}_{2}$} & \multicolumn{2}{|c|}{$\mathrm{T}_{3}$} \\
\hline & 1,38923 & 1,39101 & 0,45474 & 0,51799 & 0,25137 & 0,35180 \\
\hline $\begin{array}{c}\text { Error } \\
(\%)\end{array}$ & \multicolumn{2}{|c|}{0,13} & \multicolumn{2}{|c|}{13,91} & \multicolumn{2}{|c|}{39,95} \\
\hline
\end{tabular}

Tabla 5.2.28: Comparación periodos de vibración sin disipadores, obtenidos con IDARC y LumpST, pórtico pa6pz2-1

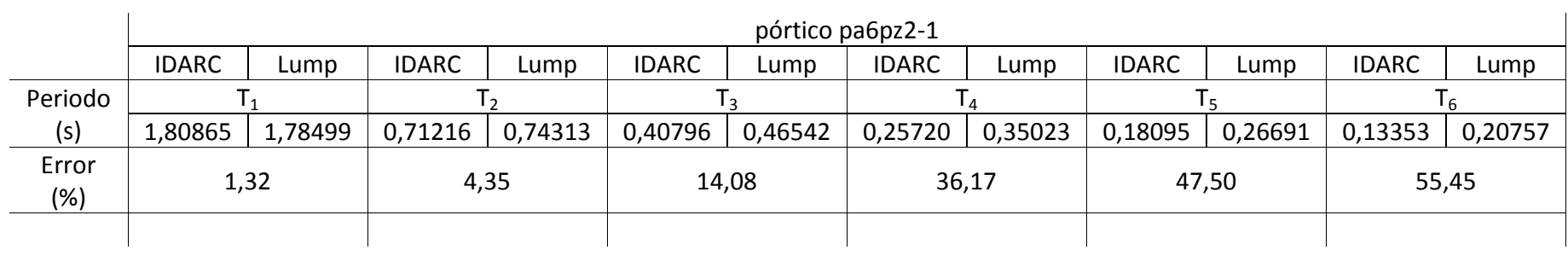

\subsubsection{Periodos de vibración con disipadores obtenidos con el programa LumpST}

- Pórtico pa2pz2-1

Peso de una planta: $P=[118,75+(162,5 \times 3)+118,75] K N=725 K N$

Masa de una planta: $m=\frac{725000 \mathrm{~N}}{981 \mathrm{~cm} / \mathrm{s}^{2}}=739,04 \mathrm{Ns}^{2} / \mathrm{cm}$

Rigidez de cada una de las plantas:

$$
\begin{aligned}
& \text { Planta 3a: }{ }_{s} k_{3}=(55000 \mathrm{~N} / \mathrm{cm}) \times 5=275000 \mathrm{~N} / \mathrm{cm} \\
& \text { Planta 2a: }{ }_{s} k_{2}=(68000 \mathrm{~N} / \mathrm{cm}) \times 5=340000 \mathrm{~N} / \mathrm{cm} \\
& \text { Planta 1a: }{ }_{s} k_{1}=(99000 \mathrm{~N} / \mathrm{cm}) \times 5=495000 \mathrm{~N} / \mathrm{cm}
\end{aligned}
$$


Fichero model.dat programa LumpST:

$1,1,4,739.04,0,99000,2.3, \quad 0.01$

$2,1,4, \quad 0 ., \quad 0,495000,0.4883,0.01$

$3,2,1,739.04,0,68000,3.2, \quad 0.01$

$4,2,1, \quad 0 ., \quad 0,340000,0.5937,0.01$

$5,3,2,739.04,0,55000, \quad 2.2, \quad 0.01$

$6,3,2, \quad 0 ., \quad 0,275000,0.4454,0.01$

Las frecuencias para cada modo de vibración expresadas en (rad/s) para el pórtico pa2pz2-1 son:

Tabla 5.2.29: Frecuencias con disipadores expresadas en (rad/s) obtenidas con LumpST para el pórtico pa2pz2-1

\begin{tabular}{c|c} 
Modo & Frcuencia $(\mathrm{rad} / \mathrm{s})$ \\
\hline 1 & $.1126 \mathrm{D}+02$ \\
\hline 2 & $.2936 \mathrm{D}+02$ \\
\hline 3 & $.4257 \mathrm{D}+02$ \\
\hline
\end{tabular}

Obtenidas las frecuencias, podemos calcular los periodos de vibración para cada uno de los modos, mediante la siguiente ecuación:

Siendo:

$$
{ }_{s} f_{i}=\frac{{ }_{s} w_{i}}{2 \pi} \Rightarrow{ }_{s} T_{i}=\frac{1}{{ }_{s} f_{i}}
$$

${ }_{s} f_{i}$ : Frecuencia con disipador para un determinado modo de vibración expresada en $(\mathrm{Hz})$

${ }_{s} w_{i}$ : Frecuencia con disipador para un determinado modo de vibración expresada en ( $\mathrm{rad} / \mathrm{s}$ )

${ }_{s} T_{i}$ : Periodo de vibración con disipador para un determinado modo de vibración expresado en(s)

- Modo 1:

$$
\begin{aligned}
& { }_{s} w_{1}=11,26 \mathrm{rad} / \mathrm{s} \\
& { }_{s} f_{1}=\frac{{ }_{s} w_{1}}{2 \pi}=\frac{11,26}{2 \pi}=1,79208 \mathrm{~Hz} \Rightarrow{ }_{s} T_{1}=\frac{1}{{ }_{s} f_{1}}=\frac{1}{1,79208} \Rightarrow{ }_{s} T_{1}=0,55801 \mathrm{~s}
\end{aligned}
$$

- Modo 2:

$$
\begin{aligned}
& { }_{s} w_{2}=29,36 \mathrm{rad} / \mathrm{s} \\
& { }_{s} f_{2}=\frac{{ }_{s} w_{2}}{2 \pi}=\frac{29,36}{2 \pi}=4,67279 \mathrm{~Hz} \Rightarrow{ }_{s} T_{2}=\frac{1}{{ }_{s} f_{2}}=\frac{1}{4,67279} \Rightarrow{ }_{s} T_{2}=0,21400 \mathrm{~s}
\end{aligned}
$$

- Modo 3:

$$
\begin{aligned}
& { }_{s} w_{3}=42,57 \mathrm{rad} / \mathrm{s} \\
& { }_{s} f_{3}=\frac{{ }_{s} w_{3}}{2 \pi}=\frac{42,57}{2 \pi}=6,77522 \mathrm{~Hz} \Rightarrow{ }_{s} T_{3}=\frac{1}{{ }_{s} f_{3}}=\frac{1}{6,77522} \Rightarrow{ }_{s} T_{3}=0,14760 \mathrm{~s}
\end{aligned}
$$


Tabla 5.2.30: Frecuencias y periodos de vibración con disipadores para el pórtico pa2pz2-1

\begin{tabular}{c|c|c} 
& \multicolumn{2}{|c}{ Pórtico pa2pz2-1 } \\
\hline Modo & $\begin{array}{c}\text { Frecuencia } \\
(\mathrm{Hz})\end{array}$ & $\begin{array}{c}\text { Periodo } \\
(\mathrm{s})\end{array}$ \\
\hline 1 & 1,79208 & 0,55801 \\
\hline 2 & 4,67279 & 0,21400 \\
\hline 3 & 6,77522 & 0,14760 \\
\hline $\begin{array}{c}\text { Periodo fund. } \\
{ }_{\mathrm{s}} \mathrm{T}_{1}(\mathrm{~s})\end{array}$
\end{tabular}

- Pórtico pa4pz2-1

Peso de una planta: $P=[139,56+(196,625 \times 3)+139,56] K N=868,995 K N$

Masa de una planta: $m=\frac{868995 \mathrm{~N}}{981 \mathrm{~cm} / \mathrm{s}^{2}}=885,82 \mathrm{Ns}^{2} / \mathrm{cm}$

Rigidez de cada una de las plantas:

$$
\begin{aligned}
& \text { Planta 3a: }{ }_{s} k_{3}=(77000 \mathrm{~N} / \mathrm{cm}) \times 5=385000 \mathrm{~N} / \mathrm{cm} \\
& \text { Planta 2a: }{ }_{s} k_{2}=(91000 \mathrm{~N} / \mathrm{cm}) \times 5=455000 \mathrm{~N} / \mathrm{cm} \\
& \text { Planta 1ㅁ: }{ }_{s} k_{1}=(95000 \mathrm{~N} / \mathrm{cm}) \times 5=475000 \mathrm{~N} / \mathrm{cm}
\end{aligned}
$$

Fichero model.dat programa LumpST:

1,1,4, 885.82, 0, 95000, 3.3., $\quad 0.01$

$2,1,4, \quad 0 ., \quad 0,475000,0.0001,0.01$

$3,2,1,885.82,0,91000,2.4, \quad 0.01$

$4,2,1, \quad 0 ., \quad 0,455000,0.1502,0.01$

$5,3,2,885.82,0,77000,1.65, \quad 0.01$

$6,3,2, \quad 0 ., \quad 0,385000,0.0001,0.01$

Las frecuencias para cada modo de vibración expresadas en (rad/s) para el pórtico pa4pz2-1 son:

Tabla 5.2.31: Frecuencias con disipadores expresadas en ( $\mathrm{rad} / \mathrm{s}$ ) obtenidas con LumpST para el pórtico pa4pz2-1

\begin{tabular}{c|c} 
Modo & Frcuencia (rad/s) \\
\hline 1 & $.1106 \mathrm{D}+02$ \\
\hline 2 & $.2971 \mathrm{D}+02$ \\
\hline 3 & $.4375 \mathrm{D}+02$ \\
\hline
\end{tabular}

De igual forma, obtenidas las frecuencias, podemos calcular los periodos de vibración para cada uno de los modos, mediante la ecuación (5.2.3):

- Modo 1:

$$
\begin{aligned}
& { }_{s} w_{1}=11,06 \mathrm{rad} / \mathrm{s} \\
& { }_{s} f_{1}=\frac{{ }_{s} w_{1}}{2 \pi}=\frac{11,06}{2 \pi}=1,76025 \mathrm{~Hz} \Rightarrow{ }_{s} T_{1}=\frac{1}{{ }_{s} f_{1}}=\frac{1}{1,76025} \Rightarrow{ }_{s} T_{1}=0,56810 \mathrm{~s}
\end{aligned}
$$


- Modo 2:

$$
\begin{aligned}
& { }_{s} w_{2}=29,71 \mathrm{rad} / \mathrm{s} \\
& { }_{s} f_{2}=\frac{{ }_{s} w_{2}}{2 \pi}=\frac{29,71}{2 \pi}=4,72849 \mathrm{~Hz} \Rightarrow{ }_{s} T_{2}=\frac{1}{{ }_{s} f_{2}}=\frac{1}{4,72849} \Rightarrow{ }_{s} T_{2}=0,21148 \mathrm{~s}
\end{aligned}
$$

- Modo 3:

$$
\begin{aligned}
& { }_{s} w_{3}=43,75 \mathrm{rad} / \mathrm{s} \\
& { }_{s} f_{3}=\frac{{ }_{s} w_{3}}{2 \pi}=\frac{43,75}{2 \pi}=6,96303 \mathrm{~Hz} \Rightarrow{ }_{s} T_{3}=\frac{1}{{ }_{s} f_{3}}=\frac{1}{6,96303} \Rightarrow{ }_{s} T_{3}=0,14361 \mathrm{~s}
\end{aligned}
$$

Tabla 5.2.32: Frecuencias y periodos de vibración con disipadores para el pórtico pa4pz2-1

\begin{tabular}{c|c|c} 
& \multicolumn{2}{|c}{ Pórtico pa4pz2-1 } \\
\hline Modo & $\begin{array}{c}\text { Frecuencia } \\
(\mathrm{Hz})\end{array}$ & $\begin{array}{c}\text { Periodo } \\
(\mathrm{s})\end{array}$ \\
\hline 1 & 1.76025 & 0.56810 \\
\hline 2 & 4.72849 & 0.21148 \\
\hline 3 & 6.96303 & 0.14361 \\
\hline $\begin{array}{c}\text { Periodo fund. } \\
\mathrm{f}_{1}(\mathrm{~s})\end{array}$ & \multicolumn{2}{|c}{0.56810} \\
\hline
\end{tabular}

- Pórtico pa6pz2-1

Peso de una planta: $P=[118,75+(162,5 \times 3)+118,75] K N=725 K N$

Masa de una planta: $m=\frac{725000 \mathrm{~N}}{981 \mathrm{~cm} / \mathrm{s}^{2}}=739,04 \mathrm{Ns}^{2} / \mathrm{cm}$

Rigidez de cada una de las plantas:

$$
\begin{aligned}
& \text { Planta 6a: }{ }_{s} k_{6}=(56000 \mathrm{~N} / \mathrm{cm}) \times 5=280000 \mathrm{~N} / \mathrm{cm} \\
& \text { Planta 5a: }{ }_{s} k_{5}=(83000 \mathrm{~N} / \mathrm{cm}) \times 5=415000 \mathrm{~N} / \mathrm{cm} \\
& \text { Planta 4a: }{ }_{s} k_{4}=(113000 \mathrm{~N} / \mathrm{cm}) \times 5=565000 \mathrm{~N} / \mathrm{cm} \\
& \text { Planta 3a: }{ }_{s} k_{3}=(177000 N / \mathrm{cm}) \times 5=885000 N / \mathrm{cm} \\
& \text { Planta 2a: }{ }_{s} k_{2}=(224000 N / \mathrm{cm}) \times 5=1120000 N / \mathrm{cm} \\
& \text { Planta 1a: }{ }_{s} k_{1}=(175000 N / \mathrm{cm}) \times 5=875000 N / \mathrm{cm}
\end{aligned}
$$

Fichero model.dat programa LumpST:

$1,1,7,739.04,0,175000,0.9, \quad 0.01$

$2,1,7, \quad 0 ., \quad 0,875000,0.400,0.01$

$3,2,1,739.04,0,224000,0.7, \quad 0.01$

$4,2,1, \quad 0 ., \quad 0,1120000,0.240,0.01$

$5,3,2,739.04,0,177000,0.8,0.01$

$6,3,2, \quad 0 ., \quad 0,885000,0.240,0.01$

$7,4,3,739.04,0,113000,1.0, \quad 0.01$ 
$8,4,3, \quad 0 ., \quad 0,565000,0.365,0.01$

$9,5,4,739.04,0,83000,2.0, \quad 0.01$

$10,5,4, \quad 0 ., \quad 0,415000,0.280,0.01$

$11,6,5,739.04,0,56000,1.6,0.01$

$12,6,5, \quad 0 ., \quad 0,280000,0.260,0.01$

Las frecuencias para cada modo de vibración expresadas en (rad/s) para el pórtico pa6pz2-1 son:

Tabla 5.2.33: Frecuencias con disipadores expresadas en ( $\mathrm{rad} / \mathrm{s}$ ) obtenidas con LumpST para el pórtico pa6pz2-1

\begin{tabular}{c|c} 
Modo & Frcuencia (rad/s) \\
\hline 1 & $.8494 \mathrm{D}+01$ \\
\hline 2 & $.2063 \mathrm{D}+02$ \\
\hline 3 & $.3236 \mathrm{D}+02$ \\
\hline 4 & $.4377 \mathrm{D}+02$ \\
\hline 5 & $.5570 \mathrm{D}+02$ \\
\hline 6 & $.7390 \mathrm{D}+02$ \\
\hline
\end{tabular}

Los periodos de vibración para cada uno de los modos, los obtenemos mediante la ecuación (5.2.3):

- Modo 1:

$$
\begin{aligned}
& { }_{s} w_{1}=8,494 \mathrm{rad} / \mathrm{s} \\
& { }_{s} f_{1}=\frac{{ }_{s} w_{1}}{2 \pi}=\frac{8,494}{2 \pi}=1,35186 \mathrm{~Hz} \Rightarrow{ }_{s} T_{1}=\frac{1}{{ }_{s} f_{1}}=\frac{1}{1,35186} \Rightarrow{ }_{s} T_{1}=0,73972 s
\end{aligned}
$$

- Modo 2:

$$
\begin{aligned}
& { }_{s} w_{2}=20,63 \mathrm{rad} / \mathrm{s} \\
& { }_{s} f_{2}=\frac{{ }_{s} w_{2}}{2 \pi}=\frac{20,62}{2 \pi}=3,28337 \mathrm{~Hz} \Rightarrow{ }_{s} T_{2}=\frac{1}{{ }_{s} f_{2}}=\frac{1}{3,28337} \Rightarrow{ }_{s} T_{2}=0,30456 \mathrm{~s}
\end{aligned}
$$

- Modo 3:

$$
\begin{aligned}
& { }_{s} w_{3}=32,36 \mathrm{rad} / \mathrm{s} \\
& { }_{s} f_{3}=\frac{{ }_{s} w_{3}}{2 \pi}=\frac{32,36}{2 \pi}=5,15025 \mathrm{~Hz} \Rightarrow{ }_{s} T_{3}=\frac{1}{{ }_{s} f_{3}}=\frac{1}{5,15025} \Rightarrow{ }_{s} T_{3}=0,19416 \mathrm{~s}
\end{aligned}
$$

- Modo 4:

$$
\begin{aligned}
& { }_{s} w_{4}=43,77 \mathrm{rad} / \mathrm{s} \\
& { }_{s} f_{4}=\frac{{ }_{s} w_{4}}{2 \pi}=\frac{43,77}{2 \pi}=6,96621 \mathrm{~Hz} \Rightarrow{ }_{s} T_{4}=\frac{1}{{ }_{s} f_{4}}=\frac{1}{6,96621} \Rightarrow{ }_{s} T_{4}=0,14355 \mathrm{~s}
\end{aligned}
$$

- Modo 5:

$$
\begin{aligned}
& { }_{s} w_{5}=55,70 \mathrm{rad} / \mathrm{s} \\
& { }_{s} f_{5}=\frac{{ }_{s} w_{5}}{2 \pi}=\frac{55,70}{2 \pi}=8,86493 \mathrm{~Hz} \Rightarrow{ }_{s} T_{5}=\frac{1}{{ }_{s} f_{5}}=8,86493 \Rightarrow{ }_{s} T_{5}=0,11280 \mathrm{~s}
\end{aligned}
$$

- Modo 6:

$$
{ }_{s} w_{6}=73,90 \mathrm{rad} / \mathrm{s}
$$




$$
{ }_{s} f_{6}=\frac{{ }_{s} w_{6}}{2 \pi}=\frac{73,90}{2 \pi}=11,76155 \mathrm{~Hz} \Rightarrow{ }_{s} T_{6}=\frac{1}{{ }_{s} f_{6}}=\frac{1}{11,76155} \Rightarrow{ }_{s} T_{6}=0,08502 s
$$

Tabla 5.2.34: Frecuencias y periodos de vibración con disipadores para el pórtico pa6pz2-1

\begin{tabular}{c|c|c} 
& \multicolumn{2}{|c}{ Pórtico pa4pz2-1 } \\
\hline Modo & $\begin{array}{c}\text { Frecuencia } \\
(\mathrm{Hz})\end{array}$ & $\begin{array}{c}\text { Periodo } \\
(\mathrm{s})\end{array}$ \\
\hline 1 & 1,35186 & 0,73972 \\
\hline 2 & 3,28337 & 0,30456 \\
\hline 3 & 5,15025 & 0,19416 \\
\hline 4 & 6,96621 & 0,14355 \\
\hline 5 & 8,86493 & 0,11280 \\
\hline 6 & 11,76155 & 0,08502 \\
\hline \multicolumn{3}{|c}{0,73972} \\
\hline $\begin{array}{c}\text { Periodo fund. } \\
\mathrm{f}_{1}(\mathrm{~s})\end{array}$
\end{tabular}

5.2.5.5. Tabla resumen frecuencias y periodos de vibración con disipadores para cada uno de los pórticos

Tabla 5.2.35: Frecuencias y periodos de vibración con disipadores para cada uno de los pórticos

\begin{tabular}{|c|c|c|c|c|c|c|}
\hline & \multicolumn{2}{|c|}{ Pórtico pa2pz2-1 } & \multicolumn{2}{|c|}{ Pórtico pa4pz2-1 } & \multicolumn{2}{|c|}{ Pórtico pa6pz2-1 } \\
\hline Modo & $\begin{array}{c}\text { Frecuencia } \\
(\mathrm{Hz})\end{array}$ & $\begin{array}{l}\text { Periodo } \\
\text { (s) }\end{array}$ & $\begin{array}{c}\text { Frecuencia } \\
(\mathrm{Hz})\end{array}$ & $\begin{array}{l}\text { Periodo } \\
\text { (s) }\end{array}$ & $\begin{array}{c}\text { Frecuencia } \\
(\mathrm{Hz})\end{array}$ & $\begin{array}{l}\text { Periodo } \\
\text { (s) }\end{array}$ \\
\hline 1 & 1,79208 & 0,55801 & 1.76025 & 0.56810 & 1,35186 & 0,73972 \\
\hline 2 & 4,67279 & 0,21400 & 4.72849 & 0.21148 & 3,28337 & 0,30456 \\
\hline 3 & 6,77522 & 0,14760 & 6.96303 & 0.14361 & 5,15025 & 0,19416 \\
\hline 4 & --- & --- & --- & --- & 6,96621 & 0,14355 \\
\hline 5 & --- & --- & --- & --- & 8,86493 & 0,11280 \\
\hline 6 & --- & --- & --- & --- & 11,76155 & 0,08502 \\
\hline $\begin{array}{c}\text { Periodo fund. } \\
{ }_{s} T_{1}(s)\end{array}$ & \multicolumn{2}{|c|}{0,55801} & \multicolumn{2}{|c|}{0,56810} & \multicolumn{2}{|c|}{0,73972} \\
\hline
\end{tabular}

5.2.5.6 Tabla resumen de los periodos fundamentales de vibración.

Tabla 5.2.36: Periodos fundamentales de vibración

\begin{tabular}{c|c|c|c|}
\hline $\begin{array}{c}\text { Periodos } \\
\text { fundamentales }\end{array}$ & $\begin{array}{c}\text { Pórtico pa2pz2-1 } \\
\text { (3 plantas) }\end{array}$ & $\begin{array}{c}\text { Pórtico pa4pz2-1 } \\
\text { (3 plantas) }\end{array}$ & $\begin{array}{c}\text { Pórtico pa6pz2-1 } \\
\text { (6 plantas) }\end{array}$ \\
\hline Sin disipador $\mathrm{f}_{1}(\mathrm{~s})$ & $1,366 \approx \mathbf{1 , 4}$ & $1,391 \approx \mathbf{1 , 4}$ & $1,785 \approx \mathbf{1 , 8}$ \\
\hline Con disipador $\mathrm{T}_{1}(\mathrm{~s})$ & $0,558 \approx \mathbf{0 , 5}$ & $0,568 \approx \mathbf{0 , 5}$ & $0,740 \approx \mathbf{0 , 7}$ \\
\hline & & &
\end{tabular}




\subsection{AUTOMATIZACIÓN DEL PROGRAMA LUMPST CON MATLAB}

Debido al gran número de iteraciones que hay que realizar, para obtener los desplazamientos máximos absolutos entre plantas de los pórticos analizados y con ellos ajustar los desplazamientos de fluencia de los disipadores, obtenidos en el apartado 5.4 de este capítulo, se ha decidido automatizar el programa LumpST mediante la aplicación del módulo de optimización de Matlab, para agilizar el cálculo.

Para ello, se han considerado dos métodos, cuyo esquema de funcionamiento se expone en la Fig. 5.3.1 y a los que denominaremos, problema directo y problema inverso.

En el problema directo, partimos de los datos de entrada del edificio y los desplazamientos de fluencia de los disipadores de cada una de las plantas. Con este método, el usuario elige el número de iteraciones que quiere obtener, así como el intervalo de las mismas.

El inverso, es un método de optimización numérica (pattern search) que se puede utilizar en funciones que no son continuas o diferenciables. Tales métodos de optimización también se conocen como métodos de búsqueda.

Se parte de la solución deseada, es decir, del desplazamiento objetivo de cada una de las plantas, y se obtienen los desplazamientos de fluencia de los disipadores. Consiste en un algoritmo de minimización, en el que la función objetivo $f$, dada por la ecuación (5.3.1), que tendiendo a cero busca la solución más óptima.

$$
f=\frac{1}{2} \sum_{i=1}^{\text {Nplantas }}\left(D E S P_{\text {LumpST }_{i}}-D_{E S P_{\text {DESEADOS }_{i}}}\right)^{2}
$$

Siendo:

$f$ : Función objetivo

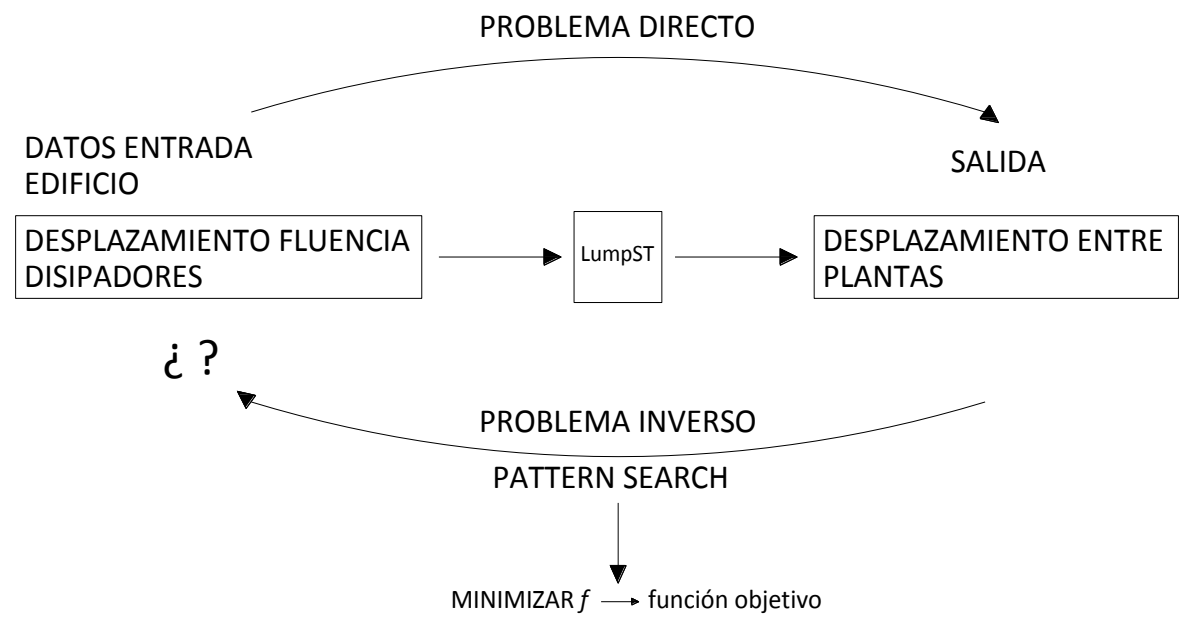

Fig. 5.3.1: Esquema de funcionamiento del problema directo e inverso 


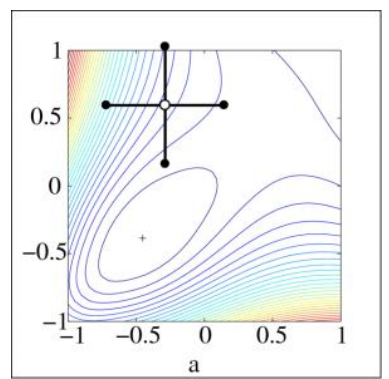

Fig. 5.3.2: Ejemplo de la convergencia de un método de búsqueda directa en la función Broyden

El patrón de búsqueda Pattern search (PS) es una familia de métodos de optimización numérica que no requieren el gradiente del problema a optimizar, es decir, es un método que para hallar el máximo o mínimo, no necesita calcular la derivada. Por lo tanto PS se pueden utilizar en funciones que no son continuas o diferenciables. Tales métodos de optimización también se conocen como métodos de búsqueda, derivada libre o caja negra.

El nombre, pattern search, fue dado por Hooke y Jeeves. Una variante de PS sencilla se atribuye a Fermi y Metropolis, cuando trabajaban en el Laboratorio Nacional Los Álamos como se describe por Davidon que resume el algoritmo de la siguiente manera:

"Variaban un parámetro teórico paso a paso, variando la magnitud, y cuando no se conseguía el objetivo, aumentaban o disminuían uno de los parámetros mejorando el ajuste a los datos experimentales, a la mitad del tamaño del paso y repetían el proceso hasta que el intervalo de pasos tienda hacia un infinitesimal".

\subsubsection{Secuencia de comandos en el problema directo para un pórtico de tres plantas}

$$
\text { clear all; }
$$

Se abre el fichero de salida: 'SalidaDesp.txt'

$$
\text { fichsal=fopen ('SalidaDesp.txt', 'w') ; }
$$

Imprime la siguiente línea en el fichero de salida: “Desp. P1 Desp. P2 Desp. P3 Planta DMax Dmin” fprintf(fichsal,'Desp. P1 Desp. P2 Desp. P3 Planta DMax Dmin\n');

Se introducen los datos correspondientes a la altura de cada planta en $\mathrm{cm}$.
$\div$ Datos 3
$\mathrm{H}(1)=300 ;$ \%Altura Planta 3
$\mathrm{H}(2)=300 ;$ oAltura Planta 2
$\mathrm{H}(3)=450 ;$ oltura Planta 1

I I, JJ, KK son los contenedores de los desplazamientos de fluencia de cada una de las plantas.

$I=0.3: 0.001: 0.9 \rightarrow$ Desplazamiento de fluencia de los disipadores de la 1a planta.

Desde 0.3 hasta 0.9 cada 0.001

$\mathrm{J}=0.3: 0.001: 0.9 \rightarrow$ Desplazamiento de fluencia de los disipadores de la $2 \underline{a}$ planta.

Desde 0.3 hasta 0.9 cada 0.001

$\mathrm{K}=0.3: 0.001: 0.9 \rightarrow$ Desplazamiento de fluencia de los disipadores de la 3a planta.

Desde 0.3 hasta 0.9 cada 0.001 
Hemos diseñado una subrutina en la se ejecuta un bucle de cálculo, que variando diferentes parámetros conseguimos el fin perseguido.

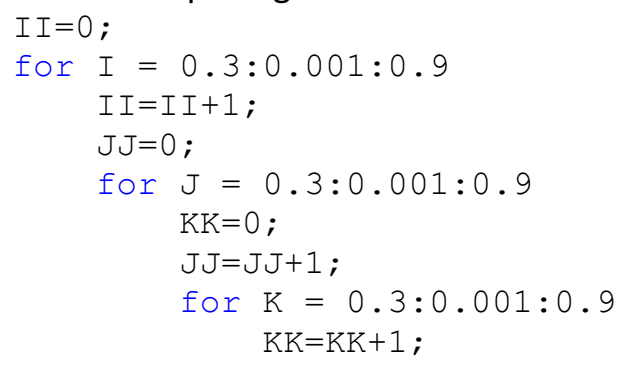

Se crea un fichero MODEL.dat con los datos introducidos para cada una de las plantas, estos son: el número de planta; número de masa superior; número de masa inferior; la masa expresada en $\left(\mathrm{Ns}^{2} / \mathrm{cm}\right)$; la rigidez expresada en $(\mathrm{N} / \mathrm{cm})$; desplazamiento de fluencia del pórtico expresado en $(\mathrm{cm})$; y la rigidez en la zona poselástica.

Además, escribe en el fichero un número de tipo real flotante $\%$ correspondiente al desplazamiento de fluencia del disipador expresado en $(\mathrm{cm})$, que es el que irá variando en cada iteración.

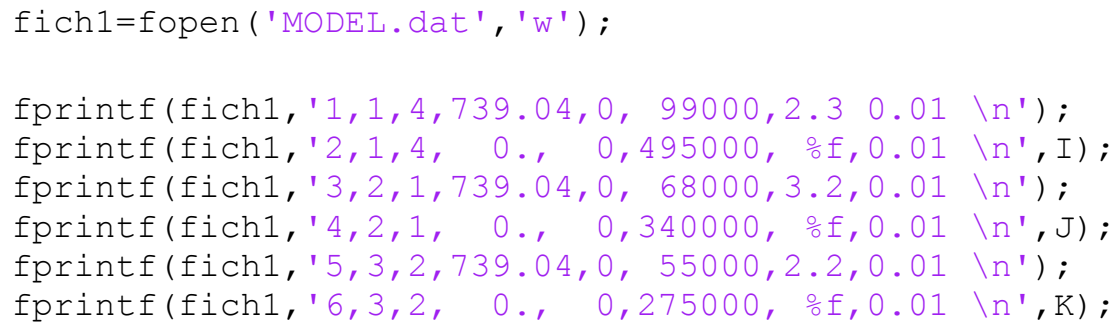

Se cierra el fichero ( $f i c h 1)$

fclose (fich1);

El comando dos ejecuta LumpST.exe, éste pide los siguientes datos por teclado: amortiguamiento para el modo n; amortiguamiento para el modo m; nombre del acelerograma a utilizar; factor de escala para la aceleración del suelo. Para ello, se hace un redireccionamiento de la entrada mediante el fichero de texto redir. txt que incluye los datos que nos pide el programa LumpST: 0.050 .05 '158xa(2).dat' 0.2245 EOF!

$$
\text { dos ('LumpsT.exe < redir.txt') }
$$

De todos los ficheros que se generan, sólo abriremos el fichero 'RESUMEN1. DAT', que es el fichero donde se encuentran los datos que estamos buscando, es decir, $D_{\max }$, el desplazamiento máximo entre plantas y $D_{\min }$, desplazamiento mínimo entre plantas.

fich1=fopen ('RESUMEN1.DAT', 'r' ) ;

El programa lee la primera línea como basura.

$\begin{array}{lllllll}\text { fscanf (fich1, [' } & \text { STORY } & \text { Dmax } & \text { Tdmax } & \text { Dmin } & \text { Tdmin } \\ \text { Amax } & \text { n+pEND1 } & \text { n-mEND1 } & \text { Wi/Wp1 } & \text { n-rem1 } & \text { n-tot1 } & \text { u-avEND1 } \\ \text { n-avEND1 } & \text { n/uEND1 } & \text { n-avDFM1 } & \text { n/uDFM1 } & \text { emax1 } & \text { Qmin1 } & \text { Qmaxx1 } \\ \text { ALFAmax1 } & \text { n+pEND2 } & \text { n-mEND2 } & \text { Wi/Wp2 } & \text { n-rem2 } & \text { n-tot2 } & \text { u-avEND2 } \\ \text { n-avEND2 } & \text { n/uEND2 } & \text { n-avDFM2 } & \text { n/uDFM2 } & \text { Qmax2 } & \text { Qmin2 } & \text { Qmaxx2 } \\ \text { ALFAmax2 } & \text { Einput } & \text { ALFAy1 } & \text { deltay1 } & \text { ALFAy2 } & \text { deltay2 } & \text { KsKf } \\ \text { ALFAmtot']) } & & & & & \end{array}$

Una vez que el programa lee la primera línea como basura, presenta los resultados de las tres primeras líneas, estando los de cada planta en una sola linea. 
for $M M=1: 3$

A partir de aquí, la variable auxiliar (aux) lee los primeros 4 datos que son los que nos interesan y los guarda: STORY Dmax Tdmax Dmin $\operatorname{aux}(M M, 1: 4)=f \operatorname{scanf}\left(\mathrm{fich} 1,{ }^{\circ} \circ \lg { }^{\prime}, 4\right)$;

En este punto del flujo, escanea el fichero ( $\mathrm{fich} 1$ ) y lo lee. Como ya se ha comentado, de los 41 datos, los 37 últimos son basura y sólo nos quedamos con los cuatro primeros: STORY Dmax Tdmax Dmin

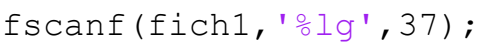

Los resultados se presentan de la forma:

resultado ( II, JJ, KK, MM, 1:4)=aux (MM, 1:4);

Leída esta línea, la comprueba. Utilizando como criterio de comprobación el de dimensionamiento de los disipadores, que obligamos a que el desplazamiento máximo entre plantas, sea el 0,5\% en valor absoluto de la altura entre plantas, y con una tolerancia de $\pm 10 \%$.

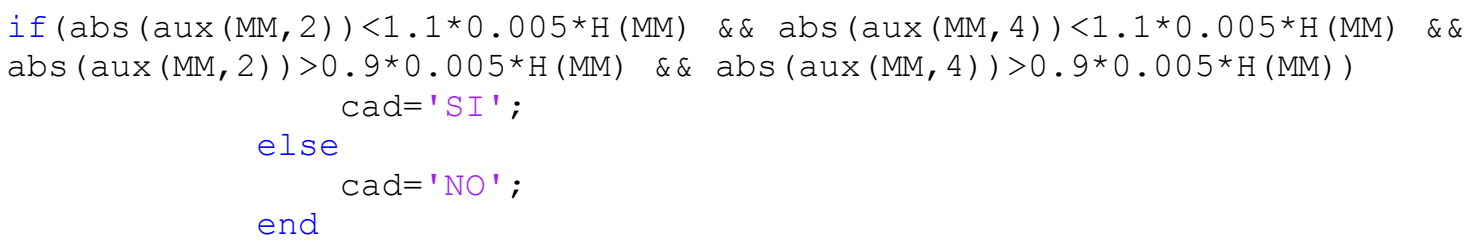

Por último imprime el fichero de salida, cuyo formato es el siguiente: Desplazamientos de fluencia; número de planta; desplazamientos relativos máximos y mínimos. Imprime SI o NO, según hallamos o no obtenido el resultado deseado. $Y$ acaba el programa.

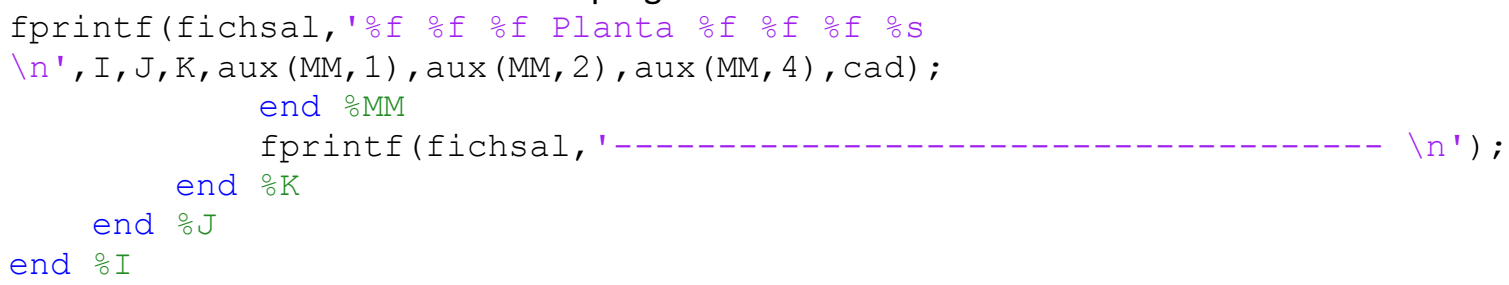

\subsubsection{Secuencia de comandos en el problema inverso para un pórtico de tres plantas}

Consta de dos ficheros Matlab:

1) run

clear;

Valor de la función objetivo.

global FUNCEVAL;

Número de iteraciones que se han hecho.

global II;

Solución.

global Sol;

Desplazamiento de fluencia.

global dflu; 


\section{Primera iteración.}

$$
\mathrm{II}=1 \text {; }
$$

$\mathrm{x} 0=\left[\begin{array}{lll}0.0001 & 0.0001 & 0.0001\end{array}\right] ;$

$\mathrm{SOl}=\left[\begin{array}{lll}1 & 1 & 1\end{array}\right]$;

dflu=[lll 11$]$;

FUNCEVAL $=1$;

$\mathrm{A}=\left[\begin{array}{llllllllll}1 & 0 & 0 ; & 0 & 1 & 0 ; & 0 & 0 & 1\end{array}\right] ;$

$\mathrm{b}=[1.5 ; 1.5 ; 1.5] ;$

$l b=[0.0 ; 0.0 ; 0.0] ;$

[ $\mathrm{x}$ fval] =patternsearch (@lump, x0,A, b, [], [] , lb) ;

save('dflu', 'Sol', 'FUNCEVAL', 'II')

Siendo $\mathrm{x} 0$ los valores iniciales de desplazamiento de fluencia del disipador, en este caso hemos considerado, $0.0001 \mathrm{~cm}$ en las tres plantas.

A es una matriz unidad de $3 \times 3$ y b los valores finales de desplazamiento de fluencia del disipador, $1.5 \mathrm{~cm}$ en las tres plantas.

$$
\left[\begin{array}{lll}
1 & 0 & 0 \\
0 & 1 & 0 \\
0 & 0 & 1
\end{array}\right]\left[\begin{array}{l}
d f_{P 3} \\
d f_{P 2} \\
d f_{P 1}
\end{array}\right]<\left[\begin{array}{l}
1,5 \\
1,5 \\
1,5
\end{array}\right]
$$

Donde $d f$ es el desplazamiento de fluencia del disipador para cada una de las plantas.

Ib es la frontera mínima del desplazamiento de fluencia de los disipadores, $0.0 \mathrm{~cm}$ en las tres plantas. patternsearch como se ha comentado, es la función de optimización, busca los desplazamientos de fluencia de los disipadores.

\section{2) lump}

Función que dados los desplazamientos de fluencia, obtiene los valores de la función objetivo [ f ] function $[f]=\operatorname{lump}(\operatorname{despf})$

Valor de la función objetivo.

global FUNCEVAL;

Número de iteraciones que se han hecho.

global II;

Solución.

global Sol;

Desplazamiento de fluencia.

global dflu;

Se abre el fichero de salida: 'SalidaDesp.txt'

fichsal=fopen('SalidaDesp.txt', 'w');

Imprime la siguiente línea en el fichero de salida: “Desp. P1 Desp. P2 Desp. P3 Planta DMax Dmin” fprintf(fichsal,'Desp. P1 Desp. P2 Desp. P3 Planta DMax Dmin\n');

Se introducen los datos correspondientes a la altura de cada planta en $\mathrm{cm}$. $\because$ Datos 

$\mathrm{H}(1)=300 ;$;Altura Planta 3
$\mathrm{H}(2)=300 ;$ \%Altura Planta 2
$\mathrm{H}(3)=450$; $\frac{\circ}{\mathrm{A}} \mathrm{ltura}$ Planta 1

Se crea un fichero MODEL.dat con los datos introducidos para cada una de las plantas, estos son: el número de planta; número de masa superior; número de masa inferior; la masa expresada en $\left(\mathrm{Ns}^{2} / \mathrm{cm}\right)$; la rigidez expresada en $(\mathrm{N} / \mathrm{cm})$; desplazamiento de fluencia del pórtico expresado en $(\mathrm{cm}) ;$ y la rigidez en la zona poselástica.

Además, escribe en el fichero un número de tipo real flotante $\mathrm{f}$ correspondiente al desplazamiento de fluencia del disipador expresado en $(\mathrm{cm})$, que es el que irá variando en cada iteración.

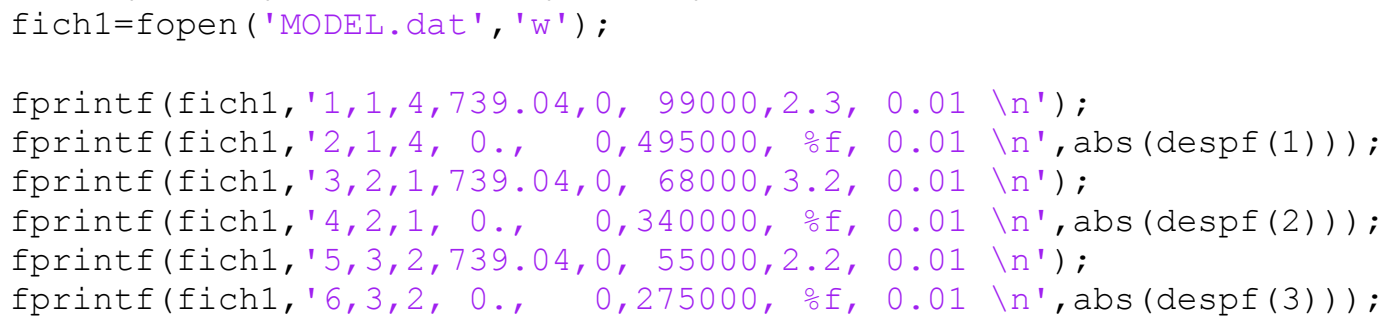

Se cierra el fichero ( $f i c h 1)$

fclose (fichl);

El comando dos ejecuta LumpST.exe, éste pide los siguientes datos por teclado: amortiguamiento para el modo n; amortiguamiento para el modo m; nombre del acelerograma a utilizar; factor de escala para la aceleración del suelo. Para ello, se hace un redireccionamiento de la entrada mediante el fichero de texto redir. txt que incluye los datos que nos pide el programa LumpST: $0.05 \quad 0.05$ '158xa(2).dat' 0.2245 EOF!

$$
\text { dos ('LumpST.exe < redir.txt') }
$$

De todos los ficheros que se generan, sólo abriremos el fichero 'RESUMEN1. DAT', que es el fichero donde se encuentran los datos que estamos buscando, es decir, $D_{\max }$, el desplazamiento máximo entre plantas y $D_{\min }$, desplazamiento mínimo entre plantas.

fich1=fopen ( 'RESUMEN1.DAT', 'r');

El programa lee la primera línea como basura.

\begin{tabular}{|c|c|c|c|c|c|c|}
\hline \multicolumn{2}{|c|}{ fscanflfichl, [' } & STORY & Dmax & Tdmax & Dmin & Tdmin \\
\hline Amax & $\mathrm{n}+\mathrm{pEND} 1$ & $\mathrm{n}-\mathrm{mEND} 1$ & Wi/Wp1 & $\mathrm{n}-r e m 1$ & $n-\operatorname{tot} 1$ & $u-a v E N D 1$ \\
\hline $\mathrm{n}-\mathrm{avEND} 1$ & $\mathrm{n} / \mathrm{uEND} 1$ & $\mathrm{n}-\mathrm{avDFM1}$ & $\mathrm{n} / \mathrm{UDFMI}$ & Qmax1 & Qmin1 & Qmaxx1 \\
\hline ALFAmax 1 & $\mathrm{n}+\mathrm{pEND} 2$ & $n-m E N D 2$ & Wi /Wp2 & $\mathrm{n}$-rem2 & $n-\operatorname{tot} 2$ & $u-a v E N D 2$ \\
\hline $\mathrm{n}-\mathrm{avEND} 2$ & $\mathrm{n} / \mathrm{uEND} 2$ & $\mathrm{n}$-avDFM2 & $\mathrm{n} / \mathrm{UDFM} 2$ & Qmax2 & Qmin2 & Qmaxx2 \\
\hline ALFAmax 2 & Einput & ALFAy1 & deltay1 & ALFAy2 & deltay2 & KsKf \\
\hline
\end{tabular}

La variable auxiliar aux $(1: 3,1: 4)$ es una matriz de 3 filas $x 4$ columnas, con todos sus elementos igual a 0 , desde la fila 1 a la 3 , y desde la columna 1 a la 4 .

$$
\operatorname{aux}(1: 3,1: 4)=0 \text {; }
$$

Una vez que el programa lee la primera línea como basura, presenta los resultados de las tres primeras líneas, estando los de cada planta en una sola linea.

$$
\text { for } \mathrm{MM}=1: 3
$$

A partir de aquí, la variable auxiliar (aux) lee los primeros 4 datos que son los que nos interesan y los guarda: STORY Dmax Tdmax Dmin

$$
\operatorname{aux}(\mathrm{MM}, 1: 4)=f \operatorname{scanf}\left(\text { fich } 1,{ }^{\prime} \circ \lg { }^{\prime}, 4\right) \text {; }
$$


En este punto del flujo, escanea el fichero ( $f i c h 1$ ) y lo lee. Como ya se ha comentado, de los 41 datos, los 37 últimos son basura y sólo nos quedamos con los cuatro primeros: STORY Dmax Tdmax Dmin

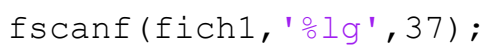

A continuación, busca el resultado considerando el criterio de dimensionamiento de los disipadores, y el valor máximo absoluto.

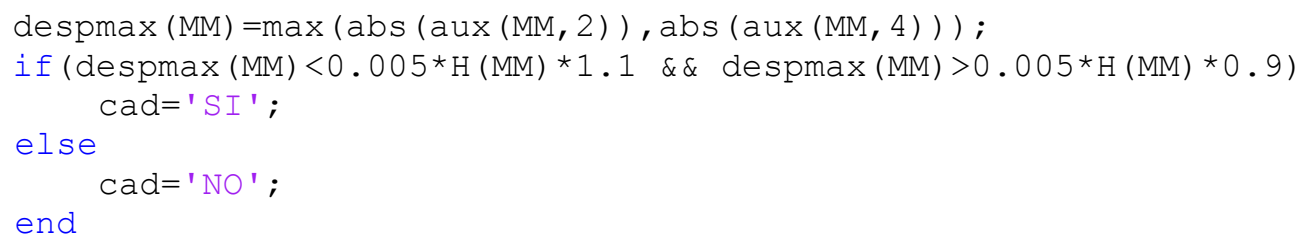

Por último imprime el fichero de salida, obteniendo así los resultados.

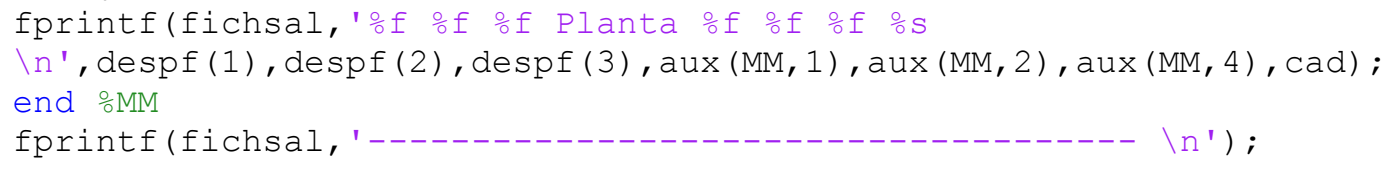


f es la función objetivo que hay que minimizar. Cuando los desplazamientos relativos entre plantas se acercan a los deseados, los valores de entrada son los correctos. Si el desplazamiento relativo de la primera planta es $2,25 \mathrm{~cm}$, el desplazamiento relativo de la segunda planta $1,5 \mathrm{~cm}$ y el desplazamiento relativo de la tercera planta $1,5 \mathrm{~cm}$, f es mínimo.

FUNCEVAL (II) =f； guarda en esta variable (vector) para cada iteración el valor de la función. 


\subsection{DIMENSIONAMIENTO DE LOS DISIPADORES}

En la Fig. 5.4.1 a) se muestra una estructura formada por tres forjados y 4 pilares por pórtico. La estructura porticada con disipadores se puede descomponer en suma de una parte flexible que es el propio pórtico sin disipadores, y una rígida compuesta que son los disipadores de energía.

Los pilares los supondremos axialmente indeformables y el forjado infinitamente rígido en su plano (hipótesis de diafragma rígido). Para modelizar la estructura supondremos que la masa de cada forjado está concentrada en un punto situado en su centro de masas, tal y como se muestra en la Fig. 5.4.1 b). En estas condiciones, los grados de libertad de cada forjado serán los de un sólido rígido en su plano.

Cuanto mayor sea el número de grados de libertad adoptados, mayor será el grado de aproximación a la respuesta real que obtengamos, pero también se incrementará la complejidad. En general, para simplificar, conviene elegir únicamente aquellos grados de libertad en los cuales esperamos que se produzca la respuesta principal de la estructura. Por tanto consideraremos cada masa con un grado de libertad, siendo éste el desplazamiento horizontal del punto másico.

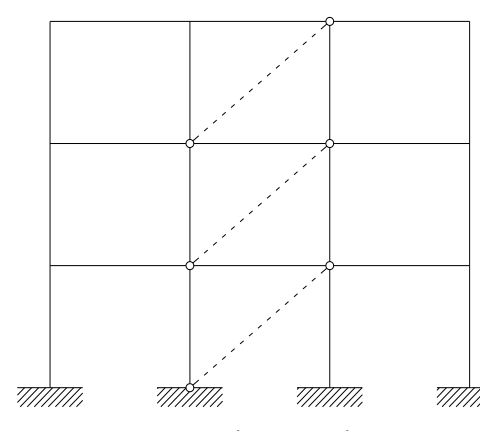

estructura sismorresistente

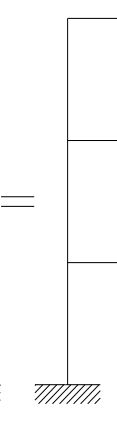$$
\text { (1) }
$$

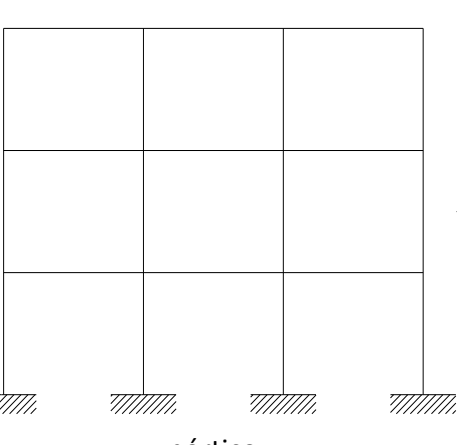

pórtico

a)

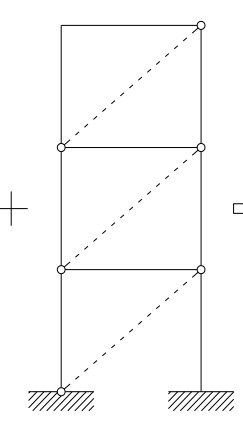

sistema disipativo

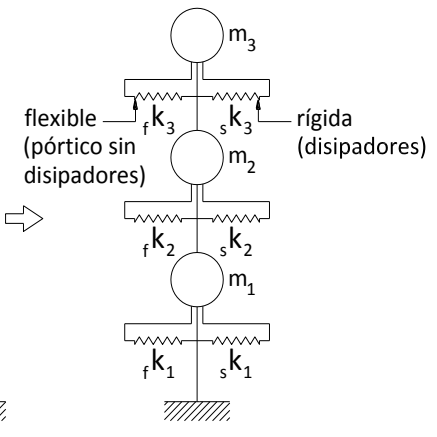

b)

Fig. 5.4.1: Subsistemas estructurales de una estructura sismorresistente con disipadores de energía

\subsubsection{Criterio para establecer la rigidez lateral de los disipadores}

El criterio para establecer la rigidez lateral de los disipadores, es el siguiente:

Siendo:

$$
{ }_{s} k_{i}=5 \times{ }_{f} k_{i}
$$

${ }_{s} k_{i}$ : Rigidez del disipador por planta.

${ }_{f} k_{i}$ : Rigidez sin disipador.

El valor de 5 empleado en la ecuación (5.4.1) es un valor mínimo recomendado en la literatura para que plastifique antes el disipador que el pórtico principal [4].

\subsubsection{Criterio para fijar la resistencia lateral de los disipadores}

El criterio que emplearemos para fijar la resistencia lateral de los disipadores de energía es que el desplazamiento máximo entre plantas, sea igual al $\mathbf{0 , 5 \%}$ en valor absoluto de la altura entre plantas. 
Se adopta este valor (con una tolerancia de $\pm \mathbf{1 0 \%}$ ) para garantizar que el pórtico principal se mantiene en régimen básicamente elástico (sin daños).

Para conseguir el objetivo, tendremos que ir variando de forma iterativa la resistencia de fluencia de los disipadores, ${ }_{s} Q_{y j}$. Con el programa LumpST empleado en este estudio, esto se hace actuando sobre el desplazamiento de fluencia ${ }_{s} \delta_{y j}$. Para realizar estas iteraciones aplicaremos un programa de optimización con Matlab, ampliamente detallado en el apartado 5.3 de este capítulo.

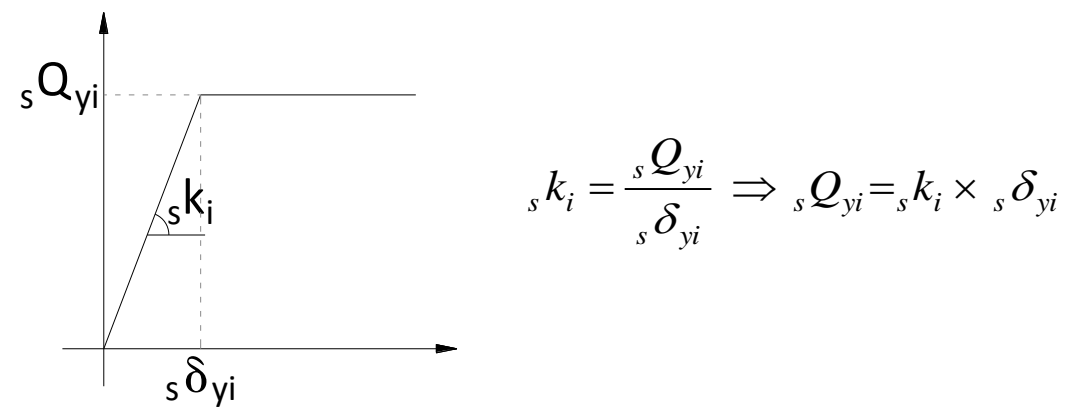

Fig. 5.4.2: Curva Desplazamiento de fluencia - Resistencia lateral del disipador

Siendo:

${ }_{s} Q_{y i}:$ Resistencia de fluencia del disipador por planta.

${ }_{s} k_{i} \quad:$ Rigidez del disipador por planta.

${ }_{s} \delta_{y i}:$ Desplazamiento de fluencia del disipador por planta.

\subsubsection{Obtención de los desplazamientos de fluencia de los disipadores y desplazamientos máximos} absolutos entre plantas

Para la obtención de los desplazamientos de fluencia de los disipadores y desplazamientos máximos absolutos entre plantas, consideraremos únicamente aquellos acelerogramas cuyo espectro de energía introducida expresado en forma de pseudovelocidad equivalente, de un valor elevado. En los cálculos dinámicos se emplea un amortiguamiento del $5 \%$.

En los subapartados siguientes, detallamos la obtención de estos parámetros para cada uno de los pórticos analizados.

\subsubsection{Pórtico pa2pz2-1 (3 plantas)}

- Rigidez disipador:

Planta 3a: ${ }_{s} k_{3}=(55000 \mathrm{~N} / \mathrm{cm}) \times 5=275000 \mathrm{~N} / \mathrm{cm}$

Planta 2a: ${ }_{s} k_{2}=(68000 \mathrm{~N} / \mathrm{cm}) \times 5=340000 \mathrm{~N} / \mathrm{cm}$

Planta 1a: ${ }_{s} k_{1}=(99000 \mathrm{~N} / \mathrm{cm}) \times 5=495000 \mathrm{~N} / \mathrm{cm}$

- Desplazamientos de fluencia del pórtico:

Planta 3a: ${ }_{f} \delta_{3}=2,2 \mathrm{~cm}$ 
Planta 2a: ${ }_{f} \delta_{2}=3,2 \mathrm{~cm}$

Planta 1: ${ }_{f} \delta_{1}=2,3 \mathrm{~cm}$

- Desplazamientos objetivo entre plantas:

Para que el desplazamiento máximo entre plantas, sea el $0,5 \%$ en valor absoluto de la altura entre plantas.

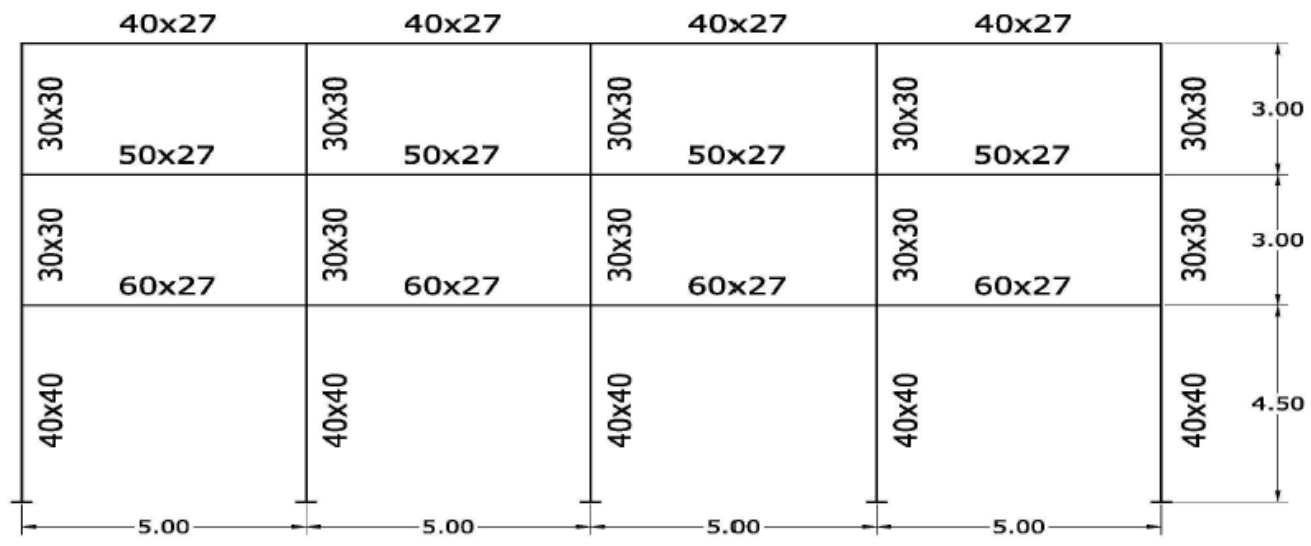

Fig. 5.4.3: Pórtico pa2pz2-1

Altura Planta 3a : $3,0 \mathrm{~m}=300 \mathrm{~cm} \Rightarrow \frac{0,5}{100} \times 300=\mathbf{1 , 5 0} \mathrm{cm}$

Altura Planta 2a: $3,0 m=300 \mathrm{~cm} \Rightarrow \frac{0,5}{100} \times 300=\mathbf{1 , 5 0} \mathrm{cm}$

Altura Planta 1a : $4,5 m=450 \mathrm{~cm} \Rightarrow \frac{0,5}{100} \times 450=\mathbf{2 , 2 5} \mathrm{cm}$

Tolerancia: $10 \%$

Planta 3a: $\frac{10}{100} \times 1,50=0,150 \Rightarrow\left\{\begin{array}{l}1,50+0,150=1,650 \mathrm{~cm} \\ 1,50-0,150=1,350 \mathrm{~cm}\end{array}\right.$

Planta 2a: $\frac{10}{100} \times 1,50=0,150 \Rightarrow\left\{\begin{array}{l}1,50+0,150=1,650 \mathrm{~cm} \\ 1,50-0,150=1,350 \mathrm{~cm}\end{array}\right.$

Planta 1a: $\frac{10}{100} \times 2,25=0,225 \Rightarrow\left\{\begin{array}{l}2,25+0,225=2,475 \mathrm{~cm} \\ 2,25-0,025=2,025 \mathrm{~cm}\end{array}\right.$

- Obtención desplazamiento de fluencia del disipador y desplazamientos máximos y mínimos entre plantas:

Fichero model.dat programa LumpST:

1,1,4,739.04,0, 99000, 2.3, 0.01

$2,1,4, \quad 0 ., \quad 0,495000$, desp. flu. disip., 0.01 
$3,2,1,739.04,0,68000,3.2, \quad 0.01$

$4,2,1, \quad 0 ., \quad 0,340000$, desp. flu. disip., 0.01

$5,3,2,739.04,0,55000,2.2, \quad 0.01$

$6,3,2, \quad 0 ., \quad 0,275000$, desp. flu. disip., 0.01

\subsection{Suelo Tipo I (Campo lejano a la falla)}

- Acelerograma №1 (Friuli) $\Delta=\mathbf{2 7} \mathrm{Km}$ (Factor de escala: 0.5717)

Tabla 5.4.1: Desplazamientos de fluencia del disipador y desplazamientos máximos absolutos entre plantas, pórtico pa2pz2-1, acelerograma no1, suelo tipo I, campo lejano

\begin{tabular}{c|c|c|c|c|c} 
& \multicolumn{5}{|c|}{ Input de energía: $\mathrm{V}_{\mathrm{E}}=20,94 \mathrm{~cm} / \mathrm{s}$} \\
\hline Planta & $\begin{array}{c}\text { Desplaz. fluencia } \\
\text { disipador } \\
(\mathrm{cm})\end{array}$ & $\begin{array}{c}\text { Desplaz. Máximo } \\
\text { entre plantas } \\
(\mathrm{cm})\end{array}$ & $\begin{array}{c}\text { Desplaz. Mínimo } \\
\text { entre plantas } \\
(\mathrm{cm})\end{array}$ & $\begin{array}{c}\text { Desplaz. Máximo } \\
\text { absoluto } \\
(\mathrm{cm})\end{array}$ & $\begin{array}{c}\text { Desplaz. objetivo } \\
(\mathrm{cm})\end{array}$ \\
\hline 1 a & 0.0030 & 0.982 & -1.712 & $\mathbf{1 . 7 1 2}$ & $2.025 \div 2.475$ \\
\hline $2^{\mathrm{a}}$ & 0.0478 & 0.956 & -1.570 & $\mathbf{1 . 5 7 0}$ & $1.350 \div 1.650$ \\
\hline 3 a & 0.00008 & 1.150 & -0.985 & $\mathbf{1 . 1 5 0}$ & $1.350 \div 1.650$ \\
\hline & & & & &
\end{tabular}

- Acelerograma №4 (Montenegro) $\Delta=65 \mathrm{Km}$ (Factor de escala: 0.9102)

Tabla 5.4.2: Desplazamientos de fluencia del disipador y desplazamientos máximos absolutos entre plantas, pórtico pa2pz2-1, acelerograma $\mathrm{n}=4$, suelo tipo I, campo lejano

\begin{tabular}{c|c|c|c|c|c}
\multicolumn{5}{|c|}{ Input de energía: $\bigvee_{\mathrm{E}}=34,95 \mathrm{~cm} / \mathrm{s}$} \\
Planta & $\begin{array}{c}\text { Desplaz. fluencia } \\
\text { disipador } \\
(\mathrm{cm})\end{array}$ & $\begin{array}{c}\text { Desplaz. Máximo } \\
\text { entre plantas } \\
(\mathrm{cm})\end{array}$ & $\begin{array}{c}\text { Desplaz. Mínimo } \\
\text { entre plantas } \\
(\mathrm{cm})\end{array}$ & $\begin{array}{c}\text { Desplaz. Máximo } \\
\text { absoluto } \\
(\mathrm{cm})\end{array}$ & $\begin{array}{c}\text { Desplaz. objetivo } \\
(\mathrm{cm})\end{array}$ \\
\hline 1 a & 0.0003 & 1.922 & -1.869 & $\mathbf{1 . 9 2 2}$ & $2.025 \div 2.475$ \\
\hline $2^{\underline{a}}$ & 0.2384 & 0.757 & -1.001 & $\mathbf{1 . 0 0 1}$ & $1.350 \div 1.650$ \\
\hline 3 a & 0.0880 & 1.109 & -1.181 & $\mathbf{1 . 1 8 1}$ & $1.350 \div 1.650$ \\
\hline & & & & &
\end{tabular}

- Acelerograma №5 (Campano Lucano) $\Delta=32 \mathrm{Km}$ (Factor de escala: 0.9431)

Tabla 5.4.3: Desplazamientos de fluencia del disipador y desplazamientos máximos absolutos entre plantas, pórtico pa2pz2-1, acelerograma no5, suelo tipo I, campo lejano

\begin{tabular}{c|c|c|c|c|c} 
& \multicolumn{5}{|c|}{ Input de energía: $\mathrm{V}_{\mathrm{E}}=39,04 \mathrm{~cm} / \mathrm{s}$} \\
Planta & $\begin{array}{c}\text { Desplaz. fluencia } \\
\text { disipador } \\
(\mathrm{cm})\end{array}$ & $\begin{array}{c}\text { Desplaz. Máximo } \\
\text { entre plantas } \\
(\mathrm{cm})\end{array}$ & $\begin{array}{c}\text { Desplaz. Mínimo } \\
\text { entre plantas } \\
(\mathrm{cm})\end{array}$ & $\begin{array}{c}\text { Desplaz. Máximo } \\
\text { absoluto } \\
(\mathrm{cm})\end{array}$ & $\begin{array}{c}\text { Desplaz. objetivo } \\
(\mathrm{cm})\end{array}$ \\
\hline 1a $^{\text {a }}$ & 0.0226 & 2.340 & -1.083 & $\mathbf{2 . 3 4 0}$ & $2.025 \div 2.475$ \\
\hline 3a & 0.0402 & 1.532 & -1.013 & $\mathbf{1 . 5 3 2}$ & $1.350 \div 1.650$ \\
\hline & 0.0001 & 0.491 & -1.117 & $\mathbf{1 . 1 1 7}$ & $1.350 \div 1.650$ \\
\hline
\end{tabular}

Las Fig. 5.4.4, 5.4 .5 y 5.4.6 muestran los desplazamientos máximos en valor absoluto experimentados por cada planta, para cada uno de los terremotos analizados en el suelo tipo I, tipo II y tipo III respectivamente del pórtico pa2pz2-1. 
También se representa el desplazamiento objetivo igual al 0,5\% en valor absoluto de la altura entre plantas con una tolerancia de $\pm 10 \%$. Los desplazamientos máximos en valor absoluto que quedan dentro de ésta franja de desplazamientos objetivo, son los que cumplen el criterio anteriormente definido en el apartado 5.4.2.

En las Fig. 5.4.8, 5.4.9 y 5.4.10 representaremos estos desplazamientos para suelo tipo I, suelo tipo II y tipo III respectivamente y para el pórtico pa4pz2-1.

Y por último, en las Fig. 5.4.12, 5.4.13 y 5.4.14, los desplazamientos máximos en valor absoluto para el pórtico pa6pz2-1 y para cada tipo de suelo.

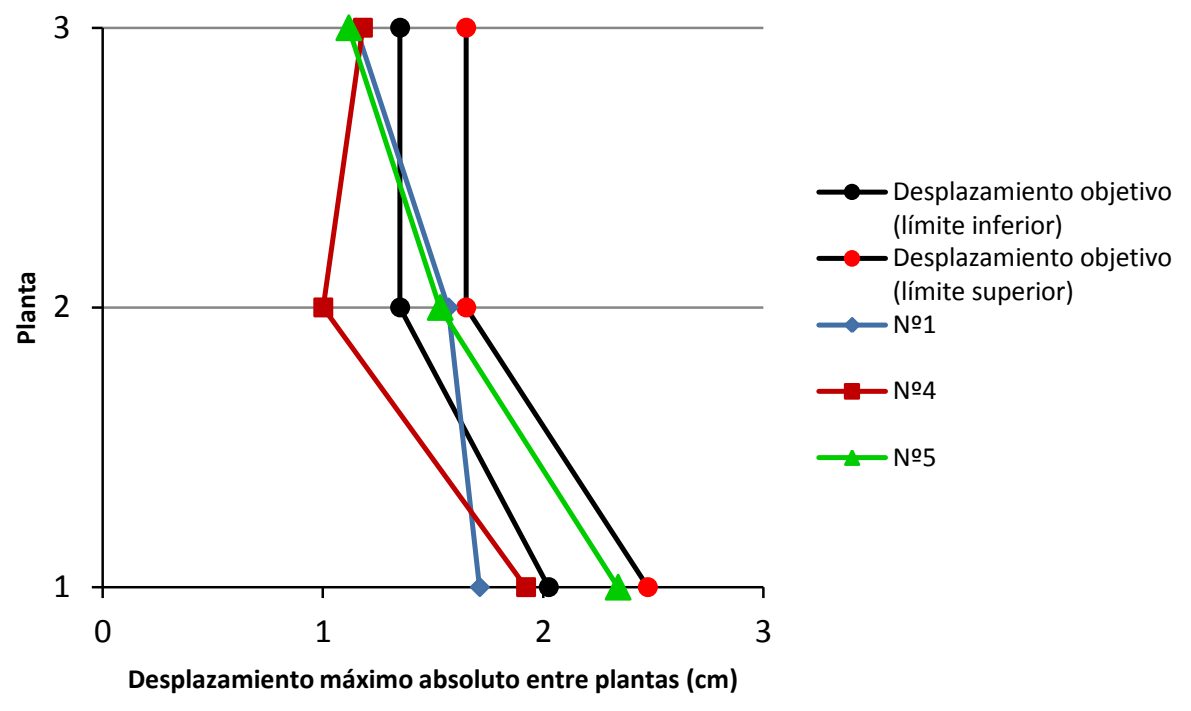

Fig. 5.4.4: Desplazamientos máximos absolutos entre plantas, pórtico pa2pz2-1, suelo tipo I (muy duro)

\subsection{Suelo Tipo II (Campo cercano a la falla)}

- Acelerograma №1 (Friuli aftershock) $\Delta=3 \mathrm{Km}$ (Factor de escala: 1.2554)

Tabla 5.4.4: Desplazamientos de fluencia del disipador y desplazamientos máximos absolutos entre plantas, pórtico pa2pz2-1, acelerograma no1, suelo tipo II, campo cercano

\begin{tabular}{|c|c|c|c|c|c|}
\hline \multicolumn{5}{|c|}{ Input de energía: $V_{E}=28,93 \mathrm{~cm} / \mathrm{s}$} & \multirow[b]{2}{*}{$\begin{array}{c}\text { Desplaz. objetivo } \\
(\mathrm{cm})\end{array}$} \\
\hline Planta & $\begin{array}{l}\text { Desplaz. fluencia } \\
\text { disipador } \\
(\mathrm{cm}) \\
\end{array}$ & $\begin{array}{c}\text { Desplaz. Máximo } \\
\text { entre plantas } \\
(\mathrm{cm})\end{array}$ & $\begin{array}{c}\text { Desplaz. Mínimo } \\
\text { entre plantas } \\
(\mathrm{cm})\end{array}$ & $\begin{array}{c}\text { Desplaz. Máximo } \\
\text { absoluto } \\
(\mathrm{cm})\end{array}$ & \\
\hline 1모 & 0.0008 & 0.886 & -1.495 & 1.495 & $2.025 \div 2.475$ \\
\hline 2 a & 0.0356 & 0.552 & -1.317 & 1.317 & $1.350 \div 1.650$ \\
\hline $3 \underline{a}$ & 0.0001 & 1.104 & -1.606 & 1.606 & $1.350 \div 1.650$ \\
\hline
\end{tabular}


- Acelerograma №14 (Montenegro aftershock) $\Delta=8 \mathrm{Km}$ (Factor de escala: 1.9662 )

Tabla 5.4.5: Desplazamientos de fluencia del disipador y desplazamientos máximos absolutos entre plantas, pórtico pa2pz2-1, acelerograma no14, suelo tipo II, campo cercano

\begin{tabular}{|c|c|c|c|c|c|}
\hline \multicolumn{5}{|c|}{ Input de energía: $V_{E}=116,08 \mathrm{~cm} / \mathrm{s}$} & \multirow[b]{2}{*}{$\begin{array}{c}\text { Desplaz. objetivo } \\
(\mathrm{cm})\end{array}$} \\
\hline Planta & $\begin{array}{l}\text { Desplaz. fluencia } \\
\text { disipador } \\
\text { (cm) }\end{array}$ & $\begin{array}{l}\text { Desplaz. Máximo } \\
\text { entre plantas } \\
(\mathrm{cm})\end{array}$ & $\begin{array}{l}\text { Desplaz. Mínimo } \\
\text { entre plantas } \\
\text { (cm) }\end{array}$ & $\begin{array}{l}\text { Desplaz. Máximo } \\
\text { absoluto } \\
\text { (cm) }\end{array}$ & \\
\hline 1 a & 0.4883 & 2.535 & -1.697 & 2.535 & $2.025 \div 2.475$ \\
\hline $2 \underline{a}$ & 0.5937 & 1.965 & -1.350 & 1.965 & $1.350 \div 1.650$ \\
\hline 3 & 0.4454 & 1.648 & -0.918 & 1.648 & $1.350 \div 1.650$ \\
\hline
\end{tabular}

\subsection{Suelo Tipo II (Campo lejano a la falla)}

- Acelerograma №2 (Friuli aftershock) $\Delta=\mathbf{1 6 K m}$ (Factor de escala: 1.7929)

Tabla 5.4.6: Desplazamientos de fluencia del disipador y desplazamientos máximos absolutos entre plantas, pórtico pa2pz2-1, acelerograma no2, suelo tipo II, campo lejano

\begin{tabular}{|c|c|c|c|c|c|}
\hline & \multicolumn{4}{|c|}{ Input de energía: $V_{\mathrm{E}}=30,80 \mathrm{~cm} / \mathrm{s}$} & \multirow[b]{2}{*}{$\begin{array}{l}\text { Desplaz. objetivo } \\
(\mathrm{cm})\end{array}$} \\
\hline Planta & $\begin{array}{l}\text { Desplaz. fluencia } \\
\text { disipador } \\
\text { (cm) }\end{array}$ & $\begin{array}{l}\text { Desplaz. Máximo } \\
\text { entre plantas } \\
(\mathrm{cm})\end{array}$ & $\begin{array}{l}\text { Desplaz. Mínimo } \\
\text { entre plantas } \\
\text { (cm) }\end{array}$ & $\begin{array}{c}\text { Desplaz. Máximo } \\
\text { absoluto } \\
(\mathrm{cm})\end{array}$ & \\
\hline 1a & 0.0180 & 0.864 & -1.780 & 1.780 & $2.025 \div 2.475$ \\
\hline $2 \underline{a}$ & 0.1270 & 0.411 & -1.209 & 1.209 & $1.350 \div 1.650$ \\
\hline $3 \underline{a}$ & 0.0420 & 1.393 & -1.411 & 1.411 & $1.350 \div 1.650$ \\
\hline
\end{tabular}

- Acelerograma №12 (Montenegro aftershock) $\Delta=33 \mathrm{Km}$ (Factor de escala: 1.1610$)$

Tabla 5.4.7: Desplazamientos de fluencia del disipador y desplazamientos máximos absolutos entre plantas, pórtico pa2pz2-1, acelerograma $\mathrm{n}$-12, suelo tipo II, campo lejano

\begin{tabular}{|c|c|c|c|c|c|}
\hline & \multicolumn{4}{|c|}{ Input de energía: $V_{E}=55,34 \mathrm{~cm} / \mathrm{s}$} & \multirow[b]{2}{*}{$\begin{array}{l}\text { Desplaz. objetivo } \\
(\mathrm{cm})\end{array}$} \\
\hline Planta & $\begin{array}{l}\text { Desplaz. fluencia } \\
\text { disipador } \\
\text { (cm) }\end{array}$ & $\begin{array}{l}\text { Desplaz. Máximo } \\
\text { entre plantas } \\
(\mathrm{cm})\end{array}$ & $\begin{array}{l}\text { Desplaz. Mínimo } \\
\text { entre plantas } \\
\text { (cm) }\end{array}$ & $\begin{array}{c}\text { Desplaz. Máximo } \\
\text { absoluto } \\
\text { (cm) }\end{array}$ & \\
\hline $1 \underline{a}$ & 0.9473 & 2.072 & -1.799 & 2.072 & $2.025 \div 2.475$ \\
\hline 2a & 1.5000 & 1.472 & -1.353 & 1.472 & $1.350 \div 1.650$ \\
\hline $3 \underline{a}$ & 2.0000 & 1.591 & -1.205 & 1.591 & $1.350 \div 1.650$ \\
\hline
\end{tabular}




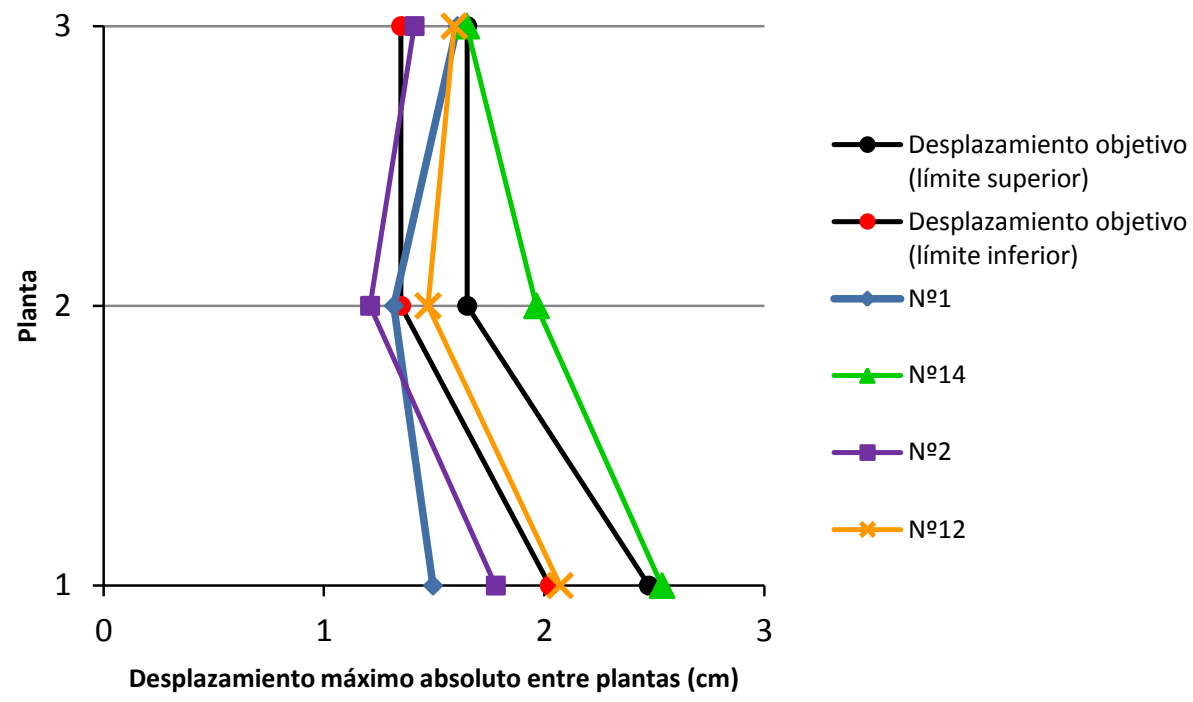

Fig. 5.4.5: Desplazamientos máximos absolutos entre plantas, pórtico pa2pz2-1, suelo tipo II (duro)

\subsection{Suelo Tipo III (Campo cercano a la falla)}

- Acelerograma №2 (Friuli aftershock) $\Delta=\mathbf{1 0 K m}$ (Factor de escala: 1.1564)

Tabla 5.4.8: Desplazamientos de fluencia del disipador y desplazamientos máximos absolutos entre plantas, pórtico pa2pz2-1, acelerograma no2, suelo tipo III, campo cercano

\begin{tabular}{|c|c|c|c|c|c|}
\hline \multicolumn{6}{|c|}{ Input de energía: $V_{E}=72,56 \mathrm{~cm} / \mathrm{s}$} \\
\hline Planta & $\begin{array}{l}\text { Desplaz. fluencia } \\
\text { disipador } \\
(\mathrm{cm})\end{array}$ & $\begin{array}{l}\text { Desplaz. Máximo } \\
\text { entre plantas } \\
\text { (cm) }\end{array}$ & $\begin{array}{l}\text { Desplaz. Mínimo } \\
\text { entre plantas } \\
\text { (cm) }\end{array}$ & $\begin{array}{c}\text { Desplaz. Máximo } \\
\text { absoluto } \\
(\mathrm{cm})\end{array}$ & $\begin{array}{c}\text { Desplaz. objetivo } \\
(\mathrm{cm})\end{array}$ \\
\hline 1aㅗ & 0.7227 & 2.468 & -1.856 & 2.468 & $2.025 \div 2.475$ \\
\hline $2 \underline{a}$ & 0.9375 & 1.625 & -1.419 & 1.625 & $1.350 \div 1.650$ \\
\hline $3 \underline{a}$ & 0.6250 & 1.606 & -1.483 & 1.606 & $1.350 \div 1.650$ \\
\hline
\end{tabular}

- Acelerograma №6 (Kalamata aftershock) $\Delta=3 \mathrm{Km}$ (Factor de escala: 1.1098)

Tabla 5.4.9: Desplazamientos de fluencia del disipador y desplazamientos máximos absolutos entre plantas, pórtico pa2pz2-1, acelerograma no6, suelo tipo III, campo cercano

\begin{tabular}{c|c|c|c|c|c} 
Planta & $\begin{array}{c}\text { Desplaz. fluencia } \\
\text { disipador } \\
(\mathrm{cm})\end{array}$ & $\begin{array}{c}\text { Desplaz. Máximo } \\
\text { entre plantas } \\
(\mathrm{cm})\end{array}$ & $\begin{array}{c}\text { Desplaz. Mínimo } \\
\text { entre plantas } \\
(\mathrm{cm})\end{array}$ & $\begin{array}{c}\text { Desplaz. Máximo } \\
\text { absoluto } \\
(\mathrm{cm})\end{array}$ & $\begin{array}{c}\text { Desplaz. objetivo } \\
(\mathrm{cm})\end{array}$ \\
\hline 1 a & 0.0001 & 1.298 & -2.319 & $\mathbf{2 . 3 1 9}$ & $2.025 \div 2.475$ \\
\hline $2^{\text {a }}$ & 0.1824 & 0.325 & -1.541 & $\mathbf{1 . 5 4 1}$ & $1.350 \div 1.650$ \\
\hline 3 a & 0.0450 & 1.004 & -1.517 & $\mathbf{1 . 5 1 7}$ & $1.350 \div 1.650$ \\
\hline & & & & &
\end{tabular}


- Acelerograma №12 (Duzce 1) $\Delta=8 \mathrm{Km}$ (Factor de escala: 0.7067)

Tabla 5.4.10: Desplazamientos de fluencia del disipador y desplazamientos máximos absolutos entre plantas, pórtico pa2pz2-1, acelerograma no12, suelo tipo III, campo cercano

\begin{tabular}{c|c|c|c|c|c}
\multicolumn{5}{|c|}{ Input de energía: $\mathrm{V}_{\mathrm{E}}=91,96 \mathrm{~cm} / \mathrm{s}$} \\
Planta & $\begin{array}{c}\text { Desplaz. fluencia } \\
\text { disipador } \\
(\mathrm{cm})\end{array}$ & $\begin{array}{c}\text { Desplaz. Máximo } \\
\text { entre plantas } \\
(\mathrm{cm})\end{array}$ & $\begin{array}{c}\text { Desplaz. Mínimo } \\
\text { entre plantas } \\
(\mathrm{cm})\end{array}$ & $\begin{array}{c}\text { Desplaz. Máximo } \\
\text { absoluto } \\
(\mathrm{cm})\end{array}$ & $\begin{array}{c}\text { Desplaz. objetivo } \\
(\mathrm{cm})\end{array}$ \\
\hline $1^{\underline{a}}$ & 0.1914 & 2.400 & -2.400 & $\mathbf{2 . 4 0 0}$ & $2.025 \div 2.475$ \\
\hline $2^{\underline{a}}$ & 0.4150 & 1.599 & -1.184 & $\mathbf{1 . 5 9 9}$ & $1.350 \div 1.650$ \\
\hline & 0.2158 & 1.553 & -1.165 & $\mathbf{1 . 5 5 3}$ & $1.350 \div 1.650$ \\
\hline & & & & &
\end{tabular}

\subsection{Suelo Tipo III (Campo lejano a la falla)}

- Acelerograma №3 (Montenegro) $\Delta=\mathbf{2 4 K m}$ (Factor de escala: 0.9077)

Tabla 5.4.11: Desplazamientos de fluencia del disipador y desplazamientos máximos absolutos entre plantas, pórtico pa2pz2-1, acelerograma no3, suelo tipo III, campo lejano Input de energía: $V_{E}=37,80 \mathrm{~cm} / \mathrm{s}$

\begin{tabular}{c|c|c|c|c|c} 
Planta & $\begin{array}{c}\text { Desplaz. fluencia } \\
\text { disipador } \\
(\mathrm{cm})\end{array}$ & $\begin{array}{c}\text { Desplaz. Máximo } \\
\text { entre plantas } \\
(\mathrm{cm})\end{array}$ & $\begin{array}{c}\text { Desplaz. Mínimo } \\
\text { entre plantas } \\
(\mathrm{cm})\end{array}$ & $\begin{array}{c}\text { Desplaz. Máximo } \\
\text { absoluto } \\
(\mathrm{cm})\end{array}$ & $\begin{array}{c}\text { Desplaz. objetivo } \\
(\mathrm{cm})\end{array}$ \\
\hline 1 a & 0.0001 & 2.138 & -1.684 & $\mathbf{2 . 1 3 8}$ & $2.025 \div 2.475$ \\
\hline $2^{\underline{a}}$ & 0.1167 & 1.571 & -1.306 & $\mathbf{1 . 5 7 1}$ & $1.350 \div 1.650$ \\
\hline a & 0.0001 & 1.421 & -1.387 & $\mathbf{1 . 4 2 1}$ & $1.350 \div 1.650$ \\
\hline & & & & &
\end{tabular}

- Acelerograma №14 (Ano Liosia) $\Delta=\mathbf{1 4 K m}$ (Factor de escala: 0.8168)

Tabla 5.4.12: Desplazamientos de fluencia del disipador y desplazamientos máximos absolutos entre plantas, pórtico pa2pz2-1, acelerograma no14, suelo tipo III, campo lejano

\begin{tabular}{c|c|c|c|c|c} 
& \multicolumn{5}{|c|}{ Input de energía: $V_{\mathrm{E}}=21,99 \mathrm{~cm} / \mathrm{s}$} \\
Planta & $\begin{array}{c}\text { Desplaz. fluencia } \\
\text { disipador } \\
(\mathrm{cm})\end{array}$ & $\begin{array}{c}\text { Desplaz. Máximo } \\
\text { entre plantas } \\
(\mathrm{cm})\end{array}$ & $\begin{array}{c}\text { Desplaz. Mínimo } \\
\text { entre plantas } \\
(\mathrm{cm})\end{array}$ & $\begin{array}{c}\text { Desplaz. Máximo } \\
\text { absoluto } \\
(\mathrm{cm})\end{array}$ & $\begin{array}{c}\text { Desplaz. objetivo } \\
(\mathrm{cm})\end{array}$ \\
\hline 1a & 0.0001 & 0.692 & -1.853 & $\mathbf{1 . 8 5 3}$ & $2.025 \div 2.475$ \\
\hline 2a & 0.0357 & 0.998 & -1.563 & $\mathbf{1 . 5 6 3}$ & $1.350 \div 1.650$ \\
\hline 3a & 0.0001 & 1.475 & -0.975 & $\mathbf{1 . 4 7 5}$ & $1.350 \div 1.650$ \\
\hline & & & & &
\end{tabular}




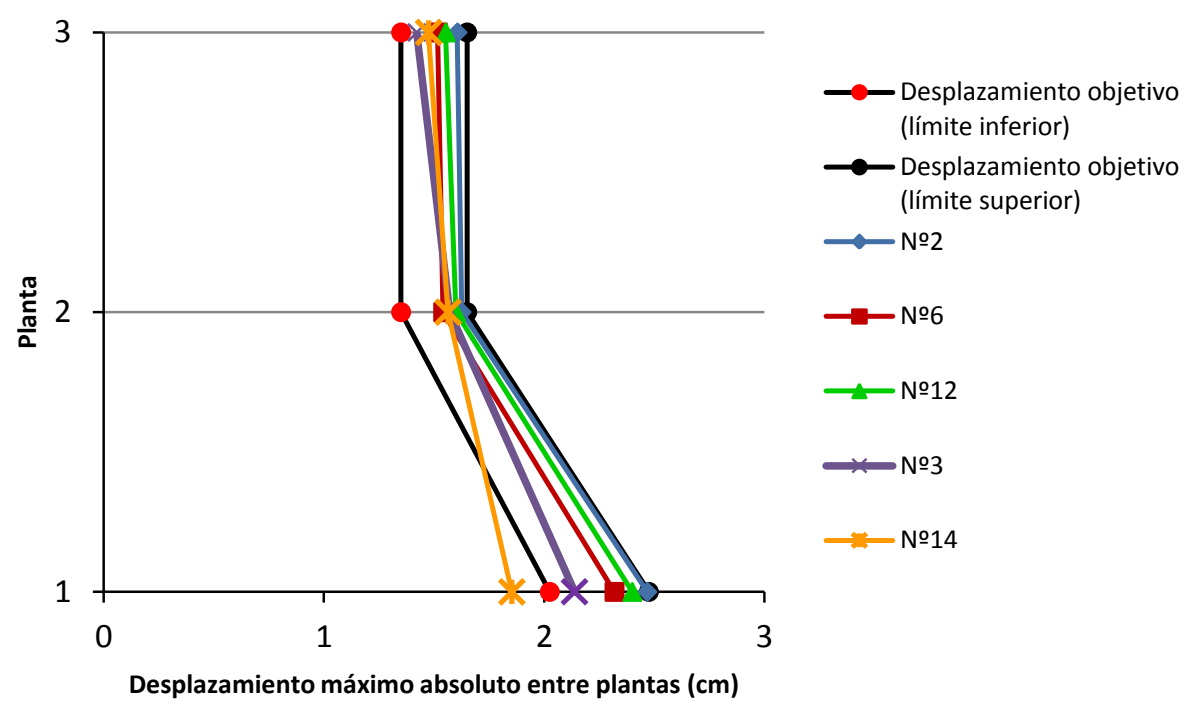

Fig. 5.4.6: Desplazamientos máximos absolutos entre plantas, pórtico pa2pz2-1, suelo tipo III (medio)

\subsubsection{Pórtico pa4pz2-1 (3 plantas)}

- Rigidez disipador:

Planta 3a: ${ }_{s} k_{3}=(77000 \mathrm{~N} / \mathrm{cm}) \times 5=385000 \mathrm{~N} / \mathrm{cm}$

Planta 2a: ${ }_{s} k_{2}=(91000 \mathrm{~N} / \mathrm{cm}) \times 5=455000 \mathrm{~N} / \mathrm{cm}$

Planta 1ạ: ${ }_{s} k_{1}=(95000 \mathrm{~N} / \mathrm{cm}) \times 5=475000 \mathrm{~N} / \mathrm{cm}$

- Desplazamientos de fluencia del pórtico:

Planta 3a : ${ }_{f} \delta_{3}=1,65 \mathrm{~cm}$

Planta 2a: ${ }_{f} \delta_{2}=2,4 \mathrm{~cm}$

Planta 1ㄹ: ${ }_{f} \delta_{1}=3,3 \mathrm{~cm}$

- Desplazamientos objetivo entre plantas:

Para que el desplazamiento máximo entre plantas, sea el $0,5 \%$ en valor absoluto de la altura entre plantas. 


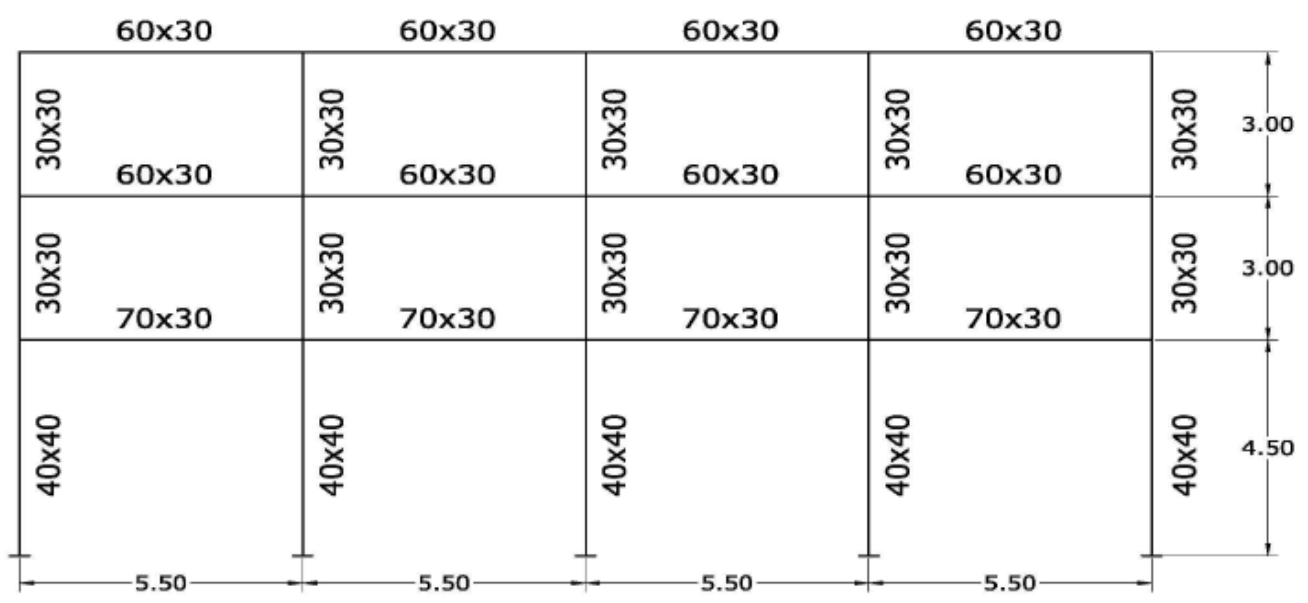

Fig. 5.4.7: Pórtico pa4pz2-1

Altura Planta 3ạ: $3,0 \mathrm{~m}=300 \mathrm{~cm} \Rightarrow \frac{0,5}{100} \times 300=\mathbf{1 , 5 0} \mathrm{cm}$

Altura Planta 2a: $3,0 \mathrm{~m}=300 \mathrm{~cm} \Rightarrow \frac{0,5}{100} \times 300=\mathbf{1 , 5 0} \mathrm{cm}$

Altura Planta 1ạ: $4,5 m=450 \mathrm{~cm} \Rightarrow \frac{0,5}{100} \times 450=\mathbf{2 , 2 5} \mathrm{cm}$

Tolerancia: $10 \%$

$$
\begin{aligned}
& \text { Planta 3a: } \frac{10}{100} \times 1,50=0,150 \Rightarrow\left\{\begin{array}{l}
1,50+0,150=1,650 \mathrm{~cm} \\
1,50-0,150=1,350 \mathrm{~cm}
\end{array}\right. \\
& \text { Planta 2a: } \frac{10}{100} \times 1,50=0,150 \Rightarrow\left\{\begin{array}{l}
1,50+0,150=1,650 \mathrm{~cm} \\
1,50-0,150=1,350 \mathrm{~cm}
\end{array}\right. \\
& \text { Planta 1a: } \frac{10}{100} \times 2,25=0,225 \Rightarrow\left\{\begin{array}{l}
2,25+0,225=2,475 \mathrm{~cm} \\
2,25-0,025=2,025 \mathrm{~cm}
\end{array}\right.
\end{aligned}
$$

- Obtención desplazamiento de fluencia del disipador y desplazamientos máximos y mínimos entre plantas:

Fichero model.dat programa LumpST:

$1,1,4,885.82,0,95000,3.3, \quad 0.01$

$2,1,4, \quad 0 ., \quad 0,475000$, desp. flu. disip., 0.01

$3,2,1,885.82,0,91000,2.4, \quad 0.01$

$4,2,1, \quad 0 ., \quad 0,455000$, desp. flu. disip., 0.01

$5,3,2,885.82,0,77000,1.65, \quad 0.01$

6,3,2, 0., 0, 385000, desp. flu. disip., 0.01 


\subsection{Suelo Tipo I (Campo lejano a la falla)}

- Acelerograma №1 (Friuli) $\Delta=\mathbf{2 7} \mathrm{Km}$ (Factor de escala: 0.5717 )

Tabla 5.4.13: Desplazamientos de fluencia del disipador y desplazamientos máximos absolutos entre plantas, pórtico pa4pz2-1, acelerograma no1, suelo tipo I, campo lejano

\begin{tabular}{c|c|c|c|c|c}
\hline \multicolumn{5}{|c|}{ Input de energía: $V_{\mathrm{E}}=18,73 \mathrm{~cm} / \mathrm{s}$} \\
Planta & $\begin{array}{c}\text { Desplaz. fluencia } \\
\text { disipador } \\
(\mathrm{cm})\end{array}$ & $\begin{array}{c}\text { Desplaz. Máximo } \\
\text { entre plantas } \\
(\mathrm{cm})\end{array}$ & $\begin{array}{c}\text { Desplaz. Mínimo } \\
\text { entre plantas } \\
(\mathrm{cm})\end{array}$ & $\begin{array}{c}\text { Desplaz. Máximo } \\
\text { absoluto } \\
(\mathrm{cm})\end{array}$ & $\begin{array}{c}\text { Desplaz. objetivo } \\
(\mathrm{cm})\end{array}$ \\
\hline 1 a & $0.1305^{*} 1.0 \mathrm{e}-003$ & 1.178 & -2.084 & $\mathbf{2 . 0 8 4}$ & $2.025 \div 2.475$ \\
\hline $2^{\mathrm{a}}$ & $0.1000^{*} 1.0 \mathrm{e}-003$ & 1.044 & -1.663 & $\mathbf{1 . 6 6 3}$ & $1.350 \div 1.650$ \\
\hline 3 a & $0.0981^{*} 1.0 \mathrm{e}-003$ & 0.905 & -1.026 & $\mathbf{1 . 0 2 6}$ & $1.350 \div 1.650$ \\
\hline & & & & &
\end{tabular}

- Acelerograma №4 (Montenegro) $\Delta=65 \mathrm{Km}$ (Factor de escala: 0.9102)

Tabla 5.4.14: Desplazamientos de fluencia del disipador y desplazamientos máximos absolutos entre plantas, pórtico pa4pz2-1, acelerograma no4, suelo tipo I, campo lejano

\begin{tabular}{|c|c|c|c|c|c|}
\hline \multicolumn{5}{|c|}{ Input de energía: $V_{E}=63,42 \mathrm{~cm} / \mathrm{s}$} & \multirow[b]{2}{*}{$\begin{array}{c}\text { Desplaz. objetivo } \\
(\mathrm{cm})\end{array}$} \\
\hline Planta & $\begin{array}{l}\text { Desplaz. fluencia } \\
\text { disipador } \\
\text { (cm) }\end{array}$ & $\begin{array}{l}\text { Desplaz. Máximo } \\
\text { entre plantas } \\
(\mathrm{cm})\end{array}$ & $\begin{array}{l}\text { Desplaz. Mínimo } \\
\text { entre plantas } \\
\text { (cm) }\end{array}$ & $\begin{array}{l}\text { Desplaz. Máximo } \\
\text { absoluto } \\
(\mathrm{cm})\end{array}$ & \\
\hline $1 \underline{a}$ & 1.0625 & 1.872 & -1.268 & 1.872 & $2.025 \div 2.475$ \\
\hline $2^{\mathrm{a}}$ & 1.2500 & 1.177 & -1.064 & 1.177 & $1.350 \div 1.650$ \\
\hline $3 \underline{a}$ & 0.6504 & 1.437 & -0.922 & 1.437 & $1.350 \div 1.650$ \\
\hline
\end{tabular}

- Acelerograma №5 (Campano Lucano) $\Delta=32 \mathrm{Km}$ (Factor de escala: 0.9431)

Tabla 5.4.15: Desplazamientos de fluencia del disipador y desplazamientos máximos absolutos entre plantas, pórtico pa4pz2-1, acelerograma $n \div 5$, suelo tipo I, campo lejano

\begin{tabular}{|c|c|c|c|c|c|}
\hline & \multicolumn{4}{|c|}{ Input de energía: $V_{E}=41,76 \mathrm{~cm} / \mathrm{s}$} & \multirow[b]{2}{*}{$\begin{array}{l}\text { Desplaz. objetivo } \\
(\mathrm{cm})\end{array}$} \\
\hline Planta & $\begin{array}{l}\text { Desplaz. fluencia } \\
\text { disipador } \\
\text { (cm) }\end{array}$ & $\begin{array}{l}\text { Desplaz. Máximo } \\
\text { entre plantas } \\
(\mathrm{cm})\end{array}$ & $\begin{array}{l}\text { Desplaz. Mínimo } \\
\text { entre plantas } \\
\text { (cm) }\end{array}$ & $\begin{array}{l}\text { Desplaz. Máximo } \\
\text { absoluto } \\
\text { (cm) }\end{array}$ & \\
\hline 1aㅗ & $0.8267 * 1.0 \mathrm{e}-003$ & 2.248 & -1.464 & 2.248 & $2.025 \div 2.475$ \\
\hline 2a & $0.1019 * 1.0 \mathrm{e}-003$ & 0.811 & -1.314 & 1.314 & $1.350 \div 1.650$ \\
\hline 3a & $0.1000 * 1.0 \mathrm{e}-003$ & 0.442 & -1.149 & 1.149 & $1.350 \div 1.650$ \\
\hline
\end{tabular}




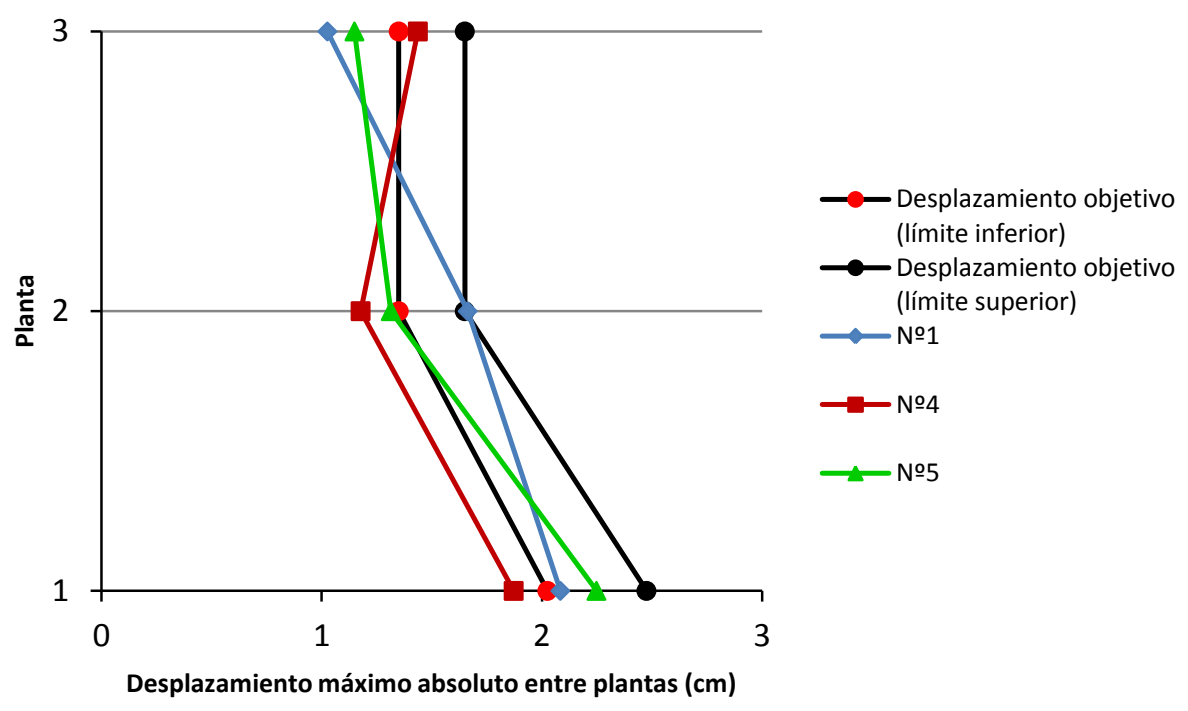

Fig. 5.4.8: Desplazamientos máximos absolutos entre plantas, pórtico pa4pz2-1, suelo tipo I (muy duro)

\subsection{Suelo Tipo II (Campo cercano a la falla)}

- Acelerograma №1 (Friuli aftershock) $\Delta=3 \mathrm{Km}$ (Factor de escala: 1.2554)

Tabla 5.4.16: Desplazamientos de fluencia del disipador y desplazamientos máximos absolutos entre plantas, pórtico pa4pz2-1, acelerograma no1, suelo tipo II, campo cercano

Input de energía: $V_{\mathrm{E}}=26,53 \mathrm{~cm} / \mathrm{s}$

\begin{tabular}{c|c|c|c|c|c} 
& \multicolumn{5}{|c|}{ Input de energía: $\mathrm{V}_{\mathrm{E}}=26,53 \mathrm{~cm} / \mathrm{s}$} \\
\hline Planta & $\begin{array}{c}\text { Desplaz. fluencia } \\
\text { disipador } \\
(\mathrm{cm})\end{array}$ & $\begin{array}{c}\text { Desplaz. Máximo } \\
\text { entre plantas } \\
(\mathrm{cm})\end{array}$ & $\begin{array}{c}\text { Desplaz. Mínimo } \\
\text { entre plantas } \\
(\mathrm{cm})\end{array}$ & $\begin{array}{c}\text { Desplaz. Máximo } \\
\text { absoluto } \\
(\mathrm{cm})\end{array}$ & $\begin{array}{c}\text { Desplaz. objetivo } \\
(\mathrm{cm})\end{array}$ \\
\hline 1 a & 0.0011 & 1.399 & -1.529 & $\mathbf{1 . 5 2 9}$ & $2.025 \div 2.475$ \\
\hline 2 a & 0.0060 & 1.033 & -1.241 & $\mathbf{1 . 2 4 1}$ & $1.350 \div 1.650$ \\
\hline 3 a & 0.0011 & 1.220 & -1.454 & $\mathbf{1 . 4 5 4}$ & $1.350 \div 1.650$ \\
\hline & & & & &
\end{tabular}

- Acelerograma №14 (Montenegro aftershock) $\Delta=8 \mathrm{Km}$ (Factor de escala: 1.9662)

Tabla 5.4.17: Desplazamientos de fluencia del disipador y desplazamientos máximos absolutos entre plantas, pórtico pa4pz2-1, acelerograma no14, suelo tipo II, campo cercano

\begin{tabular}{c|c|c|c|c|c}
\multicolumn{5}{|c}{} & \multicolumn{5}{|c|}{ Input de energía: $V_{\mathrm{E}}=32,89 \mathrm{~cm} / \mathrm{s}$} \\
Planta & $\begin{array}{c}\text { Desplaz. fluencia } \\
\text { disipador } \\
(\mathrm{cm})\end{array}$ & $\begin{array}{c}\text { Desplaz. Máximo } \\
\text { entre plantas } \\
(\mathrm{cm})\end{array}$ & $\begin{array}{c}\text { Desplaz. Mínimo } \\
\text { entre plantas } \\
(\mathrm{cm})\end{array}$ & $\begin{array}{c}\text { Desplaz. Máximo } \\
\text { absoluto } \\
(\mathrm{cm})\end{array}$ & $\begin{array}{c}\text { Desplaz. objetivo } \\
\text { (cm) }\end{array}$ \\
\hline $1^{\underline{a}}$ & 0.0001 & 1.382 & -2.234 & $\mathbf{2 . 2 3 5}$ & $2.025 \div 2.475$ \\
\hline $2^{\underline{a}}$ & 0.1502 & 0.293 & -1.487 & $\mathbf{1 . 4 8 7}$ & $1.350 \div 1.650$ \\
\hline $3^{\underline{a}}$ & 0.0001 & 1.016 & -1.504 & $\mathbf{1 . 5 0 4}$ & $1.350 \div 1.650$ \\
\hline & & & & &
\end{tabular}




\subsection{Suelo Tipo II (Campo lejano a la falla)}

- Acelerograma №2 (Friuli aftershock) $\Delta=\mathbf{1 6 K m}$ (Factor de escala: 1.7929$)$

Tabla 5.4.18: Desplazamientos de fluencia del disipador y desplazamientos máximos absolutos entre plantas, pórtico pa4pz2-1, acelerograma no2, suelo tipo II, campo lejano

\begin{tabular}{|c|c|c|c|c|c|}
\hline \multicolumn{5}{|c|}{ Input de energía: $V_{E}=49,04 \mathrm{~cm} / \mathrm{s}$} & \multirow[b]{2}{*}{$\begin{array}{c}\text { Desplaz. objetivo } \\
(\mathrm{cm})\end{array}$} \\
\hline Planta & $\begin{array}{l}\text { Desplaz. fluencia } \\
\text { disipador } \\
(\mathrm{cm})\end{array}$ & $\begin{array}{c}\text { Desplaz. Máximo } \\
\text { entre plantas } \\
(\mathrm{cm})\end{array}$ & $\begin{array}{l}\text { Desplaz. Mínimo } \\
\text { entre plantas } \\
(\mathrm{cm})\end{array}$ & $\begin{array}{c}\text { Desplaz. Máximo } \\
\text { absoluto } \\
\text { (cm) }\end{array}$ & \\
\hline 1a & 1.1719 & 2.145 & -1.079 & 2.145 & $2.025 \div 2.475$ \\
\hline $2 \underline{a}$ & 1.3750 & 1.276 & -0.959 & 1.276 & $1.350 \div 1.650$ \\
\hline 3aㅗ & 0.6606 & 1.437 & -0.709 & 1.437 & $1.350 \div 1.650$ \\
\hline
\end{tabular}

- Acelerograma №12 (Montenegro aftershock) $\Delta=33 \mathrm{Km}$ (Factor de escala: 1.1610)

Tabla 5.4.19: Desplazamientos de fluencia del disipador y desplazamientos máximos absolutos entre plantas, pórtico pa4pz2-1, acelerograma no12, suelo tipo II, campo lejano

\begin{tabular}{|c|c|c|c|c|c|}
\hline & \multicolumn{4}{|c|}{ Input de energía: $V_{E}=56,30 \mathrm{~cm} / \mathrm{s}$} & \multirow[b]{2}{*}{$\begin{array}{l}\text { Desplaz. objetivo } \\
(\mathrm{cm})\end{array}$} \\
\hline Planta & $\begin{array}{l}\text { Desplaz. fluencia } \\
\text { disipador } \\
(\mathrm{cm})\end{array}$ & $\begin{array}{c}\text { Desplaz. Máximo } \\
\text { entre plantas } \\
(\mathrm{cm})\end{array}$ & $\begin{array}{c}\text { Desplaz. Mínimo } \\
\text { entre plantas } \\
(\mathrm{cm})\end{array}$ & $\begin{array}{c}\text { Desplaz. Máximo } \\
\text { absoluto } \\
(\mathrm{cm})\end{array}$ & \\
\hline 1 a & 1.3125 & 2.251 & -2.051 & 2.251 & $2.025 \div 2.475$ \\
\hline $2 \underline{a}$ & 1.3604 & 1.501 & -1.267 & 1.501 & $1.350 \div 1.650$ \\
\hline $3 \underline{a}$ & 1.0703 & 1.500 & -0.973 & 1.500 & $1.350 \div 1.650$ \\
\hline
\end{tabular}

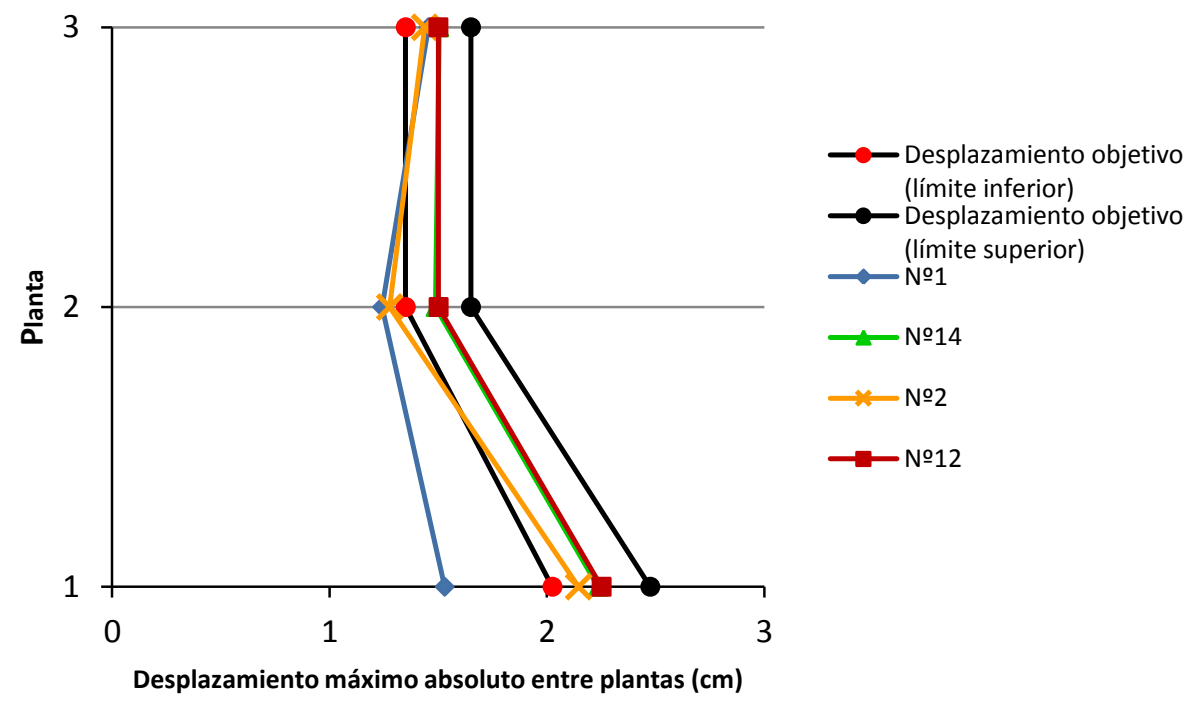

Fig. 5.4.9: Desplazamientos máximos absolutos entre plantas, pórtico pa4pz2-1, suelo tipo II (duro) 


\subsection{Suelo Tipo III (Campo cercano a la falla)}

- Acelerograma №2 (Friuli aftershock) $\Delta=10 \mathrm{Km}$ (Factor de escala: 1.1564 )

Tabla 5.4.20: Desplazamientos de fluencia del disipador y desplazamientos máximos absolutos entre plantas, pórtico pa4pz2-1, acelerograma n2, suelo tipo III, campo cercano

\begin{tabular}{|c|c|c|c|c|c|}
\hline \multicolumn{5}{|c|}{ Input de energía: $V_{\mathrm{E}}=74,02 \mathrm{~cm} / \mathrm{s}$} & \multirow[b]{2}{*}{$\begin{array}{c}\text { Desplaz. objetivo } \\
(\mathrm{cm})\end{array}$} \\
\hline Planta & $\begin{array}{l}\text { Desplaz. fluencia } \\
\text { disipador } \\
(\mathrm{cm})\end{array}$ & $\begin{array}{c}\text { Desplaz. Máximo } \\
\text { entre plantas } \\
(\mathrm{cm})\end{array}$ & $\begin{array}{c}\text { Desplaz. Mínimo } \\
\text { entre plantas } \\
(\mathrm{cm})\end{array}$ & $\begin{array}{c}\text { Desplaz. Máximo } \\
\text { absoluto } \\
(\mathrm{cm})\end{array}$ & \\
\hline $1 \underline{\underline{a}}$ & 0.9121 & 2.443 & -2.158 & 2.443 & $2.025 \div 2.475$ \\
\hline $2 \underline{a}$ & 0.7659 & 1.689 & -1.470 & 1.689 & $1.350 \div 1.650$ \\
\hline $3 \underline{a}$ & 0.4571 & 1.591 & -1.402 & 1.591 & $1.350 \div 1.650$ \\
\hline
\end{tabular}

- Acelerograma №6 (Kalamata aftershock) $\Delta=3 \mathrm{Km}$ (Factor de escala: 1.1098$)$

Tabla 5.4.21: Desplazamientos de fluencia del disipador y desplazamientos máximos absolutos entre plantas, pórtico pa4pz2-1, acelerograma n-6, suelo tipo III, campo cercano

\begin{tabular}{|c|c|c|c|c|c|}
\hline \multicolumn{5}{|c|}{ Input de energía: $V_{E}=42,61 \mathrm{~cm} / \mathrm{s}$} & \multirow[b]{2}{*}{$\begin{array}{c}\text { Desplaz. objetivo } \\
(\mathrm{cm})\end{array}$} \\
\hline Planta & $\begin{array}{l}\text { Desplaz. fluencia } \\
\text { disipador } \\
(\mathrm{cm})\end{array}$ & $\begin{array}{c}\text { Desplaz. Máximo } \\
\text { entre plantas } \\
(\mathrm{cm})\end{array}$ & $\begin{array}{l}\text { Desplaz. Mínimo } \\
\text { entre plantas } \\
(\mathrm{cm})\end{array}$ & $\begin{array}{c}\text { Desplaz. Máximo } \\
\text { absoluto } \\
(\mathrm{cm})\end{array}$ & \\
\hline $1 \underline{a}$ & 0.0802 & 1.822 & -2.247 & 2.247 & $2.025 \div 2.475$ \\
\hline $2 \underline{a}$ & 0.1319 & 1.508 & -1.374 & 1.508 & $1.350 \div 1.650$ \\
\hline 3a & 0.0879 & 1.504 & -0.938 & 1.504 & $1.350 \div 1.650$ \\
\hline
\end{tabular}

- Acelerograma №12 (Duzce 1) $\Delta=\mathbf{8 K m}$ (Factor de escala: 0.7067)

Tabla 5.4.22: Desplazamientos de fluencia del disipador y desplazamientos máximos absolutos entre plantas, pórtico pa4pz2-1, acelerograma no12, suelo tipo III, campo cercano

\begin{tabular}{c|c|c|c|c|c}
\multicolumn{5}{|c|}{ Input de energía: $\mathrm{V}_{\mathrm{E}}=93,84 \mathrm{~cm} / \mathrm{s}$} \\
\hline Planta & $\begin{array}{c}\text { Desplaz. fluencia } \\
\text { disipador } \\
(\mathrm{cm})\end{array}$ & $\begin{array}{c}\text { Desplaz. Máximo } \\
\text { entre plantas } \\
(\mathrm{cm})\end{array}$ & $\begin{array}{c}\text { Desplaz. Mínimo } \\
\text { entre plantas } \\
(\mathrm{cm})\end{array}$ & $\begin{array}{c}\text { Desplaz. Máximo } \\
\text { absoluto } \\
(\mathrm{cm})\end{array}$ & $\begin{array}{c}\text { Desplaz. objetivo } \\
(\mathrm{cm})\end{array}$ \\
\hline 1 a & 0.3633 & 2.333 & -1.780 & $\mathbf{2 . 3 3 3}$ & $2.025 \div 2.475$ \\
\hline 2 a & 0.3633 & 1.642 & -0.869 & $\mathbf{1 . 6 4 2}$ & $1.350 \div 1.650$ \\
\hline 3 a & 0.1640 & 1.544 & -1.334 & $\mathbf{1 . 5 4 4}$ & $1.350 \div 1.650$ \\
\hline & & & & &
\end{tabular}

\subsection{Suelo Tipo III (Campo lejano a la falla)}

- Acelerograma №3 (Montenegro) $\Delta=\mathbf{2 4 K m}$ (Factor de escala: 0.9077 )

Tabla 5.4.23: Desplazamientos de fluencia del disipador y desplazamientos máximos absolutos entre plantas, pórtico pa4pz2-1, acelerograma no3, suelo tipo III, campo lejano

\begin{tabular}{|c|c|c|c|c|c|}
\hline \multicolumn{5}{|c|}{ Input de energía: $V_{E}=121,70 \mathrm{~cm} / \mathrm{s}$} & \multirow[b]{2}{*}{$\begin{array}{c}\text { Desplaz. objetivo } \\
(\mathrm{cm})\end{array}$} \\
\hline Planta & $\begin{array}{l}\text { Desplaz. fluencia } \\
\text { disipador } \\
\text { (cm) }\end{array}$ & $\begin{array}{l}\text { Desplaz. Máximo } \\
\text { entre plantas } \\
(\mathrm{cm})\end{array}$ & $\begin{array}{l}\text { Desplaz. Mínimo } \\
\text { entre plantas } \\
\text { (cm) }\end{array}$ & $\begin{array}{l}\text { Desplaz. Máximo } \\
\text { absoluto } \\
\text { (cm) }\end{array}$ & \\
\hline $1 \underline{\underline{a}}$ & 0.4526 & 2.517 & -2.223 & 2.517 & $2.025 \div 2.475$ \\
\hline $2 \underline{a}$ & 0.3970 & 1.903 & -1.669 & 1.903 & $1.350 \div 1.650$ \\
\hline 3a & 0.1934 & 1.448 & -1.653 & 1.653 & $1.350 \div 1.650$ \\
\hline
\end{tabular}


- Acelerograma №14 (Ano Liosia) $\Delta=\mathbf{1 4 K m}$ (Factor de escala: 0.8168)

Tabla 5.4.24: Desplazamientos de fluencia del disipador y desplazamientos máximos absolutos entre plantas, pórtico pa4pz2-1, acelerograma no14, suelo tipo III, campo lejano

\begin{tabular}{|c|c|c|c|c|c|}
\hline \multirow[b]{2}{*}{ Planta } & \multicolumn{4}{|c|}{ Input de energía: $V_{E}=23,02 \mathrm{~cm} / \mathrm{s}$} & \multirow[b]{2}{*}{$\begin{array}{l}\text { Desplaz. objetivo } \\
(\mathrm{cm})\end{array}$} \\
\hline & $\begin{array}{l}\text { Desplaz. fluencia } \\
\text { disipador } \\
(\mathrm{cm})\end{array}$ & $\begin{array}{c}\text { Desplaz. Máximo } \\
\text { entre plantas } \\
(\mathrm{cm})\end{array}$ & $\begin{array}{l}\text { Desplaz. Mínimo } \\
\text { entre plantas } \\
(\mathrm{cm})\end{array}$ & $\begin{array}{c}\text { Desplaz. Máximo } \\
\text { absoluto } \\
\text { (cm) }\end{array}$ & \\
\hline $1 \underline{a}$ & 0.0123 & 1.752 & -2.223 & 2.223 & $2.025 \div 2.475$ \\
\hline $2^{\mathrm{a}}$ & 0.0001 & 1.121 & -1.564 & 1.564 & $1.350 \div 1.650$ \\
\hline $3 \underline{a}$ & 0.0001 & 1.390 & -0.996 & 1.390 & $1.350 \div 1.650$ \\
\hline
\end{tabular}

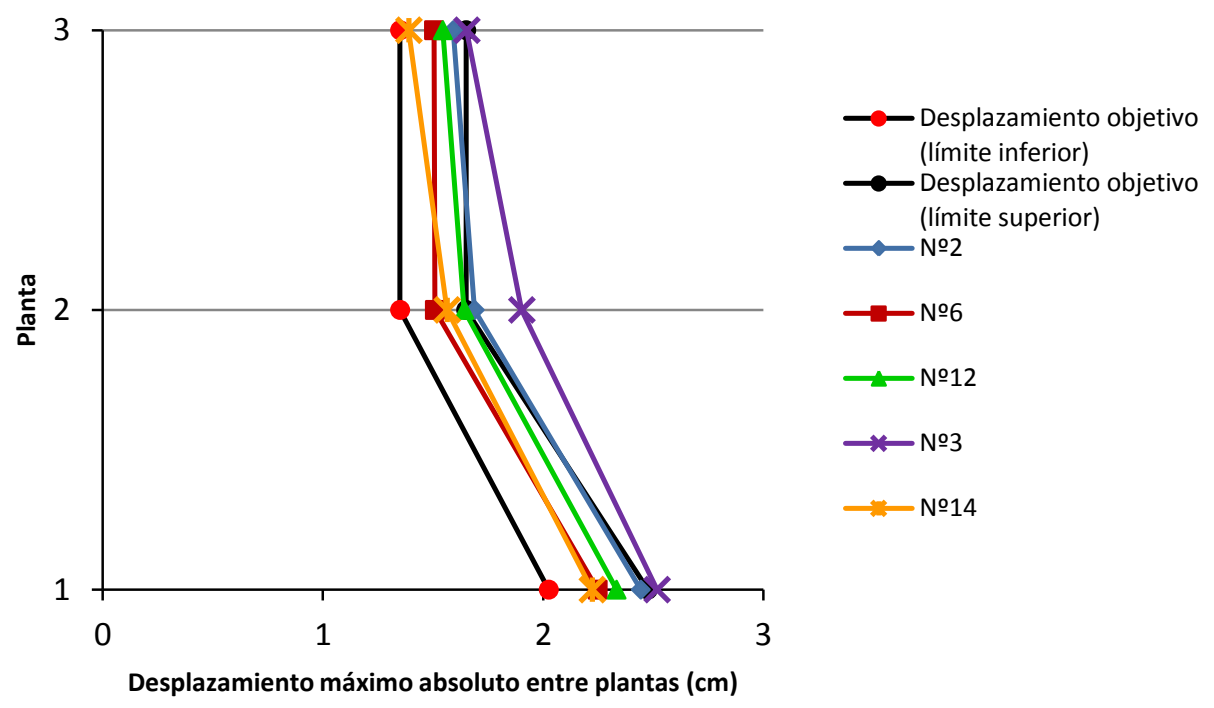

Fig. 5.4.10: Desplazamientos máximos absolutos entre plantas, pórtico pa4pz2-1, suelo tipo III (medio)

\subsubsection{Pórtico pa6pz2-1 (6 plantas)}

- Rigidez disipador:

Planta 6a: ${ }_{s} k_{6}=(56000 \mathrm{~N} / \mathrm{cm}) \times 5=280000 \mathrm{~N} / \mathrm{cm}$

Planta 5a: ${ }_{s} k_{5}=(83000 \mathrm{~N} / \mathrm{cm}) \times 5=415000 \mathrm{~N} / \mathrm{cm}$

Planta 4ạ: ${ }_{s} k_{4}=(113000 \mathrm{~N} / \mathrm{cm}) \times 5=565000 \mathrm{~N} / \mathrm{cm}$

Planta 3a: ${ }_{s} k_{3}=(177000 \mathrm{~N} / \mathrm{cm}) \times 5=885000 \mathrm{~N} / \mathrm{cm}$

Planta 2a: ${ }_{s} k_{2}=(224000 \mathrm{~N} / \mathrm{cm}) \times 5=1120000 \mathrm{~N} / \mathrm{cm}$

Planta 1a : ${ }_{s} k_{1}=(175000 \mathrm{~N} / \mathrm{cm}) \times 5=875000 \mathrm{~N} / \mathrm{cm}$

- Desplazamientos de fluencia del pórtico:

Planta 6a: ${ }_{f} \delta_{6}=1,6 \mathrm{~cm}$

Planta 5a: ${ }_{f} \delta_{5}=2,0 \mathrm{~cm}$

Planta 4모 : ${ }_{f} \delta_{4}=1,0 \mathrm{~cm}$

Planta 3a: ${ }_{f} \delta_{3}=0,8 \mathrm{~cm}$ 
Planta 2a: ${ }_{f} \delta_{2}=0,7 \mathrm{~cm}$

Planta 1ㄹ: ${ }_{f} \delta_{1}=0,9 \mathrm{~cm}$

- Desplazamientos objetivo entre plantas:

Para que el desplazamiento máximo entre plantas, sea el $0,5 \%$ en valor absoluto de la altura entre plantas.

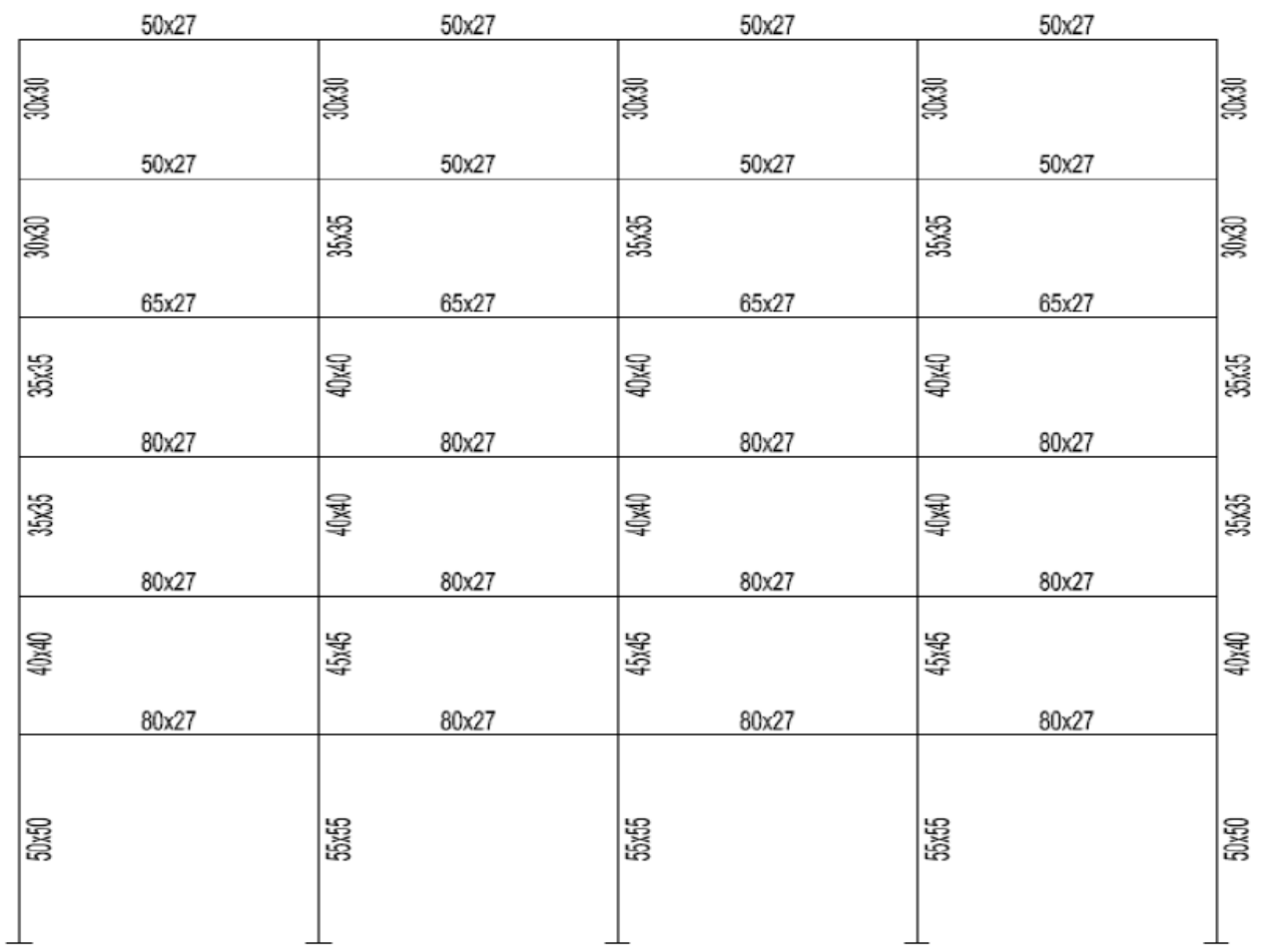

Fig. 5.4.11: Pórtico pa6pz2-1

Altura Planta 6ạ: $3,0 m=300 \mathrm{~cm} \Rightarrow \frac{0,5}{100} \times 300=\mathbf{1 , 5 0} \mathrm{cm}$

Altura Planta 5: $3,0 m=300 \mathrm{~cm} \Rightarrow \frac{0,5}{100} \times 300=\mathbf{1 , 5 0} \mathrm{cm}$

Altura Planta 4르 : $3,0 m=300 \mathrm{~cm} \Rightarrow \frac{0,5}{100} \times 300=\mathbf{1 , 5 0} \mathrm{cm}$

Altura Planta 3a: $3,0 \mathrm{~m}=300 \mathrm{~cm} \Rightarrow \frac{0,5}{100} \times 300=\mathbf{1 , 5 0} \mathrm{cm}$

Altura Planta 2a: $3,0 m=300 \mathrm{~cm} \Rightarrow \frac{0,5}{100} \times 300=\mathbf{1 , 5 0} \mathrm{cm}$

Altura Planta 1a: $4,5 m=450 \mathrm{~cm} \Rightarrow \frac{0,5}{100} \times 450=\mathbf{2 , 2 5} \mathrm{cm}$

Tolerancia: $10 \%$ : 


$$
\begin{aligned}
& \text { Planta 6a : } \frac{10}{100} \times 1,50=0,150 \Rightarrow\left\{\begin{array}{l}
1,50+0,150=1,650 \mathrm{~cm} \\
1,50-0,150=1,350 \mathrm{~cm}
\end{array}\right. \\
& \text { Planta 5a: } \frac{10}{100} \times 1,50=0,150 \Rightarrow\left\{\begin{array}{l}
1,50+0,150=1,650 \mathrm{~cm} \\
1,50-0,150=1,350 \mathrm{~cm}
\end{array}\right. \\
& \text { Planta 4a: } \frac{10}{100} \times 1,50=0,150 \Rightarrow\left\{\begin{array}{l}
1,50+0,150=1,650 \mathrm{~cm} \\
1,50-0,150=1,350 \mathrm{~cm}
\end{array}\right. \\
& \text { Planta 3a: } \frac{10}{100} \times 1,50=0,150 \Rightarrow\left\{\begin{array}{l}
1,50+0,150=1,650 \mathrm{~cm} \\
1,50-0,150=1,350 \mathrm{~cm}
\end{array}\right. \\
& \text { Planta 2a }: \frac{10}{100} \times 1,50=0,150 \Rightarrow\left\{\begin{array}{l}
1,50+0,150=1,650 \mathrm{~cm} \\
1,50-0,150=1,350 \mathrm{~cm}
\end{array}\right. \\
& \text { Planta 1a: } \frac{10}{100} \times 2,25=0,225 \Rightarrow\left\{\begin{array}{l}
2,25+0,225=2,475 \mathrm{~cm} \\
2,25-0,025=2,025 \mathrm{~cm}
\end{array}\right.
\end{aligned}
$$

- Obtención desplazamiento de fluencia del disipador y desplazamientos máximos y mínimos entre plantas:

Fichero model.dat programa LumpST:

$1,1,7,739.04,0,175000,0.9, \quad 0.01$

$2,1,7, \quad 0 ., \quad 0,875000$, desp. flu. disip., 0.01

3,2,1, 739.04,0, 224000, 0.7, $\quad 0.01$

$4,2,1, \quad 0 ., \quad 0,1120000$, desp. flu. disip.,0.01

$5,3,2,739.04,0,177000,0.8, \quad 0.01$

$6,3,2, \quad 0 ., \quad 0,885000$, desp. flu. disip., 0.01

$7,4,3,739.04,0,113000,1.0, \quad 0.01$

$8,4,3, \quad 0 ., \quad 0,565000$, desp. flu. disip.,0.01

$9,5,4,739.04,0,83000,2.0, \quad 0.01$

$10,5,4, \quad 0 ., \quad 0,415000$, desp. flu. disip.,0.01

$11,6,5,739.04,0,56000,1.6, \quad 0.01$

$12,6,5, \quad 0 ., \quad 0,280000$, desp. flu. disip.,0.01 


\subsection{Suelo Tipo I (Campo lejano a la falla)}

- Acelerograma №4 (Montenegro) $\Delta=65 \mathrm{Km}$ (Factor de escala: 0.9102)

Tabla 5.4.25: Desplazamientos de fluencia del disipador y desplazamientos máximos absolutos entre plantas, pórtico pa6pz2-1, acelerograma no4, suelo tipo I, campo lejano

\begin{tabular}{|c|c|c|c|c|c|}
\hline \multicolumn{5}{|c|}{ Input de energía: $V_{\mathrm{E}}=59,80 \mathrm{~cm} / \mathrm{s}$} & \multirow[b]{2}{*}{$\begin{array}{l}\text { Desplaz. objetivo } \\
(\mathrm{cm})\end{array}$} \\
\hline Planta & $\begin{array}{l}\text { Desplaz. fluencia } \\
\text { disipador } \\
(\mathrm{cm}) \\
\end{array}$ & $\begin{array}{c}\text { Desplaz. Máximo } \\
\text { entre plantas } \\
(\mathrm{cm})\end{array}$ & $\begin{array}{c}\text { Desplaz. Mínimo } \\
\text { entre plantas } \\
(\mathrm{cm})\end{array}$ & $\begin{array}{c}\text { Desplaz. Máximo } \\
\text { absoluto } \\
(\mathrm{cm})\end{array}$ & \\
\hline $1 \underline{a}$ & 0.610 & 1.856 & -1.040 & 1.856 & $2.025 \div 2.475$ \\
\hline 2 a & 0.470 & 0.803 & -0.500 & 0.803 & $1.350 \div 1.650$ \\
\hline $3 \underline{a}$ & 0.570 & 0.645 & -0.554 & 0.645 & $1.350 \div 1.650$ \\
\hline $4 \underline{a}$ & 0.720 & 1.020 & -1.269 & 1.269 & $1.350 \div 1.650$ \\
\hline $5 \underline{a}$ & 1.200 & 1.115 & -1.005 & 1.115 & $1.350 \div 1.650$ \\
\hline 6a & 1.100 & 0.959 & -1.472 & 1.472 & $1.350 \div 1.650$ \\
\hline
\end{tabular}

- Acelerograma №5 (Campano Lucano) $\Delta=32 \mathrm{Km}$ (Factor de escala: 0.9431)

Tabla 5.4.26: Desplazamientos de fluencia del disipador y desplazamientos máximos absolutos entre plantas, pórtico pa6pz2-1, acelerograma no5, suelo tipo I, campo lejano

\begin{tabular}{|c|c|c|c|c|c|}
\hline \multirow[b]{2}{*}{ Planta } & \multicolumn{4}{|c|}{ Input de energía: $V_{E}=32,89 \mathrm{~cm} / \mathrm{s}$} & \multirow[b]{2}{*}{$\begin{array}{l}\text { Desplaz. objetivo } \\
(\mathrm{cm})\end{array}$} \\
\hline & $\begin{array}{l}\text { Desplaz. fluencia } \\
\text { disipador } \\
\text { (cm) }\end{array}$ & $\begin{array}{l}\text { Desplaz. Máximo } \\
\text { entre plantas } \\
(\mathrm{cm})\end{array}$ & $\begin{array}{l}\text { Desplaz. Mínimo } \\
\text { entre plantas } \\
\text { (cm) }\end{array}$ & $\begin{array}{l}\text { Desplaz. Máximo } \\
\text { absoluto } \\
\text { (cm) }\end{array}$ & \\
\hline 1a & 0.0250 & 2.467 & -0.977 & 2.467 & $2.025 \div 2.475$ \\
\hline $2 \underline{a}$ & 0.0100 & 1.023 & -0.714 & 1.023 & $1.350 \div 1.650$ \\
\hline $3 \underline{a}$ & 0.0100 & 0.914 & -0.792 & 0.914 & $1.350 \div 1.650$ \\
\hline $4 \underline{a}$ & 0.0100 & 1.340 & -1.009 & 1.340 & $1.350 \div 1.650$ \\
\hline $5 \underline{a}$ & 0.0160 & 0.998 & -1.014 & 1.014 & $1.350 \div 1.650$ \\
\hline 6ạ & 0.00009 & 0.460 & -1.219 & 1.219 & $1.350 \div 1.650$ \\
\hline
\end{tabular}

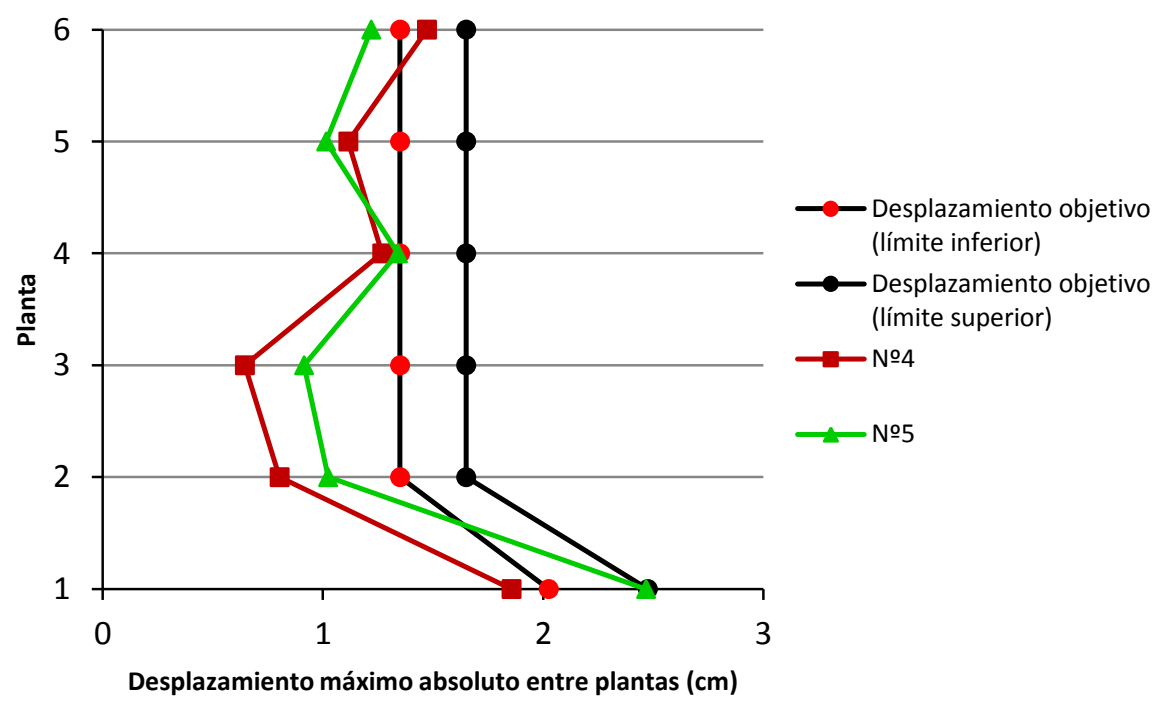

Fig. 5.4.12: Desplazamientos máximos absolutos entre plantas, pórtico pa6pz2-1, suelo tipo I (muy duro) 


\subsection{Suelo Tipo II (Campo cercano a la falla)}

\section{- Acelerograma №14 (Montenegro aftershock) $\Delta=8 \mathrm{Km}$ (Factor de escala: 1.9662)}

Tabla 5.4.27: Desplazamientos de fluencia del disipador y desplazamientos máximos absolutos entre plantas, pórtico pa6pz2-1, acelerograma no14, suelo tipo II, campo lejano

\begin{tabular}{|c|c|c|c|c|c|}
\hline \multirow[b]{2}{*}{ № } & \multicolumn{4}{|c|}{ Input de energía: $V_{E}=98,48 \mathrm{~cm} / \mathrm{s}$} & \multirow[b]{2}{*}{$\begin{array}{l}\text { Desplaz. objetivo } \\
(\mathrm{cm})\end{array}$} \\
\hline & $\begin{array}{l}\text { Desplaz. fluencia } \\
\text { disipador } \\
(\mathrm{cm})\end{array}$ & $\begin{array}{c}\text { Desplaz. Máximo } \\
\text { entre plantas } \\
(\mathrm{cm})\end{array}$ & $\begin{array}{l}\text { Desplaz. Mínimo } \\
\text { entre plantas } \\
(\mathrm{cm})\end{array}$ & $\begin{array}{c}\text { Desplaz. Máximo } \\
\text { absoluto } \\
\text { (cm) }\end{array}$ & \\
\hline $1 \underline{a}$ & 0.400 & 0.544 & -2.295 & 2.295 & $2.025 \div 2.475$ \\
\hline $2 \underline{a}$ & 0.240 & 0.456 & -1.552 & 1.552 & $1.350 \div 1.650$ \\
\hline 3a & 0.240 & 0.861 & -1.352 & 1.352 & $1.350 \div 1.650$ \\
\hline $4 \underline{a}$ & 0.365 & 1.637 & -1.038 & 1.637 & $1.350 \div 1.650$ \\
\hline 5a & 0.280 & 1.645 & -1.410 & 1.645 & $1.350 \div 1.650$ \\
\hline 6a & 0.260 & 1.570 & -1.219 & 1.570 & $1.350 \div 1.650$ \\
\hline
\end{tabular}

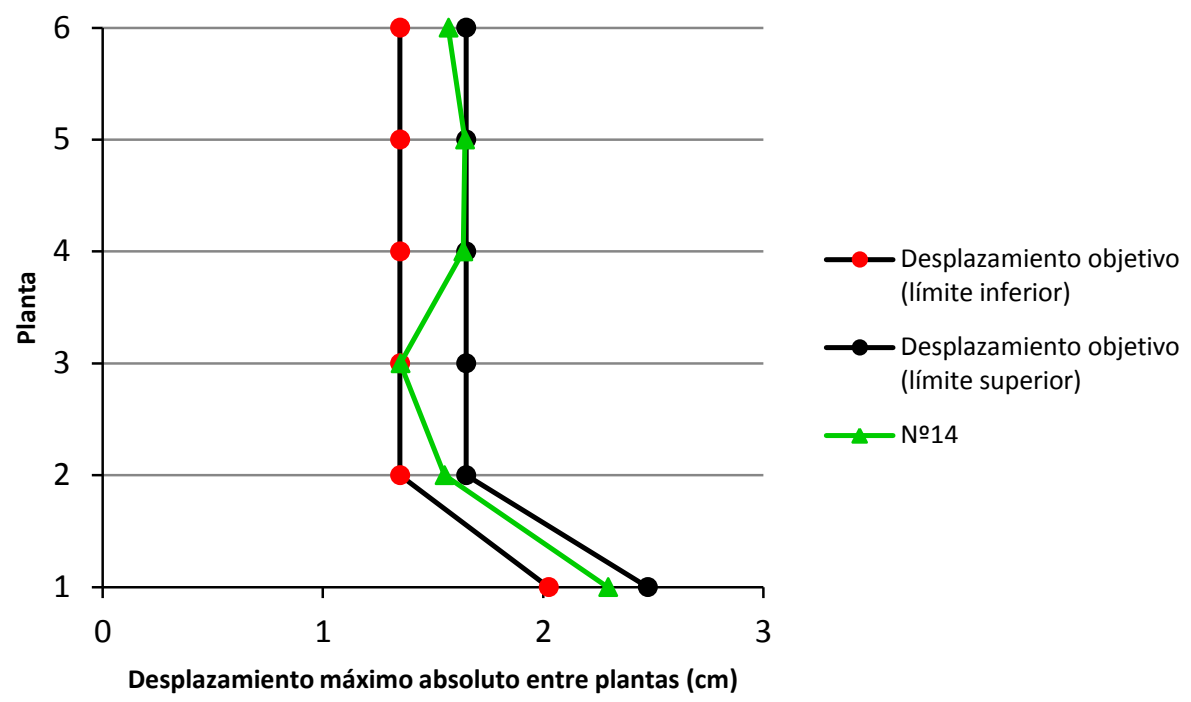

Fig. 5.4.13: Desplazamientos máximos absolutos entre plantas, pórtico pa6pz2-1, suelo tipo II (duro)

\subsection{Suelo Tipo III (Campo cercano a la falla)}

- Acelerograma №2 (Friuli aftershock) $\Delta=\mathbf{1 0 K m}$ (Factor de escala: 1.1564)

Tabla 5.4.28: Desplazamientos de fluencia del disipador y desplazamientos máximos absolutos entre plantas, pórtico pa6pz2-1, acelerograma no2, suelo tipo III, campo cercano

\begin{tabular}{|c|c|c|c|c|c|}
\hline \multicolumn{5}{|c|}{ Input de energía: $V_{E}=40,26 \mathrm{~cm} / \mathrm{s}$} & \multirow[b]{2}{*}{$\begin{array}{l}\text { Desplaz. objetivo } \\
\text { (cm) }\end{array}$} \\
\hline Planta & $\begin{array}{l}\text { Desplaz. fluencia } \\
\text { disipador } \\
(\mathrm{cm})\end{array}$ & $\begin{array}{c}\text { Desplaz. Máximo } \\
\text { entre plantas } \\
(\mathrm{cm})\end{array}$ & $\begin{array}{c}\text { Desplaz. Mínimo } \\
\text { entre plantas } \\
(\mathrm{cm})\end{array}$ & $\begin{array}{c}\text { Desplaz. Máximo } \\
\text { absoluto } \\
(\mathrm{cm})\end{array}$ & \\
\hline $1 \underline{a}$ & 0.02700 & 1.013 & -2.033 & 2.033 & $2.025 \div 2.475$ \\
\hline $2 \underline{a}$ & 0.00390 & 0.722 & -1.213 & 1.213 & $1.350 \div 1.650$ \\
\hline $3 \underline{a}$ & 0.00004 & 0.782 & -1.135 & 1.135 & $1.350 \div 1.650$ \\
\hline $4 \underline{a}$ & 0.04300 & 0.796 & -1.554 & 1.554 & $1.350 \div 1.650$ \\
\hline 5a & 0.06900 & 1.521 & -1.572 & 1.572 & $1.350 \div 1.650$ \\
\hline 6a & 0.11000 & 1.407 & -1.137 & 1.407 & $1.350 \div 1.650$ \\
\hline
\end{tabular}


- Acelerograma №6 (Kalamata aftershock) $\Delta=3 \mathrm{Km}$ (Factor de escala: 1.1098 )

Tabla 5.4.29: Desplazamientos de fluencia del disipador y desplazamientos máximos absolutos entre plantas, pórtico pa6pz2-1, acelerograma no6, suelo tipo III, campo cercano

\begin{tabular}{|c|c|c|c|c|c|}
\hline & \multicolumn{4}{|c|}{ Input de energía: $V_{E}=65,35 \mathrm{~cm} / \mathrm{s}$} & \multirow[b]{2}{*}{$\begin{array}{c}\text { Desplaz. objetivo } \\
(\mathrm{cm})\end{array}$} \\
\hline Planta & $\begin{array}{l}\text { Desplaz. fluencia } \\
\text { disipador } \\
(\mathrm{cm})\end{array}$ & $\begin{array}{l}\text { Desplaz. Máximo } \\
\text { entre plantas } \\
(\mathrm{cm})\end{array}$ & $\begin{array}{c}\text { Desplaz. Mínimo } \\
\text { entre plantas } \\
(\mathrm{cm})\end{array}$ & $\begin{array}{c}\text { Desplaz. Máximo } \\
\text { absoluto } \\
(\mathrm{cm})\end{array}$ & \\
\hline $1 \underline{a}$ & 0.776 & 0.474 & -2.108 & 2.108 & $2.025 \div 2.475$ \\
\hline 2 & 0.496 & 0.769 & -1.335 & 1.335 & $1.350 \div 1.650$ \\
\hline 3 a & 0.706 & 1.333 & -0.584 & 1.333 & $1.350 \div 1.650$ \\
\hline $4 \underline{a}$ & 1.490 & 1.410 & -0.809 & 1.410 & $1.350 \div 1.650$ \\
\hline $5 \underline{a}$ & 1.615 & 1.633 & -0.871 & 1.633 & $1.350 \div 1.650$ \\
\hline $6 \underline{a}$ & 1.900 & 1.934 & -0.944 & 1.934 & $1.350 \div 1.650$ \\
\hline
\end{tabular}

- Acelerograma №12 (Duzce 1) $\Delta=8 \mathrm{Km}$ (Factor de escala: 0.7067)

Tabla 5.4.30: Desplazamientos de fluencia del disipador y desplazamientos máximos absolutos entre plantas, pórtico pa6pz2-1, acelerograma no12, suelo tipo III, campo cercano

\begin{tabular}{|c|c|c|c|c|c|}
\hline \multicolumn{5}{|c|}{ Input de energía: $V_{E}=81,21 \mathrm{~cm} / \mathrm{s}$} & \multirow[b]{2}{*}{$\begin{array}{l}\text { Desplaz. objetivo } \\
(\mathrm{cm})\end{array}$} \\
\hline Planta & $\begin{array}{l}\text { Desplaz. fluencia } \\
\text { disipador } \\
(\mathrm{cm})\end{array}$ & $\begin{array}{c}\text { Desplaz. Máximo } \\
\text { entre plantas } \\
(\mathrm{cm})\end{array}$ & $\begin{array}{c}\text { Desplaz. Mínimo } \\
\text { entre plantas } \\
(\mathrm{cm})\end{array}$ & $\begin{array}{c}\text { Desplaz. Máximo } \\
\text { absoluto } \\
(\mathrm{cm})\end{array}$ & \\
\hline 1a & 0.1810 & 0.785 & -2.649 & 2.649 & $2.025 \div 2.475$ \\
\hline $22^{a}$ & 0.0940 & 0.613 & -1.671 & 1.671 & $1.350 \div 1.650$ \\
\hline $3 \underline{a}$ & 0.0721 & 0.748 & -1.991 & 1.991 & $1.350 \div 1.650$ \\
\hline $4 \underline{a}$ & 0.0900 & 1.214 & -1.350 & 1.350 & $1.350 \div 1.650$ \\
\hline $5 \underline{a}$ & 0.1100 & 1.334 & -1.261 & 1.334 & $1.350 \div 1.650$ \\
\hline 6a & 0.0900 & 1.547 & -1.266 & 1.547 & $1.350 \div 1.650$ \\
\hline
\end{tabular}

\subsection{Suelo Tipo III (Campo lejano a la falla)}

- Acelerograma №3 (Montenegro) $\Delta=\mathbf{2 4 K m}$ (Factor de escala: 0.9077)

Tabla 5.4.31: Desplazamientos de fluencia del disipador y desplazamientos máximos absolutos entre plantas, pórtico pa6pz2-1, acelerograma no3, suelo tipo III, campo lejano

\begin{tabular}{|c|c|c|c|c|c|}
\hline \multicolumn{5}{|c|}{ Input de energía: $V_{\mathrm{E}}=94,10 \mathrm{~cm} / \mathrm{s}$} & \multirow[b]{2}{*}{$\begin{array}{c}\text { Desplaz. objetivo } \\
(\mathrm{cm})\end{array}$} \\
\hline Planta & $\begin{array}{l}\text { Desplaz. fluencia } \\
\text { disipador } \\
\text { (cm) }\end{array}$ & $\begin{array}{c}\text { Desplaz. Máximo } \\
\text { entre plantas } \\
(\mathrm{cm})\end{array}$ & $\begin{array}{c}\text { Desplaz. Mínimo } \\
\text { entre plantas } \\
(\mathrm{cm})\end{array}$ & $\begin{array}{c}\text { Desplaz. Máximo } \\
\text { absoluto } \\
(\mathrm{cm})\end{array}$ & \\
\hline 1a & 0.980 & 2.335 & -0.736 & 2.335 & $2.025 \div 2.475$ \\
\hline $2 \underline{a}$ & 0.690 & 1.571 & -0.465 & 1.571 & $1.350 \div 1.650$ \\
\hline $3 \underline{a}$ & 0.780 & 1.573 & -0.663 & 1.573 & $1.350 \div 1.650$ \\
\hline $4 \underline{a}$ & 1.110 & 1.221 & -1.622 & 1.622 & $1.350 \div 1.650$ \\
\hline 5a & 1.300 & 1.300 & -1.645 & 1.645 & $1.350 \div 1.650$ \\
\hline 6a & 1.700 & 1.216 & -1.706 & 1.706 & $1.350 \div 1.650$ \\
\hline
\end{tabular}




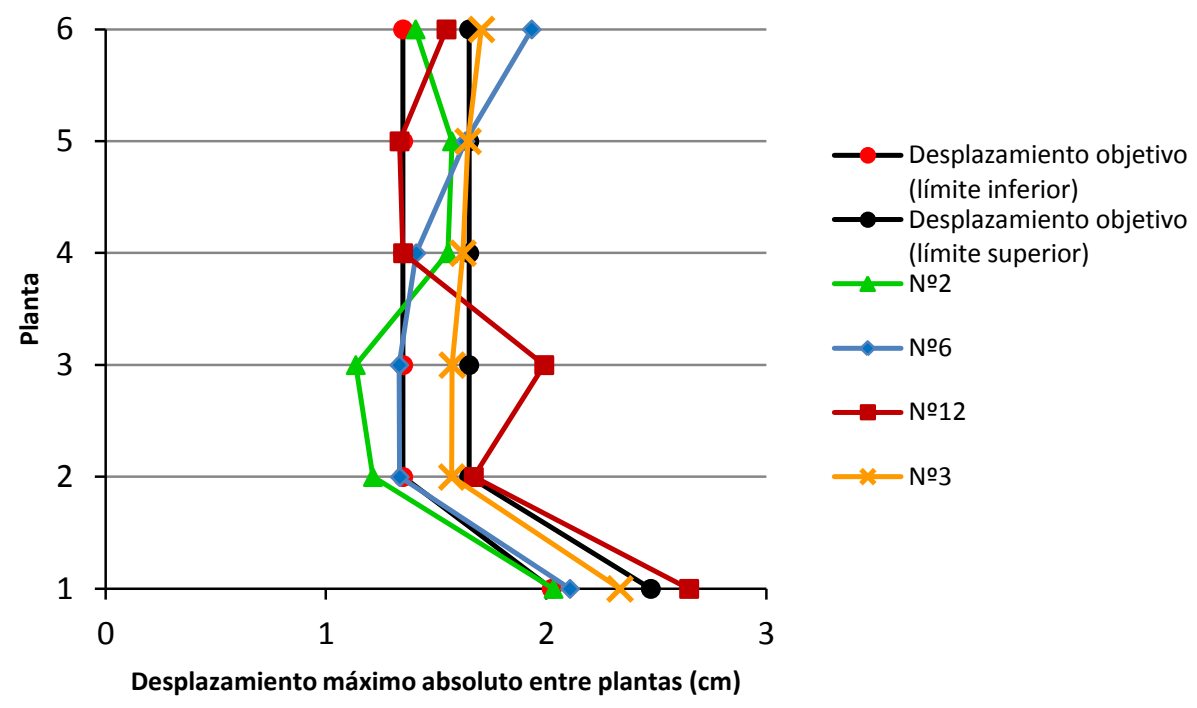

Fig. 5.4.14: Desplazamientos máximos absolutos entre plantas, pórtico pa6pz2-1, suelo tipo III (medio)

Tabla 5.4.32: Desplazamientos de fluencia del disipador, desplazamiento máximo absoluto entre plantas, input de energía expresado en forma de pseudovelocidad equivalente para cada acelerograma

\begin{tabular}{|c|c|c|c|c|c|c|}
\hline Pórtico & Tipo de suelo & Acelerograma & Planta & $\begin{array}{l}\text { Desplaz. } \\
\text { fluencia } \\
\text { disipador } \\
{ }_{s} \delta_{i}(\mathrm{~cm})\end{array}$ & $\begin{array}{l}\text { Desplaz. máximo } \\
\text { absoluto entre } \\
\text { plantas } \\
(\mathrm{cm})\end{array}$ & $\begin{array}{l}\text { Input de } \\
\text { energía } \\
V_{E}(\mathrm{~cm} / \mathrm{s})\end{array}$ \\
\hline \multirow{9}{*}{ pa2pz2-1 } & \multirow{3}{*}{$\begin{array}{c}\text { I } \\
\text { Campo lejano }\end{array}$} & $\begin{array}{c}\text { №1 } \\
\text { (Friuli) }\end{array}$ & $\begin{array}{l}1 \mathrm{a} \\
2 \underline{a} \\
3 \underline{a}\end{array}$ & $\begin{array}{c}0.0030 \\
0.0478 \\
0.00008 \\
\end{array}$ & $\begin{array}{l}1.712 \\
1.570 \\
1.150 \\
\end{array}$ & 20,94 \\
\hline & & $\begin{array}{c}\text { №4 } \\
\text { (Montenegro) }\end{array}$ & $\begin{array}{l}1 \mathrm{a} \\
2 \underline{\mathrm{a}} \\
3 \mathrm{a}\end{array}$ & $\begin{array}{l}0.0003 \\
0.2384 \\
0.0880 \\
\end{array}$ & $\begin{array}{l}1.922 \\
1.001 \\
1.181 \\
\end{array}$ & 34,95 \\
\hline & & $\begin{array}{c}\text { №5 } \\
\text { (Campano } \\
\text { Lucano) }\end{array}$ & $\begin{array}{l}1 \mathrm{a} \\
2 \mathrm{a} \\
3 \mathrm{a}\end{array}$ & $\begin{array}{l}0.0226 \\
0.0402 \\
0.0001\end{array}$ & $\begin{array}{l}2.340 \\
1.532 \\
1.117\end{array}$ & 39,04 \\
\hline & \multirow{2}{*}{$\begin{array}{c}\text { II } \\
\text { Campo cercano }\end{array}$} & $\begin{array}{c}\text { №1 } \\
\text { (Friuli } \\
\text { aftershock) }\end{array}$ & $\begin{array}{l}10 \\
2 \underline{a} \\
3 \underline{a}\end{array}$ & $\begin{array}{l}0.0008 \\
0.0356 \\
0.0001\end{array}$ & $\begin{array}{l}1.495 \\
1.317 \\
1.606 \\
\end{array}$ & 28,93 \\
\hline & & $\begin{array}{c}\text { №14 } \\
\text { (Montenegro } \\
\text { aftershock) }\end{array}$ & $\begin{array}{l}1 \text { a } \\
2 \underline{a} \\
3 \underline{a}\end{array}$ & $\begin{array}{l}0.4883 \\
0.5937 \\
0.4454\end{array}$ & $\begin{array}{l}2.535 \\
1.965 \\
1.648\end{array}$ & 116,08 \\
\hline & \multirow{2}{*}{$\begin{array}{c}\text { II } \\
\text { Campo lejano }\end{array}$} & $\begin{array}{c}\text { №2 } \\
\text { (Friuli } \\
\text { aftershock) }\end{array}$ & $\begin{array}{l}19 \\
2^{\mathrm{a}} \\
3^{\mathrm{a}} \\
\end{array}$ & $\begin{array}{l}0.0180 \\
0.1270 \\
0.0420 \\
\end{array}$ & $\begin{array}{l}1.780 \\
1.209 \\
1.411 \\
\end{array}$ & 30,80 \\
\hline & & $\begin{array}{c}\text { №12 } \\
\text { (Montenegro } \\
\text { aftershock) }\end{array}$ & $\begin{array}{l}19 \\
2 \underline{a} \\
3 a\end{array}$ & $\begin{array}{l}0.9473 \\
1.5000 \\
2.0000\end{array}$ & $\begin{array}{l}2.072 \\
1.472 \\
1.591 \\
\end{array}$ & 55,34 \\
\hline & \multirow{2}{*}{$\begin{array}{c}\text { III } \\
\text { Campo cercano }\end{array}$} & $\begin{array}{c}\text { №2 } \\
\text { (Friuli } \\
\text { aftershock) }\end{array}$ & $\begin{array}{l}1 \mathrm{a} \\
2 \underline{a} \\
3 \underline{a}\end{array}$ & $\begin{array}{l}0.7227 \\
0.9375 \\
0.6250\end{array}$ & $\begin{array}{l}2.468 \\
1.625 \\
1.606\end{array}$ & 72,56 \\
\hline & & $\begin{array}{c}\text { №6 } \\
\text { (Kalamata } \\
\text { aftershock) }\end{array}$ & $\begin{array}{l}1 \mathrm{a} \\
2 \underline{a} \\
3 \underline{a}\end{array}$ & $\begin{array}{l}0.0001 \\
0.1824 \\
0.0450\end{array}$ & $\begin{array}{l}2.319 \\
1.541 \\
1.517\end{array}$ & 31,37 \\
\hline
\end{tabular}




\begin{tabular}{|c|c|c|c|c|c|c|}
\hline & & $\begin{array}{c}\text { №12 } \\
\text { (Duzce 1) }\end{array}$ & $\begin{array}{l}1 \underline{a} \\
2 \underline{a} \\
3 \underline{a}\end{array}$ & $\begin{array}{l}0.1914 \\
0.4150 \\
0.2158 \\
\end{array}$ & $\begin{array}{l}2.400 \\
1.599 \\
1.553 \\
\end{array}$ & 91,96 \\
\hline & \multirow{2}{*}{$\begin{array}{c}\text { III } \\
\text { Campo lejano }\end{array}$} & $\begin{array}{c}\text { №3 } \\
\text { (Montenegro) }\end{array}$ & $\begin{array}{l}1 \underline{a} \\
2 \underline{a} \\
3 \underline{a}\end{array}$ & $\begin{array}{l}0.0001 \\
0.1167 \\
0.0001\end{array}$ & $\begin{array}{l}2.138 \\
1.571 \\
1.421\end{array}$ & 37,80 \\
\hline & & $\begin{array}{c}\text { №14 } \\
\text { (Ano Liosia) }\end{array}$ & $\begin{array}{l}1 \mathrm{a} \\
2 \mathrm{a} \\
3 \mathrm{a}\end{array}$ & $\begin{array}{l}0.0001 \\
0.0357 \\
0.0001 \\
\end{array}$ & $\begin{array}{l}1.853 \\
1.563 \\
1.475\end{array}$ & 21,99 \\
\hline \multirow{12}{*}{ pa4pz2-1 } & \multirow{3}{*}{$\stackrel{\text { I }}{\text { Campo lejano }}$} & $\begin{array}{c}\text { №1 } \\
\text { (Friuli) }\end{array}$ & $\begin{array}{l}1 \mathrm{a} \\
2 \underline{a} \\
3 \underline{a}\end{array}$ & $\begin{array}{l}0.1305 * 1.0 \mathrm{e}-003 \\
0.1000 * 1.0 \mathrm{e}-003 \\
0.0981 * 1.0 \mathrm{e}-003\end{array}$ & $\begin{array}{l}2.084 \\
1.663 \\
1.026\end{array}$ & 18,73 \\
\hline & & $\begin{array}{c}\text { №4 } \\
\text { (Montenegro) }\end{array}$ & $\begin{array}{l}1 \underline{a} \\
2 \underline{a} \\
3 \underline{a}\end{array}$ & $\begin{array}{l}1.0625 \\
1.2500 \\
0.6504 \\
\end{array}$ & $\begin{array}{l}1.872 \\
1.177 \\
1.437 \\
\end{array}$ & 63,42 \\
\hline & & $\begin{array}{c}\text { №5 } \\
\text { (Campano } \\
\text { Lucano) }\end{array}$ & $\begin{array}{l}1 \underline{a} \\
2 \underline{a} \\
3 \underline{a}\end{array}$ & $\begin{array}{l}0.8267 * 1.0 \mathrm{e}-003 \\
0.1019 * 1.0 \mathrm{e}-003 \\
0.1000 * 1.0 \mathrm{e}-003\end{array}$ & $\begin{array}{l}2.248 \\
1.314 \\
1.149 \\
\end{array}$ & 41,76 \\
\hline & II & $\begin{array}{c}\text { №1 } \\
\text { (Friuli } \\
\text { aftershock) }\end{array}$ & $\begin{array}{l}1 \underline{a} \\
2 \underline{a} \\
3 \underline{a}\end{array}$ & $\begin{array}{l}0.0011 \\
0.0060 \\
0.0011 \\
\end{array}$ & $\begin{array}{l}1.529 \\
1.241 \\
1.454 \\
\end{array}$ & 26,53 \\
\hline & Campo cercano & $\begin{array}{c}\text { №14 } \\
\text { (Montenegro } \\
\text { aftershock) }\end{array}$ & $\begin{array}{l}1 \underline{a} \\
2 \underline{a} \\
3 \underline{a}\end{array}$ & $\begin{array}{l}0.0001 \\
0.1502 \\
0.0001 \\
\end{array}$ & $\begin{array}{l}2.2350 \\
1.4870 \\
1.5040 \\
\end{array}$ & 32,89 \\
\hline & II & $\begin{array}{c}\text { №2 } \\
\text { (Friuli } \\
\text { aftershock) }\end{array}$ & $\begin{array}{l}1 \underline{a} \\
2 \underline{a} \\
3 \underline{a}\end{array}$ & $\begin{array}{l}1.1719 \\
1.3750 \\
0.6606 \\
\end{array}$ & $\begin{array}{l}2.145 \\
1.276 \\
1.437 \\
\end{array}$ & 49,04 \\
\hline & Campo lejano & $\begin{array}{c}\text { №12 } \\
\text { (Montenegro } \\
\text { aftershock) }\end{array}$ & $\begin{array}{l}1 \underline{a} \\
2 \underline{a} \\
3 \underline{a}\end{array}$ & $\begin{array}{l}1.3125 \\
1.3604 \\
1.0703\end{array}$ & $\begin{array}{l}2.251 \\
1.501 \\
1.500\end{array}$ & 56,30 \\
\hline & & $\begin{array}{c}\text { №2 } \\
\text { (Friuli } \\
\text { aftershock) }\end{array}$ & $\begin{array}{l}1 \mathrm{a} \\
2 \underline{a} \\
3 \underline{a}\end{array}$ & $\begin{array}{l}0.9121 \\
0.7659 \\
0.4571 \\
\end{array}$ & $\begin{array}{l}2.443 \\
1.689 \\
1.591 \\
\end{array}$ & 74,02 \\
\hline & $\begin{array}{c}\text { III } \\
\text { Campo cercano }\end{array}$ & $\begin{array}{c}\text { №6 } \\
\text { (Kalamata } \\
\text { aftershock) }\end{array}$ & $\begin{array}{l}1 \underline{a} \\
2 \underline{a} \\
3 \underline{a}\end{array}$ & $\begin{array}{l}0.0802 \\
0.1319 \\
0.0879 \\
\end{array}$ & $\begin{array}{l}2.247 \\
1.508 \\
1.504 \\
\end{array}$ & 42,61 \\
\hline & & $\begin{array}{c}\text { №12 } \\
\text { (Duzce 1) }\end{array}$ & $\begin{array}{l}1 \underline{a} \\
2 \underline{a} \\
3 \underline{a}\end{array}$ & $\begin{array}{l}0.3633 \\
0.3633 \\
0.1640 \\
\end{array}$ & $\begin{array}{l}2.333 \\
1.642 \\
1.544 \\
\end{array}$ & 93,84 \\
\hline & III & $\begin{array}{c}\text { №3 } \\
\text { (Montenegro) }\end{array}$ & $\begin{array}{l}1 \underline{a} \\
2 \underline{a} \\
3 \underline{a}\end{array}$ & $\begin{array}{l}0.4526 \\
0.3970 \\
0.1934 \\
\end{array}$ & $\begin{array}{l}2.517 \\
1.903 \\
1.653 \\
\end{array}$ & 121,70 \\
\hline & Campo lejano & $\begin{array}{c}\text { №14 } \\
\text { (Ano Liosia) }\end{array}$ & $\begin{array}{l}1 \underline{a} \\
2 \underline{a} \\
3 a\end{array}$ & $\begin{array}{l}0.0123 \\
0.0001 \\
0.0001\end{array}$ & $\begin{array}{l}2.223 \\
1.564 \\
1.390\end{array}$ & 23,02 \\
\hline pa6pz2-1 & $\begin{array}{c}\text { I } \\
\text { Campo lejano }\end{array}$ & $\begin{array}{c}\text { №4 } \\
\text { (Montenegro) }\end{array}$ & $\begin{array}{l}1 \underline{a} \\
2 \underline{a} \\
3 a \\
4 a \\
5 \underline{a} \\
6 \underline{a}\end{array}$ & $\begin{array}{l}0.610 \\
0.470 \\
0.570 \\
0.720 \\
1.200 \\
1.100\end{array}$ & $\begin{array}{l}1.856 \\
0.803 \\
0.645 \\
1.269 \\
1.115 \\
1.472\end{array}$ & 59,80 \\
\hline & & $\begin{array}{c}\text { №5 } \\
\text { (Campano } \\
\text { Lucano) }\end{array}$ & $\begin{array}{l}1 \underline{a} \\
2 \underline{a} \\
3 a \\
4 \underline{a} \\
\end{array}$ & $\begin{array}{l}0.0250 \\
0.0100 \\
0.0100 \\
0.0100\end{array}$ & $\begin{array}{l}2.467 \\
1.023 \\
0.914 \\
1.340\end{array}$ & 32,89 \\
\hline
\end{tabular}




\begin{tabular}{|c|c|c|c|c|c|c|}
\hline & & & $\begin{array}{l}5 \underline{a} \\
6 \underline{a}\end{array}$ & $\begin{array}{c}0.0160 \\
0.00009\end{array}$ & $\begin{array}{l}1.014 \\
1.219\end{array}$ & \\
\hline & & & 1a & 0.400 & 2.295 & \\
\hline & & & $2 \underline{a}$ & 0.240 & 1.552 & \\
\hline & ॥ & №14 & 3 a & 0.240 & 1.352 & \\
\hline & Campo cercano & (IVIontenegro & 4ạ & 0.365 & 1.637 & 98,48 \\
\hline & & & 5 a & 0.280 & 1.645 & \\
\hline & & & 6a & 0.260 & 1.570 & \\
\hline & & & 1a & 0.02700 & 2.033 & \\
\hline & & & $2 \underline{a}$ & 0.00390 & 1.213 & \\
\hline & & (Eriuli & $3 \underline{a}$ & 0.00004 & 1.135 & 2026 \\
\hline & & aftershock) & $4 \underline{a}$ & 0.04300 & 1.554 & \\
\hline & & & $5 \underline{a}$ & 0.06900 & 1.572 & \\
\hline & & & 6a & 0.11000 & 1.407 & \\
\hline & & & 1a & 0.776 & 2.108 & \\
\hline & & & $2 \underline{a}$ & 0.496 & 1.335 & \\
\hline & III & Kalamata & 3 ạ & 0.706 & 1.333 & 6535 \\
\hline & Campo cercano & (Rtarnata & $4 \underline{a}$ & 1.490 & 1.410 & 03,35 \\
\hline & & & $5 \underline{a}$ & 1.615 & 1.633 & \\
\hline & & & $6 \underline{a}$ & 1.900 & 1.934 & \\
\hline & & & 1aa & 0.1810 & 2.649 & \\
\hline & & & $2^{\mathrm{a}}$ & 0.0940 & 1.671 & \\
\hline & & №12 & $3 \underline{a}$ & 0.0721 & 1.991 & 8121 \\
\hline & & (Duzce 1) & $4^{\underline{a}}$ & 0.0900 & 1.350 & $01,<1$ \\
\hline & & & $5 \underline{a}$ & 0.1100 & 1.334 & \\
\hline 1 & & & $6 \underline{a}$ & 0.0900 & 1.547 & \\
\hline pdop $\angle 2-1$ & & & 1a & 0.980 & 2.335 & \\
\hline & & & $2^{a}$ & 0.690 & 1.571 & \\
\hline & III & №3 & 3ạ & 0.780 & 1.573 & 010 \\
\hline & Campo lejano & (Montenegro) & 4ạ & 1.110 & 1.622 & 94,10 \\
\hline & & & $5 \underline{a}$ & 1.300 & 1.645 & \\
\hline & & & 6모 & 1.700 & 1.706 & \\
\hline
\end{tabular}

En algunos casos, es difícil ajustar los desplazamientos dentro del intervalo considerado anteriormente. Estos problemas claramente no lineales son muy complejos. La distribución de la energía que se introduce entre plantas es muy sensible a la distribución de resistencia, y esa sensibilidad es más acusada en unos sismos que en otros.

También influye el hecho de que se han considerado leyes histeréticas del tipo elásticoperfectamente plástico.

Se observa en las figuras anteriormente representadas de los desplazamientos máximos absolutos entre plantas para cada pórtico y tipo de suelo, que para el suelo tipo III se obtiene mejores resultados que para el suelo tipo I y tipo II. 


\subsection{RESISTENCIA LATERAL Y COEFICIENTES DE FUERZA CORTANTE}

\subsubsection{Resistencia lateral de los disipadores de una planta i dada}

Se expone a continuación la obtención de la resistencia de los disipadores de cada una de las plantas de los modelos estructurales, como el producto de la rigidez del disipador por el desplazamiento de fluencia de cada planta.

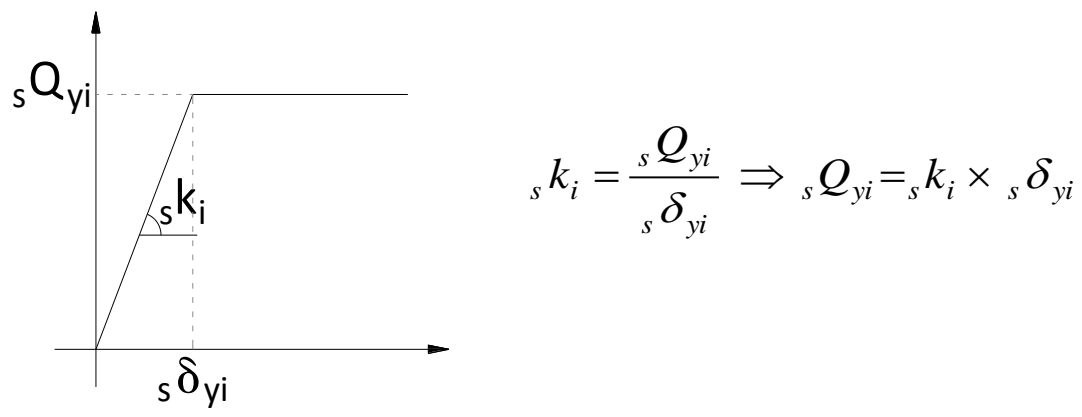

Fig. 5.5.1: Curva Desplazamiento de fluencia - Resistencia lateral del disipador

Siendo:

${ }_{s} Q_{y i}$ : Resistencia lateral aportada por los disipadores de una determinada planta i.

${ }_{s} k_{i} \quad$ : Rigidez lateral aportada por los disipadores de una determinada planta i.

${ }_{s} \delta_{y i}$ : Desplazamiento de fluencia de los disipadores de una determinada planta i.

\subsubsection{Coeficiente de fuerza cortante de fluencia de los disipadores de una planta i dada}

El coeficiente cortante de fluencia de los disipadores de una planta i se define como sigue:

Siendo:

$$
{ }_{s} \alpha_{i}=\frac{{ }_{s} Q_{y i}}{\sum_{\mathrm{j}=\mathrm{i}}^{\mathrm{j}=\mathrm{N}} m_{j} g}
$$

${ }_{s} \alpha_{i}$ : Coeficiente de fuerza cortante de fluencia de los disipadores de una determinada planta i.

$\sum_{\mathrm{j}=\mathrm{i}}^{\mathrm{j}=\mathrm{N}} m_{j} g$ : Peso de todas las plantas por encima de la planta i considerara.

\subsubsection{Resistencia lateral aportada por la parte flexible (pórtico) cuando los disipadores empiezan a} plastificar

La resistencia lateral en parte flexible (pórtico), cuando la estructura se mueve lateralmente un desplazamiento ${ }_{s} \delta_{y i}$, se obtiene simplemente como sigue:

Siendo:

$$
{ }_{f} Q_{\text {máxi }}={ }_{f} k_{i} \times{ }_{s} \delta_{y i}
$$

$$
\begin{aligned}
& { }_{f} Q_{\text {máxi }} \text { : Resistencia lateral aportada por la parte flexible (pórtico) cuando los disipadores } \\
& \quad \text { empiezan a plastifica. } \\
& { }_{f} k_{i} \quad: \text { Rigidez por planta sin disipador. }
\end{aligned}
$$


${ }_{s} \delta_{y i} \quad$ : Desplazamiento de fluencia del disipador por planta.

5.5.4. Coeficiente de fuerza cortante en la parte flexible (pórtico) cuando los disipadores empiezan a plastificar

El coeficiente cortante en el pórtico cuando los disipadores empiezan a plastificar se obtiene mediante la ecuación:

$$
{ }_{f} \alpha_{i}=\frac{{ }_{f} Q_{\text {máxi }}}{\sum_{\mathrm{j}=\mathrm{i}}^{\mathrm{j}=\mathrm{N}} m_{j} g}
$$

Siendo:

${ }_{f} \alpha_{i}$ : Coeficiente de fluencia cortante sin disipador de una determinada planta.

\subsubsection{Coeficiente de fuerza cortante total de planta}

El coeficiente de fuerza cortante total se obtiene sumando el coeficiente de fluencia cortante del disipador y el coeficiente de fuerza cortante en la parte flexible (pórtico) cuando los disipadores empiezan a plastificar.

$$
\alpha_{i}={ }_{s} \alpha_{i}+{ }_{f} \alpha_{i}
$$

Siendo:

$\alpha_{i}$ : Coeficiente de fuerza cortante total de una determinada planta.

5.5.6. Obtención de los parámetros definidos en los apartados 5.5.1 a 5.5.5 para cada uno de los pórticos

\subsubsection{Pórtico pa2pz2-1 (3 plantas)}

Masas por planta $m_{i}$

La estructura se puede idealizar con un modelo de tres masas concentradas (situadas en el centro de masas del forjado), tal como indica la Figura 5.5.2.

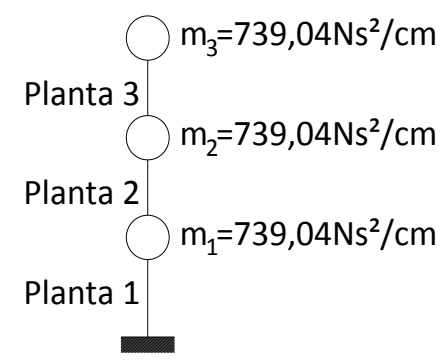

Fig. 5.5.2: Idealización de un modelo de masas del pórtico pa2pz2-1

Rigidez del disipador

Rigidez disipador $=5 \times$ Rigidez sin disipador 
Planta 3a: ${ }_{s} k_{3}=(55000 \mathrm{~N} / \mathrm{cm}) \times 5=275000 \mathrm{~N} / \mathrm{cm}$

Planta 2a: ${ }_{s} k_{2}=(68000 \mathrm{~N} / \mathrm{cm}) \times 5=340000 \mathrm{~N} / \mathrm{cm}$

Planta 1a : ${ }_{s} k_{1}=(99000 \mathrm{~N} / \mathrm{cm}) \times 5=495000 \mathrm{~N} / \mathrm{cm}$

\subsection{Suelo Tipo I (Campo lejano a la falla)}

\section{- Acelerograma №1 (Friuli) $\Delta=\mathbf{2 7} \mathrm{Km}$}

Desplazamiento de fluencia del disipador ${ }_{s} \delta_{i}$

Planta 3a: ${ }_{s} \delta_{3}=0,00008 \mathrm{~cm}$

Planta 2a: ${ }_{s} \delta_{2}=0,04780 \mathrm{~cm}$

Planta 1: ${ }_{s} \delta_{1}=0,00300 \mathrm{~cm}$

Resistencia lateral de los disipadores de una planta i dada ${ }_{s} Q_{i}$

Planta 3a: ${ }_{s} Q_{3}={ }_{s} k_{3} \times{ }_{s} \delta_{3}=275000 \mathrm{~N} / \mathrm{cm} \times 0,00008 \mathrm{~cm} \Rightarrow{ }_{s} Q_{3}=22 \mathrm{~N}$

Planta 2a: ${ }_{s} Q_{2}={ }_{s} k_{2} \times{ }_{s} \delta_{2}=340000 \mathrm{~N} / \mathrm{cm} \times 0,04780 \mathrm{~cm} \Rightarrow{ }_{s} Q_{2}=16252 \mathrm{~N}$

Planta 1a: ${ }_{s} Q_{1}={ }_{s} k_{1} \times{ }_{s} \delta_{1}=495000 \mathrm{~N} / \mathrm{cm} \times 0,00300 \mathrm{~cm} \Rightarrow \underline{{ }_{s} Q_{1}=1485 \mathrm{~N}}$

Coeficiente de fuerza cortante de fluencia de los disipadores de una planta i dada ${ }_{s} \alpha_{i}$

$$
\text { Planta 3a: }{ }_{s} \alpha_{3}=\frac{{ }_{s} Q_{3}(N)}{m_{3}\left(\frac{N s^{2}}{c m}\right) \times g\left(\frac{c m}{s^{2}}\right)}=\frac{22}{739,04 \times 981} \Rightarrow \quad \underline{{ }_{s} \alpha_{3}=3,034 e^{-05}}
$$

Planta 2: ${ }_{s} \alpha_{2}=\frac{{ }_{s}(N)}{\left(m_{2}+m_{3}\right)\left(\frac{N s^{2}}{c m}\right) \times g\left(\frac{c m}{s^{2}}\right)}=\frac{16252}{(2 \times 739,04) \times 981} \Rightarrow \quad \underline{{ }_{s} \alpha_{2}=0,011}$

$$
\text { Planta 1a: }{ }_{s} \alpha_{1}=\frac{{ }_{s} Q_{1}(N)}{\left(m_{1}+m_{2}+m_{3}\right)\left(\frac{N s^{2}}{c m}\right) \times g\left(\frac{c m}{s^{2}}\right)}=\frac{1485}{(3 \times 739,04) \times 981} \Rightarrow{ }_{s} \alpha_{1}=6,828 e^{-04}
$$

Resistencia lateral aportada por la parte flexible (pórtico) cuando los disipadores empiezan a plastificar ${ }_{f} Q_{\text {máxi }}$

Planta 3a: ${ }_{f} Q_{\text {máx } 3}={ }_{f} k_{3} \times{ }_{s} \delta_{3}=55000 \mathrm{~N} / \mathrm{cm} \times 0,00008 \mathrm{~cm} \Rightarrow{ }_{f} Q_{\text {máx } 3}=4,4 \mathrm{~N}$

Planta 2a: ${ }_{f} Q_{\text {máx } 2}={ }_{f} k_{2} \times{ }_{s} \delta_{2}=68000 \mathrm{~N} / \mathrm{cm} \times 0,04780 \mathrm{~cm} \Rightarrow{ }_{f} Q_{\text {máx } 2}=3250,4 \mathrm{~N}$

Planta 1ạ: ${ }_{f} Q_{\text {máx } 1}={ }_{f} k_{1} \times{ }_{s} \delta_{1}=99000 \mathrm{~N} / \mathrm{cm} \times 0,00300 \mathrm{~cm} \Rightarrow{ }_{f} Q_{\text {máx } 1}=297 \mathrm{~N}$ 
Coeficiente cortante en la parte flexible (pórtico) cuando los disipadores empiezan a plastificar ${ }_{f} \alpha_{i}$

Planta 3a: ${ }_{f} \alpha_{3}=\frac{{ }_{f} Q_{\text {máx } 3}(N)}{m_{3}\left(\frac{N s^{2}}{\mathrm{~cm}}\right) \times g\left(\frac{\mathrm{cm}}{\mathrm{s}^{2}}\right)}=\frac{4,4}{739,04 \times 981} \Rightarrow$

$\underline{{ }_{f}} \alpha_{3}=6,069 e^{-06}$

Planta 2a: ${ }_{f} \alpha_{2}=\frac{{ }_{f} Q_{\max 2}(N)}{\left(m_{2}+m_{3}\right)\left(\frac{N s^{2}}{c m}\right) \times g\left(\frac{c m}{s^{2}}\right)}=\frac{3250,4}{(2 \times 739,04) \times 981} \Rightarrow \quad \underline{{ }_{f} \alpha_{2}=2,242 e^{-03}}$

Planta 1a: ${ }_{f} \alpha_{1}=\frac{{ }_{f} Q_{\text {máx } 1}(N)}{\left(m_{1}+m_{2}+m_{3}\right)\left(\frac{N s^{2}}{c m}\right) \times g\left(\frac{c m}{s^{2}}\right)}=\frac{297}{(3 \times 739,04) \times 981} \Rightarrow{ }_{f} \alpha_{1}=1,365 e^{-04}$

Coeficiente de fuerza cortante total de planta $\alpha_{i}$

Planta 3a: $\alpha_{3}={ }_{s} \alpha_{3}+{ }_{f} \alpha_{3}=3,034 e^{-05}+6,069 e^{-06} \Rightarrow \frac{\alpha_{3}=3,641 e^{-05}}{\alpha_{2}=0,013}$
Planta 2a: $\alpha_{2}={ }_{s} \alpha_{2}+{ }_{f} \alpha_{2}=0,011+2,242 e^{-03} \Rightarrow \frac{\alpha_{1}=8,193 e^{-04}}{\alpha_{1}}$

\section{- Acelerograma №4 (Montenegro) $\Delta=65 \mathrm{Km}$}

Desplazamiento de fluencia del disipador ${ }_{s} \delta_{i}$

Planta 3a: ${ }_{s} \delta_{3}=0,0880 \mathrm{~cm}$

Planta 2a: ${ }_{s} \delta_{2}=0,2384 \mathrm{~cm}$

Planta 1a : ${ }_{s} \delta_{1}=0,0003 \mathrm{~cm}$

Resistencia lateral de los disipadores de una planta i dada ${ }_{s} Q_{i}$

Planta 3a: ${ }_{s} Q_{3}={ }_{s} k_{3} \times{ }_{s} \delta_{3}=275000 \mathrm{~N} / \mathrm{cm} \times 0,0880 \mathrm{~cm} \Rightarrow{ }_{s} Q_{3}=24200 \mathrm{~N}$

Planta 2a: ${ }_{s} Q_{2}={ }_{s} k_{2} \times{ }_{s} \delta_{2}=340000 \mathrm{~N} / \mathrm{cm} \times 0,2384 \mathrm{~cm} \Rightarrow{ }_{s} Q_{2}=81056 \mathrm{~N}$

Planta 1ㅁ: ${ }_{s} Q_{1}={ }_{s} k_{1} \times{ }_{s} \delta_{1}=495000 \mathrm{~N} / \mathrm{cm} \times 0,0003 \mathrm{~cm} \Rightarrow \underline{{ }_{s} Q_{1}=148,5 \mathrm{~N}}$

Coeficiente de fuerza cortante de fluencia de los disipadores de una planta i dada ${ }_{s} \alpha_{i}$

Planta 3a: ${ }_{s} \alpha_{3}=\frac{{ }_{s} Q_{3}(N)}{m_{3}\left(\frac{N s^{2}}{c m}\right) \times g\left(\frac{c m}{s^{2}}\right)}=\frac{24200}{739,04 \times 981} \Rightarrow \quad \underline{{ }_{s} \alpha_{3}=0,033}$ 
Planta 2a: ${ }_{s} \alpha_{2}=\frac{{ }_{s} Q_{2}(N)}{\left(m_{2}+m_{3}\right)\left(\frac{N s^{2}}{c m}\right) \times g\left(\frac{c m}{s^{2}}\right)}=\frac{81056}{(2 \times 739,04) \times 981} \Rightarrow \quad \underline{{ }_{s} \alpha_{2}=0,056}$

Planta 1ạ: ${ }_{s} \alpha_{1}=\frac{{ }_{s}(N)}{\left(m_{1}+m_{2}+m_{3}\right)\left(\frac{N s^{2}}{c m}\right) \times g\left(\frac{c m}{s^{2}}\right)}=\frac{148,5}{(3 \times 739,04) \times 981} \Rightarrow \underline{{ }_{s}} \alpha_{1}=6,828 e^{-05}$

Resistencia lateral aportada por la parte flexible (pórtico) cuando los disipadores empiezan a plastificar ${ }_{f} Q_{\text {máxi }}$

Planta 3a: ${ }_{f} Q_{\text {máx } 3}={ }_{f} k_{3} \times{ }_{s} \delta_{3}=55000 \mathrm{~N} / \mathrm{cm} \times 0,0880 \mathrm{~cm} \Rightarrow{ }_{f} Q_{\text {máx } 3}=4840 \mathrm{~N}$

Planta 2a: ${ }_{f} Q_{\text {máx } 2}={ }_{f} k_{2} \times{ }_{s} \delta_{2}=68000 \mathrm{~N} / \mathrm{cm} \times 0,2384 \mathrm{~cm} \Rightarrow{ }_{f} Q_{\text {máx } 2}=16211,2 \mathrm{~N}$

Planta 1ạ: ${ }_{f} Q_{\text {máx1 }}={ }_{f} k_{1} \times{ }_{s} \delta_{1}=99000 \mathrm{~N} / \mathrm{cm} \times 0,0003 \mathrm{~cm} \Rightarrow{ }_{f} Q_{\text {máx } 1}=29,7 N$

Coeficiente cortante en la parte flexible (pórtico) cuando los disipadores empiezan a plastificar ${ }_{f} \alpha_{i}$

Planta 3a: ${ }_{f} \alpha_{3}=\frac{{ }_{f} Q_{\text {máx } 3}(N)}{m_{3}\left(\frac{N s^{2}}{c m}\right) \times g\left(\frac{c m}{s^{2}}\right)}=\frac{4840}{739,04 \times 981} \Rightarrow \quad \underline{{ }_{f} \alpha_{3}=6,676 e^{-03}}$

Planta 2a: ${ }_{f} \alpha_{2}=\frac{{ }_{f} Q_{\text {máx } 2}(N)}{\left(m_{2}+m_{3}\right)\left(\frac{N s^{2}}{c m}\right) \times g\left(\frac{c m}{s^{2}}\right)}=\frac{16211,2}{(2 \times 739,04) \times 981} \Rightarrow \quad \underline{{ }_{f}} \alpha_{2}=0,011$

Planta 1ㄹ: ${ }_{f} \alpha_{1}=\frac{{ }_{f} Q_{\text {máx }}(N)}{\left(m_{1}+m_{2}+m_{3}\right)\left(\frac{N s^{2}}{c m}\right) \times g\left(\frac{c m}{s^{2}}\right)}=\frac{29,7}{(3 \times 739,04) \times 981} \Rightarrow \underline{{ }_{f} \alpha_{1}=1,365 e^{-05}}$

Coeficiente de fuerza cortante total de planta $\alpha_{i}$

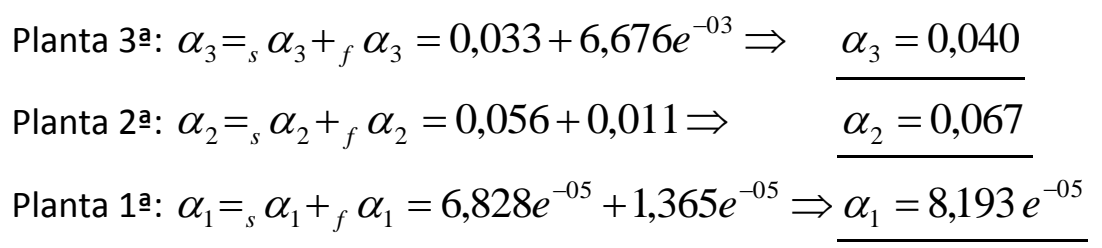

\section{- Acelerograma №5 (Campano Lucano) $\Delta=32 \mathrm{Km}$}

Desplazamiento de fluencia del disipador ${ }_{s} \delta_{i}$

Planta 3a: ${ }_{s} \delta_{3}=0,0001 \mathrm{~cm}$

Planta 2a: ${ }_{s} \delta_{2}=0,0402 \mathrm{~cm}$ 
Planta 1ㄹ: $\delta_{1}=0,0226 \mathrm{~cm}$

Resistencia lateral de los disipadores de una planta i dada ${ }_{s} Q_{i}$

Planta 3a: ${ }_{s} Q_{3}={ }_{s} k_{3} \times{ }_{s} \delta_{3}=275000 \mathrm{~N} / \mathrm{cm} \times 0,0001 \mathrm{~cm} \Rightarrow{ }_{s} Q_{3}=27,5 \mathrm{~N}$

Planta 2a: ${ }_{s} Q_{2}={ }_{s} k_{2} \times{ }_{s} \delta_{2}=340000 \mathrm{~N} / \mathrm{cm} \times 0,0402 \mathrm{~cm} \Rightarrow{ }_{s} Q_{2}=13668 \mathrm{~N}$

Planta 1ㄹ: ${ }_{s} Q_{1}={ }_{s} k_{1} \times{ }_{s} \delta_{1}=495000 \mathrm{~N} / \mathrm{cm} \times 0,0226 \mathrm{~cm} \Rightarrow{ }_{s} Q_{1}=11187 \mathrm{~N}$

Coeficiente de fuerza cortante de fluencia de los disipadores de una planta i dada ${ }_{s} \alpha_{i}$

Planta 3a: ${ }_{s} \alpha_{3}=\frac{{ }_{s} Q_{3}(N)}{m_{3}\left(\frac{N s^{2}}{c m}\right) \times g\left(\frac{c m}{s^{2}}\right)}=\frac{27,5}{739,04 \times 981} \Rightarrow \quad \underline{{ }_{s} \alpha_{3}=3,793 e^{-05}}$

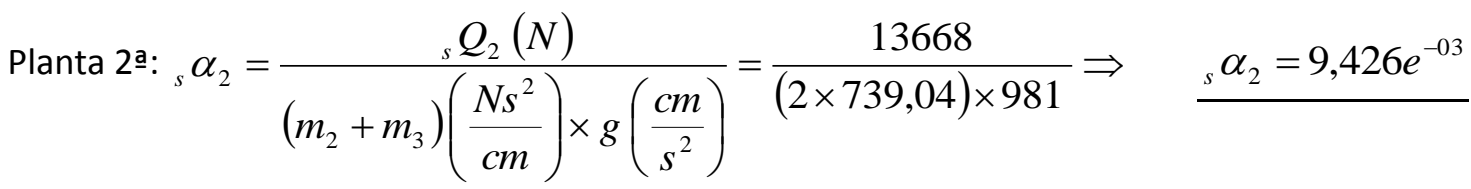
Planta 1ㄹ: ${ }_{s} \alpha_{1}=\frac{{ }_{s}(N)}{\left(m_{1}+m_{2}+m_{3}\right)\left(\frac{N s^{2}}{c m}\right) \times g\left(\frac{c m}{s^{2}}\right)}=\frac{11187}{(3 \times 739,04) \times 981} \Rightarrow \underline{{ }_{s} \alpha_{1}=5,143 e^{-03}}$

Resistencia lateral aportada por la parte flexible (pórtico) cuando los disipadores empiezan a plastificar ${ }_{f} Q_{\text {máxi }}$

Planta 3a: ${ }_{f} Q_{\operatorname{máx3}}={ }_{f} k_{3} \times{ }_{s} \delta_{3}=55000 \mathrm{~N} / \mathrm{cm} \times 0,0001 \mathrm{~cm} \Rightarrow{ }_{f} Q_{\text {máx } 3}=5,5 \mathrm{~N}$

Planta 2a: ${ }_{f} Q_{\text {máx } 2}={ }_{f} k_{2} \times{ }_{s} \delta_{2}=68000 \mathrm{~N} / \mathrm{cm} \times 0,0402 \mathrm{~cm} \Rightarrow \overline{{ }_{f} Q_{\text {máx } 2}=2733}, 6 \mathrm{~N}$

Planta 1ㄹ: ${ }_{f} Q_{\text {máx } 1}={ }_{f} k_{1} \times{ }_{s} \delta_{1}=99000 \mathrm{~N} / \mathrm{cm} \times 0,0226 \mathrm{~cm} \Rightarrow{ }_{f} Q_{\text {máx } 1}=2237,4 N$

Coeficiente cortante en la parte flexible (pórtico) cuando los disipadores empiezan a plastificar ${ }_{f} \alpha_{i}$

Planta 3a: ${ }_{f} \alpha_{3}=\frac{{ }_{f} Q_{\text {máx }}(N)}{m_{3}\left(\frac{N s^{2}}{c m}\right) \times g\left(\frac{c m}{s^{2}}\right)}=\frac{5,5}{739,04 \times 981} \Rightarrow \quad \underline{{ }_{f} \alpha_{3}=7,586 e^{-06}}$

Planta 2"a: ${ }_{f} \alpha_{2}=\frac{{ }_{f} Q_{\text {máx }}(N)}{\left(m_{2}+m_{3}\right)\left(\frac{N s^{2}}{c m}\right) \times g\left(\frac{c m}{s^{2}}\right)}=\frac{2733,6}{(2 \times 739,04) \times 981} \Rightarrow \quad \underline{{ }_{f} \alpha_{2}=1,885 e^{-03}}$

Planta 1a: ${ }_{f} \alpha_{1}=\frac{{ }_{f} Q_{\text {máx } 1}(N)}{\left(m_{1}+m_{2}+m_{3}\right)\left(\frac{N s^{2}}{c m}\right) \times g\left(\frac{c m}{s^{2}}\right)}=\frac{2237,4}{(3 \times 739,04) \times 981} \Rightarrow{ }_{f} \alpha_{1}=1,029 e^{-03}$ 
Coeficiente de fuerza cortante total de planta $\alpha_{i}$

Planta 3ạ: $\alpha_{3}={ }_{s} \alpha_{3}+{ }_{f} \alpha_{3}=3,793 e^{-05}+7,586 e^{-06} \Rightarrow \alpha_{3}=4,552 e^{-05}$

Planta 2a: $\alpha_{2}={ }_{s} \alpha_{2}+{ }_{f} \alpha_{2}=9,426 e^{-03}+1,885 e^{-03} \Rightarrow \alpha_{2}=0,011$

Planta 1: $\alpha_{1}={ }_{s} \alpha_{1}+{ }_{f} \alpha_{1}=5,143 e^{-03}+1,029 e^{-03} \Rightarrow \underline{\alpha_{1}=6,172 e^{-03}}$

\section{Representación gráfica}

El resultado de estos cálculos queda representado en las figuras definidas a continuación. La resistencia lateral de los disipadores; el coeficiente de fuerza cortante de fluencia de los disipadores; La resistencia lateral aportada por la parte flexible cuando los disipadores empiezan a plastificar; el coeficiente cortante en la parte flexible cuando los disipadores empiezan a plastificar; y el coeficiente de fuerza cortante total, de cada planta y para cada uno de los pórticos analizados y tipo de suelo.

Por lo general, aquellos terremotos que presenten un mayor input de energía expresado en forma de velocidad equivalente $V_{\mathrm{E}}$ necesitarán más cortante.

En las figuras 5.5.3 a 5.5.7, el terremoto de Montenegro es el que presenta mayor resistencia lateral en la segunda planta puesto que es el que tiene mayor desplazamiento de fluencia en dicha planta.

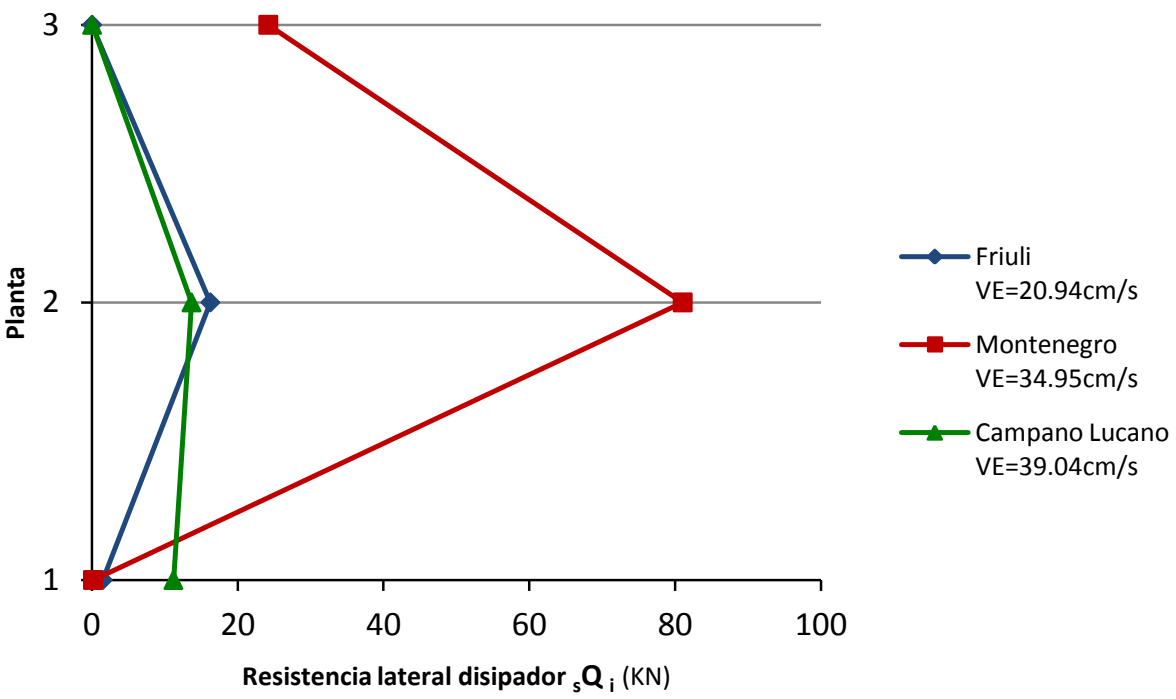

Fig. 5.5.3: Resistencia lateral de los disipadores de cada planta i, pórtico, pa2pz2-1, suelo tipo I, campo lejano 


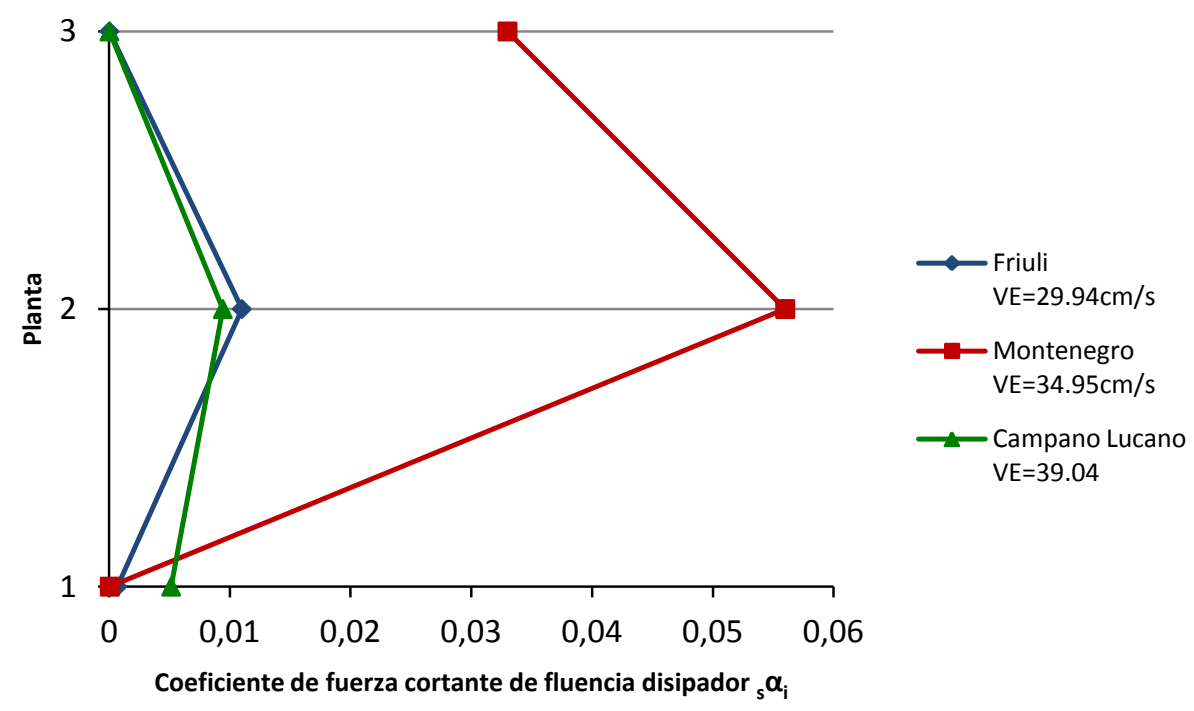

Fig. 5.5.4: Coeficiente de fuerza cortante de fluencia de los disipadores de cada planta i, pórtico pa2pz2-1, suelo tipo I, campo lejano

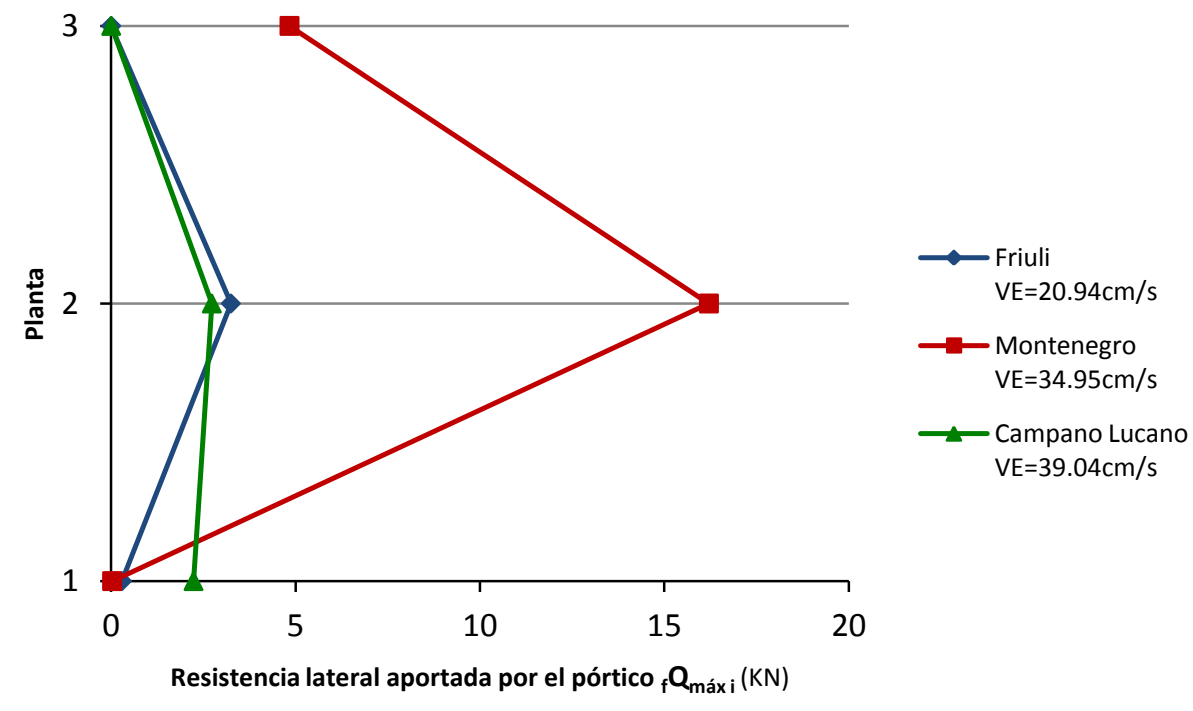

Fig. 5.5.5: Resistencia lateral aportada por la parte flexible (pórtico) cuando los disipadores empiezan a plastificar, pórtico pa2pz2-1, suelo tipo I, campo lejano 


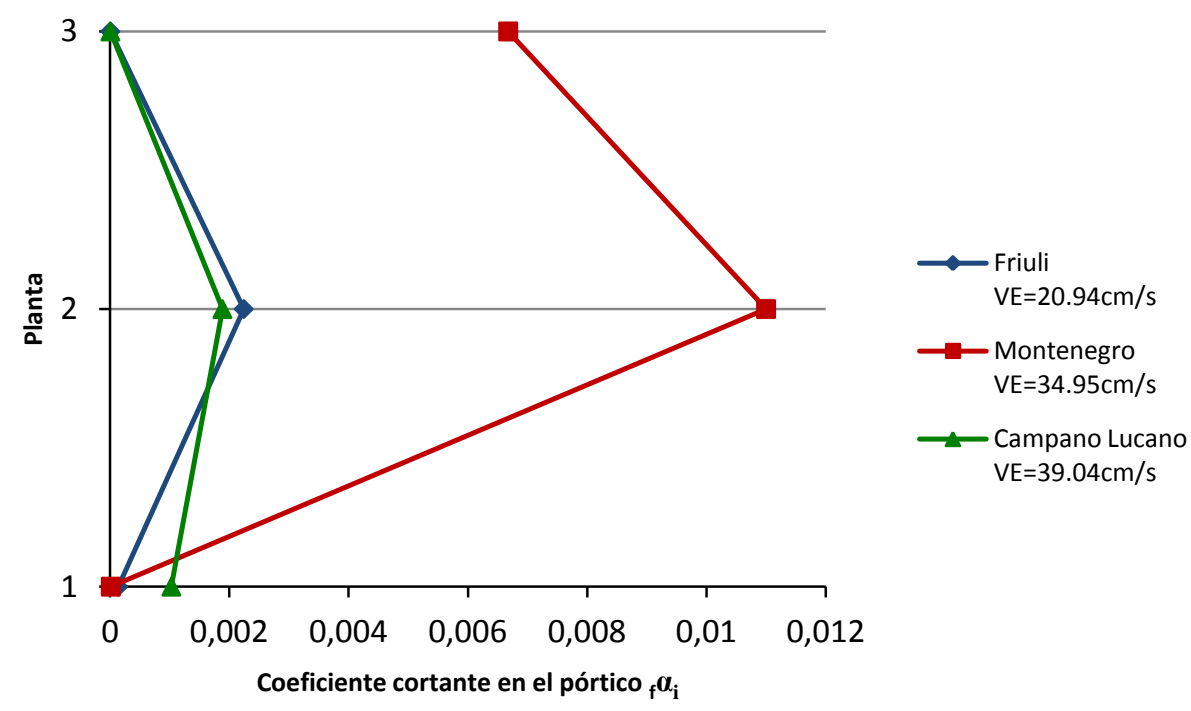

Fig. 5.5.6: Coeficiente de fuerza cortante en la parte flexible (pórtico) cuando los disipadores empiezan a plastificar, pórtico pa2pz2-1, suelo tipo I, campo lejano

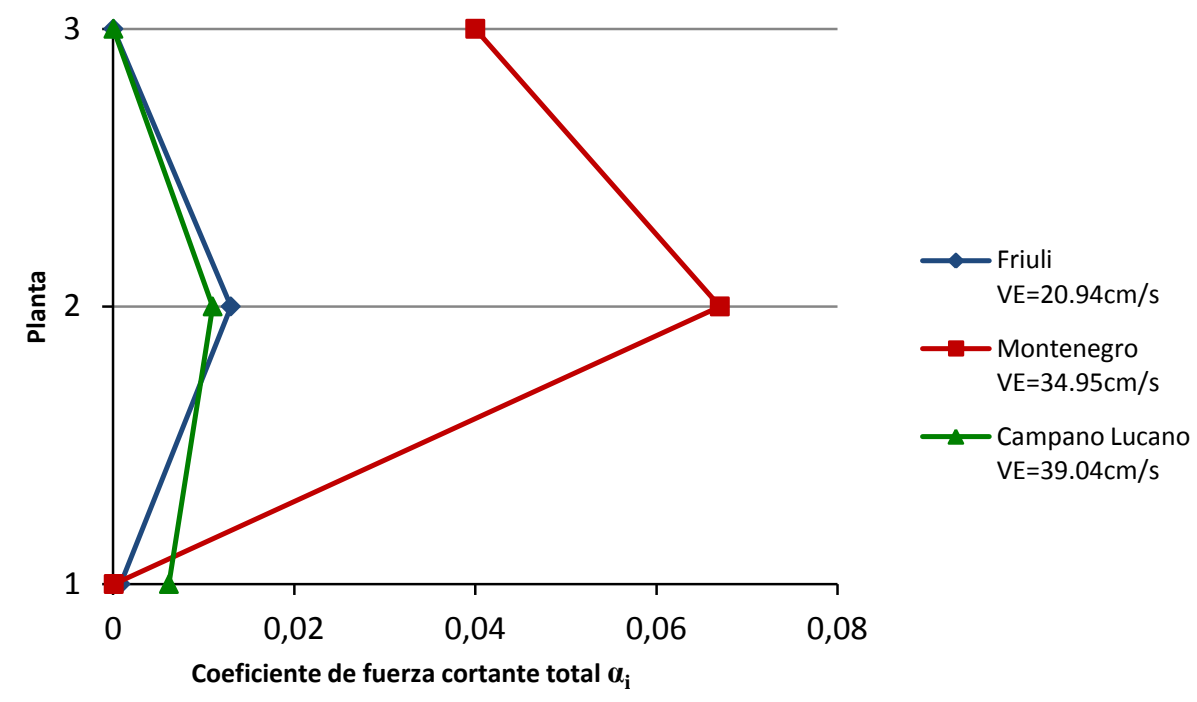

Fig. 5.5.7: Coeficiente de fuerza cortante total de planta, pórtico pa2pz2-1, suelo tipo I, campo lejano

\subsection{Suelo Tipo II (Campo cercano a la falla)}

\section{- Acelerograma №1 (Friuli aftershock) $\Delta=3 \mathrm{Km}$}

Desplazamiento de fluencia del disipador ${ }_{s} \delta_{i}$

Planta 3a: ${ }_{s} \delta_{3}=0,0001 \mathrm{~cm}$

Planta 2a: ${ }_{s} \delta_{2}=0,0356 \mathrm{~cm}$

Planta 1ạ: ${ }_{s} \delta_{1}=0,0008 \mathrm{~cm}$ 
Resistencia lateral de los disipadores de una planta i dada ${ }_{s} Q_{i}$

Planta 3a : ${ }_{s} Q_{3}={ }_{s} k_{3} \times{ }_{s} \delta_{3}=275000 \mathrm{~N} / \mathrm{cm} \times 0,0001 \mathrm{~cm} \Rightarrow{ }_{s} Q_{3}=27,5 \mathrm{~N}$

Planta 2a: ${ }_{s} Q_{2}={ }_{s} k_{2} \times{ }_{s} \delta_{2}=340000 \mathrm{~N} / \mathrm{cm} \times 0,0356 \mathrm{~cm} \Rightarrow{ }_{s} Q_{2}=12104 \mathrm{~N}$

Planta 1ㅁ: ${ }_{s} Q_{1}={ }_{s} k_{1} \times{ }_{s} \delta_{1}=495000 \mathrm{~N} / \mathrm{cm} \times 0,0008 \mathrm{~cm} \Rightarrow \underline{{ }_{s} Q_{1}=396 \mathrm{~N}}$

Coeficiente de fuerza cortante de fluencia de los disipadores de una planta i dada ${ }_{s} \alpha_{i}$

Planta 3a: ${ }_{s} \alpha_{3}=\frac{{ }_{s} Q_{3}(N)}{m_{3}\left(\frac{N s^{2}}{c m}\right) \times g\left(\frac{c m}{s^{2}}\right)}=\frac{27,5}{739,04 \times 981} \Rightarrow \quad \underline{{ }_{s} \alpha_{3}=3,793 e^{-05}}$

Planta 2 ${ }_{s}^{\mathrm{a}}{ }_{s} \alpha_{2}=\frac{{ }_{s}(N)}{\left(m_{2}+m_{3}\right)\left(\frac{N s^{2}}{c m}\right) \times g\left(\frac{c m}{s^{2}}\right)}=\frac{12104}{(2 \times 739,04) \times 981} \Rightarrow \quad \underline{{ }_{s}} \alpha_{2}=8,348 e^{-03}$

Planta 1ㄹ: ${ }_{s} \alpha_{1}=\frac{{ }_{s}(N)}{\left(m_{1}+m_{2}+m_{3}\right)\left(\frac{N s^{2}}{c m}\right) \times g\left(\frac{c m}{s^{2}}\right)}=\frac{396}{(3 \times 739,04) \times 981} \Rightarrow \underline{{ }_{s} \alpha_{1}=1,821 e^{-04}}$

Resistencia lateral aportada por la parte flexible (pórtico) cuando los disipadores empiezan a plastificar ${ }_{f} Q_{\text {máxi }}$

Planta 3a: ${ }_{f} Q_{\text {máx } 3}={ }_{f} k_{3} \times{ }_{s} \delta_{3}=55000 \mathrm{~N} / \mathrm{cm} \times 0,0001 \mathrm{~cm} \Rightarrow{ }_{f} Q_{\text {máx } 3}=5,5 \mathrm{~N}$

Planta 2a: : ${ }_{f} Q_{\text {máx } 2}={ }_{f} k_{2} \times{ }_{s} \delta_{2}=68000 \mathrm{~N} / \mathrm{cm} \times 0,0356 \mathrm{~cm} \Rightarrow{ }_{f} Q_{\text {máx } 2}=2420,8 \mathrm{~N}$

Planta 1à: ${ }_{f} Q_{\text {máx1 }}={ }_{f} k_{1} \times{ }_{s} \delta_{1}=99000 \mathrm{~N} / \mathrm{cm} \times 0,0008 \mathrm{~cm} \Rightarrow{ }_{f} Q_{\text {máx } 1}=79,2 N$

Coeficiente cortante en la parte flexible (pórtico) cuando los disipadores empiezan a plastificar ${ }_{f} \alpha_{i}$

Planta 3a: ${ }_{f} \alpha_{3}=\frac{{ }_{f} Q_{m a ́ x 3}(N)}{m_{3}\left(\frac{N s^{2}}{c m}\right) \times g\left(\frac{c m}{s^{2}}\right)}=\frac{5,5}{739,04 \times 981} \Rightarrow \quad \underline{{ }_{f} \alpha_{3}=7,586 e^{-06}}$

Planta 2a: ${ }_{f} \alpha_{2}=\frac{{ }_{f} Q_{\text {máx }}(N)}{\left(m_{2}+m_{3}\right)\left(\frac{N s^{2}}{c m}\right) \times g\left(\frac{c m}{s^{2}}\right)}=\frac{2420,8}{(2 \times 739,04) \times 981} \Rightarrow \quad \underline{{ }_{f} \alpha_{2}=1,669 e^{-03}}$

Planta 1ㄹ: ${ }_{f} \alpha_{1}=\frac{{ }_{f} Q_{\text {máx } 1}(N)}{\left(m_{1}+m_{2}+m_{3}\right)\left(\frac{N s^{2}}{c m}\right) \times g\left(\frac{c m}{s^{2}}\right)}=\frac{79,2}{(3 \times 739,04) \times 981} \Rightarrow{ }_{f} \alpha_{1}=3,641 e^{-05}$ 
Coeficiente de fuerza cortante total de planta $\alpha_{i}$

$$
\begin{aligned}
& \text { Planta 3a: } \alpha_{3}={ }_{s} \alpha_{3}+{ }_{f} \alpha_{3}=3,793 e^{-05}+7,586 e^{-06} \Rightarrow \alpha_{3}=4,552 e^{-05} \\
& \text { Planta 2a: } \alpha_{2}={ }_{s} \alpha_{2}+{ }_{f} \alpha_{2}=8,348 e^{-03}+1,669 e^{-03} \Rightarrow \underline{\alpha_{2}=0,010} \\
& \text { Planta 1a: } \alpha_{1}={ }_{s} \alpha_{1}+{ }_{f} \alpha_{1}=1,821 e^{-04}+3,641 e^{-05} \Rightarrow \underline{\alpha_{1}=2,185 e^{-04}}
\end{aligned}
$$

\section{- Acelerograma №14 (Montenegro aftershock) $\Delta=8 \mathrm{Km}$}

Desplazamiento de fluencia del disipador ${ }_{s} \delta_{i}$

Planta 3a: ${ }_{s} \delta_{3}=0,4454 \mathrm{~cm}$

Planta 2 ${ }^{\mathrm{a}}:{ }_{s} \delta_{2}=0,5937 \mathrm{~cm}$

Planta 1ㄹ: ${ }_{s} \delta_{1}=0,4883 \mathrm{~cm}$

Resistencia lateral de los disipadores de una planta i dada ${ }_{s} Q_{i}$

Planta 3a: ${ }_{s} Q_{3}={ }_{s} k_{3} \times{ }_{s} \delta_{3}=275000 \mathrm{~N} / \mathrm{cm} \times 0,4454 \mathrm{~cm} \Rightarrow{ }_{s} Q_{3}=122485 \mathrm{~N}$

Planta 2a: ${ }_{s} Q_{2}={ }_{s} k_{2} \times{ }_{s} \delta_{2}=340000 \mathrm{~N} / \mathrm{cm} \times 0,5937 \mathrm{~cm} \Rightarrow{ }_{s} Q_{2}=201858 \mathrm{~N}$

Planta 1ạ: ${ }_{s} Q_{1}={ }_{s} k_{1} \times{ }_{s} \delta_{1}=495000 \mathrm{~N} / \mathrm{cm} \times 0,4883 \mathrm{~cm} \Rightarrow{ }_{s} Q_{1}=241708,5 \mathrm{~N}$

Coeficiente de fuerza cortante de fluencia de los disipadores de una planta i dada ${ }_{s} \alpha_{i}$

$$
\text { Planta 3a: }{ }_{s} \alpha_{3}=\frac{{ }_{s} Q_{3}(N)}{m_{3}\left(\frac{N s^{2}}{c m}\right) \times g\left(\frac{c m}{s^{2}}\right)}=\frac{122485}{739,04 \times 981} \Rightarrow \quad \underline{{ }_{s} \alpha_{3}=0,169}
$$

Planta 2 : ${ }_{s} \alpha_{2}=\frac{{ }_{s}(N)}{\left(m_{2}+m_{3}\right)\left(\frac{N s^{2}}{c m}\right) \times g\left(\frac{c m}{s^{2}}\right)}=\frac{201858}{(2 \times 739,04) \times 981} \Rightarrow \quad{ }_{s} \alpha_{2}=0,139$

$$
\text { Planta 1ä: } \alpha_{s}=\frac{{ }_{s} Q_{1}(N)}{\left(m_{1}+m_{2}+m_{3}\right)\left(\frac{N s^{2}}{c m}\right) \times g\left(\frac{c m}{s^{2}}\right)}=\frac{241708,5}{(3 \times 739,04) \times 981} \Rightarrow \underline{{ }_{s} \alpha_{1}=0,111}
$$

Resistencia lateral aportada por la parte flexible (pórtico) cuando los disipadores empiezan a plastificar ${ }_{f} Q_{\text {máxi }}$

$$
\begin{aligned}
& \text { Planta 3a: : }{ }_{f} Q_{\text {máx3 }}={ }_{f} k_{3} \times{ }_{s} \delta_{3}=55000 \mathrm{~N} / \mathrm{cm} \times 0,4454 \mathrm{~cm} \Rightarrow{ }_{f} Q_{\text {máx } 3}=24497 \mathrm{~N} \\
& \text { Planta 2a: }{ }_{f} Q_{\text {máx } 2}={ }_{f} k_{2} \times{ }_{s} \delta_{2}=68000 \mathrm{~N} / \mathrm{cm} \times 0,5937 \mathrm{~cm} \Rightarrow{ }_{f} Q_{\text {máx } 2}=40371,6 N \\
& \text { Planta 1ㄹ: }{ }_{f} Q_{\text {maxx }}={ }_{f} k_{1} \times{ }_{s} \delta_{1}=99000 \mathrm{~N} / \mathrm{cm} \times 0,4883 \mathrm{~cm} \Rightarrow{ }_{f} Q_{\max 1}=48341,7 \mathrm{~N}
\end{aligned}
$$


Coeficiente cortante en la parte flexible (pórtico) cuando los disipadores empiezan a plastificar ${ }_{f} \alpha_{i}$

Planta 3ạ: ${ }_{f} \alpha_{3}=\frac{{ }_{f} Q_{\operatorname{máxx}}(N)}{m_{3}\left(\frac{N s^{2}}{c m}\right) \times g\left(\frac{c m}{s^{2}}\right)}=\frac{24497}{739,04 \times 981} \Rightarrow \quad \underline{{ }_{f} \alpha_{3}=0,034}$

Planta 2a: : ${ }_{f} \alpha_{2}=\frac{{ }_{f} Q_{\text {máx } 2}(N)}{\left(m_{2}+m_{3}\right)\left(\frac{N s^{2}}{c m}\right) \times g\left(\frac{c m}{s^{2}}\right)}=\frac{40371,6}{(2 \times 739,04) \times 981} \Rightarrow \quad \underline{{ }_{f} \alpha_{2}=0,028}$

Planta 1ㄹ: ${ }_{f} \alpha_{1}=\frac{{ }_{f} Q_{\operatorname{máx} 1}(N)}{\left(m_{1}+m_{2}+m_{3}\right)\left(\frac{N s^{2}}{c m}\right) \times g\left(\frac{c m}{s^{2}}\right)}=\frac{48341,7}{(3 \times 739,04) \times 981} \Rightarrow \underline{{ }_{f}} \alpha_{1}=0,022$

Coeficiente de fuerza cortante total de planta $\alpha_{i}$

Planta 3a: $\alpha_{3}={ }_{s} \alpha_{3}+{ }_{f} \alpha_{3}=0,169+0,034 \Rightarrow \underline{\alpha_{3}=0,203}$

Planta 2a: $\alpha_{2}={ }_{s} \alpha_{2}+{ }_{f} \alpha_{2}=0,139+0,028 \Rightarrow \underline{\alpha_{2}=0,167}$

Planta 1ㄹ: $\alpha_{1}={ }_{s} \alpha_{1}+{ }_{f} \alpha_{1}=0,111+0,022 \Rightarrow \underline{\alpha_{1}=0,133}$

Representación gráfica

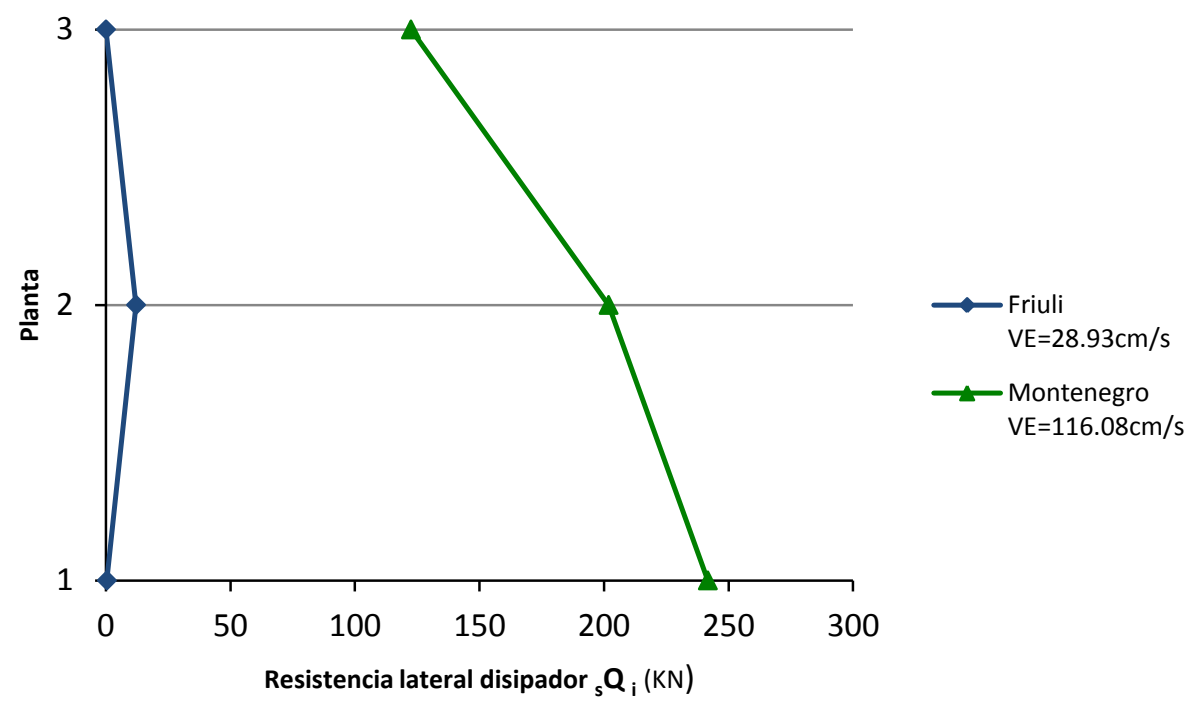

Fig. 5.5.8: Resistencia lateral de los disipadores de cada planta i, pórtico, pa2pz2-1, suelo tipo II, campo cercano 


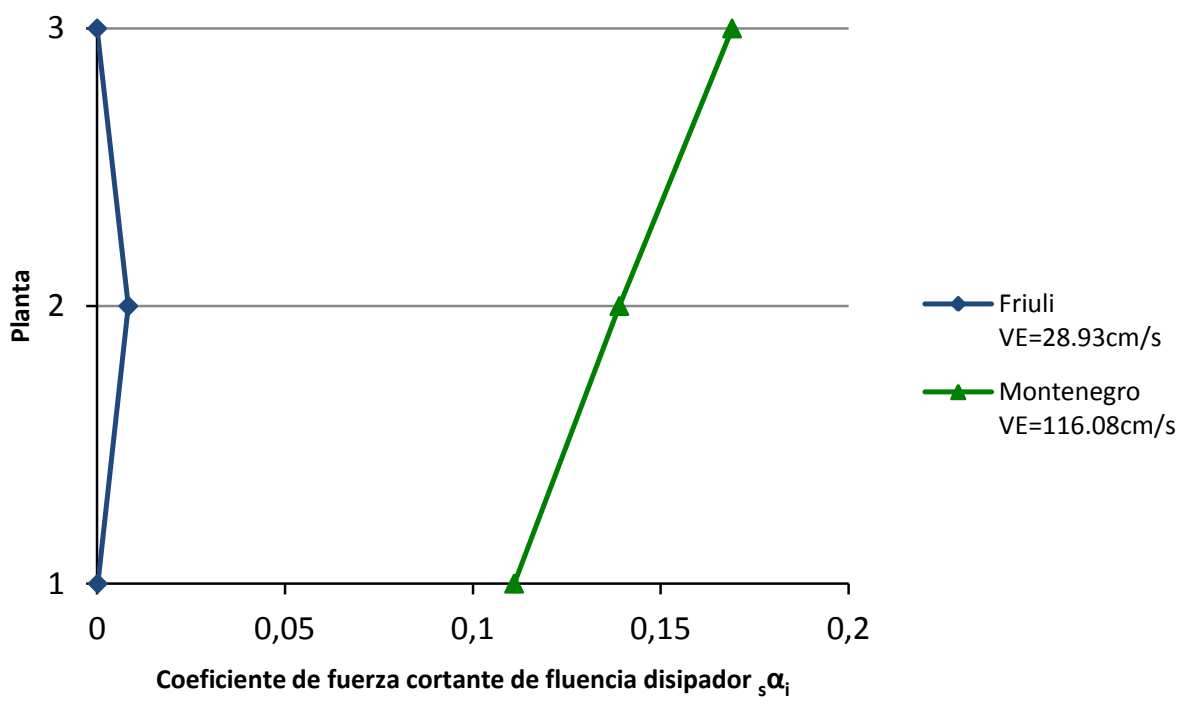

Fig. 5.5.9: Coeficiente de fuerza cortante de fluencia de los disipadores de cada planta i, pórtico pa2pz2-1, suelo tipo II, campo cercano

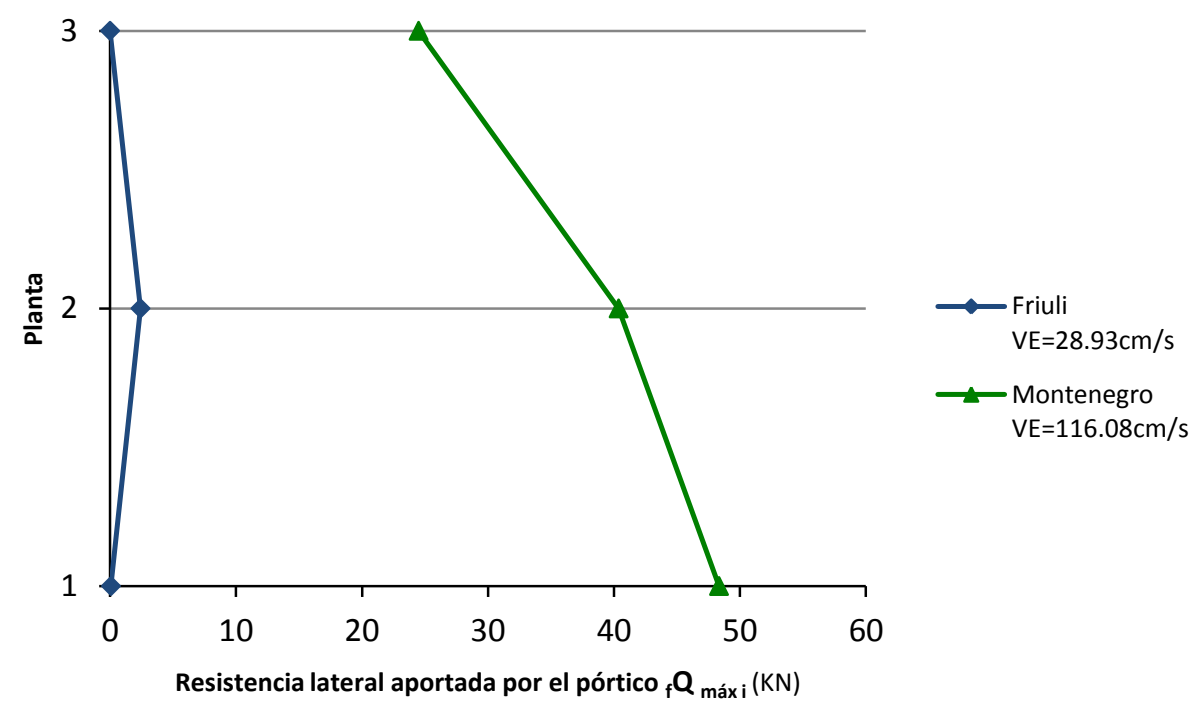

Fig. 5.5.10: Resistencia lateral aportada por la parte flexible (pórtico) cuando los disipadores empiezan a plastificar, pórtico pa2pz2-1, suelo tipo II, campo cercano 


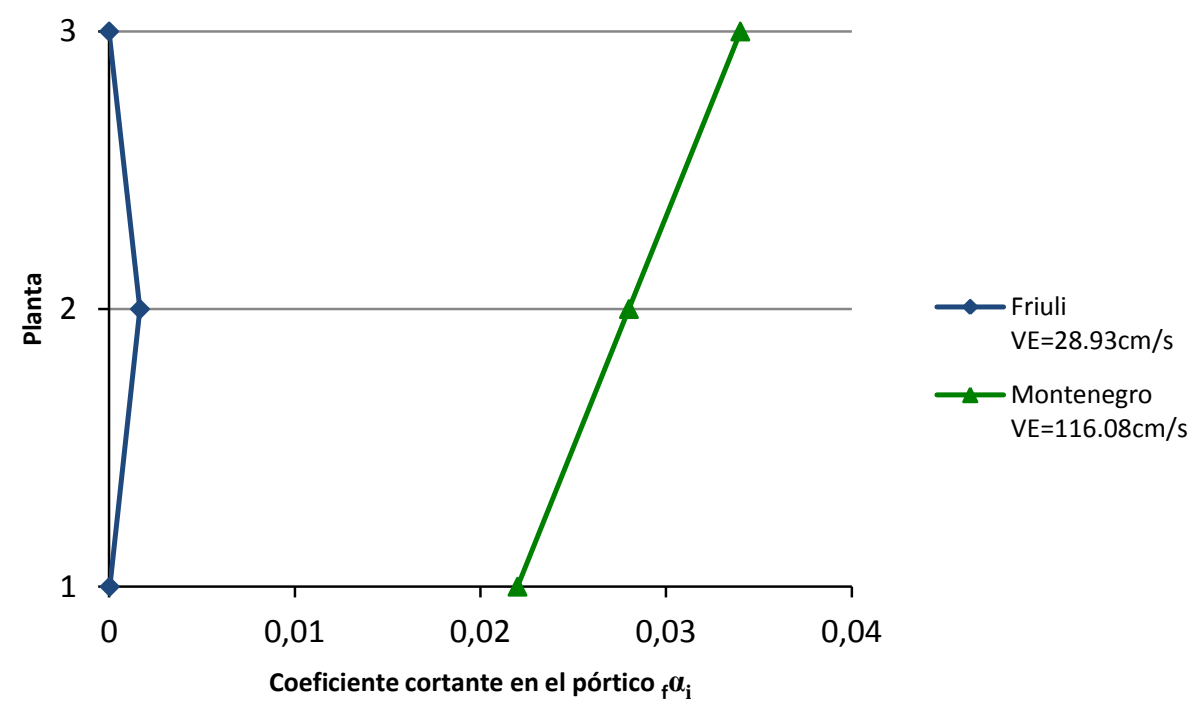

Fig. 5.5.11: Coeficiente de fuerza cortante en la parte flexible (pórtico) cuando los disipadores empiezan a plastificar, pórtico pa2pz2-1, suelo tipo II, campo cercano

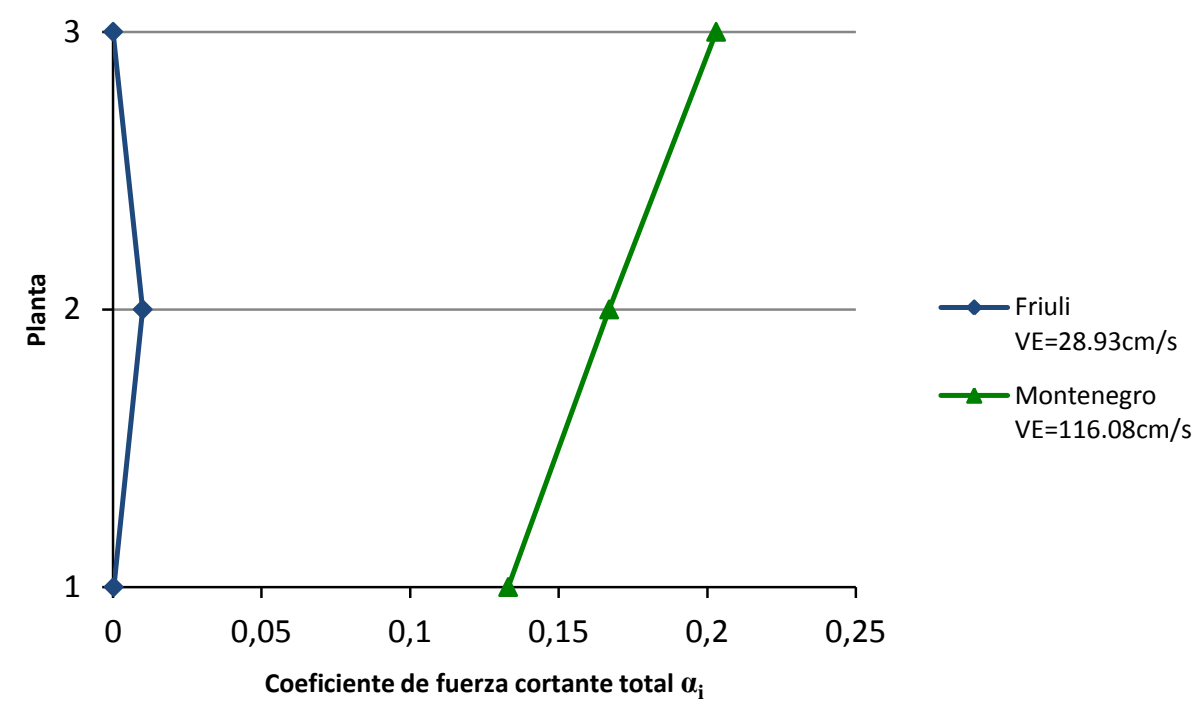

Fig. 5.5.12: Coeficiente de fuerza cortante total de planta, pórtico pa2pz2-1, suelo tipo II, campo cercano

\subsection{Suelo Tipo II (Campo lejano a la falla)}

\section{- Acelerograma №2 (Friuli aftershock) $\Delta=16 \mathrm{Km}$}

Desplazamiento de fluencia del disipador ${ }_{s} \delta_{i}$

Planta 3a: ${ }_{s} \delta_{3}=0,0420 \mathrm{~cm}$

Planta 2a: ${ }_{s} \delta_{2}=0,1270 \mathrm{~cm}$

Planta 1ㄹ: ${ }_{s} \delta_{1}=0,0180 \mathrm{~cm}$ 
Resistencia lateral de los disipadores de una planta i dada ${ }_{s} Q_{i}$

Planta 3a: ${ }_{s} Q_{3}={ }_{s} k_{3} \times{ }_{s} \delta_{3}=275000 \mathrm{~N} / \mathrm{cm} \times 0,0420 \mathrm{~cm} \Rightarrow{ }_{s} Q_{3}=11550 \mathrm{~N}$

Planta 2a: ${ }_{s} Q_{2}={ }_{s} k_{2} \times{ }_{s} \delta_{2}=340000 \mathrm{~N} / \mathrm{cm} \times 0,1270 \mathrm{~cm} \Rightarrow{ }_{s} Q_{2}=43180 \mathrm{~N}$

Planta 1ㅁ: ${ }_{s} Q_{1}={ }_{s} k_{1} \times{ }_{s} \delta_{1}=495000 \mathrm{~N} / \mathrm{cm} \times 0,0180 \mathrm{~cm} \Rightarrow \underline{{ }_{s} Q_{1}=8910 \mathrm{~N}}$

Coeficiente de fuerza cortante de fluencia de los disipadores de una planta i dada ${ }_{s} \alpha_{i}$

Planta 3a: ${ }_{s} \alpha_{3}=\frac{{ }_{s} Q_{3}(N)}{m_{3}\left(\frac{N s^{2}}{\mathrm{~cm}}\right) \times g\left(\frac{\mathrm{cm}}{\mathrm{s}^{2}}\right)}=\frac{11550}{739,04 \times 981} \Rightarrow \quad \underline{{ }_{s} \alpha_{3}=0,016}$

Planta 2a: ${ }_{s} \alpha_{2}=\frac{{ }_{s} Q_{2}(N)}{\left(m_{2}+m_{3}\right)\left(\frac{N s^{2}}{c m}\right) \times g\left(\frac{c m}{s^{2}}\right)}=\frac{43180}{(2 \times 739,04) \times 981} \Rightarrow \quad \underline{{ }_{s}} \alpha_{2}=0,030$

Planta 1ㄹ: ${ }_{s} \alpha_{1}=\frac{{ }_{s}(N)}{\left(m_{1}+m_{2}+m_{3}\right)\left(\frac{N s^{2}}{c m}\right) \times g\left(\frac{c m}{s^{2}}\right)}=\frac{8910}{(3 \times 739,04) \times 981} \Rightarrow \underline{{ }_{s} \alpha_{1}=4,096 e^{-03}}$

Resistencia lateral aportada por la parte flexible (pórtico) cuando los disipadores empiezan a plastificar ${ }_{f} Q_{\text {máxi }}$

Planta 3á: ${ }_{f} Q_{\text {máx } 3}={ }_{f} k_{3} \times{ }_{s} \delta_{3}=55000 \mathrm{~N} / \mathrm{cm} \times 0,0420 \mathrm{~cm} \Rightarrow{ }_{f} Q_{\text {máx } 3}=2310 \mathrm{~N}$

Planta 2a: ${ }_{f} Q_{\text {máx } 2}={ }_{f} k_{2} \times{ }_{s} \delta_{2}=68000 \mathrm{~N} / \mathrm{cm} \times 0,1270 \mathrm{~cm} \Rightarrow{ }_{f} Q_{\text {máx } 2}=8636 \mathrm{~N}$

Planta 1ㄹ: ${ }_{f} Q_{\text {máx1 }}={ }_{f} k_{1} \times{ }_{s} \delta_{1}=99000 \mathrm{~N} / \mathrm{cm} \times 0,0180 \mathrm{~cm} \Rightarrow{ }_{f} Q_{\text {máx } 1}=1782 N$

Coeficiente cortante en la parte flexible (pórtico) cuando los disipadores empiezan a plastificar ${ }_{f} \alpha_{i}$

Planta 3a: ${ }_{f} \alpha_{3}=\frac{{ }_{f} Q_{\operatorname{maxx}}(N)}{m_{3}\left(\frac{N s^{2}}{c m}\right) \times g\left(\frac{c m}{s^{2}}\right)}=\frac{2310}{739,04 \times 981} \Rightarrow \quad \underline{{ }_{f} \alpha_{3}=3,186 e^{-03}}$

Planta 2"a: ${ }_{f} \alpha_{2}=\frac{{ }_{f} Q_{\operatorname{máx} 2}(N)}{\left(m_{2}+m_{3}\right)\left(\frac{N s^{2}}{c m}\right) \times g\left(\frac{c m}{s^{2}}\right)}=\frac{8636}{(2 \times 739,04) \times 981} \Rightarrow \quad \underline{{ }_{f} \alpha_{2}=5,956 e^{-03}}$

Planta 1ạ: ${ }_{f} \alpha_{1}=\frac{{ }_{f} Q_{\text {máx } 1}(N)}{\left(m_{1}+m_{2}+m_{3}\right)\left(\frac{N s^{2}}{c m}\right) \times g\left(\frac{c m}{s^{2}}\right)}=\frac{1782}{(3 \times 739,04) \times 981} \Rightarrow \underline{{ }_{f}} \alpha_{1}=8,193 e^{-04}$ 
Coeficiente de fuerza cortante total de planta $\alpha_{i}$

$$
\begin{aligned}
& \text { Planta 3a: } \alpha_{3}={ }_{s} \alpha_{3}+{ }_{f} \alpha_{3}=0,016+3,186 e^{-03} \Rightarrow \frac{\alpha_{3}=0,019}{\alpha_{2}=0,036} \\
& \text { Planta 2a: } \alpha_{2}={ }_{s} \alpha_{2}+{ }_{f} \alpha_{2}=0,030+5,956 e^{-03} \Rightarrow \frac{\alpha_{1}}{\alpha_{1}=4,915 e^{-03}} \\
& \text { Planta 1: } \alpha_{1}={ }_{s} \alpha_{1}+{ }_{f} \alpha_{1}=4,096 e^{-03}+8,193 e^{-04}
\end{aligned}
$$

\section{- Acelerograma №12 (Montenegro aftershock) $\Delta=33 \mathrm{Km}$}

Desplazamiento de fluencia del disipador ${ }_{s} \delta_{i}$

Planta 3a: ${ }_{s} \delta_{3}=2,0000 \mathrm{~cm}$

Planta 2 ${ }^{\mathrm{a}}:{ }_{s} \delta_{2}=1,5000 \mathrm{~cm}$

Planta 1ㄹ: $\delta_{1}=0,9473 \mathrm{~cm}$

Resistencia lateral de los disipadores de una planta i dada ${ }_{s} Q_{i}$

Planta 3a: ${ }_{s} Q_{3}={ }_{s} k_{3} \times{ }_{s} \delta_{3}=275000 \mathrm{~N} / \mathrm{cm} \times 2,0000 \mathrm{~cm} \Rightarrow{ }_{s} Q_{3}=550000 \mathrm{~N}$

Planta 2a: ${ }_{s} Q_{2}={ }_{s} k_{2} \times{ }_{s} \delta_{2}=340000 \mathrm{~N} / \mathrm{cm} \times 1,5000 \mathrm{~cm} \Rightarrow{ }_{s} Q_{2}=510000 \mathrm{~N}$

Planta 1: ${ }_{s} Q_{1}={ }_{s} k_{1} \times{ }_{s} \delta_{1}=495000 \mathrm{~N} / \mathrm{cm} \times 0,9473 \mathrm{~cm} \Rightarrow \overline{{ }_{s} Q_{1}=468913,5 \mathrm{~N}}$

Coeficiente de fuerza cortante de fluencia de los disipadores de una planta i dada ${ }_{s} \alpha_{i}$

Planta 3a: ${ }_{s} \alpha_{3}=\frac{{ }_{s} Q_{3}(N)}{m_{3}\left(\frac{N s^{2}}{c m}\right) \times g\left(\frac{c m}{s^{2}}\right)}=\frac{550000}{739,04 \times 981} \Rightarrow \quad \quad \quad \quad{ }_{s} \alpha_{3}=0,759$

Planta 2ㄹ: ${ }_{s} \alpha_{2}=\frac{{ }_{s}(N)}{\left(m_{2}+m_{3}\right)\left(\frac{N s^{2}}{c m}\right) \times g\left(\frac{c m}{s^{2}}\right)}=\frac{510000}{(2 \times 739,04) \times 981} \Rightarrow \quad{ }_{s} \alpha_{2}=0,352$

Planta 1ạ: ${ }_{s} \alpha_{1}=\frac{{ }_{s}(N)}{\left(m_{1}+m_{2}+m_{3}\right)\left(\frac{N s^{2}}{c m}\right) \times g\left(\frac{c m}{s^{2}}\right)}=\frac{468913,5}{(3 \times 739,04) \times 981} \Rightarrow \underline{{ }_{s}} \alpha_{1}=0,215$

Resistencia lateral aportada por la parte flexible (pórtico) cuando los disipadores empiezan a plastificar ${ }_{f} Q_{\text {máxi }}$

Planta 3a: ${ }_{f} Q_{\text {máx3 }}={ }_{f} k_{3} \times{ }_{s} \delta_{3}=55000 \mathrm{~N} / \mathrm{cm} \times 2,0000 \mathrm{~cm} \Rightarrow{ }_{f} Q_{\text {máx3 }}=110000 \mathrm{~N}$

Planta 2ä: ${ }_{f} Q_{\text {máx } 2}={ }_{f} k_{2} \times{ }_{s} \delta_{2}=68000 \mathrm{~N} / \mathrm{cm} \times 1,5000 \mathrm{~cm} \Rightarrow{ }_{f} Q_{\text {máx } 2}=102000 \mathrm{~N}$

Planta 1: ${ }_{f} Q_{\text {máx } 1}={ }_{f} k_{1} \times{ }_{s} \delta_{1}=99000 \mathrm{~N} / \mathrm{cm} \times 0,9473 \mathrm{~cm} \Rightarrow{ }_{f} Q_{\text {máx } 1}=93782,7 \mathrm{~N}$ 
Coeficiente cortante en la parte flexible (pórtico) cuando los disipadores empiezan a plastificar ${ }_{f} \alpha_{i}$

Planta 3a: ${ }_{f} \alpha_{3}=\frac{{ }_{f} Q_{\text {máx } 3}(N)}{m_{3}\left(\frac{N s^{2}}{c m}\right) \times g\left(\frac{c m}{s^{2}}\right)}=\frac{110000}{739,04 \times 981} \Rightarrow \quad \underline{{ }_{f} \alpha_{3}=0,152}$

Planta 2ä: ${ }_{f} \alpha_{2}=\frac{{ }_{f} Q_{\operatorname{máx} 2}(N)}{\left(m_{2}+m_{3}\right)\left(\frac{N s^{2}}{c m}\right) \times g\left(\frac{c m}{s^{2}}\right)}=\frac{102000}{(2 \times 739,04) \times 981} \Rightarrow \quad \underline{{ }_{f}} \alpha_{2}=0,070$

Planta 1ㄹ: ${ }_{f} \alpha_{1}=\frac{{ }_{f} Q_{m a ́ x 1}(N)}{\left(m_{1}+m_{2}+m_{3}\right)\left(\frac{N s^{2}}{c m}\right) \times g\left(\frac{c m}{s^{2}}\right)}=\frac{93782,7}{(3 \times 739,04) \times 981} \Rightarrow \underline{{ }_{f}} \alpha_{1}=0,043$

Coeficiente de fuerza cortante total de planta $\alpha_{i}$

Planta 3a: $\alpha_{3}={ }_{s} \alpha_{3}+{ }_{f} \alpha_{3}=0,759+0,152 \Rightarrow \underline{\alpha_{3}=0,911}$

Planta 2a: $\alpha_{2}={ }_{s} \alpha_{2}+{ }_{f} \alpha_{2}=0,352+0,070 \Rightarrow \underline{\alpha_{2}=0,422}$

Planta 1ㄹ: $\alpha_{1}={ }_{s} \alpha_{1}+{ }_{f} \alpha_{1}=0,215+0,043 \Rightarrow \alpha_{1}=0,258$

\section{Representación gráfica}

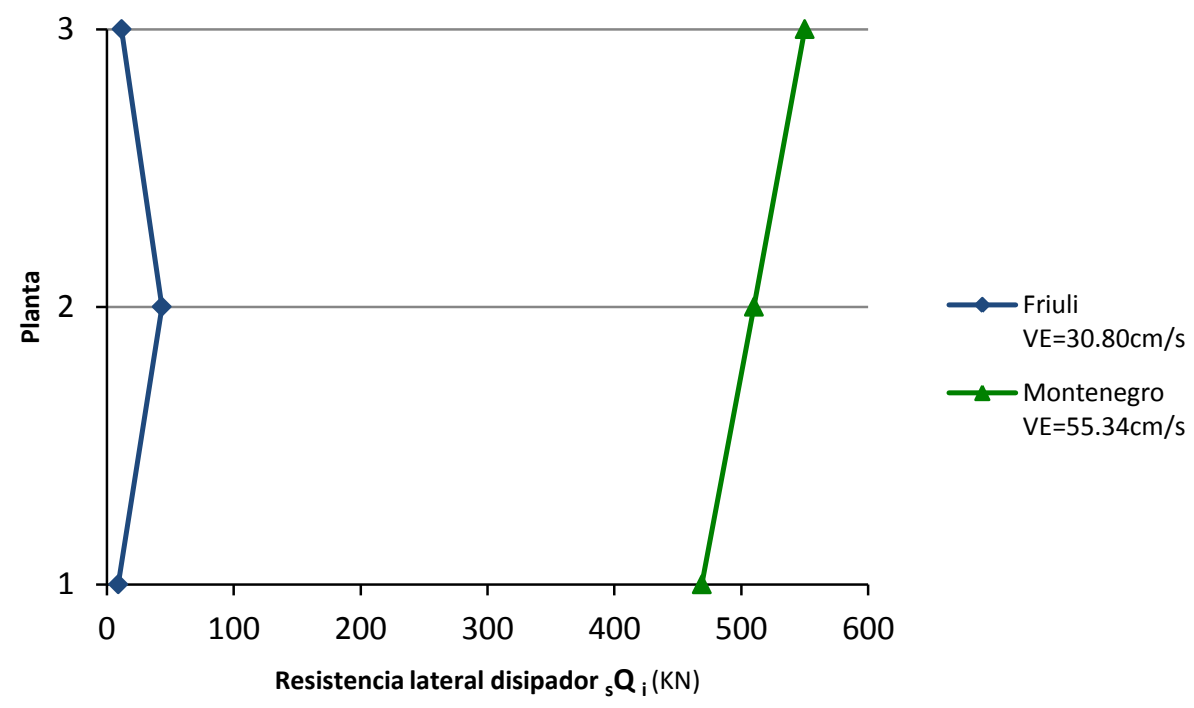

Fig. 5.5.13: Resistencia lateral de los disipadores de cada planta i, pórtico, pa2pz2-1, suelo tipo II, campo lejano 


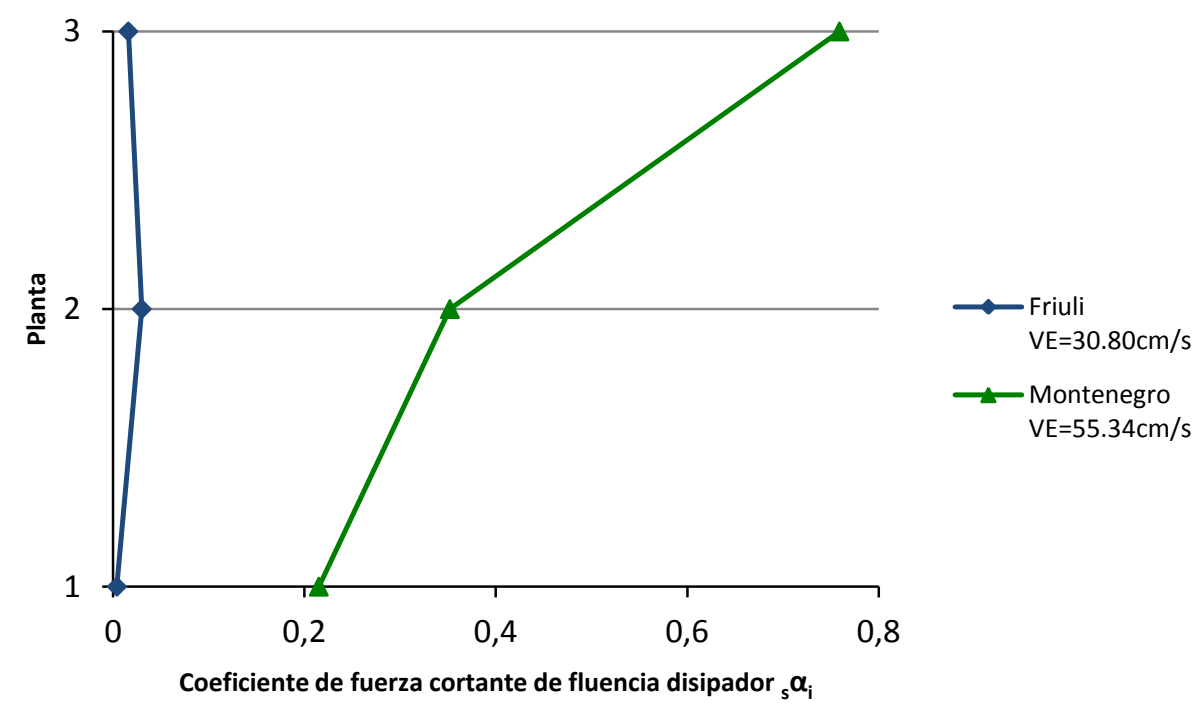

Fig. 5.5.14: Coeficiente de fuerza cortante de fluencia de los disipadores de cada planta i, pórtico pa2pz2-1, suelo tipo II, campo lejano

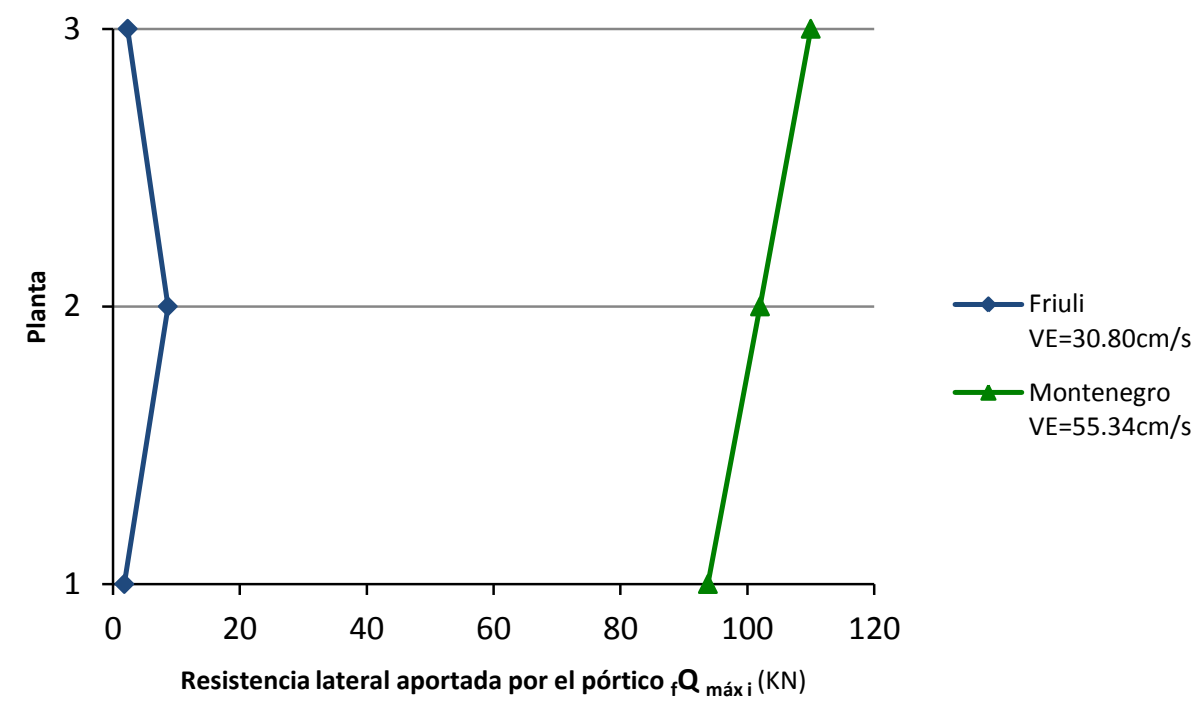

Fig. 5.5.15: Resistencia lateral aportada por la parte flexible (pórtico) cuando los disipadores empiezan a plastificar, pórtico pa2pz2-1, suelo tipo II, campo lejano 


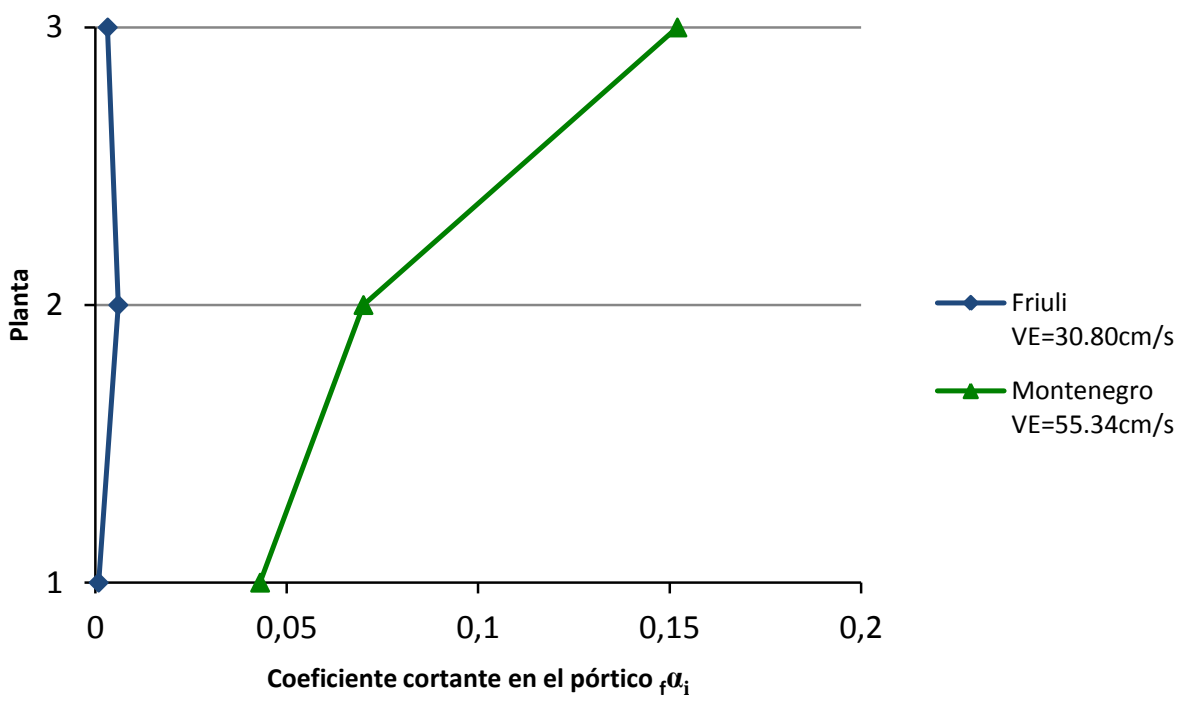

Fig. 5.5.16: Coeficiente de fuerza cortante en la parte flexible (pórtico) cuando los disipadores empiezan a plastificar, pórtico pa2pz2-1, suelo tipo II, campo lejano

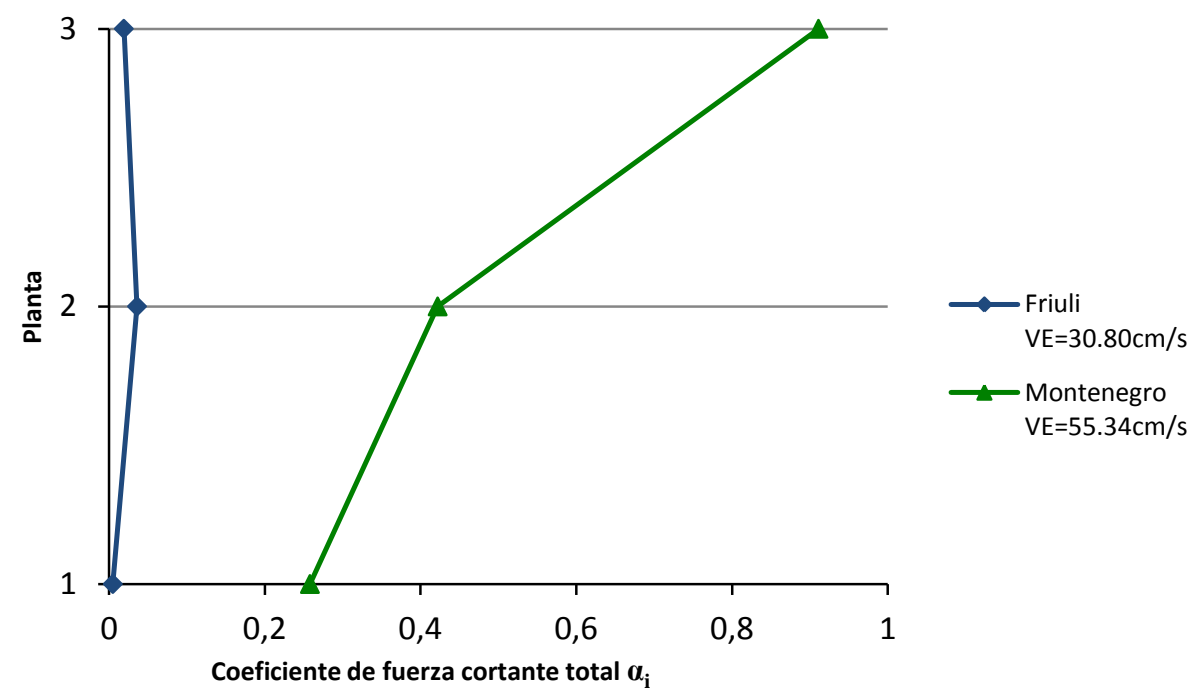

Fig. 5.5.17: Coeficiente de fuerza cortante total de planta, pórtico pa2pz2-1, suelo tipo II, campo lejano

\subsection{Suelo Tipo III (Campo cercano a la falla)}

\section{- Acelerograma №2 (Friuli aftershock) $\Delta=10 \mathrm{Km}$}

Desplazamiento de fluencia del disipador ${ }_{s} \delta_{i}$

Planta 3a: ${ }_{s} \delta_{3}=0,6250 \mathrm{~cm}$

Planta 2a: : ${ }_{s} \delta_{2}=0,9375 \mathrm{~cm}$

Planta 1ㄹ: ${ }_{s} \delta_{1}=0,7227 \mathrm{~cm}$ 
Resistencia lateral de los disipadores de una planta i dada ${ }_{s} Q_{i}$

Planta 3a: ${ }_{s} Q_{3}={ }_{s} k_{3} \times{ }_{s} \delta_{3}=275000 \mathrm{~N} / \mathrm{cm} \times 0,6250 \mathrm{~cm} \Rightarrow{ }_{s} Q_{3}=171875 \mathrm{~N}$

Planta 2a: ${ }_{s} Q_{2}={ }_{s} k_{2} \times{ }_{s} \delta_{2}=340000 \mathrm{~N} / \mathrm{cm} \times 0,9375 \mathrm{~cm} \Rightarrow{ }_{s} Q_{2}=318750 \mathrm{~N}$

Planta 1ㄹ: ${ }_{s} Q_{1}={ }_{s} k_{1} \times{ }_{s} \delta_{1}=495000 \mathrm{~N} / \mathrm{cm} \times 0,7227 \mathrm{~cm} \Rightarrow{ }_{s} Q_{1}=357736,5 \mathrm{~N}$

Coeficiente de fuerza cortante de fluencia de los disipadores de una planta i dada ${ }_{s} \alpha_{i}$

Planta 3a: ${ }_{s} \alpha_{3}=\frac{{ }_{s} Q_{3}(N)}{m_{3}\left(\frac{N s^{2}}{c m}\right) \times g\left(\frac{c m}{s^{2}}\right)}=\frac{171875}{739,04 \times 981} \Rightarrow \quad \underline{{ }_{s} \alpha_{3}=0,237}$

Planta 2 ${ }_{s} \alpha_{2}=\frac{{ }_{s} Q_{2}(N)}{\left(m_{2}+m_{3}\right)\left(\frac{N s^{2}}{c m}\right) \times g\left(\frac{c m}{s^{2}}\right)}=\frac{318750}{(2 \times 739,04) \times 981} \Rightarrow \quad{ }_{s} \alpha_{2}=0,220$

Planta 1ä: $\alpha_{s}=\frac{{ }_{s} Q_{1}(N)}{\left(m_{1}+m_{2}+m_{3}\right)\left(\frac{N s^{2}}{c m}\right) \times g\left(\frac{c m}{s^{2}}\right)}=\frac{357736,5}{(3 \times 739,04) \times 981} \Rightarrow \underline{{ }_{s}} \alpha_{1}=0,164$

Resistencia lateral aportada por la parte flexible (pórtico) cuando los disipadores empiezan a plastificar ${ }_{f} Q_{\text {máxi }}$

Planta 3a: ${ }_{f} Q_{\text {máx } 3}={ }_{f} k_{3} \times{ }_{s} \delta_{3}=55000 \mathrm{~N} / \mathrm{cm} \times 0,6250 \mathrm{~cm} \Rightarrow{ }_{f} Q_{\text {máx3 }}=34375 \mathrm{~N}$

Planta 2a: ${ }_{f} Q_{\text {máx } 2}={ }_{f} k_{2} \times{ }_{s} \delta_{2}=68000 \mathrm{~N} / \mathrm{cm} \times 0,9375 \mathrm{~cm} \Rightarrow{ }_{f} Q_{\text {máx }}=63750 \mathrm{~N}$

Planta 1ㄹ: ${ }_{f} Q_{\text {máx1 }}={ }_{f} k_{1} \times{ }_{s} \delta_{1}=99000 \mathrm{~N} / \mathrm{cm} \times 0,7227 \mathrm{~cm} \Rightarrow \overline{{ }_{f} Q_{\text {máx } 1}=71547,3 \mathrm{~N}}$

Coeficiente cortante en la parte flexible (pórtico) cuando los disipadores empiezan a plastificar ${ }_{f} \alpha_{i}$

Planta 3a: ${ }_{f} \alpha_{3}=\frac{{ }_{f} Q_{\text {máx } 3}(N)}{m_{3}\left(\frac{N s^{2}}{c m}\right) \times g\left(\frac{c m}{s^{2}}\right)}=\frac{34375}{739,04 \times 981} \Rightarrow \quad \underline{{ }_{f} \alpha_{3}=0,047}$

Planta 2ㄹ: ${ }_{f} \alpha_{2}=\frac{{ }_{f} Q_{\text {máx }}(N)}{\left(m_{2}+m_{3}\right)\left(\frac{N s^{2}}{c m}\right) \times g\left(\frac{c m}{s^{2}}\right)}=\frac{63750}{(2 \times 739,04) \times 981} \Rightarrow \quad \underline{{ }_{f} \alpha_{2}=0,044}$

Planta 1ạ: ${ }_{f} \alpha_{1}=\frac{{ }_{f} Q_{\operatorname{máxx}}(N)}{\left(m_{1}+m_{2}+m_{3}\right)\left(\frac{N s^{2}}{c m}\right) \times g\left(\frac{c m}{s^{2}}\right)}=\frac{71547,3}{(3 \times 739,04) \times 981} \Rightarrow \underline{{ }_{f}} \alpha_{1}=0,033$ 
Coeficiente de fuerza cortante total de planta $\alpha_{i}$

Planta 3a: $\alpha_{3}={ }_{s} \alpha_{3}+{ }_{f} \alpha_{3}=0,237+0,047 \Rightarrow \alpha_{3}=0,284$

Planta 2a: $\alpha_{2}={ }_{s} \alpha_{2}+{ }_{f} \alpha_{2}=0,220+0,044 \Rightarrow \alpha_{2}=0,264$

Planta 1a: $\alpha_{1}={ }_{s} \alpha_{1}+{ }_{f} \alpha_{1}=0,164+0,033 \Rightarrow \alpha_{1}=0,197$

\section{- Acelerograma №6 (Kalamata aftershock) $\Delta=3 \mathrm{Km}$}

Desplazamiento de fluencia del disipador ${ }_{s} \delta_{i}$

Planta 3a: ${ }_{s} \delta_{3}=0,0450 \mathrm{~cm}$

Planta 2a: ${ }_{s} \delta_{2}=0,1824 \mathrm{~cm}$

Planta 1: ${ }_{s} \delta_{1}=0,0001 \mathrm{~cm}$

Resistencia lateral de los disipadores de una planta i dada ${ }_{s} Q_{i}$

Planta 3a: ${ }_{s} Q_{3}={ }_{s} k_{3} \times{ }_{s} \delta_{3}=275000 \mathrm{~N} / \mathrm{cm} \times 0,0450 \mathrm{~cm} \Rightarrow{ }_{s} Q_{3}=12375 \mathrm{~N}$

Planta 2 ${ }^{\mathrm{a}}:{ }_{s} Q_{2}={ }_{s} k_{2} \times{ }_{s} \delta_{2}=340000 \mathrm{~N} / \mathrm{cm} \times 0,1824 \mathrm{~cm} \Rightarrow{ }_{s} Q_{2}=62016 \mathrm{~N}$

Planta 1a: ${ }_{s} Q_{1}={ }_{s} k_{1} \times{ }_{s} \delta_{1}=495000 \mathrm{~N} / \mathrm{cm} \times 0,0001 \mathrm{~cm} \Rightarrow{ }_{s} Q_{1}=49,5 \mathrm{~N}$

Coeficiente de fuerza cortante de fluencia de los disipadores de una planta i dada ${ }_{s} \alpha_{i}$

Planta 3a: ${ }_{s} \alpha_{3}=\frac{{ }_{s} Q_{3}(N)}{m_{3}\left(\frac{N s^{2}}{c m}\right) \times g\left(\frac{c m}{s^{2}}\right)}=\frac{12375}{739,04 \times 981} \Rightarrow \quad \underline{{ }_{s} \alpha_{3}=0,017}$

Planta 2a: ${ }_{s} \alpha_{2}=\frac{{ }_{s}(N)}{\left(m_{2}+m_{3}\right)\left(\frac{N s^{2}}{c m}\right) \times g\left(\frac{c m}{s^{2}}\right)}=\frac{62016}{(2 \times 739,04) \times 981} \Rightarrow \quad \underline{{ }_{s} \alpha_{2}=0,043}$

Planta 1ㄹ: ${ }_{s} \alpha_{1}=\frac{{ }_{s} Q_{1}(N)}{\left(m_{1}+m_{2}+m_{3}\right)\left(\frac{N s^{2}}{c m}\right) \times g\left(\frac{c m}{s^{2}}\right)}=\frac{49,5}{(3 \times 739,04) \times 981} \Rightarrow \underline{{ }_{s} \alpha_{1}=2,276 e^{-05}}$

Resistencia lateral aportada por la parte flexible (pórtico) cuando los disipadores empiezan a plastificar ${ }_{f} Q_{\text {máxi }}$

Planta 3ạ: ${ }_{f} Q_{\text {máx } 3}={ }_{f} k_{3} \times{ }_{s} \delta_{3}=55000 \mathrm{~N} / \mathrm{cm} \times 0,0450 \mathrm{~cm} \Rightarrow{ }_{f} Q_{\text {máx3 }}=2475 \mathrm{~N}$

Planta 2a: ${ }_{f} Q_{\text {máx } 2}={ }_{f} k_{2} \times{ }_{s} \delta_{2}=68000 \mathrm{~N} / \mathrm{cm} \times 0,1824 \mathrm{~cm} \Rightarrow{ }_{f} Q_{\text {máx } 2}=12403,2 N$

Planta 1ㄹ: ${ }_{f} Q_{m a ́ x 1}={ }_{f} k_{1} \times{ }_{s} \delta_{1}=99000 \mathrm{~N} / \mathrm{cm} \times 0,0001 \mathrm{~cm} \Rightarrow{ }_{f} Q_{\text {máx } 1}=9,9 N$ 
Coeficiente cortante en la parte flexible (pórtico) cuando los disipadores empiezan a plastificar ${ }_{f} \alpha_{i}$

Planta 3a: ${ }_{f} \alpha_{3}=\frac{{ }_{f} Q_{\text {máx3 }}(N)}{m_{3}\left(\frac{N s^{2}}{c m}\right) \times g\left(\frac{c m}{s^{2}}\right)}=\frac{2475}{739,04 \times 981} \Rightarrow \quad \underline{{ }_{f} \alpha_{3}=3,414 e^{-03}}$

Planta 2ㄹ: ${ }_{f} \alpha_{2}=\frac{{ }_{f} Q_{\text {máx }}(N)}{\left(m_{2}+m_{3}\right)\left(\frac{N s^{2}}{c m}\right) \times g\left(\frac{c m}{s^{2}}\right)}=\frac{12403,2}{(2 \times 739,04) \times 981} \Rightarrow \quad \underline{{ }_{f} \alpha_{2}=8,554 e^{-03}}$

Planta 1ㄹ: ${ }_{f} \alpha_{1}=\frac{{ }_{f} Q_{\text {máx } 1}(N)}{\left(m_{1}+m_{2}+m_{3}\right)\left(\frac{N s^{2}}{c m}\right) \times g\left(\frac{c m}{s^{2}}\right)}=\frac{9,9}{(3 \times 739,04) \times 981} \Rightarrow{ }_{f} \alpha_{1}=4,552 e^{-06}$

Coeficiente de fuerza cortante total de planta $\alpha_{i}$

Planta 3a: $\alpha_{3}={ }_{s} \alpha_{3}+{ }_{f} \alpha_{3}=0,017+3,414 e^{-03} \Rightarrow \frac{\alpha_{3}=0,020}{\alpha_{2}=0,051}$
Planta 2a: $\alpha_{2}={ }_{s} \alpha_{2}+{ }_{f} \alpha_{2}=0,043+8,554 e^{-03} \Rightarrow \frac{\alpha_{2}}{\alpha_{1}=2,731 e^{-05}}$
Planta 1a: $\alpha_{1}={ }_{s} \alpha_{1}+{ }_{f} \alpha_{1}=2,276 e^{-05}+4,552 e^{-06}$

\section{- Acelerograma №12 (Duzce 1) $\Delta=8 \mathrm{Km}$}

Desplazamiento de fluencia del disipador ${ }_{s} \delta_{i}$

Planta 3a: ${ }_{s} \delta_{3}=0,2158 \mathrm{~cm}$

Planta 2a: ${ }_{s} \delta_{2}=0,4150 \mathrm{~cm}$

Planta 1ㄹ: ${ }_{s} \delta_{1}=0,1914 \mathrm{~cm}$

Resistencia lateral de los disipadores de una planta i dada ${ }_{s} Q_{i}$

Planta 3a: ${ }_{s} Q_{3}={ }_{s} k_{3} \times{ }_{s} \delta_{3}=275000 \mathrm{~N} / \mathrm{cm} \times 0,2158 \mathrm{~cm} \Rightarrow{ }_{s} Q_{3}=59345 \mathrm{~N}$

Planta 2a: ${ }_{s} Q_{2}={ }_{s} k_{2} \times{ }_{s} \delta_{2}=340000 \mathrm{~N} / \mathrm{cm} \times 0,4150 \mathrm{~cm} \Rightarrow{ }_{s} Q_{2}=141100 \mathrm{~N}$

Planta 1a: ${ }_{s} Q_{1}={ }_{s} k_{1} \times{ }_{s} \delta_{1}=495000 \mathrm{~N} / \mathrm{cm} \times 0,1914 \mathrm{~cm} \Rightarrow{ }_{s} Q_{1}=94743 \mathrm{~N}$

Coeficiente de fuerza cortante de fluencia de los disipadores de una planta i dada ${ }_{s} \alpha_{i}$

Planta 3a: ${ }_{s} \alpha_{3}=\frac{{ }_{s} Q_{3}(N)}{m_{3}\left(\frac{N s^{2}}{c m}\right) \times g\left(\frac{c m}{s^{2}}\right)}=\frac{59345}{739,04 \times 981} \Rightarrow \quad \underline{{ }_{s} \alpha_{3}=0,082}$

Planta 2 : ${ }_{s} \alpha_{2}=\frac{{ }_{s}(N)}{\left(m_{2}+m_{3}\right)\left(\frac{N s^{2}}{c m}\right) \times g\left(\frac{c m}{s^{2}}\right)}=\frac{141100}{(2 \times 739,04) \times 981} \Rightarrow \quad \underline{{ }_{s} \alpha_{2}=0,097}$ 
Planta 1ㄹ: $\alpha_{s}=\frac{{ }_{s} Q_{1}(N)}{\left(m_{1}+m_{2}+m_{3}\right)\left(\frac{N s^{2}}{c m}\right) \times g\left(\frac{c m}{s^{2}}\right)}=\frac{94743}{(3 \times 739,04) \times 981} \Rightarrow \underline{{ }_{s}} \alpha_{1}=0,043$

Resistencia lateral aportada por la parte flexible (pórtico) cuando los disipadores empiezan a plastificar ${ }_{f} Q_{\text {máxi }}$

Planta 3a: ${ }_{f} Q_{\text {máx3 }}={ }_{f} k_{3} \times{ }_{s} \delta_{3}=55000 \mathrm{~N} / \mathrm{cm} \times 0,2158 \mathrm{~cm} \Rightarrow{ }_{f} Q_{\operatorname{máx} 3}=11869 \mathrm{~N}$

Planta 2a: ${ }_{f} Q_{\text {máx } 2}={ }_{f} k_{2} \times{ }_{s} \delta_{2}=68000 \mathrm{~N} / \mathrm{cm} \times 0,4150 \mathrm{~cm} \Rightarrow{ }_{f} Q_{\max 2}=28220 \mathrm{~N}$

Planta 1ㄹ: ${ }_{f} Q_{\text {maxx }}={ }_{f} k_{1} \times{ }_{s} \delta_{1}=99000 \mathrm{~N} / \mathrm{cm} \times 0,1914 \mathrm{~cm} \Rightarrow{ }_{f} Q_{\max 1}=18948,6 \mathrm{~N}$

Coeficiente cortante en la parte flexible (pórtico) cuando los disipadores empiezan a plastificar ${ }_{f} \alpha_{i}$

Planta 3a: ${ }_{f} \alpha_{3}=\frac{{ }_{f} Q_{\text {máx } 3}(N)}{m_{3}\left(\frac{N s^{2}}{c m}\right) \times g\left(\frac{c m}{s^{2}}\right)}=\frac{11869}{739,04 \times 981} \Rightarrow \quad \underline{{ }_{f} \alpha_{3}=0,016}$

Planta 2ä: ${ }_{f} \alpha_{2}=\frac{{ }_{f} Q_{\text {máx }}(N)}{\left(m_{2}+m_{3}\right)\left(\frac{N s^{2}}{c m}\right) \times g\left(\frac{c m}{s^{2}}\right)}=\frac{28220}{(2 \times 739,04) \times 981} \Rightarrow \quad \underline{{ }_{f}} \alpha_{2}=0,019$

Planta 1ạ: ${ }_{f} \alpha_{1}=\frac{{ }_{f} Q_{\text {máx1 }}(N)}{\left(m_{1}+m_{2}+m_{3}\right)\left(\frac{N s^{2}}{c m}\right) \times g\left(\frac{c m}{s^{2}}\right)}=\frac{18948,6}{(3 \times 739,04) \times 981} \Rightarrow \underline{{ }_{f}} \alpha_{1}=8,712 e^{-03}$

Coeficiente de fuerza cortante total de planta $\alpha_{i}$

Planta 3a: $\alpha_{3}={ }_{s} \alpha_{3}+{ }_{f} \alpha_{3}=0,082+0,016 \Rightarrow \underline{\alpha_{3}=0,098}$

Planta 2 2 : $\alpha_{2}={ }_{s} \alpha_{2}+{ }_{f} \alpha_{2}=0,097+0,019 \Rightarrow \alpha_{2}=0,116$

Planta 1ạ: $\alpha_{1}={ }_{s} \alpha_{1}{ }_{f} \alpha_{1}=0,043+8,712 e^{-03} \Rightarrow \alpha_{1}=0,052$ 
Representación gráfica

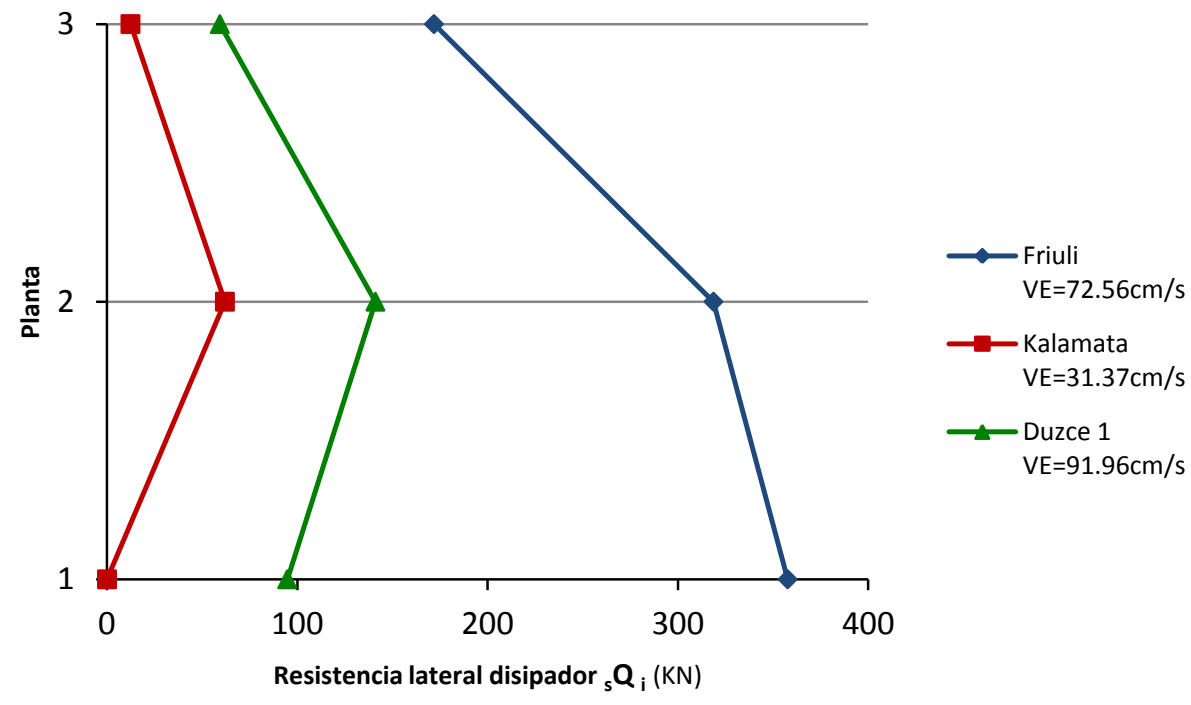

Fig. 5.5.18: Resistencia lateral de los disipadores de cada planta i, pórtico pa2pz2-1, suelo tipo III, campo cercano

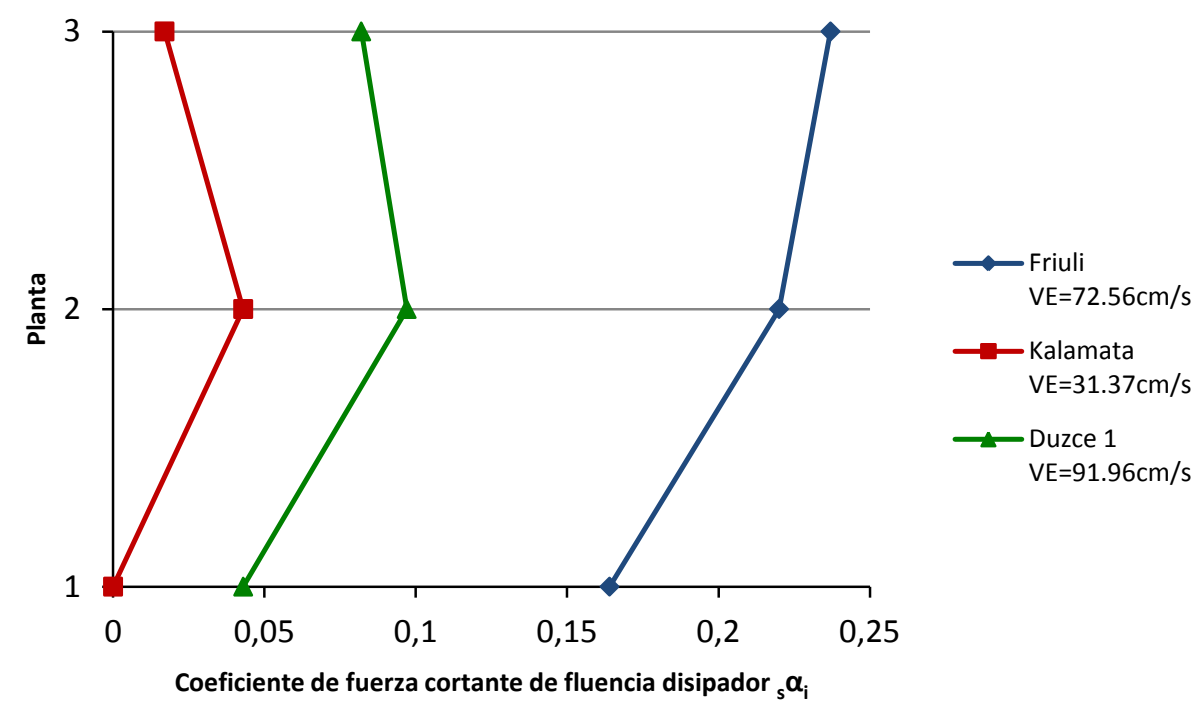

Fig. 5.5.19: Coeficiente de fuerza cortante de fluencia de los disipadores de cada planta i, pórtico pa2pz2-1, suelo tipo III, campo cercano 


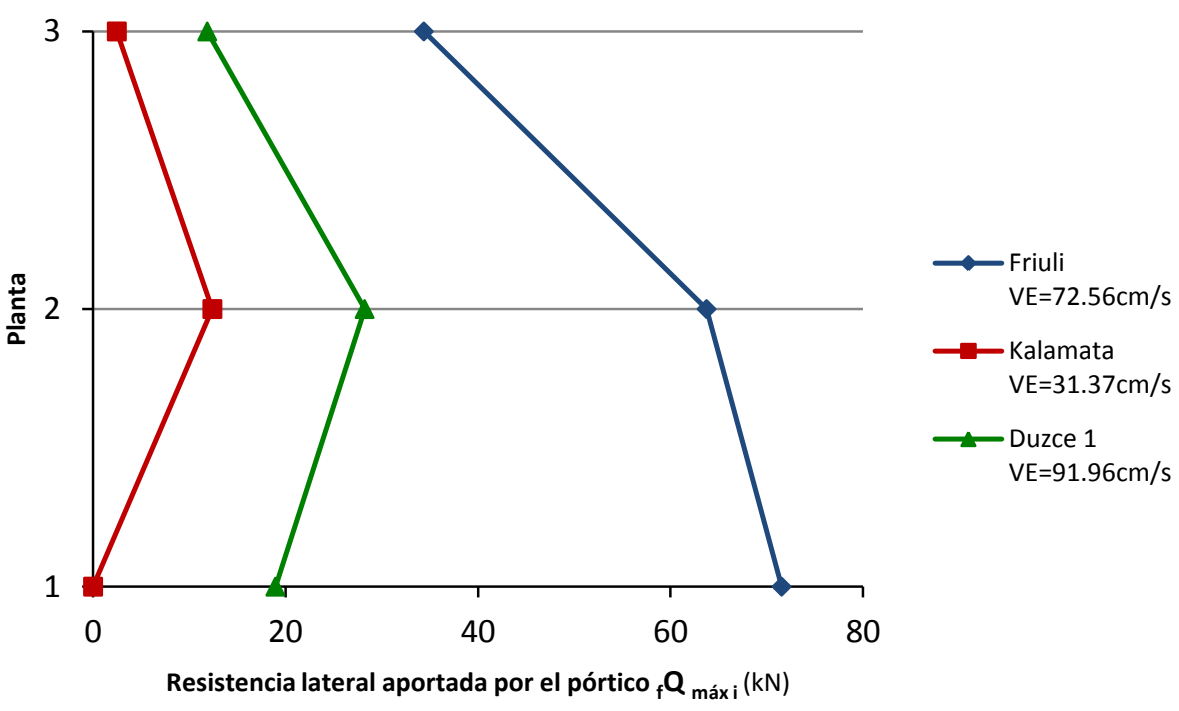

Fig. 5.5.20: Resistencia lateral aportada por la parte flexible (pórtico) cuando los disipadores empiezan a plastificar, pórtico pa2pz2-1, suelo tipo III, campo cercano

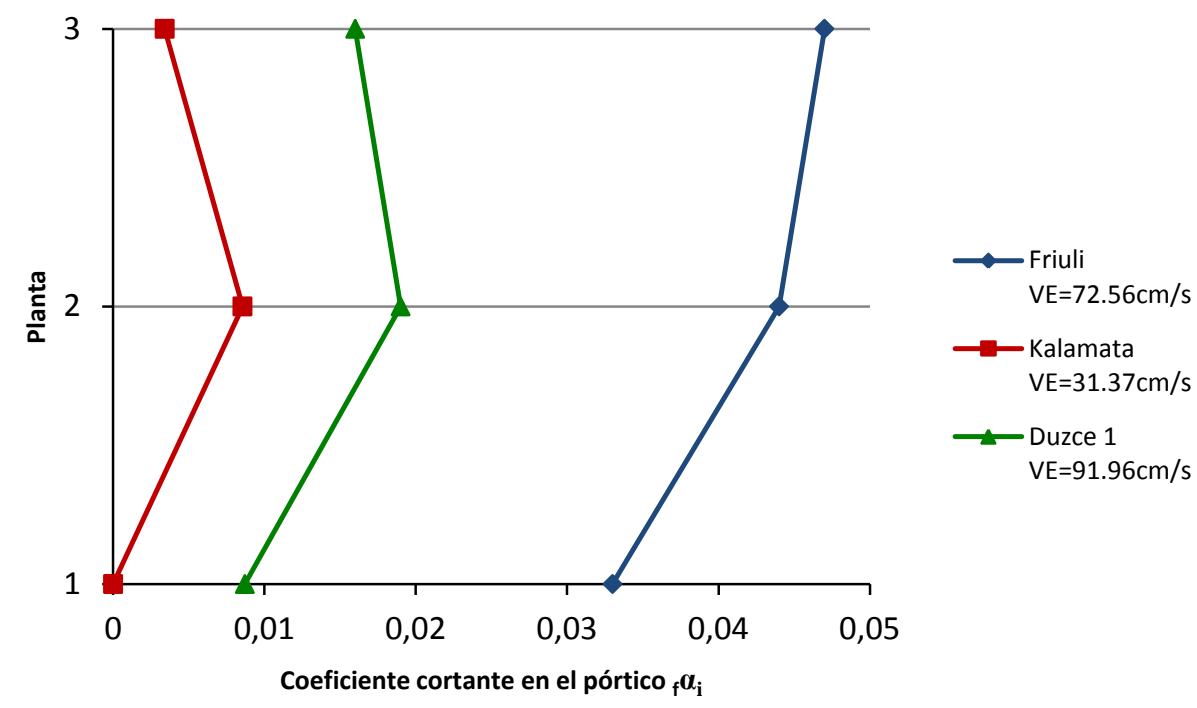

Fig. 5.5.21: Coeficiente de fuerza cortante en la parte flexible (pórtico) cuando los disipadores empiezan a plastificar, pórtico pa2pz2-1, suelo tipo III, campo cercano 


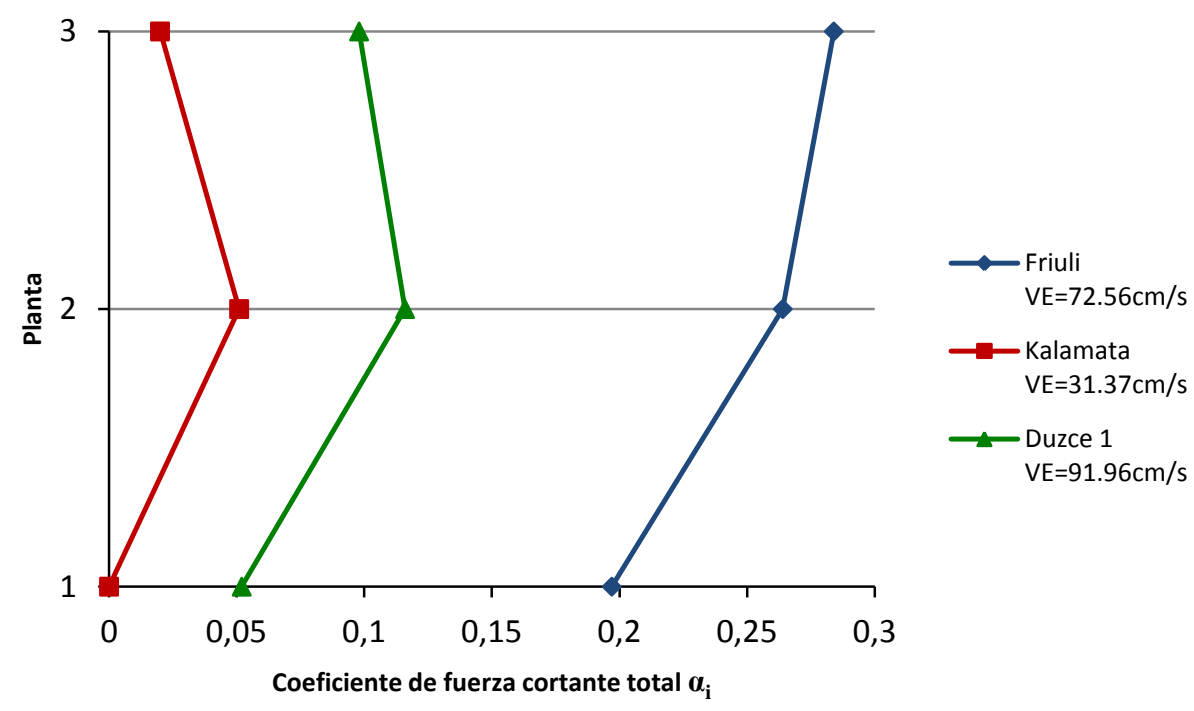

Fig. 5.5.22: Coeficiente de fuerza cortante total de planta, pórtico pa2pz2-1, suelo tipo III, campo cercano

\subsection{Suelo Tipo III (Campo lejano a la falla)}

\section{- Acelerograma №3 (Montenegro) $\Delta=24 \mathrm{Km}$}

Desplazamiento de fluencia del disipador ${ }_{s} \delta_{i}$

Planta 3a: ${ }_{s} \delta_{3}=0,0001 \mathrm{~cm}$

Planta 2a: ${ }_{s} \delta_{2}=0,1167 \mathrm{~cm}$

Planta 1ㄹ: ${ }_{s} \delta_{1}=0,0001 \mathrm{~cm}$

Resistencia lateral de los disipadores de una planta i dada ${ }_{s} Q_{i}$

Planta 3a: ${ }_{s} Q_{3}={ }_{s} k_{3} \times{ }_{s} \delta_{3}=275000 \mathrm{~N} / \mathrm{cm} \times 0,0001 \mathrm{~cm} \Rightarrow{ }_{s} Q_{3}=27,5 \mathrm{~N}$

Planta 2a: ${ }_{s} Q_{2}={ }_{s} k_{2} \times{ }_{s} \delta_{2}=340000 \mathrm{~N} / \mathrm{cm} \times 0,1167 \mathrm{~cm} \Rightarrow{ }_{s} Q_{2}=39678 \mathrm{~N}$

Planta 1ㄹ: ${ }_{s} Q_{1}={ }_{s} k_{1} \times{ }_{s} \delta_{1}=495000 \mathrm{~N} / \mathrm{cm} \times 0,0001 \mathrm{~cm} \Rightarrow{ }_{s} Q_{1}=49,5 \mathrm{~N}$

Coeficiente de fuerza cortante de fluencia de los disipadores de una planta i dada ${ }_{s} \alpha_{i}$

Planta 3a: ${ }_{s} \alpha_{3}=\frac{{ }_{s} Q_{3}(N)}{m_{3}\left(\frac{N s^{2}}{c m}\right) \times g\left(\frac{c m}{s^{2}}\right)}=\frac{27,5}{739,04 \times 981} \Rightarrow \quad \underline{{ }_{s} \alpha_{3}=3,793 e^{-05}}$

Planta 2: ${ }_{s} \alpha_{2}=\frac{{ }_{s} Q_{2}(N)}{\left(m_{2}+m_{3}\right)\left(\frac{N s^{2}}{c m}\right) \times g\left(\frac{c m}{s^{2}}\right)}=\frac{39678}{(2 \times 739,04) \times 981} \Rightarrow \quad \underline{{ }_{s} \alpha_{2}=0,027}$

Planta 1ㄹ: ${ }_{s} \alpha_{1}=\frac{{ }_{s}(N)}{\left(m_{1}+m_{2}+m_{3}\right)\left(\frac{N s^{2}}{c m}\right) \times g\left(\frac{c m}{s^{2}}\right)}=\frac{49,5}{(3 \times 739,04) \times 981} \Rightarrow \underline{{ }_{s} \alpha_{1}=2,276 e^{-05}}$ 
Resistencia lateral aportada por la parte flexible (pórtico) cuando los disipadores empiezan a plastificar ${ }_{f} Q_{\text {máxi }}$

Planta 3a: ${ }_{f} Q_{\text {máx } 3}={ }_{f} k_{3} \times{ }_{s} \delta_{3}=55000 \mathrm{~N} / \mathrm{cm} \times 0,0001 \mathrm{~cm} \Rightarrow{ }_{f} Q_{\text {máx } 3}=5,5 \mathrm{~N}$

Planta 2a: : ${ }_{f} Q_{\text {máx } 2}={ }_{f} k_{2} \times{ }_{s} \delta_{2}=68000 \mathrm{~N} / \mathrm{cm} \times 0,1167 \mathrm{~cm} \Rightarrow{ }_{f} Q_{\text {máx } 2}=7935,6 \mathrm{~N}$

Planta 1ㄹ: ${ }_{f} Q_{m a ́ x 1}={ }_{f} k_{1} \times{ }_{s} \delta_{1}=99000 \mathrm{~N} / \mathrm{cm} \times 0,0001 \mathrm{~cm} \Rightarrow \underline{{ }_{f} Q_{\text {máx } 1}=9,9 N}$

Coeficiente cortante en la parte flexible (pórtico) cuando los disipadores empiezan a plastificar ${ }_{f} \alpha_{i}$

Planta 3a: ${ }_{f} \alpha_{3}=\frac{{ }_{f} Q_{\text {máx } 3}(N)}{m_{3}\left(\frac{N s^{2}}{c m}\right) \times g\left(\frac{c m}{s^{2}}\right)}=\frac{5,5}{739,04 \times 981} \Rightarrow \quad \underline{{ }_{f} \alpha_{3}=7,586 e^{-06}}$

Planta 2a : ${ }_{f} \alpha_{2}=\frac{{ }_{f} Q_{\max 2}(N)}{\left(m_{2}+m_{3}\right)\left(\frac{N s^{2}}{c m}\right) \times g\left(\frac{c m}{s^{2}}\right)}=\frac{7935,6}{(2 \times 739,04) \times 981} \Rightarrow \quad \underline{{ }_{f}} \alpha_{2}=5,473 e^{-03}$

Planta 1ㄹ: ${ }_{f} \alpha_{1}=\frac{{ }_{f} Q_{\text {máx } 1}(N)}{\left(m_{1}+m_{2}+m_{3}\right)\left(\frac{N s^{2}}{c m}\right) \times g\left(\frac{c m}{s^{2}}\right)}=\frac{9,9}{(3 \times 739,04) \times 981} \Rightarrow{ }_{f} \alpha_{1}=4,552 e^{-06}$

Coeficiente de fuerza cortante total de planta $\alpha_{i}$

Planta 3a: $\alpha_{3}={ }_{s} \alpha_{3}+{ }_{f} \alpha_{3}=3,793 e^{-05}+7,586 e^{-06} \Rightarrow \underline{\alpha_{3}=4,552 e^{-05}}$

Planta 2 ${ }^{\mathrm{a}}: \alpha_{2}={ }_{s} \alpha_{2}+{ }_{f} \alpha_{2}=0,027+5,473 e^{-03} \Rightarrow \quad \underline{\alpha_{2}=0,032}$

Planta 1ㅁ: $\alpha_{1}={ }_{s} \alpha_{1}+{ }_{f} \alpha_{1}=2,276 e^{-05}+4,552 e^{-06} \Rightarrow \underline{\alpha_{1}=2,731 e^{-05}}$

\section{- Acelerograma №14 (Ano Liosia) $\Delta=14 \mathrm{Km}$}

Desplazamiento de fluencia del disipador ${ }_{s} \delta_{i}$

Planta 3a: ${ }_{s} \delta_{3}=0,0001 \mathrm{~cm}$

Planta 2: ${ }_{s} \delta_{2}=0,0357 \mathrm{~cm}$

Planta 1ㄹ: ${ }_{s} \delta_{1}=0,0001 \mathrm{~cm}$

Resistencia lateral de los disipadores de una planta i dada ${ }_{s} Q_{i}$

Planta 3a: ${ }_{s} Q_{3}={ }_{s} k_{3} \times{ }_{s} \delta_{3}=275000 \mathrm{~N} / \mathrm{cm} \times 0,0001 \mathrm{~cm} \Rightarrow{ }_{s} Q_{3}=27,5 \mathrm{~N}$

Planta 2a: ${ }_{s} Q_{2}={ }_{s} k_{2} \times{ }_{s} \delta_{2}=340000 \mathrm{~N} / \mathrm{cm} \times 0,0357 \mathrm{~cm} \Rightarrow \overline{{ }_{s} Q_{2}=12138 \mathrm{~N}}$

Planta 1à: ${ }_{s} Q_{1}={ }_{s} k_{1} \times{ }_{s} \delta_{1}=495000 \mathrm{~N} / \mathrm{cm} \times 0,0001 \mathrm{~cm} \Rightarrow{ }_{s} Q_{1}=49,5 \mathrm{~N}$ 
Coeficiente de fuerza cortante de fluencia de los disipadores de una planta i dada ${ }_{s} \alpha_{i}$

Planta 3a: ${ }_{s} \alpha_{3}=\frac{Q_{3}(N)}{m_{3}\left(\frac{N s^{2}}{\mathrm{~cm}}\right) \times g\left(\frac{\mathrm{cm}}{\mathrm{s}^{2}}\right)}=\frac{27,5}{739,04 \times 981} \Rightarrow$

$\underline{{ }_{s} \alpha_{3}=3,793 e^{-05}}$

Planta 2: ${ }_{s} \alpha_{2}=\frac{{ }_{s}(N)}{\left(m_{2}+m_{3}\right)\left(\frac{N s^{2}}{c m}\right) \times g\left(\frac{\mathrm{cm}}{s^{2}}\right)}=\frac{12138}{(2 \times 739,04) \times 981} \Rightarrow \quad \underline{{ }_{s} \alpha_{2}=8,371 e^{-03}}$

Planta 1ㄹ: $\alpha_{s}=\frac{{ }_{s} Q_{1}(N)}{\left(m_{1}+m_{2}+m_{3}\right)\left(\frac{N s^{2}}{c m}\right) \times g\left(\frac{c m}{s^{2}}\right)}=\frac{49,5}{(3 \times 739,04) \times 981} \Rightarrow \underline{{ }_{s}} \alpha_{1}=2,276 e^{-05}$

Resistencia lateral aportada por la parte flexible (pórtico) cuando los disipadores empiezan a plastificar ${ }_{f} Q_{\text {máxi }}$

Planta 3a: : $Q_{\text {máx } 3}={ }_{f} k_{3} \times{ }_{s} \delta_{3}=55000 \mathrm{~N} / \mathrm{cm} \times 0,0001 \mathrm{~cm} \Rightarrow{ }_{f} Q_{\text {máx3 }}=5,5 \mathrm{~N}$

Planta 2a: ${ }_{f} Q_{\text {máx } 2}={ }_{f} k_{2} \times{ }_{s} \delta_{2}=68000 \mathrm{~N} / \mathrm{cm} \times 0,0357 \mathrm{~cm} \Rightarrow{ }_{f} Q_{\text {máx } 2}=2427,6 \mathrm{~N}$

Planta 1ㄹ: ${ }_{f} Q_{m a ́ x 1}={ }_{f} k_{1} \times{ }_{s} \delta_{1}=99000 \mathrm{~N} / \mathrm{cm} \times 0,0001 \mathrm{~cm} \Rightarrow{ }_{f} Q_{\text {máx } 1}=9,9 \mathrm{~N}$

Coeficiente cortante en la parte flexible (pórtico) cuando los disipadores empiezan a plastificar ${ }_{f} \alpha_{i}$

Planta 3a: ${ }_{f} \alpha_{3}=\frac{{ }_{f} Q_{\text {máx3 }}(N)}{m_{3}\left(\frac{N s^{2}}{\mathrm{~cm}}\right) \times g\left(\frac{\mathrm{cm}}{\mathrm{s}^{2}}\right)}=\frac{5,5}{739,04 \times 981} \Rightarrow \quad \underline{{ }_{f} \alpha_{3}=7,586 e^{-06}}$

Planta 2a : ${ }_{f} \alpha_{2}=\frac{{ }_{f} Q_{\text {máx } 2}(N)}{\left(m_{2}+m_{3}\right)\left(\frac{N s^{2}}{c m}\right) \times g\left(\frac{c m}{s^{2}}\right)}=\frac{2427,6}{(2 \times 739,04) \times 981} \Rightarrow \quad \underline{{ }_{f}} \alpha_{2}=1,674 e^{-03}$

Planta 1a: ${ }_{f} \alpha_{1}=\frac{{ }_{f} Q_{\text {máx } 1}(N)}{\left(m_{1}+m_{2}+m_{3}\right)\left(\frac{N s^{2}}{c m}\right) \times g\left(\frac{c m}{s^{2}}\right)}=\frac{9,9}{(3 \times 739,04) \times 981} \Rightarrow{ }_{f} \alpha_{1}=4,552 e^{-06}$

Coeficiente de fuerza cortante total de planta $\alpha_{i}$

Planta 3a: $\alpha_{3}={ }_{s} \alpha_{3}+{ }_{f} \alpha_{3}=3,793 e^{-05}+7,586 e^{-06} \Rightarrow \underline{\alpha_{3}=4,552 e^{-05}}$

Planta 2a : $\alpha_{2}={ }_{s} \alpha_{2}+{ }_{f} \alpha_{2}=8,371 e^{-03}+1,674 e^{-03} \Rightarrow \alpha_{2}=0,010$

Planta 1ㄹ: $\alpha_{1}={ }_{s} \alpha_{1}+{ }_{f} \alpha_{1}=2,276 e^{-05}+4,552 e^{-06} \Rightarrow \underline{\alpha_{1}=2,731 e^{-05}}$ 
Representación gráfica

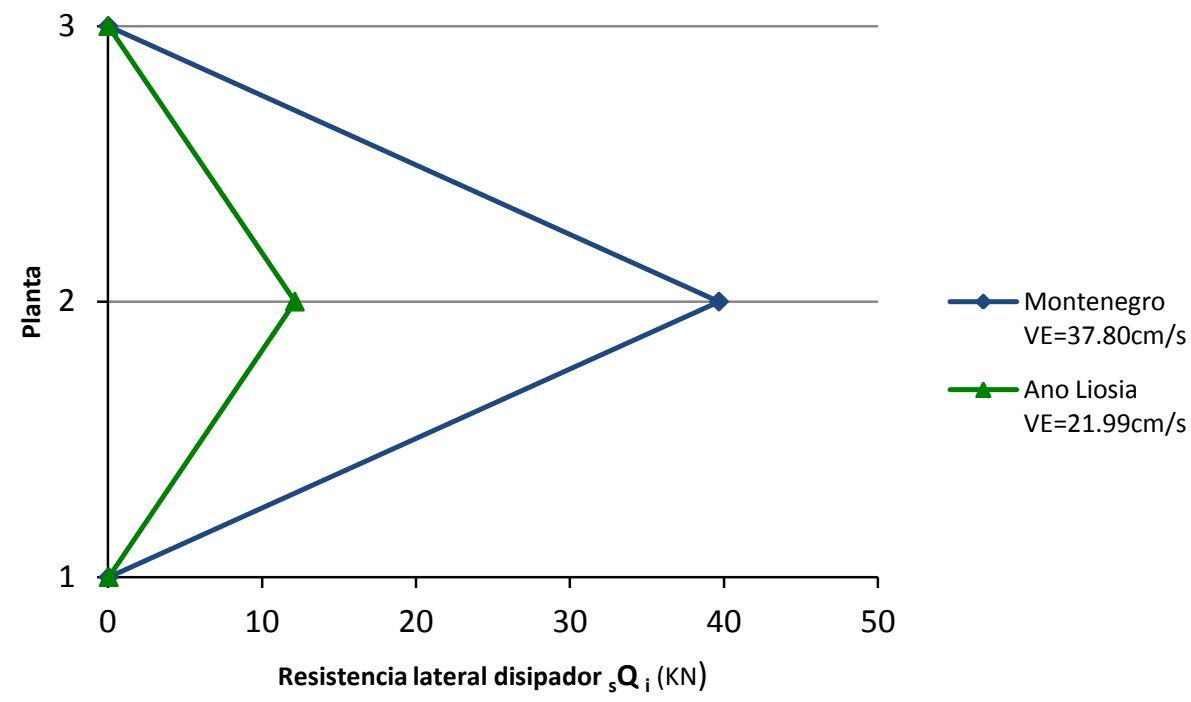

Fig. 5.5.23: Resistencia lateral de los disipadores de cada planta i, pórtico pa2pz2-1, suelo tipo III, campo lejano

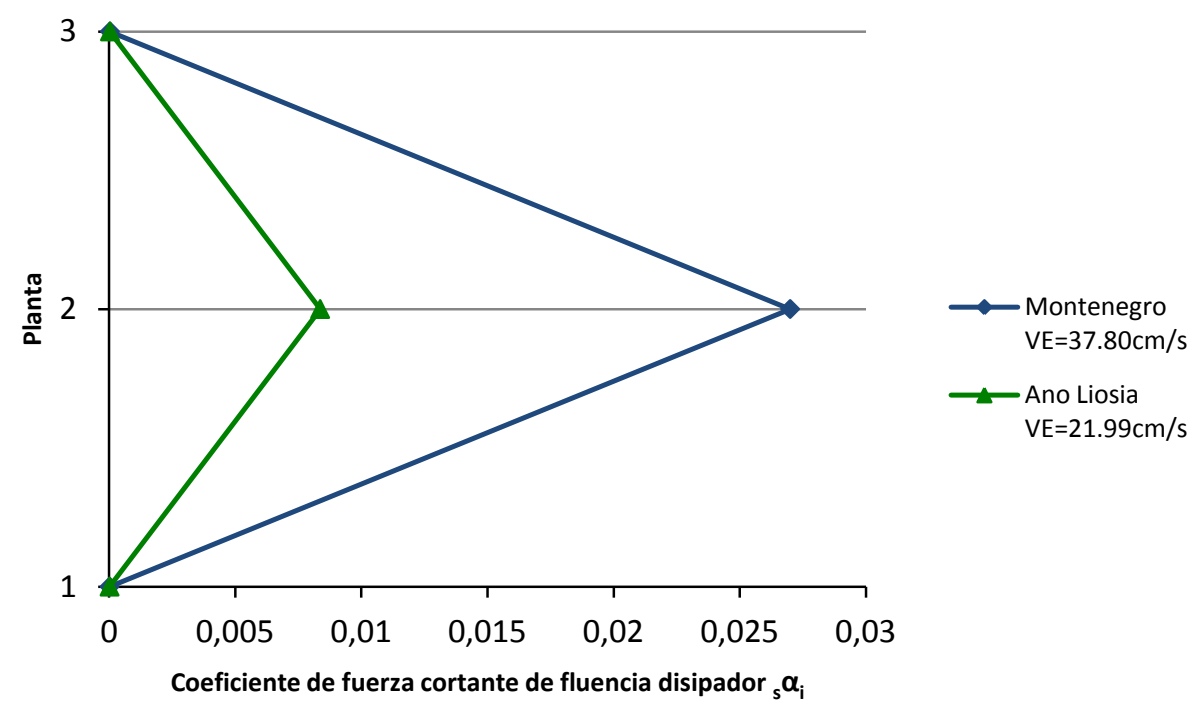

Fig. 5.5.24: Coeficiente de fuerza cortante de fluencia de los disipadores de cada planta i, pórtico pa2pz2-1, suelo tipo III, campo lejano 


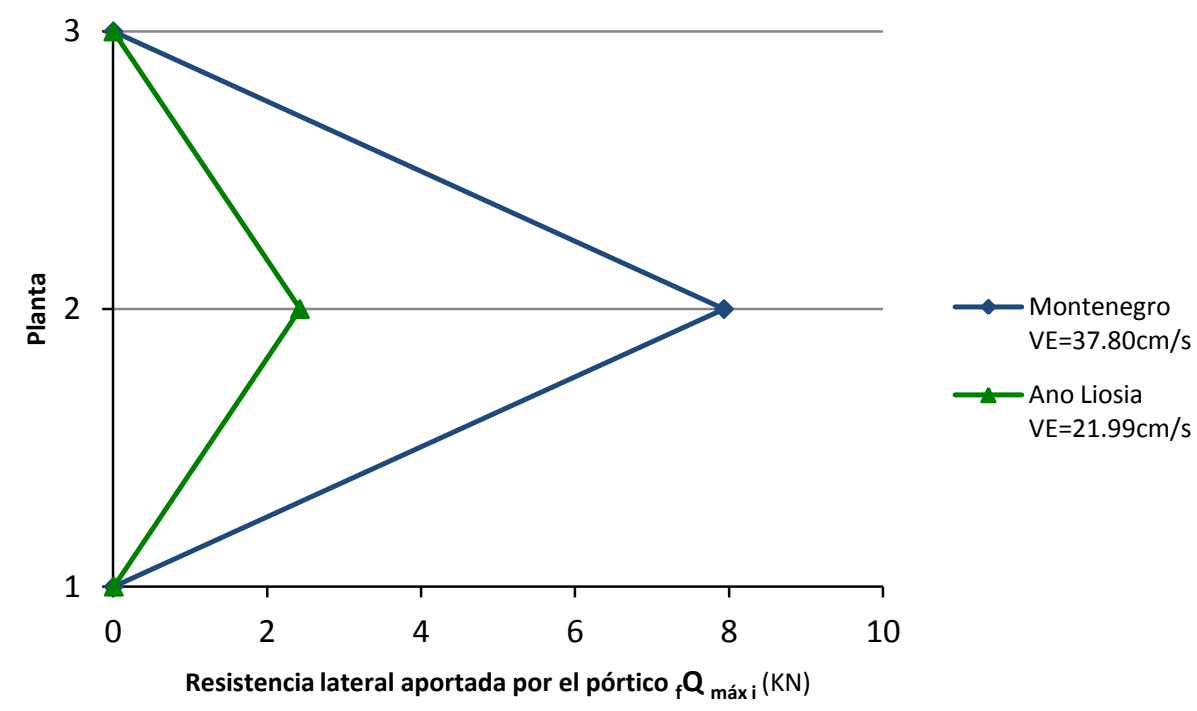

Fig. 5.5.25: Resistencia lateral aportada por la parte flexible (pórtico) cuando los disipadores empiezan a plastificar, pórtico pa2pz2-1, suelo tipo III, campo lejano

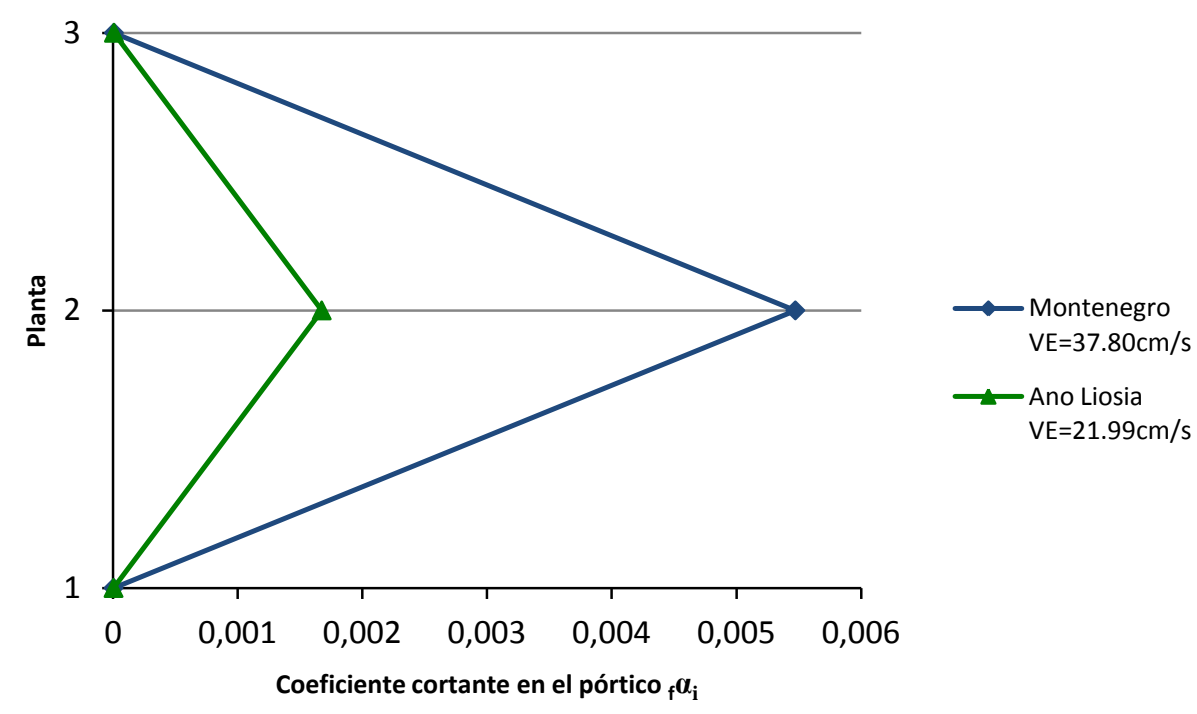

Fig. 5.5.26: Coeficiente de fuerza cortante en la parte flexible (pórtico) cuando los disipadores empiezan a plastificar, pórtico pa2pz2-1, suelo tipo III, campo lejano 


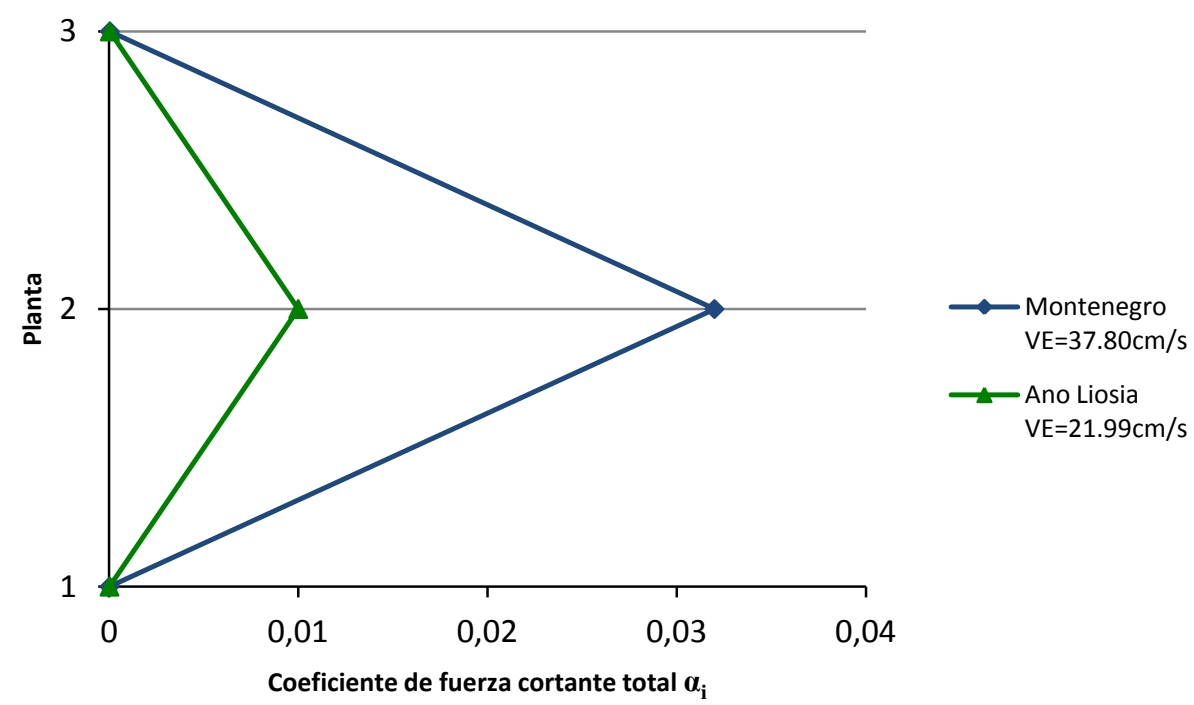

Fig. 5.9.27: Coeficiente de fuerza cortante total de planta, pórtico pa2pz2-1, suelo tipo III, campo lejano

\subsubsection{Pórtico pa4pz2-1 (3 plantas)}

Masas por planta $m_{i}$

La estructura se puede idealizar con un modelo de tres masas concentradas (situadas en el centro de masas del forjado), tal como indica la Figura 5.5.28.

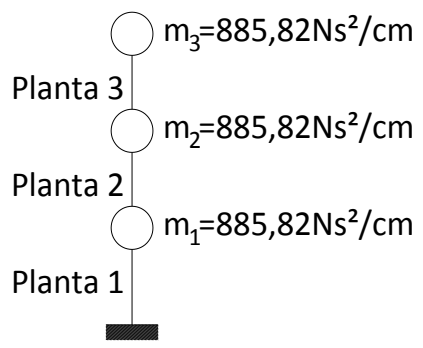

Fig. 5.5.28: Idealización de un modelo de masas del pórtico pa4pz2-1

Rigidez del disipador

Rigidez disipador $=5 \times$ Rigidez sin disipador

Planta 3a: ${ }_{s} k_{3}=(77000 \mathrm{~N} / \mathrm{cm}) \times 5=385000 \mathrm{~N} / \mathrm{cm}$

Planta 2a: ${ }_{s} k_{2}=(91000 \mathrm{~N} / \mathrm{cm}) \times 5=455000 \mathrm{~N} / \mathrm{cm}$

Planta 1ㄹ: ${ }_{s} k_{1}=(95000 \mathrm{~N} / \mathrm{cm}) \times 5=475000 \mathrm{~N} / \mathrm{cm}$

\subsection{Suelo Tipo I (Campo lejano a la falla)}

\section{- Acelerograma №1 (Friuli) $\Delta=\mathbf{2 7} \mathrm{Km}$}

Desplazamiento de fluencia del disipador ${ }_{s} \delta_{i}$

Planta 3a: ${ }_{s} \delta_{3}=0,0981 \times 1.0 \mathrm{e}-003 \mathrm{~cm}=0,0000981 \mathrm{~cm}$ 
Planta 2a: ${ }_{s} \delta_{2}=0,1000 \times 1.0 \mathrm{e}-003 \mathrm{~cm}=0,0001000 \mathrm{~cm}$

Planta 1à : $\delta_{s}=0,1305 \times 1.0 \mathrm{e}-003 \mathrm{~cm}=0,0001305 \mathrm{~cm}$

Resistencia lateral de los disipadores de una planta i dada ${ }_{s} Q_{i}$

Planta 3a: ${ }_{s} Q_{3}={ }_{s} k_{3} \times{ }_{s} \delta_{3}=385000 \mathrm{~N} / \mathrm{cm} \times 0,0000981 \mathrm{~cm} \Rightarrow{ }_{s} Q_{3}=37,77 \mathrm{~N}$

Planta 2: ${ }_{s} Q_{2}={ }_{s} k_{2} \times{ }_{s} \delta_{2}=455000 \mathrm{~N} / \mathrm{cm} \times 0,0001000 \mathrm{~cm} \Rightarrow{ }_{s} Q_{2}=45,5 \mathrm{~N}$

Planta 1ㄹ: ${ }_{s} Q_{1}={ }_{s} k_{1} \times{ }_{s} \delta_{1}=475000 \mathrm{~N} / \mathrm{cm} \times 0,0001305 \mathrm{~cm} \Rightarrow \overline{{ }_{s} Q_{1}=61,99 N}$

Coeficiente de fuerza cortante de fluencia de los disipadores de una planta i dada ${ }_{s} \alpha_{i}$

Planta 3a: ${ }_{s} \alpha_{3}=\frac{{ }_{s} Q_{3}(N)}{m_{3}\left(\frac{N s^{2}}{c m}\right) \times g\left(\frac{\mathrm{cm}}{\mathrm{s}^{2}}\right)}=\frac{37,77}{885,82 \times 981} \Rightarrow \quad \underline{{ }_{s} \alpha_{3}=4,346 e^{-05}}$

Planta 2: ${ }_{s} \alpha_{2}=\frac{{ }_{s} Q_{2}(N)}{\left(m_{2}+m_{3}\right)\left(\frac{N s^{2}}{c m}\right) \times g\left(\frac{\mathrm{cm}}{\mathrm{s}^{2}}\right)}=\frac{44,5}{(2 \times 885,82) \times 981} \Rightarrow \quad{ }_{s} \alpha_{2}=2,560 e^{-05}$

Planta 1ㄹ: ${ }_{s} \alpha_{1}=\frac{{ }_{s} Q_{1}(N)}{\left(m_{1}+m_{2}+m_{3}\right)\left(\frac{N s^{2}}{c m}\right) \times g\left(\frac{c m}{s^{2}}\right)}=\frac{61,99}{(3 \times 885,82) \times 981} \Rightarrow \underline{{ }_{s}} \alpha_{1}=2,378 e^{-05}$

Resistencia lateral aportada por la parte flexible (pórtico) cuando los disipadores empiezan a plastificar ${ }_{f} Q_{\text {máxi }}$

Planta 3ạ: ${ }_{f} Q_{\text {máx } 3}={ }_{f} k_{3} \times{ }_{s} \delta_{3}=77000 \mathrm{~N} / \mathrm{cm} \times 0,0000981 \mathrm{~cm} \Rightarrow{ }_{f} Q_{\text {máx } 3}=7,55 \mathrm{~N}$

Planta 2a: ${ }_{f} Q_{\max 2}={ }_{f} k_{2} \times{ }_{s} \delta_{2}=91000 \mathrm{~N} / \mathrm{cm} \times 0,0001000 \mathrm{~cm} \Rightarrow{ }_{f} Q_{\max 2}=9,10 \mathrm{~N}$

Planta 1ㄹ: ${ }_{f} Q_{\text {máx } 1}={ }_{f} k_{1} \times{ }_{s} \delta_{1}=95000 \mathrm{~N} / \mathrm{cm} \times 0,0001305 \mathrm{~cm} \Rightarrow{ }_{f} Q_{\text {máx } 1}=12,40 \mathrm{~N}$

Coeficiente cortante en la parte flexible (pórtico) cuando los disipadores empiezan a plastificar ${ }_{f} \alpha_{i}$

Planta 3ㄹ: ${ }_{f} \alpha_{3}=\frac{{ }_{f} Q_{\operatorname{máx3}}(N)}{m_{3}\left(\frac{N s^{2}}{c m}\right) \times g\left(\frac{c m}{s^{2}}\right)}=\frac{7,55}{885,82 \times 981} \Rightarrow \quad \underline{{ }_{f} \alpha_{3}=8,688 e^{-06}}$

Planta 2a: ${ }_{f} \alpha_{2}=\frac{{ }_{f} Q_{\text {máx }}(N)}{\left(m_{2}+m_{3}\right)\left(\frac{N s^{2}}{c m}\right) \times g\left(\frac{c m}{s^{2}}\right)}=\frac{9,1}{(2 \times 885,82) \times 981} \Rightarrow \quad \underline{{ }_{f} \alpha_{2}=5,236 e^{-06}}$

Planta 1ㄹ: ${ }_{f} \alpha_{1}=\frac{{ }_{f} Q_{\text {máx } 1}(N)}{\left(m_{1}+m_{2}+m_{3}\right)\left(\frac{N s^{2}}{c m}\right) \times g\left(\frac{c m}{s^{2}}\right)}=\frac{12,40}{(3 \times 885,82) \times 981} \Rightarrow \underline{{ }_{f}} \alpha_{1}=4,756 e^{-06}$ 
Coeficiente de fuerza cortante total de planta $\alpha_{i}$

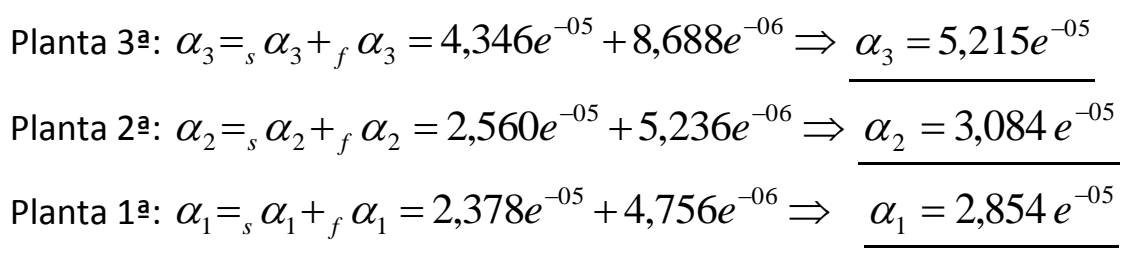

\section{- Acelerograma №4 (Montenegro) $\Delta=65 \mathrm{Km}$}

Desplazamiento de fluencia del disipador ${ }_{s} \delta_{i}$

Planta 3ㄹ: ${ }_{s} \delta_{3}=0,6504 \mathrm{~cm}$

Planta 2ㄹ: ${ }_{s} \delta_{2}=1,2500 \mathrm{~cm}$

Planta 1a: ${ }_{s} \delta_{1}=1,0625 \mathrm{~cm}$

Resistencia lateral de los disipadores de una planta i dada ${ }_{s} Q_{i}$

Planta 3a: ${ }_{s} Q_{3}={ }_{s} k_{3} \times{ }_{s} \delta_{3}=385000 \mathrm{~N} / \mathrm{cm} \times 0,6504 \mathrm{~cm} \Rightarrow{ }_{s} Q_{3}=250404 \mathrm{~N}$

Planta 2a: ${ }_{s} Q_{2}={ }_{s} k_{2} \times \delta_{2}=455000 \mathrm{~N} / \mathrm{cm} \times 1,2500 \mathrm{~cm} \Rightarrow{ }_{s} Q_{2}=568750 \mathrm{~N}$

Planta 1ㅁ: ${ }_{s} Q_{1}={ }_{s} k_{1} \times{ }_{s} \delta_{1}=475000 \mathrm{~N} / \mathrm{cm} \times 1,0625 \mathrm{~cm} \Rightarrow{ }_{s} Q_{1}=504687,5 \mathrm{~N}$

Coeficiente de fuerza cortante de fluencia de los disipadores de una planta i dada ${ }_{s} \alpha_{i}$

Planta 3a: ${ }_{s} \alpha_{3}=\frac{{ }_{s} Q_{3}(N)}{m_{3}\left(\frac{N s^{2}}{c m}\right) \times g\left(\frac{c m}{s^{2}}\right)}=\frac{250404}{885,82 \times 981} \Rightarrow \quad \underline{{ }_{s} \alpha_{3}=0,288}$

Planta 2 ${ }^{\mathrm{a}}:{ }_{s} \alpha_{2}=\frac{{ }_{s}(N)}{\left(m_{2}+m_{3}\right)\left(\frac{N s^{2}}{\mathrm{~cm}}\right) \times g\left(\frac{\mathrm{cm}}{\mathrm{s}^{2}}\right)}=\frac{568750}{(2 \times 885,82) \times 981} \Rightarrow \quad \underline{{ }_{s}} \alpha_{2}=0,327$

Planta 1ㄹ: ${ }_{s} \alpha_{1}=\frac{{ }_{s} Q_{1}(N)}{\left(m_{1}+m_{2}+m_{3}\right)\left(\frac{N s^{2}}{c m}\right) \times g\left(\frac{c m}{s^{2}}\right)}=\frac{504687,5}{(3 \times 885,82) \times 981} \Rightarrow \underline{{ }_{s}} \alpha_{1}=0,193$

Resistencia lateral aportada por la parte flexible (pórtico) cuando los disipadores empiezan a plastificar ${ }_{f} Q_{\text {máxi }}$

Planta 3a: ${ }_{f} Q_{\max 3}={ }_{f} k_{3} \times{ }_{s} \delta_{3}=77000 \mathrm{~N} / \mathrm{cm} \times 0,6504 \mathrm{~cm} \Rightarrow{ }_{f} Q_{\max x 3}=50080,8 \mathrm{~N}$

Planta 2a: ${ }_{f} Q_{\text {máx } 2}={ }_{f} k_{2} \times{ }_{s} \delta_{2}=91000 \mathrm{~N} / \mathrm{cm} \times 1,2500 \mathrm{~cm} \Rightarrow{ }_{f} Q_{\operatorname{máx} 2}=113750 \mathrm{~N}$

Planta 1ạ: ${ }_{f} Q_{\text {máx1 }}={ }_{f} k_{1} \times{ }_{s} \delta_{1}=95000 \mathrm{~N} / \mathrm{cm} \times 1,0625 \mathrm{~cm} \Rightarrow \overline{{ }_{f} Q_{\text {máx1 }}=100937,5 \mathrm{~N}}$ 
Coeficiente cortante en la parte flexible (pórtico) cuando los disipadores empiezan a plastificar ${ }_{f} \alpha_{i}$

Planta 3a: ${ }_{f} \alpha_{3}=\frac{{ }_{f} Q_{\text {máx3 }}(N)}{m_{3}\left(\frac{N s^{2}}{c m}\right) \times g\left(\frac{c m}{s^{2}}\right)}=\frac{50080,8}{885,82 \times 981} \Rightarrow \quad \underline{{ }_{f} \alpha_{3}=0,058}$

Planta 2ä: ${ }_{f} \alpha_{2}=\frac{{ }_{f} Q_{\text {máx } 2}(N)}{\left(m_{2}+m_{3}\right)\left(\frac{N s^{2}}{c m}\right) \times g\left(\frac{c m}{s^{2}}\right)}=\frac{113750}{(2 \times 885,82) \times 981} \Rightarrow \quad \underline{{ }_{f}} \alpha_{2}=0,065$

Planta 1ㄹ: ${ }_{f} \alpha_{1}=\frac{{ }_{f} Q_{m a ́ x 1}(N)}{\left(m_{1}+m_{2}+m_{3}\right)\left(\frac{N s^{2}}{c m}\right) \times g\left(\frac{c m}{s^{2}}\right)}=\frac{100937,5}{(3 \times 885,82) \times 981} \Rightarrow \underline{{ }_{f}} \alpha_{1}=0,039$

Coeficiente de fuerza cortante total de planta $\alpha_{i}$

Planta 3: $\alpha_{3}={ }_{s} \alpha_{3}+{ }_{f} \alpha_{3}=0,288+0,058 \Rightarrow \underline{\alpha_{3}=0,346}$

Planta 2a: $\alpha_{2}={ }_{s} \alpha_{2}+{ }_{f} \alpha_{2}=0,327+0,065 \Rightarrow \underline{\alpha_{2}=0,392}$

Planta 1: $\alpha_{1}={ }_{s} \alpha_{1}+{ }_{f} \alpha_{1}=0,193+0,039 \Rightarrow \underline{\alpha_{1}=0,232}$

\section{- Acelerograma №5 (Campano Lucano) $\Delta=32 \mathrm{Km}$}

Desplazamiento de fluencia del disipador ${ }_{s} \delta_{i}$

Planta 3a: ${ }_{s} \delta_{3}=0,0001000 \mathrm{~cm}$

Planta 2a: ${ }_{s} \delta_{2}=0,0001019 \mathrm{~cm}$

Planta 1ㄹ: ${ }_{s} \delta_{1}=0,0008267 \mathrm{~cm}$

Resistencia lateral de los disipadores de una planta i dada ${ }_{s} Q_{i}$

Planta 3a: ${ }_{s} Q_{3}={ }_{s} k_{3} \times{ }_{s} \delta_{3}=385000 \mathrm{~N} / \mathrm{cm} \times 0,0001000 \mathrm{~cm} \Rightarrow{ }_{s} Q_{3}=38,50 \mathrm{~N}$

Planta 2 ${ }^{\mathrm{a}}:{ }_{s} Q_{2}={ }_{s} k_{2} \times{ }_{s} \delta_{2}=455000 \mathrm{~N} / \mathrm{cm} \times 0,0001019 \mathrm{~cm} \Rightarrow{ }_{s} Q_{2}=46,36 \mathrm{~N}$

Planta 1a: ${ }_{s} Q_{1}={ }_{s} k_{1} \times{ }_{s} \delta_{1}=475000 \mathrm{~N} / \mathrm{cm} \times 0,0008267 \mathrm{~cm} \Rightarrow \overline{{ }_{s} Q_{1}=395,68 \mathrm{~N}}$

Coeficiente de fuerza cortante de fluencia de los disipadores de una planta i dada ${ }_{s} \alpha_{i}$

Planta 3a: ${ }_{s} \alpha_{3}=\frac{{ }_{s} Q_{3}(N)}{m_{3}\left(\frac{N s^{2}}{c m}\right) \times g\left(\frac{\mathrm{cm}}{s^{2}}\right)}=\frac{38,50}{885,82 \times 981} \Rightarrow \quad \underline{{ }_{s} \alpha_{3}=4,430 e^{-05}}$

Planta 2: ${ }_{s} \alpha_{2}=\frac{{ }_{s}(N)}{\left(m_{2}+m_{3}\right)\left(\frac{N s^{2}}{c m}\right) \times g\left(\frac{c m}{s^{2}}\right)}=\frac{46,36}{(2 \times 885,82) \times 981} \Rightarrow \quad{ }_{s} \alpha_{2}=2,215 e^{-05}$ 
Planta 1:a: ${ }_{s} \alpha_{1}=\frac{{ }_{s} Q_{1}(N)}{\left(m_{1}+m_{2}+m_{3}\right)\left(\frac{N s^{2}}{c m}\right) \times g\left(\frac{c m}{s^{2}}\right)}=\frac{395,68}{(3 \times 885,82) \times 981} \Rightarrow{ }_{s} \alpha_{1}=1,518 e^{-04}$

Resistencia lateral aportada por la parte flexible (pórtico) cuando los disipadores empiezan a plastificar ${ }_{f} Q_{\text {máxi }}$

Planta 3a: : $Q_{f} Q_{\text {máx } 3}={ }_{f} k_{3} \times{ }_{s} \delta_{3}=77000 \mathrm{~N} / \mathrm{cm} \times 0,0001000 \mathrm{~cm} \Rightarrow{ }_{f} Q_{\text {máx }}=7,7 \mathrm{~N}$

Planta 2a: ${ }_{f} Q_{\operatorname{maxx} 2}={ }_{f} k_{2} \times{ }_{s} \delta_{2}=91000 \mathrm{~N} / \mathrm{cm} \times 0,0001019 \mathrm{~cm} \Rightarrow \overline{{ }_{f} Q_{\text {máx } 2}=9,92 \mathrm{~N}}$

Planta 1à: ${ }_{f} Q_{\text {máx1 }}={ }_{f} k_{1} \times{ }_{s} \delta_{1}=95000 \mathrm{~N} / \mathrm{cm} \times 0,0008267 \mathrm{~cm} \Rightarrow \overline{{ }_{f} Q_{\text {máx } 1}=78,54 \mathrm{~N}}$

Coeficiente cortante en la parte flexible (pórtico) cuando los disipadores empiezan a plastificar ${ }_{f} \alpha_{i}$

Planta 3a: ${ }_{f} \alpha_{3}=\frac{{ }_{f} Q_{\operatorname{máx3}}(N)}{m_{3}\left(\frac{N s^{2}}{\mathrm{~cm}}\right) \times g\left(\frac{\mathrm{cm}}{\mathrm{s}^{2}}\right)}=\frac{7,7}{885,82 \times 981} \Rightarrow \quad \underline{{ }_{f} \alpha_{3}=8,861 e^{-06}}$

Planta 2a: ${ }_{f} \alpha_{2}=\frac{{ }_{f} Q_{\text {máx } 2}(N)}{\left(m_{2}+m_{3}\right)\left(\frac{N s^{2}}{c m}\right) \times g\left(\frac{c m}{s^{2}}\right)}=\frac{9,92}{(2 \times 885,82) \times 981} \Rightarrow \quad \underline{{ }_{f}} \alpha_{2}=5,708 e^{-06}$

Planta 1a: ${ }_{f} \alpha_{1}=\frac{{ }_{f} Q_{\text {máx } 1}(N)}{\left(m_{1}+m_{2}+m_{3}\right)\left(\frac{N s^{2}}{c m}\right) \times g\left(\frac{c m}{s^{2}}\right)}=\frac{78,54}{(3 \times 885,82) \times 981} \Rightarrow \underline{{ }_{f}} \alpha_{1}=3,013 e^{-05}$

Coeficiente de fuerza cortante total de planta $\alpha_{i}$

Planta 3a: $\alpha_{3}={ }_{s} \alpha_{3}+{ }_{f} \alpha_{3}=4,430 e^{-05}+8,861 e^{-06} \Rightarrow \underline{\alpha_{3}=5,316 e^{-05}}$
Planta 2a: $\alpha_{2}={ }_{s} \alpha_{2}{ }_{f} \alpha_{2}=2,215 e^{-05}+5,708 e^{-06} \Rightarrow \underline{\alpha_{2}=2,786 e^{-05}}$
Planta 1ㄹ: $\alpha_{1}={ }_{s} \alpha_{1}+{ }_{f} \alpha_{1}=1,518 e^{-04}+3,013 e^{-05} \Rightarrow \underline{\alpha_{1}=1,819 e^{-04}}$ 
Representación gráfica

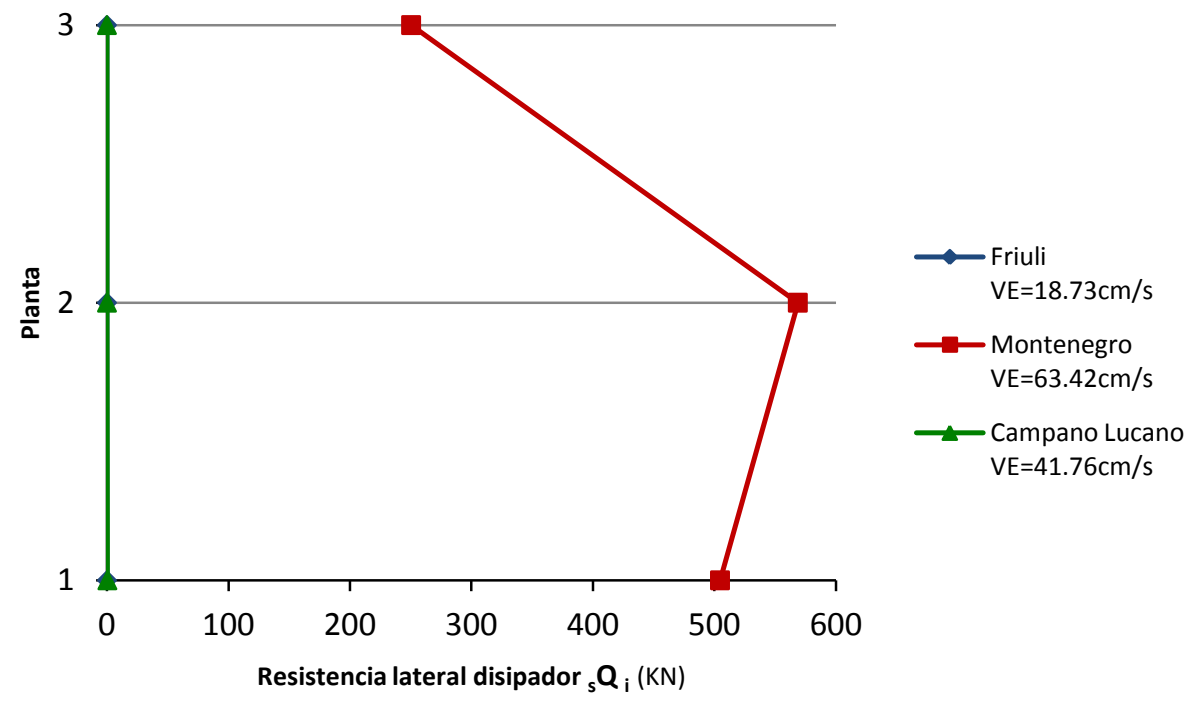

Fig. 5.5.29: Resistencia lateral de los disipadores de cada planta i, pórtico pa4pz2-1, suelo tipo l, campo lejano

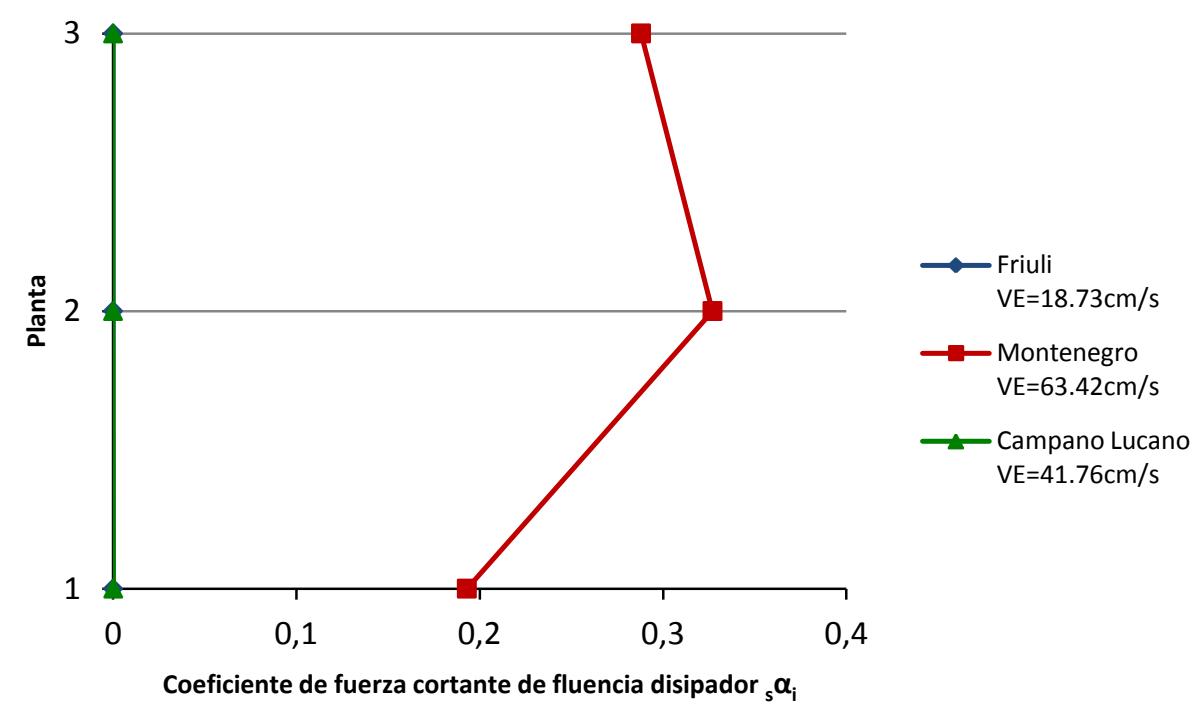

Fig. 5.5.30: Coeficiente de fuerza cortante de fluencia de los disipadores de cada planta i, pórtico pa4pz2-1, suelo tipo I, campo lejano 


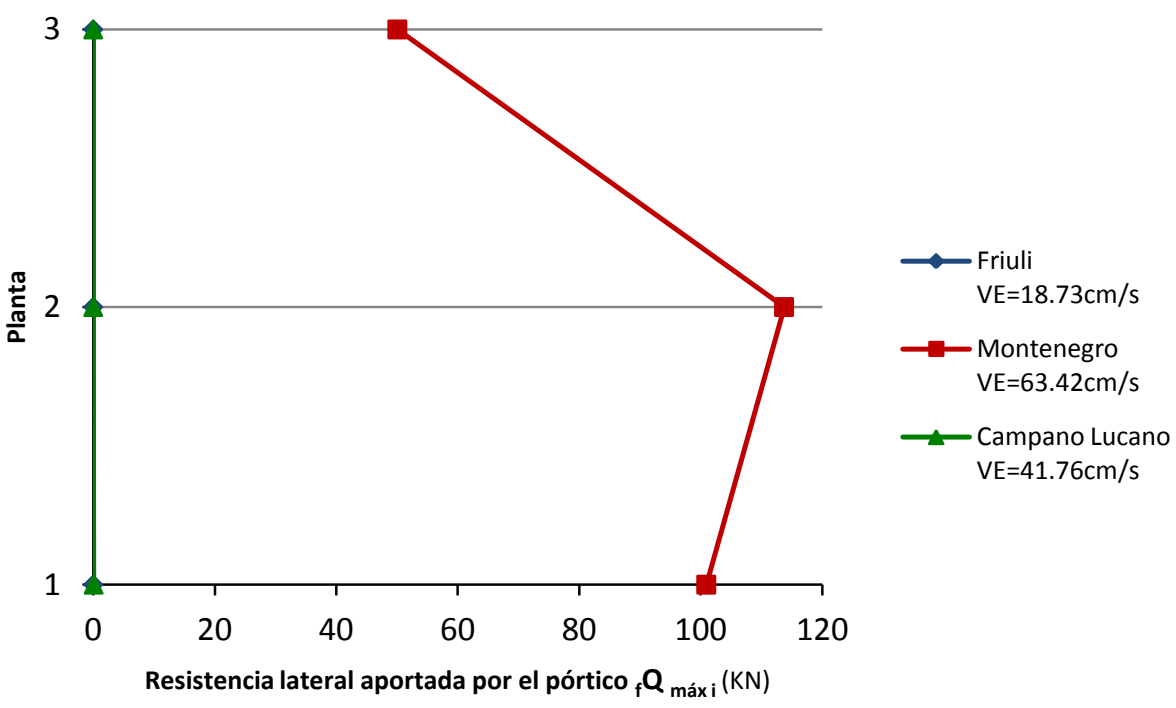

Fig. 5.5.31: Resistencia lateral aportada por la parte flexible (pórtico) cuando los disipadores empiezan a plastificar, pórtico pa4pz2-1, suelo tipo I, campo lejano

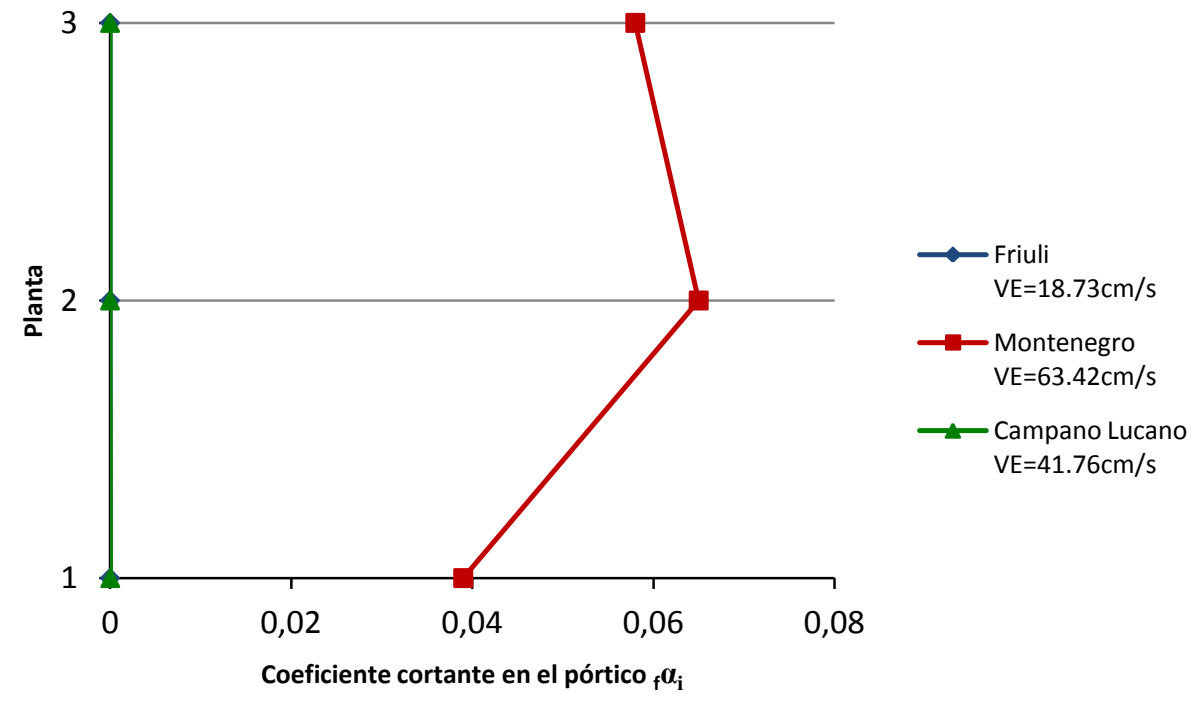

Fig. 5.5.32: Coeficiente de fuerza cortante en la parte flexible (pórtico) cuando los disipadores empiezan a plastificar, pórtico pa4pz2-1, suelo tipo I, campo lejano 


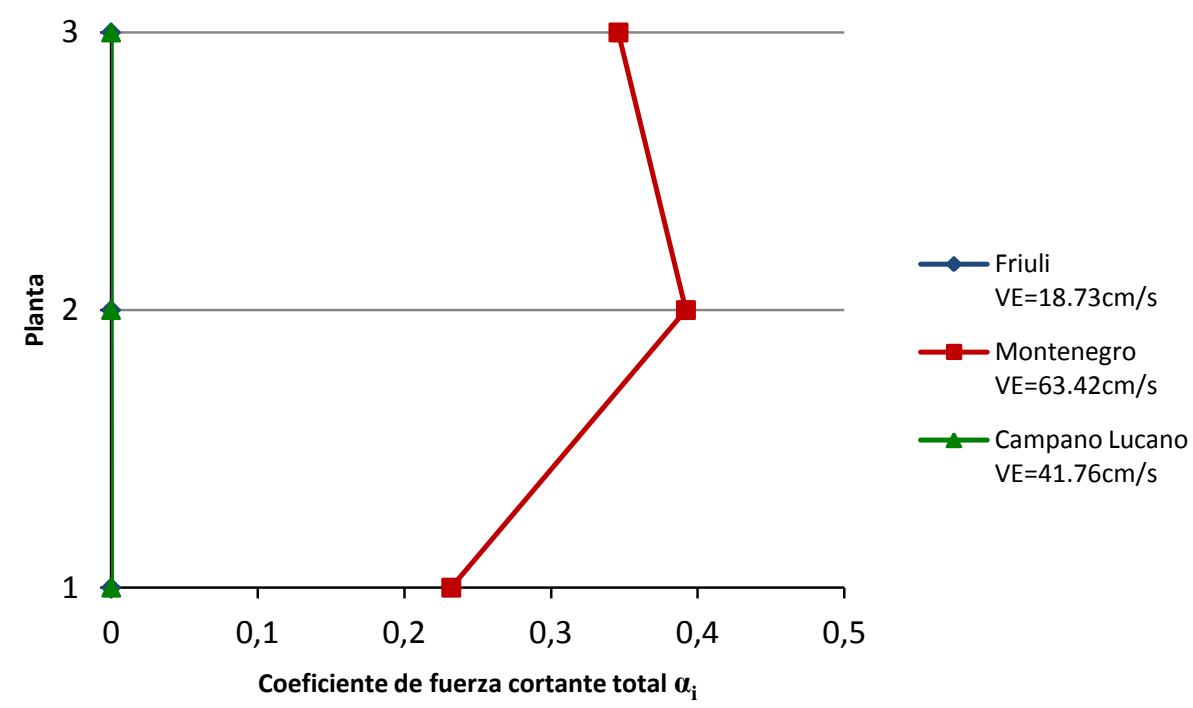

Fig. 5.5.33: Coeficiente de fuerza cortante total de planta, pórtico pa4pz2-1, suelo tipo I, campo lejano

\subsection{Suelo Tipo II (Campo cercano a la falla)}

\section{- Acelerograma №1 (Friuli aftershock) $\Delta=3 \mathrm{Km}$}

Desplazamiento de fluencia del disipador ${ }_{s} \delta_{i}$

Planta 3a: ${ }_{s} \delta_{3}=0,0011 \mathrm{~cm}$

Planta 2a: ${ }_{s} \delta_{2}=0,0060 \mathrm{~cm}$

Planta 1: ${ }_{s} \delta_{1}=0,0011 \mathrm{~cm}$

Resistencia lateral de los disipadores de una planta i dada ${ }_{s} Q_{i}$

Planta 3a: ${ }_{s} Q_{3}={ }_{s} k_{3} \times{ }_{s} \delta_{3}=385000 \mathrm{~N} / \mathrm{cm} \times 0,0011 \mathrm{~cm} \Rightarrow{ }_{s} Q_{3}=423,5 \mathrm{~N}$

Planta 2: ${ }_{s} Q_{2}={ }_{s} k_{2} \times{ }_{s} \delta_{2}=455000 \mathrm{~N} / \mathrm{cm} \times 0,0060 \mathrm{~cm} \Rightarrow{ }_{s} Q_{2}=2730 \mathrm{~N}$

Planta 1a: ${ }_{s} Q_{1}={ }_{s} k_{1} \times{ }_{s} \delta_{1}=475000 \mathrm{~N} / \mathrm{cm} \times 0,0011 \mathrm{~cm} \Rightarrow \overline{{ }_{s} Q_{1}=522,5 \mathrm{~N}}$

Coeficiente de fuerza cortante de fluencia de los disipadores de una planta i dada ${ }_{s} \alpha_{i}$

Planta 3a: ${ }_{s} \alpha_{3}=\frac{{ }_{s} Q_{3}(N)}{m_{3}\left(\frac{N s^{2}}{c m}\right) \times g\left(\frac{c m}{s^{2}}\right)}=\frac{423,5}{885,82 \times 981} \Rightarrow \quad \underline{{ }_{s} \alpha_{3}=4,873 e^{-04}}$

Planta 2 ${ }^{\mathrm{a}}:{ }_{s} \alpha_{2}=\frac{{ }_{s} Q_{2}(N)}{\left(m_{2}+m_{3}\right)\left(\frac{N s^{2}}{c m}\right) \times g\left(\frac{c m}{s^{2}}\right)}=\frac{2730}{(2 \times 885,82) \times 981} \Rightarrow \quad \underline{{ }_{s} \alpha_{2}=1,571 e^{-03}}$

Planta 1ㄹ: $\alpha_{s}=\frac{{ }_{s} Q_{1}(N)}{\left(m_{1}+m_{2}+m_{3}\right)\left(\frac{N s^{2}}{c m}\right) \times g\left(\frac{c m}{s^{2}}\right)}=\frac{522,5}{(3 \times 885,82) \times 981} \Rightarrow \underline{{ }_{s}} \alpha_{1}=2,004 e^{-04}$ 
Resistencia lateral aportada por la parte flexible (pórtico) cuando los disipadores empiezan a plastificar ${ }_{f} Q_{\text {máxi }}$

Planta 3a: ${ }_{f} Q_{\text {máx } 3}={ }_{f} k_{3} \times{ }_{s} \delta_{3}=77000 \mathrm{~N} / \mathrm{cm} \times 0,0011 \mathrm{~cm} \Rightarrow{ }_{f} Q_{\text {máx } 3}=84,7 \mathrm{~N}$

Planta 2ㄹ: ${ }_{f} Q_{\text {máx } 2}={ }_{f} k_{2} \times{ }_{s} \delta_{2}=91000 \mathrm{~N} / \mathrm{cm} \times 0,0060 \mathrm{~cm} \Rightarrow{ }_{f} Q_{\text {máx } 2}=546 \mathrm{~N}$

Planta 1ạ: ${ }_{f} Q_{\text {máx1 }}={ }_{f} k_{1} \times{ }_{s} \delta_{1}=95000 \mathrm{~N} / \mathrm{cm} \times 0,0011 \mathrm{~cm} \Rightarrow \overline{{ }_{f} Q_{\text {máx } 1}=104,5 N}$

Coeficiente cortante en la parte flexible (pórtico) cuando los disipadores empiezan a plastificar ${ }_{f} \alpha_{i}$

Planta 3a: ${ }_{f} \alpha_{3}=\frac{{ }_{f} Q_{\text {máxз }}(N)}{m_{3}\left(\frac{N s^{2}}{c m}\right) \times g\left(\frac{c m}{s^{2}}\right)}=\frac{84,7}{885,82 \times 981} \Rightarrow \quad \underline{{ }_{f} \alpha_{3}=9,747 e^{-05}}$

Planta 2ㄹ: ${ }_{f} \alpha_{2}=\frac{{ }_{f} Q_{\operatorname{máx} 2}(N)}{\left(m_{2}+m_{3}\right)\left(\frac{N s^{2}}{c m}\right) \times g\left(\frac{c m}{s^{2}}\right)}=\frac{546}{(2 \times 885,82) \times 981} \Rightarrow \quad{ }_{f} \alpha_{2}=3,141 e^{-04}$

Planta 1ㄹ: ${ }_{f} \alpha_{1}=\frac{{ }_{f} Q_{\text {máx }}(N)}{\left(m_{1}+m_{2}+m_{3}\right)\left(\frac{N s^{2}}{c m}\right) \times g\left(\frac{c m}{s^{2}}\right)}=\frac{104,5}{(3 \times 885,82) \times 981} \Rightarrow{ }_{f} \alpha_{1}=4,008 e^{-05}$

Coeficiente de fuerza cortante total de planta $\alpha_{i}$

Planta 3a: $\alpha_{3}={ }_{s} \alpha_{3}+{ }_{f} \alpha_{3}=4,873 e^{-04}+9,747 e^{-05} \Rightarrow \alpha_{3}=5,848 e^{-04}$

Planta 2a: $\alpha_{2}={ }_{s} \alpha_{2}+{ }_{f} \alpha_{2}=1,571 e^{-03}+3,141 e^{-04} \Rightarrow \underline{\alpha_{2}=1,885 e^{-03}}$

Planta 1a: $\alpha_{1}={ }_{s} \alpha_{1}+{ }_{f} \alpha_{1}=2,004 e^{-04}+4,008 e^{-05} \Rightarrow \underline{\alpha_{1}=2,405 e^{-04}}$

\section{- Acelerograma №14 (Montenegro aftershock) $\Delta=8 \mathrm{Km}$}

Desplazamiento de fluencia del disipador ${ }_{s} \delta_{i}$

Planta 3a: ${ }_{s} \delta_{3}=0,0001 \mathrm{~cm}$

Planta 2ㄹ: ${ }_{s} \delta_{2}=0,1502 \mathrm{~cm}$

Planta 1ㄹ: $\delta_{1}=0,0001 \mathrm{~cm}$

Resistencia lateral de los disipadores de una planta i dada ${ }_{s} Q_{i}$

Planta 3a: ${ }_{s} Q_{3}={ }_{s} k_{3} \times{ }_{s} \delta_{3}=385000 \mathrm{~N} / \mathrm{cm} \times 0,0001 \mathrm{~cm} \Rightarrow{ }_{s} Q_{3}=38,5 \mathrm{~N}$

Planta 2ㄹ: ${ }_{s} Q_{2}={ }_{s} k_{2} \times{ }_{s} \delta_{2}=455000 \mathrm{~N} / \mathrm{cm} \times 0,1502 \mathrm{~cm} \Rightarrow \overline{Q_{2}=68341 \mathrm{~N}}$

Planta 1a: ${ }_{s} Q_{1}={ }_{s} k_{1} \times{ }_{s} \delta_{1}=475000 \mathrm{~N} / \mathrm{cm} \times 0,0001 \mathrm{~cm} \Rightarrow{ }_{s} Q_{1}=47,5 \mathrm{~N}$ 
Coeficiente de fuerza cortante de fluencia de los disipadores de una planta i dada ${ }_{s} \alpha_{i}$

Planta 3a: ${ }_{s} \alpha_{3}=\frac{Q_{3}(N)}{m_{3}\left(\frac{N s^{2}}{\mathrm{~cm}}\right) \times g\left(\frac{\mathrm{cm}}{\mathrm{s}^{2}}\right)}=\frac{38,5}{885,82 \times 981} \Rightarrow$

${ }_{s} \alpha_{3}=4,430 e^{-05}$

Planta 2 ${ }_{s} \alpha_{2}=\frac{{ }_{s}(N)}{\left(m_{2}+m_{3}\right)\left(\frac{N s^{2}}{c m}\right) \times g\left(\frac{c m}{s^{2}}\right)}=\frac{68341}{(2 \times 885,82) \times 981} \Rightarrow \quad \underline{{ }_{s} \alpha_{2}=0,039}$

Planta 1: ${ }_{s} \alpha_{1}=\frac{{ }_{s} Q_{1}(N)}{\left(m_{1}+m_{2}+m_{3}\right)\left(\frac{N s^{2}}{c m}\right) \times g\left(\frac{\mathrm{cm}}{s^{2}}\right)}=\frac{47,5}{(3 \times 885,82) \times 981} \Rightarrow{ }_{s} \alpha_{1}=1,822 e^{-05}$

Resistencia lateral aportada por la parte flexible (pórtico) cuando los disipadores empiezan a plastificar ${ }_{f} Q_{\text {máxi }}$

Planta 3a: ${ }_{f} Q_{\text {máx } 3}={ }_{f} k_{3} \times{ }_{s} \delta_{3}=77000 \mathrm{~N} / \mathrm{cm} \times 0,0001 \mathrm{~cm} \Rightarrow{ }_{f} Q_{\text {máx3 }}=7,7 \mathrm{~N}$

Planta 2a: ${ }_{f} Q_{\max 2}={ }_{f} k_{2} \times{ }_{s} \delta_{2}=91000 \mathrm{~N} / \mathrm{cm} \times 0,1502 \mathrm{~cm} \Rightarrow \overline{{ }_{f} Q_{\max 2}=13668}, 2 \mathrm{~N}$

Planta 1a: ${ }_{f} Q_{\text {máx } 1}={ }_{f} k_{1} \times{ }_{s} \delta_{1}=95000 \mathrm{~N} / \mathrm{cm} \times 0,0001 \mathrm{~cm} \Rightarrow{ }_{f} Q_{\text {máx } 1}=9,5 \mathrm{~N}$

Coeficiente cortante en la parte flexible (pórtico) cuando los disipadores empiezan a plastificar ${ }_{f} \alpha_{i}$

Planta 3a: ${ }_{f} \alpha_{3}=\frac{{ }_{f} Q_{\text {máx3 }}(N)}{m_{3}\left(\frac{N s^{2}}{\mathrm{~cm}}\right) \times g\left(\frac{\mathrm{cm}}{\mathrm{s}^{2}}\right)}=\frac{7,7}{885,82 \times 981} \Rightarrow \quad \underline{{ }_{f} \alpha_{3}=8,861 e^{-06}}$

Planta 2a: ${ }_{f} \alpha_{2}=\frac{{ }_{f} Q_{\operatorname{máx} 2}(N)}{\left(m_{2}+m_{3}\right)\left(\frac{N s^{2}}{c m}\right) \times g\left(\frac{c m}{s^{2}}\right)}=\frac{13668,2}{(2 \times 885,82) \times 981} \Rightarrow \quad \underline{{ }_{f}} \alpha_{2}=7,664 e^{-03}$

Planta 1ㄹ: ${ }_{f} \alpha_{1}=\frac{{ }_{f} Q_{\text {máx } 1}(N)}{\left(m_{1}+m_{2}+m_{3}\right)\left(\frac{N s^{2}}{c m}\right) \times g\left(\frac{c m}{s^{2}}\right)}=\frac{9,5}{(3 \times 885,82) \times 981} \Rightarrow \underline{{ }_{f}} \alpha_{1}=3,644 e^{-06}$

Coeficiente de fuerza cortante total de planta $\alpha_{i}$

Planta 3a: $\alpha_{3}={ }_{s} \alpha_{3}+{ }_{f} \alpha_{3}=4,430 e^{-05}+8,861 e^{-06} \Rightarrow \frac{\alpha_{3}=5,316 e^{-05}}{\alpha_{2}=0,047}$
Planta 2a: $\alpha_{2}={ }_{s} \alpha_{2}+{ }_{f} \alpha_{2}=0,039+7,664 e^{-03} \Rightarrow \frac{\alpha_{1}=2,186 e^{-05}}{\alpha_{1}}$ 
Representación gráfica

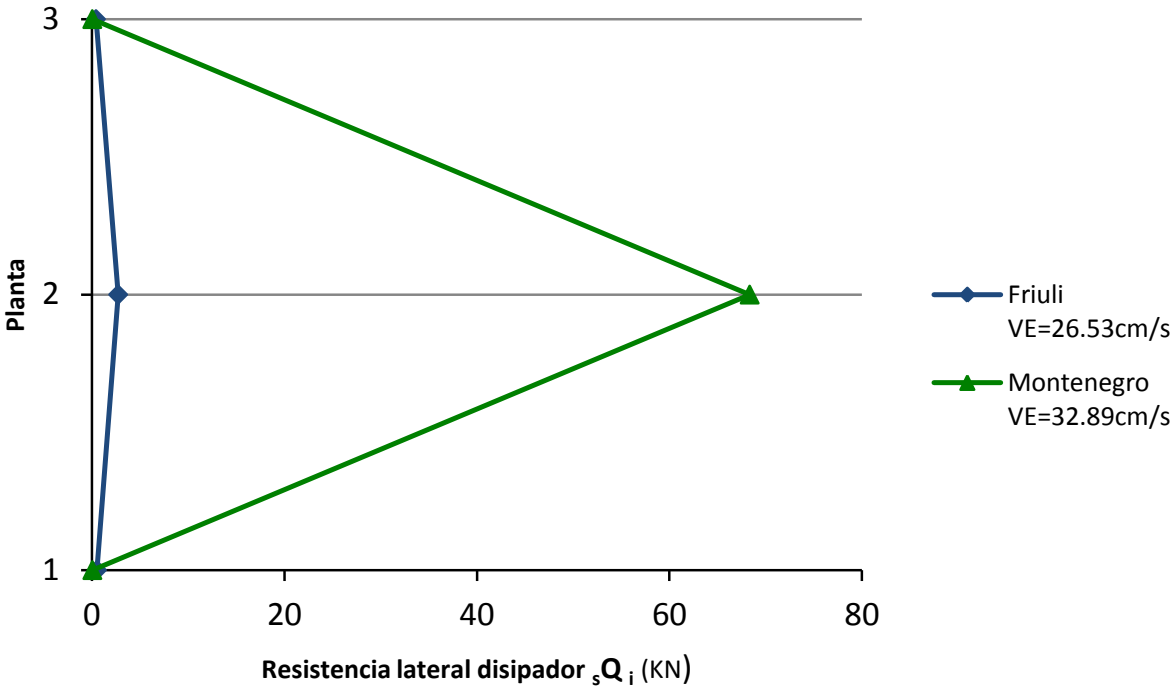

Fig. 5.5.34: Resistencia lateral de los disipadores de cada planta i, pórtico pa4pz2-1, suelo tipo II, campo cercano

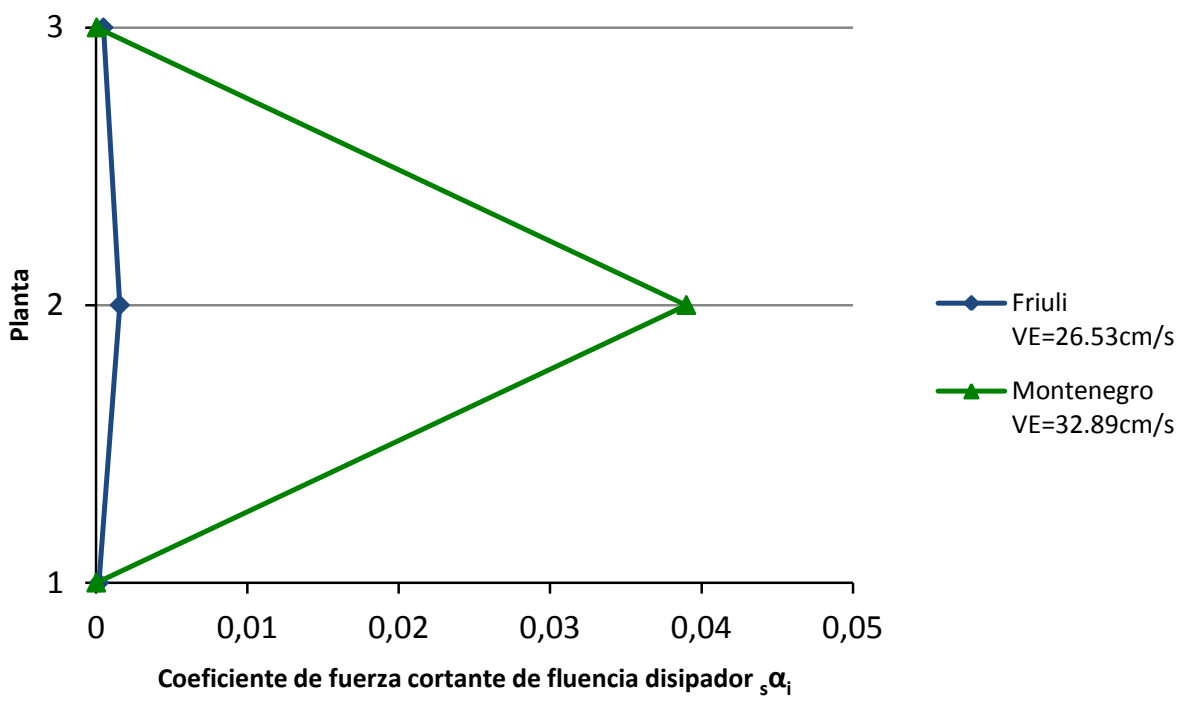

Fig. 5.5.35: Coeficiente de fuerza cortante de fluencia de los disipadores de cada planta i, pórtico pa4pz2-1, suelo tipo II, campo cercano 


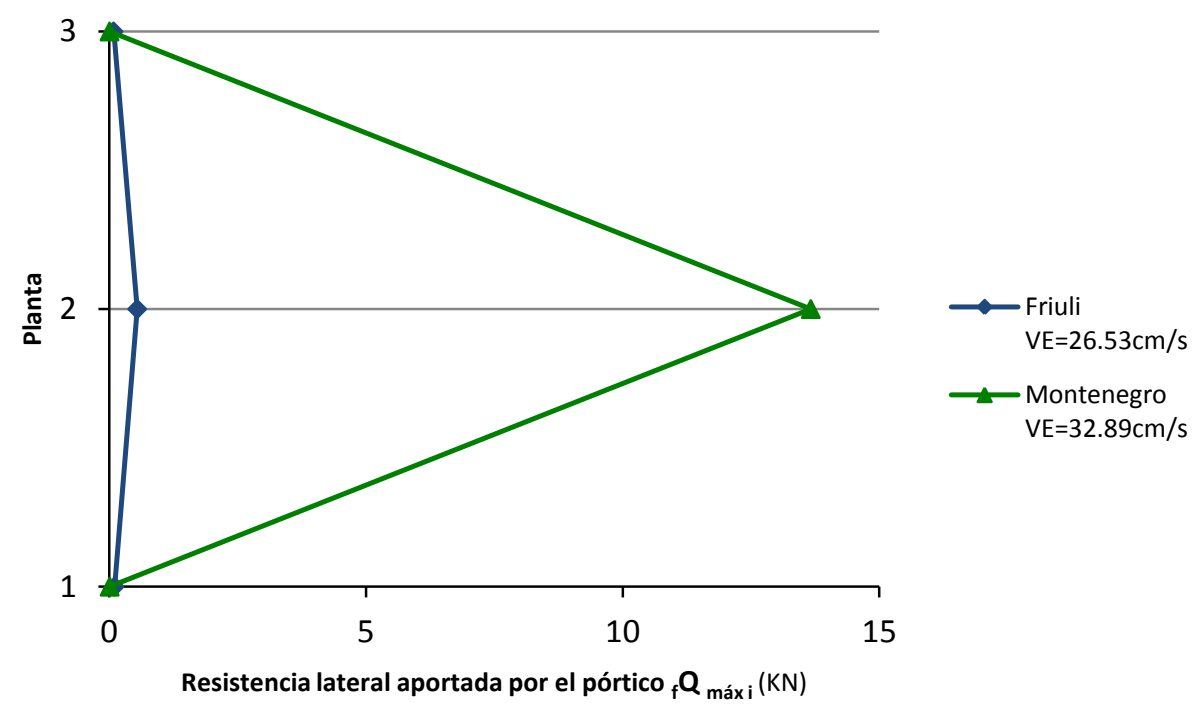

Fig. 5.5.36: Resistencia lateral aportada por la parte flexible (pórtico) cuando los disipadores empiezan a plastificar, pórtico pa4pz2-1, suelo tipo II, campo cercano

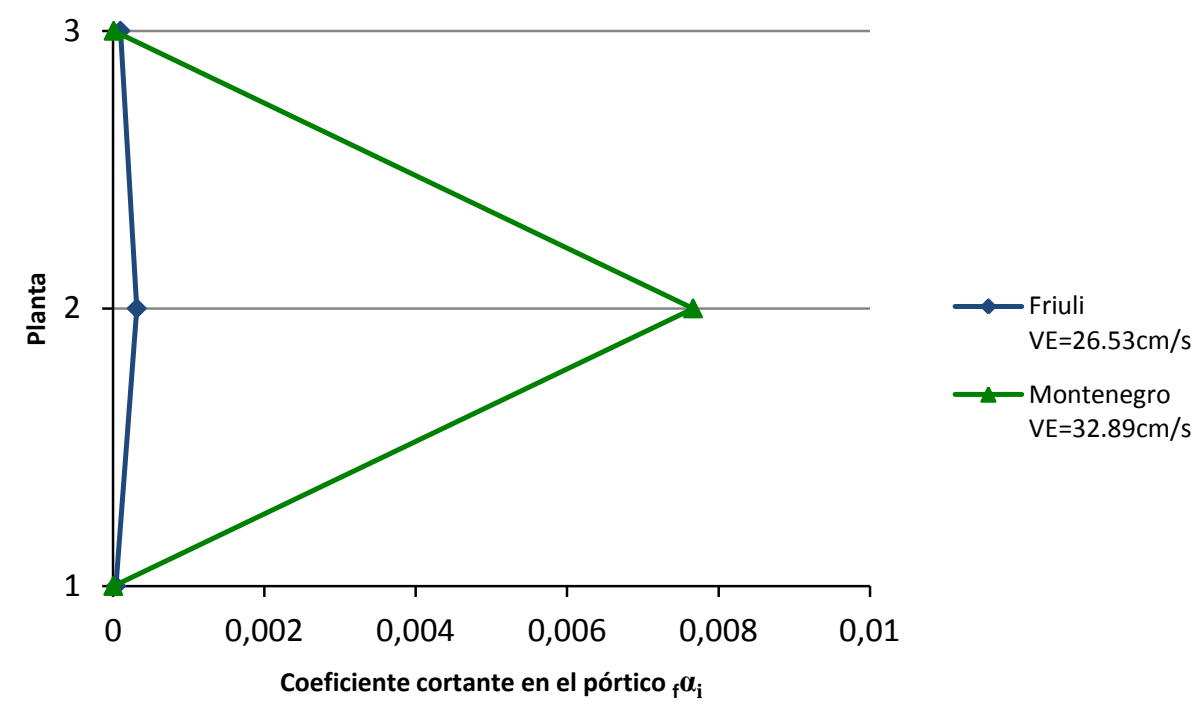

Fig. 5.5.37: Coeficiente de fuerza cortante en la parte flexible (pórtico) cuando los disipadores empiezan a plastificar, pórtico pa4pz2-1, suelo tipo II, campo cercano 


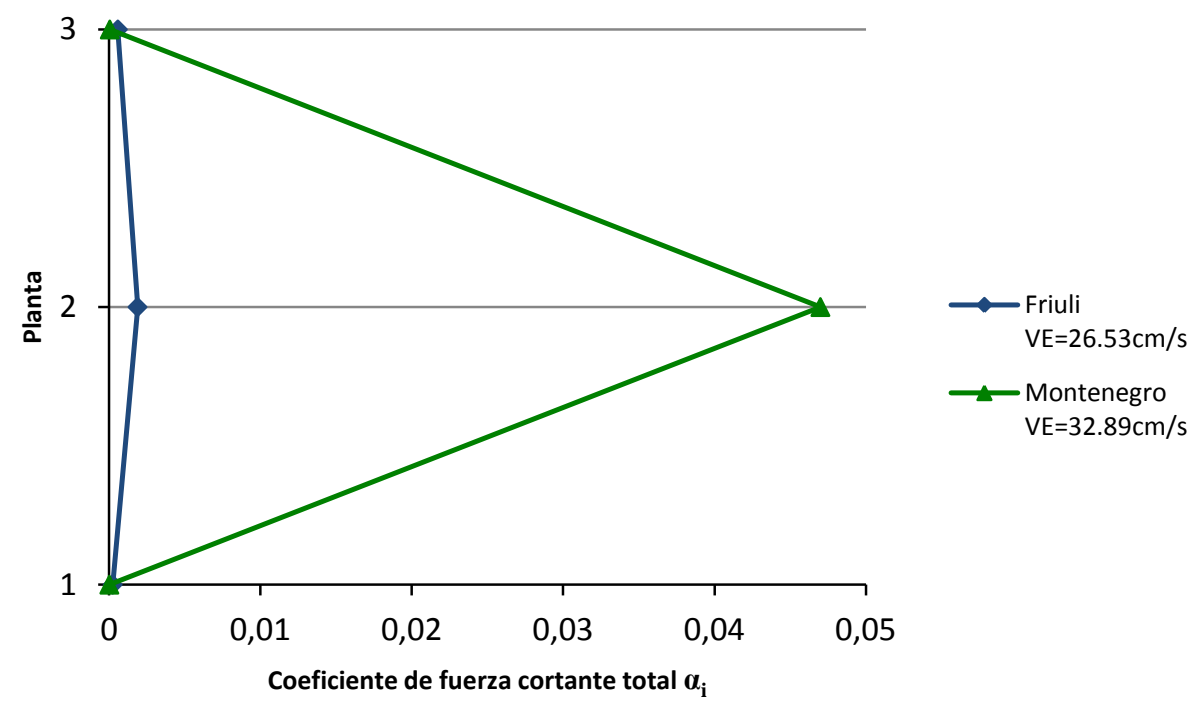

Fig. 5.5.38: Coeficiente de fuerza cortante total de planta, pórtico pa4pz2-1, suelo tipo II, campo cercano

\subsection{Suelo Tipo II (Campo lejano a la falla)}

\section{- Acelerograma №2 (Friuli aftershock) $\Delta=16 \mathrm{Km}$}

Desplazamiento de fluencia del disipador ${ }_{s} \delta_{i}$

Planta 3a: ${ }_{s} \delta_{3}=0,6606 \mathrm{~cm}$

Planta 2a: ${ }_{s} \delta_{2}=1,3750 \mathrm{~cm}$

Planta 1a: ${ }_{s} \delta_{1}=1,1719 \mathrm{~cm}$

Resistencia lateral de los disipadores de una planta i dada ${ }_{s} Q_{i}$

Planta 3a: ${ }_{s} Q_{3}={ }_{s} k_{3} \times{ }_{s} \delta_{3}=385000 \mathrm{~N} / \mathrm{cm} \times 0,6606 \mathrm{~cm} \Rightarrow{ }_{s} Q_{3}=254331 \mathrm{~N}$

Planta 2a: ${ }_{s} Q_{2}={ }_{s} k_{2} \times{ }_{s} \delta_{2}=455000 \mathrm{~N} / \mathrm{cm} \times 1,3750 \mathrm{~cm} \Rightarrow{ }_{s} Q_{2}=625625 \mathrm{~N}$

Planta 1ㄹ: ${ }_{s} Q_{1}={ }_{s} k_{1} \times{ }_{s} \delta_{1}=475000 \mathrm{~N} / \mathrm{cm} \times 1,1719 \mathrm{~cm} \Rightarrow{ }_{s} Q_{1}=556652,5 \mathrm{~N}$

Coeficiente de fuerza cortante de fluencia de los disipadores de una planta i dada ${ }_{s} \alpha_{i}$

$$
\text { Planta 3a: }{ }_{s} \alpha_{3}=\frac{{ }_{s} Q_{3}(N)}{m_{3}\left(\frac{N s^{2}}{c m}\right) \times g\left(\frac{c m}{s^{2}}\right)}=\frac{254331}{885,82 \times 981} \Rightarrow \quad \underline{{ }_{s} \alpha_{3}=0,293}
$$

Planta 2a: ${ }_{s} \alpha_{2}=\frac{{ }_{s}(N)}{\left(m_{2}+m_{3}\right)\left(\frac{N s^{2}}{\mathrm{~cm}}\right) \times g\left(\frac{\mathrm{cm}}{\mathrm{s}^{2}}\right)}=\frac{625625}{(2 \times 885,82) \times 981} \Rightarrow \quad \underline{{ }_{s} \alpha_{2}=0,360}$

Planta 1a: ${ }_{s} \alpha_{1}=\frac{{ }_{s} Q_{1}(N)}{\left(m_{1}+m_{2}+m_{3}\right)\left(\frac{N s^{2}}{c m}\right) \times g\left(\frac{c m}{s^{2}}\right)}=\frac{556652,5}{(3 \times 885,82) \times 981} \Rightarrow{ }_{s} \alpha_{1}=0,213$ 
Resistencia lateral aportada por la parte flexible (pórtico) cuando los disipadores empiezan a plastificar ${ }_{f} Q_{\text {máxi }}$

Planta 3a: ${ }_{f} Q_{\text {máx } 3}={ }_{f} k_{3} \times{ }_{s} \delta_{3}=77000 \mathrm{~N} / \mathrm{cm} \times 0,6606 \mathrm{~cm} \Rightarrow{ }_{f} Q_{\text {máx } 3}=77000,66 \mathrm{~N}$

Planta 2ä: ${ }_{f} Q_{\text {máx } 2}={ }_{f} k_{2} \times{ }_{s} \delta_{2}=91000 \mathrm{~N} / \mathrm{cm} \times 1,3750 \mathrm{~cm} \Rightarrow{ }_{f} Q_{\text {máx } 2}=125125 \mathrm{~N}$

Planta 1ạ: ${ }_{f} Q_{\text {máx } 1}={ }_{f} k_{1} \times{ }_{s} \delta_{1}=95000 \mathrm{~N} / \mathrm{cm} \times 1,1719 \mathrm{~cm} \Rightarrow \overline{{ }_{f} Q_{\text {máx } 1}=111330,5 \mathrm{~N}}$

Coeficiente cortante en la parte flexible (pórtico) cuando los disipadores empiezan a plastificar ${ }_{f} \alpha_{i}$

Planta 3ä: ${ }_{f} \alpha_{3}=\frac{{ }_{f} Q_{\text {máx } 3}(N)}{m_{3}\left(\frac{N s^{2}}{\mathrm{~cm}}\right) \times g\left(\frac{\mathrm{cm}}{\mathrm{s}^{2}}\right)}=\frac{77000,66}{885,82 \times 981} \Rightarrow \quad \underline{{ }_{f} \alpha_{3}=0,089}$

Planta 2ㄹ: ${ }_{f} \alpha_{2}=\frac{{ }_{f} Q_{\text {máx }}(N)}{\left(m_{2}+m_{3}\right)\left(\frac{N s^{2}}{c m}\right) \times g\left(\frac{c m}{s^{2}}\right)}=\frac{125125}{(2 \times 885,82) \times 981} \Rightarrow \quad \underline{{ }_{f}} \alpha_{2}=0,072$

Planta 1ㄹ: ${ }_{f} \alpha_{1}=\frac{{ }_{f} Q_{m a ́ x 1}(N)}{\left(m_{1}+m_{2}+m_{3}\right)\left(\frac{N s^{2}}{c m}\right) \times g\left(\frac{c m}{s^{2}}\right)}=\frac{111330,5}{(3 \times 885,82) \times 981} \Rightarrow \underline{{ }_{f}} \alpha_{1}=0,043$

Coeficiente de fuerza cortante total de planta $\alpha_{i}$

Planta 3a: $\alpha_{3}={ }_{s} \alpha_{3}+{ }_{f} \alpha_{3}=0,293+0,089 \Rightarrow \alpha_{3}=0,382$

Planta 2a: $\alpha_{2}={ }_{s} \alpha_{2}+{ }_{f} \alpha_{2}=0,360+0,072 \Rightarrow \underline{\alpha_{2}=0,432}$

Planta 1a: $\alpha_{1}={ }_{s} \alpha_{1}+{ }_{f} \alpha_{1}=0,213+0,043 \Rightarrow \underline{\alpha_{1}=0,256}$

\section{- Acelerograma №12 (Montenegro aftershock) $\Delta=33 \mathrm{Km}$}

Desplazamiento de fluencia del disipador ${ }_{s} \delta_{i}$

Planta 3a: ${ }_{s} \delta_{3}=1,0703 \mathrm{~cm}$

Planta 2a: ${ }_{s} \delta_{2}=1,3604 \mathrm{~cm}$

Planta 1a: ${ }_{s} \delta_{1}=1,3125 \mathrm{~cm}$

Resistencia lateral de los disipadores de una planta i dada ${ }_{s} Q_{i}$

Planta 3ㄹ: ${ }_{s} Q_{3}={ }_{s} k_{3} \times{ }_{s} \delta_{3}=385000 \mathrm{~N} / \mathrm{cm} \times 1,0703 \mathrm{~cm} \Rightarrow{ }_{s} Q_{3}=412065,5 \mathrm{~N}$

Planta 2a: ${ }_{s} Q_{2}={ }_{s} k_{2} \times{ }_{s} \delta_{2}=455000 \mathrm{~N} / \mathrm{cm} \times 1,3604 \mathrm{~cm} \Rightarrow{ }_{s} Q_{2}=618982 \mathrm{~N}$

Planta 1ㄹ: ${ }_{s} Q_{1}={ }_{s} k_{1} \times{ }_{s} \delta_{1}=475000 \mathrm{~N} / \mathrm{cm} \times 1,3125 \mathrm{~cm} \Rightarrow{ }_{s} Q_{1}=623437,5 \mathrm{~N}$ 
Coeficiente de fuerza cortante de fluencia de los disipadores de una planta i dada ${ }_{s} \alpha_{i}$

Planta 3a: ${ }_{s} \alpha_{3}=\frac{{ }_{s} Q_{3}(N)}{m_{3}\left(\frac{N s^{2}}{c m}\right) \times g\left(\frac{\mathrm{cm}}{\mathrm{s}^{2}}\right)}=\frac{412065,5}{885,82 \times 981} \Rightarrow \quad \underline{{ }_{s} \alpha_{3}=0,474}$

Planta 2: ${ }_{s} \alpha_{2}=\frac{{ }_{s}(N)}{\left(m_{2}+m_{3}\right)\left(\frac{N s^{2}}{c m}\right) \times g\left(\frac{\mathrm{cm}}{\mathrm{s}^{2}}\right)}=\frac{618982}{(2 \times 885,82) \times 981} \Rightarrow \quad{ }_{s} \alpha_{2}=0,356$

Planta 1ㄹ: $\alpha_{s}=\frac{{ }_{s} Q_{1}(N)}{\left(m_{1}+m_{2}+m_{3}\right)\left(\frac{N s^{2}}{c m}\right) \times g\left(\frac{c m}{s^{2}}\right)}=\frac{623437,5}{(3 \times 885,82) \times 981} \Rightarrow{ }_{s} \alpha_{1}=0,239$

Resistencia lateral aportada por la parte flexible (pórtico) cuando los disipadores empiezan a plastificar ${ }_{f} Q_{\text {máxi }}$

Planta 3a: : $Q_{\text {máx } 3}={ }_{f} k_{3} \times{ }_{s} \delta_{3}=77000 \mathrm{~N} / \mathrm{cm} \times 1,0703 \mathrm{~cm} \Rightarrow{ }_{f} Q_{\text {máx } 3}=82413,1 \mathrm{~N}$

Planta 2a: ${ }_{f} Q_{\text {máx } 2}={ }_{f} k_{2} \times{ }_{s} \delta_{2}=91000 \mathrm{~N} / \mathrm{cm} \times 1,3604 \mathrm{~cm} \Rightarrow{ }_{f} Q_{\operatorname{máx2}}=123796,4 \mathrm{~N}$

Planta 1ạ: ${ }_{f} Q_{\text {máx1 }}={ }_{f} k_{1} \times{ }_{s} \delta_{1}=95000 \mathrm{~N} / \mathrm{cm} \times 1,3125 \mathrm{~cm} \Rightarrow{ }_{f} Q_{\text {máx } 1}=124687,5 N$

Coeficiente cortante en la parte flexible (pórtico) cuando los disipadores empiezan a plastificar ${ }_{f} \alpha_{i}$

Planta 3a: ${ }_{f} \alpha_{3}=\frac{{ }_{f} Q_{\operatorname{máx3}}(N)}{m_{3}\left(\frac{N s^{2}}{\mathrm{~cm}}\right) \times g\left(\frac{\mathrm{cm}}{\mathrm{s}^{2}}\right)}=\frac{82413,1}{885,82 \times 981} \Rightarrow \quad \underline{{ }_{f} \alpha_{3}=0,095}$

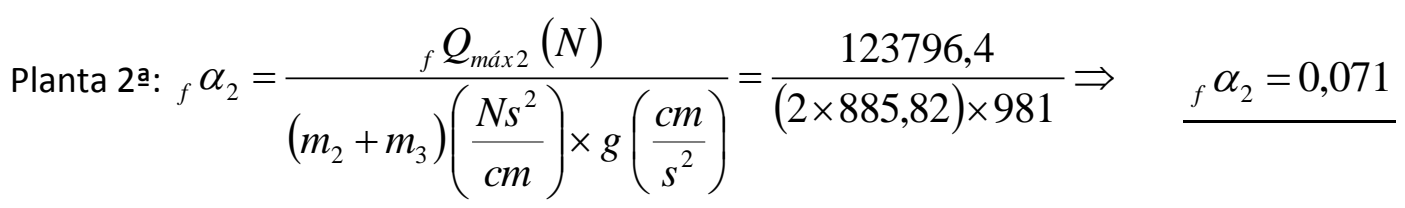

Planta 1ạ: ${ }_{f} \alpha_{1}=\frac{{ }_{f} Q_{m a ́ x 1}(N)}{\left(m_{1}+m_{2}+m_{3}\right)\left(\frac{N s^{2}}{c m}\right) \times g\left(\frac{c m}{s^{2}}\right)}=\frac{124687,5}{(3 \times 885,82) \times 981} \Rightarrow \underline{{ }_{f}} \alpha_{1}=0,048$

Coeficiente de fuerza cortante total de planta $\alpha_{i}$

Planta 3a: $\alpha_{3}={ }_{s} \alpha_{3}+{ }_{f} \alpha_{3}=0,474+0,095 \Rightarrow \alpha_{3}=0,569$

Planta 2a: $\alpha_{2}={ }_{s} \alpha_{2}+{ }_{f} \alpha_{2}=0,356+0,071 \Rightarrow \overline{\alpha_{2}=0,427}$

Planta 1a: $\alpha_{1}={ }_{s} \alpha_{1}+{ }_{f} \alpha_{1}=0,239+0,048 \Rightarrow \underline{\alpha_{1}=0,287}$ 
Representación gráfica

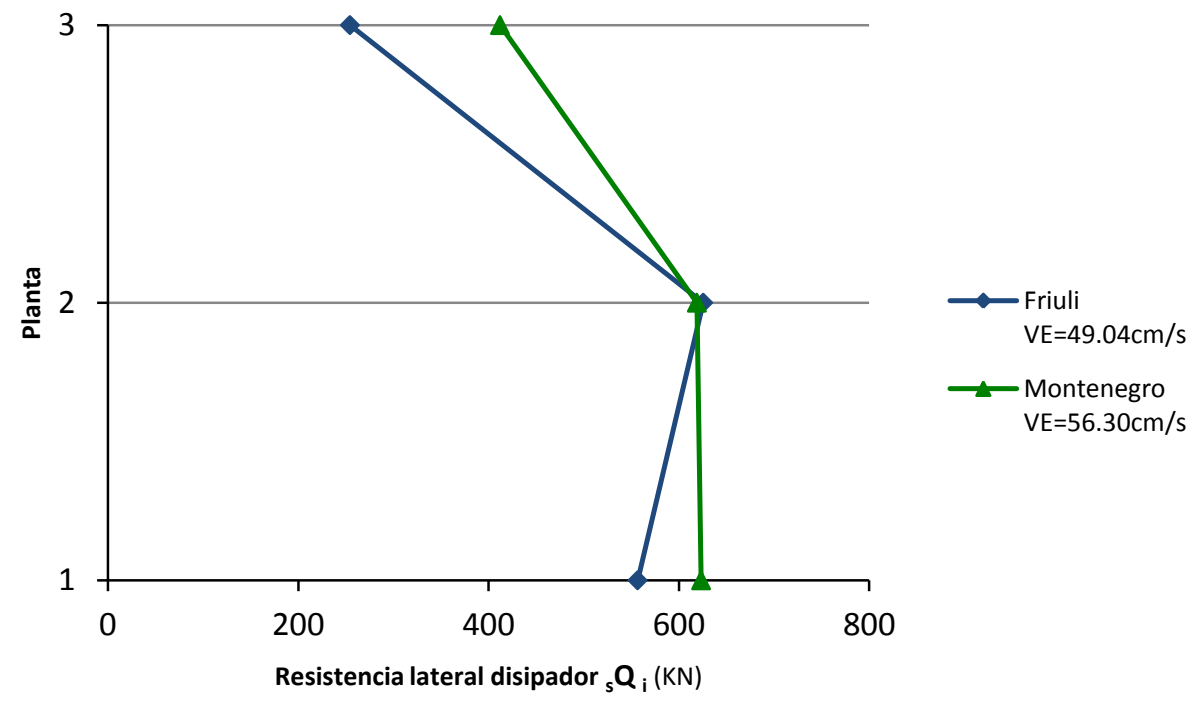

Fig. 5.5.39: Resistencia lateral de los disipadores de cada planta i, pórtico pa4pz2-1, suelo tipo II, campo lejano

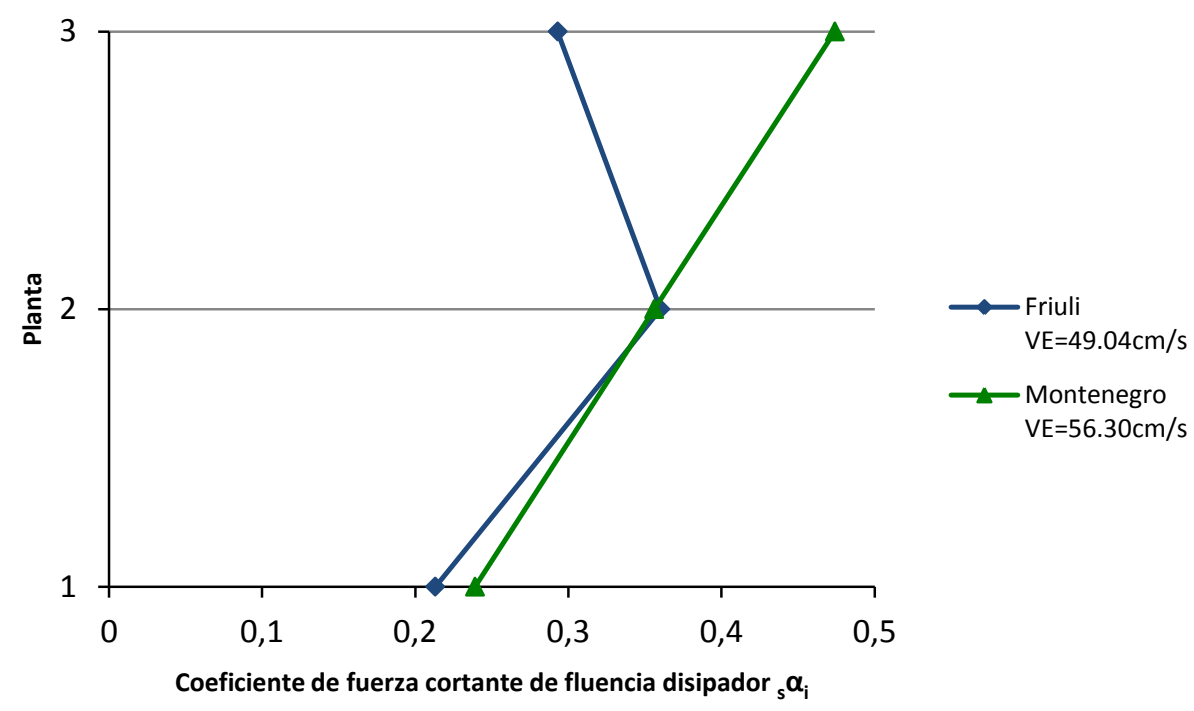

Fig. 5.5.40: Coeficiente de fuerza cortante de fluencia de los disipadores de cada planta i, pórtico pa4pz2-1, suelo tipo II, campo lejano 


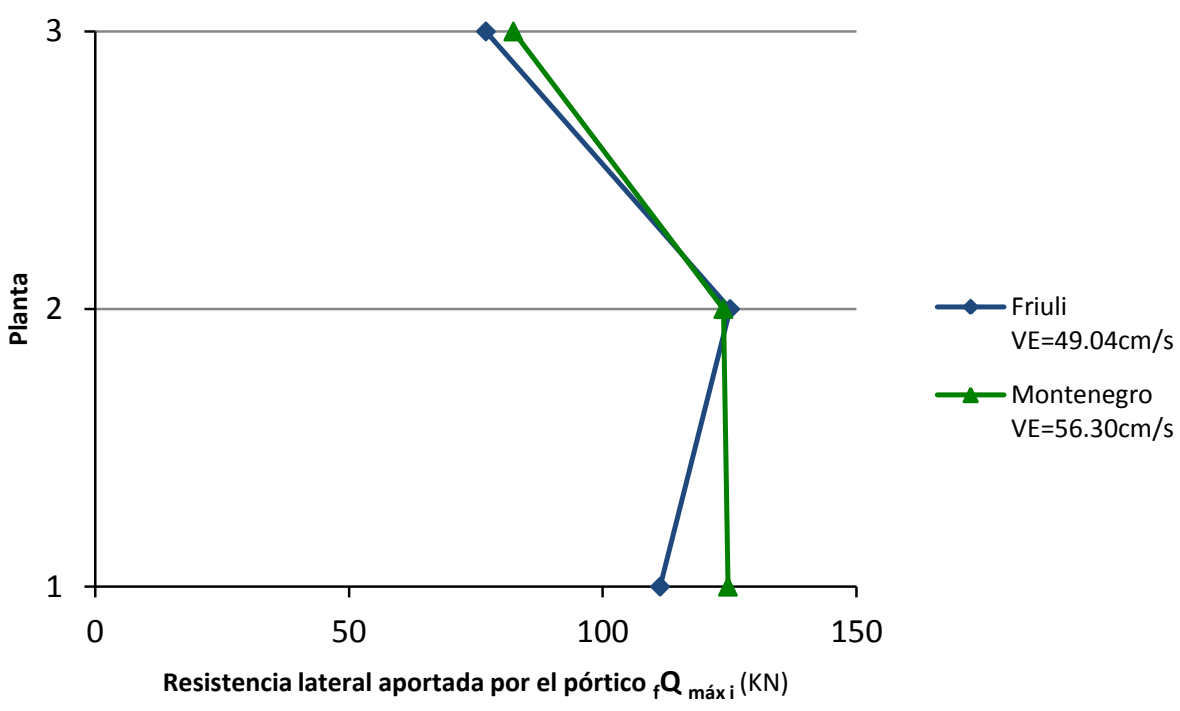

Fig. 5.5.41: Resistencia lateral aportada por la parte flexible (pórtico) cuando los disipadores empiezan a plastificar, pórtico pa4pz2-1, suelo tipo II, campo lejano

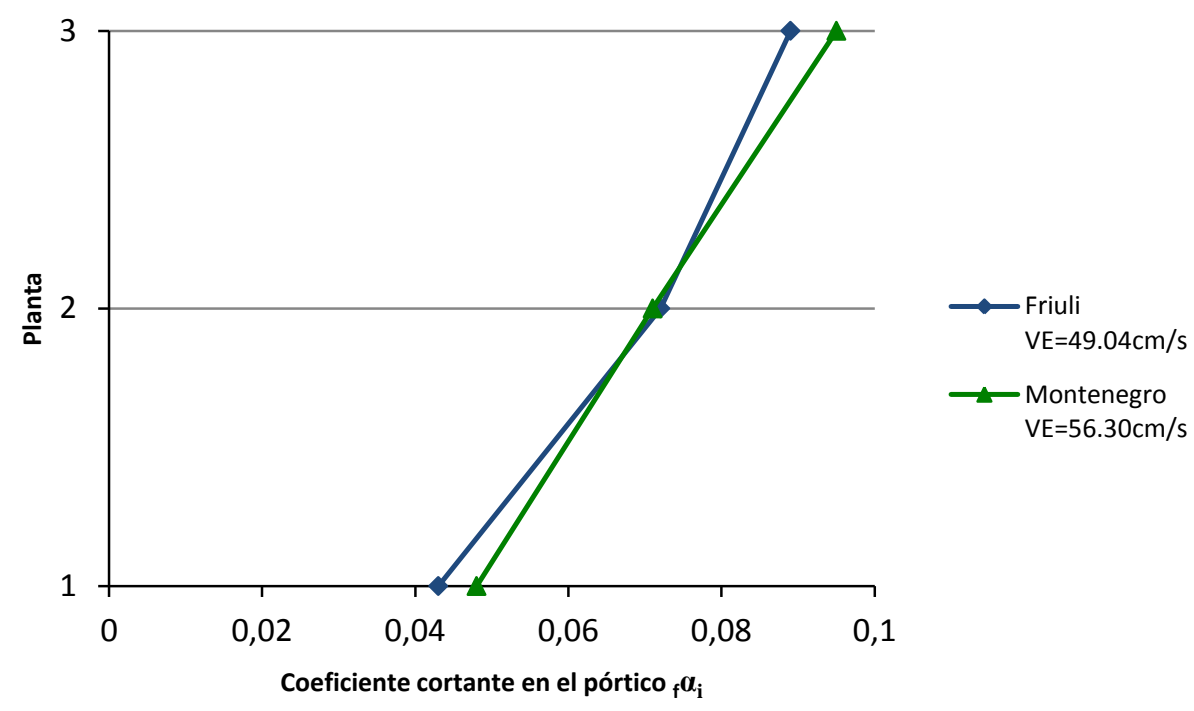

Fig. 5.5.42: Coeficiente de fuerza cortante en la parte flexible (pórtico) cuando los disipadores empiezan a plastificar, pórtico pa4pz2-1, suelo tipo II, campo lejano 


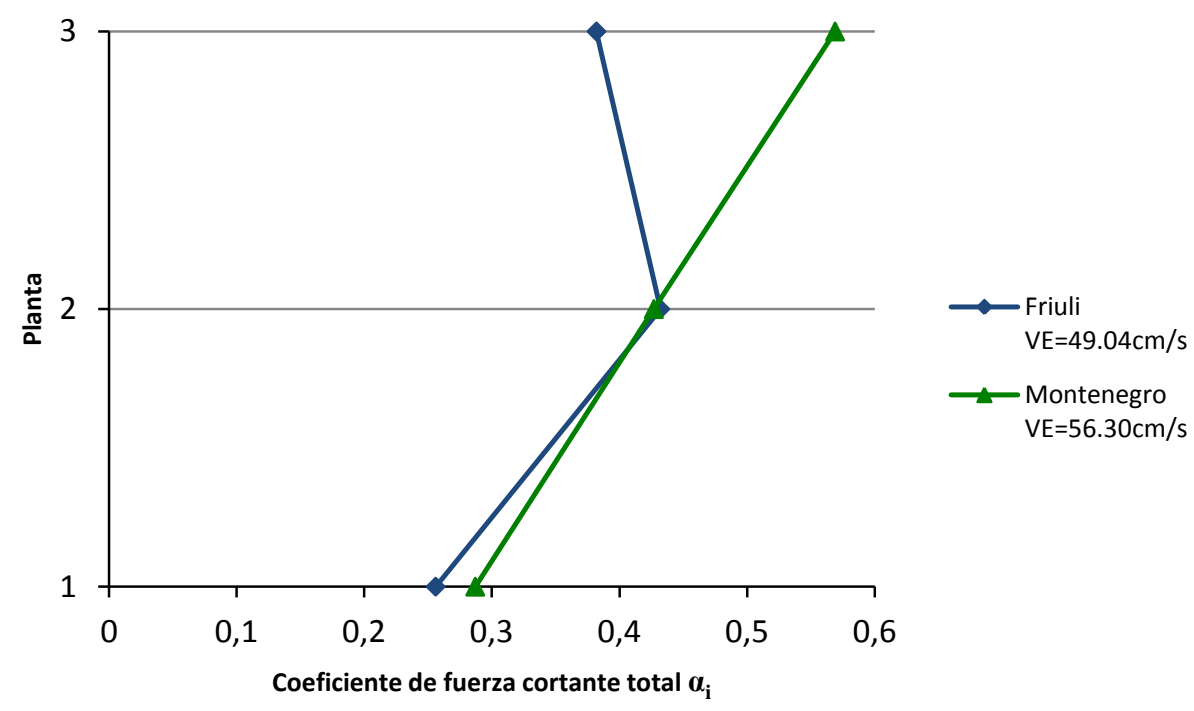

Fig. 5.5.43: Coeficiente de fuerza cortante total de planta, pórtico pa4pz2-1, suelo tipo II, campo lejano

\subsection{Suelo Tipo III (Campo cercano a la falla)}

\section{- Acelerograma №2 (Friuli aftershock) $\Delta=10 \mathrm{Km}$}

Desplazamiento de fluencia del disipador ${ }_{s} \delta_{i}$

Planta 3a: ${ }_{s} \delta_{3}=0,4571 \mathrm{~cm}$

Planta 2a: ${ }_{s} \delta_{2}=0,7659 \mathrm{~cm}$

Planta 1ㄹ: $\delta_{1}=0,9121 \mathrm{~cm}$

Resistencia lateral de los disipadores de una planta i dada ${ }_{s} Q_{i}$

Planta 3a: ${ }_{s} Q_{3}={ }_{s} k_{3} \times{ }_{s} \delta_{3}=385000 \mathrm{~N} / \mathrm{cm} \times 0,4571 \mathrm{~cm} \Rightarrow{ }_{s} Q_{3}=175983,5 \mathrm{~N}$

Planta 2a: ${ }_{s} Q_{2}={ }_{s} k_{2} \times{ }_{s} \delta_{2}=455000 \mathrm{~N} / \mathrm{cm} \times 0,7659 \mathrm{~cm} \Rightarrow \underline{{ }_{s} Q_{2}=348484,5 N}$

Planta 1ㄹ: ${ }_{s} Q_{1}={ }_{s} k_{1} \times{ }_{s} \delta_{1}=475000 \mathrm{~N} / \mathrm{cm} \times 0,9121 \mathrm{~cm} \Rightarrow{ }_{s} Q_{1}=433247,5 \mathrm{~N}$

Coeficiente de fuerza cortante de fluencia de los disipadores de una planta i dada ${ }_{s} \alpha_{i}$

Planta 3a: ${ }_{s} \alpha_{3}=\frac{{ }_{s} Q_{3}(N)}{m_{3}\left(\frac{N s^{2}}{c m}\right) \times g\left(\frac{\mathrm{cm}}{\mathrm{s}^{2}}\right)}=\frac{175983,5}{885,82 \times 981} \Rightarrow \quad \underline{{ }_{s} \alpha_{3}=0,202}$

Planta 2: ${ }_{s} \alpha_{2}=\frac{{ }_{s} Q_{2}(N)}{\left(m_{2}+m_{3}\right)\left(\frac{N s^{2}}{c m}\right) \times g\left(\frac{\mathrm{cm}}{\mathrm{s}^{2}}\right)}=\frac{348484,5}{(2 \times 885,82) \times 981} \Rightarrow \quad \underline{{ }_{s} \alpha_{2}=0,200}$

Planta 1ㄹ: $\alpha_{s}=\frac{{ }_{s} Q_{1}(N)}{\left(m_{1}+m_{2}+m_{3}\right)\left(\frac{N s^{2}}{c m}\right) \times g\left(\frac{c m}{s^{2}}\right)}=\frac{433247,5}{(3 \times 885,82) \times 981} \Rightarrow{ }_{s} \alpha_{1}=0,166$ 
Resistencia lateral aportada por la parte flexible (pórtico) cuando los disipadores empiezan a plastificar ${ }_{f} Q_{\text {máxi }}$

Planta 3a: ${ }_{f} Q_{\text {máx } 3}={ }_{f} k_{3} \times{ }_{s} \delta_{3}=77000 N / \mathrm{cm} \times 0,4571 \mathrm{~cm} \Rightarrow{ }_{f} Q_{\text {máx } 3}=35196,7 N$

Planta 2a: ${ }_{f} Q_{\text {máx } 2}={ }_{f} k_{2} \times{ }_{s} \delta_{2}=91000 \mathrm{~N} / \mathrm{cm} \times 0,7659 \mathrm{~cm} \Rightarrow{ }_{f} Q_{\operatorname{máx} 2}=69696,9 \mathrm{~N}$

Planta 1ㄹ: ${ }_{f} Q_{\text {máxx }}={ }_{f} k_{1} \times{ }_{s} \delta_{1}=95000 \mathrm{~N} / \mathrm{cm} \times 0,9121 \mathrm{~cm} \Rightarrow \overline{{ }_{f} Q_{\text {máx } 1}=86649,5 \mathrm{~N}}$

Coeficiente cortante en la parte flexible (pórtico) cuando los disipadores empiezan a plastificar ${ }_{f} \alpha_{i}$

Planta 3a: ${ }_{f} \alpha_{3}=\frac{{ }_{f} Q_{\text {máx3 }}(N)}{m_{3}\left(\frac{N s^{2}}{c m}\right) \times g\left(\frac{\mathrm{cm}}{\mathrm{s}^{2}}\right)}=\frac{35196,7}{885,82 \times 981} \Rightarrow \quad \underline{{ }_{f} \alpha_{3}=0,040}$

Planta 2a: : ${ }_{f} \alpha_{2}=\frac{{ }_{f} Q_{\text {máx }}(N)}{\left(m_{2}+m_{3}\right)\left(\frac{N s^{2}}{c m}\right) \times g\left(\frac{c m}{s^{2}}\right)}=\frac{69696,9}{(2 \times 885,82) \times 981} \Rightarrow \quad \underline{{ }_{f} \alpha_{2}=0,040}$

Planta 1ㄹ: ${ }_{f} \alpha_{1}=\frac{{ }_{f} Q_{m a ́ x 1}(N)}{\left(m_{1}+m_{2}+m_{3}\right)\left(\frac{N s^{2}}{c m}\right) \times g\left(\frac{c m}{s^{2}}\right)}=\frac{86649,5}{(3 \times 885,82) \times 981} \Rightarrow \underline{{ }_{f}} \alpha_{1}=0,033$

Coeficiente de fuerza cortante total de planta $\alpha_{i}$

Planta 3a: $\alpha_{3}={ }_{s} \alpha_{3}+{ }_{f} \alpha_{3}=0,202+0,040 \Rightarrow \alpha_{3}=0,242$

Planta 2a: $\alpha_{2}={ }_{s} \alpha_{2}+{ }_{f} \alpha_{2}=0,200+0,040 \Rightarrow \underline{\alpha_{2}=0,240}$

Planta 1: $\alpha_{1}={ }_{s} \alpha_{1}+{ }_{f} \alpha_{1}=0,166+0,033 \Rightarrow \underline{\alpha_{1}=0,199}$

\section{- Acelerograma №6 (Kalamata aftershock) $\Delta=3 \mathrm{Km}$}

Desplazamiento de fluencia del disipador ${ }_{s} \delta_{i}$

Planta 3a: ${ }_{s} \delta_{3}=0,0879 \mathrm{~cm}$

Planta 2ㄹ: ${ }_{s} \delta_{2}=0,1319 \mathrm{~cm}$

Planta 1a: ${ }_{s} \delta_{1}=0,0802 \mathrm{~cm}$

Resistencia lateral de los disipadores de una planta i dada ${ }_{s} Q_{i}$

Planta 3a: ${ }_{s} Q_{3}={ }_{s} k_{3} \times{ }_{s} \delta_{3}=385000 \mathrm{~N} / \mathrm{cm} \times 0,0879 \mathrm{~cm} \Rightarrow{ }_{s} Q_{3}=33841,5 \mathrm{~N}$

Planta 2a: ${ }_{s} Q_{2}={ }_{s} k_{2} \times{ }_{s} \delta_{2}=455000 \mathrm{~N} / \mathrm{cm} \times 0,1319 \mathrm{~cm} \Rightarrow{ }_{s} Q_{2}=60014,5 \mathrm{~N}$

Planta 1à: ${ }_{s} Q_{1}={ }_{s} k_{1} \times{ }_{s} \delta_{1}=475000 \mathrm{~N} / \mathrm{cm} \times 0,0802 \mathrm{~cm} \Rightarrow{ }_{s} Q_{1}=38095 \mathrm{~N}$ 
Coeficiente de fuerza cortante de fluencia de los disipadores de una planta i dada ${ }_{s} \alpha_{i}$

Planta 3a: ${ }_{s} \alpha_{3}=\frac{{ }_{s} Q_{3}(N)}{m_{3}\left(\frac{N s^{2}}{\mathrm{~cm}}\right) \times g\left(\frac{\mathrm{cm}}{\mathrm{s}^{2}}\right)}=\frac{33841,5}{885,82 \times 981} \Rightarrow$

Planta 2: ${ }_{s} \alpha_{2}=\frac{{ }_{s}(N)}{\left(m_{2}+m_{3}\right)\left(\frac{N s^{2}}{c m}\right) \times g\left(\frac{\mathrm{cm}}{\mathrm{s}^{2}}\right)}=\frac{60014,5}{(2 \times 885,82) \times 981} \Rightarrow \quad \underline{{ }_{s} \alpha_{2}=0,034}$

Planta 1a: ${ }_{s} \alpha_{1}=\frac{{ }_{s} Q_{1}(N)}{\left(m_{1}+m_{2}+m_{3}\right)\left(\frac{N s^{2}}{c m}\right) \times g\left(\frac{c m}{s^{2}}\right)}=\frac{38095}{(3 \times 885,82) \times 981} \Rightarrow \underline{{ }_{s} \alpha_{1}=0,015}$

Resistencia lateral aportada por la parte flexible (pórtico) cuando los disipadores empiezan a plastificar ${ }_{f} Q_{\text {máxi }}$

Planta 3a: ${ }_{f} Q_{\text {máx } 3}={ }_{f} k_{3} \times{ }_{s} \delta_{3}=77000 \mathrm{~N} / \mathrm{cm} \times 0,0879 \mathrm{~cm} \Rightarrow{ }_{f} Q_{\text {máx } 3}=6768,3 \mathrm{~N}$

Planta 2ㄹ: ${ }_{f} Q_{\text {máx } 2}={ }_{f} k_{2} \times{ }_{s} \delta_{2}=91000 \mathrm{~N} / \mathrm{cm} \times 0,1319 \mathrm{~cm} \Rightarrow{ }_{f} Q_{\operatorname{máx} 2}=12002,9 \mathrm{~N}$

Planta 1ạ: ${ }_{f} Q_{\text {máx1 }}={ }_{f} k_{1} \times{ }_{s} \delta_{1}=95000 \mathrm{~N} / \mathrm{cm} \times 0,0802 \mathrm{~cm} \Rightarrow{ }_{f} Q_{\text {máx } 1}=7619 \mathrm{~N}$

Coeficiente cortante en la parte flexible (pórtico) cuando los disipadores empiezan a plastificar ${ }_{f} \alpha_{i}$

Planta 3a: ${ }_{f} \alpha_{3}=\frac{{ }_{f} Q_{\operatorname{máx} 3}(N)}{m_{3}\left(\frac{N s^{2}}{\mathrm{~cm}}\right) \times g\left(\frac{\mathrm{cm}}{\mathrm{s}^{2}}\right)}=\frac{6768,3}{885,82 \times 981} \Rightarrow \quad \underline{{ }_{f} \alpha_{3}=7,789 e^{-03}}$

Planta 2a: ${ }_{f} \alpha_{2}=\frac{{ }_{f} Q_{\operatorname{máx} 2}(N)}{\left(m_{2}+m_{3}\right)\left(\frac{N s^{2}}{c m}\right) \times g\left(\frac{c m}{s^{2}}\right)}=\frac{12002,9}{(2 \times 885,82) \times 981} \Rightarrow \quad \underline{{ }_{f}} \alpha_{2}=6,906 e^{-03}$

Planta 1ㄹ: ${ }_{f} \alpha_{1}=\frac{{ }_{f} Q_{\text {máx } 1}(N)}{\left(m_{1}+m_{2}+m_{3}\right)\left(\frac{N s^{2}}{c m}\right) \times g\left(\frac{c m}{s^{2}}\right)}=\frac{7619}{(3 \times 885,82) \times 981} \Rightarrow \underline{{ }_{f}} \alpha_{1}=2,922 e^{-03}$

Coeficiente de fuerza cortante total de planta $\alpha_{i}$

Planta 3a: $\alpha_{3}={ }_{s} \alpha_{3}+{ }_{f} \alpha_{3}=0,039+7,789 e^{-03} \Rightarrow \alpha_{3}=0,047$

Planta 2a: $\alpha_{2}={ }_{s} \alpha_{2}+{ }_{f} \alpha_{2}=0,034+6,906 e^{-03} \Rightarrow \alpha_{2}=0,041$

Planta 1: $\alpha_{1}={ }_{s} \alpha_{1}+{ }_{f} \alpha_{1}=0,015+2,922 e^{-03} \Rightarrow \underline{\alpha_{1}=0,018}$ 


\section{- Acelerograma №12 (Duzce 1) $\Delta=8 \mathrm{Km}$}

Desplazamiento de fluencia del disipador ${ }_{s} \delta_{i}$

Planta 3a: ${ }_{s} \delta_{3}=0,1640 \mathrm{~cm}$

Planta 2a: ${ }_{s} \delta_{2}=0,3633 \mathrm{~cm}$

Planta 1a: ${ }_{s} \delta_{1}=0,3633 \mathrm{~cm}$

Resistencia lateral de los disipadores de una planta i dada ${ }_{s} Q_{i}$

Planta 3a: ${ }_{s} Q_{3}={ }_{s} k_{3} \times{ }_{s} \delta_{3}=385000 \mathrm{~N} / \mathrm{cm} \times 0,1640 \mathrm{~cm} \Rightarrow{ }_{s} Q_{3}=63140 \mathrm{~N}$

Planta 2: $:{ }_{s} Q_{2}={ }_{s} k_{2} \times{ }_{s} \delta_{2}=455000 \mathrm{~N} / \mathrm{cm} \times 0,3633 \mathrm{~cm} \Rightarrow \overline{{ }_{s} Q_{2}=165301,5 \mathrm{~N}}$

Planta 1: ${ }_{s} Q_{1}={ }_{s} k_{1} \times{ }_{s} \delta_{1}=475000 \mathrm{~N} / \mathrm{cm} \times 0,3633 \mathrm{~cm} \Rightarrow{ }_{s} Q_{1}=172567,5 \mathrm{~N}$

Coeficiente de fuerza cortante de fluencia de los disipadores de una planta i dada ${ }_{s} \alpha_{i}$

Planta 3a: ${ }_{s} \alpha_{3}=\frac{{ }_{s} Q_{3}(N)}{m_{3}\left(\frac{N s^{2}}{\mathrm{~cm}}\right) \times g\left(\frac{\mathrm{cm}}{\mathrm{s}^{2}}\right)}=\frac{63140}{885,82 \times 981} \Rightarrow \quad \underline{{ }_{s} \alpha_{3}=0,073}$

Planta 2: ${ }_{s} \alpha_{2}=\frac{{ }_{s}(N)}{\left(m_{2}+m_{3}\right)\left(\frac{N s^{2}}{c m}\right) \times g\left(\frac{\mathrm{cm}}{\mathrm{s}^{2}}\right)}=\frac{165301,5}{(2 \times 885,82) \times 981} \Rightarrow \quad{ }_{s} \alpha_{2}=0,095$

Planta 1: ${ }_{s} \alpha_{1}=\frac{{ }_{s} Q_{1}(N)}{\left(m_{1}+m_{2}+m_{3}\right)\left(\frac{N s^{2}}{c m}\right) \times g\left(\frac{c m}{s^{2}}\right)}=\frac{172567,5}{(3 \times 885,82) \times 981} \Rightarrow{ }_{s} \alpha_{1}=0,066$

Resistencia lateral aportada por la parte flexible (pórtico) cuando los disipadores empiezan a plastificar ${ }_{f} Q_{\text {máxi }}$

Planta 3ạ: ${ }_{f} Q_{\text {máx } 3}={ }_{f} k_{3} \times{ }_{s} \delta_{3}=77000 \mathrm{~N} / \mathrm{cm} \times 0,1640 \mathrm{~cm} \Rightarrow{ }_{f} Q_{\text {máx3 }}=12628 \mathrm{~N}$

Planta 2ä: ${ }_{f} Q_{\text {máx } 2}={ }_{f} k_{2} \times{ }_{s} \delta_{2}=91000 \mathrm{~N} / \mathrm{cm} \times 0,3633 \mathrm{~cm} \Rightarrow{ }_{f} Q_{\text {máx } 2}=33060,3 \mathrm{~N}$

Planta 1ㄹ: ${ }_{f} Q_{\text {máx } 1}={ }_{f} k_{1} \times{ }_{s} \delta_{1}=95000 \mathrm{~N} / \mathrm{cm} \times 0,3633 \mathrm{~cm} \Rightarrow{ }_{f} Q_{\text {máx } 1}=34513,5 \mathrm{~N}$

Coeficiente cortante en la parte flexible (pórtico) cuando los disipadores empiezan a plastificar ${ }_{f} \alpha_{i}$

Planta 3a: ${ }_{f} \alpha_{3}=\frac{{ }_{f} Q_{\text {máx3 }}(N)}{m_{3}\left(\frac{N s^{2}}{c m}\right) \times g\left(\frac{c m}{s^{2}}\right)}=\frac{12628}{885,82 \times 981} \Rightarrow \quad \underline{{ }_{f} \alpha_{3}=0,014}$

Planta 2a: ${ }_{f} \alpha_{2}=\frac{{ }_{f} Q_{\text {máx }}(N)}{\left(m_{2}+m_{3}\right)\left(\frac{N s^{2}}{c m}\right) \times g\left(\frac{c m}{s^{2}}\right)}=\frac{33060,3}{(2 \times 885,82) \times 981} \Rightarrow \quad \underline{{ }_{f} \alpha_{2}=0,019}$ 
Planta 1ㄹ: ${ }_{f} \alpha_{1}=\frac{{ }_{f} Q_{m a ́ x 1}(N)}{\left(m_{1}+m_{2}+m_{3}\right)\left(\frac{N s^{2}}{c m}\right) \times g\left(\frac{c m}{s^{2}}\right)}=\frac{34513,5}{(3 \times 885,82) \times 981} \Rightarrow \underline{{ }_{f}} \alpha_{1}=0,013$

Coeficiente de fuerza cortante total de planta $\alpha_{i}$

Planta 3a: $\alpha_{3}={ }_{s} \alpha_{3}+{ }_{f} \alpha_{3}=0,073+0,014 \Rightarrow \alpha_{3}=0,087$

Planta 2a: $\alpha_{2}={ }_{s} \alpha_{2}+{ }_{f} \alpha_{2}=0,095+0,019 \Rightarrow \alpha_{2}=0,114$

Planta 1a: $\alpha_{1}={ }_{s} \alpha_{1}+{ }_{f} \alpha_{1}=0,066+0,013 \Rightarrow \underline{\alpha_{1}=0,079}$

Representación gráfica

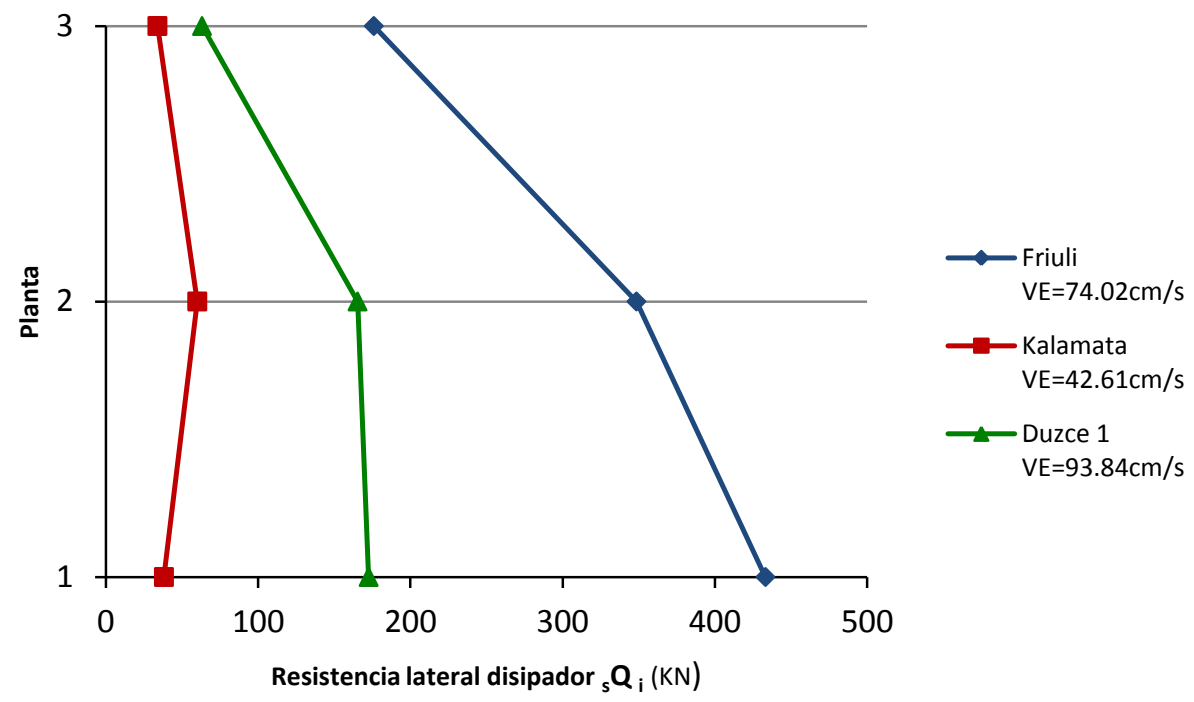

Fig. 5.5.44: Resistencia lateral de los disipadores de cada planta i, pórtico pa4pz2-1, suelo tipo III, campo cercano

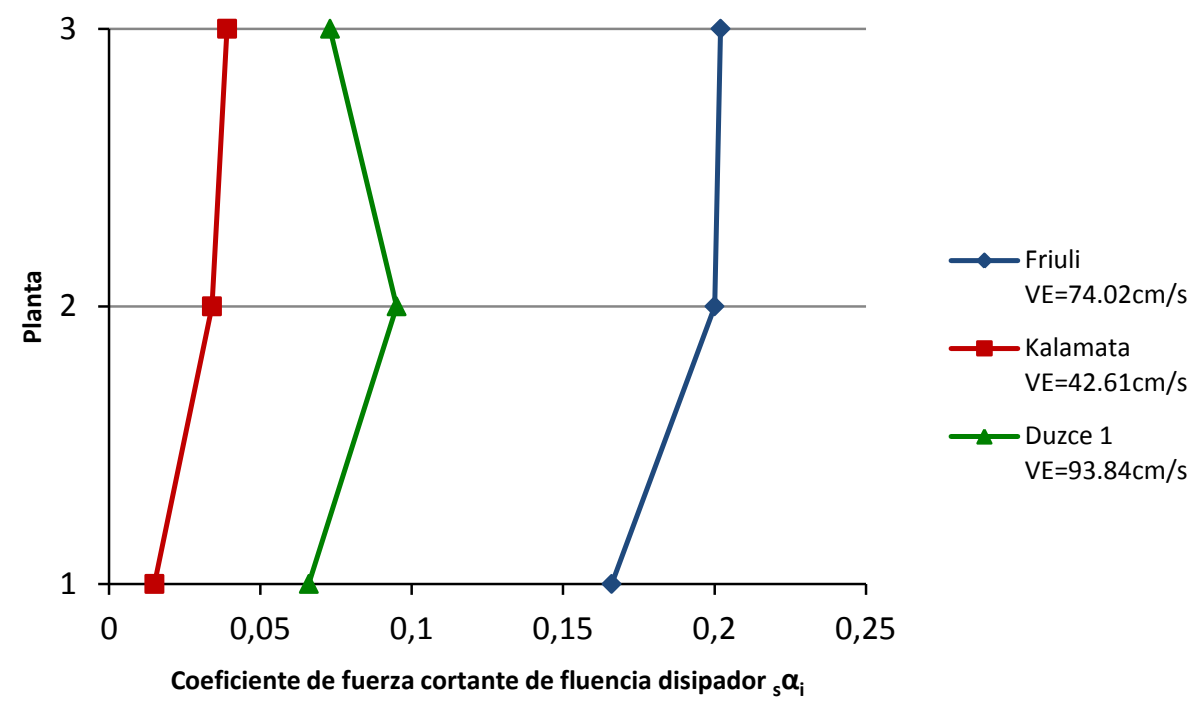

Fig. 5.5.45: Coeficiente de fuerza cortante de fluencia de los disipadores de cada planta i, pórtico pa4pz2-1, suelo tipo III, campo cercano 


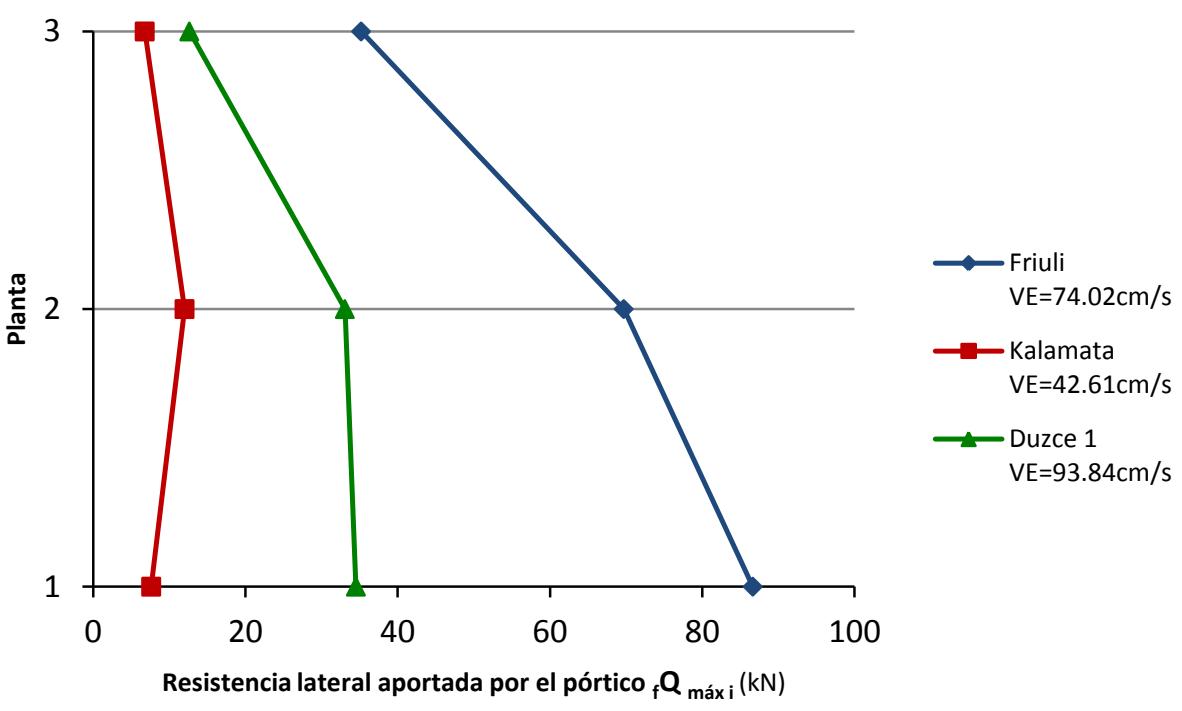

Fig. 5.5.46: Resistencia lateral aportada por la parte flexible (pórtico) cuando los disipadores empiezan a plastificar, pórtico pa4pz2-1, suelo tipo III, campo cercano

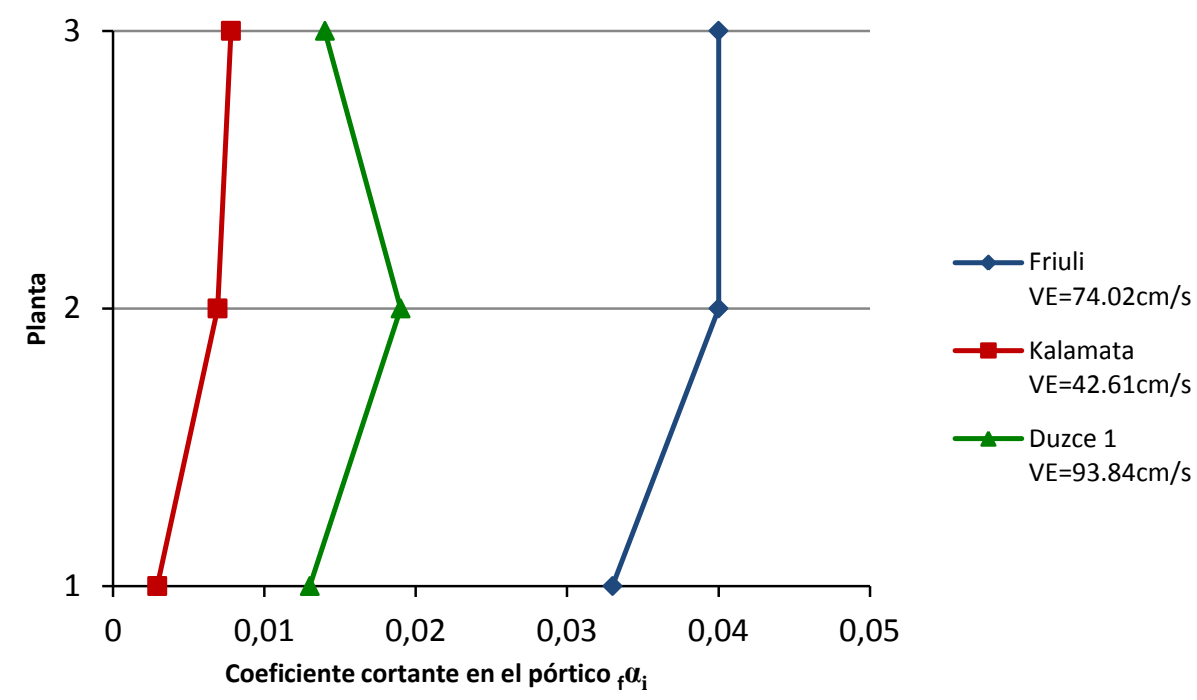

Fig. 5.9.47: Coeficiente de fuerza cortante en la parte flexible (pórtico) cuando los disipadores empiezan a plastificar, pórtico pa4pz2-1, suelo tipo III, campo cercano 


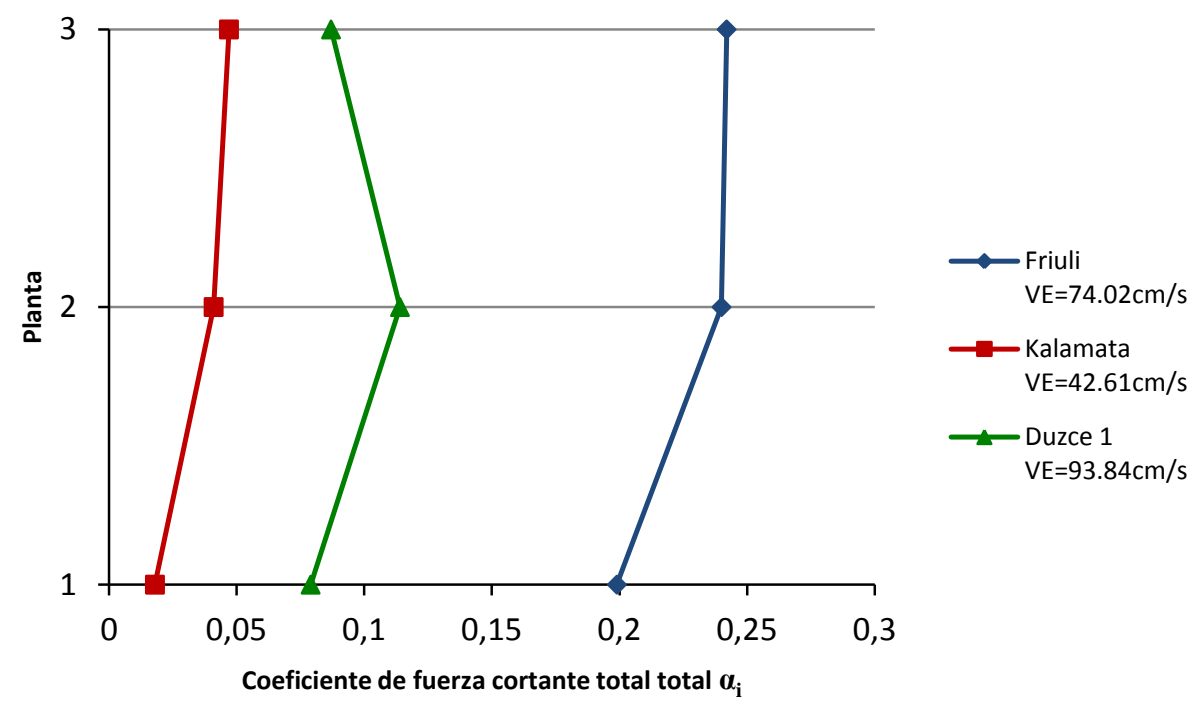

Fig. 5.5.48: Coeficiente de fuerza cortante total de planta, pórtico pa4pz2-1, suelo tipo III, campo cercano

\subsection{Suelo Tipo III (Campo lejano a la falla)}

\section{- Acelerograma №3 (Montenegro) $\Delta=24 \mathrm{Km}$}

Desplazamiento de fluencia del disipador ${ }_{s} \delta_{i}$

Planta 3a: ${ }_{s} \delta_{3}=0,1934 \mathrm{~cm}$

Planta 2a: ${ }_{s} \delta_{2}=0,3970 \mathrm{~cm}$

Planta 1a: ${ }_{s} \delta_{1}=0,4526 \mathrm{~cm}$

Resistencia lateral de los disipadores de una planta i dada ${ }_{s} Q_{i}$

Planta 3a: ${ }_{s} Q_{3}={ }_{s} k_{3} \times{ }_{s} \delta_{3}=385000 \mathrm{~N} / \mathrm{cm} \times 0,1934 \mathrm{~cm} \Rightarrow{ }_{s} Q_{3}=74459 \mathrm{~N}$

Planta 2a: ${ }_{s} Q_{2}={ }_{s} k_{2} \times{ }_{s} \delta_{2}=455000 \mathrm{~N} / \mathrm{cm} \times 0,3970 \mathrm{~cm} \Rightarrow{ }_{s} Q_{2}=180635 \mathrm{~N}$

Planta 1a: ${ }_{s} Q_{1}={ }_{s} k_{1} \times{ }_{s} \delta_{1}=475000 \mathrm{~N} / \mathrm{cm} \times 0,4526 \mathrm{~cm} \Rightarrow{ }_{s} Q_{1}=214985 \mathrm{~N}$

Coeficiente de fuerza cortante de fluencia de los disipadores de una planta i dada ${ }_{s} \alpha_{i}$

Planta 3a: ${ }_{s} \alpha_{3}=\frac{{ }_{s} Q_{3}(N)}{m_{3}\left(\frac{N s^{2}}{c m}\right) \times g\left(\frac{\mathrm{cm}}{\mathrm{s}^{2}}\right)}=\frac{74459}{885,82 \times 981} \Rightarrow \quad \underline{{ }_{s} \alpha_{3}=0,086}$

Planta 2 ${ }^{\mathrm{a}}:{ }_{s} \alpha_{2}=\frac{{ }_{s} Q_{2}(N)}{\left(m_{2}+m_{3}\right)\left(\frac{N s^{2}}{c m}\right) \times g\left(\frac{\mathrm{cm}}{\mathrm{s}^{2}}\right)}=\frac{180635}{(2 \times 885,82) \times 981} \Rightarrow \quad \underline{{ }_{s}} \alpha_{2}=0,104$

Planta 1ㄹ: $\alpha_{s}=\frac{{ }_{s} Q_{1}(N)}{\left(m_{1}+m_{2}+m_{3}\right)\left(\frac{N s^{2}}{c m}\right) \times g\left(\frac{c m}{s^{2}}\right)}=\frac{214985}{(3 \times 885,82) \times 981} \Rightarrow{ }_{s} \alpha_{1}=0,082$ 
Resistencia lateral aportada por la parte flexible (pórtico) cuando los disipadores empiezan a plastificar ${ }_{f} Q_{\text {máxi }}$

Planta 3a: ${ }_{f} Q_{\text {máx } 3}={ }_{f} k_{3} \times{ }_{s} \delta_{3}=77000 \mathrm{~N} / \mathrm{cm} \times 0,1934 \mathrm{~cm} \Rightarrow{ }_{f} Q_{\text {máx3 }}=14891,8 \mathrm{~N}$

Planta 2a: ${ }_{f} Q_{\text {máx } 2}={ }_{f} k_{2} \times{ }_{s} \delta_{2}=91000 \mathrm{~N} / \mathrm{cm} \times 0,3970 \mathrm{~cm} \Rightarrow{ }_{f} Q_{\text {máx } 2}=36127 \mathrm{~N}$

Planta 1ㄹ: ${ }_{f} Q_{m a ́ x 1}={ }_{f} k_{1} \times{ }_{s} \delta_{1}=95000 N / \mathrm{cm} \times 0,4526 \mathrm{~cm} \Rightarrow \overline{{ }_{f} Q_{\text {máx } 1}=42997 N}$

Coeficiente cortante en la parte flexible (pórtico) cuando los disipadores empiezan a plastificar ${ }_{f} \alpha_{i}$

Planta 3a: ${ }_{f} \alpha_{3}=\frac{{ }_{f} Q_{\text {máx3 }}(N)}{m_{3}\left(\frac{N s^{2}}{c m}\right) \times g\left(\frac{c m}{s^{2}}\right)}=\frac{14891,8}{885,82 \times 981} \Rightarrow \quad \underline{{ }_{f} \alpha_{3}=0,017}$

Planta 2a: ${ }_{f} \alpha_{2}=\frac{{ }_{f} Q_{m a ́ x z}(N)}{\left(m_{2}+m_{3}\right)\left(\frac{N s^{2}}{c m}\right) \times g\left(\frac{c m}{s^{2}}\right)}=\frac{36127}{(2 \times 885,82) \times 981} \Rightarrow \quad \underline{{ }_{f} \alpha_{2}=0,021}$

Planta 1ㄹ: ${ }_{f} \alpha_{1}=\frac{{ }_{f} Q_{m a ́ x 1}(N)}{\left(m_{1}+m_{2}+m_{3}\right)\left(\frac{N s^{2}}{c m}\right) \times g\left(\frac{c m}{s^{2}}\right)}=\frac{42997}{(3 \times 885,82) \times 981} \Rightarrow \underline{{ }_{f}} \alpha_{1}=0,016$

Coeficiente de fuerza cortante total de planta $\alpha_{i}$

Planta 3a: $\alpha_{3}={ }_{s} \alpha_{3}+{ }_{f} \alpha_{3}=0,086+0,017 \Rightarrow \alpha_{3}=0,103$

Planta 2a: $\alpha_{2}={ }_{s} \alpha_{2}+{ }_{f} \alpha_{2}=0,104+0,021 \Rightarrow \overline{\alpha_{2}=0,125}$

Planta 1ㅁ: $\alpha_{1}={ }_{s} \alpha_{1}+{ }_{f} \alpha_{1}=0,082+0,016 \Rightarrow \underline{\alpha_{1}=0,098}$

\section{- Acelerograma №14(Ano Liosia) $\Delta=14 \mathrm{Km}$}

Desplazamiento de fluencia del disipador ${ }_{s} \delta_{i}$

Planta 3a: ${ }_{s} \delta_{3}=0,0001 \mathrm{~cm}$

Planta 2a: ${ }_{s} \delta_{2}=0,0001 \mathrm{~cm}$

Planta 1a: ${ }_{s} \delta_{1}=0,0123 \mathrm{~cm}$

Resistencia lateral de los disipadores de una planta i dada ${ }_{s} Q_{i}$

Planta 3a: ${ }_{s} Q_{3}={ }_{s} k_{3} \times{ }_{s} \delta_{3}=385000 \mathrm{~N} / \mathrm{cm} \times 0,0001 \mathrm{~cm} \Rightarrow{ }_{s} Q_{3}=38,5 \mathrm{~N}$

Planta 2a: ${ }_{s} Q_{2}={ }_{s} k_{2} \times{ }_{s} \delta_{2}=455000 \mathrm{~N} / \mathrm{cm} \times 0,0001 \mathrm{~cm} \Rightarrow{ }_{s} Q_{2}=45,5 \mathrm{~N}$

Planta 1a: ${ }_{s} Q_{1}={ }_{s} k_{1} \times{ }_{s} \delta_{1}=475000 \mathrm{~N} / \mathrm{cm} \times 0,0123 \mathrm{~cm} \Rightarrow{ }_{s} Q_{1}=5842,5 \mathrm{~N}$ 
Coeficiente de fuerza cortante de fluencia de los disipadores de una planta i dada ${ }_{s} \alpha_{i}$

Planta 3ㄹ: ${ }_{s} \alpha_{3}=\frac{{ }_{s} Q_{3}(N)}{m_{3}\left(\frac{N s^{2}}{c m}\right) \times g\left(\frac{c m}{s^{2}}\right)}=\frac{38,5}{885,82 \times 981} \Rightarrow \quad \underline{{ }_{s} \alpha_{3}=4,430 e^{-05}}$

Planta 2ㄹ: ${ }_{s} \alpha_{2}=\frac{{ }_{s}(N)}{\left(m_{2}+m_{3}\right)\left(\frac{N s^{2}}{c m}\right) \times g\left(\frac{c m}{s^{2}}\right)}=\frac{45,5}{(2 \times 885,82) \times 981} \Rightarrow \quad{ }_{s} \alpha_{2}=2,618 e^{-05}$

Planta 1: ${ }_{s} \alpha_{1}=\frac{{ }_{s} Q_{1}(N)}{\left(m_{1}+m_{2}+m_{3}\right)\left(\frac{N s^{2}}{c m}\right) \times g\left(\frac{c m}{s^{2}}\right)}=\frac{5842,5}{(3 \times 885,82) \times 981} \Rightarrow \underline{{ }_{s} \alpha_{1}=2,241 e^{-03}}$

Resistencia lateral aportada por la parte flexible (pórtico) cuando los disipadores empiezan a plastificar ${ }_{f} Q_{\text {máxi }}$

Planta 3a: : ${ }_{f} Q_{\text {máx } 3}={ }_{f} k_{3} \times{ }_{s} \delta_{3}=77000 \mathrm{~N} / \mathrm{cm} \times 0,0001 \mathrm{~cm} \Rightarrow{ }_{f} Q_{\text {máx } 3}=7,7 \mathrm{~N}$

Planta 2a: ${ }_{f} Q_{\max 2}={ }_{f} k_{2} \times{ }_{s} \delta_{2}=91000 \mathrm{~N} / \mathrm{cm} \times 0,0001 \mathrm{~cm} \Rightarrow \overline{{ }_{f} Q_{\max 2}=9,1 \mathrm{~N}}$

Planta 1ㄹ: ${ }_{f} Q_{m a ́ x 1}={ }_{f} k_{1} \times{ }_{s} \delta_{1}=95000 \mathrm{~N} / \mathrm{cm} \times 0,0123 \mathrm{~cm} \Rightarrow{ }_{f} Q_{m a ́ x 1}=1168,5 \mathrm{~N}$

Coeficiente cortante en la parte flexible (pórtico) cuando los disipadores empiezan a plastificar ${ }_{f} \alpha_{i}$

Planta 3ạ: ${ }_{f} \alpha_{3}=\frac{{ }_{f} Q_{\text {máx } 3}(N)}{m_{3}\left(\frac{N s^{2}}{c m}\right) \times g\left(\frac{c m}{s^{2}}\right)}=\frac{7,7}{885,82 \times 981} \Rightarrow \quad \underline{{ }_{f} \alpha_{3}=8,861 e^{-06}}$

Planta 2a: ${ }_{f} \alpha_{2}=\frac{{ }_{f} Q_{\text {máx } 2}(N)}{\left(m_{2}+m_{3}\right)\left(\frac{N s^{2}}{c m}\right) \times g\left(\frac{c m}{s^{2}}\right)}=\frac{9,1}{(2 \times 885,82) \times 981} \Rightarrow \quad \underline{{ }_{f}} \alpha_{2}=5,236 e^{-06}$

Planta 1ㄹ: ${ }_{f} \alpha_{1}=\frac{{ }_{f} Q_{m a ́ x 1}(N)}{\left(m_{1}+m_{2}+m_{3}\right)\left(\frac{N s^{2}}{c m}\right) \times g\left(\frac{c m}{s^{2}}\right)}=\frac{1168,5}{(3 \times 885,82) \times 981} \Rightarrow{ }_{f} \alpha_{1}=4,482 e^{-04}$

Coeficiente de fuerza cortante total de planta $\alpha_{i}$

Planta 3a: $\alpha_{3}={ }_{s} \alpha_{3}+{ }_{f} \alpha_{3}=4,430 e^{-05}+8,861 e^{-06} \Rightarrow \underline{\alpha_{3}=5,316 e^{-05}}$

Planta 2a: $\alpha_{2}={ }_{s} \alpha_{2}+{ }_{f} \alpha_{2}=2,618 e^{-05}+5,236 e^{-06} \Rightarrow \alpha_{2}=3,142 e^{-05}$

Planta 1a: $\alpha_{1}={ }_{s} \alpha_{1}+{ }_{f} \alpha_{1}=2,241 e^{-03}+4,482 e^{-04} \Rightarrow \underline{\alpha_{1}=2,689 e^{-03}}$ 
Representación gráfica

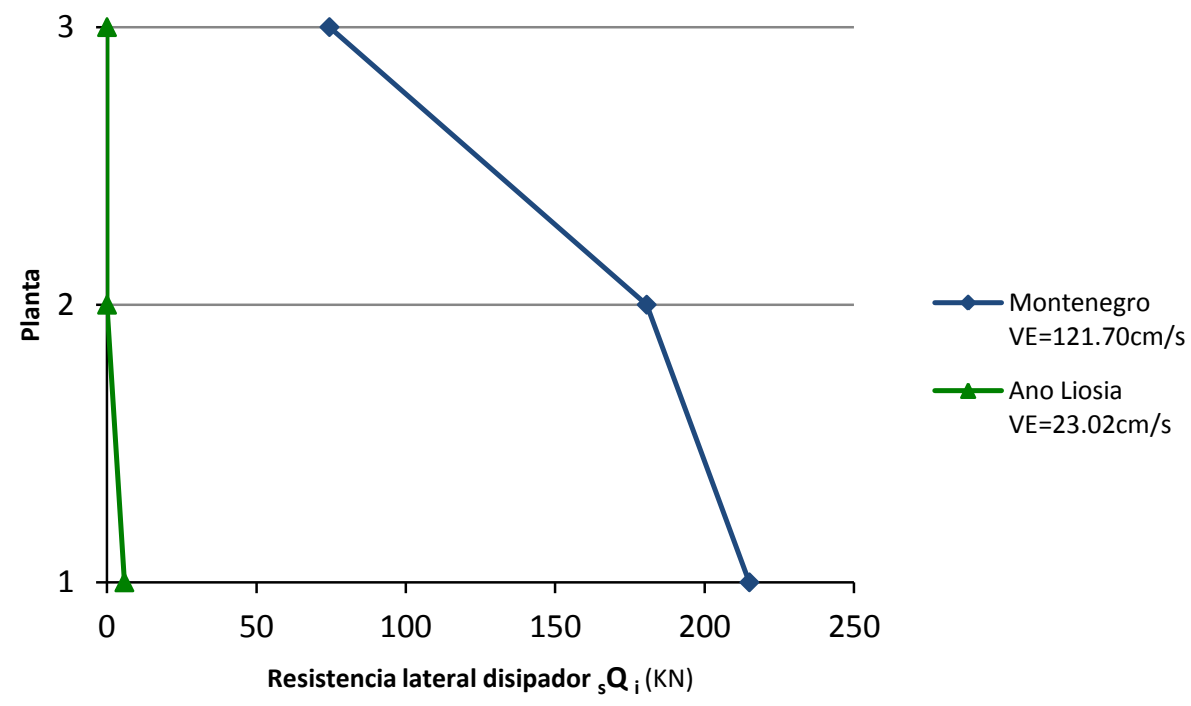

Fig. 5.5.49: Resistencia lateral de los disipadores de cada planta i, pórtico pa4pz2-1, suelo tipo III, campo lejano

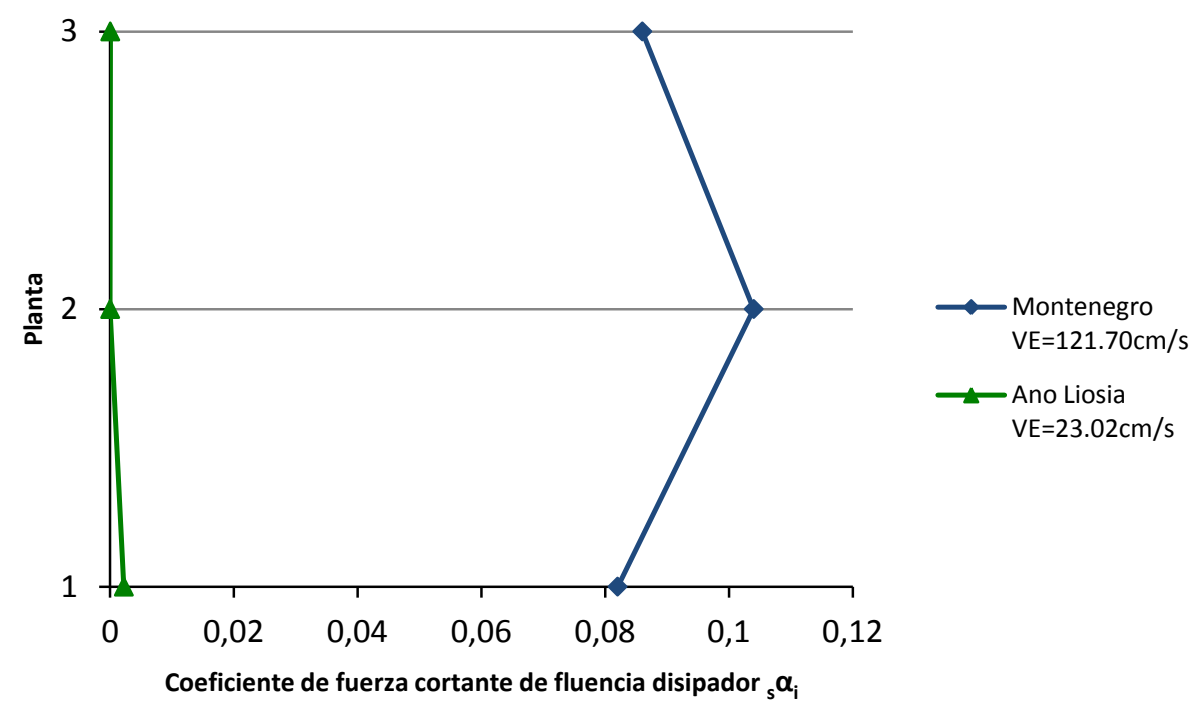

Fig. 5.5.50: Coeficiente de fuerza cortante de fluencia de los disipadores de cada planta i, pórtico pa4pz2-1, suelo tipo III, campo lejano 


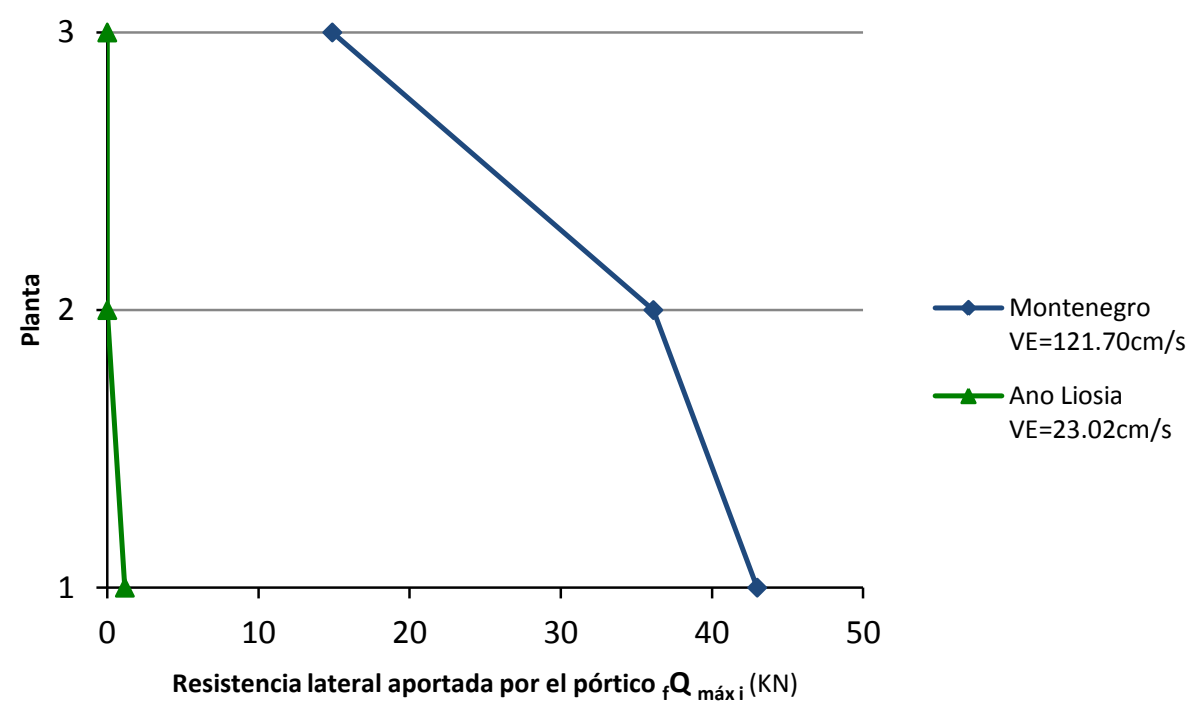

Fig. 5.5.51: Resistencia lateral aportada por la parte flexible (pórtico) cuando los disipadores empiezan a plastificar, pórtico pa4pz2-1, suelo tipo III, campo lejano

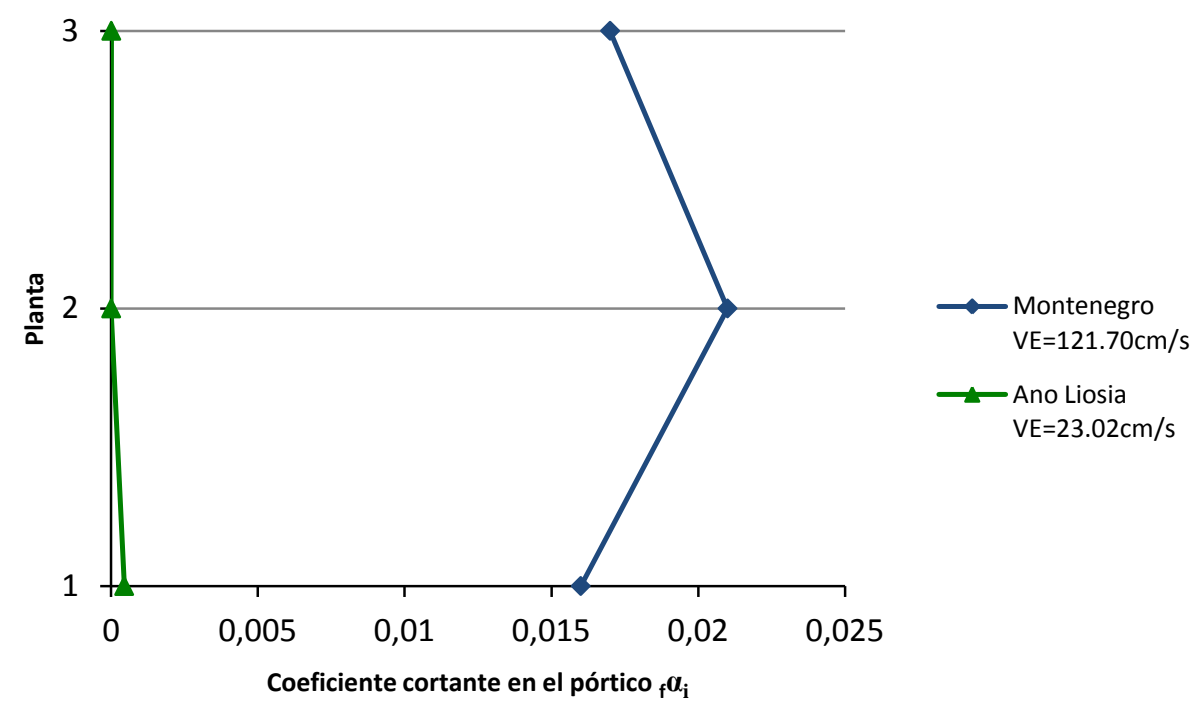

Fig. 5.5.52: Coeficiente de fuerza cortante en la parte flexible (pórtico) cuando los disipadores empiezan a plastificar, pórtico pa4pz2-1, suelo tipo III, campo lejano 


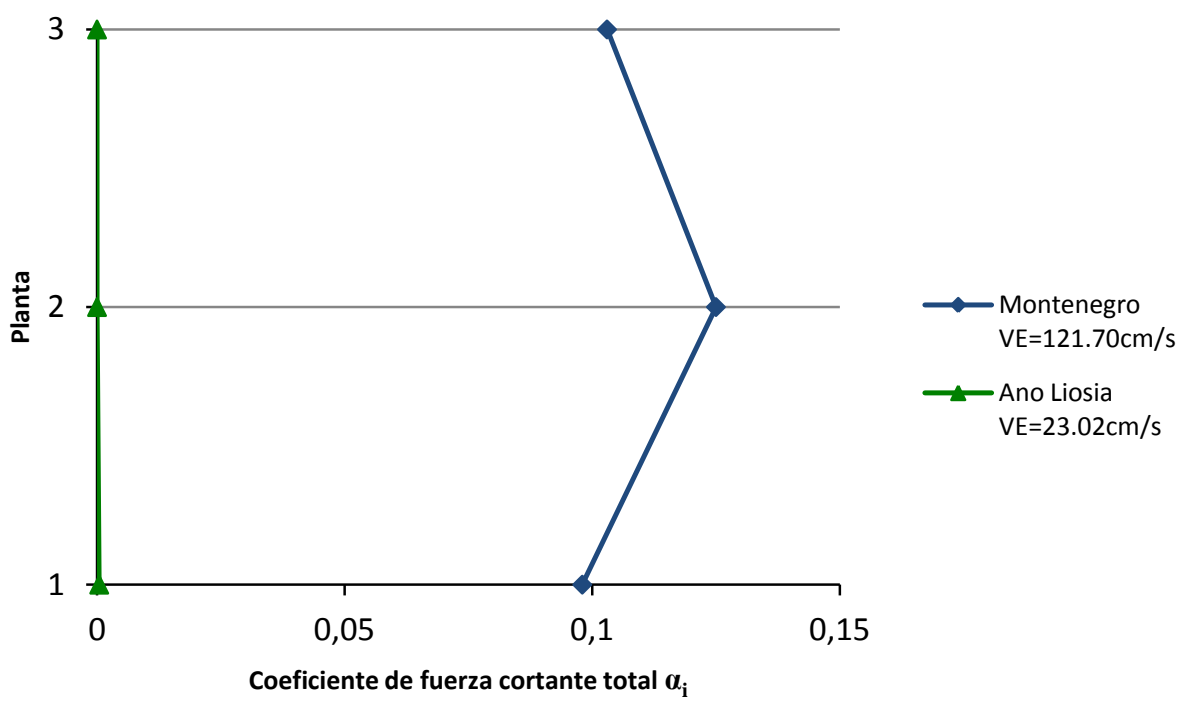

Fig. 5.5.53: Coeficiente de fuerza cortante total de planta, pórtico pa4pz2-1, suelo tipo III, campo lejano

\subsubsection{Pórtico pa6pz2-1 (6 plantas)}

\section{Masas por planta $m_{i}$}

La estructura se puede idealizar con un modelo de seis masas concentradas (situadas en el centro de masas del forjado), tal como indica la Figura 5.5.54.

$$
\text { Planta 6 } \mathrm{m}_{6}=739,04 \mathrm{Ns}^{2} / \mathrm{cm}
$$

Fig. 5.5.54: Idealización de un modelo de masas del pórtico pa6pz2-1

\section{Rigidez del disipador}

Rigidez disipador $=5 \times$ Rigidez sin disipador

Planta 6a: ${ }_{s} k_{6}=(56000 \mathrm{~N} / \mathrm{cm}) \times 5=280000 \mathrm{~N} / \mathrm{cm}$

Planta 5a: ${ }_{s} k_{5}=(83000 \mathrm{~N} / \mathrm{cm}) \times 5=415000 \mathrm{~N} / \mathrm{cm}$

Planta 4ạ: ${ }_{s} k_{4}=(113000 \mathrm{~N} / \mathrm{cm}) \times 5=565000 \mathrm{~N} / \mathrm{cm}$

Planta 3a: ${ }_{s} k_{3}=(177000 \mathrm{~N} / \mathrm{cm}) \times 5=885000 \mathrm{~N} / \mathrm{cm}$

Planta 2a: ${ }_{s} k_{2}=(224000 \mathrm{~N} / \mathrm{cm}) \times 5=1120000 \mathrm{~N} / \mathrm{cm}$ 
Planta 1ㅁ: ${ }_{s} k_{1}=(175000 \mathrm{~N} / \mathrm{cm}) \times 5=875000 \mathrm{~N} / \mathrm{cm}$

\subsection{Suelo Tipo I (Campo lejano a la falla)}

\section{- Acelerograma №4 (Montenegro) $\Delta=65 \mathrm{Km}$}

Desplazamiento de fluencia del disipador ${ }_{s} \delta_{i}$

Planta 6a: ${ }_{s} \delta_{6}=1,100 \mathrm{~cm}$

Planta 5a: ${ }_{s} \delta_{5}=1,200 \mathrm{~cm}$

Planta 4a: ${ }_{s} \delta_{4}=0,720 \mathrm{~cm}$

Planta 3a: ${ }_{s} \delta_{3}=0,570 \mathrm{~cm}$

Planta 2: ${ }_{s} \delta_{2}=0,470 \mathrm{~cm}$

Planta 1: ${ }_{s} \delta_{1}=0,610 \mathrm{~cm}$

Resistencia lateral de los disipadores de una planta i dada ${ }_{s} Q_{i}$

Planta 6a: ${ }_{s} Q_{6}={ }_{s} k_{6} \times{ }_{s} \delta_{6}=280000 \mathrm{~N} / \mathrm{cm} \times 1,100 \mathrm{~cm} \Rightarrow{ }_{s} Q_{6}=308000 \mathrm{~N}$

Planta 5a: ${ }_{s} Q_{5}={ }_{s} k_{5} \times{ }_{s} \delta_{5}=415000 \mathrm{~N} / \mathrm{cm} \times 1,200 \mathrm{~cm} \Rightarrow{ }_{s} Q_{5}=498000 \mathrm{~N}$

Planta 4a: ${ }_{s} Q_{1}={ }_{s} k_{4} \times{ }_{s} \delta_{4}=565000 \mathrm{~N} / \mathrm{cm} \times 0,720 \mathrm{~cm} \Rightarrow{ }_{s} Q_{4}=406800 \mathrm{~N}$

Planta 3a: ${ }_{s} Q_{3}={ }_{s} k_{3} \times{ }_{s} \delta_{3}=885000 \mathrm{~N} / \mathrm{cm} \times 0,570 \mathrm{~cm} \Rightarrow{ }_{s} Q_{3}=504450 \mathrm{~N}$

Planta 2a: ${ }_{s} Q_{2}={ }_{s} k_{2} \times{ }_{s} \delta_{2}=1120000 \mathrm{~N} / \mathrm{cm} \times 0,470 \mathrm{~cm} \Rightarrow{ }_{s} Q_{2}=526400 \mathrm{~N}$

Planta 1ㄹ: ${ }_{s} Q_{1}={ }_{s} k_{1} \times{ }_{s} \delta_{1}=875000 \mathrm{~N} / \mathrm{cm} \times 0,610 \mathrm{~cm} \Rightarrow{ }_{s} Q_{1}=533750 \mathrm{~N}$

Coeficiente de fuerza cortante de fluencia de los disipadores de una planta i dada ${ }_{s} \alpha_{i}$

Planta 6a: ${ }_{s} \alpha_{6}=\frac{{ }_{s} Q_{6}(N)}{m_{6}\left(\frac{N s^{2}}{c m}\right) \times g\left(\frac{c m}{s^{2}}\right)}=\frac{308000}{739,04 \times 981} \Rightarrow$

${ }_{s} \alpha_{6}=0,425$

Planta 5a: ${ }_{s} \alpha_{5}=\frac{{ }_{s}(N)}{\left(m_{5}+m_{6}\right)\left(\frac{N s^{2}}{c m}\right) \times g\left(\frac{\mathrm{cm}}{\mathrm{s}^{2}}\right)}=\frac{498000}{(2 \times 739,04) \times 981} \Rightarrow$

${ }_{s} \alpha_{5}=0,343$

Planta 4ㄹ: ${ }_{s} \alpha_{4}=\frac{{ }_{s} Q_{4}(N)}{\left(m_{4}+m_{5}+m_{6}\right)\left(\frac{N s^{2}}{c m}\right) \times g\left(\frac{c m}{s^{2}}\right)}=\frac{406800}{(3 \times 739,04) \times 981} \Rightarrow \quad \underline{{ }_{s} \alpha_{4}=0,187}$

Planta 3a: ${ }_{s} \alpha_{3}=\frac{{ }_{s} Q_{3}(N)}{\left(m_{3}+m_{4}+m_{5}+m_{6}\right)\left(\frac{N s^{2}}{c m}\right) \times g\left(\frac{\mathrm{cm}}{s^{2}}\right)}=\frac{504450}{(4 \times 739,04) \times 981} \Rightarrow$

${ }_{s} \alpha_{3}=0,174$ 
Planta 2a: ${ }_{s} \alpha_{2}=\frac{{ }_{s} Q_{2}(N)}{\left(m_{2}+m_{3}+m_{4}+m_{5}+m_{6}\right)\left(\frac{N s^{2}}{c m}\right) \times g\left(\frac{c m}{s^{2}}\right)}=\frac{526400}{(5 \times 739,04) \times 981} \Rightarrow$

Planta 1 : ${ }_{s} \alpha_{1}=\frac{{ }_{s} Q_{1}(N)}{\left(m_{1}+m_{2}+m_{3}+m_{4}+m_{5}+m_{6}\right)\left(\frac{N s^{2}}{c m}\right) \times g\left(\frac{\mathrm{cm}}{s^{2}}\right)}=\frac{533750}{(6 \times 739,04) \times 981} \Rightarrow$ ${ }_{s} \alpha_{1}=0,123$

Resistencia lateral aportada por la parte flexible (pórtico) cuando los disipadores empiezan a plastificar ${ }_{f} Q_{\text {máxi }}$

Planta 6a: ${ }_{f} Q_{\text {máx6 }}={ }_{f} k_{6} \times{ }_{s} \delta_{6}=56000 \mathrm{~N} / \mathrm{cm} \times 1,100 \mathrm{~cm} \Rightarrow{ }_{f} Q_{\text {máx6 }}=61600 \mathrm{~N}$

Planta 5a: ${ }_{f} Q_{\text {máx } 5}={ }_{f} k_{5} \times{ }_{s} \delta_{5}=83000 \mathrm{~N} / \mathrm{cm} \times 1,200 \mathrm{~cm} \Rightarrow{ }_{f} Q_{\text {máx }}=99600 \mathrm{~N}$

Planta 4ㄹ: ${ }_{f} Q_{\text {máx } 4}={ }_{f} k_{4} \times{ }_{s} \delta_{4}=113000 \mathrm{~N} / \mathrm{cm} \times 0,720 \mathrm{~cm} \Rightarrow{ }_{f} Q_{\max 4}=81360 \mathrm{~N}$

Planta 3a: ${ }_{f} Q_{\text {máx } 3}={ }_{f} k_{3} \times{ }_{s} \delta_{3}=177000 \mathrm{~N} / \mathrm{cm} \times 0,570 \mathrm{~cm} \Rightarrow{ }_{f} Q_{\text {máx } 3}=100890 \mathrm{~N}$

Planta 2a: ${ }_{f} Q_{\max 2}={ }_{f} k_{2} \times{ }_{s} \delta_{2}=224000 \mathrm{~N} / \mathrm{cm} \times 0,470 \mathrm{~cm} \Rightarrow{ }_{f} Q_{\max 2}=105280 \mathrm{~N}$

Planta 1: ${ }_{f} Q_{\text {máx } 1}={ }_{f} k_{1} \times{ }_{s} \delta_{1}=175000 \mathrm{~N} / \mathrm{cm} \times 0,610 \mathrm{~cm} \Rightarrow \overline{{ }_{f} Q_{\text {máx } 1}=106750 \mathrm{~N}}$

Coeficiente cortante en la parte flexible (pórtico) cuando los disipadores empiezan a plastificar ${ }_{f} \alpha_{i}$

Planta 6a: ${ }_{f} \alpha_{6}=\frac{{ }_{f} Q_{\text {máx6 }}(N)}{m_{6}\left(\frac{N s^{2}}{c m}\right) \times g\left(\frac{c m}{s^{2}}\right)}=\frac{61600}{739,04 \times 981} \Rightarrow$ ${ }_{f} \alpha_{6}=0,085$

Planta 5a: ${ }_{f} \alpha_{5}=\frac{{ }_{f} Q_{\text {máx }}(N)}{\left(m_{5}+m_{6}\right)\left(\frac{N s^{2}}{c m}\right) \times g\left(\frac{c m}{s^{2}}\right)}=\frac{99600}{(2 \times 739,04) \times 981} \Rightarrow$

${ }_{f} \alpha_{5}=0,069$

Planta 4ㄹ: ${ }_{f} \alpha_{4}=\frac{{ }_{f} Q_{\max x 4}(N)}{\left(m_{4}+m_{5}+m_{6}\right)\left(\frac{N s^{2}}{c m}\right) \times g\left(\frac{c m}{s^{2}}\right)}=\frac{81360}{(3 \times 739,04) \times 981} \Rightarrow \quad \underline{{ }_{f} \alpha_{4}=0,037}$ Planta 3a: ${ }_{f} \alpha_{3}=\frac{{ }_{f} Q_{\text {máx }}(N)}{\left(m_{3}+m_{4}+m_{5}+m_{6}\right)\left(\frac{N s^{2}}{\mathrm{~cm}}\right) \times g\left(\frac{\mathrm{cm}}{\mathrm{s}^{2}}\right)}=\frac{100890}{(4 \times 739,04) \times 981} \Rightarrow$

${ }_{f} \alpha_{3}=0,035$ 
Planta 2a: ${ }_{f} \alpha_{2}=\frac{{ }_{f} Q_{\text {máx }}(N)}{\left(m_{2}+m_{3}+m_{4}+m_{5}+m_{6}\right)\left(\frac{N s^{2}}{c m}\right) \times g\left(\frac{c m}{s^{2}}\right)}=\frac{105280}{(5 \times 739,04) \times 981} \Rightarrow$

Planta 1ㄹ: ${ }_{f} \alpha_{1}=\frac{{ }_{f} Q_{\text {máx }}(N)}{\left(m_{1}+m_{2}+m_{3}+m_{4}+m_{5}+m_{6}\right)\left(\frac{N s^{2}}{c m}\right) \times g\left(\frac{c m}{s^{2}}\right)}=\frac{106750}{(6 \times 739,04) \times 981} \Rightarrow$ ${ }_{f} \alpha_{1}=0,024$

Coeficiente de fuerza cortante total de planta $\alpha_{i}$

Planta 6a: $\alpha_{6}={ }_{s} \alpha_{6}+{ }_{f} \alpha_{6}=0,425+0,085 \Rightarrow \alpha_{6}=0,510$

Planta 5a: $\alpha_{5}={ }_{s} \alpha_{5}+{ }_{f} \alpha_{5}=0,343+0,069 \Rightarrow \alpha_{5}=0,412$

Planta 4a: $\alpha_{4}={ }_{s} \alpha_{4}+{ }_{f} \alpha_{4}=0,187+0,037 \Rightarrow \alpha_{4}=0,224$

Planta 3a: $\alpha_{3}={ }_{s} \alpha_{3}+{ }_{f} \alpha_{3}=0,174+0,035 \Rightarrow \alpha_{3}=0,209$

Planta 2a: $\alpha_{2}={ }_{s} \alpha_{2}+{ }_{f} \alpha_{2}=0,145+0,029 \Rightarrow \alpha_{2}=0,174$

Planta 1a: $\alpha_{1}={ }_{s} \alpha_{1}+{ }_{f} \alpha_{1}=0,123+0,024 \Rightarrow \alpha_{1}=0,147$

\section{- Acelerograma №5 (Campano Lucano) $\Delta=32 \mathrm{Km}$}

Desplazamiento de fluencia del disipador ${ }_{s} \delta_{i}$

Planta 6a: ${ }_{s} \delta_{6}=0,00009 \mathrm{~cm}$

Planta 5a: ${ }_{s} \delta_{5}=0,01600 \mathrm{~cm}$

Planta 4a : ${ }_{s} \delta_{4}=0,01000 \mathrm{~cm}$

Planta 3a: ${ }_{s} \delta_{3}=0,01000 \mathrm{~cm}$

Planta 2a: ${ }_{s} \delta_{2}=0,01000 \mathrm{~cm}$

Planta 1ä: ${ }_{s} \delta_{1}=0,02500 \mathrm{~cm}$

Resistencia lateral de los disipadores de una planta i dada ${ }_{s} Q_{i}$

Planta 6a: ${ }_{s} Q_{6}={ }_{s} k_{6} \times{ }_{s} \delta_{6}=280000 \mathrm{~N} / \mathrm{cm} \times 0,00009 \mathrm{~cm} \Rightarrow{ }_{s} Q_{6}=25,2 \mathrm{~N}$

Planta 5a: ${ }_{s} Q_{5}={ }_{s} k_{5} \times{ }_{s} \delta_{5}=415000 \mathrm{~N} / \mathrm{cm} \times 0,01600 \mathrm{~cm} \Rightarrow{ }_{s} Q_{5}=6640 \mathrm{~N}$

Planta 4a: ${ }_{s} Q_{1}={ }_{s} k_{4} \times{ }_{s} \delta_{4}=565000 \mathrm{~N} / \mathrm{cm} \times 0,01000 \mathrm{~cm} \Rightarrow{ }_{s} Q_{4}=5650 \mathrm{~N}$

Planta 3a: ${ }_{s} Q_{3}={ }_{s} k_{3} \times{ }_{s} \delta_{3}=885000 \mathrm{~N} / \mathrm{cm} \times 0,01000 \mathrm{~cm} \Rightarrow{ }_{s} Q_{3}=8850 \mathrm{~N}$

Planta 2a: ${ }_{s} Q_{2}={ }_{s} k_{2} \times{ }_{s} \delta_{2}=1120000 \mathrm{~N} / \mathrm{cm} \times 0,01000 \mathrm{~cm} \Rightarrow \overline{{ }_{s} Q_{2}=11200 \mathrm{~N}}$

Planta 1ㄹ: ${ }_{s} Q_{1}={ }_{s} k_{1} \times{ }_{s} \delta_{1}=875000 \mathrm{~N} / \mathrm{cm} \times 0,02500 \mathrm{~cm} \Rightarrow{ }_{s} Q_{1}=21875 \mathrm{~N}$ 
Coeficiente de fuerza cortante de fluencia de los disipadores de una planta i dada ${ }_{s} \alpha_{i}$

Planta 6a: ${ }_{s} \alpha_{6}=\frac{{ }_{2}(N)}{m_{6}\left(\frac{N s^{2}}{\mathrm{~cm}}\right) \times g\left(\frac{\mathrm{cm}}{\mathrm{s}^{2}}\right)}=\frac{25,2}{739,04 \times 981} \Rightarrow$

${ }_{s} \alpha_{6}=3,476 e^{-05}$

Planta 5ㄹ: ${ }_{s} \alpha_{5}=\frac{{ }_{s}(N)}{\left(m_{5}+m_{6}\right)\left(\frac{N s^{2}}{c m}\right) \times g\left(\frac{c m}{s^{2}}\right)}=\frac{6640}{(2 \times 739,04) \times 981} \Rightarrow \quad \underline{{ }_{s} \alpha_{5}=4,579 e^{-03}}$

Planta 4ㄹ: ${ }_{s} \alpha_{4}=\frac{{ }_{s} Q_{4}(N)}{\left(m_{4}+m_{5}+m_{6}\right)\left(\frac{N s^{2}}{c m}\right) \times g\left(\frac{c m}{s^{2}}\right)}=\frac{5650}{(3 \times 739,04) \times 981} \Rightarrow \quad{ }_{s} \alpha_{4}=2,598 e^{-03}$

Planta 3a: ${ }_{s} \alpha_{3}=\frac{{ }_{s} Q_{3}(N)}{\left(m_{3}+m_{4}+m_{5}+m_{6}\right)\left(\frac{N s^{2}}{c m}\right) \times g\left(\frac{\mathrm{cm}}{s^{2}}\right)}=\frac{8850}{(4 \times 739,04) \times 981} \Rightarrow$

Planta 2 ${ }^{\mathrm{a}}:{ }_{s} \alpha_{2}=\frac{{ }_{s} Q_{2}(N)}{\left(m_{2}+m_{3}+m_{4}+m_{5}+m_{6}\right)\left(\frac{N s^{2}}{\mathrm{~cm}}\right) \times g\left(\frac{\mathrm{cm}}{s^{2}}\right)}=\frac{11200}{{ }_{s} \alpha_{3}=3,052 e^{-03}}$

Planta 1ㄹ: ${ }_{s} \alpha_{1}=\frac{{ }_{s} Q_{1}(N)}{\left(m_{1}+m_{2}+m_{3}+m_{4}+m_{5}+m_{6}\right)\left(\frac{N s^{2}}{c m}\right) \times g\left(\frac{c m}{s^{2}}\right)}=\frac{21875}{(6 \times 739,04) \times 981} \Rightarrow$ $\underline{{ }_{s} \alpha_{1}=5,029 e^{-03}}$

Resistencia lateral aportada por la parte flexible (pórtico) cuando los disipadores empiezan a plastificar ${ }_{f} Q_{\text {máxi }}$

Planta 6a: ${ }_{f} Q_{\text {máx } 6}={ }_{f} k_{6} \times{ }_{s} \delta_{6}=56000 \mathrm{~N} / \mathrm{cm} \times 0,00009 \mathrm{~cm} \Rightarrow{ }_{f} Q_{\text {máx }}=5,04 N$

Planta 5a: : ${ }_{f} Q_{\text {máx } 5}={ }_{f} k_{5} \times{ }_{s} \delta_{5}=83000 \mathrm{~N} / \mathrm{cm} \times 0,01600 \mathrm{~cm} \Rightarrow{ }_{f} Q_{\operatorname{máx} 5}=1328 \mathrm{~N}$

Planta 4ạ: ${ }_{f} Q_{\text {máx } 4}={ }_{f} k_{4} \times{ }_{s} \delta_{4}=113000 \mathrm{~N} / \mathrm{cm} \times 0,01000 \mathrm{~cm} \Rightarrow{ }_{f} Q_{\text {máx } 4}=1130 \mathrm{~N}$

Planta 3a: ${ }_{f} Q_{\max 3}={ }_{f} k_{3} \times{ }_{s} \delta_{3}=177000 \mathrm{~N} / \mathrm{cm} \times 0,01000 \mathrm{~cm} \Rightarrow{ }_{f} Q_{\max 3}=1770 \mathrm{~N}$

Planta 2a: ${ }_{f} Q_{\text {máx } 2}={ }_{f} k_{2} \times{ }_{s} \delta_{2}=224000 \mathrm{~N} / \mathrm{cm} \times 0,01000 \mathrm{~cm} \Rightarrow{ }_{f} Q_{\operatorname{maxx} 2}=2240 \mathrm{~N}$

Planta 1ㄹ: ${ }_{f} Q_{\text {máxl }}={ }_{f} k_{1} \times{ }_{s} \delta_{1}=175000 \mathrm{~N} / \mathrm{cm} \times 0,02500 \mathrm{~cm} \Rightarrow \overline{{ }_{f} Q_{\text {máx } 1}=4375 \mathrm{~N}}$ 
Coeficiente cortante en la parte flexible (pórtico) cuando los disipadores empiezan a plastificar ${ }_{f} \alpha_{i}$

Planta 6a : ${ }_{f} \alpha_{6}=\frac{{ }_{f} Q_{\text {máx }}(N)}{m_{6}\left(\frac{N s^{2}}{c m}\right) \times g\left(\frac{c m}{s^{2}}\right)}=\frac{5,04}{739,04 \times 981} \Rightarrow$

$\underline{{ }_{f}} \alpha_{6}=6,952 e^{-06}$

Planta 5a: ${ }_{f} \alpha_{5}=\frac{{ }_{f} Q_{\max 5}(N)}{\left(m_{5}+m_{6}\right)\left(\frac{N s^{2}}{c m}\right) \times g\left(\frac{c m}{s^{2}}\right)}=\frac{1328}{(2 \times 739,04) \times 981} \Rightarrow$

$\underline{{ }_{f}} \alpha_{5}=9,159 e^{-04}$

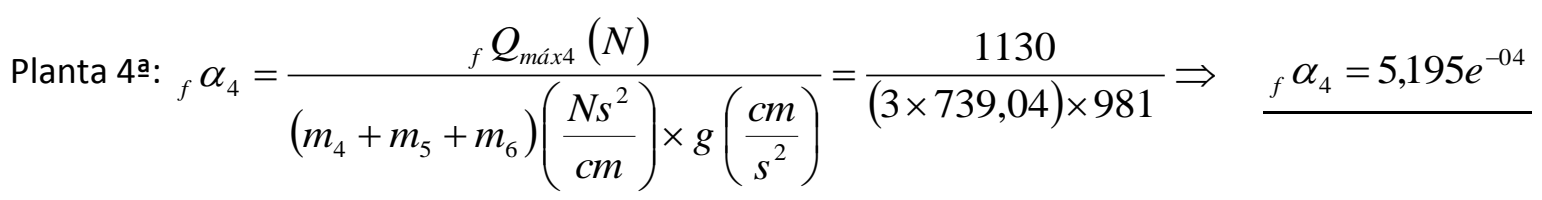
Planta 3a: ${ }_{f} \alpha_{3}=\frac{{ }_{f} Q_{\text {máx }}(N)}{\left(m_{3}+m_{4}+m_{5}+m_{6}\right)\left(\frac{N s^{2}}{c m}\right) \times g\left(\frac{\mathrm{cm}}{\mathrm{s}^{2}}\right)}=\frac{1770}{(4 \times 739,04) \times 981} \Rightarrow$

$\underline{{ }_{f} \alpha_{3}=6,103 e^{-04}}$ Planta 2a: ${ }_{f} \alpha_{2}=\frac{{ }_{f} Q_{\text {máx } 2}(N)}{\left(m_{2}+m_{3}+m_{4}+m_{5}+m_{6}\right)\left(\frac{N s^{2}}{c m}\right) \times g\left(\frac{c m}{s^{2}}\right)}=\frac{2240}{(5 \times 739,04) \times 981} \Rightarrow$ $\underline{{ }_{f}} \alpha_{2}=6,179 e^{-04}$ Planta 1ㄹ: ${ }_{f} \alpha_{1}=\frac{{ }_{f} Q_{\operatorname{maxx}}(N)}{\left(m_{1}+m_{2}+m_{3}+m_{4}+m_{5}+m_{6}\right)\left(\frac{N s^{2}}{c m}\right) \times g\left(\frac{c m}{s^{2}}\right)}=\frac{4375}{(6 \times 739,04) \times 981} \Rightarrow$ $\underline{{ }_{f}} \alpha_{1}=1,006 e^{-03}$

\section{Coeficiente de fuerza cortante total de planta $\alpha_{i}$}

\begin{tabular}{|c|c|}
\hline Planta 6a: $\alpha_{6}={ }_{s} \alpha_{6}+{ }_{f} \alpha_{6}=3,476 e^{-05}+6,952 e^{-06}=$ & $\alpha_{6}=4,171 e^{-05}$ \\
\hline Planta 5a: $\alpha_{5}={ }_{s} \alpha_{5}+{ }_{f} \alpha_{5}=4,579 e^{-03}+9,159 e^{-04} \Rightarrow$ & $\alpha_{5}=5,495 e^{-03}$ \\
\hline Planta 4a: $\alpha_{4}={ }_{s} \alpha_{4}+{ }_{f} \alpha_{4}=2,598 e^{-03}+5,195 e^{-04} \Rightarrow$ & $\alpha_{4}=3,117 e^{-03}$ \\
\hline Planta 3a: $\alpha_{3}={ }_{s} \alpha_{3}+{ }_{f} \alpha_{3}=3,052 e^{-03}+6,103 e^{-04}$ & $\alpha_{3}=3,662 e^{-03}$ \\
\hline Planta 2a: $\alpha_{2}={ }_{s} \alpha_{2}+{ }_{f} \alpha_{2}=3,090 e^{-03}+6,179 e^{-}$ & $\alpha_{2}=3,708 e^{-03}$ \\
\hline Planta 1a: $\alpha_{1}={ }_{s} \alpha_{1}+{ }_{f} \alpha_{1}=5,029 e^{-03}+1,006 e^{-03}$ & $\alpha_{1}=6,035 e^{-03}$ \\
\hline
\end{tabular}


Representación gráfica

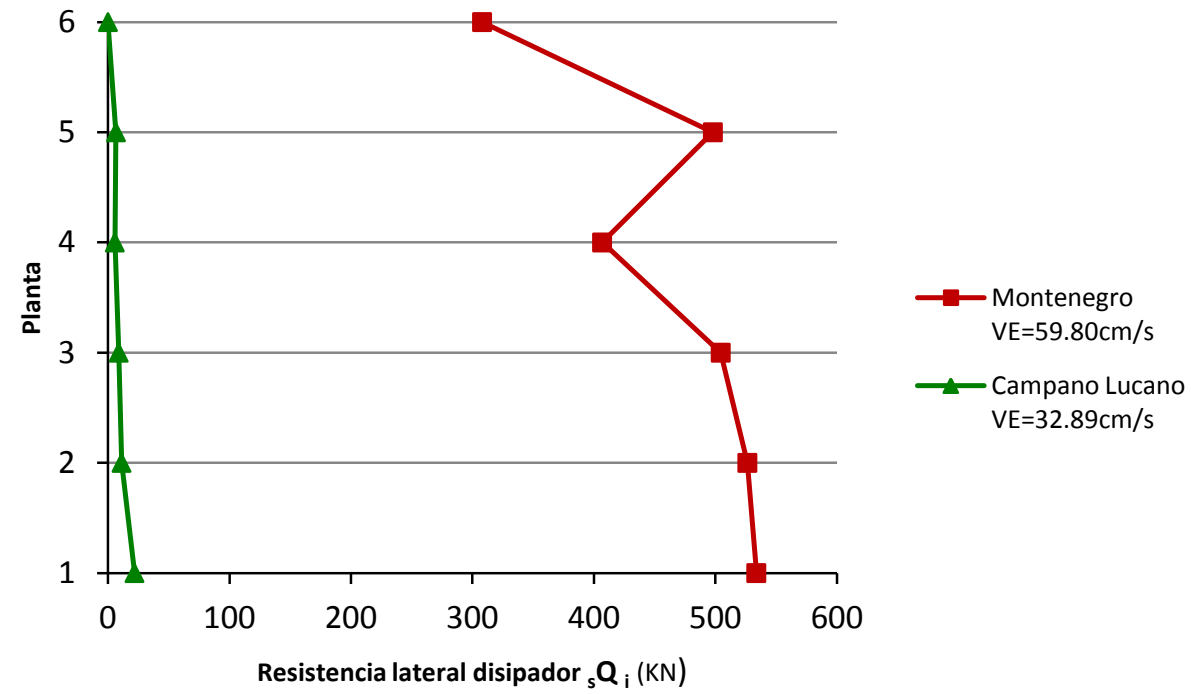

Fig. 5.5.55: Resistencia lateral de los disipadores de cada planta i, pórtico pa6pz2-1, suelo tipo I, campo lejano

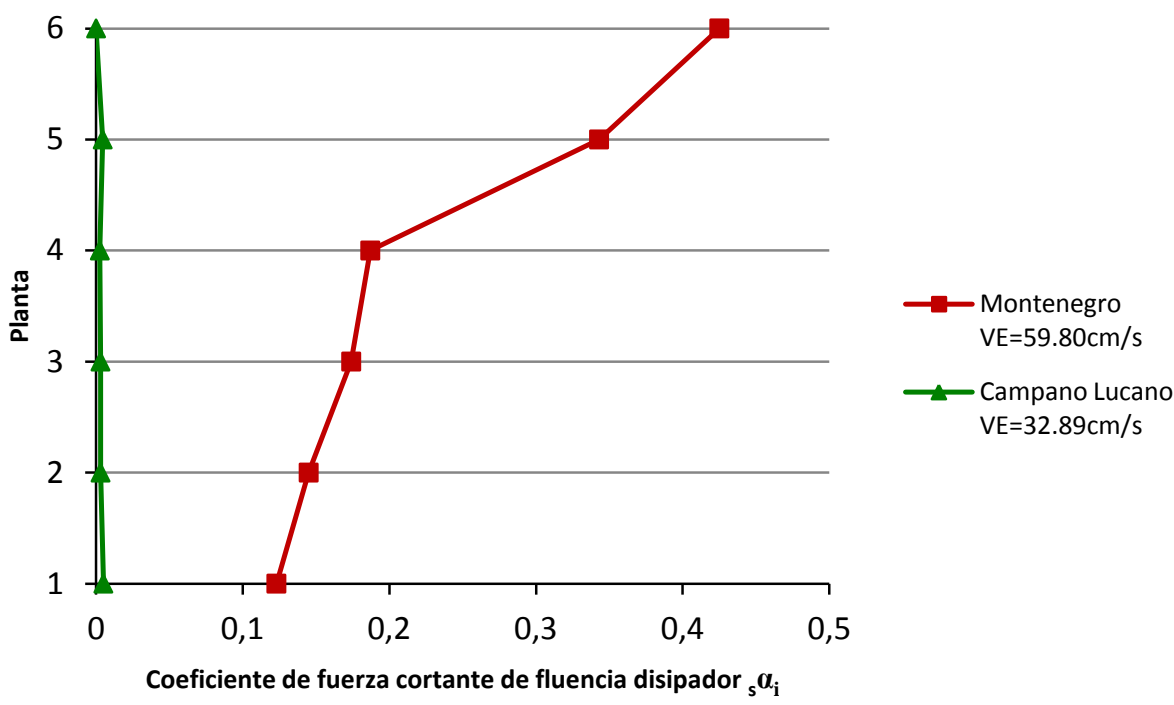

Fig. 5.5.56: Coeficiente de fuerza cortante de fluencia de los disipadores de cada planta i, pórtico pa6pz2-1, suelo tipo I, campo lejano 


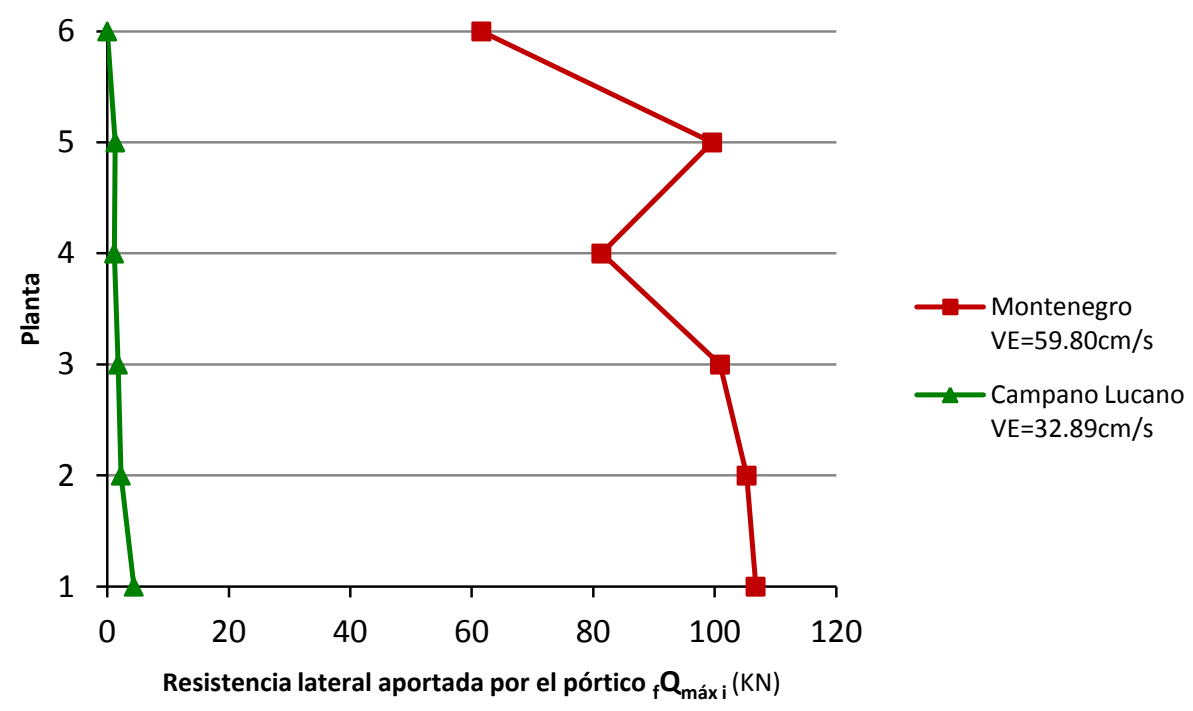

Fig. 5.5.57: Resistencia lateral aportada por la parte flexible (pórtico) cuando los disipadores empiezan a plastificar, pórtico pa6pz2-1, suelo tipo I, campo lejano

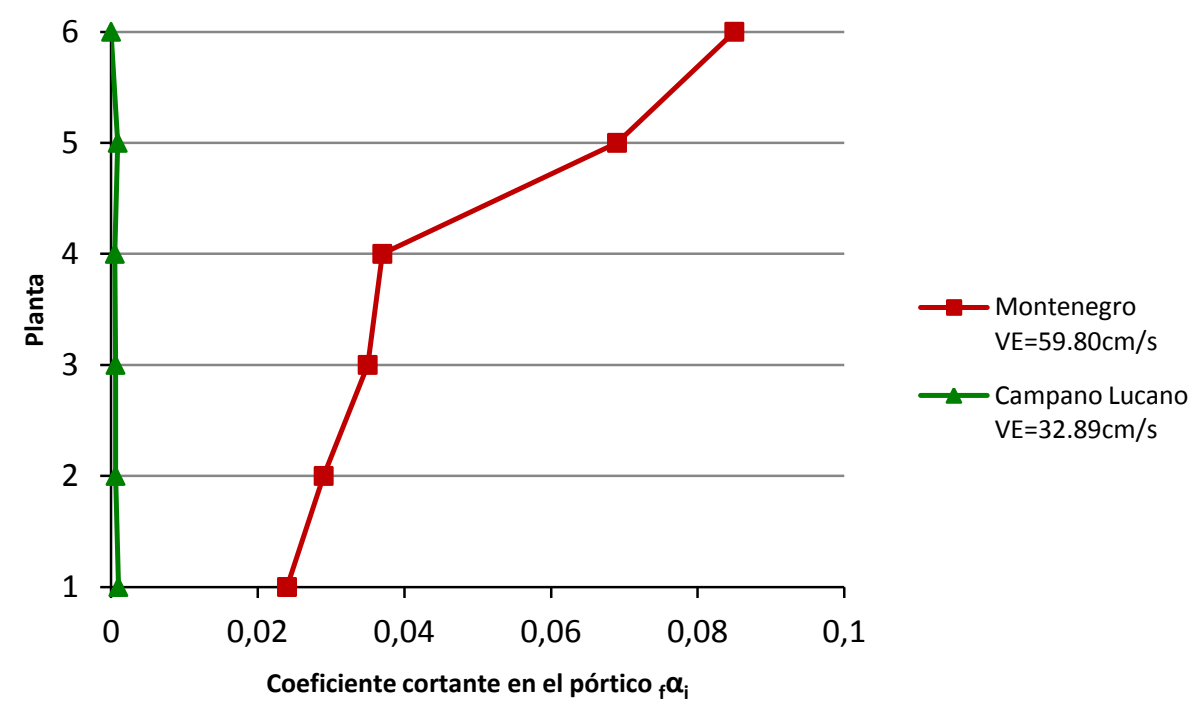

Fig. 5.5.58: Coeficiente de fuerza cortante en la parte flexible (pórtico) cuando los disipadores empiezan a plastificar, pórtico pa6pz2-1, suelo tipo I, campo lejano 


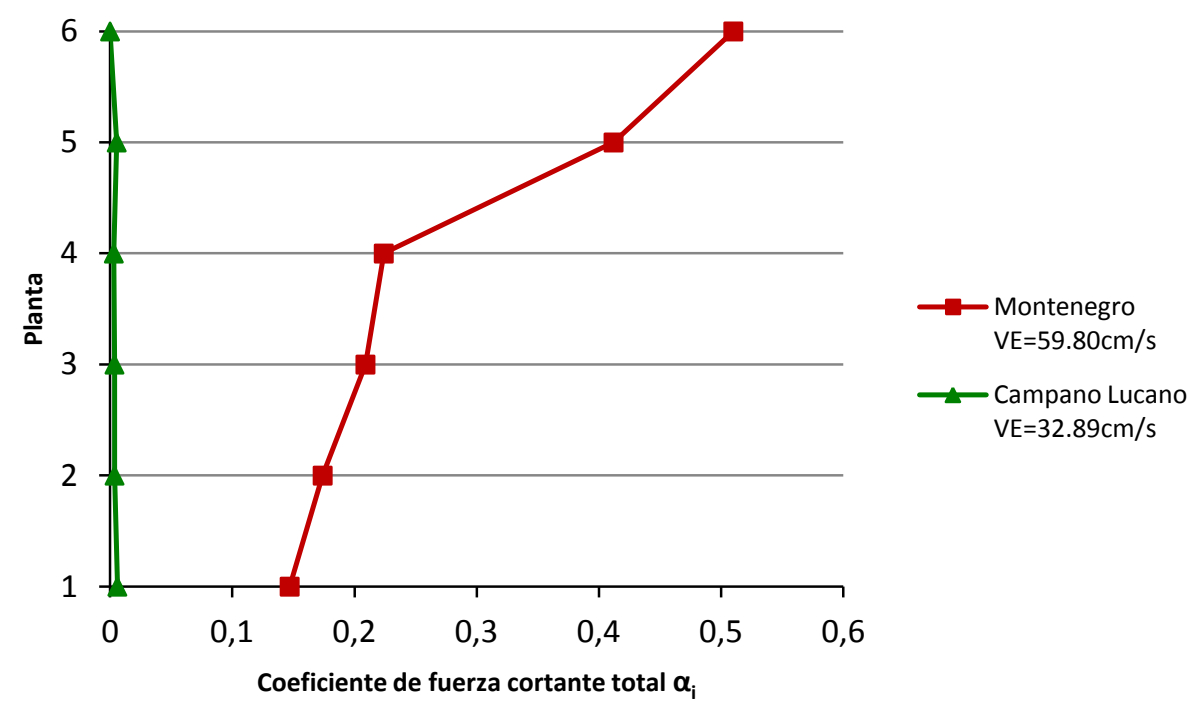

Fig. 5.5.59: Coeficiente de fuerza cortante total de planta, pórtico pa6pz2-1, suelo tipo I, campo lejano

\subsection{Suelo Tipo II (Campo cercano a la falla)}

\section{- Acelerograma №14 (Montenegro aftershock) $\Delta=8 \mathrm{Km}$}

Desplazamiento de fluencia del disipador ${ }_{s} \delta_{i}$

Planta 6a: ${ }_{s} \delta_{6}=0,260 \mathrm{~cm}$

Planta 5: ${ }_{s} \delta_{5}=0,280 \mathrm{~cm}$

Planta 4ㄹ: ${ }_{s} \delta_{4}=0,365 \mathrm{~cm}$

Planta 3a: ${ }_{s} \delta_{3}=0,240 \mathrm{~cm}$

Planta 2: ${ }_{s} \delta_{2}=0,240 \mathrm{~cm}$

Planta 1: ${ }_{s} \delta_{1}=0,400 \mathrm{~cm}$

Resistencia lateral de los disipadores de una planta i dada ${ }_{s} Q_{i}$

Planta 6a: ${ }_{s} Q_{6}={ }_{s} k_{6} \times{ }_{s} \delta_{6}=280000 \mathrm{~N} / \mathrm{cm} \times 0,260 \mathrm{~cm} \Rightarrow{ }_{s} Q_{6}=72800 \mathrm{~N}$

Planta 5a: ${ }_{s} Q_{5}={ }_{s} k_{5} \times{ }_{s} \delta_{5}=415000 \mathrm{~N} / \mathrm{cm} \times 0,280 \mathrm{~cm} \Rightarrow{ }_{s} Q_{5}=116200 \mathrm{~N}$

Planta 4a: ${ }_{s} Q_{1}={ }_{s} k_{4} \times{ }_{s} \delta_{4}=565000 \mathrm{~N} / \mathrm{cm} \times 0,365 \mathrm{~cm} \Rightarrow{ }_{s} Q_{4}=206225 \mathrm{~N}$

Planta 3a: ${ }_{s} Q_{3}={ }_{s} k_{3} \times{ }_{s} \delta_{3}=885000 \mathrm{~N} / \mathrm{cm} \times 0,240 \mathrm{~cm} \Rightarrow{ }_{s} Q_{3}=212400 \mathrm{~N}$

Planta 2a: ${ }_{s} Q_{2}={ }_{s} k_{2} \times{ }_{s} \delta_{2}=1120000 \mathrm{~N} / \mathrm{cm} \times 0,240 \mathrm{~cm} \Rightarrow{ }_{s} Q_{2}=268800 \mathrm{~N}$

Planta 1: ${ }_{s} Q_{1}={ }_{s} k_{1} \times{ }_{s} \delta_{1}=875000 \mathrm{~N} / \mathrm{cm} \times 0,400 \mathrm{~cm} \Rightarrow{ }_{s} Q_{1}=350000 \mathrm{~N}$ 
Coeficiente de fuerza cortante de fluencia de los disipadores de una planta i dada ${ }_{s} \alpha_{i}$

Planta 6a: ${ }_{s} \alpha_{6}=\frac{{ }_{s}(N)}{m_{6}\left(\frac{N s^{2}}{c m}\right) \times g\left(\frac{c m}{s^{2}}\right)}=\frac{72800}{739,04 \times 981} \Rightarrow$

${ }_{s} \alpha_{6}=0,100$

Planta 5ㄹ: $\alpha_{s}=\frac{{ }_{s}(N)}{\left(m_{5}+m_{6}\right)\left(\frac{N s^{2}}{c m}\right) \times g\left(\frac{c m}{s^{2}}\right)}=\frac{116200}{(2 \times 739,04) \times 981} \Rightarrow$

$\underline{{ }_{s}} \alpha_{5}=0,080$

Planta 4a: ${ }_{s} \alpha_{4}=\frac{{ }_{s} Q_{4}(N)}{\left(m_{4}+m_{5}+m_{6}\right)\left(\frac{N s^{2}}{c m}\right) \times g\left(\frac{c m}{s^{2}}\right)}=\frac{206225}{(3 \times 739,04) \times 981} \Rightarrow$

${ }_{s} \alpha_{4}=0,095$

Planta 3a: ${ }_{s} \alpha_{3}=\frac{{ }_{s} Q_{3}(N)}{\left(m_{3}+m_{4}+m_{5}+m_{6}\right)\left(\frac{N s^{2}}{c m}\right) \times g\left(\frac{c m}{s^{2}}\right)}=\frac{212400}{(4 \times 739,04) \times 981} \Rightarrow$

Planta 2a: ${ }_{s} \alpha_{2}=\frac{{ }_{s} Q_{2}(N)}{\left(m_{2}+m_{3}+m_{4}+m_{5}+m_{6}\right)\left(\frac{N s^{2}}{c m}\right) \times g\left(\frac{\mathrm{cm}}{s^{2}}\right)}=\frac{268800}{(5 \times 739,04) \times 981} \Rightarrow$

Planta 1ㄹ: ${ }_{s} \alpha_{1}=\frac{{ }_{s} Q_{1}(N)}{\left(m_{1}+m_{2}+m_{3}+m_{4}+m_{5}+m_{6}\right)\left(\frac{N s^{2}}{c m}\right) \times g\left(\frac{\mathrm{cm}}{s^{2}}\right)}=\frac{350000}{(6 \times 739,04) \times 981} \Rightarrow$ ${ }_{s} \alpha_{1}=0,080$

Resistencia lateral aportada por la parte flexible (pórtico) cuando los disipadores empiezan a plastificar ${ }_{f} Q_{\text {máxi }}$

Planta 6ạ: ${ }_{f} Q_{\text {máx6 }}={ }_{f} k_{6} \times{ }_{s} \delta_{6}=56000 \mathrm{~N} / \mathrm{cm} \times 0,260 \mathrm{~cm} \Rightarrow{ }_{f} Q_{\text {máx }}=14560 \mathrm{~N}$

Planta 5a: ${ }_{f} Q_{\text {máx } 5}={ }_{f} k_{5} \times{ }_{s} \delta_{5}=83000 \mathrm{~N} / \mathrm{cm} \times 0,280 \mathrm{~cm} \Rightarrow{ }_{f} Q_{\max 5}=23240 \mathrm{~N}$

Planta 4a: ${ }_{f} Q_{\text {máx } 4}={ }_{f} k_{4} \times{ }_{s} \delta_{4}=113000 \mathrm{~N} / \mathrm{cm} \times 0,365 \mathrm{~cm} \Rightarrow{ }_{f} Q_{\text {máx } 4}=41245 \mathrm{~N}$

Planta 3a: ${ }_{f} Q_{\text {máx } 3}={ }_{f} k_{3} \times{ }_{s} \delta_{3}=177000 \mathrm{~N} / \mathrm{cm} \times 0,240 \mathrm{~cm} \Rightarrow{ }_{f} Q_{\operatorname{máx3}}=42480 \mathrm{~N}$

Planta 2a: : ${ }_{f} Q_{\text {máx } 2}={ }_{f} k_{2} \times{ }_{s} \delta_{2}=224000 \mathrm{~N} / \mathrm{cm} \times 0,240 \mathrm{~cm} \Rightarrow{ }_{f} Q_{\operatorname{máx2}}=53760 \mathrm{~N}$

Planta 1ㄹ: ${ }_{f} Q_{\text {máx } 1}={ }_{f} k_{1} \times{ }_{s} \delta_{1}=175000 \mathrm{~N} / \mathrm{cm} \times 0,400 \mathrm{~cm} \Rightarrow{ }_{f} Q_{\text {máx } 1}=70000 \mathrm{~N}$ 
Coeficiente cortante en la parte flexible (pórtico) cuando los disipadores empiezan a plastificar ${ }_{f} \alpha_{i}$

Planta 6a: ${ }_{f} \alpha_{6}=\frac{{ }_{f} Q_{\text {maxx }}(N)}{m_{6}\left(\frac{N s^{2}}{c m}\right) \times g\left(\frac{c m}{s^{2}}\right)}=\frac{14560}{739,04 \times 981} \Rightarrow$

$\underline{{ }_{f}} \alpha_{6}=0,020$

Planta 5a: ${ }_{f} \alpha_{5}=\frac{{ }_{f} Q_{\operatorname{máx} 5}(N)}{\left(m_{5}+m_{6}\right)\left(\frac{N s^{2}}{c m}\right) \times g\left(\frac{\mathrm{cm}}{\mathrm{s}^{2}}\right)}=\frac{23240}{(2 \times 739,04) \times 981} \Rightarrow$

$\underline{{ }_{f} \alpha_{5}=0,016}$

Planta 4ㄹ: ${ }_{f} \alpha_{4}=\frac{{ }_{f} Q_{\text {máx }}(N)}{\left(m_{4}+m_{5}+m_{6}\right)\left(\frac{N s^{2}}{c m}\right) \times g\left(\frac{c m}{s^{2}}\right)}=\frac{41245}{(3 \times 739,04) \times 981} \Rightarrow$

$\underline{{ }_{f}} \alpha_{4}=0,019$

Planta 3a: ${ }_{f} \alpha_{3}=\frac{{ }_{f} Q_{\text {máx }}(N)}{\left(m_{3}+m_{4}+m_{5}+m_{6}\right)\left(\frac{N s^{2}}{\mathrm{~cm}}\right) \times g\left(\frac{\mathrm{cm}}{\mathrm{s}^{2}}\right)}=\frac{42480}{(4 \times 739,04) \times 981} \Rightarrow$

Planta 2ä: ${ }_{f} \alpha_{2}=\frac{{ }_{f} Q_{\max x 2}(N)}{\left(m_{2}+m_{3}+m_{4}+m_{5}+m_{6}\right)\left(\frac{N s^{2}}{c m}\right) \times g\left(\frac{c m}{s^{2}}\right)}=\frac{53760}{(5 \times 739,04) \times 981} \Rightarrow$

Planta 1a: ${ }_{f} \alpha_{1}=\frac{{ }_{f} Q_{\text {máx }}(N)}{\left(m_{1}+m_{2}+m_{3}+m_{4}+m_{5}+m_{6}\right)\left(\frac{N s^{2}}{c m}\right) \times g\left(\frac{c m}{s^{2}}\right)}=\frac{70000}{(6 \times 739,04) \times 981} \Rightarrow$ ${ }_{f} \alpha_{1}=0,016$

Coeficiente de fuerza cortante total de planta $\alpha_{i}$

Planta 6a: $\alpha_{6}={ }_{s} \alpha_{6}+{ }_{f} \alpha_{6}=0,100+0,020 \Rightarrow \alpha_{6}=0,120$

Planta 5a: $\alpha_{5}={ }_{s} \alpha_{5}+{ }_{f} \alpha_{5}=0,080+0,016 \Rightarrow \alpha_{5}=0,096$

Planta 4a: $\alpha_{4}={ }_{s} \alpha_{4}+{ }_{f} \alpha_{4}=0,095+0,019 \Rightarrow \alpha_{4}=0,114$

Planta 3a: $\alpha_{3}={ }_{s} \alpha_{3}+{ }_{f} \alpha_{3}=0,073+0,015 \Rightarrow \alpha_{3}=0,088$

Planta 2a: $\alpha_{2}={ }_{s} \alpha_{2}+{ }_{f} \alpha_{2}=0,074+0,015 \Rightarrow \underline{\alpha_{2}=0,089}$

Planta 1: $\alpha_{1}={ }_{s} \alpha_{1}+{ }_{f} \alpha_{1}=0,080+0,016 \Rightarrow \alpha_{1}=0,096$ 
Representación gráfica

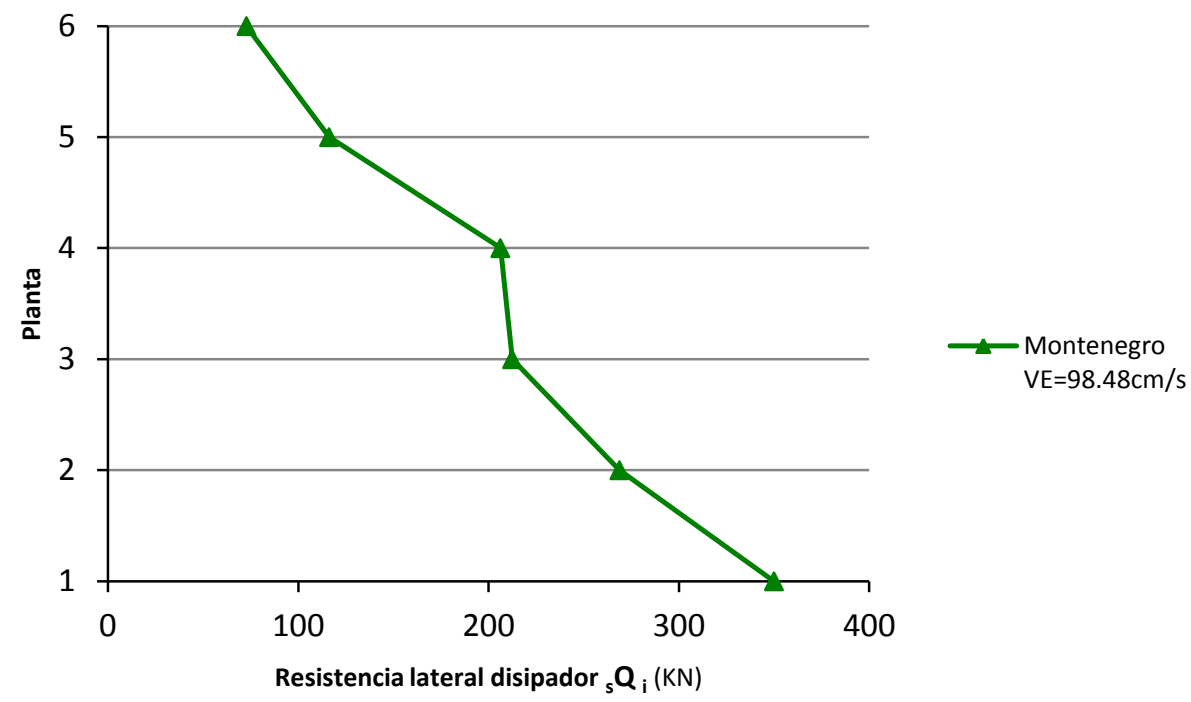

Fig. 5.5.60: Resistencia lateral de los disipadores de cada planta i, pórtico pa6pz2-1, suelo tipo II, campo cercano

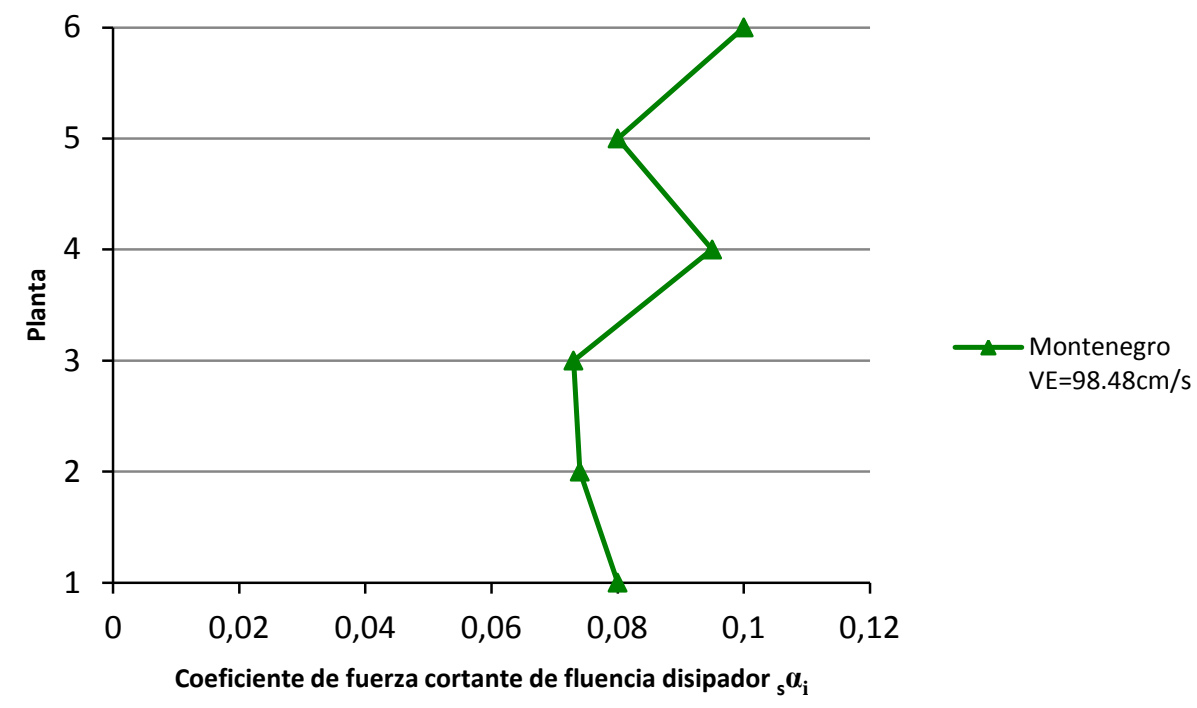

Fig. 5.5.61: Coeficiente de fuerza cortante de fluencia de los disipadores de cada planta i, pórtico pa6pz2-1, suelo tipo II, campo cercano 


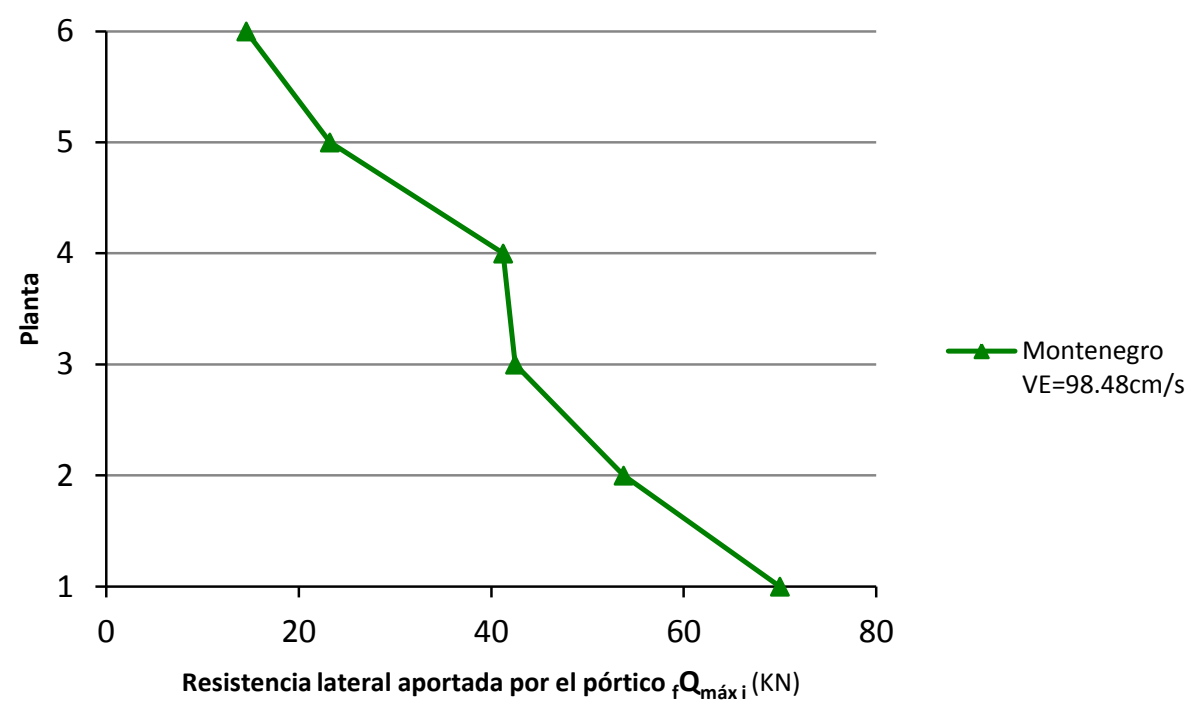

Fig. 5.5.62: Resistencia lateral aportada por la parte flexible (pórtico) cuando los disipadores empiezan a plastificar, pórtico pa6pz2-1, suelo tipo II, campo cercano

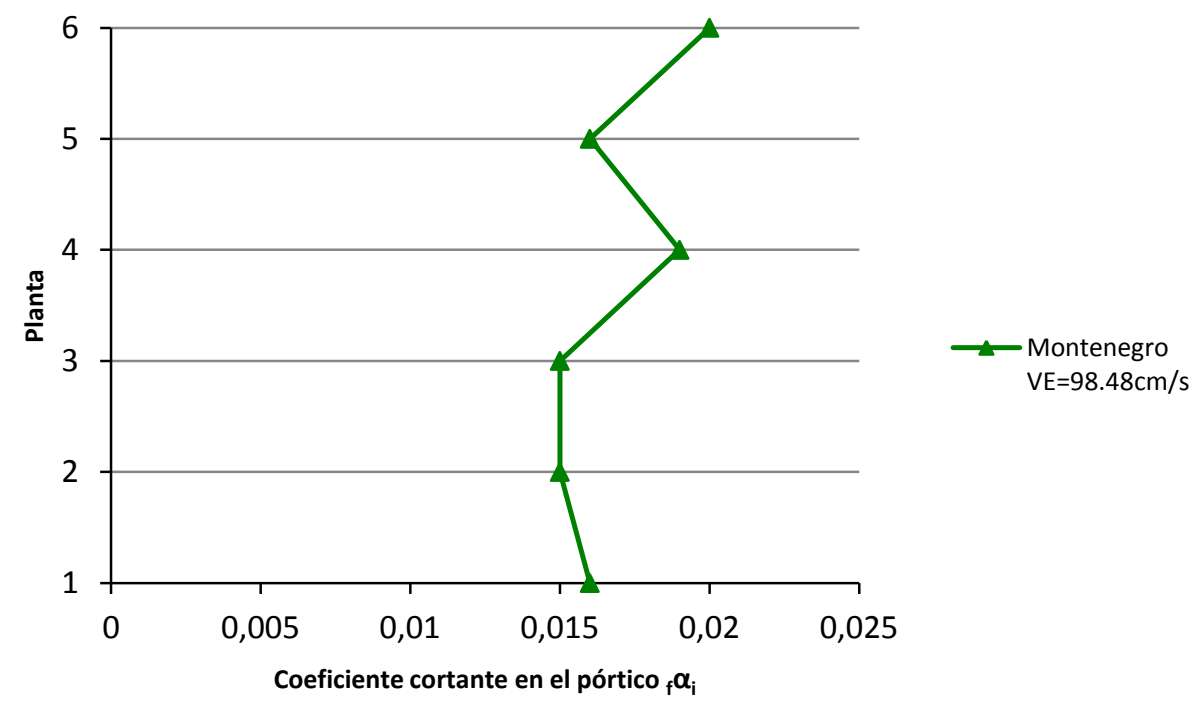

Fig. 5.5.63: Coeficiente de fuerza cortante en la parte flexible (pórtico) cuando los disipadores empiezan a plastificar, pórtico pa6pz2-1, suelo tipo II, campo cercano 


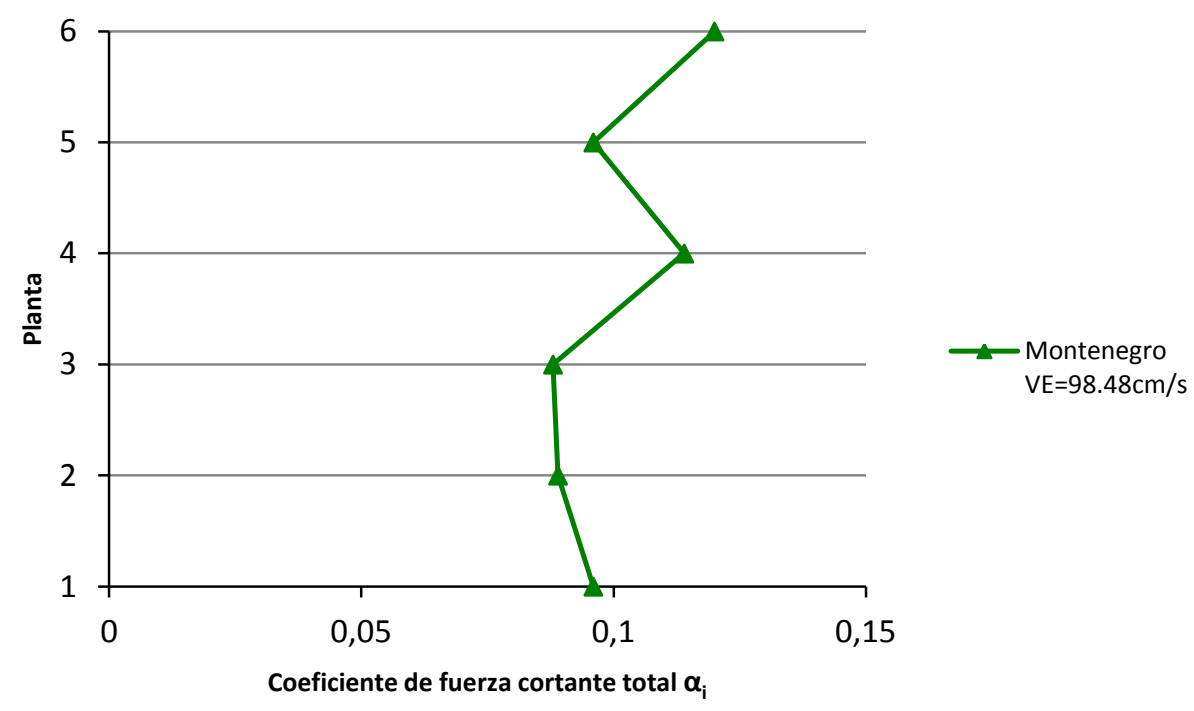

Fig. 5.5.64: Coeficiente de fuerza cortante total de planta, pórtico pa6pz2-1, suelo tipo II, campo cercano

\subsection{Suelo Tipo III (Campo cercano a la falla)}

\section{- Acelerograma №2 (Friuli aftershock) $\Delta=10 \mathrm{Km}$}

Desplazamiento de fluencia del disipador ${ }_{s} \delta_{i}$

Planta 6a: ${ }_{s} \delta_{6}=0,11000 \mathrm{~cm}$

Planta 5ㄹ: ${ }_{s} \delta_{5}=0,06900 \mathrm{~cm}$

Planta 4a : ${ }_{s} \delta_{4}=0,04300 \mathrm{~cm}$

Planta 3a: ${ }_{s} \delta_{3}=0,00004 \mathrm{~cm}$

Planta 2a: ${ }_{s} \delta_{2}=0,00390 \mathrm{~cm}$

Planta 1a: ${ }_{s} \delta_{1}=0,02700 \mathrm{~cm}$

Resistencia lateral de los disipadores de una planta i dada ${ }_{s} Q_{i}$

Planta 6a. ${ }_{s} Q_{6}={ }_{s} k_{6} \times{ }_{s} \delta_{6}=280000 \mathrm{~N} / \mathrm{cm} \times 0,11000 \mathrm{~cm} \Rightarrow \underline{{ }_{s} Q_{6}=30800 \mathrm{~N}}$

Planta 5ㅁ: ${ }_{s} Q_{5}={ }_{s} k_{5} \times{ }_{s} \delta_{5}=415000 \mathrm{~N} / \mathrm{cm} \times 0,06900 \mathrm{~cm} \Rightarrow{ }_{s} Q_{5}=28635 \mathrm{~N}$

Planta 4ㄹ: ${ }_{s} Q_{1}={ }_{s} k_{4} \times{ }_{s} \delta_{4}=565000 \mathrm{~N} / \mathrm{cm} \times 0,04300 \mathrm{~cm} \Rightarrow{ }_{s} Q_{4}=24295 \mathrm{~N}$

Planta 3a: ${ }_{s} Q_{3}={ }_{s} k_{3} \times{ }_{s} \delta_{3}=885000 \mathrm{~N} / \mathrm{cm} \times 0,00004 \mathrm{~cm} \Rightarrow{ }_{s} Q_{3}=35,4 \mathrm{~N}$

Planta 2a: ${ }_{s} Q_{2}={ }_{s} k_{2} \times{ }_{s} \delta_{2}=1120000 \mathrm{~N} / \mathrm{cm} \times 0,00390 \mathrm{~cm} \Rightarrow \overline{Q_{2}=4368 \mathrm{~N}}$

Planta 1: ${ }_{s} Q_{1}={ }_{s} k_{1} \times{ }_{s} \delta_{1}=875000 \mathrm{~N} / \mathrm{cm} \times 0,02700 \mathrm{~cm} \Rightarrow \overline{{ }_{s} Q_{1}=23625 \mathrm{~N}}$ 
Coeficiente de fuerza cortante de fluencia de los disipadores de una planta i dada ${ }_{s} \alpha_{i}$

Planta 6a: ${ }_{s} \alpha_{6}=\frac{{ }_{2}(N)}{m_{6}\left(\frac{N s^{2}}{\mathrm{~cm}}\right) \times g\left(\frac{\mathrm{cm}}{\mathrm{s}^{2}}\right)}=\frac{30800}{739,04 \times 981} \Rightarrow$

${ }_{s} \alpha_{6}=0,042$

Planta 5ㄹ: $\alpha_{s}=\frac{{ }_{s}(N)}{\left(m_{5}+m_{6}\right)\left(\frac{N s^{2}}{c m}\right) \times g\left(\frac{c m}{s^{2}}\right)}=\frac{28635}{(2 \times 739,04) \times 981} \Rightarrow$

${ }_{s} \alpha_{5}=0,020$

Planta 4a: ${ }_{s} \alpha_{4}=\frac{{ }_{s} Q_{4}(N)}{\left(m_{4}+m_{5}+m_{6}\right)\left(\frac{N s^{2}}{c m}\right) \times g\left(\frac{c m}{s^{2}}\right)}=\frac{24295}{(3 \times 739,04) \times 981} \Rightarrow$

${ }_{s} \alpha_{4}=0,011$

Planta 3a: ${ }_{s} \alpha_{3}=\frac{{ }_{s} Q_{3}(N)}{\left(m_{3}+m_{4}+m_{5}+m_{6}\right)\left(\frac{N s^{2}}{c m}\right) \times g\left(\frac{c m}{s^{2}}\right)}=\frac{35,4}{(4 \times 739,04) \times 981} \Rightarrow$

Planta 2a: ${ }_{s} \alpha_{2}=\frac{{ }_{s} Q_{2}(N)}{\left(m_{2}+m_{3}+m_{4}+m_{5}+m_{6}\right)\left(\frac{N s^{2}}{c m}\right) \times g\left(\frac{\mathrm{cm}}{s^{2}}\right)}=\frac{4368}{(5 \times 739,04) \times 981} \Rightarrow$

Planta 1ㄹ: ${ }_{s} \alpha_{1}=\frac{{ }_{s} Q_{1}(N)}{\left(m_{1}+m_{2}+m_{3}+m_{4}+m_{5}+m_{6}\right)\left(\frac{N s^{2}}{c m}\right) \times g\left(\frac{c m}{s^{2}}\right)}=\frac{23625}{(6 \times 739,04) \times 981} \Rightarrow$

${ }_{s} \alpha_{1}=5,431 e^{-03}$

Resistencia lateral aportada por la parte flexible (pórtico) cuando los disipadores empiezan a plastificar ${ }_{f} Q_{\text {máxi }}$

Planta 6a: ${ }_{f} Q_{\text {máx } 6}={ }_{f} k_{6} \times{ }_{s} \delta_{6}=56000 \mathrm{~N} / \mathrm{cm} \times 0,11000 \mathrm{~cm} \Rightarrow{ }_{f} Q_{\text {máx6 }}=6160 \mathrm{~N}$

Planta 5a: ${ }_{f} Q_{\text {máx } x}={ }_{f} k_{5} \times{ }_{s} \delta_{5}=83000 \mathrm{~N} / \mathrm{cm} \times 0,06900 \mathrm{~cm} \Rightarrow{ }_{f} Q_{\text {máx } 5}=5727 \mathrm{~N}$

Planta 4ạ: ${ }_{f} Q_{\text {máx } 4}={ }_{f} k_{4} \times{ }_{s} \delta_{4}=113000 \mathrm{~N} / \mathrm{cm} \times 0,04300 \mathrm{~cm} \Rightarrow{ }_{f} Q_{\text {máx } 4}=4859 \mathrm{~N}$

Planta 3a: ${ }_{f} Q_{\text {máx } 3}={ }_{f} k_{3} \times{ }_{s} \delta_{3}=177000 \mathrm{~N} / \mathrm{cm} \times 0,00004 \mathrm{~cm} \Rightarrow{ }_{f} Q_{\text {máx3 }}=7,08 \mathrm{~N}$

Planta 2": ${ }_{f} Q_{\max 2}={ }_{f} k_{2} \times{ }_{s} \delta_{2}=224000 \mathrm{~N} / \mathrm{cm} \times 0,00390 \mathrm{~cm} \Rightarrow \overline{{ }_{f} Q_{\max 2}=873,6 \mathrm{~N}}$

Planta 1: ${ }_{f} Q_{\text {máx } 1}={ }_{f} k_{1} \times{ }_{s} \delta_{1}=175000 \mathrm{~N} / \mathrm{cm} \times 0,02700 \mathrm{~cm} \Rightarrow{ }_{f} Q_{\text {máx } 1}=4725 \mathrm{~N}$ 
Coeficiente cortante en la parte flexible (pórtico) cuando los disipadores empiezan a plastificar ${ }_{f} \alpha_{i}$

Planta 6a: ${ }_{f} \alpha_{6}=\frac{{ }_{f} Q_{\text {máx }}(N)}{m_{6}\left(\frac{N s^{2}}{c m}\right) \times g\left(\frac{c m}{s^{2}}\right)}=\frac{6160}{739,04 \times 981} \Rightarrow$

$\underline{{ }_{f}} \alpha_{6}=8,496 e^{-03}$

Planta 5a: ${ }_{f} \alpha_{5}=\frac{{ }_{f} Q_{\max 5}(N)}{\left(m_{5}+m_{6}\right)\left(\frac{N s^{2}}{c m}\right) \times g\left(\frac{c m}{s^{2}}\right)}=\frac{5727}{(2 \times 739,04) \times 981} \Rightarrow \quad \underline{{ }_{f} \alpha_{5}=3,950 e^{-03}}$

Planta 4a: ${ }_{f} \alpha_{4}=\frac{{ }_{f} Q_{\text {max } 4}(N)}{\left(m_{4}+m_{5}+m_{6}\right)\left(\frac{N s^{2}}{c m}\right) \times g\left(\frac{c m}{s^{2}}\right)}=\frac{4859}{(3 \times 739,04) \times 981} \Rightarrow \underline{{ }_{f} \alpha_{4}=2,234 e^{-03}}$ Planta 3a: ${ }_{f} \alpha_{3}=\frac{{ }_{f} Q_{\operatorname{maxx}}(N)}{\left(m_{3}+m_{4}+m_{5}+m_{6}\right)\left(\frac{N s^{2}}{c m}\right) \times g\left(\frac{c m}{s^{2}}\right)}=\frac{7,08}{(4 \times 739,04) \times 981} \Rightarrow$

Planta 2: ${ }_{f} \alpha_{2}=\frac{{ }_{f} Q_{\operatorname{máx} 2}(N)}{\left(m_{2}+m_{3}+m_{4}+m_{5}+m_{6}\right)\left(\frac{N s^{2}}{c m}\right) \times g\left(\frac{c m}{s^{2}}\right)}=\frac{873,6}{(5 \times 739,04) \times 981} \Rightarrow$ Planta 1a: ${ }_{f} \alpha_{1}=\frac{{ }_{f} Q_{\text {máx }}(N)}{\left(m_{1}+m_{2}+m_{3}+m_{4}+m_{5}+m_{6}\right)\left(\frac{N s^{2}}{c m}\right) \times g\left(\frac{c m}{s^{2}}\right)}=\frac{\frac{{ }_{f} \alpha_{2}=2,410 e^{-04}}{4725}}{(6 \times 739,04) \times 981} \Rightarrow$ $\underline{{ }_{f}} \alpha_{1}=1,086 e^{-03}$

Coeficiente de fuerza cortante total de planta $\alpha_{i}$

Planta 6a: $\alpha_{6}={ }_{s} \alpha_{6}+{ }_{f} \alpha_{6}=0,042+8,496 e^{-03} \Rightarrow \frac{\alpha_{6}=0,050}{\alpha_{5}=0,024}$
Planta 5a: $\alpha_{5}={ }_{s} \alpha_{5}{ }_{f} \alpha_{5}=0,020+3,950 e^{-03} \Rightarrow \frac{\alpha_{4}=0,013}{\alpha_{3}=1,465 e^{-05}}$
Planta 4a: $\alpha_{4}={ }_{s} \alpha_{4}+{ }_{f} \alpha_{4}=0,011+2,234 e^{-03} \Rightarrow \underline{\alpha_{3}}$
Planta 3a: $\alpha_{3}={ }_{s} \alpha_{3}+{ }_{f} \alpha_{3}=1,221 e^{-05}+2,441 e^{-06} \Rightarrow \underline{\alpha_{2}=1,446 e^{-03}}$
Planta 2a: $\alpha_{2}={ }_{s} \alpha_{2}+{ }_{f} \alpha_{2}=1,205 e^{-03}+2,410 e^{-04}$
Planta 1a: $\alpha_{1}={ }_{s} \alpha_{1}+{ }_{f} \alpha_{1}=5,431 e^{-03}+1,086 e^{-03} \Rightarrow \underline{\alpha_{1}=6,517 e^{-03}}$ 


\section{- Acelerograma №6 (Kalamata aftershock) $\Delta=3 \mathrm{Km}$}

Desplazamiento de fluencia del disipador ${ }_{s} \delta_{i}$

Planta 6a: ${ }_{s} \delta_{6}=1,900 \mathrm{~cm}$

Planta 5a: ${ }_{s} \delta_{5}=1,615 \mathrm{~cm}$

Planta 4a: ${ }_{s} \delta_{4}=1,490 \mathrm{~cm}$

Planta 3a: ${ }_{s} \delta_{3}=0,706 \mathrm{~cm}$

Planta 2: ${ }_{s} \delta_{2}=0,496 \mathrm{~cm}$

Planta 1: ${ }_{s} \delta_{1}=0,776 \mathrm{~cm}$

Resistencia lateral de los disipadores de una planta i dada ${ }_{s} Q_{i}$

Planta 6a: ${ }_{s} Q_{6}={ }_{s} k_{6} \times{ }_{s} \delta_{6}=280000 \mathrm{~N} / \mathrm{cm} \times 1,900 \mathrm{~cm} \Rightarrow{ }_{s} Q_{6}=532000 \mathrm{~N}$

Planta 5a: ${ }_{s} Q_{5}={ }_{s} k_{5} \times{ }_{s} \delta_{5}=415000 \mathrm{~N} / \mathrm{cm} \times 1,615 \mathrm{~cm} \Rightarrow{ }_{s} Q_{5}=670225 \mathrm{~N}$

Planta 4a: ${ }_{s} Q_{1}={ }_{s} k_{4} \times{ }_{s} \delta_{4}=565000 \mathrm{~N} / \mathrm{cm} \times 1,490 \mathrm{~cm} \Rightarrow \overline{{ }_{s} Q_{4}=841850 \mathrm{~N}}$

Planta 3a: ${ }_{s} Q_{3}={ }_{s} k_{3} \times{ }_{s} \delta_{3}=885000 \mathrm{~N} / \mathrm{cm} \times 0,706 \mathrm{~cm} \Rightarrow{ }_{s} Q_{3}=624810 \mathrm{~N}$

Planta 2a: ${ }_{s} Q_{2}={ }_{s} k_{2} \times{ }_{s} \delta_{2}=1120000 \mathrm{~N} / \mathrm{cm} \times 0,496 \mathrm{~cm} \Rightarrow{ }_{s} Q_{2}=555520 \mathrm{~N}$

Planta 1a: ${ }_{s} Q_{1}={ }_{s} k_{1} \times{ }_{s} \delta_{1}=875000 \mathrm{~N} / \mathrm{cm} \times 0,776 \mathrm{~cm} \Rightarrow{ }_{s} Q_{1}=679000 \mathrm{~N}$

Coeficiente de fuerza cortante de fluencia de los disipadores de una planta i dada ${ }_{s} \alpha_{i}$

Planta 6a: ${ }_{s} \alpha_{6}=\frac{{ }_{s} Q_{6}(N)}{m_{6}\left(\frac{N s^{2}}{\mathrm{~cm}}\right) \times g\left(\frac{\mathrm{cm}}{\mathrm{s}^{2}}\right)}=\frac{532000}{739,04 \times 981} \Rightarrow$

${ }_{s} \alpha_{6}=0,734$

Planta 5ㄹ: ${ }_{s} \alpha_{5}=\frac{{ }_{s} Q_{5}(N)}{\left(m_{5}+m_{6}\right)\left(\frac{N s^{2}}{c m}\right) \times g\left(\frac{c m}{s^{2}}\right)}=\frac{670225}{(2 \times 739,04) \times 981} \Rightarrow$

${ }_{s} \alpha_{5}=0,462$

Planta 4ㄹ: ${ }_{s} \alpha_{4}=\frac{{ }_{s} Q_{4}(N)}{\left(m_{4}+m_{5}+m_{6}\right)\left(\frac{N s^{2}}{c m}\right) \times g\left(\frac{c m}{s^{2}}\right)}=\frac{841850}{(3 \times 739,04) \times 981} \Rightarrow \quad \underline{{ }_{s} \alpha_{4}=0,387}$

Planta 3a: ${ }_{s} \alpha_{3}=\frac{{ }_{s} Q_{3}(N)}{\left(m_{3}+m_{4}+m_{5}+m_{6}\right)\left(\frac{N s^{2}}{c m}\right) \times g\left(\frac{\mathrm{cm}}{s^{2}}\right)}=\frac{624810}{(4 \times 739,04) \times 981} \Rightarrow$

Planta 2ㄹ: ${ }_{s} \alpha_{2}=\frac{{ }_{s} Q_{2}(N)}{\left(m_{2}+m_{3}+m_{4}+m_{5}+m_{6}\right)\left(\frac{N s^{2}}{c m}\right) \times g\left(\frac{\mathrm{cm}}{s^{2}}\right)}=\frac{555520}{(5 \times 739,04) \times 981} \Rightarrow$ 


$$
\begin{aligned}
& \text { Planta 1ㄹ: }{ }_{s} \alpha_{1}=\frac{{ }_{s} Q_{1}(N)}{\left(m_{1}+m_{2}+m_{3}+m_{4}+m_{5}+m_{6}\right)\left(\frac{N s^{2}}{c m}\right) \times g\left(\frac{\mathrm{cm}}{s^{2}}\right)}=\frac{679000}{(6 \times 739,04) \times 981} \Rightarrow \\
& { }_{s} \alpha_{1}=0,156
\end{aligned}
$$

Resistencia lateral aportada por la parte flexible (pórtico) cuando los disipadores empiezan a plastificar ${ }_{f} Q_{\text {máxi }}$

Planta 6a: ${ }_{f} Q_{\text {máx }}={ }_{f} k_{6} \times{ }_{s} \delta_{6}=56000 \mathrm{~N} / \mathrm{cm} \times 1,900 \mathrm{~cm} \Rightarrow{ }_{f} Q_{\text {máx }}=106400 \mathrm{~N}$

Planta 5a: ${ }_{f} Q_{\text {maxx }}={ }_{f} k_{5} \times{ }_{s} \delta_{5}=83000 \mathrm{~N} / \mathrm{cm} \times 1,615 \mathrm{~cm} \Rightarrow \overline{{ }_{f} Q_{\text {max } 5}=134045 \mathrm{~N}}$

Planta 4ạ: ${ }_{f} Q_{\text {máx } 4}={ }_{f} k_{4} \times{ }_{s} \delta_{4}=113000 \mathrm{~N} / \mathrm{cm} \times 1,490 \mathrm{~cm} \Rightarrow{ }_{f} Q_{\text {máx } 4}=168370 \mathrm{~N}$

Planta 3a: ${ }_{f} Q_{\text {máx } 3}={ }_{f} k_{3} \times{ }_{s} \delta_{3}=177000 \mathrm{~N} / \mathrm{cm} \times 0,706 \mathrm{~cm} \Rightarrow \overline{{ }_{f} Q_{\text {máx3 }}=124962 \mathrm{~N}}$

Planta 2a: ${ }_{f} Q_{\text {máx } 2}={ }_{f} k_{2} \times{ }_{s} \delta_{2}=224000 \mathrm{~N} / \mathrm{cm} \times 0,496 \mathrm{~cm} \Rightarrow \overline{{ }_{f} Q_{\text {máx } 2}=111104 \mathrm{~N}}$

Planta 1a: ${ }_{f} Q_{\text {máx } 1}={ }_{f} k_{1} \times{ }_{s} \delta_{1}=175000 \mathrm{~N} / \mathrm{cm} \times 0,776 \mathrm{~cm} \Rightarrow \overline{{ }_{f} Q_{\operatorname{máx} 1}=135800 \mathrm{~N}}$

Coeficiente cortante en la parte flexible (pórtico) cuando los disipadores empiezan a plastificar ${ }_{f} \alpha_{i}$

Planta 6”: ${ }_{f} \alpha_{6}=\frac{{ }_{f} Q_{\operatorname{máx} 6}(N)}{m_{6}\left(\frac{N s^{2}}{c m}\right) \times g\left(\frac{c m}{s^{2}}\right)}=\frac{106400}{739,04 \times 981} \Rightarrow$ $\underline{{ }_{f} \alpha_{6}=0,147}$

Planta 5a: ${ }_{f} \alpha_{5}=\frac{{ }_{f} Q_{m a ́ x 5}(N)}{\left(m_{5}+m_{6}\right)\left(\frac{N s^{2}}{c m}\right) \times g\left(\frac{c m}{s^{2}}\right)}=\frac{134045}{(2 \times 739,04) \times 981} \Rightarrow$ $\underline{{ }_{f} \alpha_{5}=0,092}$

Planta 4a: ${ }_{f} \alpha_{4}=\frac{{ }_{f} Q_{m a ́ x 4}(N)}{\left(m_{4}+m_{5}+m_{6}\right)\left(\frac{N s^{2}}{c m}\right) \times g\left(\frac{\mathrm{cm}}{\mathrm{s}^{2}}\right)}=\frac{168370}{(3 \times 739,04) \times 981} \Rightarrow$
Planta 3a: ${ }_{f} \alpha_{3}=\frac{{ }_{f} Q_{m a ́ x 3}(N)}{\left(m_{3}+m_{4}+m_{5}+m_{6}\right)\left(\frac{N s^{2}}{\mathrm{~cm}}\right) \times g\left(\frac{\mathrm{cm}}{\mathrm{s}^{2}}\right)}=\frac{124962}{(4 \times 739,04) \times 981} \Rightarrow$

Planta 2": ${ }_{f} \alpha_{2}=\frac{{ }_{f} Q_{\text {máx }}(N)}{\left(m_{2}+m_{3}+m_{4}+m_{5}+m_{6}\right)\left(\frac{N s^{2}}{c m}\right) \times g\left(\frac{c m}{s^{2}}\right)}=\frac{111104}{(5 \times 739,04) \times 981} \Rightarrow$ ${ }_{f} \alpha_{2}=0,031$ 
Planta 1ㄹ: ${ }_{f} \alpha_{1}=\frac{{ }_{f} Q_{\text {máx }}(N)}{\left(m_{1}+m_{2}+m_{3}+m_{4}+m_{5}+m_{6}\right)\left(\frac{N s^{2}}{c m}\right) \times g\left(\frac{c m}{s^{2}}\right)}=\frac{135800}{(6 \times 739,04) \times 981} \Rightarrow$

${ }_{f} \alpha_{1}=0,031$

Coeficiente de fuerza cortante total de planta $\alpha_{i}$

Planta 6a: $\alpha_{6}={ }_{s} \alpha_{6}+{ }_{f} \alpha_{6}=0,734+0,147 \Rightarrow \alpha_{6}=0,881$

Planta 5a: $\alpha_{5}={ }_{s} \alpha_{5}+{ }_{f} \alpha_{5}=0,462+0,092 \Rightarrow \alpha_{5}=0,554$

Planta 4a: $\alpha_{4}={ }_{s} \alpha_{4}+{ }_{f} \alpha_{4}=0,387+0,077 \Rightarrow \alpha_{4}=0,464$

Planta 3a: $\alpha_{3}={ }_{s} \alpha_{3}+{ }_{f} \alpha_{3}=0,215+0,043 \Rightarrow \alpha_{3}=0,258$

Planta 2: $\alpha_{2}={ }_{s} \alpha_{2}+{ }_{f} \alpha_{2}=0,153+0,031 \Rightarrow \underline{\alpha_{2}=0,184}$

Planta 1a: $\alpha_{1}={ }_{s} \alpha_{1}+{ }_{f} \alpha_{1}=0,156+0,031 \Rightarrow \underline{\alpha_{1}=0,187}$

\section{- Acelerograma №12 (Duzce 1) $\Delta=8 \mathrm{Km}$}

Desplazamiento de fluencia del disipador ${ }_{s} \delta_{i}$

Planta 6a: ${ }_{s} \delta_{6}=0,0900 \mathrm{~cm}$

Planta 5a: ${ }_{s} \delta_{5}=0,1100 \mathrm{~cm}$

Planta 4a: ${ }_{s} \delta_{4}=0,0900 \mathrm{~cm}$

Planta 3a: ${ }_{s} \delta_{3}=0,0721 \mathrm{~cm}$

Planta 2: ${ }_{s} \delta_{2}=0,0940 \mathrm{~cm}$

Planta 1ㄹ: ${ }_{s} \delta_{1}=0,1810 \mathrm{~cm}$

Resistencia lateral de los disipadores de una planta i dada ${ }_{s} Q_{i}$

Planta 6a: ${ }_{s} Q_{6}={ }_{s} k_{6} \times{ }_{s} \delta_{6}=280000 \mathrm{~N} / \mathrm{cm} \times 0,0900 \mathrm{~cm} \Rightarrow{ }_{s} Q_{6}=25200 \mathrm{~N}$

Planta 5a: ${ }_{s} Q_{5}={ }_{s} k_{5} \times{ }_{s} \delta_{5}=415000 \mathrm{~N} / \mathrm{cm} \times 0,1100 \mathrm{~cm} \Rightarrow{ }_{s} Q_{5}=45650 \mathrm{~N}$

Planta 4a: ${ }_{s} Q_{1}={ }_{s} k_{4} \times{ }_{s} \delta_{4}=565000 \mathrm{~N} / \mathrm{cm} \times 0,0900 \mathrm{~cm} \Rightarrow{ }_{s} Q_{4}=50850 \mathrm{~N}$

Planta 3a: ${ }_{s} Q_{3}={ }_{s} k_{3} \times{ }_{s} \delta_{3}=885000 \mathrm{~N} / \mathrm{cm} \times 0,0721 \mathrm{~cm} \Rightarrow \overline{Q_{3}=63808,5 \mathrm{~N}}$

Planta 2a: ${ }_{s} Q_{2}={ }_{s} k_{2} \times{ }_{s} \delta_{2}=1120000 \mathrm{~N} / \mathrm{cm} \times 0,0940 \mathrm{~cm} \Rightarrow{ }_{s} Q_{2}=105280 \mathrm{~N}$

Planta 1: ${ }_{s} Q_{1}={ }_{s} k_{1} \times{ }_{s} \delta_{1}=875000 \mathrm{~N} / \mathrm{cm} \times 0,1810 \mathrm{~cm} \Rightarrow \overline{{ }_{s} Q_{1}=158375 \mathrm{~N}}$ 
Coeficiente de fuerza cortante de fluencia de los disipadores de una planta i dada ${ }_{s} \alpha_{i}$

Planta 6a: ${ }_{s} \alpha_{6}=\frac{{ }_{2}(N)}{m_{6}\left(\frac{N s^{2}}{\mathrm{~cm}}\right) \times g\left(\frac{\mathrm{cm}}{\mathrm{s}^{2}}\right)}=\frac{25200}{739,04 \times 981} \Rightarrow$

${ }_{s} \alpha_{6}=0,035$

Planta 5ㄹ: $\alpha_{s}=\frac{{ }_{s}(N)}{\left(m_{5}+m_{6}\right)\left(\frac{N s^{2}}{c m}\right) \times g\left(\frac{c m}{s^{2}}\right)}=\frac{45650}{(2 \times 739,04) \times 981} \Rightarrow$

${ }_{s} \alpha_{5}=0,031$

Planta 4a: ${ }_{s} \alpha_{4}=\frac{{ }_{s} Q_{4}(N)}{\left(m_{4}+m_{5}+m_{6}\right)\left(\frac{N s^{2}}{c m}\right) \times g\left(\frac{\mathrm{cm}}{\mathrm{s}^{2}}\right)}=\frac{50850}{(3 \times 739,04) \times 981} \Rightarrow$
Planta 3a: ${ }_{s} \alpha_{3}=\frac{{ }_{s} Q_{3}(N)}{\left(m_{3}+m_{4}+m_{5}+m_{6}\right)\left(\frac{N s^{2}}{\mathrm{~cm}}\right) \times g\left(\frac{\mathrm{cm}}{\mathrm{s}^{2}}\right)}=\frac{63808,5}{(4 \times 739,04) \times 981} \Rightarrow$

${ }_{s} \alpha_{4}=0,023$

Planta 2 : ${ }_{s} \alpha_{2}=\frac{{ }_{s} Q_{2}(N)}{\left(m_{2}+m_{3}+m_{4}+m_{5}+m_{6}\right)\left(\frac{N s^{2}}{\mathrm{~cm}}\right) \times g\left(\frac{\mathrm{cm}}{\mathrm{s}^{2}}\right)}=\frac{105280}{(5 \times 739,04) \times 981} \Rightarrow$

${ }_{s} \alpha_{3}=0,022$

Planta 1ㄹ: ${ }_{s} \alpha_{1}=\frac{{ }_{s} Q_{1}(N)}{\left(m_{1}+m_{2}+m_{3}+m_{4}+m_{5}+m_{6}\right)\left(\frac{N s^{2}}{c m}\right) \times g\left(\frac{c m}{s^{2}}\right)}=\frac{158375}{(6 \times 739,04) \times 981} \Rightarrow$

${ }_{s} \alpha_{1}=0,036$

Resistencia lateral aportada por la parte flexible (pórtico) cuando los disipadores empiezan a plastificar ${ }_{f} Q_{\text {máxi }}$

Planta 6a: ${ }_{f} Q_{\text {máx6 }}={ }_{f} k_{6} \times{ }_{s} \delta_{6}=56000 \mathrm{~N} / \mathrm{cm} \times 0,0900 \mathrm{~cm} \Rightarrow{ }_{f} Q_{\text {máx }}=5040 \mathrm{~N}$

Planta 5a: ${ }_{f} Q_{\text {máx } 5}={ }_{f} k_{5} \times{ }_{s} \delta_{5}=83000 \mathrm{~N} / \mathrm{cm} \times 0,1100 \mathrm{~cm} \Rightarrow{ }_{f} Q_{\text {máx } 5}=9130 \mathrm{~N}$

Planta 4a: ${ }_{f} Q_{\text {máx } 4}={ }_{f} k_{4} \times{ }_{s} \delta_{4}=113000 \mathrm{~N} / \mathrm{cm} \times 0,0900 \mathrm{~cm} \Rightarrow{ }_{f} Q_{\text {máx } 4}=10170 \mathrm{~N}$

Planta 3a: ${ }_{f} Q_{\text {maxx }}={ }_{f} k_{3} \times{ }_{s} \delta_{3}=177000 \mathrm{~N} / \mathrm{cm} \times 0,0721 \mathrm{~cm} \Rightarrow{ }_{f} Q_{\operatorname{maxx} 3}=12761,7 \mathrm{~N}$

Planta 2a: ${ }_{f} Q_{\max 2}={ }_{f} k_{2} \times{ }_{s} \delta_{2}=224000 \mathrm{~N} / \mathrm{cm} \times 0,0940 \mathrm{~cm} \Rightarrow{ }_{f} Q_{\max 2}=21056 \mathrm{~N}$

Planta 1ㄹ: ${ }_{f} Q_{\text {máx } 1}={ }_{f} k_{1} \times{ }_{s} \delta_{1}=175000 \mathrm{~N} / \mathrm{cm} \times 0,1810 \mathrm{~cm} \Rightarrow \underline{{ }_{f} Q_{\text {máx1 }}=31675 \mathrm{~N}}$ 
Coeficiente cortante en la parte flexible (pórtico) cuando los disipadores empiezan a plastificar ${ }_{f} \alpha_{i}$

Planta 6a: ${ }_{f} \alpha_{6}=\frac{{ }_{f} Q_{\text {máx }}(N)}{m_{6}\left(\frac{N s^{2}}{c m}\right) \times g\left(\frac{c m}{s^{2}}\right)}=\frac{5040}{739,04 \times 981} \Rightarrow$

$$
\underline{{ }_{f} \alpha_{6}=6,952 e^{-03}}
$$

Planta 5a: ${ }_{f} \alpha_{5}=\frac{{ }_{f} Q_{m a x x}(N)}{\left(m_{5}+m_{6}\right)\left(\frac{N s^{2}}{c m}\right) \times g\left(\frac{c m}{s^{2}}\right)}=\frac{9130}{(2 \times 739,04) \times 981} \Rightarrow$

$\underline{{ }_{f}} \alpha_{5}=6,296 e^{-03}$

Planta 4ㄹ: ${ }_{f} \alpha_{4}=\frac{{ }_{f} Q_{\text {máx } 4}(N)}{\left(m_{4}+m_{5}+m_{6}\right)\left(\frac{N s^{2}}{c m}\right) \times g\left(\frac{c m}{s^{2}}\right)}=\frac{10170}{(3 \times 739,04) \times 981} \Rightarrow \underline{{ }_{f}} \alpha_{4}=4,676 e^{-03}$ Planta 3a: ${ }_{f} \alpha_{3}=\frac{{ }_{f} Q_{\text {máx } 3}(N)}{\left(m_{3}+m_{4}+m_{5}+m_{6}\right)\left(\frac{N s^{2}}{c m}\right) \times g\left(\frac{c m}{s^{2}}\right)}=\frac{12761,7}{(4 \times 739,04) \times 981} \Rightarrow$

Planta 2ㄹ: ${ }_{f} \alpha_{2}=\frac{{ }_{f} Q_{\operatorname{maxx}}(N)}{\left(m_{2}+m_{3}+m_{4}+m_{5}+m_{6}\right)\left(\frac{N s^{2}}{c m}\right) \times g\left(\frac{c m}{s^{2}}\right)}=\frac{21056}{(5 \times 739,04) \times 981} \Rightarrow$ ${ }_{f} \alpha_{2}=5,808 e^{-03}$ Planta 1a: ${ }_{f} \alpha_{1}=\frac{{ }_{f} Q_{\text {máx }}(N)}{\left(m_{1}+m_{2}+m_{3}+m_{4}+m_{5}+m_{6}\right)\left(\frac{N s^{2}}{c m}\right) \times g\left(\frac{c m}{s^{2}}\right)}=\frac{31675}{(6 \times 739,04) \times 981} \Rightarrow$ ${ }_{f} \alpha_{1}=7,282 e^{-03}$

Coeficiente de fuerza cortante total de planta $\alpha_{i}$

Planta 6a: $\alpha_{6}={ }_{s} \alpha_{6}+{ }_{f} \alpha_{6}=0,035+6,952 e^{-03} \Rightarrow \alpha_{6}=0,042$

Planta 5a: $\alpha_{5}={ }_{s} \alpha_{5}+{ }_{f} \alpha_{5}=0,031+6,296 e^{-03} \Rightarrow \alpha_{5}=0,037$

Planta 4a: $\alpha_{4}={ }_{s} \alpha_{4}+{ }_{f} \alpha_{4}=0,023+4,676 e^{-03} \Rightarrow \alpha_{4}=0,028$

Planta 3a: $\alpha_{3}={ }_{s} \alpha_{3}+{ }_{f} \alpha_{3}=0,022+4,400 e^{-03} \Rightarrow \alpha_{3}=0,026$

Planta 2a: $\alpha_{2}={ }_{s} \alpha_{2}+{ }_{f} \alpha_{2}=0,029+5,808 e^{-03} \Rightarrow \alpha_{2}=0,035$

Planta 1: $\alpha_{1}={ }_{s} \alpha_{1}+{ }_{f} \alpha_{1}=0,036+7,282 e^{-03} \Rightarrow \alpha_{1}=0,043$ 
Representación gráfica

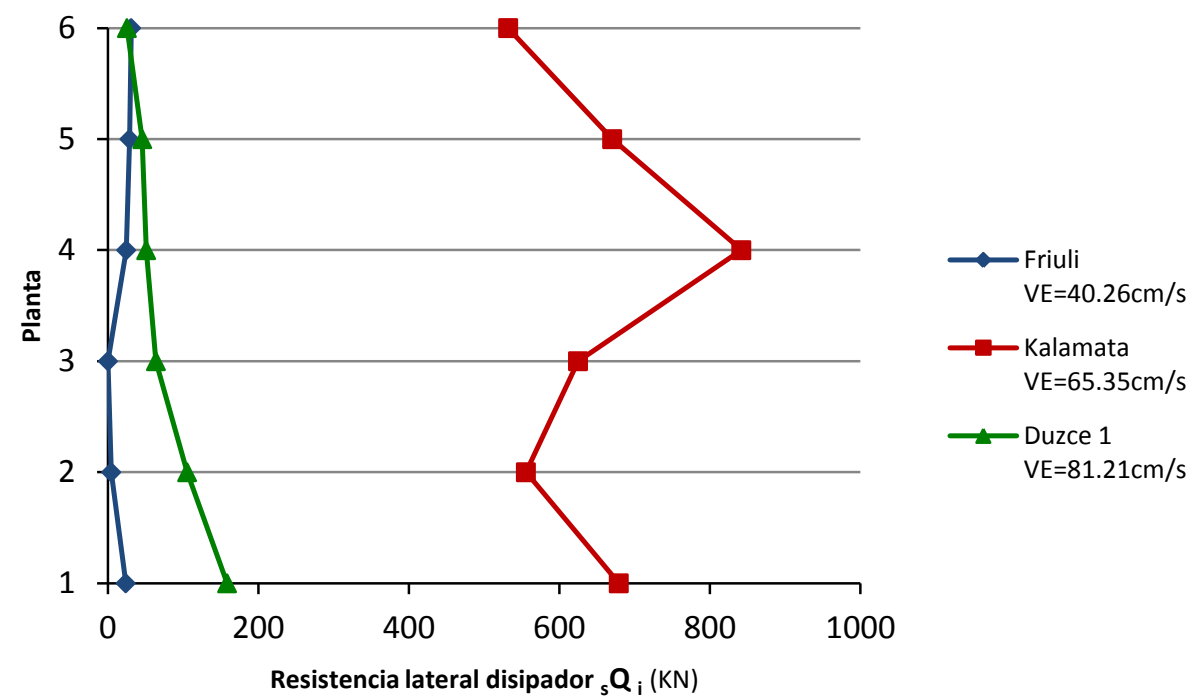

Fig. 5.5.65: Resistencia lateral de los disipadores de cada planta i, pórtico pa6pz2-1, suelo tipo III, campo cercano

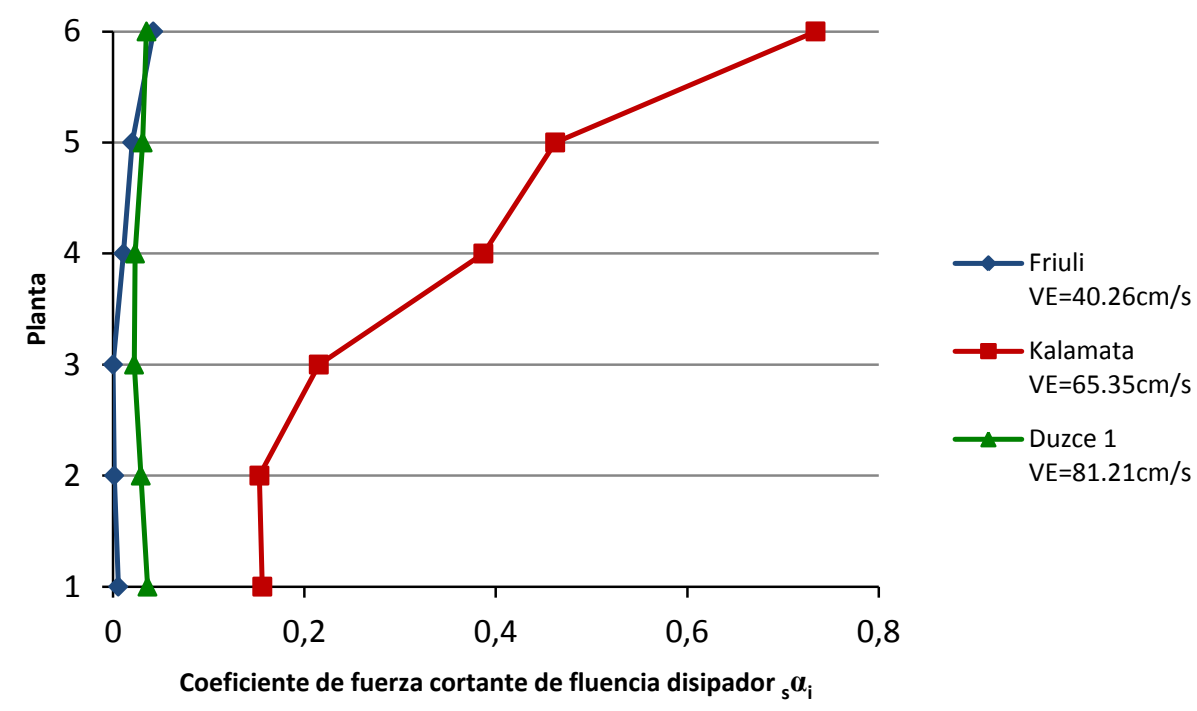

Fig. 5.5.66: Coeficiente de fuerza cortante de fluencia de los disipadores de cada planta i, pórtico pa6pz2-1, suelo tipo III, campo cercano 


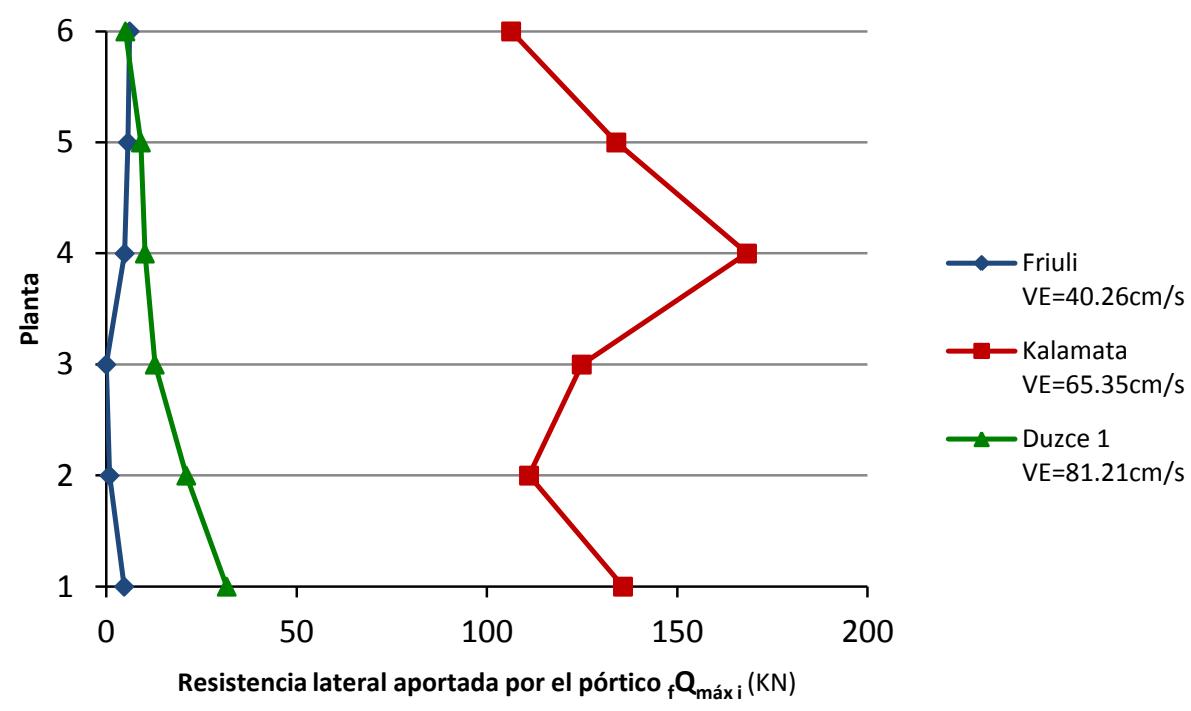

Fig. 5.5.67: Resistencia lateral aportada por la parte flexible (pórtico) cuando los disipadores empiezan a plastificar, pórtico pa6pz2-1, suelo tipo III, campo cercano

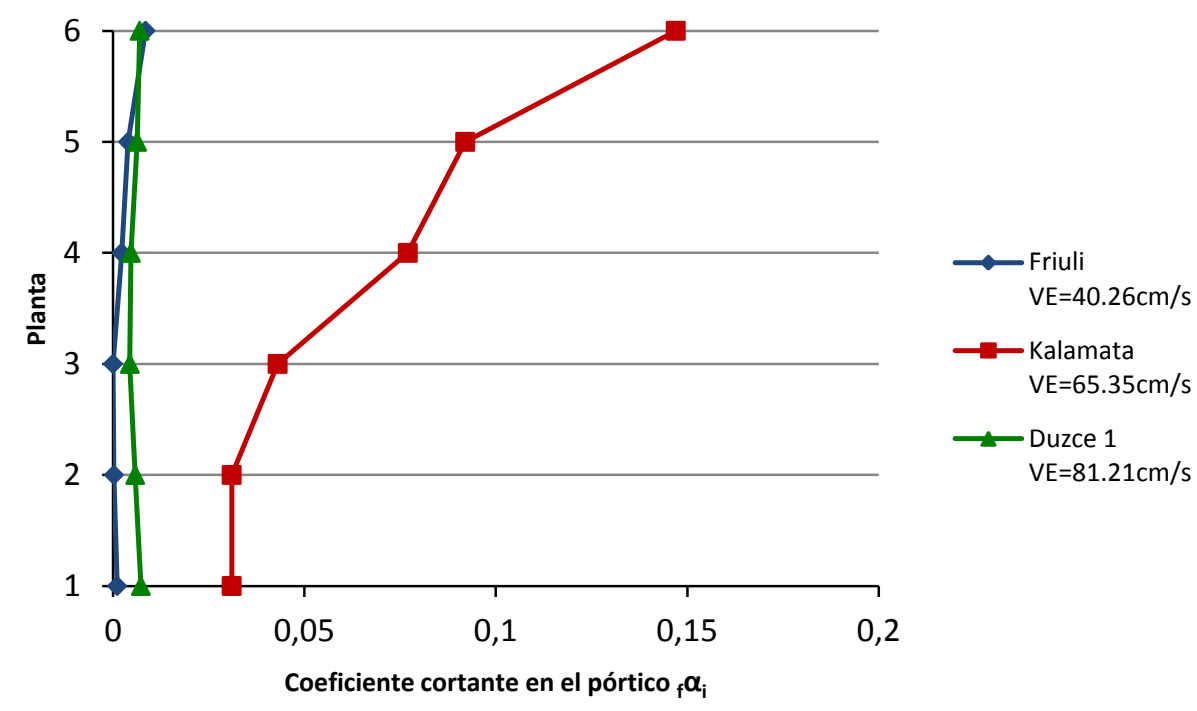

Fig. 5.5.68: Coeficiente de fuerza cortante en la parte flexible (pórtico) cuando los disipadores empiezan a plastificar, pórtico pa6pz2-1, suelo tipo III, campo cercano 


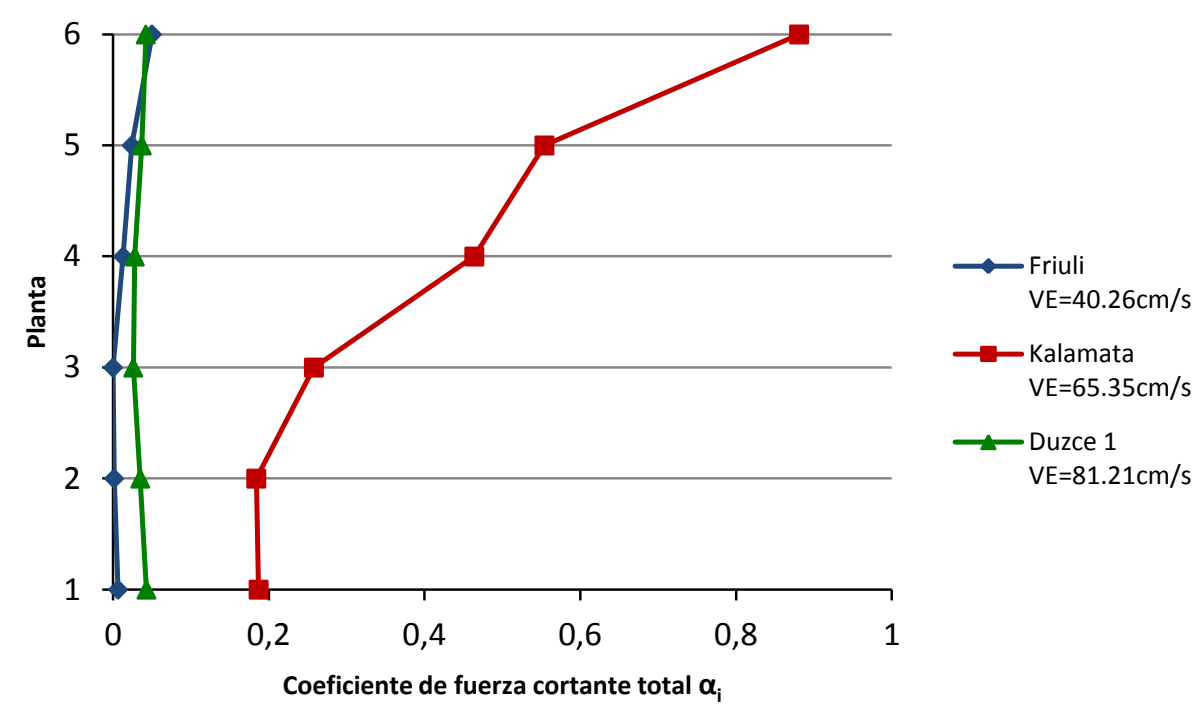

Fig. 5.5.69: Coeficiente de fuerza cortante total de planta, pórtico pa6pz2-1, suelo tipo III, campo cercano

\subsection{Suelo Tipo III (Campo lejano a la falla)}

\section{- Acelerograma №3 (Montenegro) $\Delta=24 \mathrm{Km}$}

Desplazamiento de fluencia del disipador ${ }_{s} \delta_{i}$

Planta 6a: ${ }_{s} \delta_{6}=1,700 \mathrm{~cm}$

Planta 5a: ${ }_{s} \delta_{5}=1,300 \mathrm{~cm}$

Planta 4a: ${ }_{s} \delta_{4}=1,110 \mathrm{~cm}$

Planta 3a: ${ }_{s} \delta_{3}=0,780 \mathrm{~cm}$

Planta 2a: ${ }_{s} \delta_{2}=0,690 \mathrm{~cm}$

Planta 1: ${ }_{s} \delta_{1}=0,980 \mathrm{~cm}$

Resistencia lateral de los disipadores de una planta i dada ${ }_{s} Q_{i}$

Planta 6a: ${ }_{s} Q_{6}={ }_{s} k_{6} \times{ }_{s} \delta_{6}=280000 \mathrm{~N} / \mathrm{cm} \times 1,700 \mathrm{~cm} \Rightarrow{ }_{s} Q_{6}=476000 \mathrm{~N}$

Planta 5a: ${ }_{s} Q_{5}={ }_{s} k_{5} \times{ }_{s} \delta_{5}=415000 \mathrm{~N} / \mathrm{cm} \times 1,300 \mathrm{~cm} \Rightarrow{ }_{s} Q_{5}=539500 \mathrm{~N}$

Planta 4. : ${ }_{s} Q_{1}={ }_{s} k_{4} \times{ }_{s} \delta_{4}=565000 \mathrm{~N} / \mathrm{cm} \times 1,110 \mathrm{~cm} \Rightarrow{ }_{s} Q_{4}=627150 \mathrm{~N}$

Planta 3a: ${ }_{s} Q_{3}={ }_{s} k_{3} \times{ }_{s} \delta_{3}=885000 \mathrm{~N} / \mathrm{cm} \times 0,780 \mathrm{~cm} \Rightarrow \overline{{ }_{s} Q_{3}=690300 \mathrm{~N}}$

Planta 2a: ${ }_{s} Q_{2}={ }_{s} k_{2} \times{ }_{s} \delta_{2}=1120000 \mathrm{~N} / \mathrm{cm} \times 0,690 \mathrm{~cm} \Rightarrow{ }_{s} Q_{2}=772800 \mathrm{~N}$

Planta 1ㄹ: ${ }_{s} Q_{1}={ }_{s} k_{1} \times{ }_{s} \delta_{1}=875000 \mathrm{~N} / \mathrm{cm} \times 0,980 \mathrm{~cm} \Rightarrow{ }_{s} Q_{1}=857500 \mathrm{~N}$ 
Coeficiente de fuerza cortante de fluencia de los disipadores de una planta i dada ${ }_{s} \alpha_{i}$

Planta 6a: ${ }_{s} \alpha_{6}=\frac{{ }_{2}(N)}{m_{6}\left(\frac{N s^{2}}{\mathrm{~cm}}\right) \times g\left(\frac{\mathrm{cm}}{\mathrm{s}^{2}}\right)}=\frac{476000}{739,04 \times 981} \Rightarrow$

${ }_{s} \alpha_{6}=0,656$

Planta 5a: ${ }_{s} \alpha_{5}=\frac{{ }_{s}(N)}{\left(m_{5}+m_{6}\right)\left(\frac{N s^{2}}{c m}\right) \times g\left(\frac{\mathrm{cm}}{\mathrm{s}^{2}}\right)}=\frac{539500}{(2 \times 739,04) \times 981} \Rightarrow$

${ }_{s} \alpha_{5}=0,372$

Planta 4ㅁ: ${ }_{s} \alpha_{4}=\frac{{ }_{s} Q_{4}(N)}{\left(m_{4}+m_{5}+m_{6}\right)\left(\frac{N s^{2}}{c m}\right) \times g\left(\frac{c m}{s^{2}}\right)}=\frac{627150}{(3 \times 739,04) \times 981} \Rightarrow \quad \underline{{ }_{s} \alpha_{4}=0,288}$

Planta 3a: ${ }_{s} \alpha_{3}=\frac{{ }_{s} Q_{3}(N)}{\left(m_{3}+m_{4}+m_{5}+m_{6}\right)\left(\frac{N s^{2}}{c m}\right) \times g\left(\frac{c m}{s^{2}}\right)}=\frac{690300}{(4 \times 739,04) \times 981} \Rightarrow$

Planta 2a: ${ }_{s} \alpha_{2}=\frac{{ }_{s} Q_{2}(N)}{\left(m_{2}+m_{3}+m_{4}+m_{5}+m_{6}\right)\left(\frac{N s^{2}}{\mathrm{~cm}}\right) \times g\left(\frac{\mathrm{cm}}{\mathrm{s}^{2}}\right)}=\frac{772800}{(5 \times 739,04) \times 981} \Rightarrow$

${ }_{s} \alpha_{3}=0,238$

Planta 1ㄹ: $\alpha_{s}=\frac{{ }_{s} Q_{1}(N)}{\left(m_{1}+m_{2}+m_{3}+m_{4}+m_{5}+m_{6}\right)\left(\frac{N s^{2}}{c m}\right) \times g\left(\frac{c m}{s^{2}}\right)}=\frac{857500}{(6 \times 739,04) \times 981} \Rightarrow$

${ }_{s} \alpha_{1}=0,197$

Resistencia lateral aportada por la parte flexible (pórtico) cuando los disipadores empiezan a plastificar ${ }_{f} Q_{\text {máxi }}$

Planta 6a: ${ }_{f} Q_{\text {máx } 6}={ }_{f} k_{6} \times{ }_{s} \delta_{6}=56000 \mathrm{~N} / \mathrm{cm} \times 1,700 \mathrm{~cm} \Rightarrow{ }_{f} Q_{\text {máx6 }}=95200 \mathrm{~N}$

Planta 5a: ${ }_{f} Q_{\text {máx } 5}={ }_{f} k_{5} \times{ }_{s} \delta_{5}=83000 \mathrm{~N} / \mathrm{cm} \times 1,300 \mathrm{~cm} \Rightarrow{ }_{f} Q_{\text {max } 5}=107900 \mathrm{~N}$

Planta 4a: : ${ }_{f} Q_{\text {máx } 4}={ }_{f} k_{4} \times{ }_{s} \delta_{4}=113000 \mathrm{~N} / \mathrm{cm} \times 1,110 \mathrm{~cm} \Rightarrow{ }_{f} Q_{\text {máx } 4}=125430 \mathrm{~N}$

Planta 3a: ${ }_{f} Q_{\text {máx } 3}={ }_{f} k_{3} \times{ }_{s} \delta_{3}=177000 \mathrm{~N} / \mathrm{cm} \times 0,780 \mathrm{~cm} \Rightarrow{ }_{f} Q_{\text {máx } 3}=138060 \mathrm{~N}$

Planta 2a: ${ }_{f} Q_{\text {máx } 2}={ }_{f} k_{2} \times{ }_{s} \delta_{2}=224000 \mathrm{~N} / \mathrm{cm} \times 0,690 \mathrm{~cm} \Rightarrow{ }_{f} Q_{\text {máx } 2}=154560 \mathrm{~N}$

Planta 1: ${ }_{f} Q_{\text {máx } 1}={ }_{f} k_{1} \times{ }_{s} \delta_{1}=175000 \mathrm{~N} / \mathrm{cm} \times 0,980 \mathrm{~cm} \Rightarrow \overline{{ }_{f} Q_{\text {máx } 1}=171500 \mathrm{~N}}$ 
Coeficiente cortante en la parte flexible (pórtico) cuando los disipadores empiezan a plastificar ${ }_{f} \alpha_{i}$

Planta 6a: ${ }_{f} \alpha_{6}=\frac{{ }_{f} Q_{\text {máx }}(N)}{m_{6}\left(\frac{N s^{2}}{c m}\right) \times g\left(\frac{c m}{s^{2}}\right)}=\frac{95200}{739,04 \times 981} \Rightarrow$

$\underline{{ }_{f} \alpha_{6}=0,131}$

Planta 5a: ${ }_{f} \alpha_{5}=\frac{{ }_{f} Q_{\max 5}(N)}{\left(m_{5}+m_{6}\right)\left(\frac{N s^{2}}{c m}\right) \times g\left(\frac{c m}{s^{2}}\right)}=\frac{107900}{(2 \times 739,04) \times 981} \Rightarrow$

$\underline{{ }_{f} \alpha_{5}=0,074}$

Planta 4a: ${ }_{f} \alpha_{4}=\frac{{ }_{f} Q_{\text {máx4 }}(N)}{\left(m_{4}+m_{5}+m_{6}\right)\left(\frac{N s^{2}}{c m}\right) \times g\left(\frac{c m}{s^{2}}\right)}=\frac{125430}{(3 \times 739,04) \times 981} \Rightarrow \quad \underline{{ }_{f} \alpha_{4}=0,058}$

Planta 3a: ${ }_{f} \alpha_{3}=\frac{{ }_{f} Q_{\operatorname{máx3}}(N)}{\left(m_{3}+m_{4}+m_{5}+m_{6}\right)\left(\frac{N s^{2}}{c m}\right) \times g\left(\frac{\mathrm{cm}}{s^{2}}\right)}=\frac{138060}{(4 \times 739,04) \times 981} \Rightarrow$

Planta 2": ${ }_{f} \alpha_{2}=\frac{{ }_{f} Q_{\text {máx } 2}(N)}{\left(m_{2}+m_{3}+m_{4}+m_{5}+m_{6}\right)\left(\frac{N s^{2}}{\mathrm{~cm}}\right) \times g\left(\frac{\mathrm{cm}}{\mathrm{s}^{2}}\right)}=\frac{154560}{(5 \times 739,04) \times 981} \Rightarrow$

Planta 1ㄹ: ${ }_{f} \alpha_{1}=\frac{{ }_{f} Q_{m a ́ x 1}(N)}{\left(m_{1}+m_{2}+m_{3}+m_{4}+m_{5}+m_{6}\right)\left(\frac{N s^{2}}{c m}\right) \times g\left(\frac{c m}{s^{2}}\right)}=\frac{171500}{(6 \times 739,04) \times 981} \Rightarrow$ ${ }_{f} \alpha_{1}=0,039$

Coeficiente de fuerza cortante total de planta $\alpha_{i}$

Planta 6a : $\alpha_{6}={ }_{s} \alpha_{6}+{ }_{f} \alpha_{6}=0,656+0,131 \Rightarrow \alpha_{6}=0,787$

Planta 5a: $\alpha_{5}={ }_{s} \alpha_{5}+{ }_{f} \alpha_{5}=0,372+0,074 \Rightarrow \alpha_{5}=0,446$

Planta 4a: $\alpha_{4}={ }_{s} \alpha_{4}+{ }_{f} \alpha_{4}=0,288+0,058 \Rightarrow \alpha_{4}=0,346$

Planta 3a: $\alpha_{3}={ }_{s} \alpha_{3}+{ }_{f} \alpha_{3}=0,238+0,048 \Rightarrow \alpha_{3}=0,286$

Planta 2a: $\alpha_{2}={ }_{s} \alpha_{2}+{ }_{f} \alpha_{2}=0,213+0,043 \Rightarrow \underline{\alpha_{2}=0,256}$

Planta 1: $\alpha_{1}={ }_{s} \alpha_{1}+{ }_{f} \alpha_{1}=0,197+0,039 \Rightarrow \alpha_{1}=0,236$ 
Representación gráfica

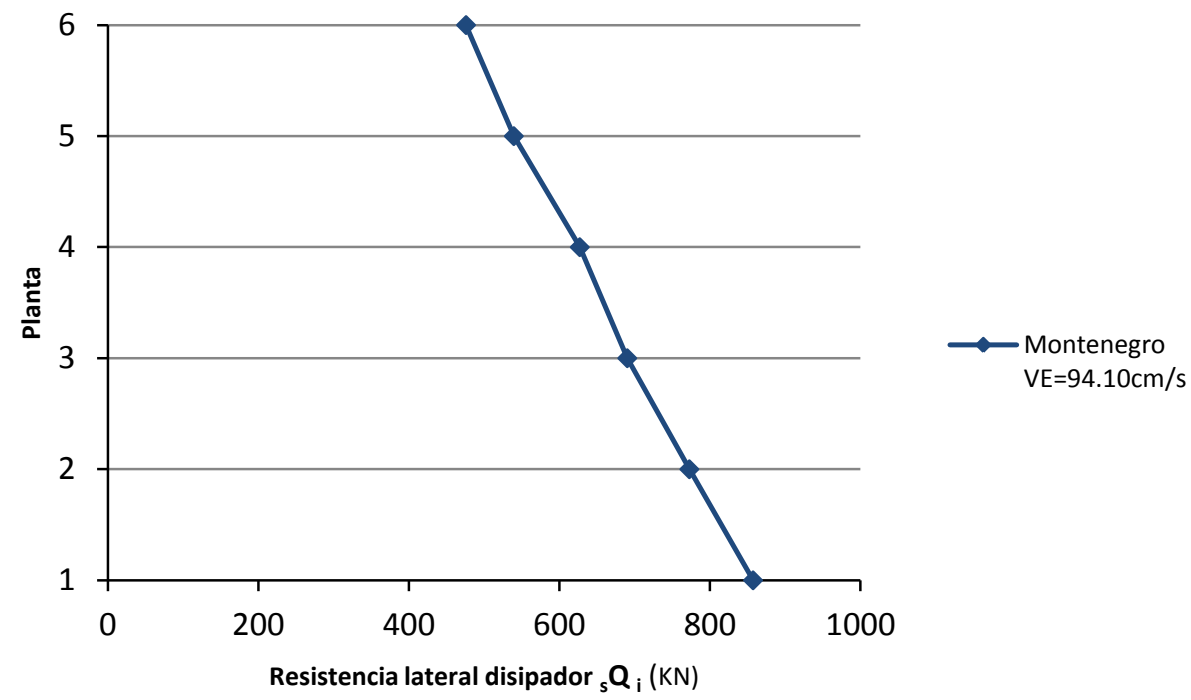

Fig. 5.5.70: Resistencia lateral de los disipadores de cada planta i, pórtico pa6pz2-1, suelo tipo III, campo lejano

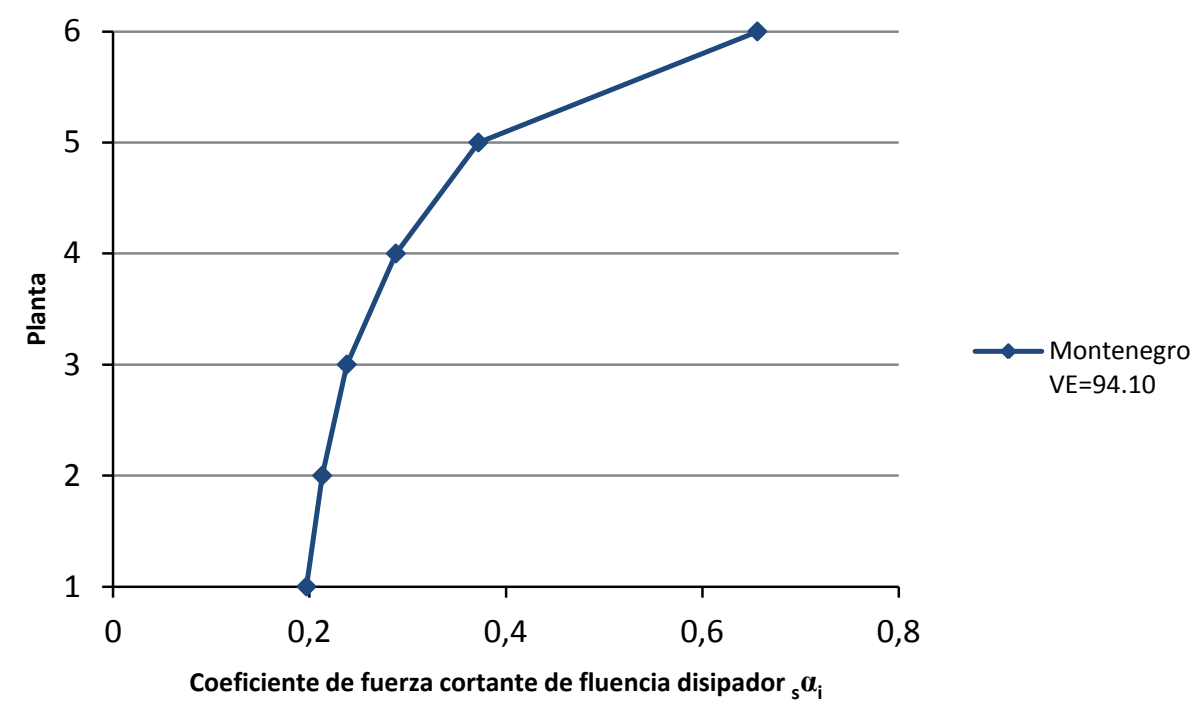

Fig. 5.5.71: Coeficiente de fuerza cortante de fluencia de los disipadores de cada planta i, pórtico pa6pz2-1, suelo tipo III, campo lejano 


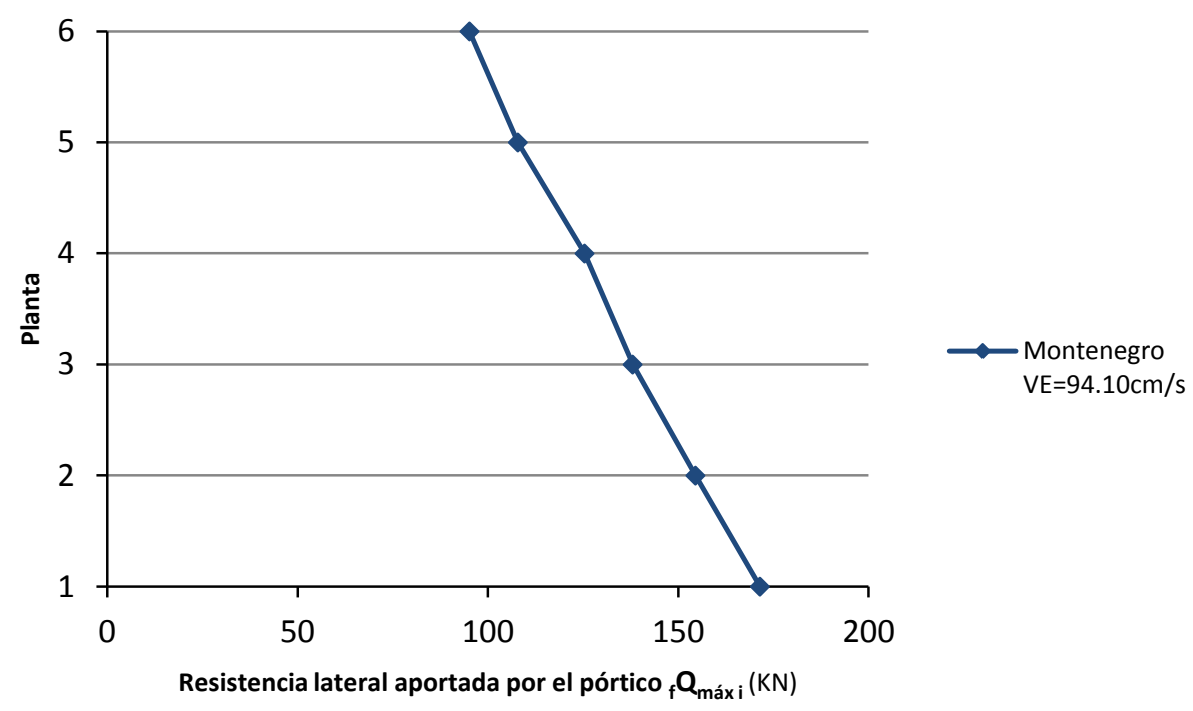

Fig. 5.5.72: Resistencia lateral aportada por la parte flexible (pórtico) cuando los disipadores empiezan a plastificar, pórtico pa6pz2-1, suelo tipo III, campo lejano

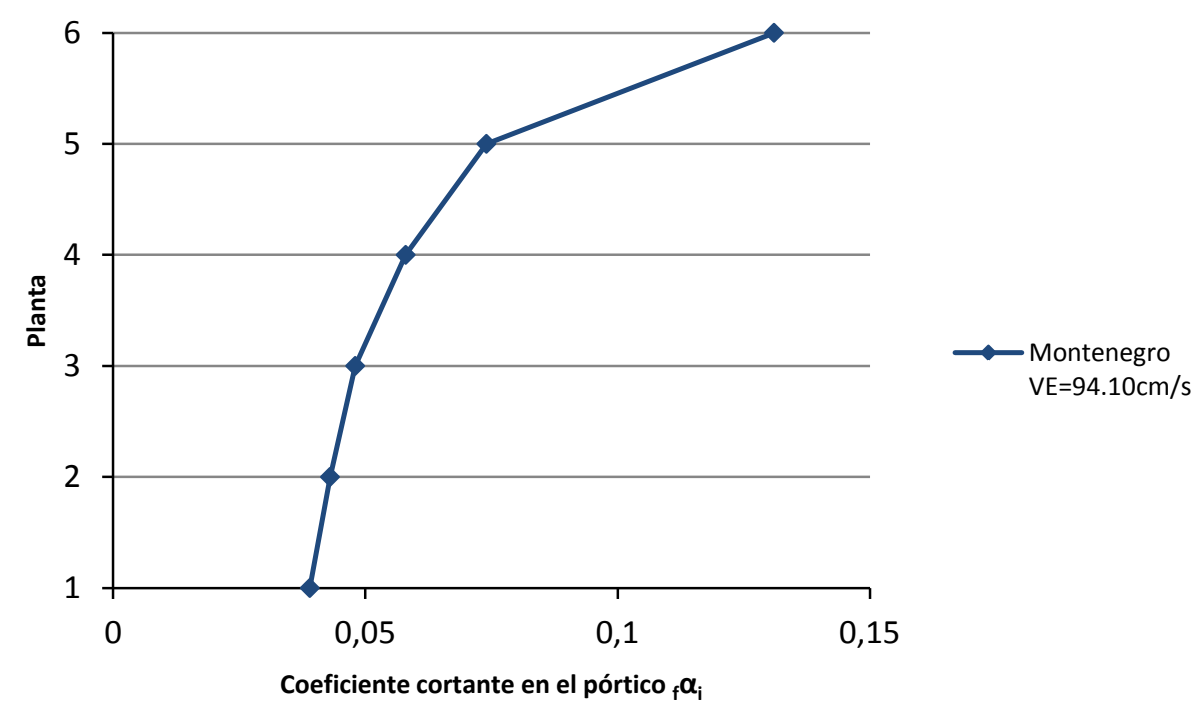

Fig. 5.5.73: Coeficiente de fuerza cortante en la parte flexible (pórtico) cuando los disipadores empiezan a plastificar, pórtico pa6pz2-1, suelo tipo III, campo lejano 


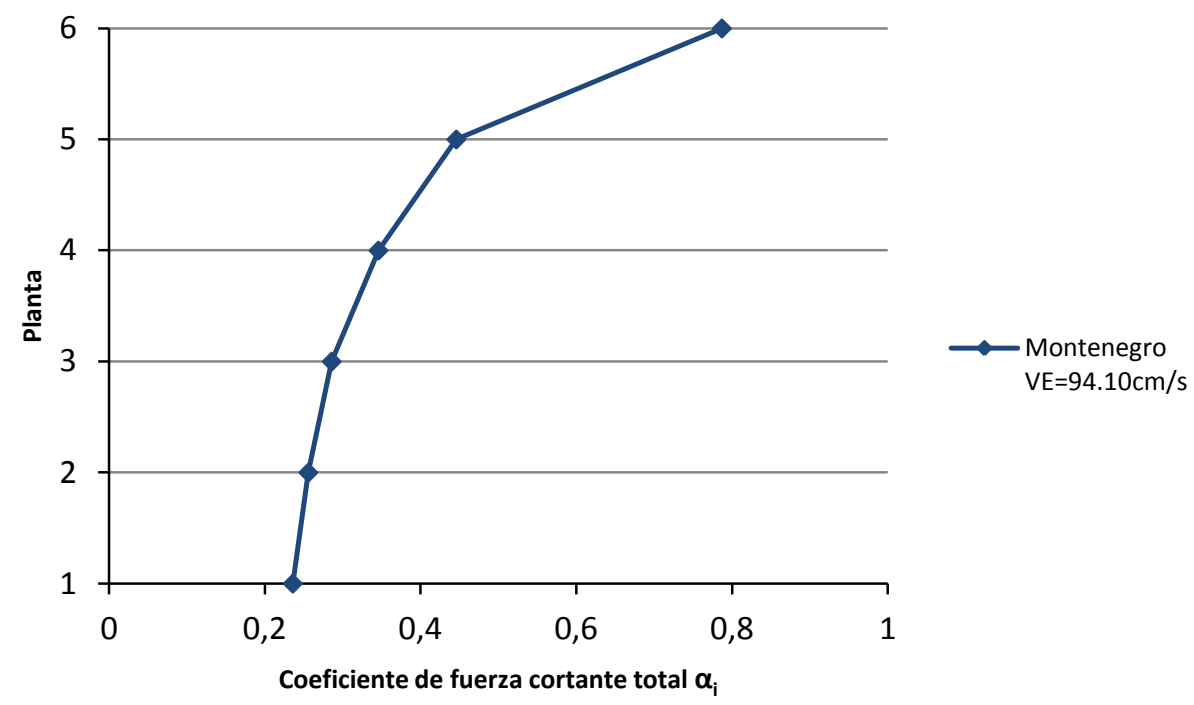

Fig. 5.5.74: Coeficiente de fuerza cortante total de planta, pórtico pa6pz2-1, suelo tipo III, campo lejano

Tabla 5.5.1: Resistencia lateral de los disipadores, coeficiente de fuerza cortante de fluencia de los disipadores, resistencia lateral aportada por la parte flexible (pórtico) cuando los disipadores empiezan a plastificar, coeficiente cortante en la parte flexible (pórtico) cuando los disipadores empiezan a plastificar, coeficiente de fuerza cortante total

\begin{tabular}{|c|c|c|c|c|c|c|c|c|}
\hline Pórtico & $\begin{array}{l}\text { Tipo de } \\
\text { suelo }\end{array}$ & Acelerograma & Planta & $\begin{array}{c}\text { Resistenc. } \\
\text { lateral } \\
\text { disipador } \\
{ }_{s} Q_{i}(\mathrm{~N})\end{array}$ & $\begin{array}{c}\text { Coefic. de } \\
\text { fuerza } \\
\text { cortante } \\
\text { fluencia } \\
\text { disipador } \\
{ }_{s} \alpha_{i}\end{array}$ & $\begin{array}{l}\text { Resistenc. } \\
\text { lateral } \\
\text { pórtico } \\
{ }_{f} Q_{\text {máx i }} \\
\text { (N) }\end{array}$ & $\begin{array}{l}\text { Coefic. } \\
\text { cortante } \\
\text { pórtico } \\
{ }_{f} \alpha_{i}\end{array}$ & $\begin{array}{c}\text { Coefic. de } \\
\text { fuerza } \\
\text { cortante } \\
\text { total } \\
\alpha_{i}\end{array}$ \\
\hline \multirow{9}{*}{ pa2pz2-1 } & \multirow{3}{*}{$\begin{array}{c}\text { I } \\
\text { Campo } \\
\text { lejano }\end{array}$} & $\begin{array}{c}\text { №1 } \\
\text { (Friuli) }\end{array}$ & $\begin{array}{l}1 \underline{a} \\
2 \underline{a} \\
3 \underline{a} \\
\end{array}$ & $\begin{array}{r}1485 \\
16252 \\
22 \\
\end{array}$ & $\begin{array}{l}6.828 e^{-04} \\
0.011 \\
3.034 e^{-05}\end{array}$ & $\begin{array}{r}297.0 \\
3250.4 \\
4.4 \\
\end{array}$ & $\begin{array}{l}1.365 \mathrm{e}^{-04} \\
2.242 \mathrm{e}^{-03} \\
6.069 \mathrm{e}^{-06}\end{array}$ & $\begin{array}{l}8.193 e^{-04} \\
0.013 \\
3.641 e^{-05} \\
\end{array}$ \\
\hline & & $\begin{array}{c}\text { No4 } \\
\text { (Montenegro) }\end{array}$ & $\begin{array}{l}19 \\
2 \underline{a} \\
3 \underline{a}\end{array}$ & $\begin{array}{r}148.5 \\
81056.0 \\
24200.0 \\
\end{array}$ & $\begin{array}{l}3.034 \mathrm{e}^{-05} \\
0.056 \\
0.033 \\
\end{array}$ & $\begin{array}{r}29.7 \\
16211.2 \\
4840.0 \\
\end{array}$ & $\begin{array}{l}1.365 \mathrm{e}^{-05} \\
0.011 \\
6.676 \mathrm{e}^{-03} \\
\end{array}$ & $\begin{array}{l}8.193 e^{-05} \\
0.067 \\
0.040 \\
\end{array}$ \\
\hline & & $\begin{array}{c}\text { №5 } \\
\text { (Campano } \\
\text { Lucano) } \\
\end{array}$ & $\begin{array}{l}1 \underline{a} \\
2 \underline{a} \\
3 \underline{a} \\
\end{array}$ & $\begin{array}{r}11187.0 \\
13668.0 \\
27.5 \\
\end{array}$ & $\begin{array}{l}5.143 \mathrm{e}^{-03} \\
9.426 \mathrm{e}^{-03} \\
3.793 \mathrm{e}^{-05}\end{array}$ & $\begin{array}{r}2237.4 \\
2733.6 \\
5.5 \\
\end{array}$ & $\begin{array}{l}1.029 \mathrm{e}^{-03} \\
1.885 \mathrm{e}^{-03} \\
7.586 \mathrm{e}^{-06}\end{array}$ & $\begin{array}{l}6.172 \mathrm{e}^{-03} \\
0.011 \\
4.552 \mathrm{e}^{-05} \\
\end{array}$ \\
\hline & \multirow{2}{*}{$\begin{array}{c}\text { Il } \\
\text { Campo } \\
\text { cercano }\end{array}$} & $\begin{array}{c}\text { №1 } \\
\text { (Friuli } \\
\text { aftershock) }\end{array}$ & $\begin{array}{l}1 \underline{a} \\
2 \underline{a} \\
3 \underline{a} \\
\end{array}$ & $\begin{array}{r}396.0 \\
12104.0 \\
27.5 \\
\end{array}$ & $\begin{array}{l}1.821 \mathrm{e}^{-04} \\
8.348 \mathrm{e}^{-03} \\
3.793 \mathrm{e}^{-05}\end{array}$ & $\begin{array}{r}79.2 \\
2420.8 \\
5.5 \\
\end{array}$ & $\begin{array}{l}3.641 \mathrm{e}^{-05} \\
1.669 \mathrm{e}^{-03} \\
7.586 \mathrm{e}^{-06}\end{array}$ & $\begin{array}{l}2.185 \mathrm{e}^{-04} \\
0.011 \\
4.552 \mathrm{e}^{-05} \\
\end{array}$ \\
\hline & & $\begin{array}{c}\text { №14 } \\
\text { (Montenegro } \\
\text { aftershock) }\end{array}$ & $\begin{array}{l}1 \underline{a} \\
2 \underline{a} \\
3 \underline{a} \\
\end{array}$ & $\begin{array}{l}241708.5 \\
201858.0 \\
122485.0 \\
\end{array}$ & $\begin{array}{l}0.111 \\
0.139 \\
0.169 \\
\end{array}$ & $\begin{array}{l}48341.7 \\
40371.6 \\
24497.0 \\
\end{array}$ & $\begin{array}{l}0.022 \\
0.028 \\
0.034 \\
\end{array}$ & $\begin{array}{l}0.133 \\
0.167 \\
0.203 \\
\end{array}$ \\
\hline & \multirow{2}{*}{$\begin{array}{c}\text { II } \\
\text { Campo } \\
\text { lejano }\end{array}$} & $\begin{array}{c}\text { №2 } \\
\text { (Friuli } \\
\text { aftershock) }\end{array}$ & $\begin{array}{l}1 \underline{a} \\
2 \underline{a} \\
3 \underline{a}\end{array}$ & $\begin{array}{r}8910.0 \\
43180.0 \\
11550.0\end{array}$ & $\begin{array}{l}4.096 \mathrm{e}^{-03} \\
0.030 \\
0.016\end{array}$ & $\begin{array}{l}1782.0 \\
8636.0 \\
2310.0\end{array}$ & $\begin{array}{l}8.193 \mathrm{e}^{-04} \\
5.956 \mathrm{e}^{-03} \\
3.186 \mathrm{e}^{-03}\end{array}$ & $\begin{array}{l}4.915 \mathrm{e}^{-03} \\
0.036 \\
0.019\end{array}$ \\
\hline & & $\begin{array}{c}\text { №12 } \\
\text { (Montenegro } \\
\text { aftershock) }\end{array}$ & $\begin{array}{l}1 \underline{a} \\
2 \underline{a} \\
3 \underline{a}\end{array}$ & $\begin{array}{l}468913.5 \\
510000.0 \\
550000.0\end{array}$ & $\begin{array}{l}0.215 \\
0.352 \\
0.759\end{array}$ & $\begin{array}{r}93782.7 \\
102000.0 \\
110000.0 \\
\end{array}$ & $\begin{array}{l}0.043 \\
0.070 \\
0.152 \\
\end{array}$ & $\begin{array}{l}0.258 \\
0.422 \\
0.911 \\
\end{array}$ \\
\hline & \multirow{2}{*}{$\begin{array}{c}\text { III } \\
\text { Campo } \\
\text { cercano }\end{array}$} & $\begin{array}{c}\mathrm{N} \text { ㅇ } \\
\text { (Friuli } \\
\text { aftershock) }\end{array}$ & $\begin{array}{l}1 \underline{a} \\
2 \underline{a} \\
3 \underline{a}\end{array}$ & $\begin{array}{l}357736.5 \\
318750.0 \\
171875.0 \\
\end{array}$ & $\begin{array}{l}0.164 \\
0.220 \\
0.237 \\
\end{array}$ & $\begin{array}{l}71547.3 \\
63750.0 \\
34375.0 \\
\end{array}$ & $\begin{array}{l}0.033 \\
0.044 \\
0.047 \\
\end{array}$ & $\begin{array}{l}0.197 \\
0.264 \\
0.284 \\
\end{array}$ \\
\hline & & $\begin{array}{c}\text { No6 } \\
\text { (Kalamata } \\
\text { aftershock) }\end{array}$ & $\begin{array}{l}1 \underline{a} \\
2 \underline{a} \\
3 \underline{a}\end{array}$ & $\begin{array}{r}49.5 \\
62016.0 \\
12375.0\end{array}$ & $\begin{array}{l}2.276 \mathrm{e}^{-05} \\
0.043 \\
0.017 \\
\end{array}$ & $\begin{array}{r}9.9 \\
12403.2 \\
2475.0 \\
\end{array}$ & $\begin{array}{l}4.552 \mathrm{e}^{-06} \\
8.554 \mathrm{e}^{-03} \\
3.414 \mathrm{e}^{-03}\end{array}$ & $\begin{array}{l}2.731 \mathrm{e}^{-05} \\
0.051 \\
0.020\end{array}$ \\
\hline
\end{tabular}


Trabajo fin de Máster en Ingeniería de Estructuras

\begin{tabular}{|c|c|c|c|c|c|c|c|c|}
\hline & & $\begin{array}{c}\text { №12 } \\
\text { (Duzce 1) }\end{array}$ & $\begin{array}{l}1 \underline{a} \\
2 \underline{a} \\
3 \underline{a}\end{array}$ & $\begin{array}{r}94743.0 \\
141100.0 \\
59345.0\end{array}$ & $\begin{array}{l}0.043 \\
0.097 \\
0.082\end{array}$ & $\begin{array}{l}18948.6 \\
28220.0 \\
11869.0\end{array}$ & $\begin{array}{l}8.712 e^{-03} \\
0.019 \\
0.016\end{array}$ & $\begin{array}{l}0.052 \\
0.116 \\
0.098\end{array}$ \\
\hline & \multirow{2}{*}{$\begin{array}{c}\text { III } \\
\text { Campo } \\
\text { lejano }\end{array}$} & $\begin{array}{c}\text { №3 } \\
\text { (Montenegro) }\end{array}$ & $\begin{array}{l}1 \underline{a} \\
2 \underline{a} \\
3 \underline{a}\end{array}$ & $\begin{array}{r}49.5 \\
39678.0 \\
27.5\end{array}$ & $\begin{array}{l}2.276 \mathrm{e}^{-05} \\
0.027 \\
3.793 \mathrm{e}^{-05}\end{array}$ & $\begin{array}{r}9.9 \\
7935.6 \\
5.5\end{array}$ & $\begin{array}{l}4.552 \mathrm{e}^{-06} \\
5.473 \mathrm{e}^{-03} \\
7.586 \mathrm{e}^{-06}\end{array}$ & $\begin{array}{l}2.731 \mathrm{e}^{-05} \\
0.032 \\
4.552 \mathrm{e}^{-05}\end{array}$ \\
\hline & & $\begin{array}{c}\text { №14 } \\
\text { (Ano Liosia) }\end{array}$ & $\begin{array}{l}1 \underline{a} \\
2 \underline{a} \\
3 \underline{a}\end{array}$ & $\begin{array}{r}49.5 \\
12138.0 \\
27.5\end{array}$ & $\begin{array}{l}2.276 \mathrm{e}^{-05} \\
8.371 \mathrm{e}^{-03} \\
3.793 \mathrm{e}^{-05}\end{array}$ & $\begin{array}{r}9.9 \\
2427.6 \\
5.5\end{array}$ & $\begin{array}{l}4.552 \mathrm{e}^{-06} \\
1.674 \mathrm{e}^{-03} \\
7.586 \mathrm{e}^{-06}\end{array}$ & $\begin{array}{l}2.731 \mathrm{e}^{-05} \\
0.010 \\
4.552 \mathrm{e}^{-05}\end{array}$ \\
\hline \multirow{12}{*}{ pa4pz2-1 } & \multirow{3}{*}{$\begin{array}{c}\text { I } \\
\text { Campo } \\
\text { lejano }\end{array}$} & $\begin{array}{c}\text { №1 } \\
\text { (Friuli) }\end{array}$ & $\begin{array}{l}1 \underline{a} \\
2 \underline{a} \\
3 \underline{a}\end{array}$ & $\begin{array}{l}61.99 \\
45.50 \\
37.77\end{array}$ & $\begin{array}{l}2.378 \mathrm{e}^{-05} \\
2.560 \mathrm{e}^{-05} \\
4.346 \mathrm{e}^{-05}\end{array}$ & $\begin{array}{r}12.40 \\
9.10 \\
7.55\end{array}$ & $\begin{array}{l}4.756 \mathrm{e}^{-06} \\
5.236 \mathrm{e}^{-06} \\
8.688 \mathrm{e}^{-06}\end{array}$ & $\begin{array}{l}2.854 \mathrm{e}^{-05} \\
3.084 \mathrm{e}^{-05} \\
5.215 \mathrm{e}^{-05}\end{array}$ \\
\hline & & $\begin{array}{c}\text { №4 } \\
\text { (Montenegro) }\end{array}$ & $\begin{array}{l}1 \underline{a} \\
2 \underline{a} \\
3 \underline{a}\end{array}$ & $\begin{array}{l}504687.5 \\
568750.0 \\
250404.0 \\
\end{array}$ & $\begin{array}{l}0.193 \\
0.327 \\
0.288 \\
\end{array}$ & $\begin{array}{r}100937.5 \\
113750.0 \\
50080.8 \\
\end{array}$ & $\begin{array}{l}0.039 \\
0.065 \\
0.058 \\
\end{array}$ & $\begin{array}{l}0.232 \\
0.392 \\
0.346 \\
\end{array}$ \\
\hline & & $\begin{array}{c}\text { №5 } \\
\text { (Campano } \\
\text { Lucano) }\end{array}$ & $\begin{array}{l}1 \underline{a} \\
2 \underline{a} \\
3 \underline{a}\end{array}$ & $\begin{array}{r}395.68 \\
46.36 \\
38.50 \\
\end{array}$ & $\begin{array}{l}1.518 \mathrm{e}^{-04} \\
2.215 \mathrm{e}^{-05} \\
4.430 \mathrm{e}^{-05}\end{array}$ & $\begin{array}{r}78.54 \\
9.92 \\
7.70 \\
\end{array}$ & $\begin{array}{l}3.013 \mathrm{e}^{-05} \\
5.708 \mathrm{e}^{-06} \\
8.861 \mathrm{e}^{-06}\end{array}$ & $\begin{array}{l}1.819 \mathrm{e}^{-04} \\
2.786 \mathrm{e}^{-05} \\
5.316 \mathrm{e}^{-05}\end{array}$ \\
\hline & II & $\begin{array}{c}\text { №1 } \\
\text { (Friuli } \\
\text { aftershock) }\end{array}$ & $\begin{array}{l}1 \underline{a} \\
2 \underline{a} \\
3 \underline{a} \\
\end{array}$ & $\begin{array}{r}522.5 \\
2730.0 \\
423.5 \\
\end{array}$ & $\begin{array}{l}2.004 \mathrm{e}^{-04} \\
1.571 \mathrm{e}^{-03} \\
4.873 \mathrm{e}^{-04}\end{array}$ & $\begin{array}{r}104.5 \\
546.0 \\
84.7 \\
\end{array}$ & $\begin{array}{l}4.008 \mathrm{e}^{-05} \\
3.141 \mathrm{e}^{-04} \\
9.747 \mathrm{e}^{-05}\end{array}$ & $\begin{array}{l}2.405 \mathrm{e}^{-04} \\
1.885 \mathrm{e}^{-03} \\
5.848 \mathrm{e}^{-04}\end{array}$ \\
\hline & cercano & $\begin{array}{c}\text { №14 } \\
\text { (Montenegro } \\
\text { aftershock) }\end{array}$ & $\begin{array}{l}1 \underline{a} \\
2 \underline{a} \\
3 \underline{a}\end{array}$ & $\begin{array}{r}47.5 \\
68341.0 \\
38.5 \\
\end{array}$ & $\begin{array}{l}1.822 \mathrm{e}^{-05} \\
1.571 \\
4.430 \mathrm{e}^{-05}\end{array}$ & $\begin{array}{r}9.5 \\
13668.2 \\
7.7 \\
\end{array}$ & $\begin{array}{l}3.644 \mathrm{e}^{-06} \\
7.664 \mathrm{e}^{-03} \\
8.861 \mathrm{e}^{-06}\end{array}$ & $\begin{array}{l}2.186 \mathrm{e}^{-05} \\
0.047 \\
5.316 \mathrm{e}^{-05}\end{array}$ \\
\hline & II & $\begin{array}{c}\mathrm{N} \text { o2 } \\
\text { (Friuli } \\
\text { aftershock) }\end{array}$ & $\begin{array}{l}1 \underline{a} \\
2 \underline{a} \\
3 \underline{a}\end{array}$ & $\begin{array}{l}556652.5 \\
625625.0 \\
254331.0 \\
\end{array}$ & $\begin{array}{l}0.213 \\
0.360 \\
0.293 \\
\end{array}$ & $\begin{array}{r}111330.5 \\
125125.0 \\
77000.7 \\
\end{array}$ & $\begin{array}{l}0.043 \\
0.072 \\
0.089 \\
\end{array}$ & $\begin{array}{l}0.256 \\
0.432 \\
0.382 \\
\end{array}$ \\
\hline & lejano & $\begin{array}{c}\text { №12 } \\
\text { (Montenegro } \\
\text { aftershock) }\end{array}$ & $\begin{array}{l}1 \underline{a} \\
2 \underline{a} \\
3 \underline{a}\end{array}$ & $\begin{array}{l}623437.5 \\
618982.0 \\
412065.5 \\
\end{array}$ & $\begin{array}{l}0.239 \\
0.356 \\
0.474 \\
\end{array}$ & $\begin{array}{r}124687.5 \\
123796.4 \\
82413.1 \\
\end{array}$ & $\begin{array}{l}0.048 \\
0.071 \\
0.095 \\
\end{array}$ & $\begin{array}{l}0.287 \\
0.427 \\
0.569 \\
\end{array}$ \\
\hline & & $\begin{array}{c}\mathrm{N} \text { o2 } \\
\text { (Friuli } \\
\text { aftershock) }\end{array}$ & $\begin{array}{l}1 \underline{a} \\
2 \underline{a} \\
3 \underline{a}\end{array}$ & $\begin{array}{l}433247.5 \\
348484.5 \\
175983.5 \\
\end{array}$ & $\begin{array}{l}0.166 \\
0.200 \\
0.202 \\
\end{array}$ & $\begin{array}{l}86649.5 \\
69696.9 \\
35196.7 \\
\end{array}$ & $\begin{array}{l}0.033 \\
0.040 \\
0.040 \\
\end{array}$ & $\begin{array}{l}0.199 \\
0.240 \\
0.242 \\
\end{array}$ \\
\hline & $\begin{array}{c}\text { III } \\
\text { Campo } \\
\text { cercano }\end{array}$ & $\begin{array}{c}\text { No6 } \\
\text { (Kalamata } \\
\text { aftershock) }\end{array}$ & $\begin{array}{l}1 \underline{a} \\
2 \underline{a} \\
3 \underline{a}\end{array}$ & $\begin{array}{l}38095.0 \\
60014.5 \\
33841.5 \\
\end{array}$ & $\begin{array}{l}0.015 \\
0.034 \\
0.039 \\
\end{array}$ & $\begin{array}{r}7619.0 \\
12002.9 \\
6768.3 \\
\end{array}$ & $\begin{array}{l}2.922 \mathrm{e}^{-03} \\
6.906 \mathrm{e}^{-03} \\
7.789 \mathrm{e}^{-03}\end{array}$ & $\begin{array}{l}0.018 \\
0.041 \\
0.047 \\
\end{array}$ \\
\hline & & $\begin{array}{c}\text { №12 } \\
\text { (Duzce 1) }\end{array}$ & $\begin{array}{l}1 \underline{a} \\
2 \underline{a} \\
3 \underline{a}\end{array}$ & $\begin{array}{r}172567.5 \\
165301.5 \\
63140.0\end{array}$ & $\begin{array}{l}0.066 \\
0.095 \\
0.073\end{array}$ & $\begin{array}{l}34513.5 \\
33060.3 \\
12628.0\end{array}$ & $\begin{array}{l}0.013 \\
0.019 \\
0.014 \\
\end{array}$ & $\begin{array}{l}0.079 \\
0.114 \\
0.087 \\
\end{array}$ \\
\hline & III & $\begin{array}{c}\text { №3 } \\
\text { (Montenegro) }\end{array}$ & $\begin{array}{l}1 \underline{a} \\
2 \underline{a} \\
3 \underline{a}\end{array}$ & $\begin{array}{r}214985.0 \\
180635.0 \\
74459.0 \\
\end{array}$ & $\begin{array}{l}0.082 \\
0.104 \\
0.086 \\
\end{array}$ & $\begin{array}{l}42997.0 \\
36127.0 \\
14891.8 \\
\end{array}$ & $\begin{array}{l}0.016 \\
0.021 \\
0.017 \\
\end{array}$ & $\begin{array}{l}0.098 \\
0.125 \\
0.103 \\
\end{array}$ \\
\hline & $\begin{array}{l}\text { Campo } \\
\text { lejano }\end{array}$ & $\begin{array}{c}\text { №14 } \\
\text { (Ano Liosia) }\end{array}$ & $\begin{array}{l}1 \underline{a} \\
2 \underline{a} \\
3 \underline{a}\end{array}$ & $\begin{array}{r}5842.5 \\
45.5 \\
38.5 \\
\end{array}$ & $\begin{array}{l}2.241 \mathrm{e}^{-03} \\
2.618 \mathrm{e}^{-05} \\
4.430 \mathrm{e}^{-05}\end{array}$ & $\begin{array}{r}1168.5 \\
9.1 \\
7.7 \\
\end{array}$ & $\begin{array}{l}4.482 \mathrm{e}^{-04} \\
5.236 \mathrm{e}^{-06} \\
8.861 \mathrm{e}^{-06}\end{array}$ & $\begin{array}{l}2.689 \mathrm{e}^{-03} \\
3.142 \mathrm{e}^{-05} \\
5.316 \mathrm{e}^{-05}\end{array}$ \\
\hline pa6pz2-1 & $\begin{array}{c}\text { I } \\
\text { Campo }\end{array}$ & $\begin{array}{c}\text { №4 } \\
\text { (Montenegro) }\end{array}$ & $\begin{array}{l}1 \underline{a} \\
2 \underline{a} \\
3 \underline{a} \\
4 \underline{a} \\
5 \underline{a} \\
6 \underline{a} \\
\end{array}$ & $\begin{array}{l}533750.0 \\
526400.0 \\
504450.0 \\
406800.0 \\
498000.0 \\
308000.0 \\
\end{array}$ & $\begin{array}{l}0.123 \\
0.145 \\
0.174 \\
0.187 \\
0.343 \\
0.425 \\
\end{array}$ & $\begin{array}{r}106750.0 \\
105280.0 \\
100890.0 \\
81360.0 \\
99600.0 \\
61600.0 \\
\end{array}$ & $\begin{array}{l}0.024 \\
0.029 \\
0.035 \\
0.037 \\
0.069 \\
0.085 \\
\end{array}$ & $\begin{array}{l}0.147 \\
0.174 \\
0.209 \\
0.224 \\
0.412 \\
0.510 \\
\end{array}$ \\
\hline & & $\begin{array}{c}\text { №5 } \\
\text { (Campano } \\
\text { Lucano) }\end{array}$ & $\begin{array}{l}1 \underline{a} \\
2 \underline{a} \\
3 \underline{a} \\
4 \underline{a}\end{array}$ & $\begin{array}{r}21875.0 \\
11200.0 \\
8850.0 \\
5650.0\end{array}$ & $\begin{array}{l}5.029 \mathrm{e}^{-03} \\
3.090 \mathrm{e}^{-03} \\
3.052 \mathrm{e}^{-03} \\
2.598 \mathrm{e}^{-03}\end{array}$ & $\begin{array}{l}4375.00 \\
2240.00 \\
1770.00 \\
1130.00\end{array}$ & $\begin{array}{l}1.006 \mathrm{e}^{-03} \\
6.179 \mathrm{e}^{-04} \\
6.103 \mathrm{e}^{-04} \\
5.195 \mathrm{e}^{-04}\end{array}$ & $\begin{array}{l}6.035 \mathrm{e}^{-03} \\
3.708 \mathrm{e}^{-03} \\
3.662 \mathrm{e}^{-03} \\
3.117 \mathrm{e}^{-03}\end{array}$ \\
\hline
\end{tabular}




\begin{tabular}{|c|c|c|c|c|c|c|c|}
\hline & & $\begin{array}{l}5 \underline{a} \\
6 \underline{a}\end{array}$ & $\begin{array}{r}6640.0 \\
25.2 \\
\end{array}$ & $\begin{array}{l}4.579 \mathrm{e}^{-03} \\
3.476 \mathrm{e}^{-05}\end{array}$ & $\begin{array}{r}1328.00 \\
5.04\end{array}$ & $\begin{array}{l}9.159 \mathrm{e}^{-04} \\
6.952 \mathrm{e}^{-06}\end{array}$ & $\begin{array}{l}5.495 \mathrm{e}^{-03} \\
4.171 \mathrm{e}^{-05}\end{array}$ \\
\hline \multirow{6}{*}{$\begin{array}{c}\text { II } \\
\text { Campo } \\
\text { cercano }\end{array}$} & \multirow{6}{*}{$\begin{array}{c}\text { №14 } \\
\text { (Montenegro } \\
\text { aftershock) }\end{array}$} & $1 \underline{a}$ & 350000.0 & 0.080 & 70000.0 & 0.016 & 0.096 \\
\hline & & $2^{a}$ & 268800.0 & 0.074 & 53760.0 & 0.015 & 0.089 \\
\hline & & $3 \underline{a}$ & 212400.0 & 0.073 & 42480.0 & 0.015 & 0.088 \\
\hline & & $4^{a}$ & 206225.0 & 0.095 & 41245.0 & 0.019 & 0.114 \\
\hline & & $5 \underline{a}$ & 116200.0 & 0.080 & 23240.0 & 0.016 & 0.096 \\
\hline & & 6a & 72800.0 & 0.100 & 14560.0 & 0.020 & 0.120 \\
\hline \multirow{24}{*}{$\begin{array}{c}\text { III } \\
\text { Campo } \\
\text { cercano }\end{array}$} & \multirow{6}{*}{$\begin{array}{c}\mathrm{N} 02 \\
\text { (Friuli } \\
\text { aftershock) }\end{array}$} & 1a & 23625.0 & $5.431 e^{-03}$ & 4725.00 & $1.086 \mathrm{e}^{-03}$ & $6.517 e^{-03}$ \\
\hline & & $2 \underline{a}$ & 4368.0 & $1.205 \mathrm{e}^{-03}$ & 873.60 & $2.410 \mathrm{e}^{-04}$ & $1.446 e^{-03}$ \\
\hline & & $3 \underline{a}$ & 35.4 & $1.221 \mathrm{e}^{-05}$ & 7.08 & $2.441 \mathrm{e}^{-06}$ & $1.465 e^{-05}$ \\
\hline & & $4 \underline{a}$ & 24295.0 & 0.011 & 4859.00 & $2.234 \mathrm{e}^{-03}$ & 0.013 \\
\hline & & $5^{\underline{a}}$ & 28635.0 & 0.020 & 5727.00 & $3.950 e^{-03}$ & 0.024 \\
\hline & & $6 \underline{a}$ & 30800.0 & 0.042 & 6160.00 & $8.496 e^{-03}$ & 0.050 \\
\hline & \multirow{6}{*}{$\begin{array}{c}\mathrm{N} \text { 을 } \\
\text { (Friuli } \\
\text { aftershock) }\end{array}$} & $1 \underline{a}$ & 23625.0 & $5.431 \mathrm{e}^{-03}$ & 4725.00 & $1.086 \mathrm{e}^{-03}$ & $6.517 e^{-03}$ \\
\hline & & $2 \underline{a}$ & 4368.0 & $1.205 \mathrm{e}^{-03}$ & 873.60 & $2.410 \mathrm{e}^{-04}$ & $1.446 e^{-03}$ \\
\hline & & $3 \underline{a}$ & 35.4 & $1.221 \mathrm{e}^{-05}$ & 7.08 & $2.441 \mathrm{e}^{-06}$ & $1.465 e^{-05}$ \\
\hline & & $4 \underline{a}$ & 24295.0 & 0.011 & 4859.00 & $2.234 \mathrm{e}^{-03}$ & 0.013 \\
\hline & & $5 \underline{a}$ & 28635.0 & 0.020 & 5727.00 & $3.950 e^{-03}$ & 0.024 \\
\hline & & 6a & 30800.0 & 0.042 & 6160.00 & $8.496 \mathrm{e}^{-03}$ & 0.050 \\
\hline & \multirow{6}{*}{$\begin{array}{c}\text { №6 } \\
\text { (Kalamata } \\
\text { aftershock) }\end{array}$} & $1 \mathrm{a}$ & 679000.0 & 0.156 & 135800.0 & 0.031 & 0.187 \\
\hline & & $2^{\mathrm{a}}$ & 555520.0 & 0.153 & 111104.0 & 0.031 & 0.184 \\
\hline & & $3 \underline{a}$ & 624810.0 & 0.215 & 124962.0 & 0.043 & 0.258 \\
\hline & & $4 \underline{a}$ & 841850.0 & 0.387 & 168370.0 & 0.077 & 0.464 \\
\hline & & $5 \underline{a}$ & 670225.0 & 0.462 & 134045.0 & 0.092 & 0.554 \\
\hline & & 6a & 532000.0 & 0.734 & 106400.0 & 0.147 & 0.881 \\
\hline & \multirow{6}{*}{$\begin{array}{c}\text { №12 } \\
\text { (Duzce 1) }\end{array}$} & $1 \underline{a}$ & 158375.0 & 0.036 & 31675.0 & $7.282 \mathrm{e}^{-03}$ & 0.043 \\
\hline & & $2 \underline{a}$ & 105280.0 & 0.029 & 21056.0 & $5.808 e^{-03}$ & 0.035 \\
\hline & & $3 \underline{a}$ & 63808.5 & 0.022 & 12761.7 & $4.400 \mathrm{e}^{-03}$ & 0.026 \\
\hline & & $4 \underline{a}$ & 50850.0 & 0.023 & 10170.0 & $4.676 \mathrm{e}^{-03}$ & 0.028 \\
\hline & & $5^{\underline{a}}$ & 45650.0 & 0.031 & 9130.0 & $6.296 \mathrm{e}^{-03}$ & 0.037 \\
\hline & & 6a & 25200.0 & 0.035 & 5040.0 & $6.952 e^{-03}$ & 0.042 \\
\hline \multirow{6}{*}{$\begin{array}{c}\text { III } \\
\text { Campo } \\
\text { lejano }\end{array}$} & \multirow{6}{*}{$\begin{array}{c}\text { №3 } \\
\text { (Montenegro) }\end{array}$} & $1 \underline{a}$ & 857500.0 & 0.197 & 171500.0 & 0.039 & 0.236 \\
\hline & & $2 \underline{a}$ & 772800.0 & 0.213 & 154560.0 & 0.043 & 0.256 \\
\hline & & $3 \underline{a}$ & 690300.0 & 0.238 & 138060.0 & 0.048 & 0.286 \\
\hline & & $4 \underline{a}$ & 627150.0 & 0.288 & 125430.0 & 0.058 & 0.346 \\
\hline & & $5 \underline{a}$ & 539500.0 & 0.372 & 107900.0 & 0.074 & 0.446 \\
\hline & & $6 \underline{a}$ & 476000.0 & 0.656 & 95200.0 & 0.131 & 0.787 \\
\hline
\end{tabular}




\subsection{COMPARACIÓN DE LAS DISTRIBUCIONES DE RESISTENCIA OBTENIDAS CON FÓRMULAS PROPUESTAS EN LA LITERATURA}

En este apartado se comparan las distribuciones de resistencia lateral total requeridas en cada planta de la estructura para que satisfaga el requisito de desplazamiento máximo entre plantas establecido (0.5\%), con la distribución óptima propuesta por Akiyama (1985). En estas distribuciones la resistencia lateral total se ha expresado en forma de coeficiente de fuerza cortante $\alpha_{i}$. La distribución de resistencia entre las plantas viene dada por los cocientes $\alpha_{i} / \alpha_{1}$ de cada planta.

Aquí, $\alpha_{1}$ es el coeficiente de fuerza cortante total de la planta más baja.

La distribución óptima del coeficiente de fuerza cortante de fluencia fue definida por Akiyama como aquella que hace que la energía de deformación plástica acumulada, normalizada por el desplazamiento y la fuerza de fluencia de la planta, valga lo mismo en todas ellas. La distribución óptima de Akiyama viene dada por las siguientes expresiones:

Para $x \leq 0,2$ :

$$
\alpha_{\text {iótima }} / \alpha_{1}=1+0,5 x
$$

Para $x>0,2$ :

$$
\alpha_{\text {ióptima }} / \alpha_{1}=1+1,5927 x-11,852 x^{2}+42,583 x^{3}-59,48 x^{4}+30,16 x^{5}
$$

Siendo $x=(i-1) / N$, donde $i$ es el número de la planta y $N$ el número total de plantas.

5.6.1 Distribución óptima del coeficiente de fuerza cortante de fluencia definida por Akiyama $\alpha_{i} / \alpha_{1}$ para una estructura de tres plantas

Planta 3a:

$$
\begin{aligned}
& x=(i-1) / N=(3-1) / 3=0,667>0,2 \\
& \alpha_{3} / \alpha_{1}=1+1,5927(3-1) / 3-11,852[(3-1) / 3]^{2}+42,583[(3-1) / 3]^{3}-59,48[(3-1) / 3]^{4}+30,16[(3-1) / 3]^{5} \Rightarrow \\
& \alpha_{3} / \alpha_{1}=1,634
\end{aligned}
$$

Planta 2a:

$$
\begin{aligned}
& x=(i-1) / N=(2-1) / 3=0,333>0,2 \\
& \alpha_{2} / \alpha_{1}=1+1,5927(2-1) / 3-11,852[(2-1) / 3]^{2}+42,583[(2-1) / 3]^{3}-59,48[(2-1) / 3]^{4}+30,16[(2-1) / 3]^{5} \Rightarrow \\
& \alpha_{2} / \alpha_{1}=1,181
\end{aligned}
$$

Planta 1:

$$
\begin{aligned}
& x=(i-1) / N=(1-1) / 3=0<0,2 \\
& \alpha_{1} / \alpha_{1}=1+0,5(1-1) / 3 \Rightarrow \underline{\alpha_{1} / \alpha_{1}=1}
\end{aligned}
$$

5.6.2 Distribución de resistencia entre plantas para el pórtico pa2pz2-1, suelo tipo I, campo lejano

$$
\alpha_{i} / \alpha_{1}
$$




\section{- Acelerograma №1 (Friuli) $\Delta=\mathbf{2 7} \mathrm{Km}$}

Coeficiente de fuerza cortante total de planta $\alpha_{i}$

Planta 3a: $\alpha_{3}=3,641 e^{-05}$

Planta 2a: $\alpha_{2}=0,013$

Planta 1: $\alpha_{1}=8,193 e^{-04}$

Distribución de resistencia entre plantas $\alpha_{i} / \alpha_{1}$

Planta 3a: $\alpha_{3} / \alpha_{1}=3,641 e^{-05} / 8,193 e^{-04} \Rightarrow \alpha_{3} / \alpha_{1}=0,044$

Planta 2a: $\alpha_{2} / \alpha_{1}=0,013 / 8,193 e^{-04} \Rightarrow \quad \alpha_{2} / \alpha_{1}=15,867$

Planta 1a: $\quad \alpha_{1} / \alpha_{1}=1,000$

\section{- Acelerograma №4 (Montenegro) $\Delta=65 \mathrm{Km}$}

Coeficiente de fuerza cortante total de planta $\alpha_{i}$

Planta 3a: $\alpha_{3}=0,040$

Planta 2a: $\alpha_{2}=0,067$

Planta 1: $\alpha_{1}=8,193 e^{-05}$

Distribución de resistencia entre plantas $\alpha_{i} / \alpha_{1}$

Planta 3a: $\alpha_{3} / \alpha_{1}=0,040 / 8,193 e^{-05} \Rightarrow \alpha_{3} / \alpha_{1}=488,222$

Planta 2a: $\alpha_{2} / \alpha_{1}=0,067 / 8,193 e^{-05} \Rightarrow \alpha_{2} / \alpha_{1}=817,771$

Planta 1a:

$$
\alpha_{1} / \alpha_{1}=1,000
$$

- Acelerograma №5 (Campano Lucano) $\Delta=32 \mathrm{Km}$

Coeficiente de fuerza cortante total de planta $\alpha_{i}$

Planta 3a: $\alpha_{3}=4,552 e^{-05}$

Planta 2a: $\alpha_{2}=0,011$

Planta 1: $\alpha_{1}=6,172 e^{-03}$

Distribución de resistencia entre plantas $\alpha_{i} / \alpha_{1}$

Planta 3a: $\alpha_{3} / \alpha_{1}=4,552 e^{-05} / 6,172 e^{-03} \Rightarrow \alpha_{3} / \alpha_{1}=7,375 e^{-03}$

Planta 2a: $\alpha_{2} / \alpha_{1}=0,011 / 6,172 e^{-03} \Rightarrow \quad \alpha_{2} / \alpha_{1}=1,782$

Planta 1:

$$
\alpha_{1} / \alpha_{1}=1,000
$$

\section{Representación gráfica}

En las figuras 5.6 .1 a 5.6 .14 se representa la distribución de resistencia entre plantas $\alpha_{i} / \alpha_{1}$, siendo $\alpha_{i}$ el coeficiente de fuerza cortante total de planta obtenido en el apartado 5.5 de este capítulo y $\alpha_{1}$ el coeficiente de fuerza cortante total de la planta más baja. Sobre estas figuras se dibuja la distribución óptima de Akiyama que viene dada por las expresiones (5.6.1) y (5.6.2). 


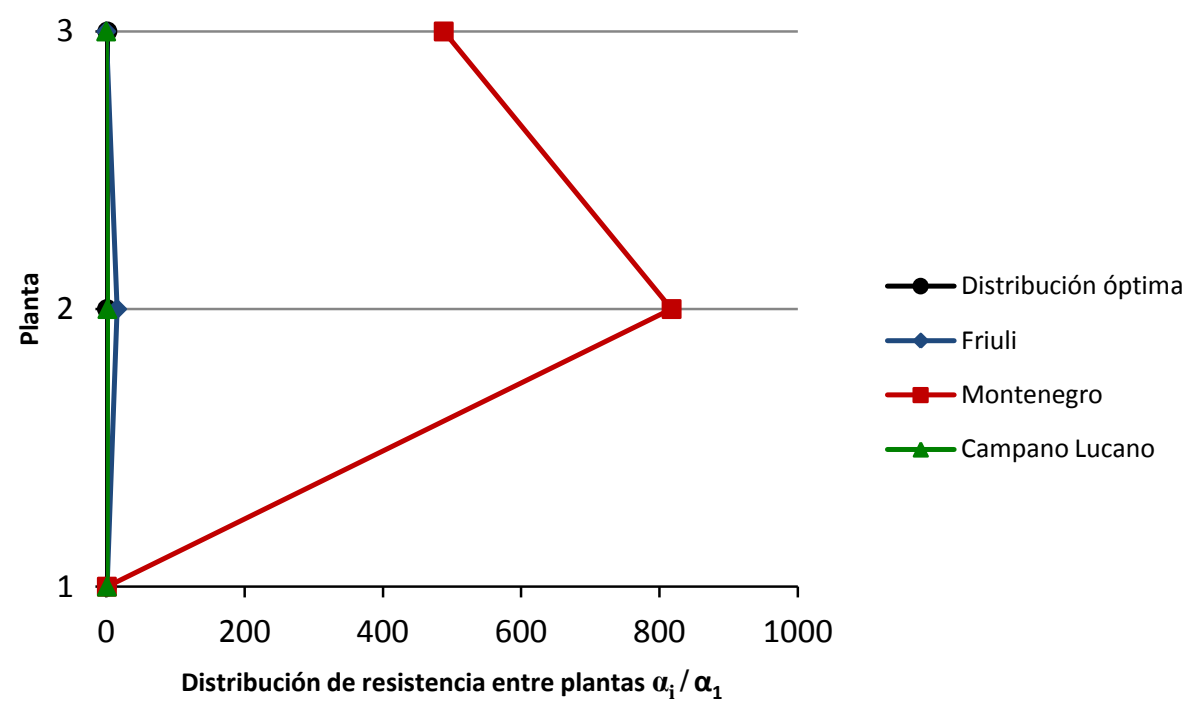

Fig. 5.6.1: Comparación de las distribuciones de resistencia, pórtico pa2pz2-1, suelo tipo I, campo lejano

5.6.3 Distribución de resistencia entre plantas para el pórtico pa2pz2-1, suelo tipo II, campo cercano $\alpha_{i} / \alpha_{1}$

\section{- Acelerograma №1 (Friuli aftershock) $\Delta=\mathbf{3 K m}$}

Coeficiente de fuerza cortante total de planta $\alpha_{i}$

Planta 3a: $\alpha_{3}=4,552 e^{-05}$

Planta 2: $\alpha_{2}=0,010$

Planta 1: $\alpha_{1}=2,185 e^{-04}$

Distribución de resistencia entre plantas $\alpha_{i} / \alpha_{1}$

Planta 3a: $\alpha_{3} / \alpha_{1}=4,552 e^{-05} / 2,185 e^{-04} \Rightarrow \alpha_{3} / \alpha_{1}=0,208$

Planta 2 ${ }^{\mathrm{a}}: \alpha_{2} / \alpha_{1}=0,010 / 2,185 e^{-04} \Rightarrow \quad \alpha_{2} / \alpha_{1}=45,766$

Planta 1a: $\quad \alpha_{1} / \alpha_{1}=1,000$

\section{- Acelerograma №14 (Montenegro aftershock) $\Delta=8 \mathrm{Km}$}

Coeficiente de fuerza cortante total de planta $\alpha_{i}$

Planta 3a: $\alpha_{3}=0,203$

Planta 2a: $\alpha_{2}=0,167$

Planta 1a: $\alpha_{1}=0,133$

Distribución de resistencia entre plantas $\alpha_{i} / \alpha_{1}$

Planta 3ạ: $\alpha_{3} / \alpha_{1}=0,203 / 0,133 \Rightarrow \alpha_{3} / \alpha_{1}=1,526$

Planta 2a: $\alpha_{2} / \alpha_{1}=0,167 / 0,133 \Rightarrow \alpha_{2} / \alpha_{1}=1,256$

Planta 1a:

$$
\alpha_{1} / \alpha_{1}=1,000
$$


Representación gráfica

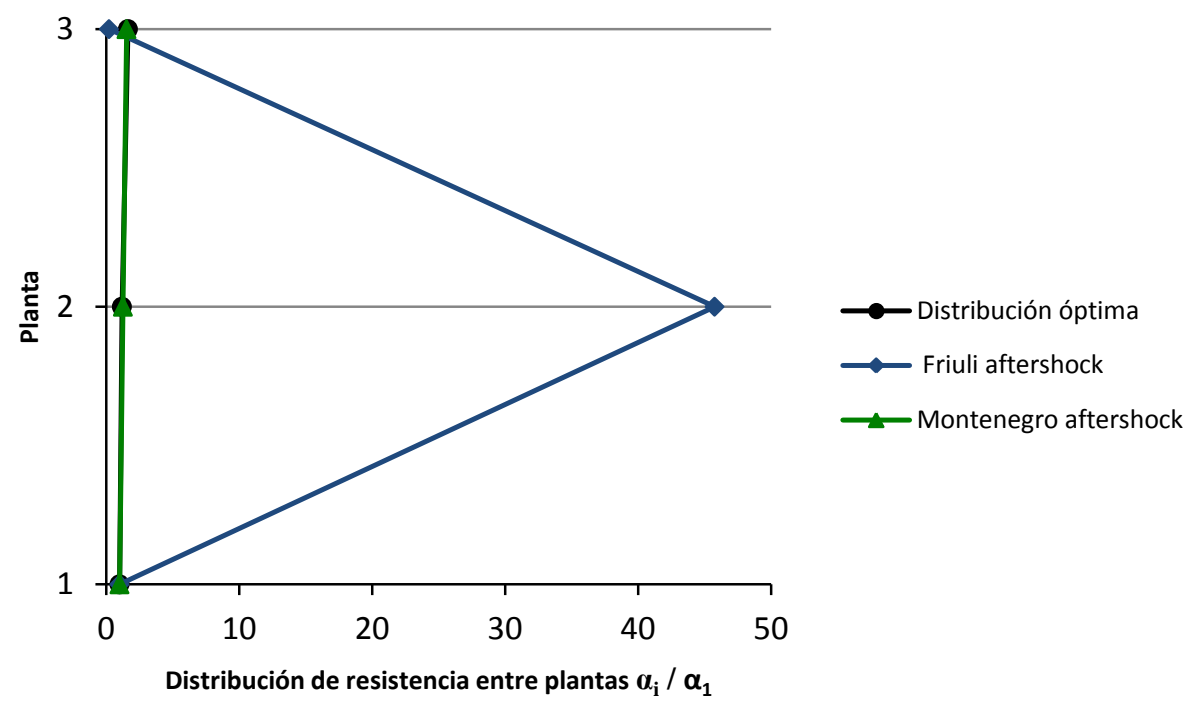

Fig. 5.6.2: Comparación de las distribuciones de resistencia, pórtico pa2pz2-1, suelo tipo II, campo cercano

5.6.4 Distribución de resistencia entre plantas para el pórtico pa2pz2-1, suelo tipo II, campo lejano $\alpha_{i} / \alpha_{1}$

- Acelerograma №2 (Friuli aftershock) $\Delta=\mathbf{1 6 K m}$

Coeficiente de fuerza cortante total de planta $\alpha_{i}$

Planta 3a: $\alpha_{3}=0,019$

Planta 2a: $\alpha_{2}=0,036$

Planta 1: $\alpha_{1}=4,915 e^{-03}$

Distribución de resistencia entre plantas $\alpha_{i} / \alpha_{1}$

Planta 3a: $\alpha_{3} / \alpha_{1}=0,019 / 4,915 e^{-03} \Rightarrow \alpha_{3} / \alpha_{1}=3,866$

Planta 2a: $\alpha_{2} / \alpha_{1}=0,036 / 4,915 e^{-03} \Rightarrow \alpha_{2} / \alpha_{1}=7,324$

Planta 1a:

$\alpha_{1} / \alpha_{1}=1,000$

\section{- Acelerograma №12 (Montenegro aftershock) $\Delta=33 \mathrm{Km}$}

Coeficiente de fuerza cortante total de planta $\alpha_{i}$

Planta 3ㄹ: $\alpha_{3}=0,911$

Planta 2a: $\alpha_{2}=0,422$

Planta 1a: $\alpha_{1}=0,258$

Distribución de resistencia entre plantas $\alpha_{i} / \alpha_{1}$

Planta 3a: $\alpha_{3} / \alpha_{1}=0,911 / 0,258 \Rightarrow \alpha_{3} / \alpha_{1}=3,531$

Planta 2a: $\alpha_{2} / \alpha_{1}=0,422 / 0,258 \Rightarrow \alpha_{2} / \alpha_{1}=1,636$

Planta 1:

$$
\alpha_{1} / \alpha_{1}=1,000
$$


Representación gráfica

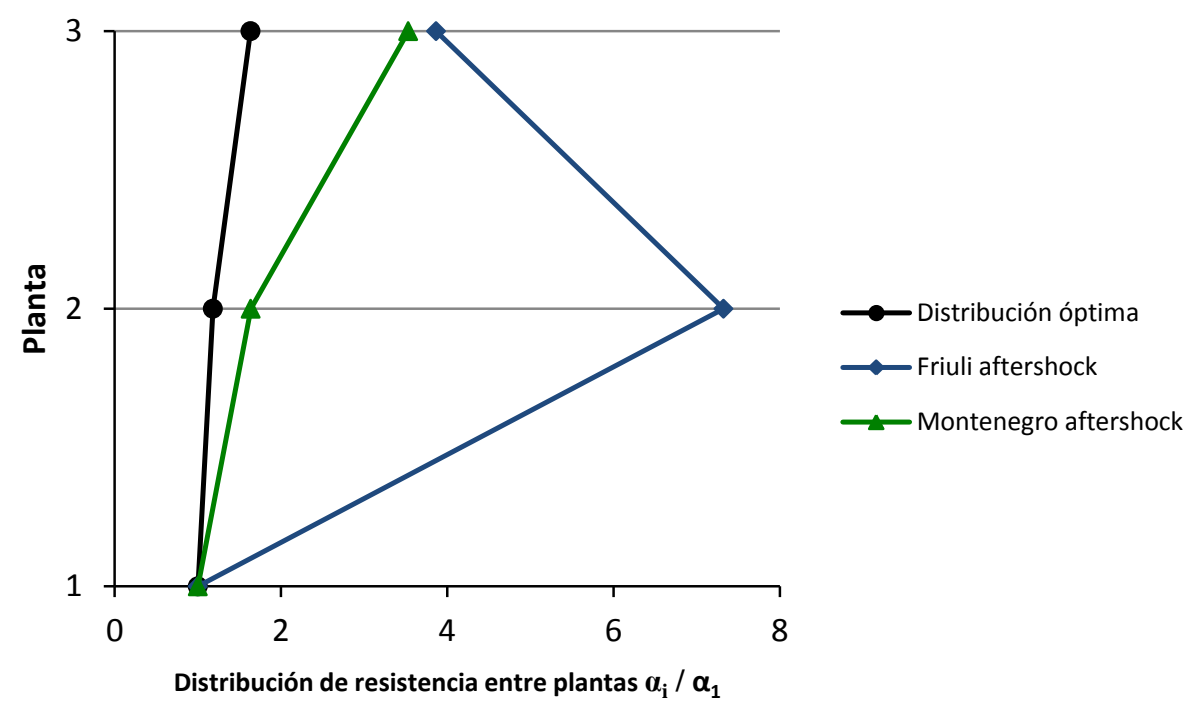

Fig. 5.6.3: Comparación de las distribuciones de resistencia, pórtico pa2pz2-1, suelo tipo II, campo lejano

5.6.5 Distribución de resistencia entre plantas para el pórtico pa2pz2-1, suelo tipo III, campo cercano $\alpha_{i} / \alpha_{1}$

- Acelerograma №2 (Friuli aftershock) $\Delta=\mathbf{1 0 K m}$

Coeficiente de fuerza cortante total de planta $\alpha_{i}$

Planta 3a: $\alpha_{3}=0,284$

Planta 2a: $\alpha_{2}=0,264$

Planta 1ㄹ: $\alpha_{1}=0,197$

Distribución de resistencia entre plantas $\alpha_{i} / \alpha_{1}$

Planta 3a: $\alpha_{3} / \alpha_{1}=0,284 / 0,197 \Rightarrow \alpha_{3} / \alpha_{1}=1,442$

Planta 2a: $\alpha_{2} / \alpha_{1}=0,264 / 0,197 \Rightarrow \alpha_{2} / \alpha_{1}=1,340$

Planta 1ạ:

$$
\alpha_{1} / \alpha_{1}=1,000
$$

\section{- Acelerograma №6 (Kalamata aftershock) $\Delta=3 \mathrm{Km}$}

Coeficiente de fuerza cortante total de planta $\alpha_{i}$

Planta 3a: $\alpha_{3}=0,020$

Planta 2a: $\alpha_{2}=0,051$

Planta 1a: $\alpha_{1}=2,731 e^{-05}$

Distribución de resistencia entre plantas $\alpha_{i} / \alpha_{1}$

Planta 3a: $\alpha_{3} / \alpha_{1}=0,020 / 2,731 e^{-05} \Rightarrow \alpha_{3} / \alpha_{1}=732,332$

Planta 2a: $\alpha_{2} / \alpha_{1}=0,051 / 2,731 e^{-05} \Rightarrow \alpha_{2} / \alpha_{1}=1867,448$

Planta 1a:

$$
\alpha_{1} / \alpha_{1}=1,000
$$


- Acelerograma №12 (Duzce 1) $\Delta=8 \mathrm{Km}$

Coeficiente de fuerza cortante total de planta $\alpha_{i}$

Planta 3a: $\alpha_{3}=0,098$

Planta 2a: $\alpha_{2}=0,116$

Planta 1: $\alpha_{1}=0,052$

Distribución de resistencia entre plantas $\alpha_{i} / \alpha_{1}$

Planta 3a: $\alpha_{3} / \alpha_{1}=0,098 / 0,052 \Rightarrow \alpha_{3} / \alpha_{1}=1,885$

Planta 2a: $\alpha_{2} / \alpha_{1}=0,116 / 0,052 \Rightarrow \alpha_{2} / \alpha_{1}=2,231$

Planta 1: $\alpha_{1} / \alpha_{1}=1,000$

Representación gráfica

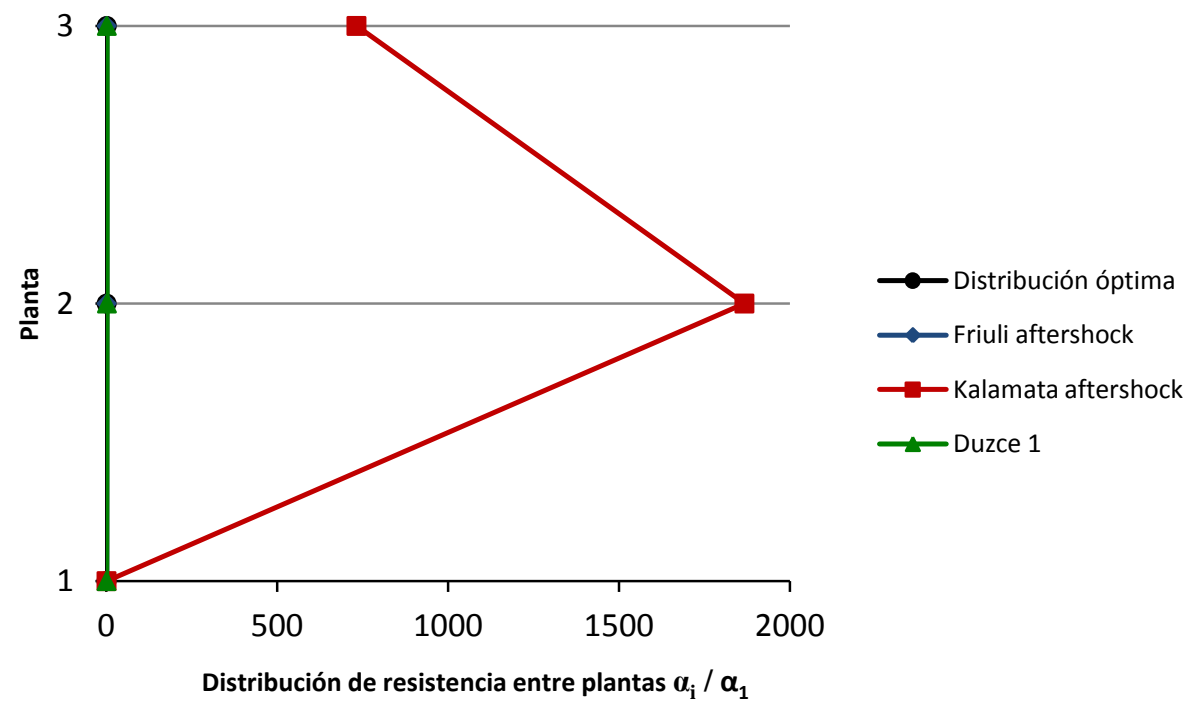

Fig. 5.6.4: Comparación de las distribuciones de resistencia, pórtico pa2pz2-1, suelo tipo III, campo cercano

5.6.6 Distribución de resistencia entre plantas para el pórtico pa2pz2-1, suelo tipo III, campo lejano $\alpha_{i} / \alpha_{1}$

\section{- Acelerograma №3 (Montenegro) $\Delta=\mathbf{2 4 K m}$}

Coeficiente de fuerza cortante total de planta $\alpha_{i}$

Planta 3a: $\alpha_{3}=4,552 e^{-05}$

Planta 2: $\alpha_{2}=0,032$

Planta 1a: $\alpha_{1}=2,731 e^{-05}$

Distribución de resistencia entre plantas $\alpha_{i} / \alpha_{1}$

Planta 3a: $\alpha_{3} / \alpha_{1}=4,552 e^{-05} / 2,731 e^{-05} \Rightarrow \alpha_{3} / \alpha_{1}=1,667$

Planta 2a: $\alpha_{2} / \alpha_{1}=0,032 / 2,731 e^{-05} \Rightarrow \quad \alpha_{2} / \alpha_{1}=1171,732$

Planta 1:

$\alpha_{1} / \alpha_{1}=1,000$ 


\section{- Acelerograma №14 (Ano Liosia) $\Delta=14 \mathrm{Km}$}

Coeficiente de fuerza cortante total de planta $\alpha_{i}$

Planta 3a: $\alpha_{3}=4,552 e^{-05}$

Planta 2: $\alpha_{2}=0,010$

Planta 1a: $\alpha_{1}=2,731 e^{-05}$

Distribución de resistencia entre plantas $\alpha_{i} / \alpha_{1}$

Planta 3a: $\alpha_{3} / \alpha_{1}=4,552 e^{-05} / 2,731 e^{-05} \Rightarrow \alpha_{3} / \alpha_{1}=1,667$

Planta 2a: $\alpha_{2} / \alpha_{1}=0,010 / 2,731 e^{-05} \Rightarrow \quad \alpha_{2} / \alpha_{1}=366,166$

Planta 1:

$$
\alpha_{1} / \alpha_{1}=1,000
$$

Representación gráfica

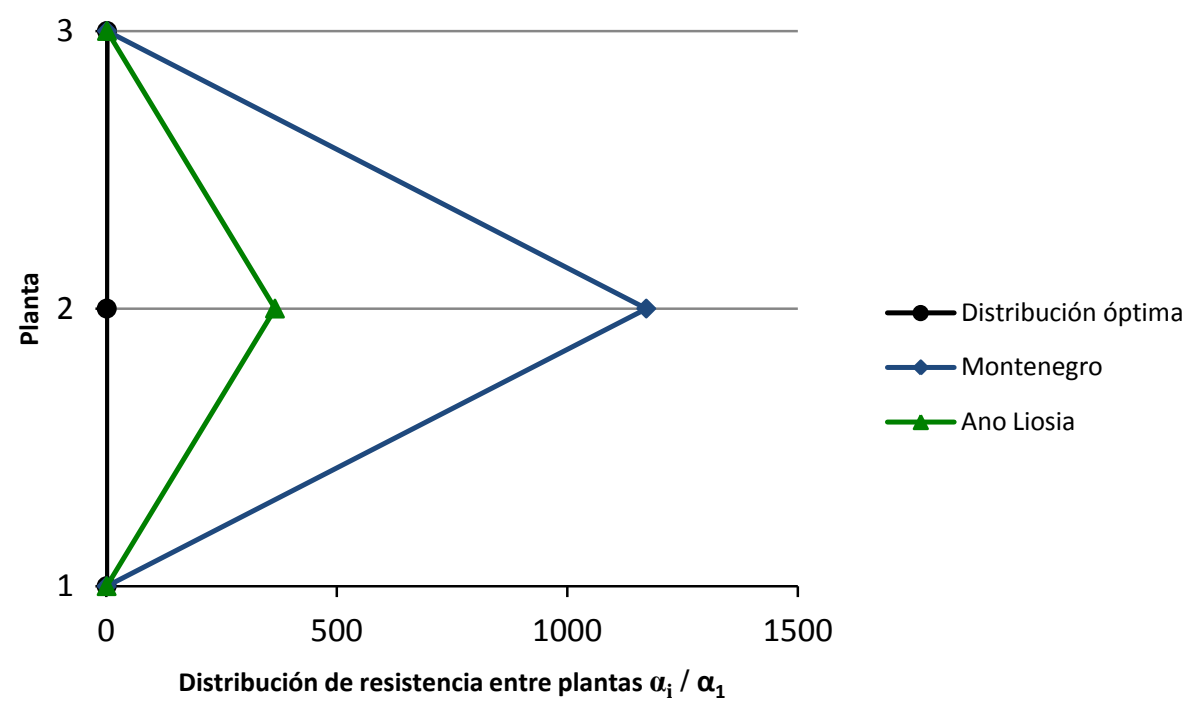

Fig. 5.6.5: Comparación de las distribuciones de resistencia, pórtico pa2pz2-1, suelo tipo III, campo lejano

5.6.7 Distribución de resistencia entre plantas para el pórtico pa4pz2-1, suelo tipo I, campo lejano $\alpha_{i} / \alpha_{1}$

\section{- Acelerograma №1 (Friuli) $\Delta=\mathbf{2 7} \mathrm{Km}$}

Coeficiente de fuerza cortante total de planta $\alpha_{i}$

Planta 3a: $\alpha_{3}=5,215 e^{-05}$

Planta 2a: $\alpha_{2}=3,084 e^{-05}$

Planta 1: $\alpha_{1}=2,854 e^{-05}$

Distribución de resistencia entre plantas $\alpha_{i} / \alpha_{1}$

Planta 3a: $\alpha_{3} / \alpha_{1}=5,215 e^{-05} / 2,854 e^{-05} \Rightarrow \alpha_{3} / \alpha_{1}=1,827$

Planta 2a: $\alpha_{2} / \alpha_{1}=3,084 e^{-05} / 2,854 e^{-05} \Rightarrow \alpha_{2} / \alpha_{1}=1,080$

Planta 1:

$$
\alpha_{1} / \alpha_{1}=1,000
$$


- Acelerograma №4 (Montenegro) $\Delta=65 \mathrm{Km}$

Coeficiente de fuerza cortante total de planta $\alpha_{i}$

Planta 3a: $\alpha_{3}=0,346$

Planta 2a: $\alpha_{2}=0,392$

Planta 1: $\alpha_{1}=0,232$

Distribución de resistencia entre plantas $\alpha_{i} / \alpha_{1}$

Planta 3a: $\alpha_{3} / \alpha_{1}=0,346 / 0,232 \Rightarrow \alpha_{3} / \alpha_{1}=1,491$

Planta 2a: $\alpha_{2} / \alpha_{1}=0,392 / 0,232 \Rightarrow \alpha_{2} / \alpha_{1}=1,690$

Planta 1a: $\quad \alpha_{1} / \alpha_{1}=1,000$

\section{- Acelerograma №5 (Campano Lucano) $\Delta=32 \mathrm{Km}$}

Coeficiente de fuerza cortante total de planta $\alpha_{i}$

Planta 3a: $\alpha_{3}=5,316 e^{-05}$

Planta 2: $\alpha_{2}=2,786 e^{-05}$

Planta 1: $\alpha_{1}=1,819 e^{-04}$

Distribución de resistencia entre plantas $\alpha_{i} / \alpha_{1}$

Planta 3a: $\alpha_{3} / \alpha_{1}=5,316 e^{-05} / 1,819 e^{-04} \Rightarrow \alpha_{3} / \alpha_{1}=0,292$

Planta 2a : $\alpha_{2} / \alpha_{1}=2,786 e^{-05} / 1,819 e^{-04} \Rightarrow \alpha_{2} / \alpha_{1}=0,153$

Planta 1: $\alpha_{1} / \alpha_{1}=1,000$

Representación gráfica

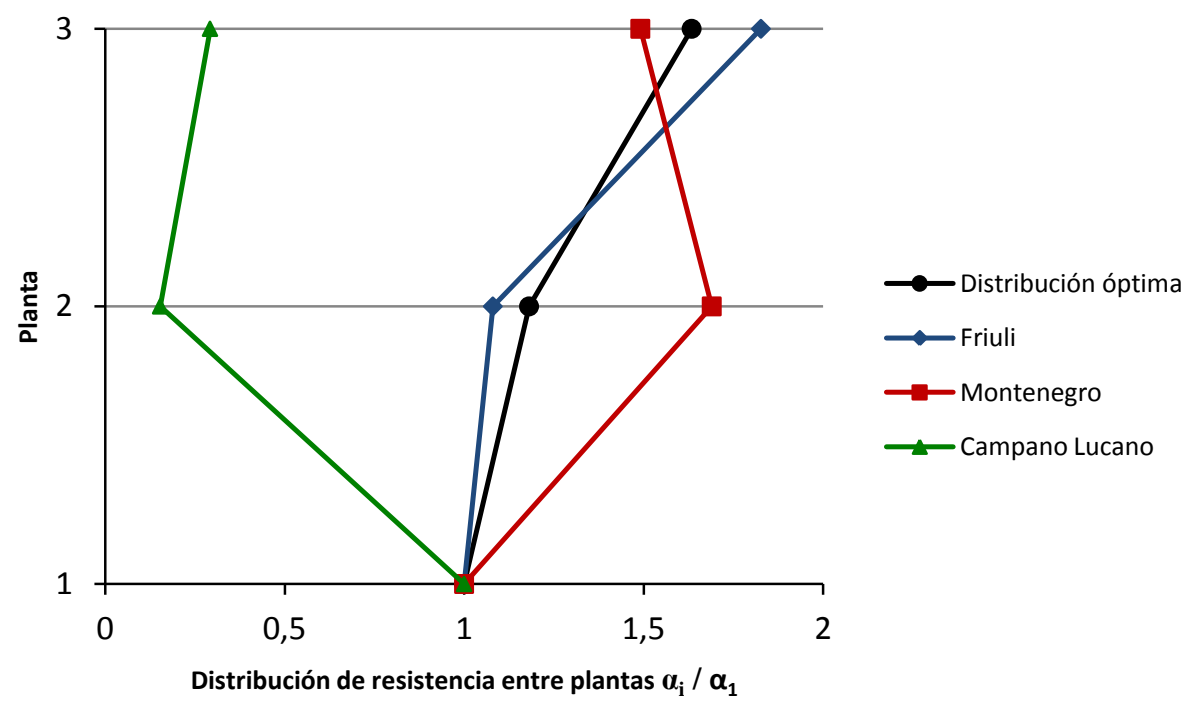

Fig. 5.6.6: Comparación de las distribuciones de resistencia, pórtico pa4pz2-1, suelo tipo I, campo lejano 
5.6.8 Distribución de resistencia entre plantas para el pórtico pa4pz2-1, suelo tipo II, campo cercano $\alpha_{i} / \alpha_{1}$

\section{- Acelerograma №1 (Friuli aftershock) $\Delta=\mathbf{3} \mathrm{Km}$}

Coeficiente de fuerza cortante total de planta $\alpha_{i}$

Planta 3a: $\alpha_{3}=5,848 e^{-04}$

Planta 2a: $\alpha_{2}=1,885 e^{-03}$

Planta 1: $\alpha_{1}=2,405 e^{-04}$

Distribución de resistencia entre plantas $\alpha_{i} / \alpha_{1}$

Planta 3a: $\alpha_{3} / \alpha_{1}=5,848 e^{-04} / 2,405 e^{-04} \Rightarrow \alpha_{3} / \alpha_{1}=2,432$

Planta 2a : $\alpha_{2} / \alpha_{1}=1,885 e^{-03} / 2,405 e^{-04} \Rightarrow \alpha_{2} / \alpha_{1}=7,838$

Planta 1:

$$
\alpha_{1} / \alpha_{1}=1,000
$$

\section{- Acelerograma №14 (Montenegro aftershock) $\Delta=8 \mathrm{Km}$}

Coeficiente de fuerza cortante total de planta $\alpha_{i}$

Planta 3a: $\alpha_{3}=5,316 e^{-05}$

Planta 2: $\alpha_{2}=0,047$

Planta 1: $\alpha_{1}=2,186 e^{-05}$

Distribución de resistencia entre plantas $\alpha_{i} / \alpha_{1}$

Planta 3a: $\alpha_{3} / \alpha_{1}=5,316 e^{-05} / 2,186 e^{-05} \Rightarrow \alpha_{3} / \alpha_{1}=2,432$

Planta 2a: $\alpha_{2} / \alpha_{1}=0,047 / 2,186 e^{-05} \Rightarrow \quad \alpha_{2} / \alpha_{1}=2150,046$

Planta 1a:

$\alpha_{1} / \alpha_{1}=1,000$

Representación gráfica

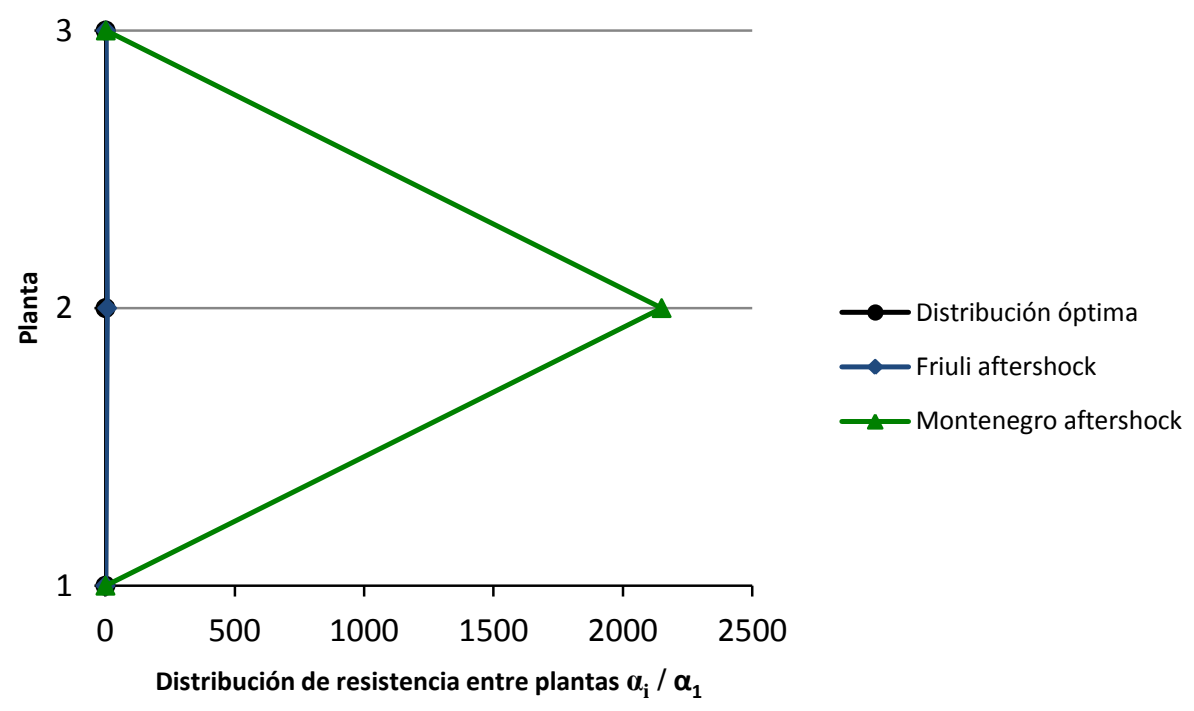

Fig. 5.6.7: Comparación de las distribuciones de resistencia, pórtico pa4pz2-1, suelo tipo II, campo cercano 
5.6.9 Distribución de resistencia entre plantas para el pórtico pa4pz2-1, suelo tipo II, campo lejano $\alpha_{i} / \alpha_{1}$

- Acelerograma №2 (Friuli aftershock) $\Delta=\mathbf{1 6 K m}$

Coeficiente de fuerza cortante total de planta $\alpha_{i}$

Planta 3a: $\alpha_{3}=0,382$

Planta 2a: $\alpha_{2}=0,432$

Planta 1a: $\alpha_{1}=0,256$

Distribución de resistencia entre plantas $\alpha_{i} / \alpha_{1}$

Planta 3a: $\alpha_{3} / \alpha_{1}=0,382 / 0,256 \Rightarrow \alpha_{3} / \alpha_{1}=1,492$

Planta 2a: $\alpha_{2} / \alpha_{1}=0,432 / 0,256 \Rightarrow \alpha_{2} / \alpha_{1}=1,687$

Planta 1:

$$
\alpha_{1} / \alpha_{1}=1,000
$$

\section{- Acelerograma №12 (Montenegro aftershock) $\Delta=33 \mathrm{Km}$}

Coeficiente de fuerza cortante total de planta $\alpha_{i}$

Planta 3a: $\alpha_{3}=0,569$

Planta 2: $\alpha_{2}=0,427$

Planta 1ㄹ: $\alpha_{1}=0,287$

Distribución de resistencia entre plantas $\alpha_{i} / \alpha_{1}$

Planta 3a: $\alpha_{3} / \alpha_{1}=0,569 / 0,287 \Rightarrow \alpha_{3} / \alpha_{1}=1,982$

Planta 2: $\alpha_{2} / \alpha_{1}=0,427 / 0,287 \Rightarrow \alpha_{2} / \alpha_{1}=1,488$

Planta 1: $\alpha_{1} / \alpha_{1}=1,000$

Representación gráfica

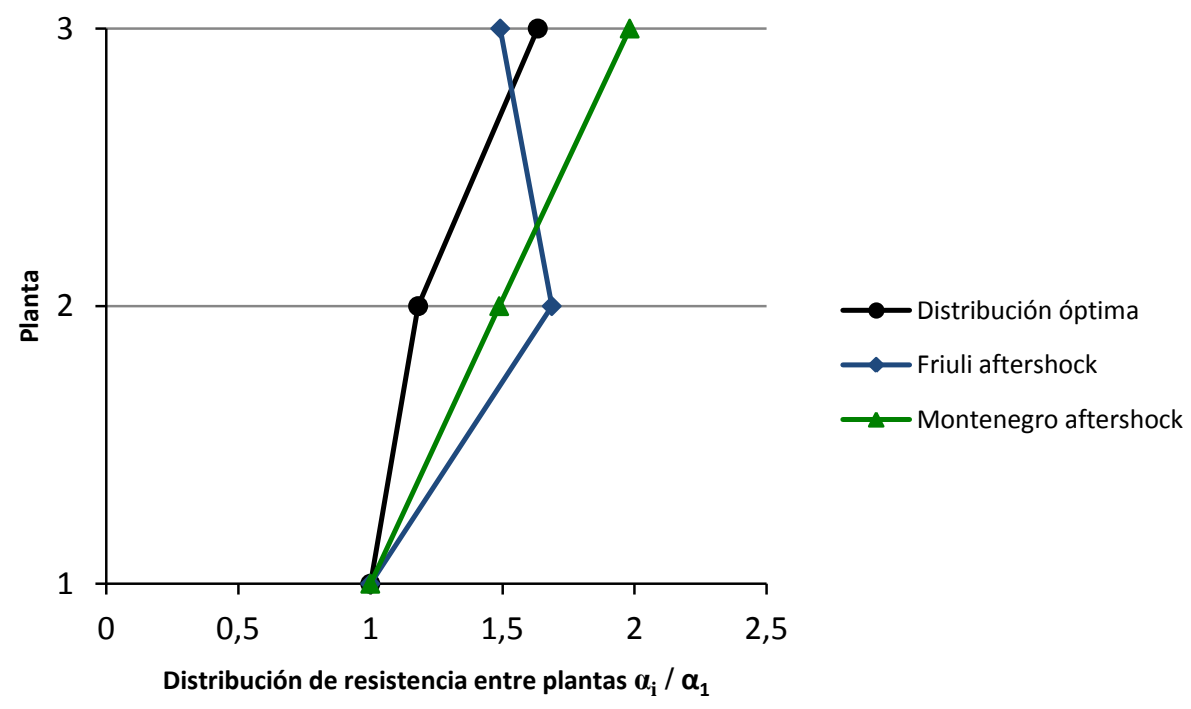

Fig. 5.6.8: Comparación de las distribuciones de resistencia, pórtico pa4pz2-1, suelo tipo II, campo lejano 
5.6.10 Distribución de resistencia entre plantas para el pórtico pa4pz2-1, suelo tipo III, campo cercano $\alpha_{i} / \alpha_{1}$

\section{- Acelerograma №2 (Friuli aftershock) $\Delta=\mathbf{1 0} \mathrm{Km}$}

Coeficiente de fuerza cortante total de planta $\alpha_{i}$

Planta 3ㄹ: $\alpha_{3}=0,242$

Planta 2a: $\alpha_{2}=0,240$

Planta 1: $\alpha_{1}=0,199$

Distribución de resistencia entre plantas $\alpha_{i} / \alpha_{1}$

Planta 3a: $\alpha_{3} / \alpha_{1}=0,242 / 0,199 \Rightarrow \alpha_{3} / \alpha_{1}=1,216$

Planta 2a: $\alpha_{2} / \alpha_{1}=0,240 / 0,199 \Rightarrow \alpha_{2} / \alpha_{1}=1,206$

Planta 1a:

$$
\alpha_{1} / \alpha_{1}=1,000
$$

\section{- Acelerograma №6 (Kalamata aftershock) $\Delta=3 \mathrm{Km}$}

Coeficiente de fuerza cortante total de planta $\alpha_{i}$

Planta 3a: $\alpha_{3}=0,047$

Planta 2a: $\alpha_{2}=0,041$

Planta 1: $\alpha_{1}=0,018$

Distribución de resistencia entre plantas $\alpha_{i} / \alpha_{1}$

Planta 3a: $\alpha_{3} / \alpha_{1}=0,047 / 0,018 \Rightarrow \alpha_{3} / \alpha_{1}=2,611$

Planta 2a: $\alpha_{2} / \alpha_{1}=0,041 / 0,018 \Rightarrow \alpha_{2} / \alpha_{1}=2,278$

Planta 1: $\alpha_{1} / \alpha_{1}=1,000$

\section{- Acelerograma №12 (Duzce 1) $\Delta=8 \mathrm{Km}$}

Coeficiente de fuerza cortante total de planta $\alpha_{i}$

Planta 3ạ: $\alpha_{3}=0,087$

Planta 2a: $\alpha_{2}=0,114$

Planta 1: $\alpha_{1}=0,079$

Distribución de resistencia entre plantas $\alpha_{i} / \alpha_{1}$

Planta 3a: $\alpha_{3} / \alpha_{1}=0,087 / 0,079 \Rightarrow \alpha_{3} / \alpha_{1}=1,101$

Planta 2 : $\alpha_{2} / \alpha_{1}=0,114 / 0,079 \Rightarrow \alpha_{2} / \alpha_{1}=1,443$

Planta 1: $\alpha_{1} / \alpha_{1}=1,000$ 
Representación gráfica

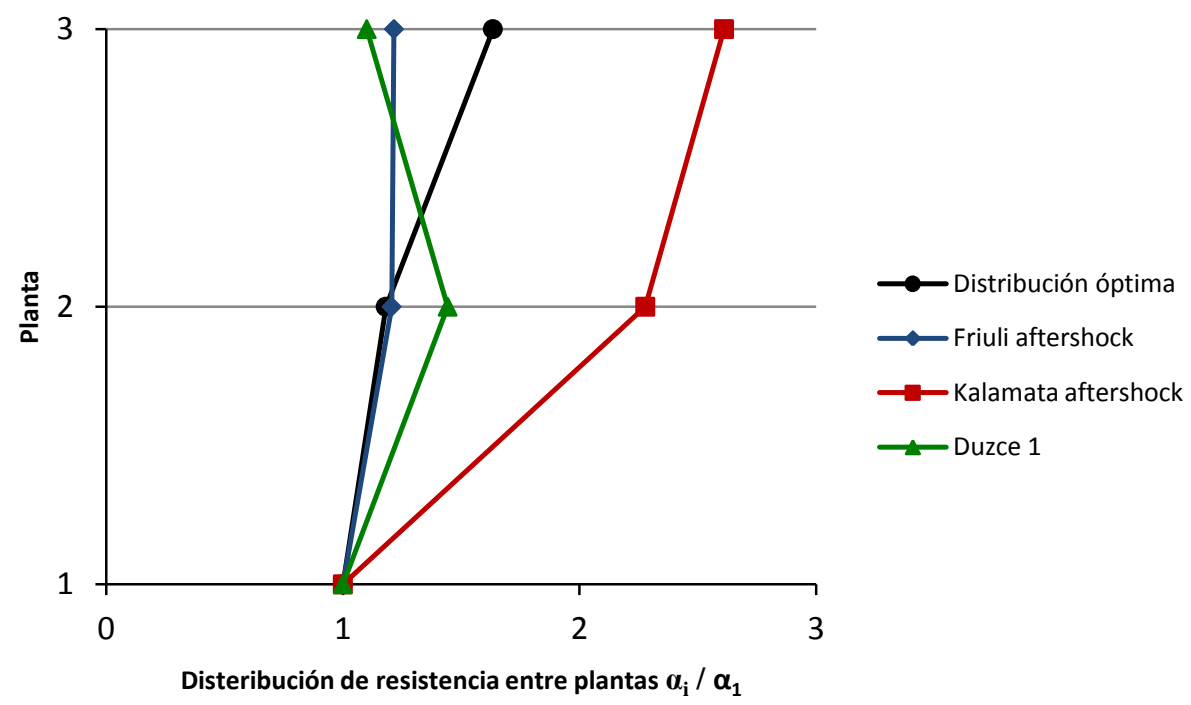

Fig. 5.6.9: Comparación de las distribuciones de resistencia, pórtico pa4pz2-1, suelo tipo III, campo cercano

5.6.11 Distribución de resistencia entre plantas para el pórtico pa4pz2-1, suelo tipo III, campo lejano $\alpha_{i} / \alpha_{1}$

- Acelerograma №3 (Montenegro) $\Delta=\mathbf{2 4 K m}$

Coeficiente de fuerza cortante total de planta $\alpha_{i}$

Planta 3ㄹ: $\alpha_{3}=0,103$

Planta 2a: $\alpha_{2}=0,125$

Planta 1a: $\alpha_{1}=0,098$

Distribución de resistencia entre plantas $\alpha_{i} / \alpha_{1}$

Planta 3a: $\alpha_{3} / \alpha_{1}=0,103 / 0,098 \Rightarrow \alpha_{3} / \alpha_{1}=1,051$

Planta 2a: $\alpha_{2} / \alpha_{1}=0,125 / 0,098 \Rightarrow \alpha_{2} / \alpha_{1}=1,275$

Planta 1: $\alpha_{1} / \alpha_{1}=1,000$

\section{- Acelerograma №14 (Ano Liosia) $\Delta=14 \mathrm{Km}$}

Coeficiente de fuerza cortante total de planta $\alpha_{i}$

Planta 3a: $\alpha_{3}=5,316 e^{-05}$

Planta 2a: $\alpha_{2}=3,142 e^{-05}$

Planta 1: $\alpha_{1}=2,689 e^{-03}$

Distribución de resistencia entre plantas $\alpha_{i} / \alpha_{1}$

Planta 3a: $\alpha_{3} / \alpha_{1}=5,316 e^{-05} / 2,689 e^{-03} \Rightarrow \alpha_{3} / \alpha_{1}=0,020$

Planta 2 $2^{\mathrm{a}}: \alpha_{2} / \alpha_{1}=3,142 e^{-05} / 2,689 e^{-03} \Rightarrow \alpha_{2} / \alpha_{1}=0,012$

Planta 1a:

$$
\alpha_{1} / \alpha_{1}=1,000
$$


Representación gráfica

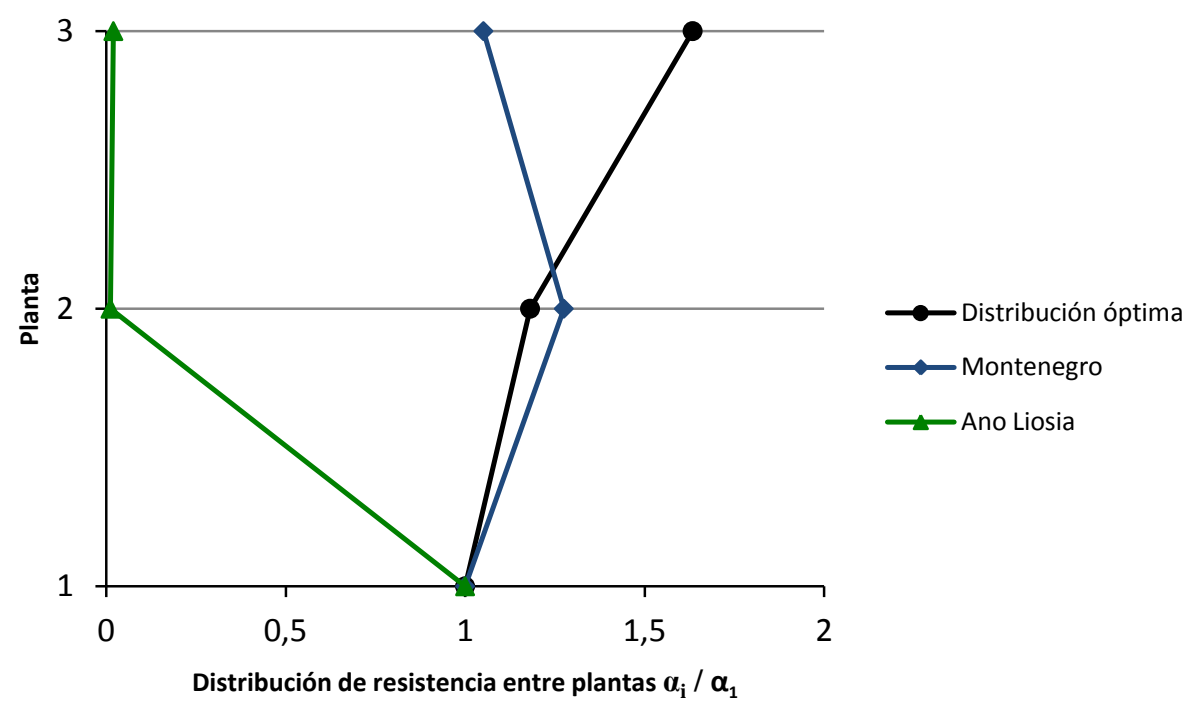

Fig. 5.6.10: Comparación de las distribuciones de resistencia, pórtico pa4pz2-1, suelo tipo III, campo lejano

5.6.12 Distribución óptima del coeficiente de fuerza cortante de fluencia definida por Akiyama $\alpha_{i} / \alpha_{1}$ para una estructura de seis plantas

Planta 6a:

$$
\begin{aligned}
& x=(i-1) / N=(6-1) / 6=0,833>0,2 \\
& \alpha_{6} / \alpha_{1}=1+1,5927(6-1) / 6-11,852[(6-1) / 6]^{2}+42,583[(6-1) / 6]^{3}-59,48[(6-1) / 6]^{4}+30,16[(6-1) / 6]^{5} \Rightarrow \\
& \alpha_{6} / \alpha_{1}=2,176
\end{aligned}
$$

Planta 5a:

$$
\begin{aligned}
& x=(i-1) / N=(5-1) / 6=0,667>0,2 \\
& \alpha_{5} / \alpha_{1}=1+1,5927(5-1) / 6-11,852[(5-1) / 6]^{2}+42,583[(5-1) / 6]^{3}-59,48[(5-1) / 6]^{4}+30,16[(5-1) / 6]^{5} \Rightarrow \\
& \alpha_{5} / \alpha_{1}=1,634
\end{aligned}
$$

\section{Planta 4a:}

$$
\begin{aligned}
& x=(i-1) / N=(4-1) / 6=0,5>0,2 \\
& \alpha_{4} / \alpha_{1}=1+1,5927(4-1) / 6-11,852[(4-1) / 6]^{2}+42,583[(4-1) / 6]^{3}-59,48[(4-1) / 6]^{4}+30,16[(4-1) / 6]^{5} \Rightarrow \\
& \alpha_{4} / \alpha_{1}=1,381
\end{aligned}
$$

Planta 3a:

$$
\begin{aligned}
& x=(i-1) / N=(3-1) / 6=0,333>0,2 \\
& \alpha_{3} / \alpha_{1}=1+1,5927(3-1) / 6-11,852[(3-1) / 6]^{2}+42,583[(3-1) / 6]^{3}-59,48[(3-1) / 6]^{4}+30,16[(3-1) / 6]^{5} \Rightarrow \\
& \alpha_{3} / \alpha_{1}=1,181
\end{aligned}
$$




\section{Planta 2a:}

$$
\begin{aligned}
& x=(i-1) / N=(2-1) / 6=0,167<0,2 \\
& \alpha_{2} / \alpha_{1}=1+0,5(2-1) / 6 \Rightarrow \alpha_{2} / \alpha_{1}=1,083
\end{aligned}
$$

\section{Planta 1a:}

$$
\begin{aligned}
& x=(i-1) / N=(1-1) / 6=0<0,2 \\
& \alpha_{1} / \alpha_{1}=1+0,5(1-1) / 6 \Rightarrow \alpha_{1} / \alpha_{1}=1
\end{aligned}
$$

5.6.13 Distribución de resistencia entre plantas para el pórtico pa6pz2-1, suelo tipo I, campo lejano $\alpha_{i} / \alpha_{1}$

\section{- Acelerograma №4 (Montenegro) $\Delta=65 \mathrm{Km}$}

Coeficiente de fuerza cortante total de planta $\alpha_{i}$

Planta 6a: $\alpha_{6}=0,510$

Planta 5a: $\alpha_{5}=0,412$

Planta 4a: $\alpha_{4}=0,224$

Planta 3a: $\alpha_{3}=0,209$

Planta 2a: $\alpha_{2}=0,174$

Planta 1a: $\alpha_{1}=0,147$

Distribución de resistencia entre plantas $\alpha_{i} / \alpha_{1}$

Planta 6a: $\alpha_{6} / \alpha_{1}=0,510 / 0,147 \Rightarrow \alpha_{6} / \alpha_{1}=3,469$

Planta 5a: $\alpha_{5} / \alpha_{1}=0,412 / 0,147 \Rightarrow \alpha_{5} / \alpha_{1}=2,803$

Planta 4a: $\alpha_{4} / \alpha_{1}=0,224 / 0,147 \Rightarrow \alpha_{4} / \alpha_{1}=1,524$

Planta 3a: $\alpha_{3} / \alpha_{1}=0,209 / 0,147 \Rightarrow \alpha_{3} / \alpha_{1}=1,422$

Planta 2a: $\alpha_{2} / \alpha_{1}=0,174 / 0,147 \Rightarrow \alpha_{2} / \alpha_{1}=1,184$

Planta 1ㄹ:

$$
\alpha_{1} / \alpha_{1}=1,000
$$

\section{- Acelerograma №5 (Campano Lucano) $\Delta=32 \mathrm{Km}$}

Coeficiente de fuerza cortante total de planta $\alpha_{i}$

Planta 6a: $\alpha_{6}=4,171 e^{-05}$

Planta 5a: $\alpha_{5}=5,495 e^{-03}$

Planta 4a: $\alpha_{4}=3,117 e^{-03}$

Planta 3a: $\alpha_{3}=3,662 e^{-03}$

Planta 2a: $\alpha_{2}=3,708 e^{-03}$

Planta 1a: $\alpha_{1}=6,035 e^{-03}$ 
Distribución de resistencia entre plantas $\alpha_{i} / \alpha_{1}$

Planta 6ạ: $\alpha_{6} / \alpha_{1}=4,171 e^{-05} / 6,035 e^{-03} \Rightarrow \alpha_{6} / \alpha_{1}=6,911 e^{-03}$

Planta 5ㅁ: $\alpha_{5} / \alpha_{1}=5,495 e^{-03} / 6,035 e^{-03} \Rightarrow \alpha_{5} / \alpha_{1}=0,910$

Planta 4a: $\alpha_{4} / \alpha_{1}=3,117 e^{-03} / 6,035 e^{-03} \Rightarrow \alpha_{4} / \alpha_{1}=0,516$

Planta 3a: $\alpha_{3} / \alpha_{1}=3,662 e^{-03} / 6,035 e^{-03} \Rightarrow \alpha_{3} / \alpha_{1}=0,607$

Planta 2a: $\alpha_{2} / \alpha_{1}=3,708 e^{-03} / 6,035 e^{-03} \Rightarrow \alpha_{2} / \alpha_{1}=0,614$

Planta 1:

$$
\alpha_{1} / \alpha_{1}=1,000
$$

Representación gráfica

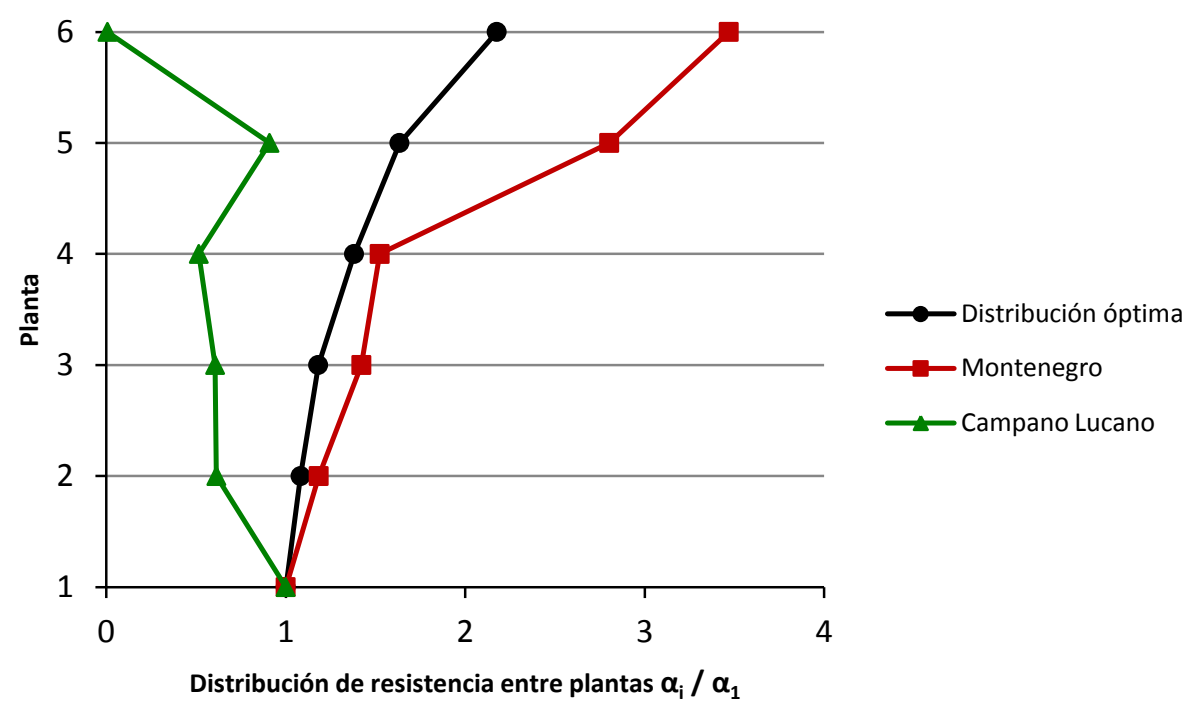

Fig. 5.6.11: Comparación de las distribuciones de resistencia, pórtico pa6pz2-1, suelo tipo I, campo lejano

5.6.14 Distribución de resistencia entre plantas para el pórtico pa6pz2-1, suelo tipo II, campo cercano $\alpha_{i} / \alpha_{1}$

\section{- Acelerograma №14 (Montenegro aftershock) $\Delta=8 \mathrm{Km}$}

Coeficiente de fuerza cortante total de planta $\alpha_{i}$

Planta 6a: $\alpha_{6}=0,120$

Planta 5a: $\alpha_{5}=0,096$

Planta 4a: $\alpha_{4}=0,114$

Planta 3: $\alpha_{3}=0,088$

Planta 2: $\alpha_{2}=0,089$

Planta 1르: $\alpha_{1}=0,096$

Distribución de resistencia entre plantas $\alpha_{i} / \alpha_{1}$

Planta 6ạ: $\alpha_{6} / \alpha_{1}=0,120 / 0,096 \Rightarrow \alpha_{6} / \alpha_{1}=1,250$ 
Planta 5a: $\alpha_{5} / \alpha_{1}=0,096 / 0,096 \Rightarrow \alpha_{5} / \alpha_{1}=1,000$

Planta 4a: $\alpha_{4} / \alpha_{1}=0,114 / 0,096 \Rightarrow \alpha_{4} / \alpha_{1}=1,187$

Planta 3a: $\alpha_{3} / \alpha_{1}=0,088 / 0,096 \Rightarrow \alpha_{3} / \alpha_{1}=0,917$

Planta 2a: $\alpha_{2} / \alpha_{1}=0,089 / 0,096 \Rightarrow \alpha_{2} / \alpha_{1}=0,927$

Planta 1:

$$
\alpha_{1} / \alpha_{1}=1,000
$$

Representación gráfica

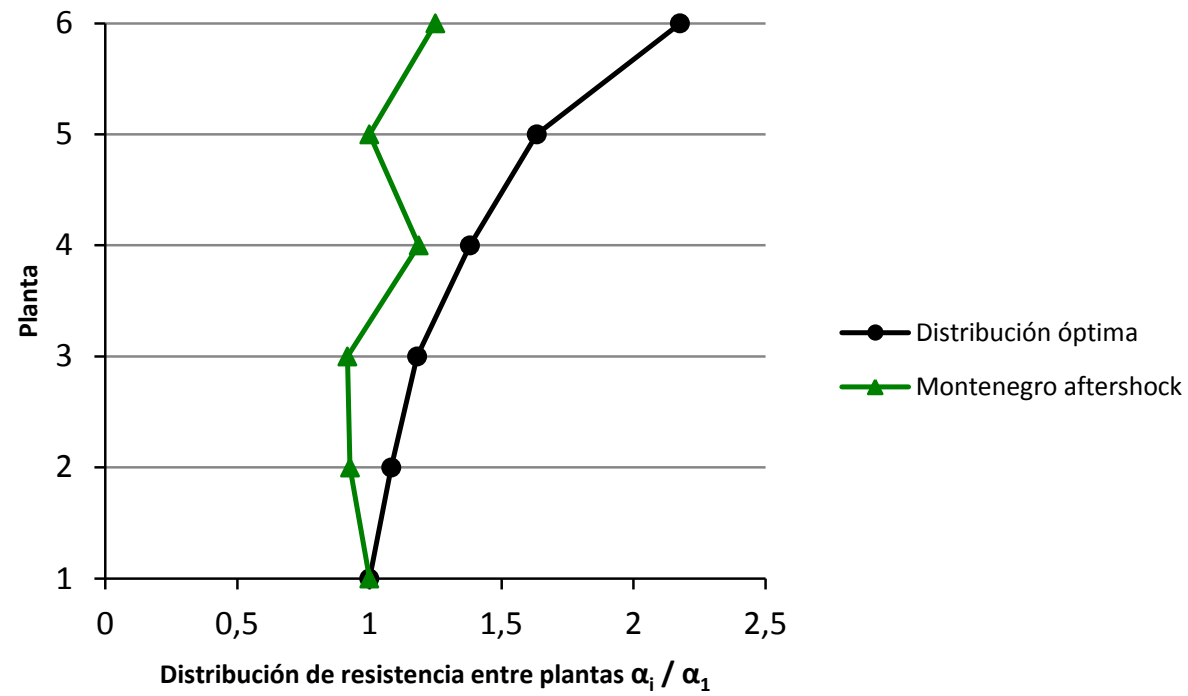

Fig. 5.6.12: Comparación de las distribuciones de resistencia, pórtico pa6pz2-1, suelo tipo II, campo cercano

5.6.15 Distribución de resistencia entre plantas para el pórtico pa6pz2-1, suelo tipo III, campo cercano $\alpha_{i} / \alpha_{1}$

\section{- Acelerograma №2 (Friuli aftershock) $\Delta=10 \mathrm{Km}$}

Coeficiente de fuerza cortante total de planta $\alpha_{i}$

Planta 6a: $\alpha_{6}=0,050$

Planta 5a : $\alpha_{5}=0,024$

Planta 4a: $\alpha_{4}=0,013$

Planta 3a: $\alpha_{3}=1,465 e^{-05}$

Planta 2: $\alpha_{2}=1,446 e^{-03}$

Planta 1: $\alpha_{1}=6,517 e^{-03}$

Distribución de resistencia entre plantas $\alpha_{i} / \alpha_{1}$

Planta 6a: $\alpha_{6} / \alpha_{1}=0,050 / 6,517 e^{-03} \Rightarrow \quad \alpha_{6} / \alpha_{1}=7,672$

Planta 5a: $\alpha_{5} / \alpha_{1}=0,024 / 6,517 e^{-03} \Rightarrow \quad \alpha_{5} / \alpha_{1}=3,683$

Planta 4ạ: $\alpha_{4} / \alpha_{1}=0,013 / 6,517 e^{-03} \Rightarrow \quad \alpha_{4} / \alpha_{1}=1,995$ 
Planta 3a: $\alpha_{3} / \alpha_{1}=1,465 e^{-05} / 6,517 e^{-03} \Rightarrow \alpha_{3} / \alpha_{1}=2,248 e^{-03}$

Planta 2a: $\alpha_{2} / \alpha_{1}=1,446 e^{-03} / 6,517 e^{-03} \Rightarrow \alpha_{2} / \alpha_{1}=0,222$

Planta 1:

$$
\alpha_{1} / \alpha_{1}=1,000
$$

- Acelerograma №6 (Kalamata aftershock) $\Delta=3 \mathrm{Km}$

Coeficiente de fuerza cortante total de planta $\alpha_{i}$

Planta 6a: $\alpha_{6}=0,881$

Planta 5a: $\alpha_{5}=0,554$

Planta 4a: $\alpha_{4}=0,464$

Planta 3a: $\alpha_{3}=0,258$

Planta 2a: $\alpha_{2}=0,184$

Planta 1: $\alpha_{1}=0,187$

Distribución de resistencia entre plantas $\alpha_{i} / \alpha_{1}$

Planta 6a: $\alpha_{6} / \alpha_{1}=0,881 / 0,187 \Rightarrow \alpha_{6} / \alpha_{1}=4,711$

Planta 5a: $\alpha_{5} / \alpha_{1}=0,554 / 0,187 \Rightarrow \alpha_{5} / \alpha_{1}=2,962$

Planta 4a: $\alpha_{4} / \alpha_{1}=0,464 / 0,187 \Rightarrow \alpha_{4} / \alpha_{1}=2,481$

Planta 3a: $\alpha_{3} / \alpha_{1}=0,258 / 0,187 \Rightarrow \alpha_{3} / \alpha_{1}=1,380$

Planta 2: $\alpha_{2} / \alpha_{1}=0,184 / 0,187 \Rightarrow \alpha_{2} / \alpha_{1}=0,984$

Planta 1:

$$
\alpha_{1} / \alpha_{1}=1,000
$$

\section{- Acelerograma №12 (Duzce 1) $\Delta=8 \mathrm{Km}$}

Coeficiente de fuerza cortante total de planta $\alpha_{i}$

Planta 6a: $\alpha_{6}=0,042$

Planta 5a: $\alpha_{5}=0,037$

Planta 4a: $\alpha_{4}=0,028$

Planta 3a: $\alpha_{3}=0,026$

Planta 2 $\stackrel{\mathrm{a}}{\mathrm{a}} \alpha_{2}=0,035$

Planta 1a: $\alpha_{1}=0,043$

Distribución de resistencia entre plantas $\alpha_{i} / \alpha_{1}$

Planta 6a: $\alpha_{6} / \alpha_{1}=0,042 / 0,043 \Rightarrow \alpha_{6} / \alpha_{1}=0,977$

Planta 5a: $\alpha_{5} / \alpha_{1}=0,037 / 0,043 \Rightarrow \alpha_{5} / \alpha_{1}=0,860$

Planta 4a: $\alpha_{4} / \alpha_{1}=0,028 / 0,043 \Rightarrow \alpha_{4} / \alpha_{1}=0,651$

Planta 3a: $\alpha_{3} / \alpha_{1}=0,026 / 0,043 \Rightarrow \alpha_{3} / \alpha_{1}=0,605$

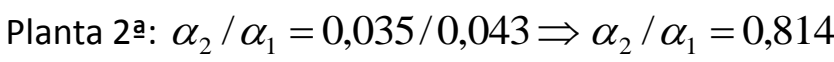

Planta 1:

$$
\alpha_{1} / \alpha_{1}=1,000
$$


Representación gráfica

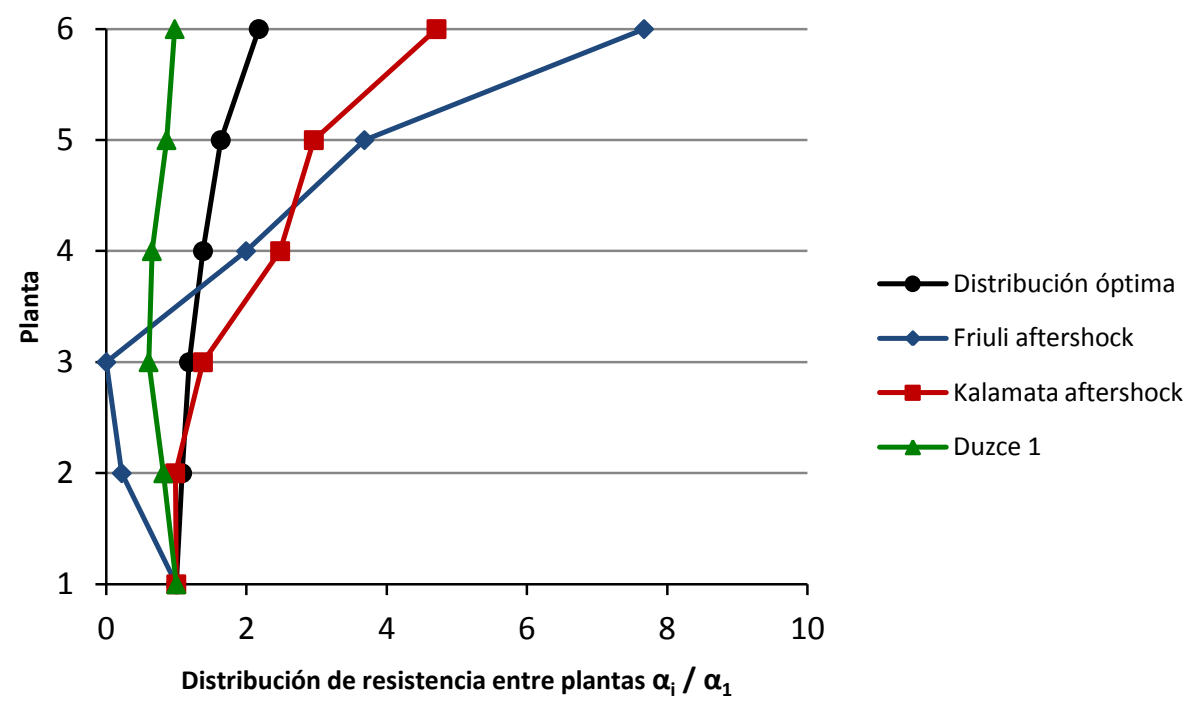

Fig. 5.6.13: Comparación de las distribuciones de resistencia, pórtico pa6pz2-1, suelo tipo III, campo cercano

5.6.16 Distribución de resistencia entre plantas para el pórtico pa6pz2-1, suelo tipo III, campo lejano $\alpha_{i} / \alpha_{1}$

\section{- Acelerograma №3 (Montenegro) $\Delta=\mathbf{2 4 K m}$}

Coeficiente de fuerza cortante total de planta $\alpha_{i}$

Planta 6a: $\alpha_{6}=0,787$

Planta 5: $\alpha_{5}=0,446$

Planta 4ㄹ: $\alpha_{4}=0,346$

Planta 3a: $\alpha_{3}=0,286$

Planta 2: $\alpha_{2}=0,256$

Planta 1ㅁ: $\alpha_{1}=0,236$

Distribución de resistencia entre plantas $\alpha_{i} / \alpha_{1}$

Planta 6a: $\alpha_{6} / \alpha_{1}=0,787 / 0,236 \Rightarrow \alpha_{6} / \alpha_{1}=3,335$

Planta 5a: $\alpha_{5} / \alpha_{1}=0,446 / 0,236 \Rightarrow \alpha_{5} / \alpha_{1}=1,890$

Planta 4a: $\alpha_{4} / \alpha_{1}=0,346 / 0,236 \Rightarrow \alpha_{4} / \alpha_{1}=1,466$

Planta 3a: $\alpha_{3} / \alpha_{1}=0,286 / 0,236 \Rightarrow \alpha_{3} / \alpha_{1}=1,212$

Planta 2a: $\alpha_{2} / \alpha_{1}=0,256 / 0,236 \Rightarrow \alpha_{2} / \alpha_{1}=1,085$

Planta 1a:

$$
\alpha_{1} / \alpha_{1}=1,000
$$


Representación gráfica

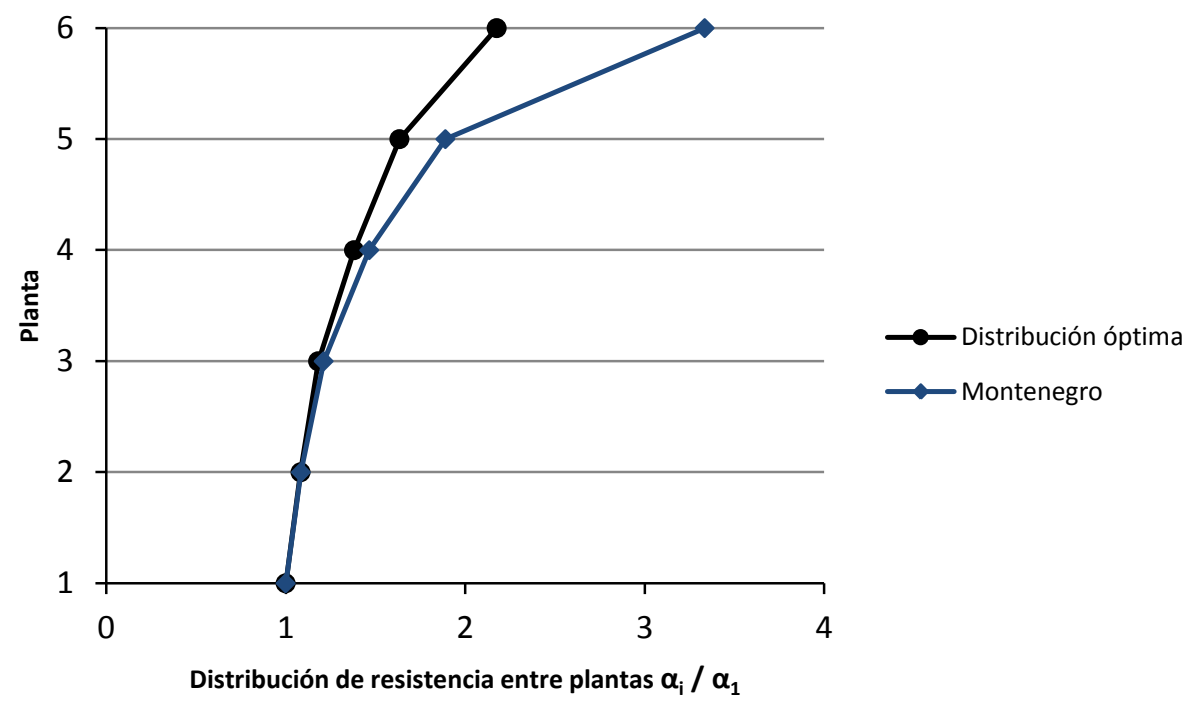

Fig. 5.6.14: Comparación de las distribuciones de resistencia, pórtico pa6pz2-1, suelo tipo III, campo lejano

\subsubsection{Valoración general}

Se observa que las curvas obtenidas en este estudio se alejan bastante de la distribución propuesta por Akiyama para los acelerogramas: №4 Montenegro, campo lejano (2a y 3a planta) Fig. 5.6.1; №1 Friuli aftershock, campo cercano (2a planta) Fig. 5.6.2; №6 Kalamata aftershock, campo cercano (2 y 3 a planta) Fig. 5.6.4; №3 Montenegro, campo lejano (2a planta) Fig. 5.6.5; №14 Ano Liosia, campo lejano (2a planta) Fig. 5.6 .5 y por último el № 14 Montenegro aftershock, campo cercano (2 aplanta) Fig. 5.6.7.

Las mayores desviaciones entre la distribución obtenida y la propuesta por Akiyama se producen en sismos que requieren unos coeficientes cortantes basales extremadamente pequeño y por lo tanto su comportamiento no se puede considerar representativo. En los sismos que requieren un cortante basal mayor (digamos superior a 0.05 aproximadamente), las distribuciones obtenidas tienden a acercarse a la óptima plateada por Akiyama.

Tabla 5.6.1: Distribución de resistencia entre plantas para cada pórtico y tipo de suelo y distribución óptima del coeficiente de fuerza cortante de fluencia propuesta por Akiyama 


\begin{tabular}{|c|c|c|c|c|c|}
\hline Pórtico & $\begin{array}{l}\text { Tipo de } \\
\text { suelo }\end{array}$ & Acelerograma & Planta & $\begin{array}{c}\text { Distribución de } \\
\text { resistencia entre } \\
\text { plantas } \\
\alpha_{i} / \alpha_{1}\end{array}$ & $\begin{array}{l}\text { Distribución óptima } \\
\text { del coeficiente de } \\
\text { fuerza cortante de } \\
\text { fluencia (Akiyama) } \\
\quad \alpha_{\text {ióptima }} / \alpha_{1}\end{array}$ \\
\hline \multirow{12}{*}{ pa2pz2-1 } & \multirow{3}{*}{$\begin{array}{c}\text { I } \\
\text { Campo } \\
\text { lejano }\end{array}$} & $\begin{array}{c}\text { №1 } \\
\text { (Friuli) }\end{array}$ & $\begin{array}{l}1{ }^{\underline{a}} \\
2 \underline{a} \\
3 \underline{a}\end{array}$ & $\begin{array}{c}1.000 \\
15.867 \\
0.044\end{array}$ & $\begin{array}{l}1.000 \\
1.181 \\
1.634\end{array}$ \\
\hline & & $\begin{array}{c}\text { №4 } \\
\text { (Montenegro) }\end{array}$ & $\begin{array}{l}19 \\
2 \underline{a} \\
3 a\end{array}$ & $\begin{array}{c}1.000 \\
817.771 \\
488.222\end{array}$ & $\begin{array}{l}1.000 \\
1.181 \\
1.634\end{array}$ \\
\hline & & $\begin{array}{c}\text { №5 } \\
\text { (Campano } \\
\text { Lucano) }\end{array}$ & $\begin{array}{l}19 \\
2 \underline{a} \\
3 \underline{a}\end{array}$ & $\begin{array}{c}1.000 \\
1.782 \\
7.375 \mathrm{e}^{-03}\end{array}$ & $\begin{array}{l}1.000 \\
1.181 \\
1.634\end{array}$ \\
\hline & \multirow{2}{*}{$\begin{array}{c}\text { II } \\
\text { Campo } \\
\text { cercano }\end{array}$} & $\begin{array}{c}\text { №1 } \\
\text { (Friuli } \\
\text { aftershock) }\end{array}$ & $\begin{array}{l}1 \underline{a} \\
2 \underline{a} \\
3 \underline{a}\end{array}$ & $\begin{array}{c}1.000 \\
45.766 \\
0.208 \\
\end{array}$ & $\begin{array}{l}1.000 \\
1.181 \\
1.634 \\
\end{array}$ \\
\hline & & $\begin{array}{c}\text { №14 } \\
\text { (Montenegro } \\
\text { aftershock) } \\
\end{array}$ & $\begin{array}{l}10 \\
2 \underline{a} \\
3 \underline{a}\end{array}$ & $\begin{array}{l}1.000 \\
1.256 \\
1.526 \\
\end{array}$ & $\begin{array}{l}1.000 \\
1.181 \\
1.634\end{array}$ \\
\hline & \multirow{2}{*}{$\begin{array}{c}\text { II } \\
\text { Campo } \\
\text { lejano }\end{array}$} & $\begin{array}{c}\text { №2 } \\
\text { (Friuli } \\
\text { aftershock) }\end{array}$ & $\begin{array}{l}19 \\
2 \underline{a} \\
3 \underline{a} \\
\end{array}$ & $\begin{array}{l}3.866 \\
7.324 \\
0.044 \\
\end{array}$ & $\begin{array}{l}1.000 \\
1.181 \\
1.634\end{array}$ \\
\hline & & $\begin{array}{c}\text { №12 } \\
\text { (Montenegro } \\
\text { aftershock) }\end{array}$ & $\begin{array}{l}19 \\
2^{a} \\
3^{a}\end{array}$ & $\begin{array}{l}1.000 \\
1.636 \\
3.531\end{array}$ & $\begin{array}{l}1.000 \\
1.181 \\
1.634\end{array}$ \\
\hline & \multirow{3}{*}{$\begin{array}{c}\text { III } \\
\text { Campo } \\
\text { cercano }\end{array}$} & $\begin{array}{c}\text { №2 } \\
\text { (Friuli } \\
\text { aftershock) }\end{array}$ & $\begin{array}{l}1{ }^{a} \\
2 \underline{a} \\
3 a \\
\end{array}$ & $\begin{array}{l}1.000 \\
1.340 \\
1.442 \\
\end{array}$ & $\begin{array}{l}1.000 \\
1.181 \\
1.634 \\
\end{array}$ \\
\hline & & $\begin{array}{c}\text { №6 } \\
\text { (Kalamata } \\
\text { aftershock) }\end{array}$ & $\begin{array}{l}10 \\
2 \underline{a} \\
3 \underline{a}\end{array}$ & $\begin{array}{c}1.000 \\
1867.448 \\
732.332 \\
\end{array}$ & $\begin{array}{l}1.000 \\
1.181 \\
1.634 \\
\end{array}$ \\
\hline & & $\begin{array}{c}\text { №12 } \\
\text { (Duzce 1) }\end{array}$ & $\begin{array}{l}1{ }^{\underline{a}} \\
2^{a} \\
3^{a}\end{array}$ & $\begin{array}{l}1.000 \\
2.231 \\
1.885\end{array}$ & $\begin{array}{l}1.000 \\
1.181 \\
1.634 \\
\end{array}$ \\
\hline & \multirow{2}{*}{$\begin{array}{l}\text { III } \\
\text { Campo } \\
\text { lejano }\end{array}$} & $\begin{array}{c}\text { №3 } \\
\text { (Montenegro) }\end{array}$ & $\begin{array}{l}1 \underline{a} \\
2^{\underline{a}} \\
3^{\underline{a}}\end{array}$ & $\begin{array}{c}1.000 \\
1171.732 \\
1.667\end{array}$ & $\begin{array}{l}1.000 \\
1.181 \\
1.634\end{array}$ \\
\hline & & $\begin{array}{c}\text { №14 } \\
\text { (Ano Liosia) }\end{array}$ & $\begin{array}{l}1 \mathrm{a} \\
2^{\mathrm{a}} \\
3^{\mathrm{a}}\end{array}$ & $\begin{array}{c}1.000 \\
366.166 \\
1.667\end{array}$ & $\begin{array}{l}1.000 \\
1.181 \\
1.634\end{array}$ \\
\hline \multirow{4}{*}{ pa4pz2-1 } & \multirow{3}{*}{$\begin{array}{l}\text { I } \\
\text { Campo } \\
\text { lejano }\end{array}$} & $\begin{array}{c}\text { №1 } \\
\text { (Friuli) }\end{array}$ & $\begin{array}{l}1 \underline{a} \\
2^{\underline{a}} \\
3^{\underline{a}}\end{array}$ & $\begin{array}{l}1.000 \\
1.080 \\
1.827\end{array}$ & $\begin{array}{l}1.000 \\
1.181 \\
1.634\end{array}$ \\
\hline & & $\begin{array}{c}\text { №4 } \\
\text { (Montenegro) }\end{array}$ & $\begin{array}{l}1 \text { a } \\
2 \underline{a} \\
3 \underline{a}\end{array}$ & $\begin{array}{l}1.000 \\
1.690 \\
1.491 \\
\end{array}$ & $\begin{array}{l}1.000 \\
1.181 \\
1.634 \\
\end{array}$ \\
\hline & & $\begin{array}{c}\text { №5 } \\
\text { (Campano } \\
\text { Lucano) }\end{array}$ & $\begin{array}{l}1 \underline{a} \\
2 \underline{a} \\
3 \underline{a}\end{array}$ & $\begin{array}{l}1.000 \\
0.153 \\
0.292\end{array}$ & $\begin{array}{l}1.000 \\
1.181 \\
1.634\end{array}$ \\
\hline & $\begin{array}{c}\text { II } \\
\text { Campo } \\
\text { cercano }\end{array}$ & $\begin{array}{c}\text { №1 } \\
\text { (Friuli } \\
\text { aftershock) }\end{array}$ & $\begin{array}{l}10 \\
2 \underline{a} \\
3 \underline{a}\end{array}$ & $\begin{array}{l}1.000 \\
7.838 \\
2.432 \\
\end{array}$ & $\begin{array}{l}1.000 \\
1.181 \\
1.634\end{array}$ \\
\hline
\end{tabular}




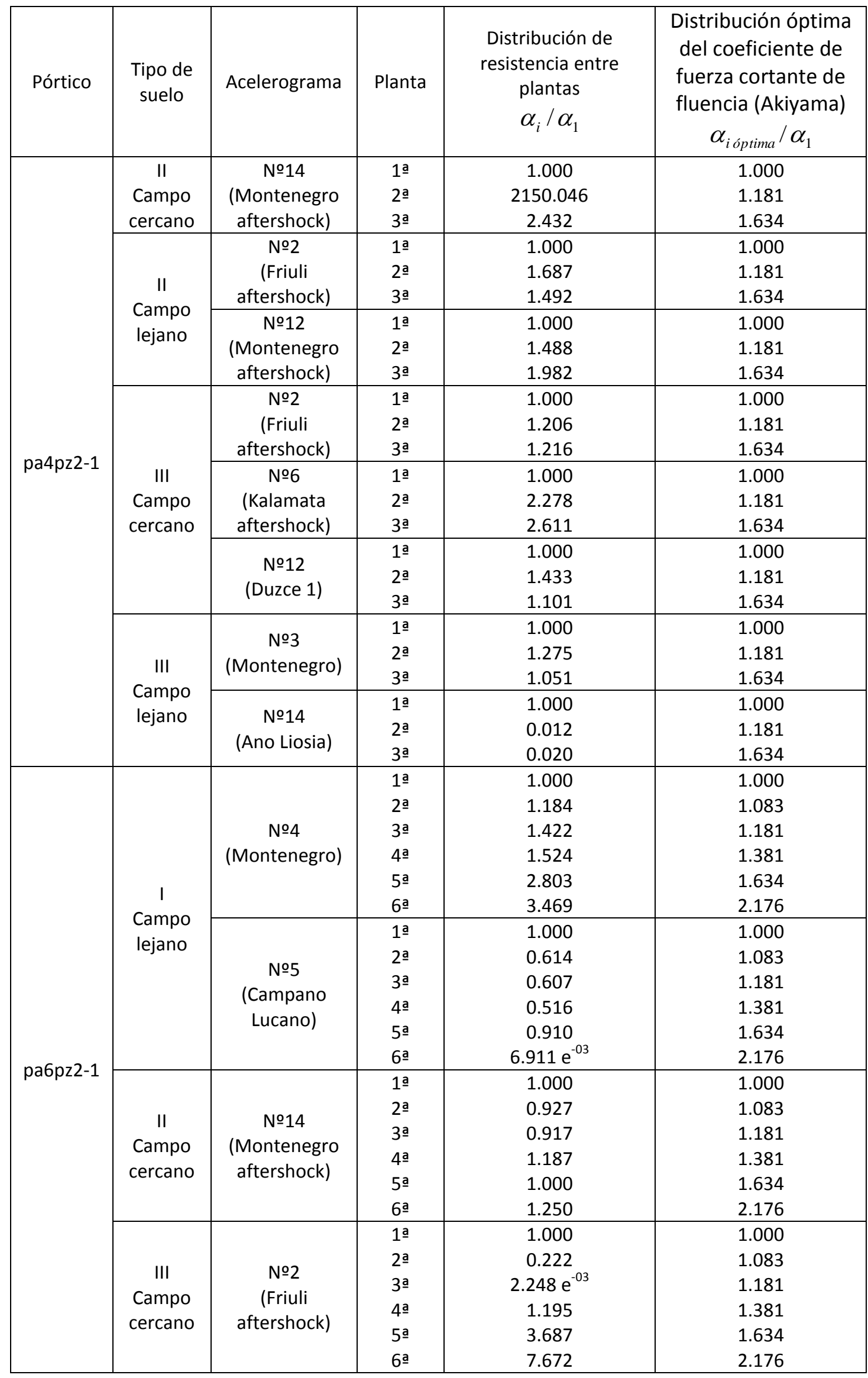




\begin{tabular}{|c|c|c|c|c|c|}
\hline Pórtico & $\begin{array}{l}\text { Tipo de } \\
\text { suelo }\end{array}$ & Acelerograma & Planta & $\begin{array}{c}\text { Distribución de } \\
\text { resistencia entre } \\
\text { plantas } \\
\alpha_{i} / \alpha_{1}\end{array}$ & $\begin{array}{c}\text { Distribución óptima } \\
\text { del coeficiente de } \\
\text { fuerza cortante de } \\
\text { fluencia (Akiyama) } \\
\alpha_{\text {ioptima }} / \alpha_{1}\end{array}$ \\
\hline \multirow{18}{*}{ pa6pz2-1 } & \multirow{12}{*}{$\begin{array}{c}\text { III } \\
\text { Campo } \\
\text { cercano }\end{array}$} & \multirow{6}{*}{$\begin{array}{c}\text { №6 } \\
\text { (Kalamata } \\
\text { aftershock) }\end{array}$} & 1 a & 1.000 & 1.000 \\
\hline & & & $2 \underline{a}$ & 0.984 & 1.083 \\
\hline & & & 3 a & 1.380 & 1.181 \\
\hline & & & $4 \underline{a}$ & 2.481 & 1.381 \\
\hline & & & $5 \underline{a}$ & 2.962 & 1.634 \\
\hline & & & $6 \underline{a}$ & 4.711 & 2.176 \\
\hline & & \multirow{6}{*}{$\begin{array}{c}\text { №12 } \\
\text { (Duzce 1) }\end{array}$} & $1 \underline{a}$ & 1.000 & 1.000 \\
\hline & & & $2^{a}$ & 0.814 & 1.083 \\
\hline & & & 3 a & 0.605 & 1.181 \\
\hline & & & $4 \underline{a}$ & 0.651 & 1.381 \\
\hline & & & $5 \underline{a}$ & 0.860 & 1.634 \\
\hline & & & $6 \underline{a}$ & 0.977 & 2.176 \\
\hline & \multirow{6}{*}{$\begin{array}{c}\text { III } \\
\text { Campo } \\
\text { lejano }\end{array}$} & \multirow{6}{*}{$\begin{array}{c}\mathrm{N} N \mathrm{3} \\
\text { (Montenegro) }\end{array}$} & $1 \underline{a}$ & 1.000 & 1.000 \\
\hline & & & $2^{a} \underline{a}$ & 1.085 & 1.083 \\
\hline & & & 3 a & 1.212 & 1.181 \\
\hline & & & $4 \underline{a}$ & 1.466 & 1.381 \\
\hline & & & $5 \underline{a}$ & 1.890 & 1.634 \\
\hline & & & $6 \underline{a}$ & 3.335 & 2.176 \\
\hline
\end{tabular}




\section{CAPÍTULO 6 \\ CONCLUSIONES}

\subsection{RESUMEN DEL TRABAJO REALIZADO}

El objetivo general de este trabajo ha sido investigar el uso de los disipadores de energía de tipo histerético para reacondicionar sísmicamente estructuras porticadas existentes de hormigón armado con vigas planas. Concretamente, el trabajo se ha centrado en determinar la resistencia mínima que deberían tener los disipadores de energía histeréticos colocados en cada una de las plantas de estructuras porticadas existentes con vigas planas a reacondicionar sísmicamente, para que bajo el terremoto de proyecto sean capaces de limitar los desplazamientos máximos entre plantas a unos valores preestablecidos. Como objetivo de desplazamiento máximo entre plantas se fijó el 0,5\%, que es un valor que garantiza que la estructura principal se va a mantener básicamente dentro del dominio elástico, y por lo tanto sin daños.

Se han definido tres prototipos de pórticos con vigas planas de estructuras existentes de hormigón armado, construidos en los años 70-90, empleados con mucha frecuencia en países del área mediterránea como España. Estos prototipos han servido de base para aplicar la estrategia de reacondicionamiento sísmico con disipadores de energía de tipo histerético. La metodología aplicada ha consistido en varias partes que se describen brevemente a continuación.

En primer lugar, se ha elegido un conjunto de terremotos históricos de diferentes características y registrados en distintos tipos de suelo, y se han escalado con un determinado criterio para que representen la sismicidad de la región donde se han supuesto ubicadas las estructuras estudiadas. En esta investigación se seleccionó inicialmente un total de 18 terremotos históricos registrados en Europa.

En segundo lugar, los tres prototipos seleccionados se dimensionaron y modelizaron para poder ser analizados con el programa de análisis no lineal en dos dimensiones IDARC versión 7, que permite realizar tanto cálculos dinámicos directos como cálculos estáticos no lineales aplicando el método del empuje incremental (pushover analysis).

Se realizaron cálculos estáticos no lineales aplicando el método del empuje incremental (pushover analysis) con control en fuerzas y empleando el primer modo de vibración para obtener la curva de capacidad global de cada pórtico. De esta forma, para cada planta de cada pórtico se obtuvo la relación entre fuerza cortante de la planta y el desplazamiento entre forjados que la delimitan. De estas últimas curvas se obtuvo la rigidez inicial y el desplazamiento de fluencia de cada planta.

A los modelos numéricos de los pórticos con vigas planas se les añadieron disipadores de energía de tipo histerético en cada planta. La determinación de la resistencia lateral requerida a los disipadores se hizo en base a cálculos dinámicos directos iterativos, modificando el desplazamiento de fluencia de los disipadores y manteniendo fija la rigidez axial del mismo, con el objetivo de que el 
desplazamiento lateral máximo de las plantas se mantuviese dentro de los límites fijados. Para realizar estas iteraciones se decidió automatizar el programa LumpST mediante la aplicación del módulo de optimización de Matlab, para agilizar los cálculos.

Obtenidos los desplazamientos de fluencia de los disipadores, se ha calculado la resistencia lateral de los disipadores de cada una de las plantas de los modelos estructurales estudiados y el coeficiente cortante de fluencia aportado por los disipadores en cada planta de cada uno de los pórticos. Se ha determinado también la resistencia lateral aportada por el pórtico principal (sin disipadores) en cada planta, y se ha expresado en forma de coeficiente de fuerza cortante (normalizando por el peso del edificio por encima de la planta considerada). Finalmente se ha obtenido el coeficiente de fluencia cortante total como suma del coeficiente de fluencia cortante aportado por los disipadores de cada planta, y el coeficiente de fuerza cortante desarrollado por el pórtico principal, con el objetivo de comparalo con distribuciones de coeficiente de fuerza cortante óptimos propuestos en la literatura.

\subsection{CONCLUSIONES}

- Con este trabajo hemos profundizado en el proyecto de estructuras porticadas con vigas planas reacondicionadas sísmicamente con disipadores de energía de tipo histerético, como solución para reducir su vulnerabilidad.

- La primera conclusión de este trabajo es que los disipadores de energía son una solución eficiente y válida para disipar la energía sísmica en estructuras existentes y controlar con ello los desplazamientos laterales.

- Se puede concluir también que los desplazamientos laterales máximos entre plantas de las estructuras analizadas son tremendamente sensibles a pequeñas variaciones de la distribución de resistencia lateral entre las distintas plantas del edificio, y dependen también fuertemente de las características del terremoto. Dentro de esta elevada sensibilidad de los desplazamientos a la resistencia lateral y a las características del terremoto, en este trabajo, los mejores ajustes a los desplazamientos objetivo se consiguieron con los terremotos registrados en suelo de tipo III (suelo medio).

- De los modelos analizados, los dos que corresponden a edificios de tres plantas (pa2pz2-1 y pa4pz2-1) y a pesar de ser similares (el pórtico pa2pz2-1 tiene un periodo fundamental de vibración sin disipador de $\mathrm{f}_{1}=1,366 \mathrm{~s}$ y con disipador $\mathrm{T}_{1}=0,558 \mathrm{~s}$ y para el pórtico pa4pz2-1 los periodos valen ${ }_{\mathrm{f}} \mathrm{T}_{1}=1,391 \mathrm{~s}$ y con disipador $\mathrm{T}_{1}=0,568 \mathrm{~s}$ ), se observa una clara diferencia en la distribución de la resistencia lateral requerida.

- Aunque los acelerogramas están escalados a una misma aceleración básica del suelo modificada por el tipo de suelo PGA, $\left(P G A=200 \mathrm{~cm} / \mathrm{s}^{2}\right.$ para el suelo tipo I, $P G A=230.5 \mathrm{~cm} / \mathrm{s}^{2}$ para el suelo tipo II y $P G A=261.4 \mathrm{~cm} / \mathrm{s}^{2}$ para el suelo tipo III), se ha comprobado que la cantidad total de energía que introducen en un misma estructura terremotos distintos escalados una misma PGA varía mucho de unos a otros. Esto apoya el hecho, bien conocido y tratado en la literatura, de que la PGA no es un parámetro adecuado para caracterizar el potencial de daño de un terremoto. El 
daño está relacionado con las deformaciones plásticas, y éstas dependen de la energía que el terremoto introduce en la estructura, más que de la PGA.

- Finalmente, se han comparado las distribuciones de resistencia lateral obtenidas de los cálculos dinámicos realizados con distribuciones óptimas propuestas en la literatura (concretamente la distribución propuesta por Akiyama en los años 80), observándose que en algunos casos el ajuste es aceptable, pero en otros se observan desviaciones importantes.

\subsection{FUTURAS LINEAS DE INVESTIGACIÓN}

Debido a la complejidad y amplitud del fenómeno tratado, este trabajo no pretende abarcar en su totalidad el problema de proyectar soluciones de reacondicionamiento sísmico de estructuras porticadas con vigas planas con disipadores de energía, y evaluar su comportamiento sísmico. Este trabajo es un primer eslabón, que abre el campo de investigación a una serie de líneas futuras entre las que se pueden citar las siguientes:

Una de las líneas de trabajo futuras a desarrollar dentro de la parte teórica sería complementar este estudio realizando un diseño probabilístico de las estructuras con disipadores.

Otra línea de investigación sería la aplicación de disipadores de energía en otros tipos de estructuras, por ejemplo de mampostería, metálicas, de hormigón armado con forjados reticulares, etc. pues en este trabajo sólo se han considerado pórticos de vigas planas de hormigón armado del tipo empleado en España durante los años 70-90. 


\section{CAPÍTULO 7 \\ REFERENCIAS}

[1] España. Ministerio de Planificación del Desarrollo (1974) Norma Sismorresistente PDS-1 Parte A.

[2] España. Ministerio de Planificación del Desarrollo (2002) Norma de Construcción Sismorresistente NCSE-02. Madrid.

[3] European Strong Motion Database - Imperial College, London; (http://www.isesd.hi.is/).

[4] Yukihiro Haranda, Hiroshi Akiyama (1998) Seismic design of flexible-stiff mixed frame with energy concentration. Engineering Structures, Vol. 20, No. 12, pp. 1039-1044.

[5] Gentry RG, Wight JK (1994) Wide beam-column connections under earthquake-type loading. Earthquake Spectra, 10(4): 675-703.

[6] LaFave JM, Wight JK (1999) Reinforced Concrete Exterior Wide Beam-Column-Slab Connections Subjected to Lateral Earthquake Loading. ACI Structural Journal, 96(4): 577-585.

[7] ACl Committee 318 (1995) Building Code Requirements for Structural Concrete (ACl-318-95) and Commentary (ACl-318R-95). American Concrete Institute: Farmington Hills (MI), USA.

[8] LaFave JM, Wight JK (2001) Reinforced Concrete Wide-Beam Construction vs. Conventional Construction: Resistance to Lateral Earthquake Loads. Earthquake Spectra, 17(3): 479-505.

[9] Benavent-Climent A (2007) Seismic behavior of RC wide beam-column connections under dynamic loading. Journal of Earthquake Engineering, 11(4): 493-511.

[10] España. Ministerio de Obras Públicas (1991) Instrucción para el proyecto y la ejecución de obras de hormigón en masa o armado (EH-91).

[11] http://en.wikipedia.org/wiki/Pattern search \%28optimization\%29

[12] M. Feriche, F. Vidal, C. Aranda. Efectos del terremoto de Lorca del 11-05-2011.

[13] España. Ministerio de Obras públicas (1994) Norma de Construcción Sismorresistente NCSE-94.

[14] Hiroshi Akiyama. Metodología de proyecto sismorresistente de edificios basada en el balance energético. Ed. Reverté S.A. 
[15] Benavent-Climent A (2010) Estructuras sismorresistentes. Edit. Maia

[16] IDARC 2D Version 7.0 Jan. 2010. USER'S GUIDE

[17] http://www.mathworks.es/help/toolbox/gads/f6010.html

[18] Benavent-Climent A (2011) An energy-based method for seismic retrofit of existing frames using hysteretic dampers. Soil Dynamics and Earthquake Engineering, pp. 1385-1396.

[19] Benavent-Climent A (2010) A brace-type seismic damper based on yielding the walls of hollow structural sections. Engineering Structures, pp. 1113-1122. 
